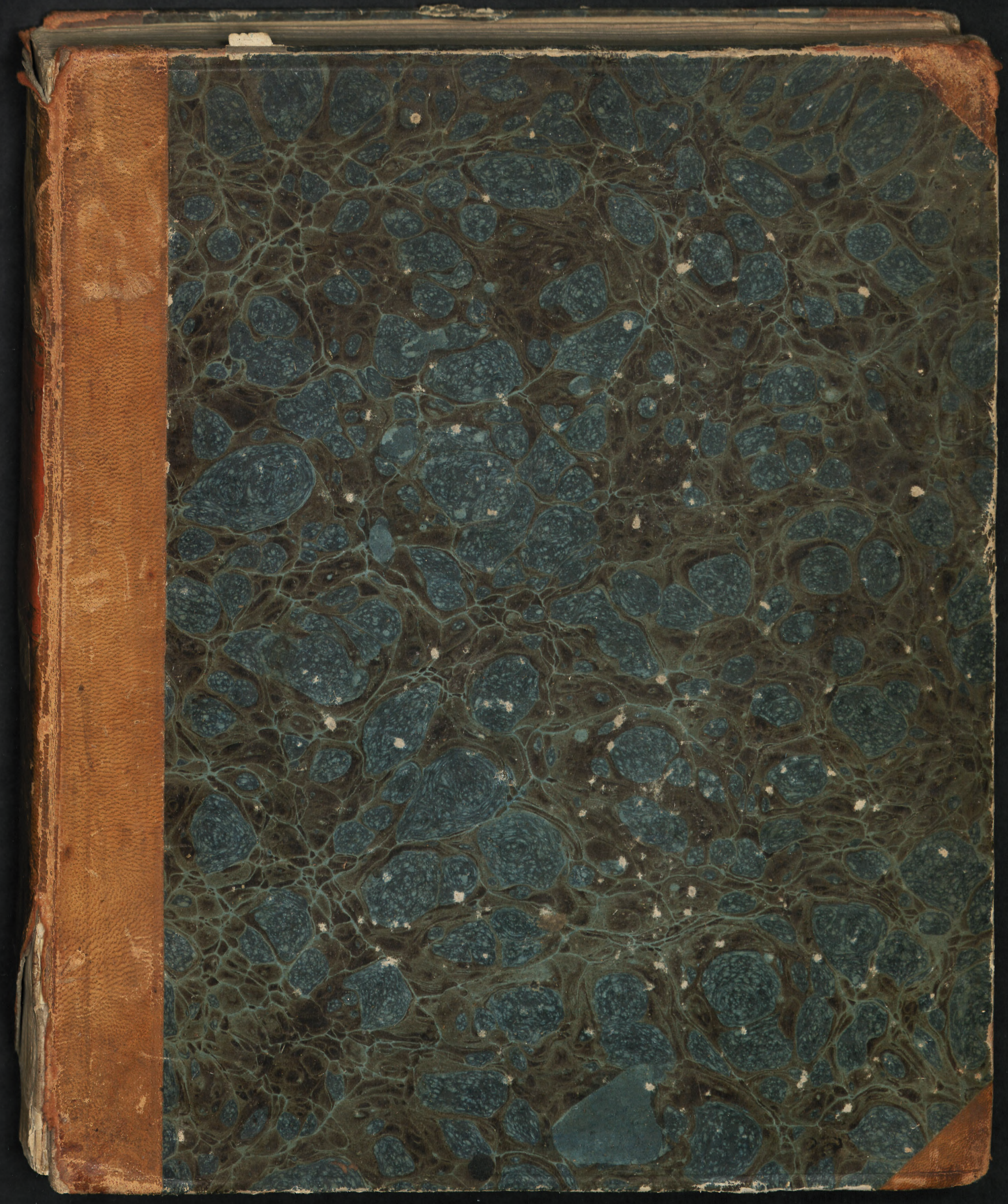




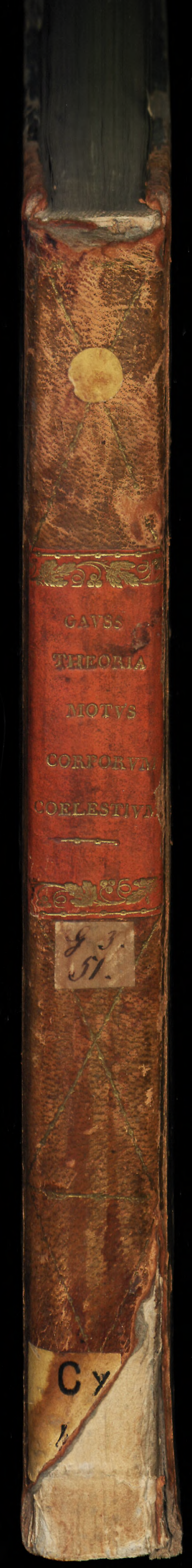




$$
\begin{array}{r}
(x+015(m) \\
40
\end{array}
$$

94 



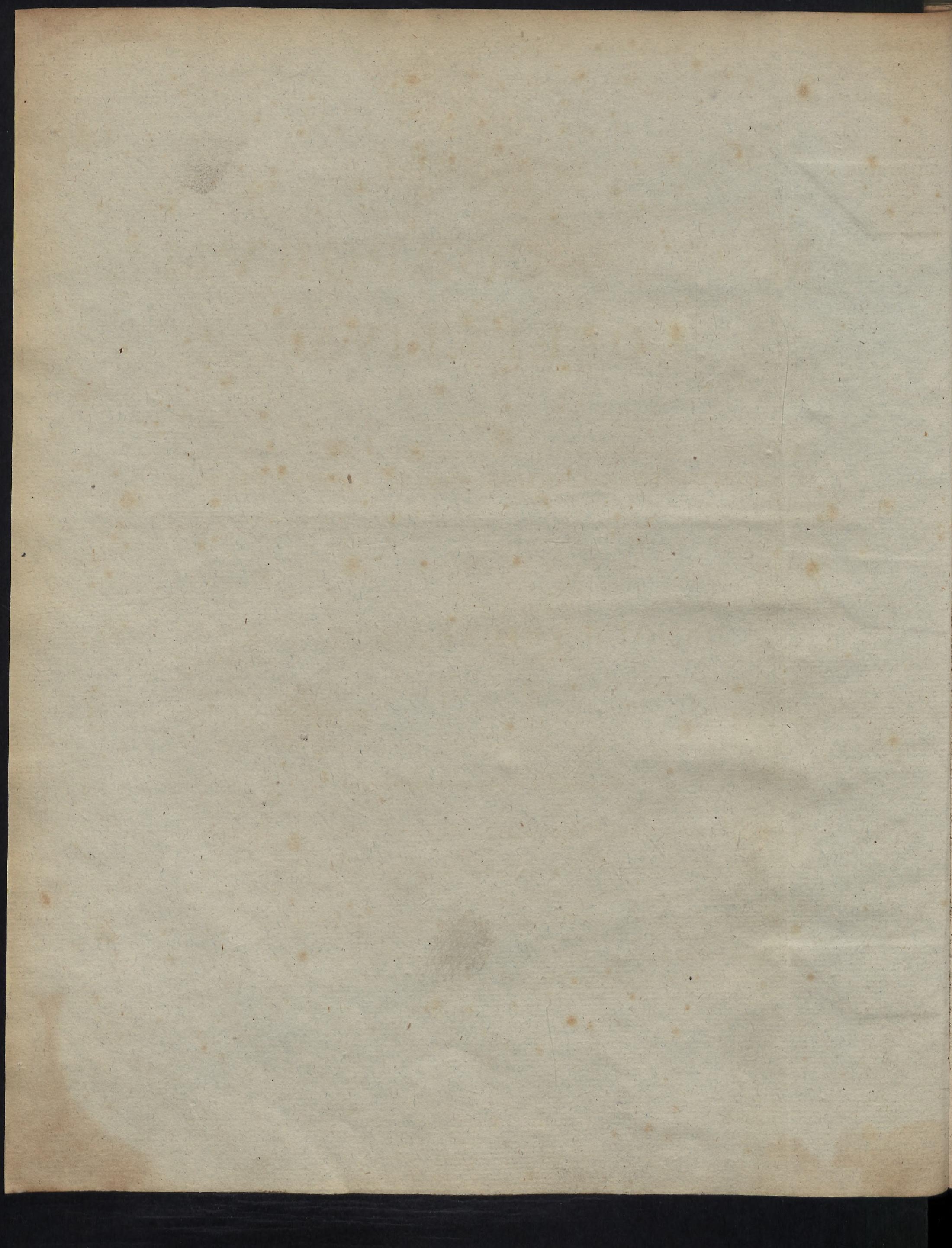




\section{T H EOR I A

\section{MOTVS CORPOR VM COELESTIVM}

I N

SECTIONIBVS CONICIS SOLEM AMBIENTIVM

A V C TOR E

CAROLO FRIDERICO GAVSS

II A BVRGI SVMTIBVS FRID. PERTHES ET I. H: BESSER 1809 .

\section{Venditur}

PARISIIs ap. Treuttel \& Würtz. S тосKноLMIAE ap. A. Wiborg.

LoNDINI ap. R. H. Evans:

Petropou $x$ ap. Klostermann.

MA D I T I ap. Sancha.

FLORENTIA ap. Molini, Landi \& Co

A. $\mathrm{S}$ × $\mathrm{E}$ OD $\mathrm{A} \mathrm{I}$ in libraria: Kunst-und Industrie-Comptoir, dicta. 



\section{P R A E F A T I O}

Detectis legibus motus planetarum Kepleri ingenio non defuerunt subsidia ad singulorum planetarum elementa ex obseruationibus eruenda. Tycho Brahe, a quo astronomia practica ad fasti. gium antea ignotum euecta erat, cunctos planetas per longam annorum seriem summa cura tantaque perseuerantia obseruauerat, vt Keplero talis thesauri dignissimo heredi seligendi tantummodo cura restaret, quae ad scopum quemuis propositum facere viderentur. Nec mediocriter subleuabant hunc laborem motus planetarum medii summa iamdudum praecisione per obseruationes antiquissimas determinati.

Astronomi, qui post Keplerum conati sunt planetarum or. bitas adiumento obseruationum recentiorum vel perfectiorum adhuc accuratius dimetiri, iisdem vel adhuc maioribus subsidiis adiuti sunt. Neque enim amplius de elementis plane incognitis eliciendis agebatur, sed nota leuiter tantum corrigenda arctioribusque limitibus circumscribenda erant.

Principium grauitationis vniuersalis a summo Nerton detectum campum plane nouum aperuit, legibusque iisdem, quibus quinque planetas regi hepler expertus fuerat, leui tantum muta- 
tione facta omnia corpora coelestia necessario obsequi debere edo. cuit, quorum quidem motus a vi Solis tantum moderentur. Scilicet obseruationum testimonio fretus hepler cuiusuis planetae orbitam ellipsem esse pronunciauerat, in qua areae circa Solem, focum alterum ellipsis occupantem, vniformiter describantur, et quidem ita, vt tempora reuolutionum in ellipsibus diuersis sint in ratione sesquialtera semiaxium maiorum. Contra Newton, principio gravitationis vniuersalis posito, a priori demonstrauit, corpora omnia a Solis vi attractiua gubernata in sectionibus conicis moueri debere, quarum quidem speciem vnam, ellipses puta, planetae nobis exhibeant, dum species reliquae, parabolae et hyperbolae, pro aeque possibilibus haberi debeant, modo adsint corpora Solis vi velocitate debita occurrentia; Solem semper focum alterum sectionis conicae tenere; areas, quas corpus idem temporibus diuersis circa Solem describat, his temporibus proportionales, areas denique a corporibus diuersis, temporibus aequalibus, circa Solem descriptas, esse in ratione subduplicata semiparametrorum orbitarum: postrema harum legum, in motu elliptico cum vltima Kepleri lege identica, ad motum parabolicum hyperbolicumque patet, ad quos haecce applicari nequit, reuolutionibus deficientibus. Iam filum repertum, quo ducente labyrinthum motuum cometarum antea inaccessum ingredi licuit. Quod tam feliciter successit, vt ominium cometarum motibus, qui quidem accurate obseruati essent, explicandis suffceret vnica hypothesis, orbitas parabolas esse. Ita systema grauitationis vniuersalis nouos analysi triumphos eosque splendidissimos parauerat; cometaeque vsque ad illum diem semper indomiti, vel si deuicti videbantur mox seditiosi et rebelles, frena sibi iniici 
passi, atque ex hostibus hospites redditi, iter suum in 'tramitibus a calculo delineatis prosequuti sunt, iisclem quibus planetae le. gibus aeternis religiose obtemperantes.

Iam in determinandis cometarum orbitis parabolicis ex obseruationibus difficultates suboriebantur longe maiores, quam in determinandis orbitis ellipticis planetarum, inde potissimum, quod cometae per breuius temporis interuallum visi delectum obserua. tionum ad haec vel illa imprimis commodarum non concedebant, sed iis vti geometram cogebant, quas fors obtulerat, ita vt methodos speciales in calculis planetarum adhibitas vix vinquam in vsum vocare licuexit. Magnus ipse Newton, primus saeculi sui geometra, problematis difficultatem haud dissimulauit, attamen, ceu exspectari poterat, ex hoc quoque certamine victor euasit. Multi post Newtoinum geometrae eidem problemati operam suam nauauerunt, varia vtique fortuna, ita tamen, vt nostris temporibus parum desiderandum relictum sit.

Verum enim vero non est praetermittendum, in hoc quoque problemate peropportune difficultatem diminüi per cognitionem vnius elementi sectionis conicae, quum per ipsam suppositionem orbitae parabolicae, axis maior infinite magnus statuatur. Quippe omnes parabolae, siquidem situs negligatur, per solam maiorem minoremue distantiam verticis a foco inter se differunt, dum sectiones conicae generaliter spectatae varietatem infinities maiorem admittant. Haud equidem aderat ratio sufficiens, cur cometarum träiectoriae absoluta praecisione parabolicae praesumerentur: quin potius infinite parum probabile censeri debet, rerum naturam vnquam tali suppositioni annuisse. Attamen quum constaret, phaenomena 
corporis coclestis in ellipsi vel hyperbola incedentis, cuius axis maior permagnus sit ratione parametri, prope perihelium perparum discrepare a motu in parabola, cui eadem verticis a foco distantia, differentiamque eo leuiorem euadere, quo maior fuerit illa ratio axis ad parametrum; porro quum experientia docuisset, inter motum obseruatum motumque in orbita parabolica computatum vix vmquam maiores differentias remanere, quam quae ipsis obseruationum erroribus (hic plerumque satis notabilibus) tuto tribui poterant: astronomi apud parabolam subsistendum esse rati sunt. Recte sane, quum omnino deessent subsidia, e quibus, num vllae quantaeue differentiae a parabola adsint, satis certo colligi potuisset. Excipere oportet cometam celebrem Halleyanum, qui ellipsem valde oblongam describens in reditu ad perihelium pluries obseruatus tempus periodicum nobis patefecit: tunc autem axi maiori inde cognito computus reliquorum elementorum vix pro difficiliori habendus est, quam determinatio orbitae parabolicae. Silentio quidem praeterire non possumus, astronomos etiam in nonnullis aliis cometis per tempus aliquanto longius obseruatis determinationem aberrationis a parabola tentauisse: attamen omnes methodi ad hunc finem propositae vel adhibitae, imnituntur suppositioni, discrepantiam a parabola haud considerabilem esse, quo pacto in illis tentaminibus ipsa parabola antea iam computata cognitionem approximatam singulorum elementorum (praeter axem maiorem vel tempus reuolutionis inde pendens) iam subministrauit, leuibus tantum mutationibus corrigendam. Praeterea fa. tendum est, omnia ista tentamina vix vnquam aliquid certi decidere valuisse, si forte cometain anni 1770 excipias. 
Quamprimum motum planetae noui anno 1781 detecti cum hypothesi parabolica conciliari non posse cognitum est, astronomi orbitam circularem illi adaptare inchoauerunt, quod negotium per calculum perfacilem ac simplicem absoluitur. Fausta quadam fortuna orbita huius planetae mediocriter tantum excentrica erat, quo pacto elementa per suppositionem illam eruta saltem approximationem qualemcunque suppeditabant, cui dein determinationem elementorum ellipticorum superstruere licuit. Accedebant plura alia peropportuna. Quippe tardus planetae motus, perparuaque orbitae ad planum eclipticae inclinatio non solum calculos longe simpliciores reddebant, methodosque speciales aliis casibus haud accommodandas in vsum vocare concedebant, sed metum quoque dissipabant, ne planeta radiis Solis immersus postea quaeritantium curas eluderet, (qui metus alias, praesertim si insuper lumen minus viuidum fuisset, vtique animos turbare potuisset), quo pacto accuratior orbitae determinatio tuto differri poterat, donec ex obseruationibus frequentioribus magisque remotis eligere liceret, quae ad. propositum maxime commodae viderentur.

In omnibus itaque casibus, vbi corporum coelestium orbitas ex obseruationibus deducere oportuit, commoda aderant quaedam haud spernenda, methodorum specialium applicationem suadentia vel saltem permittentia, quorum commodorum potissimum id erat, quod per suppositiones hypotheticas cognitionem approximatam quorundam elementorum iamiam acquirere licuerat, antequam calculus elementorum ellipticorum susciperetur. Nihilominus satis mirum videtur, problema generale

Determinare orbitam corporis coelestis, absque omni suppositione 
hypothetica, ex obseruationibus tempus haud magnum complectentibus neque adeo delectum, pro applicatione methodorum specialium, patientibus. vsque ad initium huius saeculi penitus propemodum néglectum esse, vel saltem a nemine serio ac digne tractatum, quum certe theoreticis propter difficultatem atque elegantiam sese commendare potuisset, etiamsi apud practicos de summa eius vtilitate nondum constaret. Scilicet inualuerat apud omnes opinio, impossibilem esse talem determinationem completam ex obseruationibus breuiori temporis interuallo inclusis, male sane fundata, quum nunc quidem certissimo iam euictum sit, orbitam corporis coelestis ex obseruationibus bonis paucos tantummodo dies complertentibus absque vlla suppositione hypothetica satis approximate iam determinari posse.

Incideram in quasdam ideas, quae ad solutionem problematis magni de quo dixi facere videbantur, mense Septembri a. 1801, tunc in labore plane diuerso occupatus. Haud raro in tali casu, ne nimis a grata inuestigatione distrahamur, neglectas interixe sinimus idearum associationes, quae attentius examinatae vberrimos fructus ferre potuissent. Forsan et illis ideolis eadem fortuna instabat, nisi peropportune incidissent in tempus, quo nullum sane faustius ad illas conseruandas atque fouendas eligi potuisset. Scilicet eodem circiter tempore rumor de planeta nouo Ian. 1 istius anni in specula Panormitana detecto per omnium ora volitabat, moxque ipsae obseruationes inde ab epocha illa vsque ad in Febr. ab astronomo praestantissimo Piazzi institutae ad notitiam publicam peruenerunt. Nullibi sane in annalibus astronomiae occasionem tam grauem reperimus, vixque grauior excogitari posset, ad dignitatem istius problematis luculentissime ostendendam, quam tunc in tanto 
discrimine vrgenteque necessitate, vbi omnis spes, atomum planetariam post annum fere elapsum in coelis inter innumeras stellulas reinueniendi, vnice pendebat ab orbitae cognitione satis approximata, solis illis pauculis obseruationibus superstruenda. Vmquamne opportunius experiri potuissem, ecquid valeant ideolae meae ad vsum practicum, quam si tunc istis ad determinationem orbitae Ce. reris vterer, qui planeta inter 41 illos dies geocentrice arcum trium tantummodo graduum descripserat, et post annum elapsum in coeli plaga longissime illinc remota indagari debebat? Prima haece mo thodi applicatio facta est mense Oct. 1801, primaque nox serena, vbi planeta ad noxinam numeroxuma inde deductorum quaesitus est $*$ ), transfugam obseruationibus reddidit. Tres alii planetae noui inde ab illo tempore detecti, occasiones nouas suppeditauerunt, metho. di efficaciam ac generalitatem examinandi et comprobandi.

Optabant plures astronomi, statim post reinuentionem Cerexis, vt methodos ad istos calculos adhibitas publici iuris facerem; verum obstabant plura, quominus amicis hisce sollicitationibus tune morem gererem: negotia alia, desiderium rem aliquando copiosius pertractandi, imprimisque expectatio, continuatam in hac disquisitione occupationem varias solutionis partes ad maius generalitatis, simplicitatis et elegantiae fastigium euecturam esse. Quae spes quum me haud fefellerit, non esse arbitror, cur me huius morae poeniteat. Methodi enim ab initio adhibitae identidem tot tantasque mutationes passae sunt, vt inter modum, quo olim orbita Cereris calculata est, institutionemque in hoc opere traditam vix vllum

*) Dec. 7,1801 a clar. de Zach. 
similitudinis vestigium remanserit. Quamquam vero a proposito meo alienum esset, de cunctis his disquisitionibus paullatim magis magisque perfectis narrationem completam perscribere, tamen in pluribus occasionibus, praesertim quoties de problemate quodam grauiori agebatur, methodos anteriores quoque haud omnino supprimendas esse censui. Quin potius praeter problematum principalium solutiones plurima, quae in occupatione satis longa circa motus corporum coelestium in sectionibus conicis vel propter elegan. tiam analyticam vel imprimis propter vsum practicum attentione digniora se mihi obtulerunt, in hoc opere exsequutus sum. Semper tamen vel rebus vel methodis mihi propriis maiorem curam dicaui, nota leuiter tantum, quatenusque rerum nexus postulare videbatur, attingens.

Totum itaque opus in duas partes diuiditur. In Libro primo euoluuntur relationes inter quantitates, a quibus motus corporum coelestium circa Solem secundum Kepleri leges pendet, et quidem in duabus primis Sectionibus relationes eae, vbi vnicus tantum locus per se consideratur, in Sectione tertia et quarta vero eae, vbi plures loci inter se conferuntur. Illae continent expositionem methodorum tum vulgo vsitatarum, tum potissimum aliarum illis ni fallor ad vsum practicum longe praeferendarum, per quas ab elementis cognitis ad phaenomena descenditur; hae problemata multa grauissima tractant, quae viam ad operationes inuersas sternunt. Scilicet quum ipsa phaenomena ex artificiosa intricataque quadam complicatione elementorum componantur, hanc texturae rationem penitius perspexisse oportet, antequam filorum explicationem operisque in elementa sua resolutionem cum spe successus suscipere li- 
ceàt. Compàràntur itaque in Libro primo instrumenta atque subsidia, per quae dein in Libro altero arduum hoc negotium ipsum perficitur: maxima laboris pars tunc iam in eo consistit, vt illa subsidia rite colligantur, ordine apto disponantur et in scopum propositum dirigantur.

Problemata grauiora ad maximam partem per exempla ido. nea illustrata sunt, semper quoties quidem licuit ab obseruationibus non fictis desumta. Ita non solum methodorum efficaciae maior fiducia conciliabitur, vsusque clarius ob oculos ponetur, sed id quoque cautum iri spero, vt nec minus exercitati a studio harum rerum deterreantur, quae procul dubio partem foecundissimam et pulcherrimam astronomiae theoricae constituunt.

Scripsi Gottingae d. 28 Martii 1809. 


\section{O N T E N T A}

LIBER PRIMvs. Relationes generales inter quantitates, per quas corporum coelestium motus circa Solem definiuntur.

Sectio T, Relationes ad locum simplicem in orbita spectantes. $\quad$ Pag. I.

Sectio 11. Relationes ad locum simplicem in spatio spectanter. $\quad-45$

Sectio III. Relationes inter locos plures in orbita.. $\quad-82$

Sectio IV. Relationes inter locos plures in spatio. $\quad-125$

LIBER STCVNDVS. Inuestigatio orbitarum corporum coelestium ex obseruationibus geocentricis.

Sectio I. Determinatio orbitae e tribus obseruationibus completis.

Sectio II. Determinatio orbitae e quatuor obseruationibus, quarum duae tantum completae sunt.

Sectio HI. Determinatio orbitae obseruationibus quotcunque quam proxime satisfacientis.

Sectio IV. De determinatione orbitarum, habita ratione perturbationum.

TABVLAE. 


\section{LIBER PRIMVS}

\section{RELATIONES GENERALES INTER QVANTITATES PER QVAS CORPORVM COELESTIVM MOTVS CIRCA SOLEM DEFINIVNTVR.}

\section{SECTIO PRIMA \\ Retationes ad locum simplicem in orbita spectantes.}

1.

Corporum coelestium motus in hoc opere eatenus tantum considerabimus, quatenus a Solis vi attractiua gubernantur. Excluduntur itaque ab instituto nostro omnes planetae secundarii, excluduntur perturbationes, quas primarii in se inuicem exercent, excluditur omnis motus rotatorius. Corpora mota ipsa vt puncta mathematica spectamus, motusque omnes ad normam legum sequentium fieri supponimus, quae igitur pro basi omnium disquisitionum in hoc opere sunt habendae.

I. Motus cuinsuis corporis coelestis perpetuo fit in eodem plano, in quo simul centrum Solis est situm. habens.

II. Traiectoria a corpore descripta est sectio conica focum in centro Solis

III. Motus in ista traiectoria fit ita, vt areae spatiorum in diuersis temporum interuallis circa Solem descriptorum hisce interuallis ipsis sint proportionales. Temporibus igitur et spatiis per numeros expressis, spatium quoduis per tempus intra quod describitur diuisum quotientem inuariabilem suppeditat.

IV. Pro corporibus diuersis circa Solem se mouentibus horum quotientium quadrata sunt in ratione inuersa parametrorum orbitis respondentium, atque aggregatorum massae Solis cum massis corporum motorum.

Designando itaque per $2 p$ parametrum orbitae, in qua corpus incedit, per $\mu$ quantitatem materiae huius corporis (posita massa Solis $=1$ ), per $\frac{\pi}{2} g$ aream quam 
tempore $t$ eirca Solem describit, erit $\frac{g}{t \sqrt{ } p \cdot \sqrt{ }(1+\mu)}$ numerus pro omnibus corporibus coelestibus constans. Quum igitur nihil intersit, quonam corpore ad valorem huius numeri determinandum vtamur, e motu terrae eum depromemus, cuius distantiam mediam a Sole pro vnitate distantiarum adoptabimus: vnitas temporum semper nobis erit dies medius solaris. Denotando porro per $\pi$ rationem circumferentiae circuli ad diametrum, area ellipsis integrae a terra descriptae manifesto orit $\pi \vee p$, quae igitur poni debet $=\frac{\pi}{2} g$, si pro $t$ accipitur annus sideralis, quo pacto constans nostra fit $=\frac{2 \pi}{t \sqrt{ }(1+\mu)} \cdot$ Ad valorem numericum huius constantis, in sequentibus per $k$ denotandae, explorandum, statuemus, secundum nouissimam determinationem, annum sideralem siue $t=365,2563835$, massam terrae siue $\mu=\frac{1}{35+710}=0,000002819^{2}$, vnde prodit

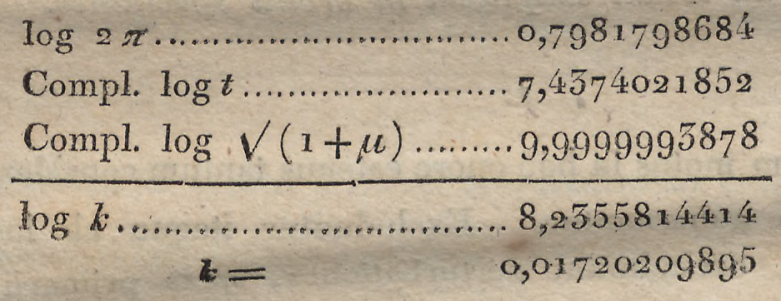

2.

Leges moda expositae ab iis, quas Keplerus naster detexit, aliter non differunt, nisi quod in forma ad omnia sectionum conicarum genera patente exhibitae sunt, actionisque corporis moti in Salem, a qua pendet factor $V(x+\mu)$, ratio est habita. Si has leges tamquam phaenomena ex innumeris atque indubiis obseruationibus depromta consideramus, geometria docebit, qualis actio in corpora circa Solem mota ab hoc exerceri debeat, vt ista phaenomena perpetuo producantur. Hoc modo inuenitur, Solis actionem in corpora ambientia perinde se exerere, ac si vis attractiua, cuius intensitas quadrato distantiae reciproce proportionalis esset, corpora versus centrum Solis propelleret. Quodsi vero vice versa a suppositione talis vis attractiuae tamquam principio proficiscimur, phaenomena illa vt consequentiae necessariae inde deriuantur. Hic leges tantum enarrauisse sufficiat, quarum nexui cum principio gratukationis hoc loco eo minus opus erit immorari, quum post summum Newton auctores plures hoc argumentum tractauerint, interque eos ill. 
Laplace in opere perfectissimo, Mecanique Celeste, tali modo, vt vihil amplius desiderandum reliquerit.

3.

Disquisitiones circa motus corporum coelestium, quatenus fiunt in sectionibus conicis, theoriam completam huius curuarum generis neutiquam postulant: quin adeo vnica aequatio generalis nobis sufficiet, cui omnia superstruantur. Et quidem maxime e re esse videtur, eam ipsam eligere, ad quam tamquam aequationem characteristicam deferimur, dum curuam secundum attractionis legem descriptam inuestigamus. Determinando scilicet quemuis corporis locum in orbita sua per distantias $x, y$ a duabus rectis in plano orbitae ductis atque in centro Solis i. e. in altero curuae foco sub angulis rectis se secantibus, et denotando insuper corporis distantian a Sole (positiue semper accipiendam) per $r$, habebimus inter $r$, $x, y$ aequationem linearem $r+\alpha x+\beta y=\gamma$, in qua $\alpha, \beta, \gamma$ quantitates constantes expriment, et quidem $\gamma$ quantitatem natura sua semper positiuam. Mutando rectarum, ad quas distantiae $x, y$ referuntur, situm per se arbitrarium, si modo sub angulis rectis se intersecare perseuerent, manifesto forma aequationis valorque ipsius $\gamma$ non mutabuntur, $\alpha$ et $\beta$ autem alios aliosque valores nanciscentur, patetque, situm illum ita determinari posse, vt $\beta$ euadat $=0, \alpha$ antem saltem non negatiua. Hoc modo scribendo pro $\alpha, \gamma$ resp. $e, p$, aequatio nostra induit formam $r+e x=p$. Recta, ad quam tunc distantiae $y$ referuntur, linea apsidum vocatur, $p$ semipa rameter, e excentricitas; sectio conica denique ellipsis, parabolae vel hyperbolae nomine distinguitur, prout $e$ vnitate minor, vnitati aequalis, vel vnitate maior est.

Ceterum facile intelligitur, situm lineąe apsidum per conditiones traditas plene determinatum esse, vnico easu excepto, vbi tum $\alpha$ tum $\beta$ iam per se erant $=0$; in hoc casu semper fit $r=p$, ad quascunque rectas distantiae $x, y$ referantur. Quoniam itaque habetur $e=0$, curua (quae erit circulus) secundum definitionem nostram ellipsium generi annumeranda est, id vero singulare habet, quod apsidum. positio prorsus arbitraria manet, siquidem istam notionem ad hunc quoque casum extendere placet.

4.

Pro distantia $x$ iam angulum $v$ introducamus, qui inter lineam apsidum et rectam a Sole ad corporis locum ductam (radium vectorem) continetur, et quidem hic angulus ab ea lineae apsidum parte vbi distantiae $x$ sunt positiuae incipiat, ver- 
susque eam regionem, quorsum motus corporis dirigitur, crescere supponatur. Hoc modo fit $x=r \cos y$, adeoque formula nostra $r=\frac{p}{1+e \cos v}$, rnde protinus deriuantur conclusiones sequentes:

I. Pro $v=0$ valor radii rectoris $r$ fit minimum, puta $=\frac{p}{1+e}:$ hoc punctum perihelium dicitur.

II. Valoribus oppositis ipsius $p$ respondent valores aequales ipsius $r$; quocirca linea apsidum sectionem conicam in duas partes aequales dirimit.

III. In ellipsi $r$ inde a $v=0$ continuo crescit, donec valoreni maximum $\frac{p}{1-e}$ assequatur in aphelio pro $v=180^{\circ}$; post aphelium eodem modo rursus decrescit, quo ante increuerat, donec pro $v=560^{\circ}$ perihelium denuo attigerit. Lineae apsidum pars perihelio hinc aphelio illinc terminata axis maior dicitur; hinc semiaxis maior, qui etiam distantia media vocatur, fit $=\frac{p}{1-e e}$; distantia puncti in medio axe iacentis (centri ellipsis) a foco erit $\frac{e p}{1-e e}=e a$, denotando per $a$ semiaxem maiorem.

IV. Contra in parabola proprie non datur aphelium, sed $r$ vltra omnes limites augetur, quo propius $v$ ad $+180^{\circ}$ vel $-180^{\circ}$ accedit. Pro $v= \pm 180^{\circ}$ valor ipsius $r$ fit infinitus, quod indicat, curuam a linea apsidum a parte perihelio opposita non secari. Quare proprie quidem loquendo de axi maiore vel centro curvae sermo esse nequit, sed secundum analyseos vsum consuetum per ampliationem formularum in ellipsi inuentarum axi maiori valor infinitus tribuitur, centrumque euruae in distantia infinita a foco collocatur.

V. In hyperbola denique $v$ inter limites adhuc arctiores coërcetur, scilicet inter $v=-\left(180^{\circ}-\psi\right)$ et $v=+\left(180^{\circ}-\psi\right)$, denotando per $\psi$ angulum, cuius cosinus $=\frac{1}{e}$. Dum enim ad hosce limites appropinquat, $r$ in infinitum crescit; si vero pro $y$ alter horum limitum ipse acciperetur, valor ipsius $r$ infinitus prodiret, quod indicat, hyperbolam a recta ad lineam apsidum angulo $180^{\circ}-\psi$ supra vel infra inclinata omnino non secari. Pro valoribus hoc modo exclusis, puta a $380^{\circ}-\psi$ vsque ad $180^{\circ}+\psi$, formula nostra ipsi $r$ valorem negatiuum assignat; recta scilicet sub tali angulo contra lineam apsidum inclinata ipsa quidem hyperbolam non secat, si vero retro producitur in alteram hyperbolae partem incidit, quam 
a prima parte omnino separatam versusque eum focum quem Sol occupat conuexam esse constat. Sed in disquisitione nostra, quae vt iam monuimus suppositioni innititur, $r$ sumi positiue, ad hanc alteram hyperbolae partem non respiciemus, in qua corpus coeleste tale tantummodo incedere posset, in quod Sol vim non attractiuam sed secundum easdem leges repulsiuam exerceret. - Proprie itaque loquendo etiam in hyperbola non datur aphelium; pro aphelii analogo id partis aucrsae punctum quod in linea apsidum iacet, et quod respondet valoribus $v=180^{\circ}, r=$ $-\frac{p}{e-1}$, haberi poterit. Quodsi ad instar ellipsis valorem expressionis $\frac{p}{1-e e}$ etiam hic, vbi negatiuus euadit, semiaxem maiorem hyperbolae dicere lubet, haec quantitas puncti modo commemorati distantiam a perihelio simulque situm ei qui in ellipsi locum habet oppositum indicat. Perinde $\frac{e p}{1-e e}$, i. e. distantia puncti inter haec duo puncta medii (centri hyperbolae) a foco, hic obtinet valorem negatiutum propter situm oppositum.

\section{5.}

Angulum 0 , qui pro parabola intra terminos $-180^{\circ}$ et $+180^{\circ}$, pro hyperbola intra $-\left(180^{\circ}-\psi\right)$ et $+\left(180^{\circ}-\psi\right)$ coërcetur, pro ellipsi vero circulum integrum periodis perpetuo renouatis percurrit, corporis moti anomaliam veram nuncupamus. Hactenus quidem omnes fere astronomi anomaliam veram in ellipsi non a perihelio sed ab aphelio inchoare solebant, contra analogiam parabolae et hyperbolae, vbi aphelium non datur adeoque a perihelio incipere oportuit: nos analogiam inter omnia sectionum conicarum genera restituere eo minus dubitauimus, quod astronomi gallici recentissimi exemplo suo iam praciuerunt.

Ceterum expressionis $r=\frac{p}{1+e \cos v}$ formam saepius aliquantulum mutare comenit; imprimis notentur formae sequentes:

$$
\begin{aligned}
& r=\frac{p}{1+e-2 e \sin \frac{x}{2} \psi^{2}}=\frac{p}{1-e+2 e \cos \frac{x}{2} y^{2}} \\
& r=\frac{p}{(1+e) \cos \frac{x}{2} v^{2}+(1-e) \sin \frac{1}{2} v^{2}} \\
& \quad \text { In parabola itaque habemus } r=\frac{p}{2 \cos \frac{1}{2} \nu^{2}} ; \text { in hyperbola expressio sequens }
\end{aligned}
$$
imprimis est commoda $r=\frac{p \cos \psi}{2 \cos \frac{\pi}{2}(\nu+\psi) \cos \frac{x}{2}(\nu-\psi)}$ : 
6.

Progredimur iam ad comparationem motus cum tempore. Statuendo vt in art. 1. spatium tempore $t$ circa Solem descriptum $=\frac{\pi}{2} g$, massam corporis moti $=\mu$, posita massa Solis $=1$, habemus $g=k t \vee p \cdot V(1+\mu)$. Differentiale spatii autem fit $=\frac{\pi}{2} r r d v$, vnde prodit $k t V p \cdot V(1+\mu)=f r r d v$, hoc integrali ita sumto, $v t$ pro $t=0$ enanescat. Haec integratio pro diuersis sectionum conicarum generibus diuerso modo tractari debet, quamobrem singula iam seorsim considerabimus, initium̃ąue ab ELLIPSI faciemus.

Quum $r$ ex per fractionem determinetur; cuius denominator e duabus partibus constat, ante omnia hoc incommodum per introductionem quantitatis novae pro $v$ auferemus. Ad hunc finem statuemus $\operatorname{tang} \frac{x}{2} v V \frac{1-e}{1+e}=\operatorname{tang} \frac{x}{2} E$, quo pacto formula vltima art. praec. pro $r$ praebet

$r=\frac{p \cos \frac{\pi}{2} E^{2}}{(1+e) \cos \frac{1}{2} v^{2}}=p\left(\frac{\cos \frac{\pi}{2} E^{2}}{1+e}+\frac{\sin \frac{\pi}{2} E^{2}}{1-e}\right)=\frac{p}{1-e e}(1-e \cos E)$.

Porro fit $\frac{d E}{\cos \frac{\pi}{2} E^{2}}=\frac{d v}{\cos \frac{r}{2} v^{2}} \vee \frac{1-e}{x+e}$, adeoque $d v=\frac{p d E}{r V(1-e e)} ;$ hinc $r r d v=\frac{r p d E}{V(x-e)}=\frac{p p}{(x-e e)^{\frac{3}{2}}}(x-e \cos E) d E$, atque integrando $k t \vee p \cdot V(1+\mu)=\frac{p p}{(x-e e)^{\frac{3}{2}}}(E-e \sin E)+$ Const.

Quodsi itaque tempus a transitu per perihelium inchoamus, vbi $v=0, E=0$ adeoque Const $=0$, habebimus, propter $\frac{p}{1-e e}=a$,

$$
E-e \sin E=\frac{k t V(1+\mu)}{a^{\frac{3}{2}}}
$$

In hac aequatione angulus auxiliaris $E$, qui anomalia excentrica dicitur, in partibus radii exprimi debet. Manifesto autem hunc angulum in gradibus etc. retinere licet, si modo etiam esin $E$ atque $\frac{k t V(1+\mu)}{a^{\frac{8}{2}}}$ eodem modo exprimantur; in minutis secundis hae quantitates exprimentur, si per numerum 206264,67 multiplicantur. Multiplicatione quantitatis posterioris supersedere possumus, si statim quantitatem $k$ in secundis expressam adhibemus, adeoque, loco valoris supra dati, statuimus $k=5548^{\prime \prime}, 18761$, cuius logarithmus $=5,5500065746$. - Hoc modo expressa quantitas $\frac{k \ell V(1+\mu)}{a^{\frac{3}{2}}}$ anomalia media vocatur, quae igitur in ratione tem- 
poris crescit, ef quidem quotidie augmento $\frac{k V(i+\mu)}{a^{\frac{3}{2}}}$, quod motus medius ciut nus dicitur. Anomaliam mediam per $\mathbb{M}$ denotabimus.

7.

In perihelio itaque anomalia vera, anomalia excentrica, et anomalia media sunt $=0$; crescente dein vera, etiam excentrica et media augentur, ita tamen, vt excentrica minor maneat quam vera, mediaque minor quam excentrica, vsque ad aphelium, vbi omnes tres simul fiunt $=180^{\circ}$; hinc vero vsque ad perihelium excentrica perpetuo est maior quam vera, mediaque maior quam excentrica, donec in perihelio omnes tres fiant $=360^{\circ}$, siue, quod eodem redit, omnes iterum $=0$. Generaliter vero patet, si anomaliae verae $v$ respondeat excentrica $E$ mediaque $M$, verae $560^{\circ}-0$ respondere excentricam $560^{\circ}-E$ atque mediam $360^{\circ}-M T$. Differentia inter anomaliam veram et mediam $\nu-M I$ aequatio centri appellatur, quae itaque a perihelio ad aphelium positita, ab aphelio ad perihelium negatiua est, in perihelio ipso autem et aphelio euanescit. Quum igitur $p$ et $M$ circulum integrum a o vsque ad $360^{\circ}$ eodem tempore percurrant, tempus reuolutionis vnius, quod et tempus periodicum dicitur, in diebus expressum inuenitur, diuidendo $560^{\circ}$ per motum diurnum $\frac{k V(1+\mu)}{a^{\frac{3}{2}}}$, vnde patet, pro corporibus dinersis circa Solem revoluentibus quadrata temporum periodicorum cubis distantiarum mediarum proportionalia esse, quatenus ipsorum massas, aut potius massarum inaequalitatem negligere liceat:

8.

Eas iam inter anomalias atque radium vectorem relationes, quae imprimis attentione dignae sunt, colligamus, quarum deductio nemini in analysi trigonometrica vel mediocriter versato difficultates obiicere poterit. Pluribus harum formularum concinnitas maior conciliatur, introducto pro $e$ angulo cuius sinus est $=e$. Quo per $\varphi$ designato, habemus $V(1-e)=\cos \varphi, V(1+e)=\cos \left(45^{\circ}-\frac{1}{2} \varphi\right) \sqrt{ } 2$, $V(1-e)=\cos \left(45^{\circ}+\frac{\pi}{2} \varphi\right) \sqrt{ } 2, V \frac{1-e}{1+e}=\operatorname{tang}\left(45^{\circ}-\frac{\pi}{2} \varphi\right), V(1+e)+V(1-e)=$ $2 \cos \frac{x}{2} \varphi, V(x+e)-V(x-e)=2 \sin \frac{x}{2} \varphi$. Ecce iam relationes praecipuas inter $a$, $p, r, e, \varphi, v, E, \mathbb{M}$. 
1. $p=a \cos \varphi^{2}$

II. $r=\frac{p}{1+e \cos \varphi}$

III. $r=a(x-e \cos E)$

IV. $\cos E=\frac{\cos v+e}{1+e \cos v}$, siue $\cos \psi=\frac{\cos E-e}{1-e \cos E}$

V. $\sin \frac{x}{2} E=\sqrt{\frac{x}{2}}(1-\cos E)=\sin \frac{x}{2} \nu V \frac{1-e}{1+e \cos \nu}=\sin \frac{x}{2} \nu \frac{r(1-e)}{p}=$ $\sin \frac{\pi}{2} v V \frac{r}{a(1+e)}$

VI. $\cos \frac{r}{2} E=\sqrt{\frac{\pi}{2}}(1+\cos E)=\cos \frac{\pi}{2} \varphi V \frac{x+e}{1+e \cos \phi} \equiv \cos \frac{r}{2} \nu V \frac{r(1+e)}{p}=$

$$
\cos \frac{x}{2} v \vee \frac{r}{a(x-e)}
$$

VII. $\operatorname{tang} \frac{\pi}{2} E=\operatorname{tang} \frac{\pi}{2} \varphi \tan g\left(45^{\circ}-\frac{\pi}{2} \varphi\right)$

VIII. $\sin E=\frac{r \sin v \cos \varphi}{p}=\frac{r \sin \psi}{a \cos \varphi}$

IX. $r \cos v=a(\cos E-e)=2 a \cos \left(\frac{r}{2} E+\frac{r}{2} \varphi+45^{\circ}\right) \cos \left(\frac{x}{2} E-\frac{r}{2} \varphi-45^{\circ}\right)$

X. $\sin \frac{x}{2}(\nu-E)=\sin \frac{x}{2} \varphi \sin \varphi \vee \frac{r}{p}=\sin \frac{x}{2} \varphi \sin E \vee \frac{a}{r}$

XI. $\sin \frac{r}{2}(v+E)=\cos \frac{r}{2} \varphi \sin v V \frac{r}{p}=\cos \frac{r}{2} \varphi \sin E V \frac{a}{r}$

XII. $M=E-e \sin E$

$$
9 .
$$

Si perpendiculum e puncto quocunque ellipsis in lineam apsidum demissum retro producitur, vsquedum circulo e centro ellipsis radio a descripto occurrat, inclinatio eius radii, qui puncto intersectionis respondet, contra lineam apsidum. (simili modo intellecta vt supra pro anomalia vera) anomaliae excentricae aequalis erit, vt nullo negotio ex aequ. IX. art. praec. deducitur. Porro patet, $r \sin v$ esse distantiam cuiusque puncti ellipsis a linea apsidum; quae quum per aequ. VIII. fiat $=a \cos \varphi \sin E$, maxima erit pro $E=90^{\circ}, i$. e. in centro ellipsis. Haecce distantia maxima, quae fit $=a \cos \varphi=\frac{p}{\cos \varphi}=\sqrt{ } a p$, semiaxis minor appellatur. In foco ellipsis, i. e. pro $\nu=90^{\circ}$, distantia ista manifesto fit $=p$, sine semiparametro aequalis. 
10.

Aequationes art. 8. omnia continent, quae ad computum anomaliae excentricae et mediae e vera, vel excentricae et verae e media requiruntur. Pro deducenda excentrica e vera vulgo formula VII. adhibetur; plerumque tamen praestat ad hunc finem aequ. X. vti, praesertim quoties excentricitas non nimis magna est, in quo casu $\boldsymbol{E}$ per X. maiori praecisione computari potest, quam per VII. Praeterea adhibita aequatione $\mathbf{X}$, logarithmus sinus $\boldsymbol{E}$, qui in XII. requiritur, protinus per aequationem VIII. habetur, quem adhibita VII. e tabulis arcessere oporteret; si igitur in illa methodo hic logarithmus etiam e tabulis desumitur, simul calculi recte instituti confirmatio hinc obtinetur. Huiusmodi calculi examina et comprobationes magni semper sunt aestimanda, quibus igitur consulere in omnibus methodis in hoc opere tradendis, vbi quidem commode fieri potest, assiduae nobis vbique curae erit. - Ad maiorem illustrationem exemplum complete calculatum adiungimus.

Data sint $y=310^{\circ} 55^{\prime} 29^{\prime \prime} 64, \varphi=14^{\circ} 12^{\prime} 1^{\prime \prime} 87, \log r=0,3307640$; quaeruntur $p, a, E$ et $M$.

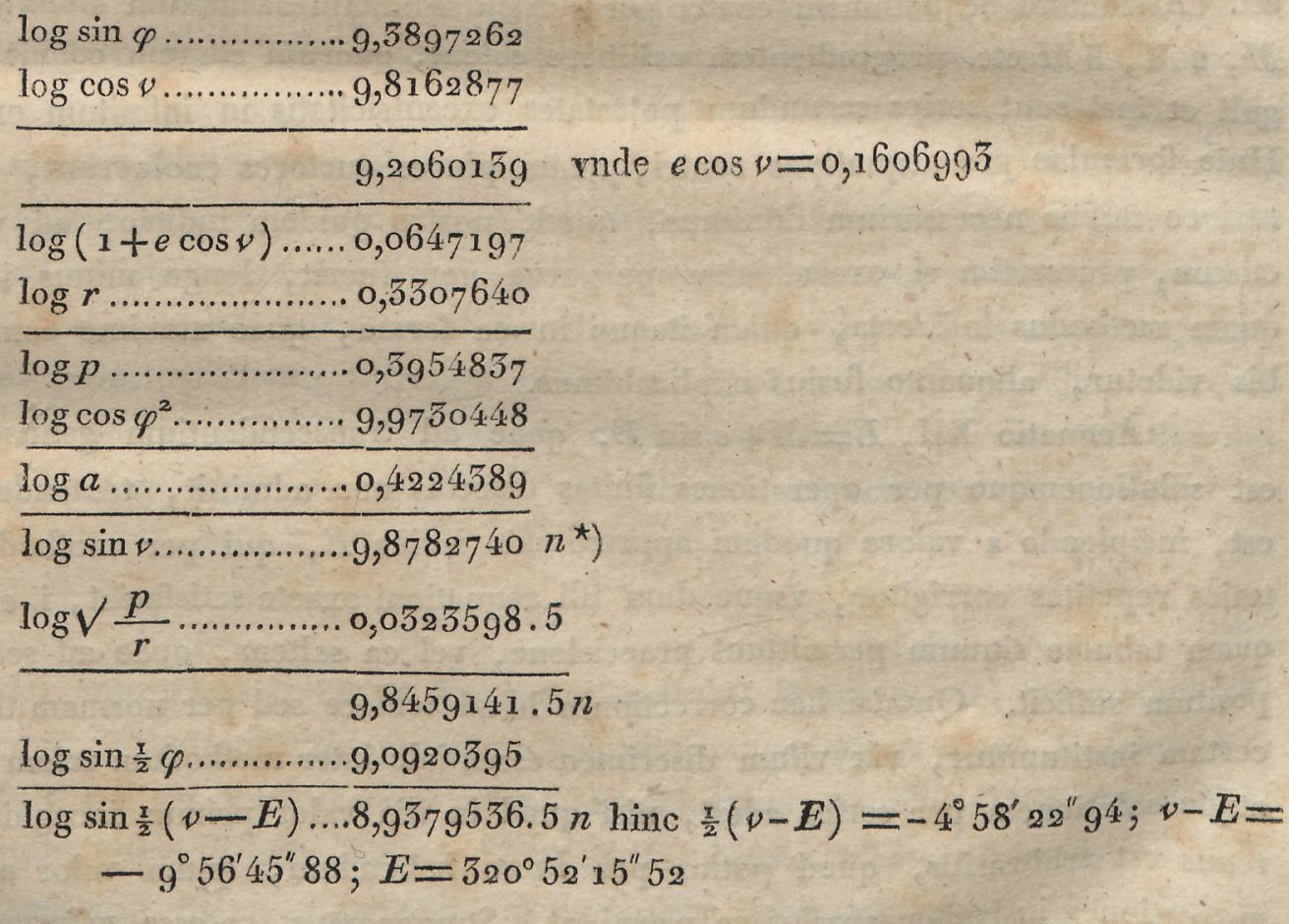

*) Litera $n$ logarithmo affixa indicat, numerum cui resfondet negatiuum esse. 
Porro

$$
\begin{aligned}
& \log e \ldots \ldots \ldots \ldots \ldots . . ., 58997262 \\
& \underline{\log 206264,7 \ldots 5,3144251} \quad \log \frac{r}{p} \sin \vartheta \ldots . .9,8135543 n \\
& \text { loge in } \sec : \ldots . . .4,7041513 \quad \log \cos \varphi \ldots \ldots \ldots \ldots .9,9865224
\end{aligned}
$$

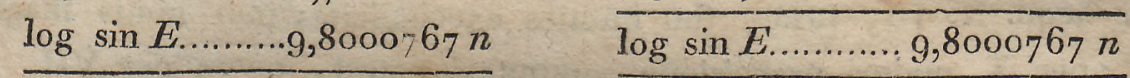

$$
\begin{aligned}
& 4,5042278 n \text { hinc } e \sin E \text { in secundis }=31932^{\prime \prime} 14=8^{\circ} 52^{\prime} 12^{\prime \prime} 14
\end{aligned}
$$

atque $I M=329^{\circ} 44^{\prime} 27^{\prime \prime} 66$. - Per formulam VII. calculus pro $E$ ita se haberet:

$$
\begin{aligned}
& \frac{x}{2} \nu=155^{\circ} 27^{\prime} 44^{\prime \prime} 82 \quad \quad \log \operatorname{tang} \frac{x}{2} y \ldots \ldots \ldots \ldots . . .9,6594579 n \\
& 45^{\circ}-\frac{\pi}{2} \varphi=37^{\circ} 53^{\prime} 59^{\prime \prime} 065 \quad \frac{\log \tan g\left(45^{\circ}-\frac{r}{2} \varphi\right) \ldots 9,8912427}{\log \operatorname{tang} \frac{\pi}{2} E \ldots \ldots \ldots \ldots 9,5507006 n}
\end{aligned}
$$

vnde $\frac{r}{2} E=160^{\circ} 26^{\prime} 7^{\prime \prime} 7^{6}$ atque $E=320^{\circ} 52^{\prime} 15^{\prime \prime} 52$ vt supra.

$$
11 .
$$

Problema inuersum, celebre sub nomine problematis Kepleri, scilicet ex anomalia media inuenire veram atque radium vectorem, longe frequentioris vsus est. Astronomi aequationem centri per seriem infinitam secundum sinus angulorum $M, 2 I M, 3 M$ etc. progredientem exhibere solent, quorum sinuum coëfficientes singuli et ipsi sunt series secundum potestates excentricitatis in infinitum excurrentes. Huic formulae pro aequatione centri, quam plures auctores euoluerunt, hic immorari eo minus necessarium duximus, quod, nostro quidem iudicio, ad vsum practicum, praesertim si excentricitas perparua non fuerit, longe minus idonea est, quam methodus indirecta, quam itaque in ea forma, quae maxime commoda nobis videtur, aliquanto fusius explicabimus.

Aequatio XII, $E=\pi+e \sin E$, quae ad transcendentium genus referenda est solutionemque per operationes finitas directas non admittit, tentando soluenda est, incipiendo a valore quodam approximato ipsius $\mathbb{E}$, qui per methodos idoneas toties repetitas corrigitur, vsque dum illi aequationi exacte satisfaciat, i. e. vel omni quam tabulae sinuum permittunt praecisione, vel ea saltem, quae ad scopum propositum sufficit. Quodsi hae correctiones haud temere sed per normam tutam atque certam instituuntur, vix vllum discrimen essentiale inter methodum talem indirectam atque solutionem per series adest, nisi quod in illa valor primus incognitae aliquatenus est arbitrarius, quod potius pro lucro habendum, quum valor apte electus correctiones insigniter accelerare permittat. Supponamus, $\varepsilon$ esse valorem approximatum ipsius $\boldsymbol{E}$, atque $x$ correctionem illi adhuc adiiciendam (in secundis expres- 
sam), ita vt valor $E=\varepsilon+x$ aequationi nostrae exacte satisfaciat. Computetur $e \sin \varepsilon$ in secundis per logarithmos, quod dum perficitur, simul e tabulis notetur variatio ipsius $\log \sin \varepsilon$ pro $x^{\prime \prime}$ variatione ipsius $\varepsilon$, atque variatio $\log e \sin \varepsilon$ pro variatione vnius vnitatis in numero $e \sin \varepsilon$; $\operatorname{sint}$ hae variationes sine respectu signorum resp. $\lambda, \mu$, vbi vix opus est monere, vtrumque logarithmum per aeque multas figuras decimales expressum supponi. Quodsi iam $\varepsilon$ ad rerum ipsius $E$ valorem tam prope iam accedit, $v t$ variationes logarithmi sinus ab $\varepsilon$ vsque ad $\varepsilon+x$, variationesque logarithmi numeri ab esin $\varepsilon$ vsque ad $e \sin (\varepsilon+x)$ pro vniformibus habere liceat, manifesto statui poterit $e \sin (\varepsilon+x)=e \sin \varepsilon \pm \frac{2 x}{\mu}$, signo superiori pro quadrante primo et quarto, inferiori pro secundo et tertio valente. Quare quum sit $\varepsilon+x=M+e \sin (\varepsilon+x)$, fit $x=\frac{\mu}{\mu \mp \lambda}(M+e \sin \varepsilon-\varepsilon)$, valorque verus ipsius $E$ siue $\varepsilon+x=M+e \sin \varepsilon \pm \frac{\lambda}{\mu \mp \lambda}(M+e \sin \varepsilon-\varepsilon)$, signis ea qua diximus ratione determinatis. Ceterum facile perspicitur, esse sine respectu signi $\mu: \lambda=1: e \cos \varepsilon$, adeoque semper $\mu>\lambda$, vnde concluditur, in quadrante primo et vltimo $M+e \sin \varepsilon$ iacere inter $\varepsilon$ atque $\varepsilon+x$, in secundo ac tertio vero $\varepsilon+x$ inter $\varepsilon$ atque $M+e \sin \varepsilon$, quae regula attentionem ad signa subleuare potest. . Si valor suppositus $\varepsilon$ nimis adhuc a vero aberrauerat, quam vt suppositionem supra traditam pro satis exacta habere liceret, certe per hanc methodum inuenietur valor multo propior, quo eadem operatio iterum adhuc, pluriesue si opus videtur, repetenda erit. Nullo vero negotio patet, si differentia valoris primi $\varepsilon$ a vero tamquam quantitas ordinis primi spectetur, errorem valoris noui ad ordinem secundum referendum fore, et per operationem iteratam ad ordinem quartum, octauum etc. deprimi. Quo minor insuper fuerit excentricitas, eo relocius correctiones successiuae convergent.

12.

Valor approximatus ipsius $E$, a quo calculus incipi possit, plerumque satis obuius erit, praesertim vbi problema pro pluribus valoribus ipsius $M$ soluendum est, e quibus quidam iam absoluti sunt. Deficientibus omnibus aliis subsidiis id saltem constat, quod $E$ inter limites $M I$ et $I M \pm e$ iacere debet (excentricitate $e$ in secundis expressa, signoque superiori in quadrante primo et secundo, inferiori in tertio et quarto accepto); quocirca pro valore initiali ipsius $E$ vel $I M$ vel valor se- 
cundum aestimationem qualemcunque anctus seu deminutus adoptari poterit. Vix opus est monere, calculum primum, quoties a valore parum accurato inchoetur, anxia praecisione haud indigere, tabulasque minores quales cel. Lalande curauit, abunde sufficere. Praeterea, vt calculi commoditati consulatur, tales semper valores pro $\varepsilon$ eligentur, quorum sinus e tabulis ipsis absque interpolatione excerpere licet; puta in minutis seu secundorum denariis completis, prout tabulae per singula minuta seu per singulos secundorum denarios progeedientes adhibentur. Ceterum. modificationes, quas haec praecepta patiuntur, si anguli secundum diuisionem novam decimalem exprimantur, quisque sponte enoluere poterit.

13.

Exemplum. Sit excentricitas eadem quae in exemplo art. 10. $M=332^{\circ} 28^{\prime} 54^{\prime \prime} 77$. Hic igitur est $\log e$ in secundis 4,7041513 , adeoque $e=50600^{\prime \prime}=14^{\circ} 3^{\prime} 20^{\prime \prime}$. Quare quum hic $E$ minor esse debeat quam $M$, statuemus ad calculum primum $\varepsilon=326^{\circ}$, vnde per tabulas minores fit

$$
\begin{aligned}
& \log \sin \varepsilon \ldots . .9,74756 n, \text { mutatio pro } 1^{\prime} \ldots . .19, \text { vade } \lambda=0,3_{2} \\
& \frac{\log e \text { in sec...4,70415 }}{4,45171 n}
\end{aligned}
$$

hinc e $\sin \varepsilon=-28295^{\prime \prime}=-7^{\circ} 51^{\prime} 35^{\prime \prime}$.

$M+e \sin \varepsilon \ldots \ldots \ldots \ldots \ldots \ldots \ldots . \ldots . \ldots . \ldots 3420$

MIutatio logarithmi pro vnitate tabulac, quae hic 10 secundis aequinalet, $\ldots \ldots 16$; vnde $\mu=1,6$

Differt $a b \varepsilon$

$$
12240=4960^{\prime \prime} \text {. Hinc } \frac{0,52}{1,28} \times 4960^{\prime \prime}=1240^{\prime \prime}
$$

$=20^{\prime} 40^{\circ}$. Quare valor correctus ipsius $E$ fit $=524^{\circ} 37^{\prime} 20^{\prime \prime}-20^{\prime} 40^{\prime \prime}=324^{\circ} 16^{\prime} 40^{\prime \prime}$, cum quo calculum secundum tabulas maiores repetemus.

$$
\begin{aligned}
& \log \sin \varepsilon \ldots . . . . .9,7663058 n \quad \lambda=29,25 \\
& \frac{\log e \ldots \ldots \ldots \ldots . . .4,7041513}{4,4704571 n} \quad \mu=147 \\
& e \sin \varepsilon=-29543^{\prime \prime}, 18=-8^{\circ} 12^{\prime} 23^{\prime \prime} 18 \\
& M+e \sin \varepsilon \ldots \ldots \ldots \ldots \ldots \ldots \ldots . . .524 \quad 16 \quad 31,59
\end{aligned}
$$

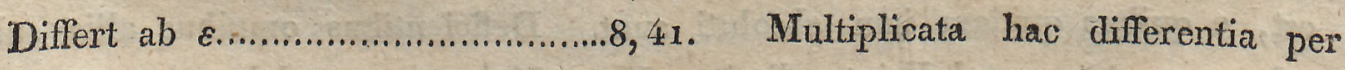
$\frac{\lambda}{\mu-\lambda}=\frac{29,25}{117,75}$, prodit $2^{\prime \prime} 09$, vnde valor denuo correctus ipsius $E=$ $524^{\circ} 16^{\prime} 3 x^{\prime \prime} 59-2^{\prime \prime} 09=324^{\circ} 16^{\prime} 29^{\prime \prime} 50$, intra o" or exactus. 
14.

Pro deriuatione anomaliae verae radiique vectoris ex anomalia excentrica aequationes art. 8. plures methodos suppeditant, e quibus praestantissimas explicabimus.

I. Secundum methodum vulgarem $v$ per aequationem VII, atque tunc $r$ per aequationem II. determinantur; hoc modo exemplum art. praec. ita se habet, retinendo pro $p$ valorem in art. 10. traditum:

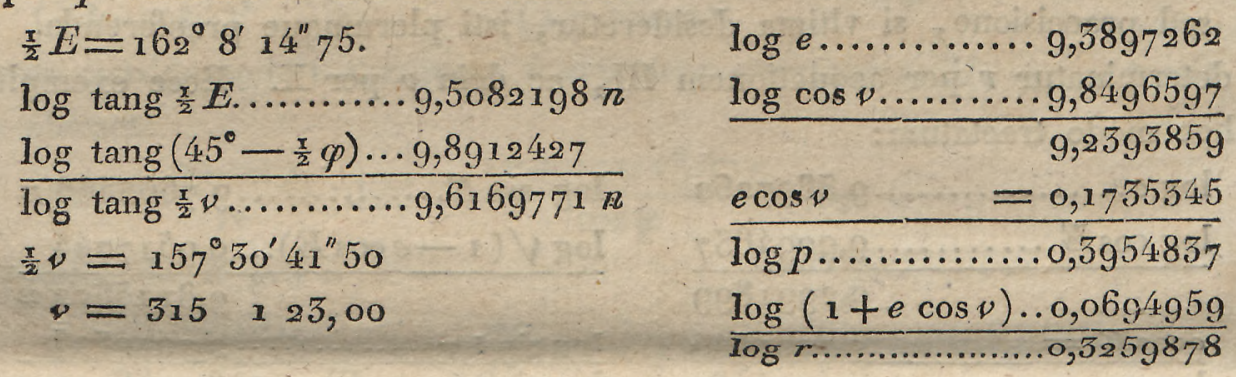

II. Breuior est methodus sequens, siquidem plures loci calculandi sunt, pro quibus logarithmos constantes quantitatum $\vee a(x+e), \sqrt{ } a(1-e)$ semel tantum computare oportet. Ex aequationibus V et VI habetur

$\sin \frac{\pi}{2} v \vee r=\sin \frac{\pi}{2} E V a(1+e)$

$\cos \frac{\pi}{2} \nu \vee r=\cos \frac{r}{2} E V a(1-e)$

vnde $\frac{\tau}{2} v$ atque $\log \sqrt{ } r$ expedite determinantur. Generaliter nimirum, quoties habetur $P \sin Q=A, P \cos Q=B$, inuenitur $Q$ per formulam $\operatorname{tang} Q=\frac{A}{B}$, atque tunc $P$ per hanc $P=\frac{A}{\sin Q}$, vel per $P=\frac{B}{\cos Q}:$ priorem adhibere praestat, quando $\sin Q$ est maior quam $\cos Q$; posteriorem, quando $\cos Q$ maior est quam $\sin Q$. Plerumque problemata, in quibus ad tales aequationes peruenitur (qualia in hoc opere frequentissime occurrent), conditionem implicant, quod $P$ esse debet quantitas positiua; tunc dubium, vtrum $Q$ inter o et $180^{\circ}$ an inter $180^{\circ}$ et $360^{\circ}$ accipere oporteat, sponte hinc tollitur. Si vero talis conditio non adest, haec determinatio arbitrio nostro relinquitur.

In exemplo nostro habemus $e=0,2453162$,

$$
\begin{array}{ll}
\log \sin \frac{x}{2} E \ldots \ldots .9,4867632 & \log \cos \frac{x}{2} E \ldots \ldots .9,9785454 n \\
\log V a(x+e) \ldots .0,2588593 & \log V a(x-e) \ldots . .0,1501020
\end{array}
$$


Hine

$$
\begin{aligned}
& \log \sin \frac{x}{2} \nu \sqrt{ } \cdot \ldots 9,7456225 \text { | vnde } \log \operatorname{tang} \frac{\pi}{2} \nu=9,6169771 \mathrm{n} \\
& \left.\log \cos \frac{x}{2} p \vee r \ldots 0,1286454 n\right\} \quad \frac{x}{2} v=157^{\circ} 30^{\prime} 41^{\prime \prime} 50 \\
& \log \cos \frac{\pi}{2} v \ldots \ldots .9,9656515 n \quad v=315 \quad 123,00 \\
& \frac{\log \sqrt{ }{ }^{\prime} \ldots \ldots \ldots . . .0,1629939}{\log r \ldots \ldots}
\end{aligned}
$$

$\log r \ldots \ldots \ldots . . ., 3259878$

III. His methodis tertiam adiicimus, quae aeque fere expedita est ac secunda, sed praecisione, si vltima desideretur, isti plerumque praeferenda. Scilicet primo determinatur $r$ per aequationem III, ac dein $v$ per $\mathrm{X}$. Ecce exemplum nostrum hoc modo tractatum:

$$
\begin{aligned}
& \log e \ldots \ldots \ldots \ldots \ldots .9,3897262 \quad \log \sin E \ldots \ldots \ldots \ldots 9,7663566 n \\
& \frac{\log \cos E \ldots \ldots \ldots \ldots 9,9094637}{9,2991899} \quad \frac{\log V(1-e \cos E) \ldots 9,9517744}{9,8145622 n} \\
& e \cos E=\quad 0,1991544 \quad \log \sin \frac{x}{2} \varphi \ldots \ldots \ldots \ldots . .9,0920395 \\
& \begin{array}{rr}
\log (1-e \cos E) \ldots 9,9055488 & \log \sin \frac{x}{2}(\nu-E) \ldots \ldots .8,9^{066017} \\
\hline & \frac{x}{2}(\nu-E)=-4^{\circ} 37^{\prime} 35^{\prime \prime} 24
\end{array} \\
& \overline{\log r \ldots \ldots \ldots \ldots \ldots .0,5259877} \quad \boldsymbol{v}-\boldsymbol{E}=-9156,48 \\
& v=515 \quad 1 \quad 25,02
\end{aligned}
$$

Ad calculum confirmandum formula VIII vel IX percommoda est, praesertim, si $v$ et $r$ per methodum tertiam determinatae sunt. Ecce calculum:

$$
\begin{array}{ll}
\log \frac{a}{r} \sin E \ldots .9,8627878 n & \log \sin E V \frac{a}{r} \ldots . .9,8145622 n \\
\frac{\log \cos \varphi \ldots \ldots .99,9865224}{9,8495102 n} & \log \cos \frac{r}{2} \varphi \ldots \ldots \ldots 9,9966567 \\
\log \sin v \ldots \ldots .9,8495102 n & \log \sin \frac{\pi}{2}(\nu+E) \ldots 9,8112189 n
\end{array}
$$

15.

Quum anomalia media $M$, vt vidimus, per $\dot{\varphi}$ et $\varphi$ complete determinata sit, sicuti $v$ per $I M$ et $\varphi$, patet, si omnes tres quantitates simul vt variabiles spectentur, inter ipsarum variationes differentiales aequationem conditionalem locum habere debere, cuius inuestigatio hand superflua erit. Differentiando primo aequationem VII art. 8, prodit $\frac{\mathrm{d} E}{\sin E}=\frac{\mathrm{d} \psi}{\sin \psi}-\frac{\mathrm{d} \varphi}{\cos \varphi}$; differentiando perinde aequationem XII, fit $d \Pi I=(1-e \cos E) \mathrm{d} E-\sin E \cos \varphi \mathrm{d} \varphi$. Eliminando ex his aequationibus differentialibus $\mathrm{d} E$, obtinemus 
$\mathrm{d} M=\frac{\sin E(1-e \cos E)}{\sin \varphi} \mathrm{d} \nu-\left(\sin E \cos \varphi+\frac{\sin E(1-e \cos E)}{\cos \varphi}\right) \mathrm{d} \varphi$

siue substituendo pro $\sin E, 1-e \cos E$ valores suos ex aequatt. VIII, III

$\mathrm{d} M=\frac{r r}{a a \cos \varphi} \mathrm{d} v-\frac{r(r+p) \sin v}{a a \cos \varphi^{2}} \mathrm{~d} \varphi$

siue denique, exprimendo vtrumque coëfficientem per $v$ et $\phi$ tantum,

$\mathrm{d} I I=\frac{\cos \varphi^{3}}{(1+e \cos v)^{2}} \mathrm{~d} v-\frac{(2+e \cos \varphi) \sin v \cos \varphi^{2}}{(1+e \cos v)^{2}} \mathrm{~d} \varphi$

Vice versa considerando tamquam functionem quantitatum $\boldsymbol{M}, \varphi$, aequatio hancce formam obtinet:

siue introducendo $E$ pro

$$
d v=\frac{a a \cos \varphi}{r r} d M+\frac{(2+e \cos v) \sin v}{\cos \varphi} d \varphi
$$

$$
\mathrm{d} v=\frac{a a \cos \varphi}{r r} \mathrm{~d} A M+\frac{a a}{r r}(2-e \cos E-e) \sin E \mathrm{~d} \varphi
$$

16.

Radius vector $r$ per $\varphi$ et $\varphi$ vel per $M$ et $\varphi$ plene nondum determinatus est, sed insuper a $p$ vel $a$ pendet; constabit igitur eius differentiale tribus membris. Per differentiationem aequationis II. art. 8. nanciscimur

$$
\frac{\mathrm{d} r}{r}=\frac{\mathrm{d} p}{p}+\frac{e \sin v}{1+e \cos v} \mathrm{~d} \psi-\frac{\cos \varphi \cos v}{1+e \cos v} \mathrm{~d} \varphi
$$

Statuendo hic $\frac{\mathrm{d} p}{p}=\frac{\mathrm{d} a}{a}-2 \operatorname{tang} \varphi \mathrm{d} \varphi($ quod sequitur e differentiatione aequ. I), exprimendoque secundum art. praec. d $\nu$ per d $M$ et $d \varphi$, prodit post debitas reductiones

$$
\begin{aligned}
& \frac{d r}{r}=\frac{d a}{a}+\frac{a}{r} \tan g \varphi \sin \psi d M-\frac{a}{r} \cos \varphi \cos \varphi d \varphi, \text { siue } \\
& \mathrm{d} r=\frac{r}{a} \mathrm{~d} a+a \tan g \varphi \sin \nu \mathrm{d} M-a \cos \varphi \cos \nu \mathrm{d} \varphi
\end{aligned}
$$

Ceterum hae formulae, sicut eae quas in art. praec. euoluimus, suppositioni imnituntur, $v, \varphi$ et $M$ siue potius $d v, d \varphi$, et $d I I$ in partibus radii exprimi. Quodsi igitur variationes angulorum $\nu, \varphi, I M$ in secundis exprimere placet: vel eas formularum partes quae $\mathrm{d} \psi, \mathrm{d} \varphi$ aut $\mathrm{d} M$ implicant, per 206264,7 diuidere oportet, vel eas, quae continent $\mathrm{d} r, \mathrm{~d} p$ aut $\mathrm{d} a$, per eundem numerum multiplicare. Formulae igitur art. praec., quae hoc respectu sunt homogeneae, mutatione opus non habebunt. 
17.

De indagatione aequationis centri maximae pauca adiecisse haud poenitebit. Prino sponte obuium est, differentiam inter anomaliam excentricam et mediam maximum esse pro $E=90^{\circ}$, vbi fit $=e$ (in gradibus etc. exprimenda); radius vector in hoc puncto est $=a$, vnde $\nu=90^{\circ}+\varphi$, adeoque aequatio centri tota $=\varphi+e$, quae tamen hic non est maximum, quoniam differentia inter $v$ et $E$ adhuc vltra $\varphi$ crescere potest. Haecce differentia fit maximum pro $\mathrm{d}(\psi-E)=0$ siue pro $\mathrm{d} v=\mathrm{d} \boldsymbol{E}$, vbi excentricitas manifesto $\mathrm{vt}$ constans spectanda est. Qua suppositione quum generaliter fiat $\frac{d v}{\sin v}=\frac{d E}{\sin E}$, patet, in eo puncto vbi differentia inter $v$ et $E$ maximum est, esse debere $\sin v=\sin E$; vnde erit, per aequatt. VIII, III, $r=a \cos \varphi, e \cos E=1-\cos \varphi$, siue $\cos E=+\operatorname{tang} \frac{x}{2} \varphi$. Perinde inuenitur $\cos v=-\operatorname{tang} \frac{x}{2} \varphi$, quapropter erit $\left.{ }^{\star}\right) v=90^{\circ}+\operatorname{arc} \sin \operatorname{tang} \frac{1}{2} \varphi, E=90^{\circ}-$ arc. $\sin \operatorname{tang} \frac{1}{2} \varphi ;$ hinc porro $\sin E=V\left(1-\operatorname{tang} \frac{x}{2} \varphi^{2}\right)=\frac{V \cos \varphi}{\cos \frac{x}{2} \varphi}$, ita vt aequatio centri tota in hoc puncto fiat $=2 \operatorname{arc} \sin \operatorname{tang} \frac{\pi}{2} \varphi+2 \sin \frac{\pi}{2} \varphi V \cos \varphi$, parte secunda in gradibus etc. expressa. - In eo denique puncto, vbi tota aequatio centri ipsa maximum est, fieri debet $\mathrm{d} v=\mathrm{d} M$, adeoque secundum art. $15, r=a \vee \cos \varphi$; hinc fit $\cos \psi=-\frac{1-\cos \varphi^{\frac{3}{2}}}{e}, \cos E=\frac{1-V \cos \varphi}{e}=\frac{1-\cos \varphi}{e(1+\sqrt{ } \cos \varphi)}=\frac{\tan g \frac{\pi}{2} \varphi}{1+V \cos \varphi}$ per quam formulam $E$ vltima praecisione determinare licet. Inuenta $E$, erit per aequ. X, XII aequatio centri $=2 \arcsin \frac{\sin \frac{1}{2} \varphi \sin E}{4}+e \sin E$. Expressioni acquationis centri maximae per seriem secundum potestates excentricitatis progredientem, quam plures auctores tradiderunt, hic non immoramur. Vt exemplum habeatur, conspectum trium maximorum, quae hic contemplati sumus, pro Iunone adiungimus, vbi excentricitas secundum elementa nouissima $=0,2554996$ supposita est.

\begin{tabular}{|c|c|c|c|c|c|}
\hline Maximum & & $E$ & $E-M$ & $v-E$ & $v-M$ \\
\hline$E-I M$ & $90^{\circ}$ & $0^{\prime} o^{\prime \prime}$ & $14^{\circ} 58^{\prime} 20^{\prime \prime} 57$ & $14^{\circ} 48^{\prime} 11^{\prime \prime} 48$ & $29^{\circ} 26^{\prime} 52^{\prime \prime} 05$ \\
\hline$v-E$ & & $32 \quad 9$ & 145054,01 & 145541,79 & $2926 \quad 35,80$ \\
\hline$p-M$ & 86 & 1440 & $14 \quad 36 \quad 27,39$ & $14 \quad 53 \quad 49,57$ & $2930 \quad 16,96$ \\
\hline
\end{tabular}

*) Ad ea maxima, quae inter aphelium et peribelium iacent, non opus, est respicere, quum manifesto ab iis, quae inter perihelium et aphelium sita sunt, in signis tantum differant. 
18.

In PARABOLA anomalia excentrica, anomalia media atque motus medius fierent $=0$; hic igitur istae notiones comparationi motus cum tempore inserrire nequeunt. Attamen in parabola angulo auxiliari ad integrandum $r \boldsymbol{r} \mathrm{d} v$ omnino opus non habemus; fit enim $r r \mathrm{~d} v=\frac{p p \mathrm{~d} v}{4 \cos \frac{\mathrm{T}}{2} v^{4}}=\frac{p p \mathrm{~d} \operatorname{tang} \frac{\mathrm{T}}{2} v}{2 \cos \frac{3}{2} v^{2}}=$

$\frac{\pi}{2} p p\left(1+\operatorname{tang} \frac{x}{2} v^{2}\right) d \operatorname{tang} \frac{x}{2} v$, adeoque $\int r r d v=\frac{x}{2} p p\left(\operatorname{tang} \frac{x}{2} v+\frac{x}{3} \operatorname{tang} \frac{x}{2} v^{3}\right)+$ Const. Si tempus a transitu per perihelium incipere supponitur, Constans fit $=0$; habetw itaque

$$
\operatorname{tang} \frac{\pi}{2} v+\frac{\pi}{3} \operatorname{tang} \frac{\pi}{2} v^{3}=\frac{2 t k \sqrt{ }(1+\mu)}{p^{\frac{3}{2}}}
$$

per quam formulam $t$ ex $v$, atque $v$ ex $t$ deriuare licet, simulac $p$ et $\mu$ sunt cognitae. Pro $p$ inter elementa parabolica radius vector in perihelio qui est $\frac{1}{2} p$ exhiberi, massaque $\mu$ omnino negligi solet. Vix certe vmquam possibile erit, massam corporis talis cuius orbita tamquam parabola computatur, determinare, reueraque omnes cometae per optimas recentissimasque obseruationes densitatem atque massam tam exiguam habere videntur, vt haec insensibilis censeri tutoque negligi possit.

19.

Solutio problematis, ex anomalia vera deducere tempus, multoque adhuc magis solutio problematis inuersi, magnopere abbreuiari potest per tabulam auxiliarem, qualis in pluribus libris astronomicis reperitur. Longe vero commodissima est tabula Barkeriana, quae etiam operi egregio cel. Olbers (Abhandlung über die leichteste und bequemste Methode die Bahn eines Cometen zu berechnen, Weimar 1797.) annexa est. Continet ea pro omnibus anomaliis veris a o vsque ad $180^{\circ}$ per singula 5 minuta valorem expressionis $75 \operatorname{tang} \frac{x}{2} p+25 \operatorname{tang} \frac{x^{\prime}}{2} t^{3}$ sub nomine motus medii. Si itaque tempus desideratur anomaliae verae $v$ respondens, diuidere oportebit motum medium e tabula argumento $v$ excerptum per $\frac{150 k}{p^{\frac{3}{2}}}$, quae quantitas motus medius diurnus dicitur; contra si e tempore anomalia vera computanda est, illud in diebus expressum per $\frac{150 k}{p^{\frac{3}{2}}}$ multiplicabitur, vt motus medius prodeat, quo anomaliam respondentem e tabula sumere licebit. Ceterum manifesto valori negatiuo ipsius $v$ motus medius tempusque idem sed negatiue sumtum respondet: eadem igitur tabula anomaliis negatiuis et positiuis perinde inseruit. Si pro $p$ 
distantia in perihelio $\frac{1}{2} p=q$ vti malumus, motus medius diurnus exprimitur per $\frac{k V_{2} 812,5}{q^{\frac{s}{2}}}$, vbi factor constans $k V_{2812,5} \mathrm{fit}=0,912279061$, ipsiusque logarithmus 9,9601277069 . - Inuenta anomalia $v$ radius vector determinabitur per formulam iam supra traditam $r=\frac{q}{\cos \frac{r}{2} \nu^{2}}$.

20.

Per differentiationem aequationis tang $\frac{x}{2} \psi+\frac{x}{3} \operatorname{tang} \frac{3}{2} \psi^{3}=2 t k p^{-\frac{3}{2}}$, si omnes quantitates $v, t, p$ cen variabiles tractantur, prodit

$$
\begin{aligned}
& \frac{d w}{2 \cos \frac{x}{2} p^{4}}=2 k p^{-\frac{3}{2}} \mathrm{~d} t-3 t k p^{-\frac{5}{2}} \mathrm{~d} p, \text { siue } \\
& \mathrm{d} v=\frac{k \sqrt{ } p}{r r} \mathrm{~d} t-\frac{3 t k}{2 r r \sqrt{ } p} \mathrm{~d} p
\end{aligned}
$$

Si variationes anomaliae $v$ in secundis expressae desiderantur, etiam ambae partes ipsius $\mathrm{d} v$ hoc modo exprimendae sunt, i. e. pro $k$ valorem in art. 6 . traditum 3548 " 188 accipere oportet. Quodsi insuper pro $p$ introducatur $\frac{r}{2} p=q$, formula ita se habebit

$$
\mathrm{d} v=\frac{k \sqrt{2 q}}{r r} \mathrm{~d} t-\frac{3 k t}{r r \sqrt{2 q} \mathrm{~d} q}
$$

vbi logarithmi, constantes adhibendi sunt $\log k \sqrt{ } 2=3,7005215724, \log 3 k \sqrt{\frac{\pi}{2}}=$ 5,8766128315 .

Porro differentiatio aequationis $r=\frac{p}{2 \cos \frac{x}{2} v^{2}}$ suppeditat $\frac{d r}{r}=\frac{d p}{p}+\operatorname{tang} \frac{x}{2} v d \psi$, situe exprimendo $d v$ per $d t$ et $d p$ $\frac{\mathrm{d} r}{r}=\left(\frac{1}{p}-\frac{5 k t \operatorname{tang} \frac{\pi}{2} \varphi}{2 r r V p}\right) \mathrm{d} p+\frac{k \sqrt{ } p \operatorname{tang} \frac{x}{2} \nu}{r r} \mathrm{~d} t$

Coëfliciens ipsius $\mathrm{d} p$, substituendo pro $t$ valorem suum per $v$ transit in $\frac{1}{p}-\frac{5 p \operatorname{tang} \frac{\pi}{2} v^{2}}{4 r r}-\frac{p \operatorname{tang} \frac{x}{2} v^{4}}{4 r r}=\frac{1}{r}\left(\frac{x}{2}+\frac{\pi}{2} \operatorname{tang} \frac{\pi}{2} v^{2}-\frac{3}{2} \sin \frac{\pi}{2} v^{2}-\frac{\pi}{2} \sin \frac{\pi}{2} v^{2} \tan g \frac{\pi}{2} v^{2}\right)$ $=\frac{\operatorname{cas} p}{2 r} ;$ coëfficiens ipsius $\mathrm{d} t$ antem fit $=\frac{k \sin v}{r \sqrt{ } p}$. Hinc prodit $\mathrm{d} r=\frac{x}{2} \cos v \mathrm{~d} p$ $+\frac{k \sin v}{\sqrt{ } p} \mathrm{~d} t$, sive introducendo $q$ pro $p$ 
$\mathrm{d} r=\cos v \mathrm{~d} q+\frac{k \sin v}{\sqrt{ } 2 q} \mathrm{~d} t$

Logarithmus constans hic adhibendus est $\log k \sqrt{\frac{x}{2}}=8,0850664456$.

21.

In HYPERBOLA $\varphi$ atque $E$ quantitates imaginariae fierent, quales si auersamur, illarum loco aliae quantitates auxiliares sunt introducendae. Angulum cuius cosinus $=\frac{1}{e}$ iam supra per $\psi$ designauimus, radiumque vectorem $=\frac{p}{2 e \cos \frac{x}{2}(\nu-\psi) \cos \frac{\pi}{2}(1+\psi)}$ inuenimus. Factores in denominatore huius fractionis, $\cos \frac{x}{2}(\nu-\psi)$ et $\cos \frac{x}{2}(\psi+\psi)$, aequales fiunt pro $\nu=0$, secundus euanescit pro valore maximo positiuo ipsius $v$, primus vero pro valore maximo negatiuo. Statuendo igitur $\frac{\cos \frac{x}{2}(v-\psi)}{\cos \frac{x}{2}(\nu+\psi)}=u$, erit $u=0$ in perihelio; crescet in infinitum, dum $v$ ad limitem suum $180^{\circ}-\psi$ appropinquat; contra decrescet in infinitum, dum $v$ ad limitem alterum $-\left(180^{\circ}-\psi\right)$ regredi supponitur: quod fiet ita, vt valoribus oppositis ipsius $v$ valores reciproci ipsius $u$, vel quod idem est valores tales quorum logarithmi oppositi sunt, respondeant.

Hic quotiens $u$ percommode in hyperbola vt quantitas auxiliaris adhibetur; aequali fere concinnitate istius vice fungi potest angulus cuius tangens $=$ $\operatorname{tang} \frac{x}{2} v V \frac{e-1}{e+1}$, quem $v t$ analogiam cum ellipsi sequamur, per $\frac{x}{2} F$ denotabimus. Hoc modo facile sequentes relationes inter quantitates $v, r, u, F$ colliguntur, vbi $a=-b$ statuimus, ita vt $b$ euadat quantitas positiua.

I. $b=p \operatorname{cotang} \psi^{2}$

II. $r=\frac{p}{1+e \cos \nu}=\frac{p \cos \psi}{2 \cos \frac{x}{2}(\nu-\psi) \cos \frac{x}{2}(\nu+\psi)}$

III. $\operatorname{tang} \frac{x}{2} F=\operatorname{tang} \frac{x}{2} \nu V \frac{e-1}{e+1}=\operatorname{tang} \frac{x}{2} \nu \operatorname{tang} \frac{x}{2} \psi=\frac{u-1}{u+x}$

IV. $u=\frac{\cos \frac{x}{2}(\nu-\psi)}{\cos \frac{x}{2}(\nu+\psi)}=\frac{1+\tan g \frac{x}{2} F}{1-\tan \frac{x}{2} F}=\operatorname{tang}\left(45^{\circ}+\frac{x}{2} F\right)$

V. $\frac{1}{\cos F}=\frac{x}{2}\left(u+\frac{1}{u}\right)=\frac{1+\cos \psi \cos \psi}{2 \cos \frac{x}{2} \cdot(\psi-\psi) \cos \frac{x}{2}(\psi+\psi)}=\frac{e+\cos \psi}{1+e \cos \psi}$

Subtrahendo $a b$ aequ. V. vtrimque 1 , prodit 
VI. $\sin \frac{x}{2} v \cdot V_{r}=\sin \frac{x}{2} F V \frac{p}{(e-1) \cos F}=\sin \frac{x}{2} F V \frac{(e+1) b}{\cos F}$

$$
=\frac{1}{2}(u-1) \sqrt{ } \frac{p}{(e-1) u}=\frac{1}{2}(u-1) \vee \frac{(e+1) b}{u}
$$

Simili modo addendo vtrimque 1 fit

VII. $\cos \frac{r}{2} v \cdot V r=\cos \frac{x}{2} F V \frac{p}{(e+1) \cos F}=\cos \frac{x}{2} F V \frac{(e-1) b}{\cos F}$

$$
=\frac{1}{2}(u+1) V \frac{p}{(e+1) u}=\frac{x}{2}(u+1) V \frac{(e-1) b}{u}
$$

Diuidendo VI per VII ad III reueniremus; multiplicatio producit

VIII. $r \sin v=p \operatorname{cotang} \psi \operatorname{tang} F=b \operatorname{tang} \psi \operatorname{tang} F$

$$
=\frac{r}{2} p \operatorname{cotang} \psi\left(u-\frac{1}{u}\right)=\frac{x}{2} b \operatorname{tang} \psi\left(u-\frac{1}{u}\right)
$$

E combinatione aequatt. II, V porro facile deducitur

IX. $r \cos v=b\left(e-\frac{1}{\cos F^{i}}\right)=\frac{x}{2} b\left(2 e-u-\frac{1}{u}\right)$.

X. $r=b\left(\frac{e}{\cos T}-1\right)=\frac{r}{2} b\left(e\left(u+\frac{1}{u}\right)-2\right)$

22.

Per differentiationem formulae IV prodit (spectando $\psi$ vt quantitatem constantem) $\frac{\mathrm{d} u}{u}=\frac{r}{2}\left(\operatorname{tang} \frac{r}{2}(\nu+\psi)-\operatorname{tang} \frac{x}{2}(v-\psi)\right) \mathrm{d} v=\frac{r \operatorname{tang} \psi}{p} \mathrm{~d} v ;$ hinc $r r \mathrm{~d} v=\frac{p r}{u \operatorname{tang} \psi} \mathrm{d} u$, siue substituendo pro $r$ valorem ex $\mathbf{X}$, $\operatorname{rrd} v=b b \operatorname{tang} \psi\left(\frac{1}{2} e\left(1+\frac{1}{u u}\right)-\frac{1}{u}\right) \mathrm{d} u$

Integrando deinde ita, vt integrale in perihelio euanescat, fit

$f r r d v=b b \operatorname{tang} \psi\left(\frac{1}{2} e\left(u-\frac{1}{u}\right)-\log u\right)=k t \sqrt{ } p \cdot V(1+\mu)=k t \tan g \psi V V b \cdot \sqrt{ }(1+\mu)$ Logarithmus hic est hyperbolicus; quodsi logarithmos e systemate Briggico vel generaliter e systemate cuius modulus $=\lambda$ adhibere placet, massaque $\mu$ (quam pro corpore in hyperbola incedente haud determinabilem esse supponere possumus) negligitur, aequatio hancce formam induit:

XI. $\frac{x}{2} \lambda e \frac{u u-1}{u}-\log u=\frac{\lambda k t}{b^{\frac{x}{2}}}$ 
siue introducendo $F$

$$
\lambda e \operatorname{tang} F-\log \operatorname{tang}\left(45^{\circ}+\frac{\gamma}{2} F\right)=\frac{\lambda k t}{b^{\frac{3}{2}}}
$$

Si logarithmos Briggicos adhiberi supponimus, habemus $\log \lambda=9,6577845115$, $\log \lambda k=7,8733657527$, sed praecisionem aliquantulum maiorem attingere licet, si logarithmi hyperbolici immediate applicantur. Tangentium logarithmi hyperbolici in pluribus tabularum collectionibus reperiuntur, e. g. in iis quas Schulze curauit, maiorique adhuc extensione in Beni. Ursini Magno Canone Triangulorum logarithmico, Colon. 1624. vbi per singula $10^{\prime \prime}$ progrediuntur. - Ceterum formula XI ostendit, valoribus reciprocis ipsius $u$, siue valoribus oppositis ipsius $F$ et $v$ respondere valores oppositos ipsius $t$, quapropter partes hyperbolae aequales a perihelioque vtrimque aequidistantes temporibus aequalibus describentur.

\section{5.}

Si pro inueniendo tempore ex anomalia vera quantitate auxiliari $u$ vti placuerit, huius valor commodissime per aequ. IV determinatur; formula dein II absque novo calculo statim dat $p$ per $r$, vel $r$ per $p$. Inuenta $u$ formula XI dabit quantitatem $\frac{\lambda k t}{b^{\frac{3}{2}}}$, quae analoga est anomaliae mediae in ellipsi et per $N$ denotabitur, vnde demanabit tempus post transitum per perihelium elapsum. Quum pars. prior ipsius $N$ puta $\frac{\lambda e(u u-1)}{2 u}$ per formulam VIII fiat $=\frac{\lambda r \sin \psi}{b \sin \psi}$, calculus duplex huius quantitatis ipsius praecisioni examinandae inseruire, aut si mauis, $N$ absque $u$ ita exhiberi potest

XII. $N=\frac{2 \operatorname{tang} \psi \sin v}{2 \cos \frac{r}{2}(\nu+\psi) \cos \frac{\pi}{2}(\nu-\psi)}-\log \frac{\cos \frac{\pi}{2}(\nu-\psi)}{\cos \frac{r}{2}(\nu+\psi)}$

Exemplum. Sit $e=1,2618820$ siue $\psi=57^{\circ} 55^{\prime} 0^{\prime \prime}, v=18^{\circ} 51^{\prime} 0^{\prime \prime}, \log r$ $=0,0335585$. Tum calculus pro $u, p, b, N, t$ ita se habet:

$$
\begin{aligned}
& \log \cos \frac{x}{2}(\nu-\psi) \ldots . .9,9941706 \text { \} hinc } \log u \ldots \ldots, 0,0491129 \\
& \left.\log \cos \frac{x}{2}(v+\psi) \ldots . .9,9450577\right\} \quad u=1,1197289 \\
& \log r \ldots \ldots \ldots \ldots \ldots \ldots \ldots, 0 . \ldots 335585
\end{aligned}
$$

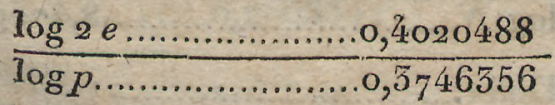

$$
\begin{aligned}
& \frac{\log \operatorname{cotang} \psi^{2} \ldots \ldots \ldots \ldots .0,2274244}{\log b \ldots \ldots \ldots} \\
& u u=1,2537928
\end{aligned}
$$




\begin{tabular}{|c|c|}
\hline 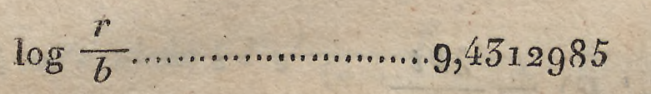 & $\begin{array}{c}\text { Calculus alter } \\
\log (u u-1) \ldots .09044795\end{array}$ \\
\hline $\log \sin y \ldots \ldots \ldots \ldots \ldots \ldots \ldots, 9,5093258$ & Compl. $\log u \ldots . .9,9508871$ \\
\hline g $\lambda \ldots \ldots \ldots \ldots \ldots \ldots \ldots \ldots \ldots \ldots, 6377843$ & $\log \lambda \ldots \ldots \ldots \ldots \ldots . . . . .9,6577845$ \\
\hline ompl. $\log \sin \psi \ldots \ldots \ldots \ldots, 0,2147509$ & $\log \frac{x}{2} e \ldots \ldots \ldots \ldots \ldots 9,7999888$ \\
\hline 8,7931595 & 8,7931595 \\
\hline \multirow{6}{*}{ 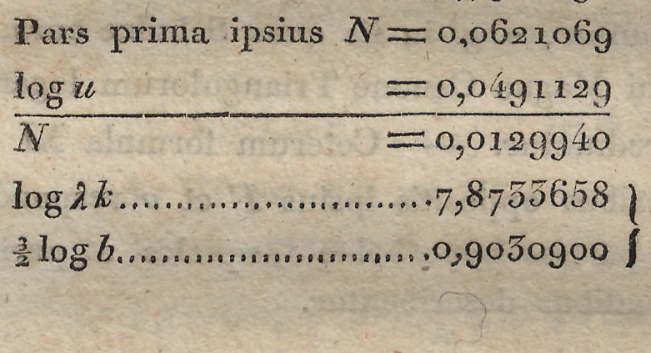 } & \\
\hline & \\
\hline & $\log N \ldots \ldots \ldots \ldots \ldots . .8,1 \times 37429$ \\
\hline & \\
\hline & Differentia ........0,9702700 \\
\hline & $\begin{array}{c}\log t \ldots \ldots \ldots \ldots \ldots, 1,1434671 \\
t=15,91448\end{array}$ \\
\hline
\end{tabular}

24.

Si calculum per logarithmos hyperbolicos exsequi constitutum est, quantitate auxiliari $F$ vti praestat, quae per aequ. III determinabitur, atque inde $N$ per XI; semiparameter e radio vectore, vel vicissim hic ex illo per formulam VIII computabitur; pars secunda ipsius $N$ duplici si lubet modo erui potest, scilicet per formulam log hyp tang $\left(45^{\circ}+\frac{x}{2} F^{\prime}\right)$, et per hanc log hyp $\cos \frac{x}{2}(v-\psi)-\log$ hyp $\cos \frac{x}{2}(v+\psi)$. Ceterum patet, quantitatem $N$ hic vbi $\lambda=1$ in ratione $1: \lambda$ maiorem enadere, quam si logarithmi Briggici adhibeantux. Ecce exemplum nostrum hoc modo tractatum:

\begin{tabular}{|c|c|}
\hline $\log \operatorname{tang} \frac{1}{2} v \ldots \ldots \ldots \ldots \ldots \ldots \ldots, 2201009$ & \\
\hline 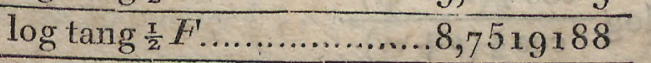 & $\frac{1}{2} F^{\prime}=5^{\circ} 13^{\prime} 58^{\prime \prime} 12$ \\
\hline 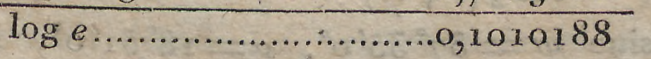 & 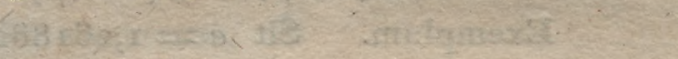 \\
\hline $\log \operatorname{tang} F \ldots \ldots \ldots \ldots \ldots \ldots, 0545566$ & \\
\hline 9,1553554 & C. $\log$ hyp $\cos \frac{x}{2}(v-\psi)=0,01342266$ \\
\hline 0,14300658 & C. $\log$ hyp $\cos \frac{1}{2}(p+\psi)=0,12650950$ \\
\hline $\log$ hyp $\operatorname{tang} 45^{\circ}+\frac{x}{2} F=0,11508666$ & Differ. $\quad=0,11508664$ \\
\hline$N=0,02991972$ & $\overline{\log N \ldots \ldots \ldots \ldots \ldots \ldots \ldots \ldots \ldots \ldots \ldots, 8,4759575}$ \\
\hline $\log k \ldots \ldots \ldots \ldots \ldots \ldots \ldots \ldots \ldots, 8,2555814$ & Differ.............................. 7,5324014 \\
\hline$\frac{3}{2} \log b \ldots \ldots \ldots \ldots \ldots \ldots \ldots \ldots \ldots, 0,9050900$ & $\log t \ldots \ldots \ldots \ldots \ldots \ldots \ldots \ldots \ldots \ldots \ldots \ldots \ldots \ldots \ldots, 1434661$ \\
\hline & 15,91445 \\
\hline
\end{tabular}


25.

Ad solutionem problematis intersi, e tempore anomaliam veram radiumque vectorem determinare, primo ex $N=\lambda k b^{-\frac{9}{2}} t$ per aequationem XI elicienda est quantitas auxiliaris $u$ vel $F$. Solutio huius aequationis transscendentis tentando perficienda erit, et per artificia uis quae in art. 11 exposuimus analoga abbreuiari poterit. Haec-antem fusius explicare supersedemus: neque enim operae pretium esse videtur, praecepta pro motu hyperbolico in coelis vix vmquam fortasse se oblaturo aeque anxie expolire ac pro motu elliptico, praetereaque omnes casus qui forte occurrere possent per methodum aliam infra tradendam absoluere licebit. Postquam I vel $u$ inuenta erit, $v$ inde per formulam III, ac dein $r$ vel per II vel per VIII determinabitur; commodius adhuc per formulas VI et VII $v$ et $r$ simul eruentur; e formulis reliquis vna alteraue pro confirmatione calculi, si lubet, in vsum vocari poterit.

26.

Exemplum. Manentibus $e$ et $b$ vt in exemplo praecedente, sit $t=65,41256$ : quaeruntur $v$ et $r$. Vtendo logarithmis Briggicis habemus $\log t \ldots \ldots \ldots \ldots . . .1,8156598$

$\frac{\log \lambda k b^{-\frac{3}{2}} \ldots \ldots .6,9702758}{\log N \ldots \ldots \ldots \ldots . .8,7859556}$, vnde $N=0,06108514$. Hinc aequationi $N=$ 2e tang $F-\log \operatorname{tang}\left(45^{\circ}+\frac{\pi}{2} F\right)$ satisfieri inuenitur per $F=25^{\circ} 24^{\prime} 27^{\prime \prime} 60$, vnde fit per formulam III

$\log \operatorname{tang} \frac{x}{2} F \ldots . . .9,5530120$

$\log \operatorname{tang} \frac{\mathrm{T}}{2} \psi \ldots . . .9,5318179$

$\log \operatorname{tang} \frac{x}{2} v \ldots \ldots 9,82119^{41}$ adeoque $\frac{x}{2} v=35^{\circ} 31^{\prime} 29^{\prime \prime} 89$. atque $u=67^{\circ} 2^{\prime} 59^{\prime \prime} 78$. Hinc porro habetur

C. $\left.\log \cos \frac{x}{2}(v+\psi) \ldots \ldots, 21374476\right)$

C. $\left.\log \cos \frac{x}{2}(v-\psi) \ldots 0,0145197\right)$

differentia. 0,1992279

$\log \operatorname{tang}\left(45^{\circ}+\frac{7}{2} F\right) \ldots .0,199^{2280}$

$\log \frac{p}{2 e}$

$\overline{\log r \ldots \ldots \ldots \ldots \ldots \ldots \ldots . . . . . . . .2008541}$

27.

prodit

Si aequatio IV ita differentiatur, vt $u, v, \psi$ simul vt variabiles tractentur, 
$\frac{d u}{u}=\frac{\sin \psi d \nu+\sin v d \psi}{2 \cos \frac{r}{2}(\nu-\psi) \cos \frac{\pi}{2}(\nu+\psi)}=\frac{r \operatorname{tang} \psi}{p} d \nu+\frac{r \sin \psi}{p \cos \psi} d \psi$

Differentiando perinde aequationem $\mathbf{X I}$, inter variationes differentiales quantitatum $u, \psi, N$ emergit relatio

$\frac{d N}{2}=\left(\frac{x}{2} e\left(1+\frac{1}{u u}\right)-\frac{1}{u}\right) d u+\frac{(u u-1) \sin \psi}{2 u \cos \psi^{2}} d \psi$, siue $\frac{d N}{\lambda}=\frac{r}{b u} d u+\frac{r \sin \psi}{b \cos \psi} d \psi$

Hinc eliminando du adiumento aequationis praecedentis obtinemus

$$
\begin{aligned}
& \frac{\mathrm{d} N}{\lambda}=\frac{r r}{b b \operatorname{tang} \psi} \mathrm{d} \psi+\left(1+\frac{r}{p}\right) \frac{r \sin \psi}{b \cos \psi} \mathrm{d} \psi, \text { size } \\
& \mathrm{d} v=\frac{b b \operatorname{tang} \psi}{\lambda r r} \mathrm{~d} N-\left(\frac{b}{r}+\frac{b}{p}\right) \frac{\sin \psi \tan \psi}{\cos \psi} \mathrm{d} \psi \\
& \quad=\frac{b b \tan g \psi}{\lambda r r} \mathrm{~d} N-\left(1+\frac{p}{r}\right) \frac{\sin \psi}{\sin \psi} \mathrm{d} \psi
\end{aligned}
$$

28.

Differentiando aequationem $\mathbf{X}$, omnibus $r, b, e, u$ pro variabilibus habitis, substituendo $\mathrm{d} e=\frac{\sin \psi}{\cos \psi^{2}} \mathrm{~d} \psi$, eliminandoque $\mathrm{d} u$ adiumento aequationis inter $\mathrm{d} N$, $d u, d \psi$ in art. praec. traditae, prodit

$$
\begin{aligned}
d r= & \frac{r}{b} d b+\frac{b b e(u u-1)}{2 \lambda u r} d N \\
& +\frac{b}{2 \cos \psi^{2}}\left\{\left(u+\frac{1}{u}\right) \sin \psi-\left(u-\frac{1}{u}\right) \sin \psi\right\} d \psi
\end{aligned}
$$

Coëfficiens ipsius $d N$ per aequ. VIII transit in $\frac{b \sin \vartheta}{2 \sin \psi}$; coëfliciens ipsius $d \psi$ autem, substituendo per aequ. IV, $u(\sin \psi-\sin \psi)=\sin (\psi-v), \frac{1}{u}(\sin \psi+\sin \psi)=$ $\sin (\psi+v)$, mutatur in $\frac{b \sin \psi \cos \psi}{\cos \psi^{2}}=\frac{p \cos \psi}{\sin \psi}$, ita vt habeatur

$$
\mathrm{d} r=\frac{r}{b} \mathrm{~d} b+\frac{b \sin \psi}{2 \sin \psi} \mathrm{d} N+\frac{p \cos \psi}{\sin \psi} \mathrm{d} \psi
$$

Quatenus porro $N$ vt functio ipsarum $b$ et $t$ spectatur, fit $\mathrm{d} N=\frac{N}{t} \mathrm{~d} t-\frac{3}{2} \cdot \frac{N}{b} \mathrm{~d} b$, quo valore substituto, $\mathrm{d} r$, ac perinde in art. praec. $\mathrm{d} v$, per $\mathrm{d} t, \mathrm{~d} b, \mathrm{~d} \psi$ expressae habebuntur. Ceterum quod supra monuimus etiam hic repetendum est, scilicet 
si angulorum $v$ et $\psi$ variationes non in partibus radii sed in secundis expressae concipiantur, vel omnes terminos qui $\mathrm{d} v, \mathrm{~d} \psi$ continent per 206264,7 diuidi, vel omnes reliquos per hunc numerum multiplicari debere.

\section{9.}

Quum quantitates auxiliares in ellipsi adhibitae $\varphi, E, M$, in hyperbola valores imaginarios obtineant, haud abs re erit, horum nexum cum quantitatibus realibus, quibus hic vsi sumus, inuestigare: apponimus itaque relationes praecipuas, vbi quantitatem imaginariam $V-1$ per $i$ denotamus.

$$
\begin{aligned}
& \sin \varphi=e=\frac{x}{\cos \psi} \\
& \operatorname{tang}\left(45^{\circ}-\frac{x}{2} \varphi\right)=V \frac{1-e}{x+e}=i V \frac{e-1}{e+x}=i \operatorname{tang} \frac{x}{2} \psi \\
& \operatorname{tang} \varphi=\frac{x}{2} \operatorname{cotang}\left(45^{\circ}-\frac{x}{2} \varphi\right)-\frac{1}{2} \operatorname{tang}\left(45^{\circ}-\frac{\pi}{2} \varphi\right)=-\frac{i}{\sin \psi} \\
& \cos \varphi=i \operatorname{tang} \psi \\
& \varphi=90^{\circ}+i \log (\sin \varphi+i \cos \varphi)=90^{\circ}-i \log \operatorname{tang}\left(45^{\circ}+\frac{x}{2} \psi\right) \\
& \operatorname{tang} \frac{1}{2} E=i \operatorname{tang} \frac{x}{2} F=\frac{i(u-1)}{u+1} \\
& \frac{1}{\sin E}=\frac{x}{2} \operatorname{cotang} \frac{x}{2} E+\frac{x}{2} \operatorname{tang} \frac{x}{2} E=-i \operatorname{cotang} F \text { siue } \\
& \sin E=i \operatorname{tang} F=\frac{i(u u-1)}{2 u} \\
& \cot E=\frac{x}{2} \operatorname{cotang} \frac{x}{2} E-\frac{x}{2} \operatorname{tang} \frac{x}{2} E=-\frac{i}{\sin F} \text { siue } \\
& \operatorname{tang} E=i \sin F=\frac{i(u u-1)}{u u+1} \\
& \cos E=\frac{1}{\cos F^{\prime}}=\frac{u u+1}{2 u} \\
& i E=\log (\cos E+i \sin E)=\log \frac{1}{u} \text { siue } \\
& E=i \log u=i \log \left(45^{\circ}+\frac{\pi}{2} F\right) \\
& M=E-e \sin E=i \log u-\frac{i e(u u-1)}{2 u}=-\frac{i N}{\lambda}
\end{aligned}
$$

Logarithmi in his formulis sunt hyperbolici. 
50.

Quum omnes quos e tabulis logarithmicis et trigonometricis depromimus numeri praecisionem absolutam non admittant, sed ad certum tantummodo gradum sint approximati, ex omnibus calculis illarum adiumento perfectis proxime tantum vera resultare possunt. In plerisque quidem casibus tabulae vulgares ad septimam figuram decimalem vsque exactae, i. e. vltra dimidiam vnitatem in figura septima excessu seu defectu numquam aberrantes a vero, praecisionem plus quam sufficientem suppeditant, ita vt errores ineuitabiles nullius plane sint momenti: nihilominus vtique fieri potest, vt errores tabularum in casibus specialibus effectum suum exserant augmentatione tanta, vt methodum alias optimam plane abdicare aliamque ei substituere cogamur. Huiusmodi casus in iis quoque calculis, quos hactenus explicauimus, occurrere potest; quamobrem ab instituto nostro haud alienum erit, disquisitiones quasdam circa gradum praecisionis, quam tabulae vulgares in illis permittunt, hic instituere. Etsi vero ad hoc argumentum calculatori practico gravissimum exhauriendum hic non sit locus, inuestigationem eo perducemus, vt ad propositum nostrum sufficiat, et a quolibet, cuius interest, vlterius expoliri et ad quasuis alias operationes extendi possit.

31.

Quilibet logarithmus, sinus, tangens etc. (aut generaliter quaelibet quantitas irrationalis e tabulis excerpta) errori obnoxius est, qui ad dimidiam vnitatem in figura vltima ascendere potest: designabimus hunc erroris limitem per $\omega$, qui itaque in tabulis vulgaribus fit $=0,00000005$. Quodsi logarithmus etc. e tabulis immediate desumi non potuit, sed per interpolationem erui debuit, error duplici canssa aliquantulum adhuc maior esse potest. Primo enim pro parte proportionali, quoties (figuram vltimam tamquam vnitatem spectando) non est integer, adoptari solet integer proxime maior vel minor: hac ratione errorem tantum non vsque ad dutplum augeri posse facile perspicitur. Ad hanc vero erroris augmentationem omnino hic non respicimus, quum nihil obstet, quominus vnam alteramue figuram decimalem parti illi proportionali affigamus, nulloque negotio pateat, logarithmum interpolatum, si pars proportionalis absolute exacta esset, errori maiori obnoxium non esse quam logarithmos in tabulis immediate expressos, quatenus quidem horum variationes tamquam vniformes considerare liceat. Erroris augmentatio altera inde nascitur, quod suppositio ista omni rigore non est vera: sed hanc quoque negligi- 
mus; quoniam effectus differentiarum secundarum altiortumque in omnibus propemodum casibus nullius plane momenti est (praesertim si pro quantitatibus trigonometricis tabulae excellentissimae quas Taylor curauit adhibentur), facilique negotio ipsius ratio haberi possit, vbi forte panllo maior enaderet. Statuemus itaque pro omnibus casibus tabularum errorem maximum ineuitabilem $=\omega$, siquidem argumentum (i. e. numerus cuius logarithmus, seu angulus cuius sinus etc. quacritur) praecisione absoluta habetur. Si vero argumentum ipsum proxime tantum innotuit, errorique maximo, cui obnoxium esse potest, respondere supponitur logarithmi etc. variatio $\omega^{\prime}$ (quam per rationem differentialium definire licet), error maximus logarithmi per tabulas computati rsque ad $\omega+\omega^{\prime}$ ascendere potest.

Vice versa, si adiumento tabularum argumentum logarithmo dato respondens computatur, error maximus ei eius variationi aequalis est, quae respondet variationi $\omega$ in logarithmo, si hic exacte datur, vel quae respondet variationi logarithmi $\omega+\omega^{\prime}$, si logarithmus ipse rsque ad $\omega^{\prime}$ erroneus esse potest. Vix opus erit monere, $\omega$ et $\omega^{\prime}$ eodem signo affici debere.

Si plures quantitates intra certos tantum limites exactae adduntur, aggregati error maximus aequalis erit aggregato singulorum errorum maximorum, uisdem signis affectorum; quare etiam in subtractione quantitatum proxime exactarum differentiae error maximus summae errorum singulorum maximorum aequalis crit. In multiplicatione vel dinisione quantitatis non absolnte exactae error maximus in eadem ratione augetur vel diminuitur vt quantitas ipsa.

\section{2.}

Progredimur iam ad applicationem hortum principiorum ad vtilissimas operationum supra explicatarum.

I. Adhibendo ad computum anomaliae verae ex anomalia excentrica in motu elliptico formilam VII art. 8, si $\varphi$ et $E$ exacte haberi supponuntur, in $\log \operatorname{tang}\left(45^{\circ}-\frac{\pi}{2} \varphi\right)$ et $\log \operatorname{tang} \frac{\pi}{2} E$ committi potest error $\omega$, adeoque in differentia $=\log \tan g \frac{x}{2} \nu$ error $2 \omega$; error maximus itaque in determinatione anguli $\frac{x}{2} v$ erit $\frac{3 \omega d \frac{x}{2} v}{d \log \operatorname{tang} \frac{\pi}{2} v}=$ $\frac{5 \omega \sin v}{2 \lambda}$, designante $\lambda$ modulum logarithmorum ad hunc calculum adhibitorum. Error itaque, cui anomalia vera $\nu$ obnoxia est, in secundis expressus fit $=$ $\frac{5 \omega \sin v}{\lambda}-206265=00712 \sin \%$, si logarithmi Briggici ad septem figuras decimales ad- 
hibentur, ita vt semper intra o" o7 de valore ipsius $v$ certi esse possimus : si tabulae minores ad quinque tantum figuras adhibentur, error vsque ad $7^{\prime \prime} 12$ ascendere posset.

II. Si $e \cos E$ adiumento logarithmorum computatur, error committi potest vsque ad $\frac{3 \omega e \cos E}{\lambda}$; eidem itaque errori obnoxia erit quantitas $1-e \cos E$ siue $\frac{r}{a}$. In computando ergo logarithmo huius quantitatis error vsque ad $(1+\delta) \omega$ ascendere potest, designando per $\delta$ quantitatem $\frac{3 e \cos E}{1-e \cos E}$ positiue sumtam: ad eundem limitem $(1+\delta) \omega$ ascendit exror in $\log r$ possibilis, siquidem $\log a$ exacte datus supponitur. Quoties excentricitas parua est, quantitas $\delta$ arctis semper limitibus coërcetur: quando vero $e$ partum differt ab $1, x-e \cos E$ perparua manet, quamdiu $E$ parua est; tunc igitur $\delta$ ad magnitudinem haud contemnendam increscere potest, quocirca in hoc casu formula III art. 8. minus idonea esset. Quantitas $\delta$ ita etiam exprimi potest $\frac{3(a-r)}{r}=\frac{3 e(\cos v+e)}{1-e e}$, quae formula adhuc clarius ostendit, quando errorem $(1+\delta) \omega$ contemnere liceat.

III. Adhibendo formulam $\mathbf{X}$ art. 8. ad computum anomaliac verae ex excentrica, $\log V \frac{a}{r}$ obnoxius exit errori $\left(\frac{\pi}{2}+\frac{T}{2} \delta\right) \omega ;$ adeoque $\log \sin \frac{\tau}{2} \varphi \sin E V \frac{a}{r}$ huic $\left(\frac{s}{2}+\frac{x}{2} \delta\right) \omega$; hinc error maximus in determinatione anguli $v-E$ vel $v$ possibilis eruitur $=\frac{\omega}{\lambda}(7+\delta)$ tang $\frac{\pi}{2}(\nu-E)$, siue in secundis expressus, si septem figurae decimales adhibentur, $=\left(0^{\prime \prime}, 66+0^{\prime \prime} 024 \delta\right) \operatorname{tang} \frac{\tau}{2}(\nu-E)$. Quoties excentricitas modica est, $\delta$ et $\operatorname{tang} \frac{x}{2}(\psi-E)$ quantitates paruae erunt, quapropter haec methodus praecisionem maiorem permittet, quam ea quam in I contemplati sumus: haecce contra methodus tunc praeferenda erit, quando excentricitas valde magna est propeque ad vnitatem accedit, vbi $\delta$ et $\operatorname{tang} \frac{x}{2}(v-E)$ valores valde considerabiles nancisci possunt. Per formulas nostras, vtra methodus alteri praeferenda sit, facile semper decidi poterit.

IV. In determinatione anomaliae mediae ex excentrica per formulam XII art. 8. error quantitatis $e \sin E$, adiumento logarithmorum computatae, adeoque etiam ipsius anomaliae $M$, rsque ad $\frac{3 \omega e \sin E}{\lambda}$ ascendere potest, qui erroris limes si in secundis expressus desideratur per $206265^{\prime \prime}$ est multiplicandus. Hinc facile concluditur, in problemate inuerso, vbi $E$ ex $M$ tentando determinatur, $E$ quantitate 
$\frac{3 \omega e \sin E}{\lambda} \cdot \frac{\mathrm{d} E}{\mathrm{~d} M} \cdot 206265^{\prime \prime}=\frac{3 \omega e a \sin E}{\lambda r} \cdot 206265^{\prime \prime}$ erroneam esse posse, etsiaequationi $E-e \sin E=I V$ omni quam tabulae permittunt praecisione satisfactum fuerit.

Anomalia vera itaque e media computata duabus rationibus erronea esse potest, siquidem mediam tamquam exacte datam consideramus, primo propter errorem in computo ipsius $\vartheta$ ex $E$ commissum, qui vt vidimus leuis semper momenti est, secundo ideo quod valor anomaliae excentricae ipse iam erroneus esse potuit. Effectus rationis posterioris definietur per productum erroris in $E$ commissi per $\frac{d v}{d E}$, quod productum fit $=\frac{3 \omega e \sin E}{\lambda} \cdot \frac{d v}{d M} \cdot 206265^{\prime \prime}=\frac{3 \omega e a \sin v}{\lambda r} \cdot 206265^{\prime \prime \prime}$ $=\left(\frac{e \sin v+\frac{x}{2} e e \sin 2 v}{x-e e}\right) 0^{\prime \prime} 0712$, si septem figurae adhibentur. Hic error, pro var loribus paruis ipsius e semper n̈odicus, pexmagnus euadene potest, quoties e abr vnitate parum differt, vti tabella sequens ostendit, quae pro quibusdam valoribus ipsius $e$ valorem maximum illius expressionis exhibet.

\begin{tabular}{c|c|c|c|c|c}
$e$ & error maximus & $e$ & error maximus & $e$ & error maximus \\
0,90 & $0^{\prime \prime} 42$ & 0,94 & $0^{\prime \prime} 73$ & 0,98 & $2^{\prime \prime 28}$ \\
0,91 & 0,48 & 0,95 & 0,89 & 0,99 & 4,59 \\
0,92 & 0,54 & 0,96 & 1,12 & 0,999 & 46,23 \\
0,93 & 0,62 & 0,97 & 1,50 & &
\end{tabular}

V. In motu hyperbolico, si v per formulam III art. 21 ex $F$ et $\psi$ exacte notis determinatur, error vsque ad $\frac{3 \omega \sin \vartheta}{\lambda} \cdot 206265^{\prime \prime}$ ascendere potest; si vero per formulam $\operatorname{tang} \frac{1}{2} \nu=\frac{(u-1) \operatorname{tang} \frac{1}{2} \psi}{u+1}$ computatur, $u$ et $\psi$ exacte notis, erroris limes triente maior erit, puta $=\frac{4 \omega \sin v}{\lambda} .206265^{\prime \prime}=0 " 00 \sin v$ pro septem figuris.

VI. Si per formulam XI art. 22 quantitas $\frac{2 k t}{b^{\frac{3}{2}}}=N$ adiumento logarithmorum Briggicorum computatur, $e$ et $u$ vel $e$ et $F$ tamquam exacte notas supponendo, pars prima obnoxia erit errori $\frac{5(u u-1) e \omega}{2 u}$, si computata est in forma $\frac{\lambda e(u-1)(u+1)}{2 u}$, vel exrori $\frac{3(u u+1) e \omega}{2 u}$, si computata est in forma $\frac{x}{2} \lambda e u$ 
$-\frac{\lambda e}{2 u}$, vel errori $3 e \omega \operatorname{tang} F$, sí computata est in forma $\lambda$ etang $F$, siquidem errorem in $\log \lambda$ vel $\log \frac{x}{2} \lambda$ commissum contemnimus. In casu primo error etiam per $5 e \omega \operatorname{tang} F$, in secundo per $\frac{5 e \omega}{\cos F}$ exprimi potest, vnde patet, in casu tertio errorem omnium semper minimum esse, in primo autem vel secundo maior erit, prout $u$ aut $\frac{1}{u}>2$ vel $<2$, sine prout $\pm F>36^{\circ} 52^{\prime}$ vel $<36^{\circ} 52^{\prime}$. Pars secunda ipsius $N$ autem semper obnoxia erit errori $\omega$.

VII. Vice versa patet, si $u$ vel $F$ ex $N$ tentando eruatur; $u$ obnoxiam fore errori $(1 \pm 5$ ew tang $F) \frac{\mathrm{d} u}{\mathrm{~d} N}$, vel huic $\left(1+\frac{5 e \omega}{\cos F}\right) \frac{\mathrm{d} u}{\mathrm{~d} N}$, prout membrum primum in valore ipsius $N$ vel in factores vel in partes resolutum adhibeatur; $F$ autem errori huic $(1 \pm 3 e \omega \tan g F) \frac{\mathrm{d} F}{\mathrm{~d} N}$. Signa superiora post perihelium, inferiora ante perihelium valent. Quodsi hic pro $\frac{\mathrm{d} u}{\mathrm{~d} N}$ rel pro $\frac{\mathrm{d} F}{\mathrm{~d} N}$ substituitur $\frac{\mathrm{d} v}{\mathrm{~d} N}$; emerget effectus huius erroris in determinationem ipsius $v$, qui igitur exit $\frac{b b \operatorname{tang} \psi(1+5 e \tan g F) \omega}{\lambda r r}$ ant $\frac{b b \operatorname{tang} \psi(1+5 e \sec F) \omega}{2 r r}$, si quantitas auxiliaris $u$ adhibita est; contra, si adhibita est $F$, ille effectus fit $=$ $\frac{b b \operatorname{tang} \psi(1+5 e \operatorname{tang} F) \omega}{2 r r}=\frac{\omega}{2}\left\{\frac{(1+e \cos \psi)^{2}}{\operatorname{tang} \psi^{3}}+\frac{5 e \sin \psi(1+e \cos \psi)}{\tan g \psi^{2}}\right\}$.

Adiicere oportet factorem $206265^{\prime \prime}$, si error in secundis exprimendus est. Manifesto hic error tunc tantum considerabilis enadere potest, quando $\psi$ est angulus parvus, siue e paullo maior quam 1 ; ecce valores maximos huius tertiae expressionis pro quibusdam valoribus ipsius $e$, si septem figurae decimales adhibentur:

\begin{tabular}{r|r}
$e$ & error maximus \\
1,3 & $0 " 34$ \\
1,2 & 0,54 \\
1,1 & 1,31 \\
1,05 & 5,05 \\
1,01 & 34,41 \\
1,001 & 1064,65
\end{tabular}


Huic errori ex erroneo valore ipsius $F$ vel $u$ orto adiicere oportet errorem in $\mathrm{V}$ determinatum, vt incertitudo totalis ipsius $v$ habeatur.

VIII. Si aequatio XI art. 22, adiumento logarithmorum hyperbolicorum solvitur, $F$ pro quantitate auxiliari achibita, effectus erroris in hac operatione possibilis in determinationem ipsius $v$ per similia ratiocinia inuenitur $=$

$$
\frac{(1+e \cos \psi)^{2} \omega^{\prime}}{\operatorname{tang} \psi^{3}} \pm \frac{3 e \sin \psi(1+e \cos \psi) \omega}{2 \operatorname{tang} \psi^{2}}
$$

vbi per $\omega^{\prime}$ incertitudinem maximam in tabulis logarithmorum hyperbolicorum designamus. Pars secunda huius expressionis identica est cum parte secunda cxpressionis in VII traditae, prima vero in ratione $2 \omega^{\prime}: \omega$ minor quam prima in illa expressione, i. e. in ratione $1: 23$, si tabulam Vrsini ad octo vbique figuras exactam sine $\omega^{\prime}=0,000000005$ supponére liceret.

33.

In is igitur sectionibus conicis, quarum excentricitas $a b$ vnitate parum differt, i. e. in ellipsibus et hyperbolis, quae ad parabolam proxime accedunt, methodi supra expositae tum pro determinatione anomaliae verae e tempore, tum pro determinatione temporis ex anomalia vera ${ }^{\star}$ ), omnem quae desiderari posset praecisionem non patiuntur: quin adeo errores ineuitabiles, crescentes dum orbita magis ad parabolae similitudinem vergit, tandem omnes limites egrederentur. Tabulae maiores ad plures quam septem figuras constructae hanc incertitudinem diminuerent quidem, sed non tollerent, nec impedirent, quominus omnes limites supcraret, simulac orbita ad parabolam nimis prope accederet. Praeterea methodi stipra traditae in hocce casu satis molestae fiunt, quoniam pars earum indirecta tentamina saepius repetitá requirit: cuius incommodi taedium vel grauius est, si tabulis maioribus operamur. Haud sane igitur superfluum erit, methodum peculiarem excolere, per quam in hoc casu incertitudinem illam euitare, sologue tabularum vulgarium adminiculo praecisionem sufficientem assequi liceat.

*) Quoniam tempus implicat factorem $a^{\frac{3}{2}}$ vel $b^{\frac{3}{2}}$, error in $M$ vel $N$ commissus eo magis angetur, quo maior fucrit $a=\frac{p}{x-e e}$, vel $b=\frac{p}{e e-1}$. 
34.

Methodus vulgaris, per quam istis incommodis remedium afferri solet, sequentibus principiis innititur. Respondeat in ellipsi vel hyperbola, cuius excentricitas $e$, semiparameter $p$ adeoque distantia in perihelio $=\frac{p}{1+e}=q$, tempori post perihelium $t$ anomalia vera $v$; respondeat porro eidem tempori in parabola, cuitus semiparameter $=2 q$, siue distantia in perihelio $=q$, anomalia vera $w$, massa $\mu$ vel vtrimque neglecta vel vtrimque aequali supposita. Tunc patet haberi

$$
\int \frac{p p d v}{(1+e \cos w)^{2}}: \int \frac{4 q q d w}{(1+\cos w)^{2}}=\sqrt{ } p: \sqrt{ } 2 q
$$

integralibus a $v=0$ et $w=0$ incipientibus, siue

$$
\int \frac{(1+e)^{\frac{3}{2}} d v}{(1+e \cos v)^{2} \sqrt{2}}=\int \frac{2 d w}{(1+\cos w)^{2}}
$$

Designando $\frac{1-e}{1+e}$ per $\alpha, \operatorname{tang} \frac{x}{2} \nu$ per $\theta$, integrale prius inuenitur $=$

$$
\sqrt{ }(1+\alpha) \cdot\left(\theta+\frac{r}{3} \theta^{3}(1-2 \alpha)-\frac{1}{5} \theta^{5}(2 \alpha-3 \alpha \alpha)+\frac{\pi}{7} \theta^{7}\left(3 \alpha \alpha-4 \alpha^{3}\right)-\text { etc. }\right)
$$

posterius $=\operatorname{tang} \frac{\pi}{2} w+\frac{\pi}{3} \operatorname{tang} \frac{\tau}{2} w^{3}$. Ex hac aequatione facile est determinare $w$ per $\alpha$ et $w$, atque $v$ per $\alpha$ et $w$, adiumento serierum infinitarum: pro $\alpha$ si magis placet introduci potest $1-e=\frac{2 \alpha}{1+\alpha}=\delta$. Quum manifesto pro $\alpha=0$ vel $\delta=0$ fiat $v=w$, hae series sequentem formam habebunt:

$$
\begin{aligned}
& w=v+\delta w^{\prime}+\delta \delta w^{\prime \prime}+\delta^{3} w^{\prime \prime}+\text { etc. } \\
& v=w+\delta w^{\prime}+\delta \delta w^{\prime \prime}+\delta^{3} w^{\prime \prime \prime}+\text { etc. }
\end{aligned}
$$

vbi $v^{\prime}, v^{\prime \prime}, v^{\prime \prime}$ etc. erunt functiones ipsius $v$, atque $w^{\prime}, w^{\prime \prime}, w^{\prime \prime}$ etc. functiones ipsius $w$. Quoties $\delta$ est quantitas perparua, hae series celeriter conuergent, paucique termini sufficient ad determinandum $w$ ex $v$, vel $v$ ex $w$. Ex $w$ inuenitur $t$, vel $w$ ex $t$ eo quem supra pro motu parabolico explicauimus modo.

\section{5.}

Expressiones analyticas trium coëfficientium primorum seriei secundae $w^{\prime}, w^{\prime \prime}, w^{q_{4}}$ Bessel noster euoluit, simulque pro valoribus numericis duorum primorum $w^{\prime}, w^{\prime \prime}$ tabulam ad singulos argumenti $w$ gradus constructam addidit (Von Zach Monatliche Correspondenz, vol. XII. p. 197.). Pro coëfficiente primo w' tabula iam ante ha- 
bebatur a Simpson computata, quae operi clar. Olbers supra laudato annexa est. In plerisque casibus hacce methodo adiumento tabulae Besselianae anomaliam veram e tempore praecisione sufficiente determinare licet: quod adhuc desiderandum relinquitur, ad haecce fere momenta reducitur:

I. In problemate inuerso, temporis puta ex anomalia vera determinatione ad methodum quasi indirectam confugere atque w ex $v$ tentando deriuare oportet. Cui incommodo vt obneniretur, series prior eodem modo tractata esse deberet ac secunda: et quum facile perspiciatur, - $v^{\prime}$ esse eandem functionem ipsius $v$, qualis $w^{\prime}$ est ipsius $w$; ita vt tabula pro $w^{\prime}$ signo tantum mutato pro $v^{\prime}$ inseruire possit, nihil iam requireretur nisi tabula pro $v^{\prime \prime}$, quo vtrumque problema aequali praecisione soluere liceat.

II. Interdum vtique occurrere possunt casus, vbi excentricitas ab vaitate parum quidem differt, ita vt methodi generales supra expositae praecisionem haud sufficientem dare videantur, nimis tamen etiamnum, quam vt in methodo peculiari modo adumbrata effectum potestatis tertiae ipsius $\delta$ altiorumque tuto contemnere liceat. In motu imprimis hyperbolico eiusmodi casus sunt possibiles, vbi, siue illas methodis adoptes siue hanc, errorem plurium secundorum euitare non possis, siquidem tabulis vulgaribus tantum ad septem figuras constructis vtaris. Etiamsi vero huiusmodi casus in praxi raro occurrant, aliquid certe deesse videri posset, si in omnibus casibus anomaliam veram intra $\mathrm{o}^{\prime \prime} 1$ aut saltem $\mathrm{o}_{2} 2$ determinare non liceret, nisi tabulae maiores consulerentur, quas tamen ad libros rariores referendas esse constat. Haud igitur prorsus superfluam visum iri speramus expositionem methodi peculiaris, qua iamdudum vsi sumus, quaeque eo etiam nomine se commendabit, quod ad excentricitates ab vnitate parum diuersas haud limitata est, sed hocce saltem respectu applicationem generalem patitur.

36.

Antequam hanc methodum exponere aggrediamur, obseruare conueniet, incertitudinem methodorum generalium supra traditarum in orbitis ad parabolae similitudinem vergentibus sponte desinere, simulac $E$ vel $F$ ad magnitudinem considerabilem increuerint, quod quidem in magnis demum a Sole distantiis fiet. Quod vt ostendamus, errorem maximum in ellipsi possibilem, quem in art. 32 , IV invenimus $\frac{3 \omega e a \sin \vartheta}{\lambda r} \cdot 206265^{\prime \prime}$ ita exhibemus $\frac{3 \omega e V(1-e e) \cdot \sin E}{2(1-e \cos E)^{2}} \cdot 206265^{\prime \prime}$, vnde 
sponte patet, errorem arctis semper limitibus circumscriptum esse, simulac $E$ valorem considerabilem acquisiuerit, siue simulac $\cos E$ ab vnitate magis recesserit, quantumuis magna sit excentricitas. Quod adhuc luculentius apparebit per tabulam. sequentem, in qua valorem numericum maximum istius formulae pro quibusdam valoribus determinatis computaumus (pro septem figuris decimalibus):

$$
\begin{array}{r|r}
E=10^{\circ} & \text { error maximus }= \\
20 & 5^{\prime \prime} 04 \\
30 & 0,76 \\
40 & 0,54 \\
50 & 0,19 \\
60 & 0,12 \\
& 0,08
\end{array}
$$

Simili modo res se habet in hyperbola, vt statim apparet, si expressio in art. 52. VII eruta sub hanc formam ponitur $\frac{\omega \cos F(\cos F+5 e \sin F) V(e e-1)}{\lambda(e-\cos F)^{2}} 206265^{\prime \prime}$.

Valores maximos huius expressionis pro quibusdam valoribus determinatis ipsius $F$ tabula sequens exhibet:

\begin{tabular}{|c|c|c|c|}
\hline$F$ & \multicolumn{2}{|c|}{$u$} & error maximus \\
\hline $10^{\circ}$ & 1,192 & 0,859 & $8^{\prime \prime} 66$ \\
20 & 1,428 & 0,700 & 1,58 \\
50 & 1,732 & 0,577 & 0,47 \\
40 & 2,144 & 0,466 & 0,22 \\
50 & 2,747 & 0,564 & 0,11 \\
60 & 3,732 & 0,268 & 0,06 \\
70 & 5,671 & 0,176 & 0,02 \\
\hline
\end{tabular}

Quoties itaque $\boldsymbol{E}$ vel $F$ vltra $40^{\circ}$ vel $50^{\circ}$ egreditur (qui tamen casus in orbitis a parabola parum discrepantibus hand facile occurret, quum corpora coelestia in talibus orbitis incedentia in tantis a Sole distantiis oculis nostris plerumque se subducant), nulla aderit ratio, cur methodum generalem deseramus. Ceterum in tali casu etiam series de quibus in art. 34. egimus nimis lente conuergerent: neutiquam igitur pro defectu methodi nunc explicandae haberi potest, quod is imprimis casibus adaptata est, vbi $E$ vel $F$ vltra valores modicos nondum excrenit. 


\section{7.}

Resumamus in motu elliptico aequationem inter anomaliam cxcentricam et tempus

$$
E-e \sin E=\frac{k t V(1+\mu)}{a^{\frac{3}{2}}}
$$

vbi $E$ in partibus radii expressam supponinus. Factorem $V(1+\mu)$ abhinc omittemus; si vmquam casus occurreret, vbi eius rationem habere in potestate operaeque pretium esset, signum $t$ non tempus ipsum post perihelium, sed hoc tempus per $V(1+\mu)$ multiplicatum exprimere deberet. Designamus porro per $q$ distantiam in perihelio, et pro $E$ et $\sin E$ introducimus quantitates $E-\sin E$ et $E-\frac{x}{x^{\circ}}(E-\sin E)=$ $\frac{9}{50} E+\frac{r}{50} \sin E$ : rationem cur has potissimum eligamus lector attentus ex sequentibus sponte deprehendet. Hoc modo aequatio nostra formam sequentem induit:

$$
(1-e)\left(\frac{9}{10} E+\frac{x}{x} \sin E\right)+\left(\frac{x}{x}+\frac{0}{x} e\right)(E-\sin E)=k t\left(\frac{1-e}{q}\right)^{\frac{a}{2}}
$$

Quatenus $E$ vt quantitas parua ordinis primi spectatur, erit $\frac{9}{10} \boldsymbol{E}+\frac{1}{x_{0}} \sin E=E-$

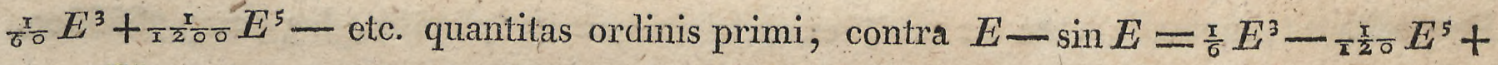
$5 \frac{x}{0}-E^{7}$ - etc. quantitas ordinis tertii. Statuendo itaque

$$
\frac{6(E-\sin E)}{\frac{9}{10} E+\frac{T}{10} \sin E}=4 A, \frac{\frac{9}{10} E+\frac{x}{10} \sin E}{2 \mathscr{V} A}=B
$$

erit $4 A=E^{2}-\frac{r}{30} E^{4}-\frac{r}{50} E^{6}-$ etc. quantitas ordinis secundi, atque $B=$ $1+\frac{3}{200} E^{4}-$ etc. ab vnitate quantitate quarti ordinis diuersa. Aequatio nostra autem hinc fit

$$
B\left(2(1-e) A^{\frac{x}{2}}+\frac{2}{x^{5}}(1+9 e) A^{\frac{y}{2}}\right)=k t\left(\frac{1-e}{q}\right)^{\frac{8}{2}}
$$

Per tabulas vulgares trigonometricas $\frac{9}{x^{\circ}} E+\frac{x}{x^{\circ} \circ} \sin E$ quidem praecisione sufficiente calculari potest, non tamen $E-\sin E$, quoties $E$ est angulus paruus: hacce igitur via quantitates $A$ et $B$ satis exacte determinare non liceret. Huic autem difficultati remedium afferret tabula peculiaris, ex qua cum argumento $E$ aut ipsum $B$ aut logarithmum ipsius $B$ excerpere possemus: subsidia ad constructionem talis tabulae necessaria cuique in analysi vel mediocriter versato facile se offerent. Adiumento aequationis

$$
\frac{9 E+\sin E}{20 B}=V A
$$

etiam $\vee A$, atque hine $t$ per formulam $[1]$ omni quae desiderari potest praecisione determinare liceret. 
Ecce specimen talis tabulae, quod saltem lentam augmentationem ipsius $\log B$ manifestabit: superfluum esset, hanc tabulam maiori extensione elaborare, infra enim tabulas formae multo commodioris descripturi sumus:

\begin{tabular}{|r|r|r|r|r|r|}
\hline$E$ & \multicolumn{1}{|r|}{$\log B$} & $E$ & $\log B$ & $E$ & $\log B$ \\
\hline $0^{\circ}$ & 0,0000000 & $25^{\circ}$ & 0,0000168 & $50^{\circ}$ & 0,0002675 \\
5 & 00 & 50 & 0549 & 55 & 5910 \\
10 & 04 & 35 & 0645 & 60 & 5526 \\
15 & 22 & 40 & 1099 & & \\
20 & 69 & 45 & 1758 & & \\
\hline
\end{tabular}

38.

Haud inutile erit, ea quae in art. praec. sunt tradita exemplo illustrare. Proposita sit anomalia vera $=100^{\circ}$, excentricitas $=0,96764567, \log q=9,7656500$. Ecce iam calculum pro $E, B, A$ et $t$ :

$\log \operatorname{tang} \frac{x}{2}, y \ldots \ldots \ldots \ldots . . . . .0,0761865$

$\log V \frac{1-e}{1+e} \ldots \ldots \ldots . . . .9,1079927$ $\overline{\log \tan g \frac{x}{2} E \ldots \ldots \ldots \ldots . . . . .9}, \overline{1841792}$, vade $\frac{x}{2} E=8^{\circ} 41^{\prime} 19^{\prime \prime} 32$, atque $E=17^{\circ} 22^{\prime} 38^{\prime \prime} 64$. Huic valori ipsius $E$ respondet $\log B=0,0000040$; porro inuenitur in partibus radii $E=0,3032928, \sin E=0,2986643$, vnde $\frac{9}{20} E+\frac{x}{20} \sin E=0,1514150$, cuius logarithmus $=9,1801689$, adeoque $\log A^{\frac{1}{2}}=9,1801649$. Hinc deducitur per formulam [1] art. praec.

$$
\begin{array}{ll}
\log \frac{2 B q^{\frac{3}{2}}}{k V(1-e)} \ldots \ldots . . .2,4589614 & \log \frac{2 B(1+9 e)}{15 k}\left(\frac{q}{1-e}\right)^{\frac{3}{2}} \ldots \ldots . .5,7601058 \\
\frac{\log A^{\frac{1}{2}} \ldots \ldots \ldots \ldots \ldots \ldots \ldots ., 1801649}{\log 45,56386}=\frac{\log A^{\frac{3}{2}} \ldots \ldots \ldots \ldots \ldots \ldots \ldots \ldots \ldots \ldots \ldots .7,5404947}{1,6591265} & \log 19,98014 \\
\frac{19,98014}{65,54400}=t &
\end{array}
$$

Tractando idem exemplum secundum methodum vulgarem, inuenitur $e \sin E$ in secundis $=59610^{\prime \prime} 79=16^{\circ} 33^{\prime} 30^{\prime \prime} 79$, vnde anomalia media $=49^{\prime} 7^{\prime \prime} 85=2947^{\prime \prime} 85$. Hinc et ex $\log k\left(\frac{1-e}{q}\right)^{\frac{3}{2}}=1,6664302$ deriuatur $t=63,54410$. Differentia, 
quae hic tantum est го $\frac{x}{0}$ б vel quadruplo maior euadere potuisset.

Ceterum patet, solo adiumento talis tabulae pro $\log B$ etiam problema inversum omni praecisione solui posse, determinando $E$ per tentamina repetita, ita vt valor ipsius $t$ inde calculatus cum proposito congruat. Sed haec operatio satis molesta foret: quamobrem iam ostendemus, quomodo tabulam auxiliarem multo commodius adornare, tentamina vaga omnino euitare, totumque calculum ad algorithmum maxime concinnum atque expeditum reducere liceat, qui nihil desiderandum relinquere videtur.

\section{9.}

Dimidiam fere partem laboris quem illa tentamina requirerent abscindi posse statim obuium est, si tabula ita adornata habeatur, ex qua $\log B$ immediate argumento $A$ desumere liceat. Tres tunc superessent operationes; prima indirecta, puta determinatio ipsius $A$, vt aequationi [1] art. 37 satisfiat; secunda, determinatio ipsius $E$ ex $A$ et $B$, quae fit directe vel per aequationem $E={ }_{2} B\left(A^{\frac{1}{2}}+\frac{x}{x^{\frac{x}{5}}} A^{\frac{3}{2}}\right)$, vel per hanc $\sin E=2 B\left(A^{\frac{x}{2}}-\frac{3}{5} A^{\frac{3}{2}}\right)$; tertia, determinatio ipsius $»$ ex $E$ pér aequ. VII. art. 8. Operationem primam ad algorithmum expeditum et a tentaminibus vagis liberum reducemus; secundam et tertiam vero in vnicam contrahemus, tabulae nostrae quantitatem nouam $C$ inserendo, quo pacto ipsa $E$ omnino opus non habebimus, simulque pro radio vectore formulam elegantem et commodam nanciscemur. Quae singula ordine suo iam persequemur.

Primo aequationem [1] ita transformabimus, vt tabulam Barkerianam ad eius solutionem adhibere liceat. Statuemus ad hunc finem $A^{\frac{x}{2}}=\operatorname{tang} \frac{x}{2} w V \frac{5-5 e}{1+9 e}$, vnde fit $75 \operatorname{tang} \frac{x}{2} w+25 \operatorname{tang} \frac{x}{2} w^{3}=\frac{75 k t V\left(\frac{x}{3}+\frac{9}{5} e\right)}{2 B q^{\frac{3}{2}}}=\frac{\alpha t}{B}$ designando constantem $\frac{75 k \sqrt{ }\left(\frac{x}{5}+\frac{9}{5} e\right)}{2 q^{\frac{3}{2}}}$ per $\alpha$. Si itaque $B$ esset cognita, w illico e tabula Barkeriana desumi posset, vbi est anomalia vera, cui respondet motus medius $\frac{\alpha t}{B}$; ex $w$ deriuabitur $A$ per formulam $A=\beta \operatorname{tang} \frac{x}{2} w^{2}$, designando constantem $\frac{5-5 e}{1+9 e}$ per $\beta$. Iam etsi $B$ demum ex $A$ per tabulam nostram auxilia- 
rem innotescat, tamen propter perparuam ipsius ab vnitate differentiam praeuidere licet, $w$ et $A$ leui tantum errore affectas prouenire posse, si ab initio diuisor $B$ ominino negligatur. Determinabimus itaque primo, leui tantum calamo, $w$ et $A$, statuendo $B=1$; cum valore approximato ipsius $A$ e tabula nostra auxiliari inueniemus ipsam $B$, cum qua eundem calculum exactius repetemus; plerumque respondebit valori sic correcto ipsius $A$ prorsus idem valor ipsius $B$, qui exproximato inuentus erat, ita vt noua operationis repetitio superflua sit, talibus casibus exeeptis, vbi valor ipsius $E$ iam valde considerabilis fuerit. Ceterum vix opus erit monere, si forte iam ab initio valor ipsins $B$ quomodocunque approximatus aliunde innotuerit (quod sempér fiet, quoties e pluribus locis haud multum ab inuicem di- stantibus computandis, vnus aut alter iam sunt absoluti). praestare, hoc staim in prima approximatione vti: hoc modo calculator scitus sacpissime ne vna quidem calculi repetitione opus habebit. Hanc celerrimam approximationem inde assecuti sumus, quod $B$ ab 1 differentia ordinis quarti tantum distat, in coëficientem perparuum numericum insuper multiplicata, quod commodum praeparatum esse iam perspicietur per introductionem quantitatum $E-\sin E, \frac{9}{T} E+\frac{x}{x} \sin E$ loco ipsarum $E, \sin E$.

40.

Quum ad operationem tertiam, puta determinationem anomaliae verae, angulus $E$ ipse non requiratur, sed tantum $\tan g \frac{x}{2} E$ siue potius $\log \operatorname{tang} \frac{x}{2} E$, operatio illa cum. secunda commode iungi posset, si tabula nostra immediate suppeditaret logarithmum quantitatis $\frac{\operatorname{tang} \frac{1}{2} E}{\sqrt{ } A}$, quae ab 1 quantitate ordinis secundi differt. Maluimus tamen tabulam nostram modo aliquantulum dinerso adornare, quo extensione minuta nihilominus interpolationem multo commodiorem assecuti sumus. Scribendo breuitatis gratia $T$ pro tang $\frac{1}{2} E^{2}$, valor ipsius $A$ in art. 57 traditus $\frac{15(E-\sin E)}{9 E+\sin E}$ facile transmutatur in

$$
A=\frac{T-\frac{6}{5} T^{2}+\frac{9}{7} T^{3}-\frac{12}{9} T^{4}+\frac{\pi 5}{1} T^{5}-\text { otc. }}{1-\frac{6}{1} T+\frac{7}{2} T^{2}-\frac{8}{3} T^{3}+\frac{9}{4} T^{4}-\text { etc. }}
$$

vbi lex progressionis obuia est. Hinc deducitur per conuersionem serierun

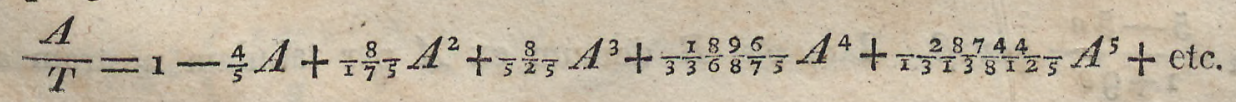


Statuendo igitur $\frac{A}{T}=1-\frac{a}{5} A+C$, erit $C$ quantitas ordinis quarti, qua in tabulam nostram recepta, ab $A$ protinus transire possumus ad $\%$ per formulam

$$
\operatorname{tang} \frac{x}{2} v=V \frac{1+e}{1-e} \cdot \sqrt{\frac{A}{1-\frac{4}{5} A+C}}=\frac{\gamma \operatorname{tang} \frac{1}{2} w}{\sqrt{\left(1-\frac{4}{5} A+C\right)}}
$$

designando per $\gamma$ constantem $\vee \frac{5+5 e}{1+9 e}$. Hoc modo simul lucramir calculum percommodum pro radio vectore. Fit enim (art. 8, VI)

$$
r=\frac{q \cos \frac{x}{2} E^{2}}{\cos \frac{x}{2} v^{2}}=\frac{q}{(1+T) \cos \frac{\pi}{2} v^{2}}=\frac{\left(1-\frac{4}{5} A+C\right) q}{\left(1+\frac{\pi}{5} A+C\right) \cos \frac{x}{2} v^{2}}
$$

41.

Nihil iam superest, nisi vt etiam problema inuersum, puta determinationem temporis ex anomalia vera, ad algorithmum expeditiorem reducamus: ad hunc finem tabulae nostrae columnam nouam pro $T$ adiecimus. Computabitur itaque primo $T$ ex $v$ per formulam $T=\frac{1-e}{1+e} \operatorname{tang} \frac{x}{2} v^{2}$; dein ex tabula nostra argumento $T$ desumetur $A$ et $\log B$, siue (quod exactius, imo etiam commodius est) $C$ et $\log B$, atque hinc $A$ per formulam $A=\frac{(1+C) T}{1+\frac{4}{5} T} ; \operatorname{tandem}$ ex $A$ et $B$ erretur $t$ per formulam [1] art. 37. Quodsi hic quoque tabulam Barkerianam in visum rocare placet, quod tamen in hoc problemate inuerso calculum minus subleuat, non opus est ad $A$ respicere, sed statim habetur

$$
\operatorname{tang} \frac{x}{2} w=\operatorname{tang} \frac{x}{2} v V \frac{1+C}{\gamma\left(1+\frac{4}{5} T\right)}
$$

atque hinc tempus $t$, multiplicando motum medium anomaliae verae $w$ in tabula Barkeriana respondentem per $\frac{B}{\alpha}$.

42.

Tabulam, qualem hactenus descripsimus, extensione idonea construximus, operique huic adiecimus (Tab. I.). Ad ellipsin sola pars prior spectat; partem alteram, quae motum hyperbolicum complectitur, infra explicabimus. Argumentum tabulae, quod est quantitas $A$, per singulas partes millesimas a o rsque ad 0,500 progreditur; sequuntur $\log B$ et $C$, quas quantitates in partibus $10000000^{\text {mis }}$, siue 
ad septem figuras decimales expressas subintelligere oportet ; cifrae enim primae, figuris significatiuis praeeuntes, suppressae sunt; columna denique quarta exhibet quantitatem $T$ primo ad 5 dein ad 6 figuras computatam, quae praecisio abunde sufficit, quxm haec columna ad eum tantummodo vsum requiratur, vt argumento $T$ valores respondentes ipsius $\log B$ et $C$ habeantur, quoties ad normam art. praec. $t$ ex $v$ determinare lubet. Quum problema inuersum, quod longe frequentioris vsus est, puta determinatio ipsius $v$ et $r$ ex $t$, omnino absque quantitatis $T$ subsidio absoluatur, quantitatem $A$ pro argumento tabulae nostrae eligere maluimus quam $T$, quae alioquin argumentum aeque fere idoneum fuisset, imo tabulae constructionem aliquantulum facilitauisset. Haud superfluum erit monere, omnes tabulae numeros ad decem figuras ab origine calculatos fuisse, septemque adeo figuris, quas hic damus, vbique tuto confidere licere; methodis autem analyticis ad hunc laborem in vsum vocatis hoc loco immǫrari non possumus, quarum explicatione copiosa nimium ab instituto nostro distraheremur. Ceterum tabulae extensio omnibus casibús, vbi methodum hactenus expositam sequi prodest, abunde sufficit, quum vltra limitem $A=0,3$, cui respondet $T=0,39_{2} 37^{4}$ siue $E=64^{\circ} 7^{\prime}$, methodis artificialibus commode vt supra ostensum est abstinere liceat.

\section{3.}

Ad maiorem disquisitionum praecedentium illustrationem exemplum calculi completi pro anomalia vera et radio vectore ex tempore adiicimus, ad quem finem numeros art. 38. resumemus. Statuimus itaque $e=0,96764567, \log q=9,7656500$, $t=65,54400$, vnde primo deducimus constantes $\log \alpha=0,5052557, \log \beta=8,2217564$, $\log \gamma=0,0028755$.

Hinc fit $\log \alpha t=2,1085102$, cui respondet in tabula Barkeri valor approximatus ipsius $w=99^{\circ} 6^{\prime}$, vnde deriuatur $A=0,229^{2} 6$, et ex tabula nostra $\log B=$ 0,0000040 . Hinc argumentum correctum quo tabulam Barkeri intrare oportet fit $=$ $\log \frac{\alpha t}{B}=2,1083062$, cui respondet $w=99^{\circ} 6^{\prime} 13^{\prime \prime} 14 ;$ dein calculus vlterior ita se habet: 
RELATIONES AD LOCVM SIMPLICEM IN ORBITA SPECTANTES.

$$
\begin{gathered}
\log \tan g \frac{x}{2} w^{2} \ldots \ldots .0,1585954 \\
\log \beta \ldots \ldots \ldots \ldots \ldots . \ldots .2217564 \\
\log A \ldots \ldots \ldots \ldots . .8,3603298 \\
A=\quad 0,02292608
\end{gathered}
$$

hinc $\log B$ perinde vt ante;

$$
C=0,0000242
$$

$$
1-\frac{4}{5} A+C=0,9816833
$$$$
1+\frac{x}{5} A+C=1,0046094
$$

$\log \operatorname{tang} \frac{\pi}{2} w \ldots \ldots \ldots \ldots \ldots \ldots \ldots . . . \ldots, 0692967$

$\log \gamma \ldots \ldots \ldots \ldots \ldots \ldots \ldots \ldots \ldots . . . . . . . . . . . . . . . .028755$

$\frac{\pi}{2}$ Comp. $\log 1-\frac{4}{3} A+C \ldots \ldots .0,0040143$

$\log \operatorname{tang} \frac{T}{2} p \ldots \ldots \ldots \ldots \ldots \ldots \ldots . \ldots, 0761865$

$$
\begin{aligned}
\frac{1}{2} v & =50^{\circ} 0^{\prime} 0^{\prime \prime} \\
v & =10000
\end{aligned}
$$

$\log q \ldots \ldots \ldots \ldots \ldots \ldots \ldots \ldots \ldots . \ldots . \ldots . \ldots . \ldots . \ldots, 656500$

2 Comp. $\log \cos \frac{x}{2} \mathfrak{v} \ldots \ldots \ldots \ldots \ldots . . . . .0,5858650$

$\log 1-\frac{4}{5} A+C$...................9,9919714

C. $\log 1+\frac{\mathrm{r}}{5} A+C \ldots \ldots \ldots \ldots \ldots . . .9,9980028$

$\overline{\log r \ldots \ldots \ldots \ldots \ldots \ldots \ldots \ldots \ldots . . . . . . . . \ldots, 159489^{2}}$

Si in hoc calculo factor $B$ omnino esset neglectus, anomalia vera errorusculo o" 1 tantum (in excessu) prodiisset affecta:

\section{4.}

Motum hyperbolicum eo breuius absoluere licebit, quoniam methodo ei quam hactenus pro motu elliptico exposuimus prorsus analoga tractandus est. Aequationem inter tempus $t$ atque quantitatem anxiliarem $u$ forma sequente exhibemus:

$(e-1)\left(\frac{x}{20}\left(u-\frac{1}{u}\right)+\frac{9}{x O} \log u\right)+\left(\frac{x}{10}+\frac{9}{10} e\right)\left(\frac{x}{2}\left(u-\frac{1}{u}\right)-\log u\right)=k t\left(\frac{e-1}{q}\right)^{\frac{5}{2}}$

vbi logarithmi sunt hyperbolici, atque $\frac{x}{20}\left(u-\frac{1}{u}\right)+\frac{9}{\frac{1}{3}} \log u$ quantitas ordinis primi, $\frac{x}{2}\left(u-\frac{x}{u}\right)-\log u$ quantitas ordinis tertii, simulac $\log u$ tamquam quantitas parua ordinis primi spectatur. Statuendo itaque

$$
\frac{6\left(\frac{x}{2}\left(u-\frac{1}{u}\right)-\log u\right)}{\frac{x}{2} 0\left(u-\frac{1}{u}\right)+\frac{9}{50} \log u}=4 A, \frac{\frac{x}{0}\left(u-\frac{1}{u}\right)+\frac{9}{50} \log u}{2 \sqrt{ } A}=B
$$

erit $A$ quantitas ordinis secundi, $B$ autem ab vnitate differentia ordinis quarti discrepabit. Aequatio nostra tunc formam sequentem induet:

$$
B\left(2(e-1) A^{\frac{\pi}{2}}+\frac{2}{x^{5}}(1+9 e) A^{\frac{3}{2}}\right)=k\left(\frac{e-1}{q}\right)^{\frac{3}{2}}
$$

quae aequationi $[1]$ art. 37 prorsus analoga est. Statuendo porro $\left(\frac{u-1}{u+1}\right)^{2}=T$, 
erit $T$ ordinis secundi, et per methodum serierum infinitarum inuenietur

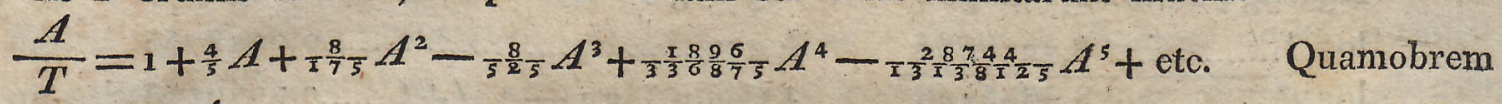
ponendo $\frac{A}{T}=1+\frac{4}{5} A+C$, erit $C$ quantitas ordinis quarti, atque $A=\frac{(1+C) T}{1-\frac{4}{5} T}$. Denique pro radio vectore ex aequ. VII art. 21 facile sequitur

$$
r=\frac{q}{(1-T) \cos \frac{1}{2} \nu^{2}}=\frac{\left(1+\frac{4}{5} A+C\right) q}{\left(1-\frac{2}{5} A+C\right) \cos \frac{1}{2} v^{2}}
$$

45.

Pars posterior tabulae primae operi huic annexae ad motum hyperbolicum spectat, vt iam supra monuimus, et pro argumento $A$ (vtrique tabulae parti communi) logarithmum ipsius $B$ atque quantitatem $C$ ad septem figuras decimales (cifris praecedentibus omissis), quantitatem $T$ vero ad quinque dein ad sex figuras sistit. Extensa est haec pars, perinde vt prior, vsque ad $A=0,500$, cui respondet $T=0,241207, u=2,930$ vel $=0,341, F= \pm 52^{\circ} 19^{\prime} ;$ vlterior extensio superflua fuisset (art. 36.)

Ecce iam ordinem calculi tum pro determinatione temporis ex anomalia vera tum pro determinatione anomaliae verae ex tempore. In problemate priori habebitur $T$ per formulam $T=\frac{e-1}{e+1} \operatorname{tang} \frac{x}{2} v^{2} ;$ ex $T$ tabula nostra dabit $\log B$ et $C$, vnde erit $A=\frac{(1+C) T}{1-\frac{4}{5} T}$; hinc tandem per formulam [2] art. praec. inuenietur $t$. In problemate posteriori computabuntur primo logarithmi constantium

$$
\begin{aligned}
& \alpha=\frac{75 k V\left(\frac{x}{5}+\frac{9}{5} e\right)}{2 q^{\frac{3}{2}}} \\
& \beta=\frac{5 e-5}{1+9 e} \\
& \gamma=\sqrt{ } \frac{5 e+5}{1+9 e}
\end{aligned}
$$

Tunc determinabitur $A$ ex $t$ prorsus eodem modo vt in motu elliptico, ita scilicet vt motui medio $\frac{a t}{B}$ in tabula Barkeri respondeat anomalia vera $w$ atque fiat $A=$ $\beta \operatorname{tang} \frac{\pi}{2} w^{2}$; eruetur scilicet primo valor approximatus ipsius $A$ neglecto vel si subsidia adsunt aestimato factore $B$; hinc tabula nostra suppeditabit valorem approximatum ipsius $B$, cum quo operatio repetetur; valor nouus ipsius $B$ hoc modo prodiens vix rmquam correctionem sensibilem passus, neque adeo noua calculi itera- 
tio necessaria erit. Correcto valore ipsius $A$ e tabula desumetur $C$, quo facto habebitur

$$
\operatorname{tang} \frac{\pi}{2} w=\frac{\gamma \operatorname{tang} \frac{x}{2} w}{\sqrt{\left(1+\frac{4}{5} A+C\right)}}, r=\frac{\left(1+\frac{4}{5} A+C\right) q}{\left(1-\frac{\pi}{5} A+C\right) \cos \frac{x}{2} w^{2}}
$$

Patet hinc, inter formulas pro motu elliptico et hyperbolico nullam omnino differentiam reperiri, si modo $\beta, A$ et $T$ in motu hyperbolico tamquam quantitates negatiuas tractemus.

\section{6.}

Motam hyperbolicum quoque aliquot exemplis illustrauisse haud inutile erit, ad quem finem numeros arit. 25,26 resumemus.

I. Data sunt $e=1,2618820, \log q=0,0201657, v=18^{\circ} 5 \mathrm{r}^{\prime} 0^{\prime \prime}:$ quaeritur $t$. Habemus

$$
\begin{aligned}
& 2 \log \operatorname{tang} \frac{T}{2} y \ldots \ldots 8,4402018 \quad \log T \ldots \ldots \ldots \ldots \ldots \ldots .7,5058375
\end{aligned}
$$

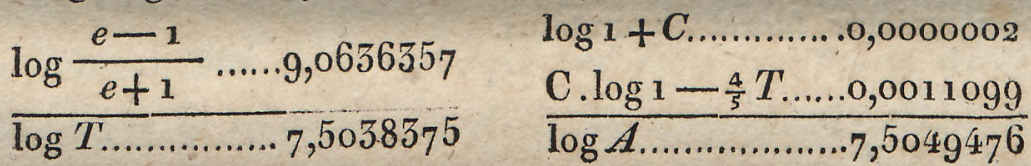

$$
\begin{aligned}
& T=0,00319034 \\
& \log B=0,0000001 \\
& C=0,0000005 \\
& \log \frac{2 B q^{\frac{3}{2}}}{k \sqrt{(e-1)}} \cdots . .2,5866444 \\
& \log \frac{2 B(1+9 e)}{15 k}\left(\frac{q}{e-1}\right)^{\frac{3}{2}} \ldots \ldots .2,8845582 \\
& \frac{\log A^{\frac{3}{2}} \ldots \ldots \ldots \ldots \ldots \ldots \ldots \ldots \ldots \ldots \ldots, \ldots \ldots \ldots \ldots \ldots \ldots}{\log 0,158605}=\ldots \\
& \frac{0,15861}{13,91445}=t
\end{aligned}
$$$$
\frac{\log A^{\frac{\pi}{2}} \ldots \ldots \ldots \ldots \ldots . . .1,7524758}{\log 15,77584=1,1591182}
$$

II. Manentibus $e$ et $q$ vt ante, datur $t=65,41236$, quaeruntur $p$ et $s$. Inuenimus logarithmos constantium

$$
\begin{aligned}
& \log \alpha=9,9758345 \\
& \log \beta=9,0251649 \\
& \log \gamma=9,9807646
\end{aligned}
$$

Porro prodit $\log \alpha t=1,7914943$, vnde per tabulam Barkeri valor approximatus ipsius $w=70^{\circ} 31^{\prime} 44^{\prime \prime}$, atque hinc $A=0,052983$. Huic $A$ in tabula nostra respondet $\log B=0,0000207 ;$ vnde $\log \frac{\alpha t}{B}=1,7914756$, valor correctus ipsius $w=70^{\circ} 31^{\prime} 56^{\prime \prime} 86$. Calculi operationes reliquae ita se habent: 


$$
\begin{aligned}
& 2 \log \operatorname{tang} \frac{x}{2} w \ldots . . .9,6989398 \quad \log \operatorname{tang} \frac{x}{2} w \ldots \ldots \ldots \ldots \ldots . . \ldots, 8494699
\end{aligned}
$$

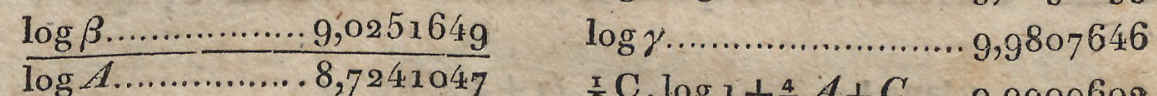

$$
\begin{aligned}
& A=\begin{array}{rr}
0,05297911 & \frac{\mathrm{T}}{2} \mathrm{C} \cdot \log 1+\frac{4}{5} A+C \ldots \ldots 9,9909602 \\
\log \operatorname{tang} \frac{x}{2} \psi \ldots \ldots \ldots \ldots \ldots . . \ldots, 8211947
\end{array} \\
& \log B \text { vt ante } \quad \frac{r}{2} \nu=33^{\circ} 3 \mathrm{i}^{\prime} 3 \mathrm{o}^{\prime \prime} 02 \\
& \bar{C}=0,0001252 \quad v=6730,04 \\
& 1+\frac{4}{5} A+C=\quad 1,0425085 \quad \log q \ldots \ldots \ldots \ldots \ldots \ldots \ldots \ldots . . \ldots, 0201657 \\
& \text {. } 1-\frac{\pi}{5} A+C=0,9895294 \quad 2 C \cdot \log \cos \frac{\pi}{2} \psi \ldots \ldots \ldots \ldots . . .0,1580578 \\
& \log 1+\frac{4}{5} A+C \ldots \ldots \ldots \ldots .0,018079^{6} \\
& \frac{\mathrm{C} . \log 1-\frac{\mathrm{T}}{3} A+C \ldots \ldots .0,0045713}{\log r \ldots \ldots \ldots \ldots \ldots \ldots \ldots \ldots . .0,2008544}
\end{aligned}
$$

Quae supra (art. 26.) inueneramus $v=67^{\circ} 2^{\prime} 59^{\prime \prime} 78, \log r=0,2008541$, minus exacta sunt, proprieque euadere debuisset $v=67^{\circ} 3^{\prime} 0^{\prime \prime} 00$, quo valore supposito valor ipsius $t$ per tabulas maiores fuerat computatus. 


\section{SECTIO SECVNDA}

\section{Relationes ad locum simplicem in spatio spectantes.}

47.

In sectione prima de motu corporum coelestium in orbitis suis actum est, nulla situs, quem hae orbitae in spatio occupant, ratione habita. Ad hunc situm determinandum, quo relationem locorum corporis coelestis ad quaeuis alia spatii puncta assignare liceat, manifesto requiritur tum situs plani in quo orbita iacet respectu cuiusdam plani cogniti (e. g. plani orbitae telluris, eclipticae), tum situs apsidum in illo plano. Quae quum commodissime ad trigonometriam sphaericam referantur, superficiem sphacricam radio arbitrario circa Solem vt centrum descriptam fingimus, in qua quoduis planum per Solem transiens circulum maximum, quaeuis autem recta e Sole ducta punctum depinget. Planis aut rectis per Solem ipsum non transeuntibus plana rectasque parallelas per Solem ducimus, circulosque maximos et puncta in sphaerae superficie his respondentia etiam illa repraesentare concipimus: potest quoque sphaera radio vt vocant infinito magno descripta supponi, in qua plana rectaeque parallelae perinde repraesentantur.

Nisi itaque planum orbitae cum plano eclipticae coincidit, circuli maximi illis planis respondentes (quos etiam simpliciter orbitam et ecliptican rocabimus) duobus punctis se intersecant, quae nodi dicuntur; in nodorum altero corpus e Sole visum e regione australi per eclipticam in borealem transibit, in altero ex hac in illam reuertet; nodus prior ascendens, posterior descendens appellatur. Nodorum situs in ecliptica per eorum distantiam ab aequinoctio vernali medio (longitudinem) secundum ordinem signorum numeratam assignamus. Sit, in Fig. $1, \delta$ nodus ascendens, $A \delta B$ pars eclipticae, $C \delta D$ pars orbitae; motus terrae et corporis coelestis fiant in directionibus ab $A$ versus $B$ et a $C$ versus $D$, patetque angulum sphaericum, quem $\delta D$ facit cum $\delta B$, a o vsque ad $180^{\circ}$ crescere posse, neque tamen vltra, quin $\delta$ nodus ascendens esse desinat: hunc angulum inclinationem orbilcue ad eclipticam dicimas. Situ plani orbitae per longitudinem nodi atque inclinationem orbitae determinato, nihil aliud iam requiritur, nisi distantia perihelii a nodo ascendente, quam secundum ipsam directionem molus numeramus, adeoque negatiuam siue inter $180^{\circ}$ et $560^{\circ}$ assumimus, quoties perihelium ab ecliptica ad austrum situm est. Notentur adhuc expressiones sequentes. Longitudo cuiusuis puncti in circulo orbitae numeratur ab eo puncto, quod retrorsum a nodo ascen- 
dente in orbita tantundem distat, quantum aequinoctium vernale ab eodem puncto retrorsum in ecliptica: hinc longitudo perihelii erit summa longitudinis nodi et distantiae periheli a nodo; longitudo verc corporis in orbita autem summa anomaliae verae et longitudinis perihelii. Denique longitudo media vocatur summa anomaliae mediae et longitudinis perihelii: haec postrema expressio manifesto in orbitis ellipticis tantum locum habere potest.

48.

Vt igitur corporis coelestis locum in spatio pro quouis temporis momento assignare liceat, sequentia in orbita elliptica nota esse oportebit.

I. Longitudo media pro quodam temporis momento arbitrario, quod epocha vocatur: eodem nomine interdum ipsa quoque longitudo designatur. Plerumque pro epocha eligitur initium alicuius anni, scilicet meridies 1. Ianuarii in anno bissextili, siue meridies 31 . Decembris anno communi praecedentis.

II. Motus medius inter certum temporis interuallum, e. g. in vno die solari medio, siue in diebus $365,365 \frac{x}{4}$ aut 56525 .

III. Semiaxis maior, qui quidem omilti posset, quoties corporis massa aut nota est aut negligi potest, quum per motzm -medium iam detur (art. 7): commoditatis tamen gratia vterque semper proferri solet.

IV. Excentricitas. V, Longitudo perihelii. VI. Longitudo nodi ascendentis. VII. Inclinatio orbitae.

Haec septem momenta vocantur elementa motus corporis.

In parabola et hyperbola tempus transitus per perihelium elementi primi vice fungetur; pro II tradentur quae in his sectionum conicarum generibus motui medio diurno analoga sunt (v. art. 19; in motu hyperbolico quantitas $2 k b^{-\frac{3}{2}}$ art. 23 ). In hyperbola elementa reliqua perinde retineri poterunt, in parabola vero, vbi axis maior infinitus atque excentricitas $=x$, loco elementi III et IV sola distantia in perihelio proferetur.

49.

Secundum vulgarem loquendi morem inclinatio orbitae, quam nos a a vsque ad $180^{\circ}$ numeramus, ad $90^{\circ}$ tantum extenditur, atque si angulus orbitae cum arcu $\$ B B$ (Fig. 1) angulum rectum egreditur, angulus orbitae cum arcu $\delta A$ (qui est illius complementum ad $180^{\circ}$ tamquam inclinatio orbitae spectatur; in tali tunc casu 
addere oportebit, motum esse retrogradum (veluti si in figura nostra $E \Omega F$ partem orbitae repraesentat), vt a casu altero vbi motus directus dicitur distinguatur. Longitudo in orbita tunc ita numerari solet, $v t$ in $\delta$ cum longitudine huius puncti in ecliptica conueniat, in directione $\delta F$ autem decrescat; punctum initiale itaque a quo longitudines contra ordinem motus numerantur in directione $\Omega F$ tantundem a $\delta$ distat, quantum aequinoctiom vernale $a b$ eodem $\delta$ in directione $\Omega A$. Quare in hoc casu longitudo perihelii erit longitudo nodi deminuta distantia perihelii a nodo. Hoc modo alteruter loquendi ysus facile in alterum conuertitur, nostrum autem ideo praetulimus, vt distinctione inter motum directum et retrogradum supersedere, et pro vtroque semper formulas easdem adhibere possemus, quum vsus vulgaris saepenumero praecepta duplicia requirat.

50.

Ratio simplicissima, puncti cuiusuis in superficie sphaerne coelestis situm respectu eclipticae determinandi, fit per ipsius distantiam ab ecliptica (latitudinem), atque distantiam puncti, vbi ecliptica a perpendiculo demisso secatur, ab aequinoctio (longitudinem). Latitudo, ab vtraque eclipticae parte vsque ad $90^{\circ}$ numerata, in regione boreali vt positiua, in australi vt negatiua spectatur. Respondeant corporis coelestis loco heliocentrico, i. e. proiectioni rectae a Sole ad corpus ductae in sphaeram coelestem, longitudo $\lambda$, latitudo $\beta$; sit porro, $u$ distantia loci heliocentrici a nodo ascendente (quae argumentum latitudinis dicitur), $i$ inclinatio orbitae, $\delta$ longitudo nodi ascendentis, habebunturque inter $i, u, \beta, \lambda-\delta$, quae quantitates erunt partes trianguli sphaerici rectanguli, relationes sequentes, quas sine vlla restrictione valere facile euincitur:
I. $\operatorname{tang}(\lambda-\Omega)=\cos i \operatorname{tang} u$
II. $\operatorname{tang} \beta=\operatorname{tang} i \sin (\lambda-\delta)$
III. $\sin \beta=\sin i \sin u$
IV. $\cos u=\cos \beta \cos (\lambda-\delta)$

Quando $i$ et $u$ sunt quantitates datae, $\lambda-\delta$ inde per. aequ. I determinabitur, ac dein $\beta$ per II vel per III, siquidem $\beta$ non nimis ad $\pm 90^{\circ}$ appropinquat; formula IV si placet ad calculi confirmationem adhiberi potest. Ceterum formulae $I$ et IV docent, $\lambda-\Omega$ et $u$ semper in eodem quadrante iacere, quoties $i$ est inter o et $90^{\circ}$; contra $\lambda-\Omega$ et $360^{\circ}-u$ ad eundem quadrantem pertinebunt, quoties $i$ est inter $90^{\circ}$ et $180^{\circ}$, siue, secundum vsum vulgarem, quoties motus est retrogradus: hine 
ambiguitas, quam determinatio ipsius $\lambda-\delta$ per tangentem secundun formulam I relinquit, sponte tollitur.

Formulae sequentes e praecedentium combinatione facile deriuantur:

V. $\sin (u-\lambda+\delta)=2 \sin \frac{\pi}{2} i^{2} \sin u \cos (\lambda-\beta)$

VI. $\sin (u-\lambda+\delta)=\operatorname{tang} \frac{x}{2} i \sin \beta \cos (\lambda-\delta)$

VII. $\sin (u-\lambda+\delta)=\operatorname{tang} \frac{\pi}{2} i \operatorname{tang} \beta \cos u$.

VIII. $\sin (u+\lambda-\delta)=2 \cos \frac{x}{2} i^{2} \sin u \cos (\lambda-\delta)$

IX. $\sin (u+\lambda-\delta)=\operatorname{cotang} \frac{\pi}{2} i \sin \beta \cos (\lambda-\delta)$

$\mathrm{X} \sin (u+\lambda-\delta)=\operatorname{cotang} \frac{\pi}{2} i \operatorname{tang} \beta \cos u$

Angulus $u-\lambda+\delta$, quoties $i$ est infra $90^{\circ}$, aut $u+\lambda-\Omega$, quoties $i$ est vltra $90^{\circ}$, secundum vsum vulgarem reductio ad eclipticam dicitur, est scilicet differentia inter longitudinem heliocentricam $\lambda$ atque longitudinem in orbita quae secundum illum. vsum est $\Omega+u,($ secundum nostrum $\Omega+u$ ). Quoties inclinatio vel parua est vel a $180^{\circ}$ parum diuersa, ista reductio tamquam quantitas secundi ordinis spectari potest, et in hoc quidem casu praestabit, $\beta$ primo per formulam III ac dein $\lambda$ per VII aut $\mathrm{X}$ computare, quo pacto praecisionem maiorem quam per formulam I assequi licebit.

Demisso perpendiculo a loco corporis coelestis in spatio ad planum eclipticae, distantia puncti intersectionis a Sole distantia curtata appellatur. Quam per $r^{\prime}$, radium vectorem autem per $r$ designando, habebimus XI. $r^{\prime}=r \cos \beta$

51.

Exempli caussa calculum in artt. 15,14 inchoatum, cuius numeros planeta Ixxionis suppeditauerat, vlterius continuabimzis. Supra inueneramus anomaliam veram $315^{\circ} x^{\prime} 23^{\prime \prime} 02$, logaxithmum radii vectoris 0,3259877 : sit iam $i=13^{\circ} 6^{\prime} 44^{\prime \prime} 10$, . distantia perihelii a nodo $=241^{\circ} 10^{\prime} 20^{\prime \prime} 57$, adeoque $u=196^{\circ} 11^{\prime} 43^{\prime \prime} 59$; denique sit $\Omega_{8}=7^{\circ} 7^{\prime} 48^{\prime \prime} 73$. Hinc habemus:

$$
\begin{aligned}
& \log \operatorname{tang} u \ldots \ldots \ldots \ldots \ldots . . . .9,4650573 \\
& \log \cos i \ldots \ldots \ldots \ldots \ldots \ldots . . . . .9,9885266 \\
& \text { log tang }(\lambda-\Omega) \ldots . .9,4515859 \\
& \lambda-\Omega=195^{\circ} 47^{\prime} 40^{\prime \prime}{ }_{25} \\
& 2=65528,98 \\
& \log r \ldots \ldots \ldots \ldots \ldots \ldots \ldots . . . . .0,5259877 \\
& \frac{\log \cos \beta \ldots \ldots \ldots \ldots \ldots . .9,9991289}{\log r^{\prime} \ldots \ldots \ldots \ldots \ldots \ldots \ldots .6,3251166}
\end{aligned}
$$

$$
\begin{aligned}
& \log \sin (\lambda-\Omega) \ldots . .9,454869 \times n \\
& \log \operatorname{tang} i \ldots \ldots \ldots \ldots \ldots, 567_{2} 505 \\
& \log \operatorname{tang} \beta \ldots \ldots \ldots \ldots . . . . . .8,8020996 n \\
& \beta=-3^{\circ} 37^{\prime} 40^{\prime \prime} 02 \\
& \log \cos \beta \ldots \ldots \ldots \ldots \ldots . .9,9991289 \\
& \frac{\log \cos (\lambda-8) \ldots . .9,9832852 n}{9,9824141 n} \\
& \log \cos u \ldots \ldots \ldots \ldots \ldots . .9,9824141 \quad n
\end{aligned}
$$


Calculus secundum formulas III, VII ita se haberet:

$$
\begin{aligned}
& \log \sin u \ldots \ldots 9,4454714 n \quad \log \tan g \frac{x}{2} i \ldots \ldots \ldots \ldots . . .9,0604259 \\
& \log \sin i \ldots \ldots .9,3557570 \quad \log \operatorname{tang} \beta \ldots \ldots \ldots \ldots \ldots ., 8020995 \text { i }
\end{aligned}
$$

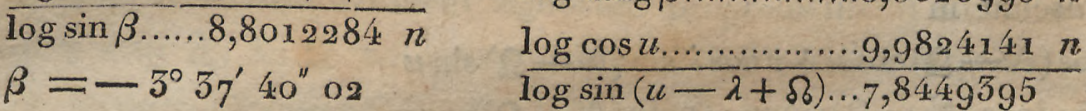

$$
\begin{aligned}
& u-\lambda+\Omega=\quad 0^{\circ} 24^{\prime} 3^{\prime \prime} 34 \\
& \lambda-\Omega=1954740,25
\end{aligned}
$$

52.

Spectando $i$ et $u$ tamquam quantitates variabiles, differentiatio aequationis III art. 50 suggerit:

$\operatorname{cotang} \beta \mathrm{d} \beta=$ cotang $i \mathrm{~d} i+\operatorname{cotang} u \mathrm{~d} u$ siue

XII. $d \beta=\sin (\lambda-\Omega) d i+\sin i \cos (\lambda-\Omega) d u$

Perinde per differentiationem aequationis $I$ obtinemus

Denique e differentiatione aequationis XI prodit

XIII. $\mathrm{d}(\lambda-\Omega)=-\operatorname{tang} \beta \cos (\lambda-\Omega) \mathrm{d} i+\frac{\cos }{\cos \beta^{2}} \mathrm{~d} u$

$\mathrm{d} r^{\prime}=\cos \beta \mathrm{d} r-r \sin \beta \mathrm{d} \beta$, siue

XIV. $\mathrm{d} r^{\prime}=\cos \beta \mathrm{d} r-r \sin \beta \sin (\lambda-\Omega) \mathrm{d} i-r \sin \beta \sin i \cos (\lambda-\Omega) \mathrm{d} u$

In hac viltima aequatione vel partes quae continent $d i$ et $\mathrm{d} u$ per $206265^{\prime \prime}$ sunt diuidendae, vel reliquae per hunc numerum multiplicandae, si mutationes ipsarum $i$ et $u$ in minutis secundis expressae supponuntur.

\section{3.}

Situs puncti cuiuscunque in spatio commodissime per distantias a tribus planis sub angulis rectis se secantibus determinatur. Assumendo pro planorum vno planum eclipticae, designandoque per $z$ distantiam corporis coelestis ab hoc plano a parte boreali positiue, $a b$ australi negatiue sumendam, manifesto habebimus $z=$ $r^{\prime} \operatorname{tang} \beta=r \sin \beta=r \sin i \sin u$. Plana duo reliqua, quae per Solem quoque ducta supponemus, in sphaera coelesti circulos maximos proiicient, qui eclipticam sub angulis rectis secabunt, quorumque adeo poli in ipsa ecliptica iacebunt et $90^{\circ}$ ab inuicem distabunt. Vtriusque plani polum istum, a cuius parte distantiae positiuae censentur, polum positiuum appellamus. Sint itaque $N$ et $N+90^{\circ}$ longitudines polorum positioorum, designenturque distantiae a planis quibus respondent respectiue per $x, y$. Tunc facile perspicietur haberi 


$$
\begin{aligned}
& x=r^{\prime} \cos (\lambda-N)=r \cos \beta \cos (\lambda-\Omega) \cos (N-\Omega)+r \cos \beta \sin (\lambda-\Omega) \sin (N-\Omega) \\
& y=r^{\prime} \sin (\lambda-N)=r \cos \beta \sin (\lambda-\Omega) \cos (N-\Omega)-r \cos \beta \cos (\lambda-\Omega) \sin (N-\Omega)
\end{aligned}
$$

qui valores transeunt in

$$
\begin{aligned}
& x=r \cos (N-\Omega) \cos u+r \cos i \sin (N-\Omega) \sin u \\
& y=r \cos i \cos (N-\Omega) \sin u-r \sin (N-\Omega) \cos u
\end{aligned}
$$

Quodsi itaque polus positiuus plani ipsarum $x$ in ipso nodo ascendente collocatur, $\mathrm{Nt}$ sit $N=\delta$, habebimus coordinatarum $x, y, z$ expressiones simplicissimas

$$
\begin{aligned}
& x=r \cos u \\
& y=r \cos i \sin u \\
& z=r \sin i \sin u
\end{aligned}
$$

Si vero haec suppositio locum non habet, tamen formulae supra datae formam aeque fere commodam nanciscuntur per introductionem quatuor quantitatum auxiliarium $a$, $6, A, B$ ita determinatarum vt habeatur

$$
\begin{aligned}
& \cos (N-\Omega)=a \sin A \\
& \cos i \sin (N-\Omega)=a \cos A \\
& -\sin (N-\Omega)=b \sin B \\
& \cos i \cos (N-\Omega)=b \cos B
\end{aligned}
$$

(vid. art. 14, II). Manifesto tunc erit

$$
\begin{aligned}
& x=r a \sin (u+A) \\
& y=r b \sin (u+B) \\
& z=r \sin i \sin u
\end{aligned}
$$

54.

Relationes motus ad eclipticam in praecc. explicatae manifesto perinde valebunt, etiamsi pro ccliptica quoduis aliud planum substituatur, si modo situs plani orbitae ad hoc planum innotuerit; expressiones longitudo et latitudo autem tunc supprimendae erunt. Offert itaque se problema: e situ cognito plani orbitae aliusque plani noui ad eclipticam deriuare situm plani orbitae ad hoc nouum planum. Sint $\pi \Omega, \Omega \Omega^{\prime}, n \Omega^{\prime}$ partes circulorum maximorum, quos planum eclipticae, planum orbitae planumque noùum in sphaera coelesti proiiciunt (Fig. 2). Vt inclinatio circuli secundi ad tertium locusque nodi ascendentis absque ambiguitate assignari possit, in circulo tertio alterutra directio eligi debebit tamquam ei analoga, quae in ecliptica est secundum ordinem signorum; sit haec in fig. nostra directio ab $n$ vorsus $\Omega^{\prime}$. Praeterea duorum hemisphaeriorum, quae circulus $n \Omega^{\prime}$ separat, alterum 
censere oportebit ánalogum haemisphaerio boreali, alterum australi: haec vero haemisphaeria sponte iam sunt distincta, quatenus id semper quasi boreale spectatur, quod in circulo secundum ordinem signorum progredienti*) a dextra est. In figura igitur nostra sunt $\Omega, n, \Omega^{\prime}$ nodi ascendentes circuli secundi in primo, tertii in primo, secundi in tertio; $180^{\circ}-n \Omega \Omega^{\prime}, \Omega n \Omega^{\prime}, n \Omega^{\prime} \Omega$ inclinationes secundi ad primum, tertii ad primum, secundi ad tertium. Pendet itaque problema nostrum a solutione trianguli sphaerici, vbi e latere vno angulisque adiacentibus reliqua sunt deducenda. Praecepta vulgaria, quae in trigonometria sphaerica pro hoc casin traduntur, tamquam abunde nota supprimimus: commodius autem methodus alia in vsum vocatur ex aequationibus quibusdam petita, quae in libris nostris trignonometricis frustra quaeruntur. Ecce has aequationes, quibus in sequentibus frequenter vtemur: designant $a, b, c$ latera trianguli sphaerici atque $A, B, C$ angulos illis resp. oppositos:

$$
\begin{aligned}
& \text { I. } \frac{\sin \frac{\pi}{2}(b-c)}{\sin \frac{r}{2} a}=\frac{\sin \frac{\pi}{2}(B-C)}{\cos \frac{\pi}{2} A} \\
& \text { II. } \frac{\sin \frac{\pi}{2}(b+c)}{\sin \frac{r}{2} a}=\frac{\cos \frac{r}{2}(B-C)}{\sin \frac{r}{2} A} \\
& \text { III. } \frac{\cos \frac{\pi}{2}(b-c)}{\cos \frac{\pi}{2} a}=\frac{\sin \frac{r}{2}(B+C)}{\cos \frac{\pi}{2} A} \\
& \text { IV. } \frac{\cos \frac{\pi}{2}(b+c)}{\cos \frac{\pi}{2} a}=\frac{\cos \frac{\pi}{2}(B+C)}{\sin \frac{\pi}{2} A}
\end{aligned}
$$

Quamquam demonstrationem harum propositionum breuitatis caussa hic praeterire oporteat, quisque tamen earum veritatem in triangulis, quorum nec latera nec anguli $180^{\circ}$ excedunt, haud difficile confirmare poterit. Quodsi quidem idea trianguli sphaerici in maxima generalitate concipitur, vt nec latera nec anguli vllis limitibus restringantur (quod plurima commoda insignia praestat, attamen quibusdam dilucidationibus praeliminaribus indiget), casus existere possunt, vbi in cunctis aequationibus praecedentibus signum mutare oportet; quoniam vero signa priora manifesto restituuntur, simulac vnus angulorum vel vnum laterum $360^{\circ}$ augetur vel diminuitur, signa, qualia tradidimus, semper tuto retinere licebit, sine e latere angulisque adiacentibus reliqua determinanda sint, siue ex angulo lateribusque adiacentibus; semper enim vel quaesitorum valores ipsi vel $560^{\circ}$ a veris diuersi hisque adeo aequiualentes per formulas nostras elicientur. Dilucidationem copiosiorem huius argumenti ad aliam occasionem nobis reseruamus: quod vero praecepta, quae tum pro

*) Puta in interiori splacerae superficie, quam figura nostra repraesentat. 
solutione problematis nostri tum in aliis occasionibus formulis istis superstruemus, in omnibus casibus generaliter valent, tantisper adiumento inductionis rigorosae, i. e. completae omnium casuum enumerationis, haud difficile comprobari poterit,

\section{5.}

Designando $v t$ supra longitudinem nodi ascendentis orbitae in ecliptica per $\Omega$, inclinationem per $i$; porro longitudinem nodi ascendentis plani noui in ecliptica per $n$, inclinationem per $\varepsilon$; distantiam nodi ascendentis orbitae in plano nouo a nodo ascendente plani, noui in eeliptica ( $\operatorname{arcum} n \Omega^{\prime}$ in Fig. 2) per $\Omega^{\prime}$, inclinationem orbitae ad planum noutm per $i^{\prime}$; denique arcum ab $\Omega$ ad $\Omega^{\prime}$ secundum directionem motus per $\Delta$ : erunt trianguli sphaerici nostri latera $\Omega-n, \Omega^{\prime}, \Delta$, angulique oppositi $i^{\prime}$, $\pi 80^{\circ}-i$, \&. Hinc erit secundum formulas art. praec.

$$
\begin{aligned}
& \sin \frac{\pi}{2} i^{\prime} \sin \frac{\pi}{2}\left(\Omega^{\prime}+\Delta\right)=\sin \frac{\pi}{2}(\Omega-n) \sin \frac{\pi}{2}(i+\varepsilon) \\
& \sin \frac{\pi}{2} i^{\prime} \cos \frac{\pi}{2}\left(\Omega^{\prime}+\Delta\right)=\cos \frac{\pi}{2}(\Omega-n) \sin \frac{\pi}{2}(i-\varepsilon) \\
& \cos \frac{\pi}{2} i^{\prime} \sin \frac{\pi}{2}\left(\Omega^{\prime}-\Delta\right)=\sin \frac{\pi}{2}(\Omega-n) \cos \frac{\pi}{2}(i+\varepsilon) \\
& \cos \frac{\pi}{2} i^{\prime} \cos \frac{\pi}{2}\left(\Omega^{\prime}-\Delta\right)=\cos \frac{1}{2}(\Omega-n) \cos \frac{\pi}{2}(i-\varepsilon)
\end{aligned}
$$

Duae primae aequationes suppeditabunt $\frac{x}{2}\left(\Omega^{\prime}+\Delta\right)$ atque $\sin \frac{x}{2} i^{\prime} ;$ duae reliquae $\frac{\pi}{2}\left(\Omega^{\prime}-\Delta\right)$ atque $\cos \frac{x}{2} i^{\prime}$; ex $\frac{x}{2}\left(\Omega^{\prime}+\Delta\right)$ et $\frac{x}{2}\left(\Omega^{\prime}-\Delta\right)$ demanabunt $\Omega^{\prime}$ et $\Delta$; ex $\sin \frac{1}{2} i^{\prime}$ aut $\cos \frac{\pi}{2} i^{\prime}$ (quorum consensus calculo confirmando inseruiet) prodibit $i^{\prime}$. Ambiguitas, vtrum $\frac{\pi}{2}\left(\Omega^{\prime}+\Delta\right)$ et $\frac{x}{2}\left(\Omega^{\prime}-\Delta\right)$ inter 0 et $180^{\circ}$ vel inter $180^{\circ}$ et $560^{\circ}$ accipere oporteat, ita tolletur, vt tum $\sin \frac{x}{2} i^{\prime}$ tum $\cos \frac{x}{2} i^{\prime}$ fiant positiui, quoninm per rei naturam $i^{\prime}$ infra $180^{\circ}$ cadere debet.

\section{6.}

Praecepta praecedentia exemplo illustranisse haud inutile erit. Sit $\Omega=$ $172^{\circ} 28^{\prime} 15^{\prime \prime} 7, i=34^{\circ} 38^{\prime} 1^{\prime \prime} 1$; porro sit planum nouum aequatori parallelum, adeoque $n=180^{\circ}$; angulum $\varepsilon$, qui erit obliquitas eclipticae, statuimus $=23^{\circ} 27^{\prime} 55^{\prime \prime} 8$. Habemus itaque

$$
\begin{aligned}
& \Omega-n=-7^{\circ} 3 x^{\prime} 46^{\prime \prime} 5 \\
& { }_{2}^{x}(\Omega-n)=-3^{\circ} 45^{\prime} 55^{\prime \prime} \times 5 \\
& i+\varepsilon=58 \quad 5 \quad 56,9 \\
& \frac{i}{2}(i+\varepsilon)=29258,45 \\
& i-\varepsilon=1110 \quad 5,3 \\
& \frac{x}{2}(i-\varepsilon)=5352,65 \\
& \log \sin \frac{\pi}{2}(8-n) \ldots 8,8173026 n \\
& \log \cos \frac{x}{2}(8-n) \ldots . .99,9990618 \\
& \log \sin \frac{x}{2}(i+\varepsilon) \ldots . . .9,6862484 \\
& \log \sin \frac{x}{2}(i-\varepsilon) \ldots \ldots 8,9881405 \\
& \log \cos \frac{\pi}{2}(i+\varepsilon) \ldots . . .999416108 \\
& \log \cos \frac{\pi}{2}(i-\&) \ldots . . .99,9979542
\end{aligned}
$$

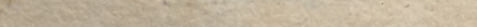


Whine fit

$\log \sin \frac{x}{2} i^{\prime} \sin \frac{\pi}{2}\left(\Omega^{\prime}+\Delta\right) \ldots 8,5055510 n$

$\log \cos \frac{\pi}{2} i^{\prime} \sin \frac{r}{2}(\Omega-\Delta), \ldots 8,7589134 x$

$\log \sin \frac{x}{2} i^{\prime} \cos \frac{x}{2}\left(\Omega^{\prime}+\Delta\right) \ldots 8,9872025$

vnde $\frac{\pi}{2}\left(\overline{\Omega+\Delta)}=541^{\circ} 49^{\prime} 19^{\prime \prime} 01\right.$

$\log \sin \frac{x}{2} i^{\prime} \ldots . . .9,0094368$

$\log \cos \frac{x}{2} i^{\prime} \cos \frac{x}{2}\left(\Omega^{\prime}-\Delta\right) \ldots 9,9969960$

vnde $\frac{1}{2}\left(\Omega^{\prime}-\Delta\right)=556^{\circ} 41^{\prime} 31^{\prime \prime} 43$

$\log \cos \frac{x}{2} i^{\prime}$

9,9977202

Obtinemus itaque $\frac{x}{2} i^{\prime}=5^{\circ} 51^{\prime} 56^{\prime \prime} 445, i^{\prime}=11^{\circ} 45^{\prime} 52^{\prime \prime} 89, \quad \Omega^{\prime}=338^{\circ} 30^{\prime} 50^{\prime \prime} 43$, $\Delta=-14^{\circ} 52^{\prime} 12^{\prime \prime} 42$. Ceterum punctum $n$ in sphaera coelesti manifesto respondet aequinoctio autumnali; quocirca distantia nodi ascendentis orbitae in aequatore ab 2equinoctio vernali (eius rectascensio) erit $158^{\circ} 30^{\prime} 50^{\prime \prime} 43$.

Ad illustrationem art. 53 hoc exemplum adhuc vlterius continuabimus, formulasque pro coordinatis respectu trium planorum per Solem transeuntium euoluemus, quorum vnum aequatori parallelum sit, duorumque reliquorum poli positiui in ascensione recta $0^{\circ}$ et $90^{\circ}$ sint siti: distantiae ab his planis sint resp. $z, x, y$. Iam si insuper distantia loci heliocentrici in sphaera coelesti a punotis $\Omega, \Omega^{\prime}$ resp. denotetur per $u, u^{\prime}$, fiet $u^{\prime}=u-\Delta=u+14^{\circ} 52^{\prime} 12^{\prime \prime} 42$, et quae in art. 53 per $i$, $N-\delta, u$ exprimebantur, hic erunt $i^{\prime}, 180^{\circ}-\Omega^{\prime}, u^{\prime}$. Sic per formulas illic datas prodit

$\begin{array}{cc}\log a \sin A \ldots . .9,9687197 n & \log b \sin B \ldots \ldots 9,5638058 \\ \frac{\log a \cos A \ldots \ldots 9,5546580 n}{\text { vnde } A=248^{\circ} 55^{\prime} 22^{\prime \prime} 97} & \frac{\log b \cos B \ldots \ldots 9,9595519 n}{\operatorname{lnde} B=158^{\circ} 5^{\prime} 54^{\prime \prime} 97} \\ \log a \ldots \ldots \ldots .9,99^{\prime} 879_{2} 3 & \log b \ldots \ldots \ldots .9,9920848\end{array}$

Habemus itaque

$$
\begin{aligned}
& x=a r \sin \left(u^{\prime}+248^{\circ} 55^{\prime} 22^{\prime \prime} 97\right)=a r \sin \left(u+263^{\circ} 47^{\prime} 35^{\prime \prime} 39\right) \\
& y=b r \sin \left(u^{\prime}+158 \quad 5 \quad 54,97\right)=b r \sin \left(u+17258 \quad 7^{\prime \prime} 59\right) \\
& z=c r \sin u^{\prime} \quad=c r \sin (u+145212,42)
\end{aligned}
$$

bi $\log c=\log \sin i^{\prime}=9,3081870$.

Alia solutio problematis hic tractati inuenitur in Fon Zach Monatliche Correspondenz B. IX. S. 585.

57.

Corporis itaque coelestis distantia a quouis plano per Solem transeunte reduci poterit ad formam $k r \sin (v+K)$, designante $v$ anomaliam veram, eritque $k$ sinus inclinationis orbitae ad hoc planum, $K$ distantia perihelii a nodo ascendente orbitae in eodem plano. Quatenus situs plani orbitae, lineaeque apsidum in $e 0$, 
nec non situs plani ad quod distantiae referuntur pro constantibus haberi possuni, etiam $k$ et $K$ constantes erunt. Frequentius tamen illa methodus in tali casu in vsum vocabitur, vbi tertia saltem suppositio non permittitur, etiamsi perturbationes negligantur, quae primam atque secundam semper aliquatenus afficiunt. Illud euenit, quoties distantiae referuntur ad aequatorem, siue ad planum aequatorem sub angulo recto in rectascensione data secans: quum enim situs aequatoris propter praecessionem aequinoctiorum insuperque propter nutationem (siquidem de vero non de medio situ sermo fuerit) mutabilis sit, in hoc casu etiam $k$ et $K$ mutationibus, lentis vtique, obnoxiae erunt. Computus harum mutationum per formulas differentiales absque difficultate eruendas absolui potest: hic vero breuitatis caussa sufficiat, variationes differentiales ipsarum $i, \Omega^{\prime}, \Delta$ apposuisse, quatenus a variationibus ipsarum $\beta-n$ atque $\varepsilon$ pendent.

$$
\begin{aligned}
& \mathrm{d} i^{\prime}=\sin \varepsilon \sin \Omega^{\prime} \mathrm{d}(\delta-n)-\cos \Omega^{\prime} \mathrm{d} \varepsilon \\
& \mathrm{d} \Omega^{\prime}=\frac{\sin i \cos \Delta}{\sin i^{\prime}} \mathrm{d}(\Omega-n)+\frac{\sin \Omega^{\prime}}{\operatorname{tang} i^{\prime}} \mathrm{d} \varepsilon \\
& \mathrm{d} \Delta=\frac{\sin \varepsilon \cos \Omega^{\prime}}{\sin i^{\prime}} \mathrm{d}(\Omega-n)+\frac{\sin \Omega^{\prime}}{\sin i^{\prime}} \mathrm{d} \varepsilon
\end{aligned}
$$

Cetcrum quoties id tantum agitur, vt plures corporis coelestis loci respectu talium planorum mutabilium calculentur, qui temporis interuallum mediocre complectuntur (e. g. vnum annum), plerumque commodissimum erit, quantitates $a, A, b, B$, $c, C$ pro duabus epochis intra quas illa cadunt reipsa calculare, ipsarumque mutationes pro singulis temporibus propositis ex illis per simplicem interpolationem eruere.

\section{8.}

Formulae nostrae pro distantiis a planis datis inuoluunt $v$ et $r$ : quoties has quantitates e tempore prius determinare oportet, partem operationum adhuc contrahere, atque sic laborom notabiliter alleuare licebit. Deriuari enim possunt illae distantiae per formulam persimplicem statim ex anomalia excentrica in ellipsi, vel e quantitate auxiliari $F$ aut $u$ in hyperbola, ita vt computo anomaliae verae radiique vectoris plane non sit opus. Mutatur scilicet expressio $k r \sin (\nu+K)$

I. pro ellipsi, retentis characteribus art. 8 , in

$a k \cos \varphi \cos K \sin E+a k \sin K(\cos E-e)$

Determinando itaque $l, L, \lambda$ per aequationes 


$$
\begin{aligned}
& a^{2} \sin K=l \sin L \\
& a k \cos \varphi \cos K=l \cos L \\
& -e a k \sin K=-e l \sin L=\lambda
\end{aligned}
$$

expressio nostra transit in $l \sin (E+L)+\lambda$, vbi $l, L, \lambda$ constantes erunt, quatenus $k, K$, e pro constantibus habere licet; sin minus, de illarum mutationibus computandis eadem valebunt, quae in art. praec. monuimus.

Exempli caussa transformationem expressionis pro $x$ in art. 56 inuenti apponimus, vbi longitudinem perihelii $=121^{\circ} 17^{\prime} 34^{\prime \prime} 4, \varphi=14^{\circ} 15^{\prime} 31^{\prime \prime} 97, \log \alpha=0,4423790$ statuimus. Fit igitur distantia perihelii a nodo ascendente in ecliptica $=308^{\circ} 49^{\prime} 20^{\prime \prime} 7=$ $t$; hinc $K=212^{\circ} 36^{\prime} 56^{\prime \prime}$ og. Habemus itaque

$$
\begin{aligned}
& \log a k \ldots \ldots \ldots \ldots . . .0,4411713 \quad \log l \sin L \ldots \ldots, 1727600 n \\
& \begin{array}{ll}
\log \sin K \ldots \ldots \ldots . . .9,7515887 n & \frac{\log l \cos L \ldots \ldots ., 5551154 n}{\log a k \cos \varphi \ldots \ldots .0,4276456}
\end{array} \\
& \log \cos K \ldots \ldots \ldots . .9,9254698 n \quad \log l=0,4316627 \\
& \log \lambda=9,5632352 \\
& \lambda=+0,5657929
\end{aligned}
$$

II. In hyperbola formula $k r \sin (\nu+K)$ secundum art. 21 transit in $\lambda+\mu \operatorname{tang} F$ $+\nu$ secans $F$, si statuitur e $b k \sin K=\lambda, \quad b k \operatorname{tang} \psi \cos K=\mu,-b k \sin K=\nu ;$ manifesto eandem expressionem etiam sub formam $\frac{n \sin (F+N)+\nu}{\cos F}$ reducere licet, Si loco ipsius $F$ quantitas auxiliaris $u$ adhibita est, expressio $k r \sin (\nu+K)$ per art. 21 transibit in $\alpha+\beta u+\frac{\gamma}{u}$, vbi $\alpha, \beta, \gamma$ determinantur per formulas

$$
\begin{aligned}
& \alpha=\lambda=e b k \sin K \\
& \beta=\frac{1}{2}(\nu+\mu)=-\frac{1}{2} e b k \sin (K-\psi) \\
& \gamma=\frac{1}{2}(\nu-\mu)=-\frac{1}{2} e b k \sin (K+\psi)
\end{aligned}
$$

III. In parabola, vbi anomalia vera e tempore immediate derinatur, nihil aliud supererit, nisi vt pro radio vectore valor suus substituatur. Denotando itaque distantiam in perihelio per $q$, expressio $k r \sin (\nu+K)$ fit $=-\frac{q k \sin (\nu+K)}{\cos \frac{2}{7} v^{2}}$.

\section{9.}

Praecepta pro determinandis distantiis a planis per Solem transeuntibus manifesto etiam ad distantias terrae applicare licet: hic vero simplicissimi tanfum casus occurrere solent. Sit $R$ distantia terrae a Sole, $L$ longitudo heliocentrica ter- 
rae (quae $180^{\circ}$ a longitudine geocentrica Solis differt), denique $X, Y, Z$ distantiae terrae a tribus planis in Sole sub angulis rectis se secantibus. Iam si

I. Planum ipsarum $Z$ est ipsa ecliptica, longitudinesque polorum planorum reliquorum, a quibus distantiae sunt $X, Y$, resp. $N$ et $N+90^{\circ}$ : erit

$$
X=R \cos (L-N), Y=R \sin (L-N), Z=0 \text {. }
$$

II. Si planum ipsarum $Z$ aequatori parallelum est, atque rectascensiones polorum planorum reliquorum, a quibus distantiae sunt $X, Y$, resp. o et $90^{\circ}$, habebimus, obliquitate eclipticae per $\varepsilon$ designata

$$
X=R \cos L, Y=R \cos \varepsilon \sin L, Z=R \sin \varepsilon \sin L
$$

Tabularum solarium recentissimarum editores, clarr. de Zach et de Lambre, latitudinis Solis rationem habere coeperunt, quae quantitas a perturbationibus reliquorum planetarum atque lunae producta vix vnum minutum secundum attingere potest. Designando latitudinem heliocentricam terrae, quae latitudini Solis semper aequalis sed signo opposito affecta erit, per $B$, habebimus:

$$
\begin{array}{l|l}
X=R \cos B \cos (L-N) & \begin{array}{l}
\text { in casu II. } \\
X=R \cos B \cos L
\end{array} \\
Y=R \cos B \sin (L-N) & Y=R \cos B \cos \varepsilon \sin L-R \sin B \sin \varepsilon \\
Z=R \sin B & Z=R \cos B \sin \varepsilon \sin L+R \sin B \cos \varepsilon
\end{array}
$$

Pro $\cos B$ hic semper tuto substitui poterit 1 , angulusque $B$ in partibus radii expressus pro $\sin B$.

Coordinatae ita inuentae ad centrum terrae referuntur: si $\xi, \eta, \xi$ sunt distantiae puncti cuiuslibet in terrae superficie a tribus planis per centrum terrae ductis iisque quae per Solem ducta erant parallelis, distantiae illius puncti a planis per Solem transeuntibus manifesto erunt $X+\xi, Y+\eta, Z+\zeta$, valores coordinatarum $\xi, \eta, \zeta$ autem pro vtroque casi facile determinantur sequenti modo. Sit $\varrho$ radius globi terrestris (siue sinus parallaxis horizontalis mediae Solis) 2 longitudo puncti sphaerae coelestis, vbi recta a terrae centro ad punctum superficiei ductum proiicitur, $\beta$ eiusdem latitudo, $\alpha$ ascensio recta, $\delta$ declinatio, eritque

$$
\begin{aligned}
& \text { in casu } \mathrm{x} \text {. } \\
& \xi=\rho \cos \beta \cos (\lambda-, N) \quad \xi=\rho \cos \delta \cos \alpha \\
& \eta=\rho \cos \beta \sin (\lambda-N) \quad \eta=\rho \cos \delta \sin \alpha \\
& \zeta=\rho \sin \beta \quad \mid \xi=\rho \sin \delta
\end{aligned}
$$


Punctum illud sphaerae coelestis manifesto respondet ipsi zenith loci in superficie (siquidem terra tamquam sphaera spectatur), quocirca ipsius ascensio recta conueniet cum ascensione recta medii coeli siue cum tempore siderali in gradus conuerso, declinatio autem cum eleuatione poli; si operae pretium esset, figurae terrestris sphaeroidicae rationem habere, pro $\delta$ eleuationem poli correctam, atque pro $\varrho$ distantiam veram loci a centro terrae accipere oporteret, quae per regulas notas eruuntur. Ex $\alpha$ et $\delta$ longitudo et latitudo $\lambda$ et $\beta$ per regulas notas infra quoque tradendas deducentur; ceterum patet, $\lambda$ conuenire cum longitudine nonagesimi, atque $90^{\circ}-\beta$ cum eiusdem altitudine.

60.

Designantibus $x, y, z$ distantias corporis coelestis a tribus planis in Sole sub angulis rectis se secantibus; $X, Y, Z$ distantias terrae (siue centri siue puncti in superficie) ab iisdem planis: patet, $x-X, y-Y, z-Z$ fore distantias corporis coelestis a tribus planis illis parallele per terram ductis, hasque distantias ad distantiam corporis a terra ipsiusque locum geocentricum *), i. e. situm proiectionis rectae a terra ad ipsum ductae in sphaera coelesti, relationem eandem habituras, quam $x, y, z$ habent ad distantiam a Sole locumque heliocentricum. Sit $\Delta$ distantia corporis coelestis a terra; concipiatur in sphaera coelesti perpendiculum a loco geocentrico ad circulum maximum, qui respondet plano distantiarum $z$, demissum, sitque $a$ distantia intersectionis a polo positiuo circuli maximi, qui respondet plano ipsarum $x$, denique sit $b$ longitudo ipsius perpendiculi siue distantia loci geocentrici a circulo maximo distantiis $z$ respondente. Tunc erit $b$ latitudo aut declinatio geocentrica, prout planum distantiarum $z$ est ecliptica aut aequator; contra $a+N$ longitudo seu ascensio recta geocentrica, si $N$ designat in casu priori longitudinem in posteriori ascensionem rectam poli plani distantiarum $x$ Quamobrem erit

$$
\begin{aligned}
& x-X=\Delta \cos b \cos a \\
& y-Y=\Delta \cos b \sin a \\
& z-Z=\Delta \sin b
\end{aligned}
$$

Duae priores aequationes dabunt $a$ atque $\Delta \cos b$; quantitas posterior (quam positiuam fieri oportet) cum aequatione tertia combinata dabit $b$ atque $\Delta$.

*) In sensu latiori: proprie enim baec expressio ad cum casum refertur, vbi recta e terrae centro ducitur. 
61.

Tradidimus in praecedentibus methodum facillimam; corporis coelestis locum geocentricum respectu eclipticae seu aequatoris, a parallaxi liberum siue ea affectum, ac perinde a nutatione libertim seu ea affectum determinandi. Quod enim attinet ad nutationem, omnis differentia in eo versabitur, vtrum aequatoris positionem mediam adoptemus an veram, adeoque, in casu priori longitudines ab aequinoctio medio, in posteriori a vero numeremus, sicuti in casu illo eclipticae obliquitas media, in hoc vera adhibenda est. Ceterum sponte elucet, quo plures abbreuiationes in calculo coordinatarum introducantur, eo plures operationes praeliminares esse instituendas: quamobrem praestantia methodi supra explicatae, coordinatas immediate ex anomalia excentrica deducendi, tunc potissimum se manifestabit, vbi multos locos geocentricos determinare oportet: contra quoties vnus tantum locus computandus esset, aut perpauci, neutiquam operae pretium foret, laborem tot quantitates auxiliares calculandi suscipere. Quin potius in tali casu methodum vulgarem haud deserere praestabit, secundum quam ex anomalia excentrica deducitur vera atque radius vector; hinc locus heliocentricus respectu eclipticae; hinc longitudo et latitudo geocentrica, atque hinc tandem rectascensio et declinatio. Ne quid igitur hic deesse videatur, duas vltimas operationes adhuc breuiter explicabimus.

62.

Sit corporis coelestis longitudo heliocentrica $\lambda$, latitudo $\beta$; longitudo geocentrica $l$, latitudo $b$, distantia a Sole $r$, a terra $\Delta$; denique terrae longitudo heliocentrica $L$, latitudo $B$, distantia a Sole $R$. Quum non statuamus $B=0$, forsnulae nostrae ad eum quoque casum applicari poterunt, vbi loci heliocentrici et geocentricus non ad eclipticam sed ad quoduis aliud planum referuntur, modo denominationes longitudinis et latitudinis supprimere oportebit: praeterea parallaxeos ratio statim haberi potest, si modo locus heliocentricus terrae non ad centrum sed. ad locum in superficie immediate refertur. Statuamus porro $r \cos \beta=r^{\prime}, \Delta \cos b=\Delta^{\prime}$, $R \cos B=R^{\prime}$. Iam referendo locum corporis coelestis atque terrae in spatio ad tria plana, quorum vnum sit ecliptica, secundumque et tertium polos suos habeant in longitudine $N$ et $N+90^{\circ}$, protinus emergent aequationes sequentes:

$$
\begin{aligned}
& r^{\prime} \cos (\lambda-N)-R^{\prime} \cos (L-N)=\Delta^{\prime} \cos (l-N) \\
& r^{\prime} \sin (\lambda-N)-R^{\prime} \sin (L-N)=\Delta^{\prime} \sin (l-N) \\
& r^{\prime} \operatorname{tang} \beta-R^{\prime} \operatorname{tang} B=\Delta^{\prime} \operatorname{tang} b
\end{aligned}
$$


vbi angulus $N$ omnino arbitrarius est. Aequatio prima et secunda statim determinabunt $l-N$ atque $\Delta^{\prime}$, vnde et ex tertia demanabit $b$; ex $b$ et $\Delta^{\prime}$ habebis $\Delta$. Iam vt labor calculi quam commodissimus euadat, angulum arbitrarium $N$ tribus modis sequentibus determinamus:

I. Statuendo $N=L$, faciemus $\frac{r^{\prime}}{R^{\prime}} \sin (\lambda-L)=P, \frac{r^{\prime}}{R^{\prime}} \cos (\lambda-L)-\lambda$ $=Q$, inuenienturque $l-L, \frac{\Delta^{\prime}}{R^{\prime}}$ atque $b$ per formulas

$$
\begin{aligned}
& \operatorname{tang}(l-L)=\frac{P}{Q} \\
& \frac{\Delta^{\prime}}{R^{\prime}}=\frac{P}{\sin (l-L)}=\frac{Q}{\cos (l-L)} \\
& \operatorname{tang} b=\frac{\frac{r^{\prime}}{R^{\prime}} \operatorname{tang} \beta-\operatorname{tang} B}{\frac{\Delta^{\prime}}{R^{\prime}}}
\end{aligned}
$$
eritque

II. Statuendo $N=\lambda$, faciemus $\frac{R^{\prime}}{r^{\prime}} \sin (\lambda-L)=P, 1-\frac{R^{\prime}}{r^{\prime}} \cos (\lambda-L)=Q$,

$$
\begin{aligned}
& \operatorname{tang}(l-\lambda)=\frac{P}{Q} \\
& \frac{\Delta^{\prime}}{r^{\prime}}=\frac{P}{\sin (l-\lambda)}=\frac{Q}{\cos (l-\lambda)} \\
& \operatorname{tang} b=\frac{\operatorname{tang} \beta-\frac{R^{\prime}}{r^{\prime}} \operatorname{tang} B}{\frac{\Delta^{\prime}}{r^{\prime}}}
\end{aligned}
$$

III. Statuendo $N=\frac{x}{2}(\lambda+L)$, inuenientur $l$ atque $\Delta^{\prime}$ per aequationes

$$
\begin{aligned}
& \operatorname{tang}\left(l-\frac{r}{2}(\lambda+L)\right)=\frac{r^{\prime}+R^{\prime}}{r^{\prime}-R^{\prime}} \operatorname{tang} \frac{r}{2}(\lambda-L) \\
& \Delta^{\prime}=\frac{\left(r^{\prime}+R^{\prime}\right) \sin \frac{x}{2}(\lambda-L)}{\sin \left(l-\frac{x}{2}(\lambda+L)\right)}=\frac{\left(r^{\prime}-R^{\prime}\right) \cos \frac{x}{2}(\lambda-L)}{\cos \left(l-\frac{r}{2}(\lambda+L)\right)}
\end{aligned}
$$

ac dein $b$ per aequationem supra datam. Logarithmus fractionis $\frac{r^{\prime}+R^{\prime}}{r^{\prime}-R^{\prime}}$ commode calculatur, si statuitur $\frac{R^{\prime}}{r^{\prime}}=\operatorname{tang} \zeta$, vnde fit $\frac{r^{\prime}+R^{\prime}}{r-R^{\prime}}=\operatorname{tang}\left(45^{\circ}+\zeta\right)$. Hoc modo methodus III ad determinationem ipsius $l$ aliquanto breuior est, quam I et II, ad operationes reliquas autem has illi praeferendas censemus. 
63.

Exempli caussa calcultum in art. $5 \mathrm{z}$ vsque ad locum heliocentricum productum viterius continuamus. Respondeat illi loco longitudo heliocentrica terrae $24^{\circ} 19^{\prime} 49^{\prime \prime} \circ 5=L$, atque $\log R=9,9980979$; latitudinem $B$ statuimus $=0$. Habemus itaque $\lambda-L=-17^{\circ} 24^{\prime} 20^{\prime \prime} 07, \log R^{\prime}=\log R$, adeoque secundum methodum II,

$$
\begin{aligned}
& \log \frac{R^{\prime}}{r^{\prime}} \ldots \ldots \ldots \ldots \ldots . . . . .9,6729813 \quad \log (1-Q) \ldots \ldots 9,6526258 \\
& \log \sin (\lambda-L) \ldots . .9,4758655 n \quad 1-Q=0,4495925 \\
& \log \cos (\lambda-L) \ldots . .9,9796445 \quad Q=0,5506075
\end{aligned}
$$

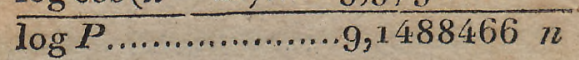

$$
\begin{aligned}
& \frac{\log Q \ldots \ldots \ldots \ldots \ldots . . . . .9,740842 \lambda}{\text { Hinc } l-\lambda=-14^{\circ} 21^{\prime} 6^{\prime \prime} 75} \quad \text { vnde } l=352^{\circ} 34^{\prime} 22^{\prime \prime} 25 \\
& \log \frac{\Delta^{\prime}}{r^{\prime}} \ldots \ldots \ldots \ldots \ldots . . . .9,7546117 \quad \text { vnde } \log \Delta^{\prime} \ldots 0,0797283 \\
& \frac{\log \operatorname{tang} \beta \ldots \ldots \ldots \ldots \ldots .8,8020996 n}{\log \operatorname{tang} b \ldots \ldots \ldots \ldots . . . .9,0474879 n} \quad \frac{\log \cos 6 \ldots \ldots .9,9973144}{\log \Delta \ldots \ldots \ldots \ldots, 0824139} \\
& b=-6^{\circ} 21^{\prime} 55^{\prime \prime} 07
\end{aligned}
$$

Secundum methodum III ex log tang $\xi=9,6729815$ habetur $\xi=25^{\circ} 13^{\prime} 6^{\prime \prime} 51$, adeoque

$$
\begin{aligned}
& \log \operatorname{tang}\left(45^{\circ}+\zeta\right) \ldots \ldots \ldots \ldots . . .0,4441091 \\
& \frac{\log \operatorname{tang} \frac{x}{2}(\lambda-L) \ldots \ldots \ldots . .9,1848938 n}{\log \operatorname{tang}\left(l-\frac{1}{2} \lambda-\frac{T}{2} L\right) \ldots 8,6290029 n}
\end{aligned}
$$

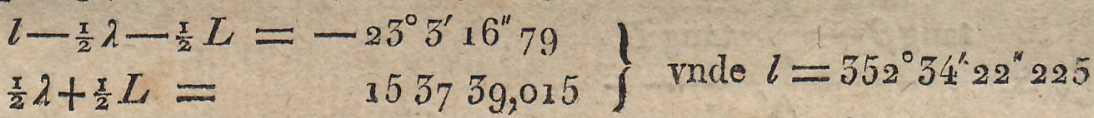

64.

Circa problema art. 62 sequentes adhuc obseruationes adiicimus.

I. Statuendo in aequatione secunda illic tradita $N=\lambda, N=L, N=l$, prodit $R^{\prime} \sin (\lambda-L)=\Delta^{\prime} \sin (l-\lambda) ; r^{\prime} \sin (\lambda-L)=\Delta^{\prime} \sin (l-L) ; r^{\prime} \sin (l-\lambda)=R^{\prime} \sin (l-L)$ aequatio prima aut secunda commode ad calculi confirmationem applicatur, si methodus I aut II. art. 62 adhibita est. Ita habetur in exemplo nostro

$$
\begin{aligned}
& \log \sin (\lambda-L) \ldots . .9,4758653 n \quad l-L=-31^{\circ} 45^{\prime}{ }_{2}^{\prime \prime} 82 \\
& \frac{\log \frac{\Delta^{\prime}}{r^{\prime}} \ldots \ldots \ldots \ldots 9,7546117}{9,77^{212536 n}} \\
& \log \sin (l-L) \ldots \ldots . .9,7212556 n
\end{aligned}
$$


II. Sol duoque in plano eclipticae puncta, quae sunt proiectiones loci corporis coelestis atque loci terrae, triangulum planum formant, cuius Iatera sunt $\Delta^{\prime}, R^{\prime}, r^{\prime}$, angulique oppositi vel $\lambda-L, l-\lambda, 180^{\circ}-l+L$, vel $L-\lambda, \lambda-l$, $180^{\circ}-L+l$ : ex hoc principio relationes in I traditae sponte sequuntur.

III. Sol, locus verus corporis coelestis in spatio, locusque verus terrae aliud triangulum formabunt, cuius latera erunt $\Delta, R, r$ : angulis itaque his resp. oppositis per $S, T, 180^{\circ}-S-T$ denotatis, erit $\frac{\sin S}{\Delta}=\frac{\sin T}{R}=\frac{\sin (S+T)}{r}$. Planum huius trianguli in sphaera coelesti circulum maximum proïciet, in quo locus heliocentricus terrae, locus heliocentricus corporis coelestis eiusdemque locus geocentricus siti erunt, et quidem ita vt distantia secundi a primo, tertii a secundo, tertii a primo, secundum eandem directionem numeratae, resp. sint $S, T, S+T$.

IV. Vel ex notis variationibus differentialibus partium trianguli plani, vel. aeque facile e formulis art. 62 sequentes aequationes differentiales deriuantur:

$$
\begin{aligned}
\mathrm{d} l= & \frac{r^{\prime} \cos (\lambda-l)}{\Delta^{\prime}} \mathrm{d} \lambda+\frac{\sin (\lambda-l)}{\Delta^{\prime}} \mathrm{d} r^{\prime} \\
\mathrm{d} \Delta^{\prime}=-r^{\prime} \sin (\lambda-l) \mathrm{d} \lambda+\cos (\lambda-l) \mathrm{d} r^{\prime} & \frac{r^{\prime} \cos b \sin b \sin (\lambda-l)}{\Delta^{\prime}} \mathrm{d} \lambda+\frac{r^{\prime} \cos b^{2}}{\Delta^{\prime} \cos \beta^{2}} \mathrm{~d} \beta+ \\
\mathrm{d} b= & \frac{\cos b^{2}}{\Delta^{\prime}}(\tan g-\cos (\lambda-l) \tan g) \mathrm{d} r^{\prime}
\end{aligned}
$$

vbi partes quae continent $\mathrm{d} r^{\prime}, \mathrm{d} \Delta^{\prime}$ per 206265 sunt multiplicandae, vel reliquae per 206265 diuidendae, si mutationes angulorum in minutis secundis exprimuntur.

V. Problema inuersum, scilicet determinatio loci heliocentrici e geocentrico problemati supra euoluto prorsus analogum est, quamobrem superfluum foret, illi amplius inhaerere. Omnes enim formulae art. 62 etiam pro illo problemate valent, si modo omnibus quantitatibus quae ad locum corporis coelestis geocentricum spectant cum analogis iis quae ad geocentricum referuntur permutatis, pro $L, B$ resp: substituitur $L+180^{\circ},-B$, siue quod idem est pro loco heliocentrico terrae geo= centricus solis accipitur.

65.

Etiamsi in eo casu, vbi ex elementis datis paucissimi tantum loci geocentrici sunt determinandi, omnia artificia supra tradita, per quae ab anomalia excentrica station ad Iongitudinem et latitudinem geocentricam, vel adeo ad rectascensio- 
nem et declinationem, transire licet, in vsum vocare vix operae pretinm sit, quoniam compendia inde demanantia a multitudine quantitatum auxiliarium antea computandarum absorberentur: semper tamen contractio reductionis ad eclipticam cum calculo longitudinis et latitudinis geocentricae lucrum haud spernendum praestabit. Si enim pro plano coordinatarum $z$ assumitur ipsa ecliptica, poli antem planorum coordinatarum $x, y$ collocantur in longitudine $\delta, 90^{\circ}+\delta$, coordinatae facillime absque vlla quantitatum auxiliarium necessitate determinantur. Habetur scilicet

$$
\begin{array}{l|l|l}
x=r \cos u & X=R^{\prime} \cos \left(L_{1}-\Omega\right) \\
y=r \cos i \sin u \\
z=r \sin i \sin u
\end{array} \quad \begin{aligned}
& x-X=\Delta^{\prime} \cos (Z-\Omega) \\
& Y=R^{\prime} \sin (L-\Omega) \\
& Z=R^{\prime} \operatorname{tang} B
\end{aligned} \quad \begin{aligned}
& y-Y=\Delta^{\prime} \sin (l-\Omega) \\
& z-Z=\Delta^{\prime} \operatorname{tang} b
\end{aligned}
$$

\begin{tabular}{|c|c|}
\hline 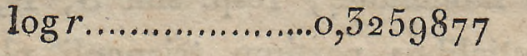 & …...9,9980979 \\
\hline $\log \cos u \ldots \ldots \ldots \ldots \ldots .9,9824141$ & $\log \cos (L-\Omega) \ldots .9,9226027 n$ \\
\hline $\log \sin u \ldots \ldots \ldots \ldots . . . .9,4454714 n$ & $\log \sin (L-86) \ldots 9,7384353 n$ \\
\hline $\log x \ldots \ldots \ldots \ldots \ldots \ldots, 3084018 n$ & $\log X \ldots \ldots \ldots \ldots \ldots \ldots, 9,9207006 n$ \\
\hline $\log r \sin u \ldots \ldots \ldots \ldots, 9,7714591$ & \\
\hline $\log \cos i \ldots \ldots \ldots \ldots . . . . .9,9885266$ & \\
\hline $\log \sin i \ldots \ldots \ldots \ldots . . .9,5557570$ & \\
\hline $\log y \ldots \ldots \ldots \ldots \ldots \ldots . ., 9,7599857$ & $\log Y \ldots \ldots \ldots \ldots \ldots, 9,7565352 n$ \\
\hline $\log z \ldots \ldots \ldots \ldots \ldots \ldots . .6,1272161$ & $Z=$ \\
\hline
\end{tabular}

Quoties $B=0$, est $R^{\prime}=R, Z=0$. Secundum has formulas exemplum nostrum numeris sequentibus absoluitur: $L-\Omega=215^{\circ} 12^{\prime} 0^{\prime \prime} 52$

Hinc fit

$$
\begin{aligned}
& \log (x-X) \ldots \ldots . .0,0795906 n \\
& \log (y-Y) \ldots \ldots . .8,4807165 n \\
& \text { vide }(l-\Omega)=181^{\circ} 26^{\prime} 33^{\prime \prime} 49 \quad \quad l= \\
& \log \Delta^{\prime} \ldots \ldots \ldots \ldots \ldots . \ldots, 0797285 \\
& \log \operatorname{tang} b \ldots \ldots \ldots \ldots . . \ldots, 0474878 n \quad b=
\end{aligned}
$$

66.

E longitudine et latitudine puncti cuiusuis in sphaera coelesti eius rectascensio et declinatio deriuantur per solutionem trianguli sphaerici, quod ab illo puncto polisque arcticis eclipticae et aequatoris formatur. Sit $\varepsilon$ obliquitas eclipticae, $l$ longitudo, $b$ latitudo, $\alpha$ ascensio recta, $\delta$ declinatio, eruntque trianguli latera $\varepsilon$, $90^{\circ}-b, 90^{\circ}-\delta$; pro angulis lateri secundo et tertio oppositis accipere licebit' $90^{\circ}+\alpha$, $90^{\circ}-l$ (siquidem trianguli sphaerici ideam maxima generalitate concipimus); angu- 
Ium tertium lateri $\&$ oppositum statuemus $=90^{\circ}-E$. Habebimus itaque per formulas art. 54 .

$$
\begin{aligned}
& \sin \left(45^{\circ}-\frac{x}{2} \delta\right) \sin \frac{x}{2}(E+\alpha)=\sin \left(45^{\circ}+\frac{\pi}{2} l\right) \sin \left(45^{\circ}-\frac{\pi}{2}(\varepsilon+b)\right) \\
& \sin \left(45^{\circ}-\frac{x}{2} \delta\right) \cos \frac{x}{2}(E+\alpha)=\cos \left(45^{\circ}+\frac{x}{2} l\right) \cos \left(45^{\circ}-\frac{x}{2}(\varepsilon-b)\right) \\
& \cos \left(45^{\circ}-\frac{x}{2} \delta\right) \sin \frac{x}{2}(E-\alpha)=\cos \left(45^{\circ}+\frac{x}{2} l\right) \sin \left(45^{\circ}-\frac{x}{2}(\varepsilon-)\right) \\
& \cos \left(45^{\circ}-\frac{x}{2} \delta\right) \cos \frac{\pi}{2}(E-\alpha)=\sin \left(45^{\circ}+\frac{1}{2} l\right) \cos \left(45^{\circ}-\frac{x}{2}(\varepsilon+b)\right)
\end{aligned}
$$

Aequationes duae primae dabunt $\frac{x}{2}(\boldsymbol{E}+\alpha)$ atque $\sin \left(45^{\circ}-\frac{x}{2} \delta\right)$; duae vltimae $\frac{x}{2}(\boldsymbol{E}-\alpha)$ atque $\cos \left(45^{\circ}-\frac{r}{2} \delta\right)$; ex $\frac{x}{2}(E+\alpha)$ et $\frac{r}{2}(E-\alpha)$ habebitur $\alpha$ simulque $E$; ex $\sin \left(45^{\circ}-\frac{x}{2} \delta\right)$ aut $\cos \left(45^{\circ}-\frac{x}{2} \delta\right)$, quorum consensus calculo confirmando inseruiet, determinabitur $45^{\circ}-\frac{1}{2} \delta$ atque hinc $\delta$. Determinatio angulorum $\frac{x}{2}(E+\alpha), \frac{1}{2}(E-\alpha)$ per tangentes suos ambiguitati non est obnoxia, quoniam tum sinus tum cosinus anguli $45^{\circ}-\frac{x}{2} \delta$ pasitiuus euadere debet.

Mutationes differentiales quantitatum $\alpha, \delta$ e mutationibus ipsarum $b, b$ secundum principia nota ita inueniuntur:

$$
\begin{aligned}
& \mathrm{d} \alpha=\frac{\sin E \cos b}{\cos \delta} \mathrm{d} l-\frac{\cos E}{\cos \delta} \mathrm{d} b \\
& \mathrm{~d} \delta=\cos E \cos b \mathrm{~d} l+\sin E \mathrm{~d} b
\end{aligned}
$$

$$
67 .
$$

Methodus alia, problema art. praec. soluendi, ex aequationibus

$\cos \varepsilon \sin l=\sin \varepsilon \operatorname{tang} b+\cos l \operatorname{tang} \alpha$

$$
\sin \delta=\cos \varepsilon \sin b+\sin \varepsilon \cos b \sin l
$$$$
\cos b \cos l=\cos \alpha \cos \delta
$$

petitur. Determinetur angulus auxiliaris $\theta$ per aequationem

$$
\begin{aligned}
& \operatorname{tang} \theta=\frac{\operatorname{tang} b}{\sin l}, \text { eritque } \\
& \operatorname{tang} \alpha=\frac{\cos (\varepsilon+\theta) \operatorname{tang} l}{\cos \theta} \\
& \operatorname{tang} \delta=\sin \alpha \operatorname{tang}(\varepsilon+\theta)
\end{aligned}
$$

quibus aequationibus ad calculi confirmationem adiici potest

$$
\cos \delta=\frac{\cos b \cos l}{\cos \alpha} \text { siue } \cos \delta=\frac{\cos (\varepsilon+\theta) \cos b \sin \tau}{\cos \theta \sin \alpha}
$$

Ambiguitas in determinatione ipsius $\propto$ per aequ. secundam eo tollitur, quiod $\cos \alpha$ et $\cos l$ eadem signa habere debent. 
Haec methodus minus expedita est, si praeter $\alpha$ et $\delta$ etiam $E$ desideratur: formula commodissima ad hunc angulum determinandun tunc erit $\cos E=\frac{\sin \varepsilon \cos \alpha}{\cos b}$ $=\frac{\sin \varepsilon \cos l}{\cos \delta}$. Sed per hanc formulam $E$ accurate computari nequit, quoties $\pm \cos E$ parum ab vnitate differt; praeterea ambiguitas remanet, vtrum $E$ inter o "et $180^{\circ}$ an inter $180^{\circ}$ et $560^{\circ}$ accipere oporteat. Incommodum prius raro vllius momenti est, praesertim, quum ad computandas rationes differentiales vltima praecisio in ralore ipsius $E$ non requiratur: ambiguitas vero illa adiumento aequationis $\cos b \cos \delta \sin E$ $=\cos \varepsilon-\sin b \sin \delta$ facile tollitur, quae ostendit $E$ inter o et $180^{\circ}$, vel inter $180^{\circ}$ et $360^{\circ}$ accipi debere, prout $\cos \varepsilon$ maior fuerit vel minor quam $\sin b \sin \delta$ : manifesto hoc examen ne necessarium quidem est, quoties alteruter angulorum $b, \delta$ limitem $66^{\circ} 32^{\prime}$ non egreditur: tune enim $\sin E$ semper fiet positiuus. Ceterum eadem aequatio in casu supra addigitato ad determinationem exactiorem ipsius $E$, si operae pretium videtur, adhiberi poterit.

\section{8.}

Solutio problematis inuersi, putä determinatio longitudinis et latitudinis ex ascensione recta et declinatione, eidem triangulo sphaerico superstruitur: formulae itaque supra traditae huic fini accommodabuntur per solam permutationem ipsius $b$ cum $\delta$, ipsiusque $l$ cum $-\alpha$. Etiam has formulas, propter vstum frequentem, hic apposuisse haud pigebit:

Secundum methodum art. 66 habemus

$$
\begin{aligned}
& \sin \left(45^{\circ}-\frac{\pi}{2} b\right) \sin \frac{\pi}{2}(E-l)=\cos \left(45^{\circ}+\frac{\gamma}{2} \alpha\right) \sin \left(45^{\circ}-\frac{x}{2}(\varepsilon+\delta)\right) \\
& \sin \left(45^{\circ}-\frac{\pi}{2} b\right) \cos \frac{\pi}{2}(E-l)=\sin \left(45^{\circ}+\frac{x}{2} \alpha\right) \cos \left(45^{\circ}-\frac{x}{2}(\varepsilon-\delta)\right) \\
& \cos \left(45^{\circ}-\frac{\pi}{2} b\right) \sin \frac{\pi}{2}(E+l)=\sin \left(45^{\circ}+\frac{\pi}{2} \alpha\right) \sin \left(45^{\circ}-\frac{x}{2}(\varepsilon-\delta)\right) \\
& \cos \left(45^{\circ}-\frac{\pi}{2} b\right) \cos \frac{\pi}{2}(E+l)=\cos \left(45^{\circ}+\frac{\pi}{2} \alpha\right) \cos \left(45^{\circ}-\frac{\pi}{2}(\varepsilon+\delta)\right)
\end{aligned}
$$

Contra ad instar methodi alterius art. 67 determinabimus angulum atuxiliarem $\zeta$ per aequationem

$$
\begin{aligned}
& \operatorname{tang} \zeta=\frac{\operatorname{tang} \delta}{\sin \alpha}, \text { eritque } \\
& \operatorname{tang} l=\frac{\cos (\zeta-\varepsilon) \operatorname{tang} \alpha}{\cos \zeta} \\
& \operatorname{tang} b=\sin l \operatorname{tang}(\zeta-\varepsilon)
\end{aligned}
$$


RELATIONES AD LOCVM STMPLICEM IN SEATIO SPFCTANTES.

Ad calculi confirmationem adiungi poterit

$$
\cos b=\frac{\cos \delta \cos \alpha}{\cos b}=\frac{\cos (\zeta-\varepsilon) \cos \delta \sin \alpha}{\cos \theta \sin l}
$$

Pro determinatione ipsius $E$ inseruient perinde $\mathrm{vt}$ in art. praec. aequationes

$$
\begin{aligned}
& \cos E=\frac{\sin \varepsilon \cos \alpha}{\cos b}=\frac{\sin \varepsilon \cos l}{\cos \delta} \\
& \cos b \cos \delta \sin E=\cos \varepsilon-\sin b \sin \delta
\end{aligned}
$$

Variationes differentiales ipsarum $l, b$ hisce formulis exhibebuntux:

$$
\begin{aligned}
& \mathrm{d} l=\frac{\sin E \cos \delta}{\cos b} \mathrm{~d} \alpha+\frac{\cos E}{\cos b} \mathrm{~d} \delta \\
& \mathrm{d} b=-\cos E \cos \delta \mathrm{d} \alpha+\sin E \mathrm{~d} \delta
\end{aligned}
$$

69.

Exempli caussa ex ascensione recta $355^{\circ} 45^{\prime} 45^{\prime \prime} 30=\alpha$, declinatione $-8^{\circ} 47^{\prime} 25^{\prime \prime}=\delta$, obliquitate eclipticae $23^{\circ} 27^{\prime} 59^{\prime \prime} 26=\varepsilon$ longitudinem et latitudinem computabimus. Est igitur $45^{\circ}+\frac{T}{2} \alpha=222^{\circ} 51^{\prime} 52^{\prime \prime} 65,45^{\circ}-\frac{1}{2}(\varepsilon+\delta)=37^{\circ} 39^{\prime} 42^{\prime \prime} 87$, $45^{\circ}-\frac{x}{2}(\varepsilon-\delta)=28^{\circ} 52^{\prime} 17^{\prime \prime} 87$; hinc porro

$\log \cos \left(45^{\circ}+\frac{x}{2} \alpha\right) \ldots \ldots \ldots \ldots \ldots . . \ldots, 8650820 n \quad \log \sin \left(45^{\circ}+\frac{\pi}{2} \alpha\right) \ldots \ldots \ldots \ldots \ldots . . .9,8326803 n$

$\log \sin \left(45^{\circ}-\frac{x}{2}(\varepsilon+\delta)\right) \ldots . .9,7860418 \quad \log \sin \left(45^{\circ}-\frac{x}{2}(\varepsilon-\delta)\right) \ldots . .9,6838112$

$\frac{\log \cos \left(45^{\circ}-\frac{\pi}{2}(\varepsilon+\delta)\right) \ldots . .9,8985222 \quad \log \cos \left(45^{\circ}-\frac{x}{2}(\varepsilon-\delta)\right) \ldots . .9,9423572}{\log \sin \left(45^{\circ}-\frac{\pi}{2} b\right) \sin \frac{x}{2}(E-l) \ldots \ldots \ldots .9,6511258 \pi}$

$\log \sin \left(45^{\circ}-\frac{r}{2} b\right) \cos \frac{x}{2}(E-l) \ldots \ldots . .9,7750375 n$

rnde $\frac{x}{2}(E-Z)=216^{\circ} 56^{\prime} 5^{\prime \prime} 59 ; \operatorname{Iog} \sin \left(45^{\circ}-\frac{x}{2} b\right)=9,8725171$

$\log \cos \left(45^{\circ}-\frac{x}{2} b\right) \sin \frac{x}{2}(E+l) \ldots \ldots . .9,5164915 n$

$\log \cos \left(45^{\circ}-\frac{\pi}{2} b\right) \cos \frac{1}{2}(E+l) \ldots \ldots .9,7656042 n$

Fnde $\frac{x}{2}(E+l)=209^{\circ} 50^{\prime} 49^{\prime \prime} 9^{4}: \log \cos \left(45^{\circ}-\frac{\pi}{2} b\right)=9,8239669$

Fit itaque $E=426^{\circ} 26^{\prime} 55^{\prime \prime} 35, l=-7^{\circ} 25^{\prime} 15^{\prime \prime} 45$, sine quod eodem redit $E=66^{\circ} 26^{\prime} 55^{\prime \prime} 35$, $l=352^{\circ} 34^{\prime} 44^{\prime \prime} 55$; angulus $45^{\circ}-\frac{x}{2} b$ e logarithmo sinus habetur $48^{\circ} 10^{\prime} 58^{\prime \prime}{ }_{12}$, e $\log -$ arithmo cosinus $48^{\circ} 10^{\prime} 58^{\prime \prime} 17$, e tangente, cuius logarithmus illorum differentia est, $38^{\circ} \times 0^{\prime} 58^{\prime \prime} 14 ;$ hinc $b=-6^{\circ} 21^{\prime} 56^{\prime \prime} 28$. 
Secundum methodum alteram calculus ita se habet:

$$
\begin{aligned}
& \log \tan g \delta \ldots . .9,1893062 n \\
& \text { C. } \log \cos \xi \ldots \ldots \ldots . . .0,3626190 \\
& \frac{\log \sin \alpha \ldots \ldots . .8,8719-92 n}{\log \tan 66 \ldots \ldots . . .0,3175270} \\
& \log \cos (\zeta-\varepsilon) \ldots \ldots .9,8789705 \\
& \zeta=64^{\circ} 17^{\prime} 6^{\prime \prime} 85 \\
& \zeta-\varepsilon=40497,57 \\
& \frac{\log \tan g \alpha \ldots \ldots \ldots . .8,8731869 n}{\log \tan g l \ldots \ldots \ldots \ldots . . . .9,1147762 n} \\
& \log \sin t \ldots \ldots \ldots \ldots \ldots . . .9,1171252 \quad n \\
& \frac{\log \operatorname{tang}(\zeta-\varepsilon) \ldots . .9,9565874}{\log \operatorname{tang} b \ldots \ldots \ldots \ldots . . .09,0475106 n} \\
& b=-6^{\circ} 21^{\prime} 56^{\prime \prime} 26
\end{aligned}
$$

\begin{tabular}{|c|c|}
\hline $\log \sin \varepsilon \ldots \ldots \ldots . . . .9,600 \pi x 44$ & $\log \sin 8 \ldots \ldots \ldots \ldots 9,6001144$ \\
\hline $\log \cos \alpha \ldots \ldots \ldots . . .9,9987924$ & $\log \cos l \ldots \ldots \ldots \ldots . . . .9,9963470$ \\
\hline C. $\log \cos b \ldots \ldots . .0,0026859$ & C. $\log \cos \delta \ldots \ldots . .0,0051513$ \\
\hline $\log \cos E \ldots \ldots \ldots \ldots . .6,60159^{2} 7$ & $\overline{\log \cos E \ldots \ldots \ldots . . . .69,6015927}$ \\
\hline vnde $E=66^{\circ} 26^{\prime} 55^{\prime \prime} 35$ & \\
\hline
\end{tabular}

Ad determinandum angulum $E$ habemus calculum duplicem:

70.

Ne quid eorum, quae ad calculum locorum geocentricorum requiruntur, hic desideretur, quaedam adhuc de parallaxi atque aberratione adiicienda sunt. Methodum quidem supra iam descripsimus, secundum quam locus parallaxi affectus, i. c. cuilibet in superficie terrae puncto respondens, immediate maximaque facilitate determinari potest: sed quum in methodo vulgari in art. 62 et sequ. tradita locus geocentricus ad terrae centrum referri soleat, in quo casu a parallaxi liber dicitur, methodum peculiarem pro determinanda parallaxi, quae est inter vtrumque locum differentia, adiicere oportebit.

Sint corporis coelestis longitudo et latitudo geocentrica respectu centri terrae $\lambda, \beta$; eaedem respectu puncti cuiusuis in superficie terrae $l, b$; distantia corporis a terrae centro $r$, a puncto superficiei $\Delta$; denique respondeat in sphaera coelesti ipsi zenith huius puncti longitudo $L$, latitado $B$, designeturque radius terrae per $R$. Sponte iam patet, omnes aequationes art. 62 etiam hic locum esse habituras; sed notabiliter contrahi poterunt, quum $R$ hic exprimat quantitatem prae $x$ et $\Delta$ tantum non enanescentem. Ceterum eaedem aequationes manifesto etiamnum valebunt, si $\lambda, l, L$ pro longitudinibus ascensiones rectas, atque $\beta, b, B$ pro latitudinibus declinationes exprimunt. In hoc casu $l-\lambda, b-\beta$ erunt parallaxes 
ascensionis rectae et declinationis, in illo vero parallaxes longitudinis et latitudinis. Quodsi iam $R$ vt quantitas primi ordinis tractatur, eiusdem ordinis erunt $l-\lambda$, $b-\beta, \Delta-r$, neglectisque ordinibus superioribus e formulis art. 62 facile derivabiur:

$$
\begin{aligned}
& \text { I. } l-\lambda=\frac{R \cos B \sin (\lambda-L)}{r \cos \beta} \\
& \text { II. } \delta-\beta=\frac{R \cos B \cos \beta}{r}(\operatorname{tang} \beta \cos (\lambda-L)-\operatorname{tang} B) \\
& \text { III. } \Delta-r=-R \cos B \sin \beta(\operatorname{cotang} \beta \cos (\lambda-L)+\operatorname{tang} B)
\end{aligned}
$$

Accipiendo angulum auxiliarem $\theta$ ita vt fiat $\operatorname{tang} \theta=\frac{\operatorname{tang} B}{\cos (\lambda-L)}$, aequationes II, III formam sequentem nanciscuntur:

$$
\begin{aligned}
& \text { II. } b-\beta=\frac{R \cos B \cos (\lambda-L) \sin (\beta-\theta)}{r \cos \theta}=\frac{R \sin B \sin (\beta-\theta)}{r \sin \theta} \\
& \text { III. } \Delta-r=-\frac{R \cos B \cos (\lambda-L) \cos (\beta-\theta)}{\cos \theta}=-\frac{R \sin B \cos (\beta-\theta)}{\sin \theta}
\end{aligned}
$$

Ceterum patet, vt in I et II $l-\lambda$ et $b-\beta$ in minutis secundis obtineantur, pro $R$ accipi debere parallaxem mediam solarem in minutis secundis expressam; in III vero pro $R$ eadem parallaxis per $206265^{\prime \prime}$ diuisa accipienda est. Tandem nullo praecisionis detrimento in valoribus parallaxium pro $r, \lambda, \beta$, adhibere licebit $\Delta, l, b_{8}$ quoties in problemate inuerso e loco parallaxi affecto locum ab eadem liberum determinare oportet.

Exemplum. Sit ascensio recta Solis pro centro terrae $220^{\circ} 46^{\prime \prime} 44^{\prime \prime} 65=\lambda$, declinatio $-15^{\circ} 49^{\prime} 43^{\prime \prime} 9^{4}=\beta$, distantia $0,9904311=r$; porro tempus sidereum in aliquo loco in terrae superficie gradibus expressa $78^{\circ} 20^{\prime} 38^{\prime \prime}=L$, loci eleuatio poli $45^{\circ} 27^{\prime} 57^{\prime \prime}=B$, parallaxis media solaris $8^{\prime \prime} 6=R$. Quaeritur locus Solis ex hoc loco visus, distantiaque ab eodem.

$$
\begin{aligned}
& \log R \ldots \ldots \ldots \ldots \ldots \ldots . . . . .0,93450 \\
& \log \cos B \ldots \ldots \ldots \ldots \ldots . . . .9,84593 \\
& \text { C. } \log r \ldots \ldots \ldots \ldots \ldots . . \ldots, 00418 \\
& \text { C. } \log \cos \beta \ldots \ldots \ldots . .0,01679 \\
& \frac{\log \sin (\lambda-L) \ldots \ldots .9,78508}{\log (l-\lambda) \ldots \ldots \ldots \ldots ., 58648} \\
& -\lambda=+3^{\prime \prime} 86 \\
& l=\quad 220^{\circ} 46^{\prime} 48^{\prime \prime} 5 x \\
& \log R \text {................. } 0,95450 \\
& \log \sin B \ldots \ldots \ldots \ldots . . .9,85299 \\
& \text { C. } \log r \ldots \ldots \ldots \ldots \ldots, 0,00418^{\text {, }} \\
& \text { C. } \log \sin \theta \ldots \ldots \ldots \ldots, 10317 \\
& \frac{\log \sin (\beta-\theta) \ldots \ldots .9,77152 n}{\log (b-\beta) \ldots \ldots \ldots \ldots, 66627 n} \\
& b-\beta=\quad-4^{\prime \prime} 64 \\
& b=-15^{\circ} 49^{\prime} 43^{\prime \prime} 86
\end{aligned}
$$




$$
\begin{aligned}
& \log \operatorname{tang} B \ldots \ldots \ldots \ldots ., 00706 \\
& \frac{\log \cos (\lambda-L) \ldots \ldots 9,89909 n}{\log \tan \theta \ldots \ldots \ldots \ldots .0,10797 n} \\
& \theta=\quad 127^{\circ} 57^{\prime \prime} o^{\prime \prime} \\
& \beta-\theta=-145^{\circ} 46^{\prime} 44^{\prime \prime}
\end{aligned}
$$

$$
\begin{aligned}
& \log (b-\beta) \ldots \ldots \ldots . .0,66627 n \\
& \log \cot (\beta-\theta) \ldots \ldots . .0,15522 \\
& \log r \ldots \ldots \ldots \ldots \ldots \ldots . \ldots, 99582 \\
& \log 1^{\prime \prime} \ldots \ldots \ldots \ldots \ldots \ldots ., 68557 \\
& \log (r-\Delta) \ldots \ldots \ldots .5,48288 n \\
& r-\Delta=-0,0000304 \\
& \Delta \quad=\quad 0,9904615
\end{aligned}
$$

7.

Aberratio fixarum, nee non pars ea aberrationis planetarum et cometarum quae soli motui terrae debetur, oritur inde, quod cum terra integra tubus mouetur, dum radius luminis ipsius axem opticum percurrit. Corporis coelestis locus obseruatus (qui et apparens seu aberratione affectus dicitur) determinatur per situm axis optici telescopii ita collocati, vt radius luminis ab illo egressus in via sua vtramque huius axis extremitatem attingat: hic autem situs diuersus est a situ vero radii luminis in spatio. Distinguamus duo temporis momenta $t$, $t^{\prime}$, vbi radius $\mathrm{lu}-$ minis extremitatem anteriorem (centrum vitri obiectiui), vbique posteriorem (focum vitri obiectiui) attingit; sint harum extremitatum loci in spatio pro momento priori $a, b$; pro posteriori $a^{\prime}, b^{\prime}$. Tunc patet, rectam $a b^{\wedge}$ esse situm verum radii in spatio, loco apparenti autem respondere rectam $a b$ vel $a^{\prime} b^{\prime}$ (quas pro parallelis habere licet): nullo porro negotio perspicitur, locum apparentem a longitudine tubi non pendere. Differentia inter situm rectarum $b^{\prime} a, b a$ est aberratio qualis pro stellis fixis locum habet: modum eam calculandi hic tamquam notum silentio transimus. Pro stellis errantibus autem ista differentia nondum est aberratio completa: planeta scilicet, dum radius ex ipso egressus ad terram descendit, locum suum ipse mutat, quapropter situs huius radii non respondet loco geocentrico vero tempore obseruationis. Supponamus, radium luminis qui tempore $t$ in tubum mpin= git tempore $T$ e planeta egressum esse; designeturque locus planetae in spatio tempore $T$ per $P$, tempore $t$ autem per $p$; denique sit $A$ locus extremitatis antecedentis axis tubi pro tempore $T$. Tunc patet

$I^{\circ}$ rectam $A P$ exhibere locum verum planetae tempore $T$.

$2^{\circ}$ rectam ap autem locum verum tempore $t$.

$3^{\prime}$ rectam $b a$ vel $b^{\prime} a^{\prime}$ locum apparentem tempore $t$ vel $t^{\prime}$ (quorum differentia ceu quantitas infinite parua spectari potest).

$4^{\circ}$ rectam $b^{\prime} a$ eundem locum apparentem ab aberratione fixarum purgatum. 
Iam puncta $P, \alpha, b^{\prime}$ in linea recta iacent, eruntque partes $P a, a b^{\prime}$ proportionales temporum interuallis $t-T, t^{\prime}-t$, siquidem motus luminis celeritate vniformi peragitur. Temporis intervallum $t^{\prime}-T$ propter immensam luminis velocitatem semper est perparuum, intra quod motum terrae tanquam rectilineum ac celcritate vniformi peractum supponere licet: sic etiam $A, a, a^{\prime}$ in directum iacebunt, partesque $A a, a a^{\prime}$ quoque interuallis $t-T, t^{\prime}-t$ proportionales erunt. Hinc $f a-$ cile concluditur, rectas $A P, b^{\prime} a^{\prime}$ esse parallelas, adeoque locum primum cum tertio identicum.

Tempus $t-T$ erit productum distantiae $P a$ in $495^{*}$, intra quod lumen percurrit distantiam mediam terrae a Sole, quam pro vnitate accepimus. In hoc calculo pro distantia $P_{a}$ etiam $P A$ vel $p a$ accipere licebit, quum differentia nulJius momenti esse possit.

Ex his principuis tres demanant methodi, planetae vel cometae locum apparentem pro quouis tempore $t$ determininandi, e quibus modo hane modo illam praeferre conueniet.

I. Subtrahatur a tempore proposito tempus intra quod lumen a planeta ad terram descendit: sic prodibit tempus reductum $T$, pro quo locus verus more solito computatus cum apparente pro $t$ identicus erit. Ad computum reductionis temporis $t-T$ distantiam a terra nouisse oportet: plerumque ad hunc finem subsidia commada non deerunt e. g. per ephemeridem rel leui tantum calamo calculatam, alioquin distantiam reram pro tempore $t$ more solito sed neglecta praecisione nimia per calculum praeliminarem determinare sufficiet.

II. Computetur pro tempore proposito $t$ locus rerus atque distantia, ex hac reductio temporis $t-T$, atque hinc adiumento motus diumi (in longitudine et latitudine vel in ascensione recta et declinatione) reductio loci veri ad tempus $T$.

III. Computetur locus heliocentricus terrae quidem pro tempore $t$ : locus heliocentricus planetae autem pro tempore $T$ : dein ex horum combinatione more solito locus geocentricus planetae, qui aberratione fixarum (per methodum notam: eruenda siue e tabulis depromenda) auctus locum apparentem quaesitum suppeditabit.

Methodis secunda, quae vulgo in vsum vocari solet, eo quidem prae reliquis se commendat, quod ad distantiam determinandam numquam opus est ealculo: duplici, attamen eo laborat incommodo, quod adhiberi nequit, nisi plures loci vicini vel calculentur vel ex obseruationibus iam innotuerint; alioguin enim motum diurnum pro dato habere non liceret. 
Incommodum, quo methodus prima et tertia premuntur, plane tollitur quoties plures loci sibi vicini calculandi sunt. Quam primum enim pro quibusdarn distantiae iam innotuerunt, percommode et praecisione sufficiente distantias proxime sequentes per subsidia trita concludere licebit. Ceterum si distantia est nota, methodus prima tertiae ideo plerumque praeferenda erit, quod aberratione fixarum opus non habet; sin vero ad calculum duplicem refugiendum est, tertia eo se commendat, quod in calculo altero locus terrae saltem retinendus est.

Sponte iam se offerunt, quae ad problema inuersum requiruntur, puta si e loco apparente verns deriuandus est. Scilicet secundum methodum I retinebis locum ipsum immutatum, sed tempus $t$, cui locus propositus vt apparens respondet, conuertes in reductum $T$, cui idem tamquam verus respondebit. Secundum methodum II retinebis tempus $t$, sed loco proposito adiicies motum intra tempus $t-T$, quasi istum ad tempus $t+(t-T)$ reducere velles. Secundum methodum III locum. propositum ab aberratione fixarum liberatum tamquam locum verum pro tempore $T$ considerabis, sed terrae locus verus tempori $t$ respondens retinendus est ac si ad istud pertineret. Vtilitas methodi tertiae in Libro secundo clarius elucebit.

Ceterum, ne quid desit, adhuc obseruamus, locum Solis ab aberratione perinde affici ac locum planetae: sed quoniam tum distantia a terra tum motus diurnus propemodum sunt constantes, aberratio ipsa semper valorem tantum non constantem obtinet motui medio solis in $495^{\prime \prime}$ aequalem, adeoque $=20^{\prime \prime}{ }_{2} 5$, quac quantitas a longitudine vera subtrahenda est vt media prodeat. Valor aberrationis exactus est in ratione composita distantiae et motus diurni, siue quod eodem redit in ratione inuersa distantiae, vnde ille valor medius in apogeo o" 34 diminuendus in perigeo tantumdem augendus esset. Ceterum tabulae nostrae solares aberrationem constantem - 20 " 25 iam includunt; quapropter ad obtinendum longitudinem veram tabulari $20^{\prime \prime} 25$ addere oportebit.

72.

Finem huic Sectioni imponent quaedam problemata, quae in determinatione orbitarum planetarum et cometarum vsum frequentem praestant. Ac primo quidem ad parallaxem reueniemus, a qua locum obseruatum liberare in art. 70 docuimus. Talis reductio ad centrum terrae, quum planetae distantiam a terra proxime saltem notam supponat, institui nequit, quoties planetae obseruati orbita omnino adhue incognita est. Attamen in hoc quoque casu finem saltem eundem assequi licet, cuius caussa reductio ad centrum terrae suscipitur, ideo scilicet, quod hoc centro in plano 
eclipticae iacente vel iacere supposito plures formulae maiorem simplicitatem et concinnitatem nanciscuntur, quam si obseruatio ad punctum extra planum eclipticae referretur. Hoc itaque respectu nihil interest, vtrum obseruatio ad centrum terrac an ad quoduis aliud punctum in plano eclipticae reducatur. Iam patet, si ad hune finem punctum intersectionis plani eclipticae cum recta a planeța ad locum verum obseruationis ducta eligatur, obseruationem ipsam nulla prorsus reductione opus habere, quum planeia ex omnibus punctis illius rectae perinde videatur *): quamobrem hoc punctum quasi locum fictum obseruationis pro vero substituere licebit. Situm illius puncti sequenti modo determinamus.

Sit corporis coelestis longitudo $\lambda$, latitudo $\beta$, distantia $\Delta$, omnia respectu loci reri obseruationis in terrae superficie, cuius zenith respondeat longitudo $l$, latitudo $b$; porro sit $\pi$ semidiameter terrae, $L$ longitudo heliocentrica centri terrae, $B$ eiusdem latitudo, $R$ eiusdem distantia a Sole; denique $L^{\prime}$ longitudo heỉiocentrica loci ficti, $R^{\prime}$ ipsius distantia a Sole, $\Delta+\delta$ ipsius distantia a corpore coelesti. Tune: nullo negotio eruentur aequationes sequentes, denotante $N$ angulum arbitrarium:

$$
\begin{aligned}
& R^{\prime} \cos \left(L^{\prime}-N\right)+\delta \cos \beta \cos (\lambda-N)=R \cos B \cos (L-N)+\pi \cos b \cos (l-N) \\
& R^{\prime} \sin \left(L^{\prime}-N\right)+\delta \cos \beta \sin (\lambda-N)=R \cos B \sin (L-N)+\pi \cos b \sin (l-N) \\
& \delta \sin \beta=R \sin B+\pi \sin b
\end{aligned}
$$

Statuendo itaque I. $(R \sin B+\pi \sin b) \operatorname{cotang} \beta=\mu$, erit

II. $R^{\prime} \cos \left(L^{\prime}-N\right)=R \cos B \cos (L-N)+\pi \cos b \cos (l-N)-\mu \cos (\lambda-N)$

III. $R^{\prime} \sin \left(L^{\prime}-N\right)=R \cos B \sin (L-N)+\pi \cos b \sin (l-N)-\mu \sin (\lambda-N)$

IV. $\delta=\frac{\mu}{\cos \beta}$

Ex aequationibus II, III determinari poterunt $R^{\prime}$ et $L^{\prime}$, ex IV internalfum temporis tempori obseruationis addendum quod erit minutis secundis $=495 \delta$.

Hae aequationes sunt exactae et generales, poteruntque tunc quoque adhiberi, vbi pro plano ecliptica aequatore substituto $L, L, l, \lambda$ designant ascensiones. rectas, $B, b, \beta$ declinationes. Sed in casu de quo hic potissimum agimus, scilicet vbi locus fictus in ecliptica situs esse debet, exiguitas quantitatum $B, \pi, L^{\prime}-L$ adhuc quandam formularum praecedentium contractionem permittit. Poterit enimx pro $\pi$ assumi parallaxis media solaris, $B$ pro $\sin B, I$ pro $\cos B$ et $\cos \left(L^{\prime}-L\right)_{x}$

*) Si vltima praccisio desideraretur, intexuallum temporis, intra quod lumen a vero loco obseruationis ad fictum sen ab hoc ad illum delabitur, tempori proposito vel addere vel inde subducere oporteret, siquidem de locis aberratione affectis agitur: sed hacc differentia vix vllius momenti: esse potest, nisi latitudo perparua fuerit. 
$L^{\prime}-L$ pro $\sin \left(L^{\prime}-L\right)$. Ita faciendo $N=L$, formulae praecedentes assumunt formam sequentem:

I. $\mu=(R \cdot B+\pi \sin b) \operatorname{cotang} \beta$

II. $R^{\prime}=R+\pi \cos b \cos (l-L)-\mu \cos (\lambda-L)$

$$
\text { III. } L^{\prime}-L=\frac{\pi \cos b \sin (l-L)-\mu \sin (\lambda-L)}{R^{\prime}}
$$

Proprie quidem hic $B, \pi, L^{\prime}-L$ in partibus radii exprimendi sunt; sed patet, si illi anguli in minutis secundis exprimantur, aequationes I, III sine mutatione retineri posse, pro II autem substitui debere

$$
R^{\prime}=R+\frac{\pi \cos b \cos (l-L)-\mu \cos (\lambda-L)}{206265^{\prime \prime}}
$$

Ceterum in formula III pro denominatore $R^{\prime}$ absque errore sensibili semper adhibere licebit $R$. Reductio temporis autem, angulis in minutis secundis expressis, fiet

$$
=\frac{493^{\prime \prime} \cdot \mu}{206265^{\prime \prime} \cdot \cos \beta} \text {. }
$$

73.

Excmplum. Sit $\lambda=554^{\circ} 44^{\prime} 54^{\prime \prime}, \beta=-4^{\circ} 59^{\prime} 32^{\prime \prime}, l=24^{\circ} 29^{\prime}, b=46^{\circ} 53^{\prime}$, $L=12^{\circ} 28^{\prime} 54^{\prime \prime}, B=+0^{\prime \prime} 49, R=0,9988839, \pi=8^{\prime \prime} 60$. Ecce iam calculum:

$$
\begin{array}{ll}
\log R \ldots \ldots \ldots \ldots . .9,99951 & \log \pi \ldots \ldots \ldots \ldots ., 93450 \\
\frac{\log B \ldots \ldots \ldots \ldots . .9,69020}{\log B R \ldots \ldots \ldots . . .6,68971} & \frac{\log \sin b \ldots \ldots \ldots 9,86550}{\log \pi \sin b \ldots \ldots ., 79780}
\end{array}
$$

Hinc $\log (B R+\pi \sin b) \ldots . . .0,83040$

$$
\begin{aligned}
& \frac{\log \operatorname{cotan} g \beta \ldots \ldots \ldots \ldots \ldots 1,05873 n}{\log \mu \ldots \ldots \ldots \ldots \ldots \ldots \ldots \ldots 1,88913 n} \\
& \log \pi \ldots \ldots \ldots \ldots \ldots . \ldots, 93450 \quad \log \mu \ldots \ldots \ldots \ldots \ldots 1,88913 n \\
& \log \cos 6 \ldots \ldots \ldots \ldots . . \ldots, 85475 \quad \log 1^{\prime \prime} \ldots \ldots \ldots \ldots \ldots . .4,68557 \\
& \begin{array}{l}
\log x^{\prime \prime} \ldots \ldots \ldots \ldots \ldots . . . \ldots 4,68557 \\
\log \cos (I-L) \ldots \ldots .9,99040
\end{array} \quad \frac{\log \cos (\lambda-L) \ldots 9,97886}{6,55356 n} \\
& \frac{\log \cos (l-L) \cdots \cdot 9,99040}{5,44520} \quad \text { numerus }-0,0003577 \\
& \text { numerus }+0,0000279
\end{aligned}
$$


Hinc colligitur $R^{\prime}=R+0,0005856=0,9992695$. Porro exit

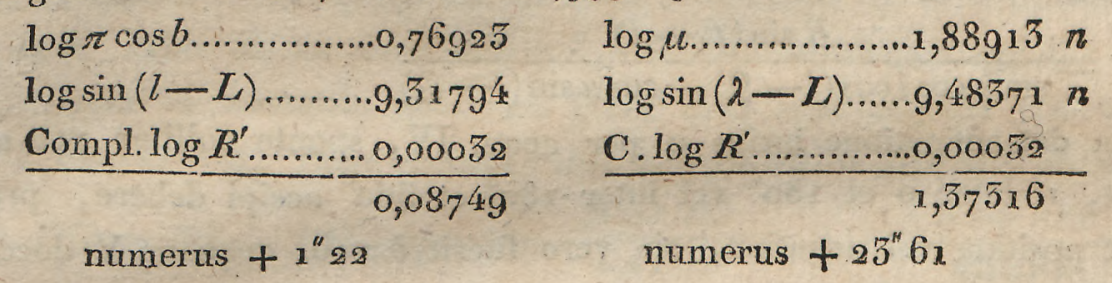

Vnde colligitur $L^{\prime}=L-22^{\prime \prime} 59$. Denique habetur

$\log \mu \ldots \ldots \ldots \ldots \ldots . .1,88915 n$
$C . \log 206265 \ldots \ldots 4,68557$
$\log 495 \ldots \ldots \ldots \ldots \ldots 2,69285$
$\frac{C \cdot \log \cos \beta \ldots \ldots \ldots . .0,00165}{9,269^{20} n}$

nullius momenti.

$9,269^{20} n$, vnde reductio temporis $=-0^{\prime \prime} 186$, adeoque

\section{4.}

Problema alind, e corporis coelestis loco geocentrico atque silu plani orbitae eius locum heliocentricum in orbita deriuare, eatenus praecedenti affine est, quod quoque ab intersectione rectae inter terram et corpus coeleste ductae cum plano positione dato pendet. Solutio commodissime petitur e formulis art. 65 , vbi characterum significatio haec erat:

$L$ longitudo terrae, $R$ distantia a Sole, latitudinem $B$ statuimus $=0$ (quum casus, vbi non est $=0$, ad hunc facile reduci possit per art. 72), vnde $R^{\prime}=R$; $z$ corporis coelestis longitudo geocentrica, $b$ latitudo, $\Delta$ distantia a terra, $r$ distantia a Sole, $u$ argumentum latitudinis, $\delta$ longitudo nodi ascendentis, $i$ inclinatio orbitae. Ita habemus aequationes

$$
\begin{aligned}
& \text { I. } r \cos u-R \cos (L-\Omega)=\Delta \cos b \cos (l-\Omega) \\
& \text { II. } r \cos i \sin u-R \sin (L-\Omega)=\Delta \cos b \sin (l-\Omega) \\
& \text { III. } r \sin i \sin u=\Delta \sin b
\end{aligned}
$$

Multiplicando aequationem I per $\sin (L-\Omega) \sin b$, II per $-\cos (L-\Omega) \sin b$, III per $-\sin (L-l) \cos b$, fit additis productis rnde $\cos u \sin (L-\Omega) \sin b-\sin u \cos i \cos (L-\Omega) \sin b-\sin u \sin i \sin (L-\not) \cos b=0$

$$
\text { IV. } \operatorname{tang} u=\frac{\sin (L-\Omega) \sin b}{\cos i \cos (L-\Omega) \sin b+\sin i \sin (L-Z) \cos b}
$$


Multiplicando autem I per $\sin (l-\Omega)$, II per $-\cos (l-\Omega)$, prodit productis additis

$$
\text { V. } r=\frac{R \sin (L-l)}{\sin u \cos i \cos (l-\Omega)-\cos u \sin (l-\Omega)}
$$

Ambiguitas in determinatione ipsius $u$ per aequ. IV, sponte tollitur per aequ. III, quae ostendit, $u$ inter o et $180^{\circ}$ vel inter $180^{\circ}$ et $560^{\circ}$ accipi debere, prout latitudo $b$ fuerit positiua vel negatiua; $\sin$ vero fuerit $b=0$, aequatio $\mathrm{V}$ docet, statui debere $u=0$ vel $u=180^{\circ}$, prout $\sin (L-l)$ et $\sin (l-\Omega)$ diuersa signa habeant, vel eadem.

Computum numericum formularum IV et $\mathrm{V}$ varïs modis per introductionem angulorum auxiliarium contrahere licet. E. $g$.

$$
\begin{aligned}
& \text { statuendo } \frac{\operatorname{tang} b \cos (L-\Omega)}{\sin (L-l)}=\operatorname{tang} A, \text { fit } \operatorname{tang} u=\frac{\sin A \operatorname{tang}(L-\delta)}{\sin (A+i)} \\
& \text { statuendo } \frac{\operatorname{tang} i \sin (L-l)}{\cos (L-\delta)}=\operatorname{tang} B, \text { fit } \operatorname{tang} u=\frac{\cos B \sin b \tan g(L-\Omega)}{\sin (B+b) \cos i}
\end{aligned}
$$

Perinde aequ. V per introductionem anguli cuius tangens $=\cos i \operatorname{tang} u$, vel $=$ $\frac{\operatorname{tang}(l-\Omega)}{\cos i}$ formam concinniorem nanciscitur. Sicuti formulam $V$ e combinatione aequationum I, II obtinuimus, per combinationem aequationum II, III ad sequentem peruenimus:

$$
r=\frac{R \sin (L-\Omega)}{\sin u(\cos i-\sin i \sin (l-\Omega) \operatorname{cotang} b)}
$$

et perinde per combinationem aequationum $I$, III ad hanc

$$
r=\frac{R \cos (l-\Omega)}{\cos u-\sin u \sin i \cos (l-\Omega) \operatorname{cotang} b}
$$

Vtramque perinde vt $\mathrm{V}$ per introductionem angulorum auxiliarium simpliciorem reddere licet. Solutiones e praecedentibus demanantes colllectae exemploque illustratae inueniuntur in Von Zach Monatliche Correspondenz Vol.V.p. 540, quapropter hic euolutione vlteriori supersedemus. - Si praeter $u$ et $r$ etiam distantia $\Delta$ desideratur, per aequationem III determinari poterit.

$$
75 .
$$

Alia solutio problematis praec. superstruitur obseruationi in art. 64 III traditae, quod locus heliocentricus terrae, geocentricus corporis coelestis eiusdemque locus heliocentricus in vno eodemque circulo maximo sphaerae sunt siti. Sint in fig. 3 illi loci resp. $T, G, H$; porro $\Omega$ locus nodi ascendentis; $\Omega T, \Omega H$ partes 
eclipticae et orbitae, GP perpendiculum ad eclipticam ex $G$ demissum, quod igitur erit $=b$. Hinc et ex arcu $P T=L-l$ determinabitur angulus $T$ atque arcus $T G$. Dein in triangulo sphaerico $\delta H T$ data sunt angulus $\Omega=i$, angulus $T$ latusque $\Omega T=L-\Omega$, vnde eruentur duo reliqua latera $\Omega H=u$ atque $T H$. Tandem erit $H G=T G-T H$ atque $r=\frac{R \sin T G}{\sin H G}, \Delta=\frac{R \sin T H}{\sin H G}$ :

76.

In art. 52 variationes differentiales longitudinis et latitudinis heliocentricae distantiaeque curtatae per variationes argumenti latitudinis $u$, inclinationis $i$ radique rectoris $r$ exprimere docuimus, posteaque (art. 64, IV) ex illis deduximus variationes longitudinis et latitudinis geocentricae, $l$ et $b$ : per combinationem itaque harum formularum $\mathrm{d} l$ et $\mathrm{d} b$ per $\mathrm{d} u, \mathrm{~d} i, \mathrm{~d} \Omega, \mathrm{d} r$ expressae habebuntur. Sed operae pretium erit ostendere, quomodo in hoc quoque calculo reductione loci heliocentrici ad eclipticam supersedere liceat, sicuti in art. 65 locum geocentricum immediate e loco heliocentrico in orbita deduximus. Vt formulae eo simpliciores euadant, latitudinem terrae negligemus, quum certe in formulis differentialibus effectum sensibilem habere nequeat. Praesto sunt itaque formulae sequentes, in quibus brevitatis caussa $\omega$ pro $l-\Omega$, nec non vt supra $\Delta^{\prime}$ pro $\Delta \cos b$ scribimus.

$$
\begin{aligned}
& \Delta^{\prime} \cos \omega=r \cos u-R \cos (L-\Omega)=\xi \\
& \Delta^{\prime} \sin \omega=r \cos i \sin u-R \sin (L-\Omega)=\eta \\
& \Delta^{\prime} \operatorname{tang} b=r \sin i \sin u=\zeta
\end{aligned}
$$

e quarum differentiatione prodit

$$
\begin{aligned}
& \cos \omega \cdot \mathrm{d} \Delta^{\prime}-\Delta^{\prime} \sin \omega \cdot \mathrm{d} \omega=\mathrm{d} \xi \\
& \sin \omega \cdot \mathrm{d} \Delta^{\prime}+\Delta^{\prime} \cos \omega \cdot \mathrm{d} \omega=\mathrm{d} \eta \\
& \operatorname{tang} b \cdot \mathrm{d} \Delta^{\prime}+\frac{\Delta}{\cos b} \mathrm{~d} b=\mathrm{d} \zeta
\end{aligned}
$$

Hine per eliminationem

$$
\begin{aligned}
& \mathrm{d} \omega=\frac{-\sin \omega \cdot \mathrm{d} \xi+\cos \omega \cdot \mathrm{d} \eta}{\Delta^{\prime}} \\
& \mathrm{d} b=\frac{-\cos \omega \sin b \cdot \mathrm{d} \xi-\sin \omega \sin b \cdot \mathrm{d} \eta+\cos b \cdot d \xi}{\Delta}
\end{aligned}
$$

$\mathrm{Si}$ in his formulis pro $\xi, \eta, \zeta$ valores sui rite substituuntur, d $\omega$ et $\mathrm{d} b$ per $d r, \mathrm{~d} u, \mathrm{~d} i, \mathrm{~d} \Omega$ expressae prodibunt; dein, propter $\mathrm{d} l=\mathrm{d} \omega+\mathrm{d} \Omega$, differentialia partialia ipsarum $l, b$ ita se habebunt: 
I. $\Delta^{\prime}\left(\frac{\mathrm{d} l}{\mathrm{~d} r}\right)=-\sin \omega \cos u+\cos \omega \sin u \cos i$

II. $\frac{\Delta^{\prime}}{r}\left(\frac{\mathrm{d} l}{\mathrm{~d} u}\right)=\sin \omega \sin u+\cos \omega \cos u \cos i$

III. $\frac{\Delta^{\prime}}{r}\left(\frac{\mathrm{d} l}{\mathrm{~d} i}\right)=-\cos \omega \sin u \sin i$

IV. $\left(\frac{\mathrm{d} l}{\mathrm{~d} \Omega}\right)=1+\frac{R}{\Delta^{\prime}} \cos (L-\Omega-\omega)=1+\frac{R}{\Delta^{\prime}} \cos (L-l)$

V. $\Delta\left(\frac{\mathrm{d} b}{\mathrm{~d} r}\right)=-\cos \omega \cos u \sin b-\sin \omega \sin u \cos i \sin b+\sin u \sin i \cos b$

VI. $\frac{\Delta}{r}\left(\frac{\mathrm{d} b}{\mathrm{~d} u}\right)=\cos \omega \sin u \sin b-\sin \omega \cos u \cos i \sin b+\cos u \sin i \cos b$

VII. $\frac{\Delta}{r}\left(\frac{\mathrm{d} b}{\mathrm{~d} i}\right)=\sin \omega \sin u \sin i \sin b+\sin u \cos i \cos b$

VIII. $\frac{\Delta}{R}\left(\frac{\mathrm{d} b}{\mathrm{~d}} \Omega\right)=\sin b \sin (L-\Omega-\omega)=\sin b \sin (L-\zeta)$

Formulae IV et VIII hic iam in forma ad calculum commodissima apparent; formulae I, III, V autem per substitutiones obuias ad formam concinniorem rediguntur, puta

$$
\begin{aligned}
& I^{\star} \cdot\left(\frac{\mathrm{d} l}{\mathrm{~d} r}\right)=\frac{R}{r \Delta^{\prime}} \sin (L-l) \\
& \text { III* }^{\star}\left(\frac{\mathrm{d} l}{\mathrm{~d} i}\right)=-\cos \omega \operatorname{tang} b \\
& \mathrm{~V}^{\star} \cdot\left(\frac{\mathrm{d} b}{\mathrm{~d} r}\right)=-\frac{R}{r \Delta} \cos (L-l) \sin b=-\frac{R}{r \Delta^{\prime}} \cos (L-l) \operatorname{tang} b
\end{aligned}
$$

Denique formulae reliquae quoque II, VI, VII per introductionem quorundam angulorum auxiliarium in formam simpliciorem abeunt: quod commodissime fit sequenti modo. Determinentur anguli auxiliares $M, N$ per formulas $\operatorname{tang} M=\frac{\tan g \omega}{\cos i}$, $\operatorname{tang} N=\sin \omega \operatorname{tang} i=\operatorname{tang} M \cos \omega \sin i . \quad$ Tunc simul fit $\frac{\cos M^{2}}{\cos N^{2}}=\frac{1+\operatorname{tang} N^{2}}{1+\operatorname{tang} M^{2}}$ $=\frac{\cos i^{2}+\sin \omega^{2} \sin i^{2}}{\cos i^{2}+\tan g \omega^{2}}=\cos \omega^{2}:$ iam quum ambiguitatem in determinatione ipsorum $M, N$ per tangentes suas remanentem ad lubitum decidere liceat, hoc ita feri posse patet, vt habeatur $\frac{\cos M}{\cos N}=+\cos \omega$, ac proin $\frac{\sin N}{\sin M}=+\sin i$. Quibus ita factis, formulae II, VI, VII transeunt in sequentes: 


$$
\begin{aligned}
& I^{\star}\left(\frac{\mathrm{d} l}{\mathrm{~d} u}\right)=\frac{r \sin \omega \cos (M-u)}{\Delta \sin M} \\
& V I^{\star}\left(\frac{\mathrm{d} b}{\mathrm{~d} u}\right)=\frac{r}{\Delta}\{\cos \omega \sin i \cos (M-u) \cos (N-b)+\sin (M-u) \sin (N-b\} \\
& V I I^{\star}\left(\frac{\mathrm{d} b}{\mathrm{~d} i}\right)=\frac{r \sin u \cos i \cos (N-b)}{\Delta \cos N}
\end{aligned}
$$

Hae transformationes respectu formularum II, VII neminem morabuntur, respectu formulae VI autem aliqua explicatio haud superflua erit. Substituendo scilicet in formula VI primo $I I-(M-u)$ prou, prodit $\frac{\Delta}{r}\left(\frac{\mathrm{d} b}{\mathrm{~d} u}\right)=$

$$
\begin{array}{r}
\cos (M I-u)\{\cos \omega \sin M \sin b-\sin \omega \cos i \cos M \sin b+\sin i \cos M \cos b\} \\
-\sin (M T-u)\{\cos \omega \cos M \sin b+\sin \omega \cos i \sin M \sin b-\sin i \sin M M \cos b
\end{array}
$$

Iam fit $\cos \omega \sin M=\cos i^{2} \cos \omega \sin M+\sin i^{2} \cos \omega \sin M=\sin \omega \cos i \cos M+$ $\sin i^{2} \cos \omega \sin M$; vnde pars prior illius expressionis transit in $\sin i \cos (\pi-u)\{\sin i \cos \omega \sin I M \sin b+\cos I M \cos b\}$

$=\sin i \cos (M-u)\{\cos \omega \sin N \sin b+\cos \omega \cos N \cos b\}$

$=\cos \omega \sin i \cos (M-u) \cos (N-b)$

Perinde fit $\cos N=\cos \omega^{2} \cos N+\sin \omega^{2} \cos N=\cos \omega \cos M+\sin \omega \cos i \sin M$; vnde expressionis pars posterior transit in

$$
-\sin (M-u)\{\cos N \sin b-\sin N \cos b\}=\sin (M-u) \sin (N-b)
$$

Hins expressio VI* protinus demanat.

Angulus auxiliaris $M$ etiam ad transformationem formulae I adhiberi potest, quo introducto assumit formam

$$
I * \star\left(\frac{d l}{d r}\right)=-\frac{\sin \omega \sin (M-u)}{\Delta^{\prime} \sin M}
$$

e cuius comparatione cum formula $I^{*}$ concluditur $-R \sin (L-\ell) \sin M=r \sin \omega \sin (M-u)$; hinc etiam formulae $I I^{*}$ forma paullo adhuc simplicior tribui potest, puta

$$
I * *\left(\frac{\mathrm{d} l}{\mathrm{~d} u}\right)=-\frac{R}{\Delta^{\prime}} \sin (L-l) \operatorname{cotang}(M-u)
$$

Vt formula $V^{*}$, adhuc magis contrahatur, angulum auxiliarem noumm in- 
troducere oportet, quod duplici modo fieri potest, scilicet statuendo vel $\operatorname{tang} P=$ $\frac{\operatorname{tang}(I M-u)}{\cos \omega \sin i}$, vel $\operatorname{tang} Q=\frac{\operatorname{tang}(N-b)}{\cos \omega \cos i}:$ quo facto emergit .

$$
\mathrm{VI}{ }^{*}\left(\frac{\mathrm{d} b}{\mathrm{~d} u}\right)=\frac{r \sin (M-u) \cos (N-b-P)}{\Delta \sin P}=\frac{r \sin (N-b) \cos (M-u-Q)}{\Delta \sin Q}
$$

Ceterum quantitates auxiliares $M, N, P, Q$ non sunt mere fictitiae, facileque, quidnam in sphaera coelesti singulis respondeat, assignare liceret: quin adeo hoc modo aequationum praecedentium plures adhuc elegantius exhiberi possent per arcus angulosue in sphaera, quibus tamên eo minus hic immoramur, quum in calculo numerico ipso formulas supra traditas superfluas reddere non valeant.

\section{$77^{\circ}$}

Iunctis is, quae in art. praec. euoluta sunt, cum ins quae in artt. 15,16 , $20,27,28$ pro singulis sectionum conicarum generibus tradidimus, omnia praesto erunt, quae ad calculum variationum differentialium loco geocentrico a variationibus singulorum elementorum inductarum requiruntur. Ad maiorem illustrationem horum praeceptorum exemplum supra in artt. 15, 14, 51, 63, 65 tractatum resumemus. Ac primo quidem ad normam art. praec. $\mathrm{d} l$ et $\mathrm{d} b$ per $\mathrm{d} r, \mathrm{~d} u, \mathrm{~d} i, \mathrm{~d} \Omega$ exprimemus, qui calculus ita se habet:

\begin{tabular}{|c|c|c|}
\hline 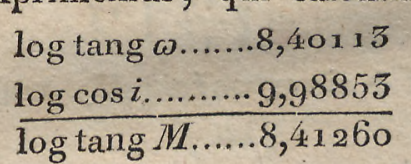 & $\begin{array}{l}\log \sin \omega \ldots \ldots . .8,40099 n \\
\frac{\log \tan g i \ldots \ldots \ldots 9,56723}{\log \operatorname{tang} N \ldots \ldots .7,76822 n}\end{array}$ & $\begin{array}{l}\log \tan g(M-u) \ldots 9,41932 n \\
\frac{\log \cos \omega \sin i \ldots \ldots \ldots 9,55562 n}{\log \tan g P \ldots \ldots \ldots \ldots 0,06570}\end{array}$ \\
\hline$M=i^{\circ} 28^{\prime} 52^{\prime \prime}$ & $N=179^{\circ} 39^{\prime} 50^{\prime \prime}$ & $P=49^{\circ} 11^{\prime} 15^{\prime \prime}$ \\
\hline $\begin{array}{c}I M-u=165178 \\
I^{*}\end{array}$ & $\begin{array}{c}N-b=186 \quad 145 \\
I^{* *}\end{array}$ & $\begin{array}{c}N-b-P=1365052 \\
\text { III* }\end{array}$ \\
\hline 1. $\sin (L-l) . .9,72125$ & $\left.{ }^{\star}{ }^{\star}\right) \ldots \ldots \ldots \ldots \ldots . . . .9,63962$ & $\log \cos \omega \ldots \ldots \ldots \ldots . . . .9,99986 n$ \\
\hline $\log R \ldots \ldots \ldots \ldots . . . .9,99810$ & 1. $\cot (M-u) . .0,58068 n$ & $\log \tan g b \ldots \ldots \ldots \ldots, 9,04749 n$ \\
\hline$\frac{C \cdot \log \Delta^{\prime} \ldots \ldots \ldots 9,92027}{\left(^{\star}\right) \ldots \ldots \ldots \ldots \ldots . . \ldots 9,65962}$ & $\log \left(\frac{\mathrm{d} l}{\mathrm{~d} u}\right) \ldots \ldots 0,22030$ & $\log \left(\frac{\mathrm{d} l}{\mathrm{~d} i}\right) \ldots \ldots \ldots \ldots, 9,04735 n$ \\
\hline$\frac{C \cdot \log r \ldots \ldots \ldots .9,67401}{\log \left(\frac{d l}{d r}\right) \ldots . .99,31363}$ & & \\
\hline
\end{tabular}


RELATIONES AD LOCVM STMPLICEM IN SPATIO SPECTANTES.

79

$$
\begin{aligned}
& \text { IV } \\
& \log \frac{R}{\Delta^{\prime}} \ldots \ldots \ldots . . .9,91837 \\
& V^{\star} \\
& \mathrm{VI}^{\star *} \\
& (* *) \ldots \ldots \ldots \ldots \ldots .9,84795 \\
& \frac{1 . \cos (I-l) \ldots .9,92956}{\left({ }^{\star *}\right) \ldots \ldots \ldots \ldots . . .9,84793} \\
& \log \tan g 6 \ldots \ldots . .9,04749 n \\
& \log \frac{r}{\Delta} \\
& \frac{C \cdot \log r \ldots \ldots \ldots 9,67401}{\log \left(\frac{d b}{d r}\right) \ldots \ldots 8,56945} \\
& \log \sin (M-u) \\
& =\log \left(\frac{d l}{d \Omega}-1\right) \\
& \frac{C \cdot \log \sin P \ldots}{\log \left(\frac{d b}{d u}\right)} \\
& \text { VII* } \\
& \text { VIII } \\
& \text { 1. } r \sin u \cos i \ldots 9,75999 n \\
& \text { (*). } \\
& 9,65962 \\
& \text { 1. } \cos (N-6) \ldots .99,99759 n \\
& \text { C. } \log \Delta \ldots \ldots . .9,91759 \\
& \frac{C \cdot \log \cos N \ldots . .0, \text { oooor } n}{\log \left(\frac{\mathrm{d} b}{\mathrm{~d} i}\right) \ldots 9,67518 n} \\
& \frac{\log \operatorname{tang} b \ldots \ldots . .9,04749 n}{\log \left(\frac{d b}{d}\right) \ldots \ldots 8,68711 n} \\
& d l=+0,20589 \mathrm{~d} r+1,66075 \mathrm{~d} u-0,1 \times 152 \mathrm{~d} i+1,70458 \mathrm{~d} \Omega \\
& \mathrm{d} b=+0,05710 \mathrm{~d} r-0,42895 \mathrm{~d} u-0,47355 \mathrm{di}-0,04865 \mathrm{~d} \Omega
\end{aligned}
$$$$
\log \cos (N-b-P) . .9,86301 n
$$

Vix necesse erit quod iam saepius monuimus hic repetere, scilicet, vel variationes $\mathrm{d} l, \mathrm{~d} b, \mathrm{~d} u, \mathrm{~d} i, \mathrm{~d} \delta$ in partibus radii exprimendas esse, vel coëfficientes ipsius d $r$ per $206265^{\prime \prime}$ multiplicandos, si illae in minutis secundis expressae concipiantur.

Designando iam longitudinem perihelii (quae in exemplo nostro est $52^{\circ} 1.8^{\prime} g^{\prime \prime} 30$ ) per $I I$ atque anomaliam veram per $v$, erit longitudo in orbita $=u+\delta=v+\Pi$, adeoque $\mathrm{d} u=\mathrm{d} y+\mathrm{d} \|-\mathrm{d} \Omega$, quo valore in formulis praecedentibus substituto, $\mathrm{d} l$ et $\mathrm{d} b$ per $\mathrm{d} r, \mathrm{~d} v, \mathrm{~d} \|, \mathrm{d} \Omega, \mathrm{d} i$ expressae habebuntur. Nihil itaque iam snperest, nisi $v t \mathrm{~d} r$ et $\mathrm{d} v$ ad normam artt. 15, 16 per variationes differentiales elementorum ellipticorum exhibeantur *).

*) Chaxacterem $M$ in calculo sequente haud amplius angulum nostrum anxiliarem exprimere, sed ( $v t$ in Sect. 1) anomaliam mediam, quisque sponte videbit. 
Erat in exemplo nostro, art. 14, $\log \frac{r}{a}=9,90355=\log \left(\frac{\mathrm{d} r}{\mathrm{~d} a}\right)$

$$
\begin{aligned}
& \log \frac{a a}{r r} \ldots \ldots \ldots \ldots . . ., 19^{2} 9^{0} \\
& \frac{\log \cos \varphi \ldots \ldots \ldots \ldots . . .9,98652}{\log \left(\frac{d}{d M}\right) \ldots \ldots .0,17942} \\
& 2-e \cos E=1,80085 \\
& \frac{e e \quad=0,06018}{1,74067}
\end{aligned}
$$

$\log \ldots \ldots \ldots \ldots \ldots \ldots . . \ldots, 2407$ ?

$\log \frac{a a}{r r} \ldots \ldots \ldots \ldots, 19^{2} 9^{\circ}$

$$
\frac{\log \sin E \ldots \ldots \ldots .9,76634 \ldots}{\log \left(\frac{d v}{d \varphi}\right) \ldots \ldots . .0,19996 n}
$$

$\log a \ldots \ldots \ldots \ldots . .0,42244$

$\log \operatorname{tang} \varphi \ldots . .9,40320$

$\underline{\log \sin 1 \ldots \ldots . .9,8493 x n}$

$\log \left(\frac{d r}{d M}\right) \ldots 9,67495 \pi$

$\log a \ldots \ldots \ldots . . .0,42244$

$\log \cos \varphi \ldots . . .9,98652$

$\log \cos \psi \ldots . . .9,84962$

$\log \left(\frac{\mathrm{d} r}{\mathrm{~d} \varphi}\right) \ldots 0,25858 \pi$

Hinc colligitur

$$
\begin{aligned}
& \mathrm{d} v=+1,51154 \mathrm{~d} M-1,58475 \mathrm{~d} \varphi \\
& \mathrm{d} r=-0,47310 \mathrm{~d} M-1,81376 \mathrm{~d} \varphi+0,80085 \mathrm{~d} a
\end{aligned}
$$

quibus valoribus in formulis praeceđentibus substitutis, prodit

$\mathrm{d} l=+2,41287 \mathrm{~d} I M-3,00527 \mathrm{~d} \varphi+0,16488 \mathrm{~d} a+1,66075 \mathrm{~d} \Pi-0,11132 \mathrm{~d} i+0,04385 \mathrm{~d} \Omega$ $\mathrm{d} b=-0,66593 \mathrm{~d} M+0,61248 \mathrm{~d} \varphi+0,02,972 \mathrm{~d} a-0,42895 \mathrm{~d} \Pi-0,47355 \mathrm{~d} i+0,58050 \mathrm{~d} \Omega$

Si tempus cui locus computatus respondet $n$ diebus ab epocha distare supponitur, longitudoque media pro epocha per $N$, motus diurnus per 7 denotatm erit $M=N+n 7-\Pi$, adeoque $\mathrm{d} M=\mathrm{d} N+n \mathrm{~d} 7-\mathrm{d} \Pi$. In exemplo nostro tempus loco computato respondens est Octobris dies $17,4 \times 507$ anni 1804 sub meridiano Parisiensi: quodsi itaque pro epocha assumitur initium anni 1805 , est $n=-74,58493$; longitudo media pro epocha ista statuta fuerat $=41^{\circ} 52^{\prime} 21^{\prime \prime} 61$, motusque diurnus $=824^{\prime \prime} 7988$. Substituto iam in formulis modo inuentis pro d $M$ valore suo, mutationes differentiales loci geocentrici per solas mutationes elementorum expressae ita se habent:

$$
\begin{gathered}
\mathrm{d} l=2,41287 \mathrm{~d} N-{ }^{7} 79,96 \mathrm{~d} 7-0,75214 \mathrm{~d} \Pi-3,00527 \mathrm{~d} \varphi+0,16488 \mathrm{~d} a \\
-0,11152 \mathrm{~d} i+0,04385 \mathrm{~d} \Omega \\
\mathrm{d} b=-0,66593 \mathrm{~d} N+49,67 \mathrm{~d} 7+0,23698 \mathrm{~d} \Pi+0,61248 \mathrm{~d} \varphi+0,02972 \mathrm{~d} a \\
-0,4,7335 \mathrm{~d} i+0,58050 \mathrm{~d} \Omega
\end{gathered}
$$


Si corporis coelestis massa vel negligitur vel saltem tamquam cognita spectatur, 7 et $a$ ab inuicem dependentes erunt, adeoque vel d 7 vel $d a$ e formulis nostris eliminare licebit. Scilicet quum per art. 6 habeatur $7 a^{\frac{3}{2}}=k V(x+\mu)$, erit $\frac{\mathrm{d} 7}{7}=-\frac{3}{2} \frac{\mathrm{d} \alpha}{a}$, in qua formula, si $\mathrm{d} 7$ in partibus radii exprimenda est, etiam 7 perinde exprimere oportebit. Ita in exemplo nostro habetur

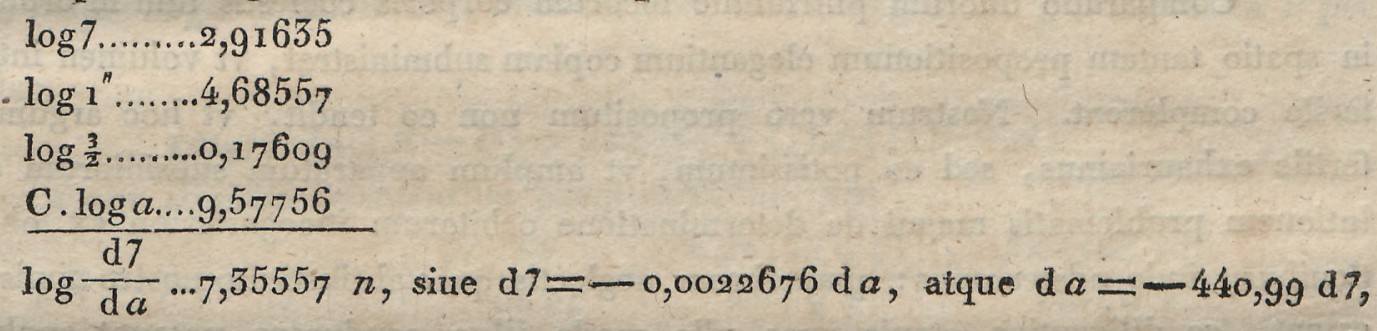
quo valore in formulis nostris substituto, tandem emergit forma vltima: $\mathrm{d} l=2,41287 \mathrm{~d} N-252,67 \mathrm{~d} 7-0,75214 \mathrm{~d} \Pi-3,00527 \mathrm{~d} \varphi-0,11152 \mathrm{~d} i+0,04585 \mathrm{~d} \Omega$ $\mathrm{d} b=-0,66595 \mathrm{~d} N+36,57 \mathrm{~d} 7+0,25698 \mathrm{~d} \Pi+0,61248 \mathrm{~d} \varphi-0,4.7355 \mathrm{~d} i+0,38050 \mathrm{~d} \Omega$ In euolutione harum formularum omnes mutationes $\mathrm{d} l, \mathrm{~d} b, \mathrm{~d} N, \mathrm{~d} 7, \mathrm{~d} \Pi, \mathrm{d} \varphi, \mathrm{d} i$, $\mathrm{d} \Omega$ in partibus radii expressas supposuimus, manifesto autem propter homogeneitatem omnium partium eaedem formulae etiamnum valebunt, si omnes illae mutationes in minutis secundis exprimuntur. 


\title{
SECTIO TERTIA
}

\author{
Relationes inter locos plures in orbita.
}

78.

Comparatio duorun pluriumue locorum corporis coelestis tum in orbita tum in spatio tantam propositionum elegantium copiam subministrat, vt volumen integrum facile complerent. Nostrum vero propositum non eo tendit, vt hoc argumentum fertile exhauriamus, sed eo potissimum, vt amplum apparatum subsidiorum ad solutionem problematis magni de determinatinne orbitarum incognitarum ex obsertationibus, inde adstruamus: quamobrem neglectis quae ab instituto nostro nimis aliena essent, feo diligeutius omnia quae vllo modo illuc conducere possunt euoluemus. Disquisitionibus ipsis quasdam propositiones trigonometricas praemittimus, ad quas, quum frequentioris vsus sint, saepius recurrere oportet.

I. Denotantibus $A, B, C$ angulos quoscunque, habetur $\sin A \sin (C-B)+\sin B \sin (A-C)+\sin C \sin (B-A)=o$ $\cos A \sin (C-B)+\cos B \sin (A-C)+\cos C \sin (B-A)=0$

II. Si duae quantitates $p, P$ ex aequationibus talibus

$$
\begin{aligned}
& p \sin (A-P)=a \\
& p \sin (B-P)=b
\end{aligned}
$$

determinandae sunt, hoc fiet generaliter adiumento formularum

$$
\begin{aligned}
& p \sin (B-A) \sin (H-P)=b \sin (H-A)-a \sin (H-B) \\
& p \sin (B-A) \cos (H-P)=b \cos (H-A)-a \cos (I-B)
\end{aligned}
$$

in quibus $H$ est angulus arbitrarius. Hinc deducuntur (art. 14, II) angulus $H-P$ atque $p \sin (B-A)$; et hinc $P$ et $p$. Plerumque conditio adiecta esse solet, vt $p$ esse debeat quantitas positiua, vnde ambiguitas in determinatione anguli $H-P$ per tangentem suam deciditur; deficiente autem illa conditione, ambiguitatem ad lubitum decidere licebit. Vt calculus commodissimus sit, angulum arbitrarium $H$ $\mathrm{vel}=A \mathrm{vel}=B \mathrm{vel}=\frac{\mathrm{r}}{2}(A+B)$ statuere conueniet. In casu prioxi aequationes ad determinandum $P$ et $p$ erunt

$$
\begin{aligned}
& p \sin (A-P)=a \\
& p \cos (A-P)=\frac{b-a \cos (B-A)}{\sin (B-A)}
\end{aligned}
$$


In casu secundo aequationes prorsus analogae erunt; in casu tertio autem

$$
\begin{aligned}
& p \sin \left(\frac{x}{2} A+\frac{1}{2} B-P\right)=\frac{b+a}{2 \cos \frac{x}{2}(B-A)} \\
& p \cos \left(\frac{r}{2} A+\frac{r}{2} B-P\right)=\frac{b-a}{2 \sin \frac{r}{2}(B-A)}
\end{aligned}
$$

Quodsi itaque angulus auxiliaris $\zeta$ introducitur, cuius tangens $=\frac{a}{b}$, inuenietur $P$ per formulam

$$
\operatorname{tang}\left(\frac{r}{2} A+\frac{\pi}{2} B-P\right)=\operatorname{tang}\left(45^{\circ}+\zeta\right) \operatorname{tang} \frac{r}{2}(B-A)
$$

ac dein $p$ per aliquam formularum praecedentium, vbi

$$
\begin{aligned}
& \frac{\pi}{2}(b+a)=\sin \left(45^{\circ}+\zeta\right) \vee \frac{a b}{\sin 2 \zeta}=\frac{a \sin \left(45^{\circ}+\zeta\right)}{\sin \zeta \sqrt{2}^{2}}=\frac{b \sin \left(45^{\circ}+\zeta\right)}{\cos \zeta \sqrt{2}} \\
& \frac{\pi}{2}(b-a)=\cos \left(45^{\circ}+\zeta\right) \vee \frac{a b}{\sin 2 \zeta}=\frac{a \cos \left(45^{\circ}+\zeta\right)}{\sin \zeta \sqrt{2}^{2}}=\frac{b \cos \left(45^{\circ}+\zeta\right)}{\sin \zeta V^{2}}
\end{aligned}
$$

III. Si $p$ et $P$ determinandae sunt ex aequationibus

$$
\begin{aligned}
& p \cos (A-P)=a \\
& p \cos (B-P)=b
\end{aligned}
$$

omnia in II. exposita statim applicari possent, si modo illic pro $A$ et $B$ vbique scriberetur $90^{\circ}+A, 90^{\circ}+B$ : sed vt vsus eo commodior sit, formulas euolutas apponere non piget. Formulae generales erunt

$$
\begin{aligned}
& p \sin (B-A) \sin (H-P)=-b \cos (H-A)+a \cos (I I-B) \\
& p \sin (B-A) \cos (H-P)=b \sin (H-A)-a \sin (I-B)
\end{aligned}
$$

Transeunt itaque, pro $H=A$ in

$$
\begin{aligned}
& p \sin (A-P)=\frac{a \cos (B-A)-b}{\sin (B-A)} \\
& p \cos (A-P)=a
\end{aligned}
$$

Pro $I I=B$, formam similem obtinent; pro $H=\frac{x}{2}(A+B)$ autem fiunt

$$
\begin{aligned}
& p \sin \left(\frac{\pi}{2} A+\frac{r}{2} B-P\right)=\frac{a-b}{2 \sin \frac{r}{2}(B-A)} \\
& p \cos \left(\frac{r}{2} A+\frac{r}{2} B-P\right)=\frac{a+b}{2 \cos \frac{1}{2}(B-A)}
\end{aligned}
$$

ita $\mathrm{vt}$ introducto angulo auxiliari $\zeta$, cuius tangens $=\frac{a}{b}$, fiat

$$
\operatorname{cotang}\left(\frac{\pi}{2} A+\frac{x}{2} B-P\right)=\operatorname{tang}\left(\zeta-45^{\circ}\right) \operatorname{tang} \frac{\frac{1}{2}}{2}(B-A)
$$


Ceterum si $p$ immediate ex $a$ et $b$ sine pracuio computo anguli $P$ determinare cupimus, habemus formulam

$$
p \sin (B-A)=V(a a+b b-2 a b \cos (B-A))
$$

tum in problemate praesente tum in II.

79.

Ad completam determinationem sectionis conicae in plano suo tria requiruntur, situs perihelii, excentricitas et semiparameter. Quae si e quantitatibus datis ab ipsis pendentibus eruenda sunt, tot data adsint oportet, vt tres aequationes ab inuicem independentes formare liceat. Quilibet radius vector magnitudine et positione datus vnam aequationem suppeditat: quamobrem ad determinationem orbitae tres radii vectores magnitudine et positione dati requiruntur; si vero duo tantum habentur, vel vnum elementum ipsum iam datum esse debet, vel saltem alia quaedam quantitas, cuj aequationem tertiam superstruere licet. Hinc oritur varietas problematum, quae iam deinceps pertractabimus.

Sint $r, r^{\prime}$ duo radii vectores, qui cum recta in plano orbitae e Sole ad lubitum ducta faciant secundum directionem motus angulos $N, N^{\prime}$; sit porro $\Pi$ angulus quem cum eadem recta facit radius vector in perihelio, ita vt radiis vectoribus $r, r^{\prime}$ respondeant anomaliae verae $N-\Pi, N^{\prime}-I I$; denique sit $e$ excentricitas, $p$ semiparameter. 'Tunc habentur aequationes

$$
\begin{aligned}
& \frac{p}{r}=1+e \cos (N-\Pi) \\
& \frac{p}{r^{\prime}}=x+e \cos \left(N^{\prime}-\Pi\right)
\end{aligned}
$$

o quibus, si insuper vna quantitatum $p, e, \| I$ data est, duas reliquas determinare licebit.

Supponamus primo, datum esse semiparametrum $p$, patetgue determinationem quant:tatum $e$ et $\Pi$ ex aequationibus

$$
\begin{aligned}
& e \cos (N-I I)=\frac{p}{r}-1 \\
& e \cos \left(N^{\prime}-\Pi\right)=\frac{p}{r^{\prime}}-1
\end{aligned}
$$


fieri posse ad normam lemmatis III in art. praec. Habemus itaque

$$
\begin{aligned}
& \operatorname{tang}(N-\Pi)=\operatorname{cotang}\left(N^{\prime}-N\right)-\frac{r\left(p-r^{\prime}\right)}{r^{\prime}(p-r) \sin \left(N^{\prime}-N\right)} \\
& \operatorname{tang}\left(\frac{r}{2} N+\frac{1}{2} N^{\prime}-\Pi\right)=\frac{\left(r^{\prime}-r\right) \operatorname{cotang} \frac{r}{2}(B-A)}{r^{\prime}+r-\frac{2 r r^{\prime}}{p}}
\end{aligned}
$$

80.

Si angulus $I I$ datus est, $p$ et $e$ determinabuntur per aequationes

$$
\begin{aligned}
& p=\frac{r r^{\prime}\left(\cos (N-\Pi)-\cos \left(N^{\prime}-I\right)\right)}{r \cos (N-\Pi)-r^{\prime} \cos \left(N^{\prime}-\Pi\right)} \\
& e=\frac{r^{\prime}-r}{r \cos (N-I I)-r^{\prime} \cos \left(N^{\prime}-\Pi\right)}
\end{aligned}
$$

Denominatorem communem in his formulis reducere licet sub formam $a \cos (A-I)$, ita vt $a$ et $A$ a $\Pi$ sint independentes. Designante scilicet $H$ angulum arbitrarium, fit

$$
r \cos (N-\Pi)-r^{\prime} \cos \left(N^{\prime}-\Pi\right)=\left\{\begin{array}{r}
\left(r \cos (N-H)-r^{\prime} \cos \left(N^{\prime}-H\right)\right) \cos (H-I I) \\
-\left(r \sin (N-H)-r^{\prime} \sin \left(N^{\prime}-H\right)\right) \sin (H-I)
\end{array}\right.
$$

adeoque $=a \cos (A-\Pi)$, si $a$ et $A$ determinantur per aequationes

$$
\begin{aligned}
& r \cos (N-H)-r^{\prime} \cos \left(N^{\prime}-H\right)=a \cos (A-H) \\
& r \sin (N-H)-r^{\prime} \sin \left(N^{\prime}-H\right)=a \sin (A-H)
\end{aligned}
$$

Hoc modo fit

$$
\begin{aligned}
& p=\frac{2 r r^{\prime} \sin \frac{x}{2}\left(N^{\prime}-N\right) \sin \left(\frac{r}{2} N+\frac{x}{2} N^{\prime}-\Pi\right)}{a \cos (A-U)} \\
& e=\frac{r^{\prime}-r}{a \cos (A-\Pi)}
\end{aligned}
$$

Hae formulae imprimis sunt commodae, quoties $p$ et e pro pluribus valoribus ipsius $\Pi$ computandae sunt, manentibus $r, r^{\prime}, N, N^{\prime}$. - Quum ad calculum quantitatum auxiliarium $a, A$ angulum $H$ ad libitum assumere liceat, e re erit statuere $H=\frac{r}{2}\left(N+N^{\prime}\right)$, quo pacto formulae abeunt in has

$$
\begin{aligned}
& \left(r^{\prime}-r\right) \cos \frac{r}{2}\left(N^{\prime}-N\right)=-a \cos \left(A-\frac{r}{2} N-\frac{\pi}{2} N^{\prime}\right) \\
& \left(r^{\prime}+r\right) \sin \frac{r}{2}\left(N^{\prime}-N\right)=-a \sin \left(A-\frac{r}{2} N-\frac{r}{2} N^{\prime}\right)
\end{aligned}
$$

Determinato itaque angulo $A$ per aequationem $\operatorname{tang}\left(A-\frac{1}{2} N-\frac{\pi}{2} N^{\prime}\right)$

$=\frac{r^{\prime}+r}{r^{\prime}-r} \operatorname{tang} \frac{r}{2}\left(N^{\prime}-N\right)$, statim habetur $e=-\frac{\cos \left(A-\frac{\pi}{2} N-\frac{1}{2} N^{\prime}\right)}{\cos \frac{r}{2}\left(N^{\prime}-N\right) \cos (A-\Pi)}$. 
Calculum logarithmi quantitatis $\frac{r^{\prime}+r}{r-r}$ per artificium saepius iam explicatum contrahere licebit.

81.

Si excentricitas $e$ data est, angulus $\Pi$ per aequationem

$$
\cos (A-\Pi)=-\frac{\cos \left(A-\frac{\pi}{2} N-\frac{\pi}{2} N^{\prime}\right)}{e \cos \frac{T}{2}\left(N^{\prime}-N\right)}
$$

inuenietur, postquam angulus auxiliaris $A$ per aequationem

$$
\operatorname{tang}\left(A-\frac{\pi}{2} N-\frac{r}{2} N^{\prime}\right)=\frac{r^{\prime}+r}{r^{\prime}-r} \operatorname{tang} \frac{r}{2}\left(N^{\prime}-N\right)
$$

deterninatus est. Ambiguitas in determinatione anguli $A-\Pi$ per ipsius cosinum remanens in natura problematis fundata est, ita vt problemati duabus solutionibus diuersis satisfieri possit, e quibus quam adoptare quamue reiicere oporteat aliunde decidendum erit, ad quem finem valor saltem approximatus ipsius $\Pi$ iam cognitus esse debet. - Postquam $\Pi$ inuentus est, $p$ vel per formulas

$$
p=r(1+e \cos (N-\Pi))=r^{\prime}\left(1+e \cos \left(N^{\prime}-\Pi\right)\right)
$$

vel per hanc computabitur

$$
p=\frac{2 r r^{\prime} e \sin \frac{x}{2}}{\left(N^{\prime}-N\right) \sin \frac{r}{2}\left(N+N^{\prime}-\Pi\right)} \frac{\pi}{r^{\prime}-r}
$$

82.

Supponamus denique, tres radios vectores $r, r^{\prime}, r^{\prime \prime}$ datos esse, qui cum recta ad lubitum e Sole in plano orbitae ducta faciant angulos $N, N^{\prime}, N^{\prime \prime}$. Habebumtur itaque, retentis signis reliquis, aequationes $(\mathrm{I})$;

$$
\begin{aligned}
& \frac{p}{r}=1+e \cos (N-I I) \\
& \frac{p}{r^{\prime}}=1+e \cos \left(N^{\prime}-I\right) \\
& \frac{p}{r^{\prime}}=1+e \cos \left(N^{\prime \prime}-I\right)
\end{aligned}
$$

e quibus $p, \pi, e$ pluribus modis diuersis elici possmnt. Si quantitatem $p$ ante reliquas computare placet, multiplicentur tres aequationes (I) resp. per $\sin \left(N^{\prime \prime}-N^{\prime}\right)$, $-\sin \left(N^{\prime \prime}-N\right), \quad \sin \left(N^{\prime}-N\right)$, fietque additis productis per lemma $I$. art. 78

$$
p=\frac{\sin \left(N^{\prime \prime}-N^{\prime}\right)-\sin \left(N^{\prime \prime}-N\right)+\sin \left(N^{\prime}-N\right)}{\frac{1}{r} \sin \left(N^{\prime \prime}-N^{\prime}\right)-\frac{1}{r^{\prime}} \sin \left(N^{\prime \prime}-N\right)+\frac{1}{r^{\prime \prime}} \sin \left(N^{\prime}-N\right)}
$$


Hace expressio propius considerari meretur. Numerator manifesto fit

$$
\begin{aligned}
& =2 \sin \frac{x}{2}\left(N^{\prime \prime}-N^{\prime}\right) \cos \frac{x}{2}\left(N^{\prime \prime}-N^{\prime}\right)-2 \sin \frac{x}{2}\left(N^{\prime \prime}-N^{\prime}\right) \cos \left(\frac{x}{2} N^{\prime \prime}+\frac{x}{2} N^{\prime}-N\right) \\
& =4 \sin \frac{1}{2}\left(N^{\prime \prime}-N^{\prime}\right) \sin \frac{x}{2}\left(N^{\prime \prime}-N\right) \sin \frac{x}{2}\left(N^{\prime}-N\right)
\end{aligned}
$$

Statuendo porro $r^{\prime} r^{\prime \prime} \sin \left(N^{\prime \prime}-N^{\prime}\right)=n, r r^{\prime \prime} \sin \left(N^{\prime \prime}-N\right)=n^{\prime}, r r^{\prime} \sin \left(N^{\prime}-N\right)=n$, patet $\frac{x}{2} n, \frac{x}{2} n^{\prime}, \frac{x}{2} n^{\prime \prime}$ esse areas triangulorum inter radium vectorem secundum et terium, inter primum et tertium, inter primum et secundum. Hinc facile perspicietur, in formula noua

$$
p=\frac{4 \sin \frac{x}{2}\left(N^{H}-N^{\prime}\right) \sin \frac{x}{2}\left(N^{n}-N\right) \sin \frac{x}{2}\left(N^{\prime}-N\right) \cdot r^{\prime} r^{\prime \prime}}{n-n^{\prime}+n^{\prime \prime}}
$$

denominatorem esse duplum areae trianguli inter trium radiorum vectorum" extremitates i. e. inter tria corporis coelestis loca in spatio contenti. Quoties haec Irca parum ab inuicem remota sunt, area ista semper erit quantitas perparua et quidem ordinis tertii, siquidem $N^{\prime}-N, N^{\prime \prime}-N^{\prime}$ vt quantitates paruae ordinis primi spectantur. Hinc simul concluditur, si quantitatum $r, r^{\prime}, r^{\prime \prime}, N, N^{\prime}, N^{\prime \prime}$ vna vel plures erroribus vtut leuibus affecti sint, in determinatione ipsius $p$ errorem permagnum illine nasci posse; quamobrem haecce ratio orbitae dimensiones eruendi magnam praecisionem numquam admittet, nisi tria loca heliocentrica interuallis considerabilibus ab inuicem distent.

Ceterum simulac semiparameter $p$ inuentus est, $e$ et $I I$ determinabuntur e combinatione duarum quarumcunque aequationum I per methodum art. 79 .

\section{3.}

Si solutionem eiusdem problematis a computo anguli $I I$ inchoare malumus, methodo sequente vtemur. Subtrahimus ab aequationum (I) secunda tertiam, a prima tertiam, a prima secundam, quo pacto tres nouas sequentes obtinemus (II):

$$
\begin{aligned}
& \frac{\frac{1}{r^{\prime}}-\frac{1}{r^{\prime \prime}}}{2 \sin \frac{r}{2}\left(N^{\prime \prime}-N^{\prime}\right)}=\frac{e}{p} \sin \left(\frac{r}{2} N^{\prime}+\frac{r}{2} N^{\prime \prime}-\Pi\right) \\
& \frac{\frac{1}{r}-\frac{1}{r^{\prime \prime}}}{2 \sin \frac{r}{2}\left(N^{\prime \prime}-N\right)}=\frac{e}{p} \sin \left(\frac{r}{2} N+\frac{r}{2} N^{\prime \prime}-\Pi\right) \\
& \frac{\frac{1}{r}-\frac{1}{r^{\prime}}}{2 \sin \frac{x}{2}\left(N^{\prime}-N\right)}=\frac{e}{p} \sin \left(\frac{r}{2} N+\frac{r}{2} N^{\prime}-\Pi\right)
\end{aligned}
$$


Duae quaecunque ex his aequationibus secundum lemma II. art. 78 . dabunt $\Pi$ et $\frac{\grave{e}}{p}$, vnde per quamlibet aequationum, (I) habebuntur etiam e et $p$. Quodsi solutionem tertiam in art. 78 , II traditam adoptamus, combinatio aequationis primae cum tertia algorithmum sequentem producit. Determinetur angulus auxiliaris $\zeta$ per aequationem

$$
\operatorname{tang} \zeta=\frac{\frac{r^{\prime}}{r}-1}{1-\frac{r^{\prime}}{r^{\prime \prime}}} \cdot \frac{\sin \frac{T}{2}\left(N^{\prime \prime}-N^{\prime}\right)}{\sin \frac{1}{2}\left(N^{\prime}-N\right)}
$$

eritque $\operatorname{tang}\left(\frac{x}{4} N+\frac{x}{2} N^{\prime}+\frac{x}{4} N^{\prime \prime}-I I\right)=\operatorname{tang}\left(45^{\circ}+\zeta\right) \operatorname{tang} \frac{x}{4}\left(N^{\prime \prime}-N\right)$

Permutando locum secundum cum primo vel tertio, duae aliae solutiones huie prorsus analogae prodibunt. Quum hac methodo adhibita formulae pro $\frac{e}{p}$ minus expeditae euadant, $e$ et $p$ per methodum art. 80 e duabus aequationum (I) eruere praestabit. Ceterum ambiguitas in determinatione ipsius $I I$ per tangentem anguli $\frac{x}{4} N+\frac{\pi}{2} N^{\prime}+\frac{t}{4} N^{\prime \prime}-I I$ ita decidi debebit, vt $e$ fiat quantitas positiua: scilicet manifestum est, pro $e$ valores oppositos prodituros esse, si pro $\|$ valores $180^{\circ}$ dinersi accipiantur. Signum ipsius $p$ autem ab hac ambiguitate non pendet, valorque ipsins $p$ negatiuus euadere nequit, nisi tria puncta data in parte hyperbolae a Sole auersa iaceant, ad quem casum legibus naturae contrarium hic non respicimus.

Quae ex applicatione methodi primae in art. 78 , II post substitutiones operosiores orirentur, in casu praesente commodius sequenti modo obtineri possunt. Multiplicetur aequationum II prima per $\cos \frac{1}{2}\left(N^{\prime \prime}-N^{\prime}\right)$, tertia per $\cos \frac{1}{2}\left(N^{\prime}-N\right)$ subtrahaturque productum posterius a priori. Tunc lemmate I art. $7^{8}$ rite applicato*) prodibit aequatio

$$
\begin{gathered}
\frac{1}{2}\left(\frac{1}{r^{\prime}}-\frac{1}{r^{\prime \prime}}\right) \operatorname{cotang} \frac{x}{2}\left(N^{\prime \prime}-N^{\prime}\right)-\frac{x}{2}\left(\frac{1}{r}-\frac{1}{r^{\prime}}\right) \operatorname{cotang} \frac{x}{2}\left(N^{\prime}-N\right) \\
=\frac{e}{p} \sin \frac{r}{2}\left(N^{\prime \prime}-N\right) \cos \left(\frac{x}{2} N+\frac{r}{2} N^{\prime \prime}-\Pi\right)
\end{gathered}
$$

Quam combinando cum aequationum II secunda innenientur $I I$ et $\frac{e}{p}$, et quidem I per formulam

*) Statuendo scilicet in formula secunda $A=\frac{\%}{2}\left(N^{\prime \prime}-N^{\prime}\right), B=\frac{\pi}{3} N+\frac{\pi}{8} N^{\prime \prime}-\Pi, C=\frac{\pi}{2}\left(N-N^{\prime}\right)$. 


$$
\operatorname{tang}\left(\frac{r}{2} \cdot N+\frac{\pi}{2} N^{\prime \prime}-I I\right)=\frac{\frac{r^{\prime}}{r}-\frac{r^{\prime}}{r^{\prime \prime}}}{\left(1-\frac{r^{\prime}}{r^{\prime \prime}}\right) \operatorname{cotang} \frac{\pi}{2}\left(N^{\prime \prime}-N\right)-\left(\frac{r^{\prime}}{r}-1\right) \operatorname{cotang} \frac{r}{2}\left(N^{\prime}-N\right)}
$$

Etiam hine duae aliae formulae prorsus analogae deriuantur, permutando locum secundum eum primo vel tertio.

84.

Quum per duos radios vectores magnitudine et positione datos, atque elemeximu orbitae vnum orbitam integram determinare liceat, per illa data etiam tempus, intra quod corpus coeleste ab vno radio vectore ad alterum mouetur, determinabile erit, siquidem corporis massam vel negligimus vel saltem tamquam cognitam spectamus: nos stipposilioni priori inhaerebimus, ad quam posterior facile reducitur. Hinc vice versa patet, duos radios vectores magnitudine et positione datos vna cum tempore, intra quod corpns coeleste spatium intermedium describit, orbitam integram determinare. Hoc vero problema, ad grauissima in theoria motus corporum coelestium referendum, hand ita facile soluitur, quum expressio temporis per elementa transscendens sit, insuperque satis complicata. Eo magis dignum est, quod omni cura tractetur: quamobrem lectoribus haud ingratum fore speramus, quod praeter solutionem post tradendam, quae nihil amplius desiderandum relinquere videtur, eam quoque obliuioni eripiendam csse censuimus, qua olim antequam ista se obtulisset frequenter vsi sumus. Problemata difficiliora semper iuuat pluribus viis aggredi, nec bonam spernere etiamsi meliorem praeferas. Ab expositione huius methodi anterionis initium facimus.

\section{5.}

Retinebimus characteres $r, r^{\prime}, N, N^{\prime}, p, e, \pi$ in eadem significatione, in qua supra accepti sunt; differentiam $N^{\prime}-N$ denotabimus per $\Delta$, tempusque intra quod corpus coeleste a loco priori ad posteriorem mouetur per $t$. Jam patet, si valor approximatus alicuius quantitatum $p, e, I I$ sit notus, etiam duas reliquas inde determinari posse, ac dein per methodos in sectione prima explicatas tempus motui a loco primo ad secundum respondens. Quod si tempori proposito $t$ aequale euadit, valor suppositus ipsius $p$, e vel $\Pi$ est ipse verus, orbitaque ipsa iam inventa; sin minus, calculus cum valore alio a primo parum diuerso repetitus docebit, quanta variatio in valore temporis variationi exiguae in valore ipsius $p, e, \Pi$ 
respondeat, vnde per simplicem interpolationem valor correctus eruetur. Cum quo si-calculus denuo repetitur, tempus emergens vel ex asse cum proposito quadrabit, vel saltem perparum ab eo differet, ita vt certe nouis correctionibus adhibitis consensum tam exactum attingere liceat, quantum tabulae logarithmicae et trigonometricae permittunt.

Problema itaque eo reductum est, vt pro eo casu, vbi orbita adhuc penitus incognita est, valorem saltem approximatum alicuius quantitatum $p, e, \Pi$ determinare doceamus. Methodum iam trademus, per quam valor ipsius $p$ tanta praecisione ernitur, vt pro paruis quidem valoribus ipsius $\Delta$ nulla amplius correctione indigeat, adeoque tota orbita per primum calculum omni iam praecisione determinetur, quam tabulae vulgares permittunt. Vix ymquam autem aliter nisi pro valoribus mediocribus ipsius $\Delta$ ad hanc methodum recurrere oportebit, quum determinationem orbitae omnino adhuc incognitae, propter problematis complicationem. nimis intricatam, vix aliter suscipere liceat, nisi per obseruationes non nimis ab inuicem distantes, aut potius tales, quibus motus heliocentricus non nimius respondet.

86.

Designando radium vectorem indefinitum seu variabilem anomaliae verae $\nu$ - II respondentem per $\rho$, exit area sectoris a corpore coelesti intra tempus $t$ descripti $=\frac{r}{2} \int \varrho \rho d \nu$, hoc integrali a $\nu=N$ vsque ad $\nu=N^{\prime}$ exenso, adeoque, accipiendo $k$ in significatione art. $6, k t \vee p=\int \varrho \varrho \mathrm{d} \nu$. Iam constat, per formulas a Cotesio euolutas, si $\varphi x$ exprimat functionem quamcunque ipsius $x$, valorem coniinuo magis approximatum integralis $\int \varphi x \cdot d x$ ab $x=u$ rsque ad $x=u+\Delta$ extensi exhiberi per formulas

$$
\begin{aligned}
& \frac{x}{2} \Delta(\varphi u+\varphi(u+\Delta)) \\
& \frac{x}{6} \Delta\left(\varphi u+4 \varphi\left(u+\frac{1}{2} \Delta\right)+\varphi(u+\Delta)\right) \\
& \frac{x}{8} \Delta\left(\varphi u+5 \varphi\left(u+\frac{\pi}{3} \Delta\right)+5 \varphi\left(u+\frac{2}{3} \Delta\right)+\varphi(u+\Delta)\right)
\end{aligned}
$$

etc.: ad institutum nostrum apud duas formulas primas subsistere sufficiet.

Per formulam itaque primam in problemate nostro habemus $\int \varrho \rho d \nu=$ $\frac{1}{2} \Delta\left(r r+r^{\prime} r^{\prime}\right)=\frac{\Delta r r^{\prime}}{\cos 2 \omega}$, si statuitur $\frac{r^{\prime}}{r}=\operatorname{tang}\left(45^{\circ}+\omega\right)$. Quamobrem valor approximatus primus ipsius $\sqrt{ } p$ erit $=\frac{\Delta r r^{\prime}}{k t \cos 2 \omega}$, quem statuemus $=3 \alpha$. 
Per formulam secundam habemus exactius $\int \varrho \rho d \nu=\frac{x}{6} \Delta\left(r r+r^{\prime} r^{\prime}+4 R R\right)$, designante $R$ radium vectorem anomaliae intermediae $\frac{r}{2} N+\frac{r}{2} N^{\prime}-\Pi$ respondentem. Iam exprimendo $p$ per $r, R, r^{\prime}, N, N+\frac{r}{2} \Delta, N+\Delta$ ad normam formulae in art. 82 traditae, inuenimus

$$
\begin{aligned}
& p=\frac{4 \sin \frac{x}{4} \Delta^{2} \sin \frac{r}{2} \Delta}{\left(\frac{1}{r}+\frac{1}{r^{\prime}}\right) \sin \frac{r}{2} \Delta-\frac{1}{R} \sin \Delta}, \text { atque hinc } \\
& \frac{\cos \frac{\pi}{2} \Delta}{R}=\frac{x}{2}\left(\frac{1}{r}+\frac{1}{r^{\prime}}\right)-\frac{2 \sin \frac{x}{4} \Delta^{2}}{p}=\frac{\cos \omega}{\sqrt{\left(r r^{\prime} \cos 2 \omega\right)}-\frac{2 \sin \frac{x}{4} \Delta^{2}}{p}}
\end{aligned}
$$

Statuendo itaque $\frac{2 \sin \frac{1}{4} \Delta^{2} V\left(r r^{\prime} \cos 2 \omega\right)}{\cos \omega}=\delta$, fit $R=\frac{\cos \frac{1}{2} \Delta V\left(r r^{\prime} \cos 2 \omega\right)}{\cos \omega\left(1-\frac{\delta}{p}\right)}$, wnde valor approximatus secundus ipsius $\sqrt{ } p$ elicitur

$$
\vee p=\alpha+\frac{2 \alpha \cos \frac{x}{2} \Delta^{2} \cos 2 \omega^{2}}{\cos \omega^{2}\left(1-\frac{\delta}{p}\right)^{2}}=\alpha+\frac{\varepsilon}{\left(1-\frac{\delta}{p}\right)^{2}}
$$

si statuitur $2 \alpha\left(\frac{\cos \frac{x}{2} \Delta \cos 2 \omega}{\cos \omega}\right)^{2}=\varepsilon$. Scribendo itaque $\pi$ pro $\vee p$, determinabitur $\pi$ per aequationem $(\pi-\alpha)\left(1-\frac{\delta}{\pi \pi}\right)^{2}=\varepsilon$, quae rite euoluta ad quintum gradum ascenderet. Statuamus $\pi=q+\mu$, ita vt sit $q$ valor approximatus ipsius $\pi$, atque $\mu$ quantitas perexigua, cuius quadrata altioresque potestates negligere liceat: Qua substitutione prodit

$$
\begin{aligned}
& (q-\alpha)\left(1-\frac{\delta}{q q}\right)^{2}+\mu\left\{\left(1-\frac{\delta}{q q}\right)^{2}+\frac{4 \delta(q-\alpha)}{q^{3}}\left(1-\frac{\delta}{q q}\right)\right\}=\varepsilon, \text { siue } \\
& \mu=\frac{\varepsilon q^{5}-(q q-\alpha q)(q q-\delta)^{2}}{(q q-\delta)\left(q^{3}+3 \delta q-4 \alpha \delta\right)}, \text { adeoque } \\
& \pi=\frac{\varepsilon q^{5}+(q q-\delta)(\alpha q q+4 \delta q-5 \alpha \delta) q}{(q q-\delta)\left(q^{3}+3 \delta q-4 \alpha \delta\right)}
\end{aligned}
$$

Iam in problemate nostro habemus valorem approximatum ipsius $\pi$, puta $=3 \alpha$, quo in formula praecedente pro $q$ substituto, prodit valor correctus

$$
\pi=\frac{243 \alpha^{4} \varepsilon+3 \alpha(9 \alpha \alpha-\delta)(9 \alpha \alpha+7 \delta)}{(9 \alpha \alpha-\delta)(27 \alpha \alpha+5 \delta)}
$$

Statuendo itaque $\frac{\delta}{27 . \alpha \alpha}=\beta, \frac{\varepsilon}{(1-3 \beta) \alpha}=\gamma$, formula induit formam hancce 
$\pi=\frac{\alpha(1+\gamma+21 \beta)}{1+5 \beta}$, omnesque operationes ad problematis solutionem necessarine in his quinque formulis continentur:
I. $\frac{r^{\prime}}{r}=\operatorname{tang}\left(45^{\circ}+\omega\right)$
II. $\frac{\Delta r r^{\prime}}{5 k t \cos 2 \omega}=\alpha$
III. $\frac{2 \sin \frac{\pi}{4} \Delta^{2} V\left(r r^{\prime} \cos 2 \omega\right)}{27 \alpha \alpha \cos \omega}=\beta$
IV. $\frac{2 \cos \frac{\pi}{2} \Delta^{2} \cos 2 \omega^{2}}{(1-5 \beta) \cos \omega^{2}}=\gamma$
V. $\frac{\alpha(1+\gamma+21 \beta)}{1+5 \beta}=\sqrt{p}$

Si quid a praecisione harum formularum remittere placet, expressiones adhuc simpliciores euoluere licebit. Scilicet faciendo $\cos \omega$ et $\cos 2 \omega=1$ et euoluendo valorem ipsius $\sqrt{ } p$ in seriem secundum potestates ipsius $\Delta$ progredientem, prodit neglectis biquadratis altioribusque potestatibus

$\checkmark p=\alpha\left(5-\frac{x}{2} \Delta \Delta+\frac{\Delta \Delta V r r^{\prime}}{18 a \alpha}\right)$

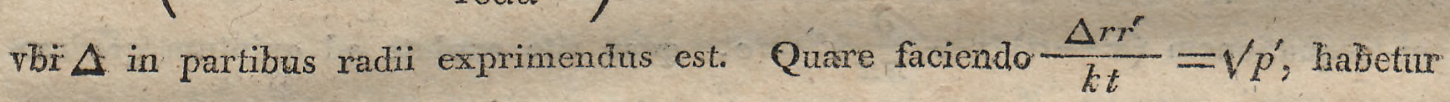

$$
\text { VI. } p=p^{\prime}\left(1-\frac{1}{3} \Delta \Delta+\frac{\Delta \Delta V r r^{\prime}}{5 p^{\prime}}\right)
$$

Simili modo explicando $\sqrt{ } p$ in seriem secundum potestates ipsius sin $\Delta$ progredientem exrergit posito $\frac{r^{\prime} \sin \Delta}{k t}=\sqrt{ } p^{\prime \prime}$

$$
\begin{aligned}
& \text { VIr. } \vee p=\left(1+\frac{\sin \Delta^{2} \sqrt{ } r r^{\prime}}{6 p^{\prime \prime}}\right) \vee p^{\prime \prime} \text {, siue } \\
& \text { VIII. } p=p^{\prime \prime}+\frac{x}{3} \sin \Delta^{2} \vee r r^{\prime}
\end{aligned}
$$

Formulae VII et VIII conueniunt cum iis, quas ill. Fuler tradidit in Theoric motus planetarum et cometarum, formula VI autem cum ea, quae in vsum vocata est in Recherches el calculs sur la vraie orbite elliptique de la comete de 1769, p. 80 .

87.

Exempla sequentia vsum praeceptorum praecedentium jllustrabunt, simulque inde gradus praecisionis aestimari poterit. 
I. Sit $\log r=0,5307640, \log r^{\prime}=0,5222259, \Delta=7^{\circ} 34^{\prime} 55^{\prime \prime} 75=27293^{\prime \prime} 75$, $i=2 x, 9559 x$ dies. Mic inuenitur $\omega=-55^{\prime} 47^{\prime \prime} 90$, vnde calculus vlterior ita se habet:

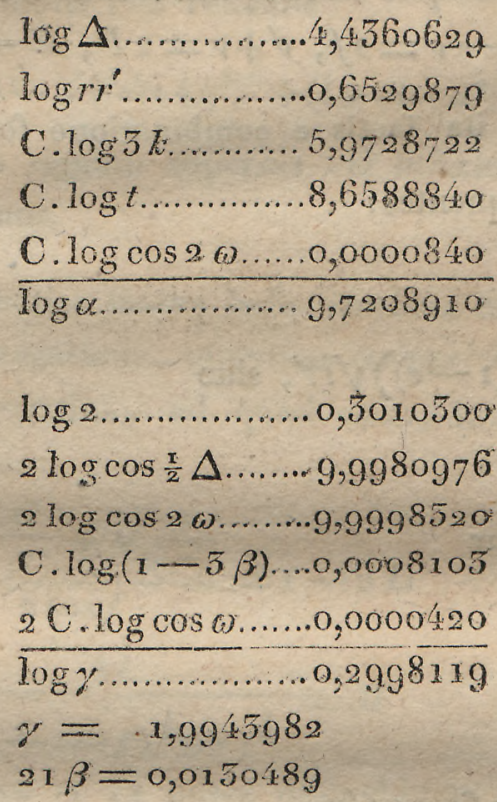

$\frac{x}{2} \log r r^{\prime} \cos 26 \ldots . .0,5264519$ ${ }_{2} \log \sin \frac{x}{4} \Delta \ldots \ldots \ldots .7,0589972$ $\log \frac{2}{27} \ldots \ldots \ldots \ldots \ldots . . . .8,8696662$ C. $\log \alpha \alpha \ldots \ldots \ldots \ldots, 5582180$

C. $\log \cos \omega \ldots \ldots . .0,0000210$ $\overline{\log \beta \ldots \ldots \ldots \ldots \ldots . .6,7933543}$ $\beta=0,0006215757$

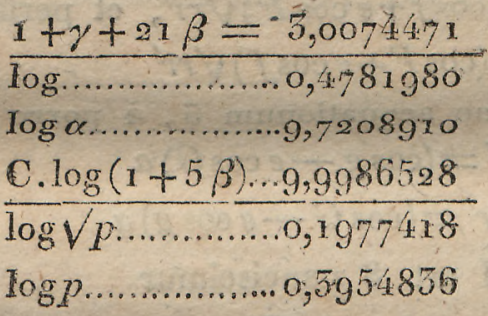

Hic valor ipsins $\log p$ vix vna vnitate in figura septima a vero differt: formula VI in hoc exemplo dat $\log p=0,3954807$; formula VII producit 0,5954780 ; denique formula VIII dat $0,3954,754$.

II. Sit $\log r=0,428279^{2}, \log r^{\prime}=0,4062035, \Delta=62^{\circ} 55^{\prime} 16^{\prime \prime} 64, t=259,88477$ dies. Hinc ervitur $\omega=-1^{\circ} 27^{\prime} 20^{\prime \prime} 14, \log \alpha=9,7182548, \beta=0,04555216, \gamma=$ $x, 68 \times 127, \log V p=0,2198027, \log p=0,4596054$, qui valor 187 vnitatibus in figura septima iusto minor est. Valor enim veras in hoe exemplo est 0,4596257 ; per formulam VI inuenitur 0,4368730 ; per formulan VH prodit 0,4159824 ; denique per formulam VIII eruitur 0,1051105 : duo postremi valores hic a vero tanium discrepant, yt ne approximationis quidem vice fungi possint.

88.

Methodi secundae expositio permultis relationibus notuis atque elegantibus enucleandis occasionem dabit: quae quum in diuersis sectionum conicarum generibus formas diuersas induant, singula seorsim tractare oportebit: ab ELLIPS I initium faciemus. 
Respondeant duobus locis anomaliae verae $w, v^{\prime}$ (e quibus $v$ sit tempore anterior), anomaliae excentricae $\mathbb{E}, \mathbb{E}^{\prime}$, radiique vectores $r, r^{\prime}$; porro sit $p$ semiparameter, $e=\sin \varphi$ excentricitas, $a$ semiaxis maior, $t$ tempus intra quod motus a loco primo ad secundum absoluitur; denique statuamus $y^{\prime}-v=2 f, v^{\prime}+v=2 F, E-E=2 g$, $E^{\prime}+E={ }_{2} G, a \cos \varphi=\frac{p}{\cos \varphi}=b$. Quibus ita factis e combinatione formularcm $\mathrm{V}$, VI art. 8 facile deducuntur aequationes sequentes:

[1] $b \sin g=\sin f \cdot V r r^{\prime}$

[2] $b \sin G=\sin F \cdot V r r^{\prime}$

$p \cos g=\left(\cos \frac{x}{2} v \cos \frac{x}{2} v^{\prime} \cdot(1+e)+\sin \frac{x}{2} v \sin \frac{x}{2} y^{\prime} \cdot(1-e)\right) V r r^{\prime}$, siue

[3] $p \cos g=(\cos f+e \cos F) V r r^{\prime}$, et perinde

[4] $p \cos G=(\cos F+e \cos f) V r r^{\prime}$

E combinatione aequationum 3,4 porro oritur

[5] $\cos f \cdot V r r^{\prime}=(\cos g-e \cos G) a$

[6] $\cos F \cdot V r r^{\prime}=(\cos G-e \cos g) a$

$E$ formula $I I$ art. 8 nanciscimur

[7] $r^{\prime}-r=2 a e \sin g \sin G$

$r^{\prime}+r=2 a-2 a e \cos g \cos G=2 a \sin g^{2}+2 \cos f \cos g V r r^{\prime}$

vade

$[8] a=\frac{r+r^{\prime}-2 \cos f \cos g V r r^{\prime}}{2 \sin g^{2}}$

Statuamus

[9] $\frac{\sqrt{\frac{r^{\prime}}{r}+V \frac{r}{r^{\prime}}}}{2 \cos f}=1+2 l$, eritque

[10] $a=\frac{2\left(l+\sin \frac{\pi}{2} g^{2}\right) \cos f \sqrt{ } r r^{\prime}}{\sin g^{2}}$

nec non $\vee a= \pm \frac{V\left(2\left(l+\sin \frac{x}{2} g^{2}\right) \cos f V r r^{\prime}\right)}{\sin g}$, vbi signum superius accipere oportet vel inferius, prout sing positiuus est vel negatiuus. - Formula XII. art. 8 nobis suppeditat aequationem

$\frac{k t}{a^{\frac{3}{2}}}=E^{\prime}-e \sin E^{\prime}-E+e \sin E=2 g-2 e \sin g \cos G=2 g-\sin 2 g+2 \cos f \sin g \frac{V r r^{\prime}}{a}$

Quodsi iam in hac aequatione pro a substituitur ipsius valor ex 10, ac breuitatis gratia ponitur 
[11] $\frac{R \cdot t}{2^{\frac{3}{2}} \cos f^{\frac{3}{2}}\left(r r^{\prime}\right)^{\frac{3}{4}}}=m$

prodit omnibus rite reductis

$[12] \pm m=\left(l+\sin \frac{7}{2} g^{2}\right)^{\frac{\pi}{2}}+\left(l+\sin \frac{r}{2} g^{2}\right)^{\frac{3}{2}}\left(\frac{2 g-\sin 2 g}{\sin g^{3}}\right)$

vbi ipsi $m$ signum superius vel inferius praefigendum est, prout sing positiuus est vel negatiuus.

Quoties motus heliocentricus est inter $180^{\circ}$ et $360^{\circ}$, siue generalius quoties $\cos f$ est negatiuus, quantitas $m$ per formulam 11 determinata euaderet imaginaria, atque $l$ negatiua, ad quod euitandum pro aequationibus 9,11 in hoc casu hasce adoptabimus:

$$
\begin{aligned}
& {\left[9^{\star}\right] \frac{V \frac{r^{\prime}}{r}+V \frac{r}{r^{\prime}}}{2 \cos f}=x-2 L} \\
& {\left[11^{\star}\right] \frac{k t}{2^{\frac{3}{2}}(-\cos f)^{\frac{3}{2}}\left(r r^{\prime}\right)^{\frac{3}{4}}}=I M}
\end{aligned}
$$

vnde pro 10 et 12 hasce obtinebimus.

$$
\begin{aligned}
& {\left[10^{*}\right] a=\frac{-2\left(L-\sin \frac{x}{2} g^{2}\right) \cos f V r r^{\prime}}{\sin g^{2}}} \\
& {\left[12^{*}\right] \pm M=-\left(L-\sin \frac{x}{2} g^{2}\right)^{\frac{x}{2}}+\left(L-\sin \frac{x}{2} g^{2}\right)^{\frac{3}{2}}\left(\frac{2 g-\sin 2 g}{\sin g^{3}}\right)}
\end{aligned}
$$

vbi signum ambiguum eodem modo determinandum est vt ante.

$$
89 .
$$

Duplex iam negotium nobis incumbit, primum, vt ex aequatione transcendente 12, quoniam solutionem directam non admittit, incognitam $g$ quam commodissime eruamus; secundum, vt ex angulo $g$ invento elementa ipsa deducamus. Quae antequam adeamus, transformationem quandam attingemus, cuius adiumento calculus quantitatis auxiliaris $\zeta$ vel $L$ expeditius absoluitur, insuperque plures formulae post euoluendae ad formam elegantiorem reducuntur.

Introducendo scilicet angulum auxiliarem $\omega$ per formulam $\stackrel{4}{v^{\prime}} \frac{r^{\prime}}{r}=$ $\operatorname{tang}\left(45^{\circ}+\omega\right)$ determinandum, fit $V \frac{r^{\prime}}{r}+V \frac{r}{r^{\prime}}=$ 
$2+\left(\operatorname{tang}\left(45^{\circ}+\omega\right)-\operatorname{cotang}\left(45^{\circ}+\omega\right)\right)^{2}=2+4 \operatorname{tang} 2 \omega^{2} ;$ vnde habetur

$l=\frac{\sin \frac{x}{2} f^{2}}{\cos f}+\frac{\operatorname{tang} 2 \omega^{2}}{\cos f}, L=-\frac{\sin \frac{x}{2} f^{2}}{\cos f}-\frac{\operatorname{tang} 2 \omega^{2}}{\cos f}$

90.

Considerabimus primo casum eum, vbi e solutione aequationis 12 valor non. nimis maguus ipsius $g$ emergit, ita $v t \frac{2 g-\sin 2 g}{\sin g^{3}}$ in scricm secundum potestates ipsius $\sin \frac{\tau}{2} g$ progredientem euoluere liceat. Numerator huius expressionis, quam per $X$ denotabimus, fit

$=\frac{32}{3} \sin \frac{\pi}{2} g^{3}-\frac{x 6}{5} \sin \frac{x}{2} g^{5}-\frac{4}{7} \sin \frac{x}{2} g^{7}-e t c$.

Denominator antem

$=8 \sin \frac{x}{2} g^{3}-12 \sin \frac{x}{2} g^{5}+3 \sin \frac{x}{2} g^{7}+$ etc.

Vnde $\mathrm{X}$ obtinet formam

$\frac{4}{3}+\frac{8}{5} \sin \frac{x}{2} g^{2}+\frac{64}{3} \frac{\sin }{\frac{\pi}{2}} g^{4}+$ etc.

Vt autem legem progressionis coëfficientium eruamus, differentiamus aequationem $X \sin g^{3}=2 g-\sin 2 g$, vnde prodit $3 X \cos g \sin g^{2}+\sin g^{3} \frac{d X}{d g}=2-2 \cos 2 g=4 \sin g^{2}$ statuenclo porro $\sin \frac{1}{2} g^{2}=x$, fit $\frac{d x}{d g}=\frac{1}{2} \sin g$, vnde concluditur $\frac{d X}{d x}=$ $\frac{8-6 X \cos g}{\sin g^{2}}=\frac{4-3 X(1-2 x)}{2 x(1-x)}$, et proin $(2 x-2 x x) \frac{d X}{d x}=4-(5-6 x) X$

Quodsi igitur statuimus

$X=\frac{4}{3}\left(1+\alpha x+\beta x x+\gamma x^{3}+\delta x^{4}+\right.$ etc. $)$

obtinemus aequationem

$\frac{8}{3}\left(\alpha x+(2 \beta-\alpha) x x+(5 \gamma-2 \beta) x^{3}+(4 \delta-5 \gamma) x^{4}+e t c\right)=(8-4 \alpha) x+(8 \alpha-4 \beta) x x$ $+(8 \beta-4 \gamma) x^{3}+(8 \gamma-4 \delta) x^{4}+$ etc.

quae identica esse debet. Hinc colligimus $\alpha=\frac{5}{5}, \beta=\frac{8}{7} \alpha, \gamma=\frac{r 0}{9} \beta, \delta=\frac{r}{3} \frac{2}{x} y$ etc., vbi lex progressionis obuia est. Habemus itaque

$X=\frac{4}{3}+\frac{4.6}{3.5} x+\frac{4 \cdot 6.8}{3.5 \cdot 7} x x+\frac{4 \cdot 6.8 \cdot 10}{3.5 \cdot 7 \cdot 9} x^{3}+\frac{4 \cdot 6 \cdot 3 \cdot 10 \cdot 12}{3.5 \cdot 7 \cdot 9 \cdot 11} x^{4}+$ etc. 
Hanc seriem transformare licet in fractionem continnam sequentem:

$$
X=\frac{\frac{\frac{4}{3}}{1-\frac{\frac{6}{3} x}{1+\frac{2}{5 \cdot 7} x}}}{\frac{1-\frac{5.8}{7 \cdot 9} x}{1-\frac{1 \cdot 4}{9 \cdot 11} x}}
$$

Lex secundum quam coëfficientes $\frac{6}{5},-\frac{2}{5 \cdot 7}, \frac{5.8}{7 \cdot 9}, \frac{1 \cdot 4}{9 \cdot 11}$ etc. progrediuntur, obuia est; scilicet terminus $n^{t u s}$ huius seriei fit pro $n$ pari $=\frac{n-5 . n}{2 n+1.2 n+3}$, pro $n$ impari autem $=\frac{n+2 \cdot n+5}{2 n+1.2 n+3}:$ vlterior huius argumenti euolutio nimis aliena esset ab instituto nostro. Quodsi iam statuimus

$$
\frac{x}{1+\frac{\frac{2}{5.7} x}{1-\frac{5.8}{\frac{7.9}{3}}}}=x-\xi
$$

fit $X=\frac{1}{\frac{3}{4}-\frac{9}{10}(x-\xi)}$, atque $\xi=x-\frac{5}{6}+\frac{10}{9 X}$, sine

$\xi=\frac{\sin g^{3}-\frac{3}{4}(2 g-\sin 2 g)\left(1-\frac{6}{5} \sin g^{2}\right)}{\frac{9}{5}(2 g-\sin 2 g)}$. Numerator huius expressionis est quantitas ordiniș septimi, denominator ordinis tertii, adeoque $\xi$ ordinis quarti, siquidem $g$ tamquam quantitas ordinis primi, siue $x$ tamquam ordinis secundi spectatur. Hinc concluditur, formulam hancee ad computum numericum exactum ipsius $\xi$ haud idoneam esse, quoties $g$ angulum non valde considerabilem exprimat: tunc autem ad, hunc finem commode adhibentur formulae sequentes, quae ab inuicem per ordinem commutatum numeratorum in coëfficientibus fractis differunt, et quarum prior e ralore supposito ipsius $x-\xi$ haud difficile deriuatur ${ }^{\star}$ ):

*) Deductio posterioris quasdam transformationes minus obuias aliaque occasione explicandas supponit. 


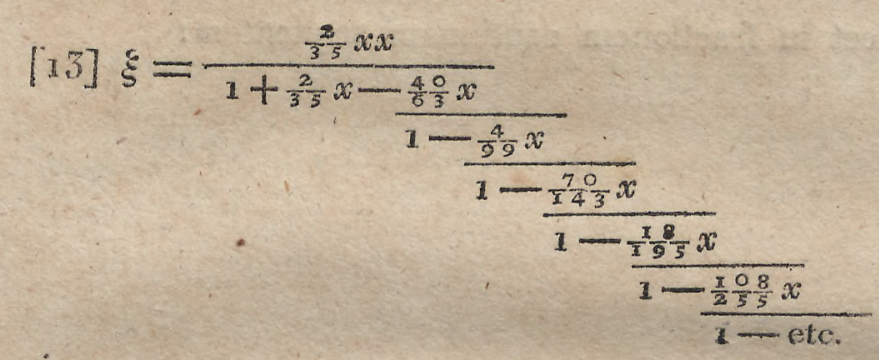

sine

$$
\xi=\frac{\frac{2}{35} x x}{1-\frac{18}{3 \frac{8}{5} x-\frac{4}{\frac{6}{3} x}}}
$$

In tabula tertia huic operi annexa pro cunctis valoribus ipsius $x$ a o vsque ad 0,3 , per singulas partes millesimas, valores respondentes ipsius $\xi$ ad septem figuras decimales computati reperiuntur. Haec tabula primo aspectu monstrat exiguitatem ipsius $\xi$ pro valoribus modicis ipsius g; ita e. $g$. pro $E^{\prime}-E=10^{\circ}$, siue $g=5^{\circ}$, vbi $x=0,00195$, fit $\xi=0,0000002$. Superfluum fuisset, tabulam adhuc vlterius continuare, quum termino vltimo $x=0,3$ respondeat $g=66^{\circ} 25^{\prime}$ siue $E^{\prime}-E=1322^{\circ} 50^{\prime}$. Ceterum tabulae columna tertia, quae valores ipsius $\xi$ valoribus negatiuis ipsins $x$ respondentes continet, infra loco suo explicabitur.

91.

Aequatio 12 , in qua, co de quo agimus casu, manifesto signum superius adoptare oportet, per introductionem quantitatis $\xi$ obtinet formam

$m=(l+x)^{\frac{x}{2}}+\frac{(l+x)^{\frac{3}{3}}}{\frac{3}{4}-\frac{9}{10}(x-\xi)}$

Statuendo itaque $V(l+x)=\frac{m}{y}$, atque

[14] $\frac{m m}{\frac{5}{6}+l+\xi}=h$, omnibus rite reductis prodit

$[15] h=\frac{(y-1) y y}{y+\frac{x}{9}}$

Quodsi itaque $h$ tamquam quantitatem cognitam spectare licet, $y$ inde per aequationem cubicam determinabitur, ac dein erit 
$[16] x=\frac{m m}{y y}-l$

Iam etiamsi $h$ implicet quantitatem adhuc incognitam $\xi$, in approximatione prims eam negligere atque pro $h$ accipere licebit $\frac{m m}{\frac{5}{6}+l}$, quoniam certe in eo de quo agimus casu $\xi$ semper est quantitas valde parua. Hinc per aequationes 15,16 elicientur $y$ et $x$; ex $x$ per tabulam III habebitur $\xi$, cuius adiumento per formulam 14 eruetur valor correctus ipsius $h$, cum quo calculus idem repetitus valores correctos ipsarum $y, x$ dabit: plerumque hi tam parum a praecedentibus different, $v t \xi$ iterum e tabula III desumta haud diuersa sit a valore primo: alioquin calculum denuo repetere oporteret, donec nullam amplius mutationem patiatur. Simulac quantitas $x$ inuenta erit, habebitur $g$ per formulam $\sin \frac{\pi}{2} g^{2}=x$.

Haec praecepta referuntur ad casum primum, vbi $\cos f$ positiuus est; in castz altero vbi negatiuus est statuimus $V(L-x)=\frac{I I T}{Y}$ atque

$\left[14^{*}\right] \frac{M M}{L-\frac{5}{6}-\xi}=H$, vnde aequatio $12^{*}$ rite reducta transit in hanc

$\left[15^{\star}\right] H=\frac{(Y+1) Y Y}{Y-\frac{x}{9}}$.

Per hanc itaque aequationem cubicam determinare licet $Y$ ex $I$, vnde rursus $x$ deriuabitur per aequationem

$\left[16^{*}\right] x=L-\frac{M M}{Y Y}$

In approximatione prima pro $I I$ accipietur valor $\frac{M M}{L-\frac{5}{6}}$; cum valore ipsius $x$ inde por aequationes $15^{*}, 16^{*}$ deriuato desumetur $\xi$ ex tabula III; hine per formulam. $14^{*}$ habebitur valor correctus ipsius $I I$, cum quo calculus codem modo repetetur. Tandem ex $x$ angulus $g$ eódem modo determinabitur $v t$ in casu primo.

92.

Quamquam aequationes $15,15^{*}$ in quibusdam easibus thes radices reales habere possint, tamen ambiguum numquam erit, quamnam in problemate nostro adoptare oporteat. Quum enim $h$ manifesto sit quantitas positiua, ex aequationum theoria facile concluditur, aequationem 15 habere radicem vnicam positiuam rol eum duabus imaginariis vel cum dnabus negatiuis: iam quum $y=\frac{m}{\sqrt{(l+x)}}$ neces- 
sario esse debeat quantitas positiua, nullam hic incertitudinem remanere patel. Quod vero attinet ad aequationem $15^{*}$, primo obseruamus, $L$ necessario esse maiorem quam 1 : quod facile probatur, si aequatio in art. 89 tradita sub formam $L=$ $1+\frac{\cos \frac{i}{2} f^{2}}{-\cos f}+\frac{\operatorname{tang} 2 \omega^{2}}{-\cos f}$ ponitur. Porro substituendo in aequatione $12^{*}$ pro $I M T$, $Y V(L-x)$, prodit $Y+1=(L-x) X$, adeoque $Y+1>(1-x) X>\frac{4}{3}+\frac{4}{5.5} x+$ $\frac{4.6}{5.5 .7} x x+\frac{4.6 .8}{5.5 .7 .9} x^{3}+$ etc. $>\frac{4}{3}$, et proin $Y>\frac{x}{3}$. Statuendo itaque $Y=\frac{r}{3}+Y^{\prime}$, nom cossario $Y^{\prime}$ erit quantitas positiua, aequatio $15^{*}$ antem hinc transit in hane $Y^{\prime 3}+2 Y^{\prime} Y^{\prime}+(1-H) Y^{\prime}+\frac{4}{2}-\frac{2}{9} H=0$, quam plures radices positiuas habere non posse ex aequationum theoria facile probatur. Hinc colligitur, aequationem $15^{\text {* }}$ vnicam radicem habituram esse maiorem quam $\frac{x}{3}^{*}$ ), quam neglectis reliquis in problemate nostro adoptare oportebit.

95.

Vt solutionem aequationis 15 pro casibus in praxi frequentissimis quantum fieri potest commodissimam reddamus, ad calcem huius operis tabulam peculiarem adiungimus (tabulam II), quac pro valoribus ipsius $h$ a o vsque ad o,6 logarithmos respondentes ipsius $y y$ ad septem figuras decimales summa cura computatos exhibet. Argumentum $h$ a o vsque ad 0,04 per singulas partes decies millesimas progreditur, quo pacto differentiae secundae ipsius $\log y y$ euanescentes sunt redditae, ita vt in hac quidem tabulae parte interpolatio simplex sufficiat. Quoniam vero tabula, si vbiuis eadem extensione gauderet, valde roluminosa euasisset, ab $h=0,04 \mathrm{vs-}$ que ad finem per singulas tantum millesimas partes progredi debuit; quamobrem in hac parte posteriori ad differentias secundas respicere oportebit, siquidem errores aliquot vnitatum in figura septima euitare cupimus. Ceterum valores minores ipsius $h$ in praxi longe sunt frequentissimi.

Solutio aequationis 15 quoties $h$ limitem tabulae egreditur, nec non solutio aequationis $15^{\star}$ sine difficultate per methodum indirectam vel per alias methodos satis cognitas perfici poterit. Ceterum haud abs re erit monere, valorem paruum ipsius $g$ cum valore negatiuo ipsitus $\cos f$ consistere non posse nisi in orbitis valde excentricis, $\mathrm{vt}$ eX aequatione 20 infra in art. $9^{5}$ tradenda sponte elucebit **).

*) Siquidem problema reuera solubile esse supponimus.

**) Ostendit ista aequatio, si $\cos f$ sit negatiuus, $\emptyset$ certe maiorem esse deberc quam $90^{\circ}-g$. 
$9^{4:}$

Tractatio aequationum $12,12^{*}$ in art. $91,92,93$ explicata, innixa est suppositioni, angulum $g$ non esse nimis magnum, certe infra limitem $66^{\circ}{ }_{2} 5^{\prime}$, vitra quem tabulam. III non extendimus. Quoties haec suppositio locum non habet, aequationes illae tantis artificiis non indigent: poterunt enim forma non mutata tutissime semper ac commodissime tentando solui. Tuto scilicet, quoniam valor expressionis $\frac{2 g-\sin 2 g}{\sin g^{3}}$, in qua $2 g$ in partibus radii exprimendum esse sponte patet, pro valoribus maioribus ipsius $g$ omni praecisione computari potest per tabulas trigonometricas, quod vtique fieri nequit, quamdiu $g$ est angulus paruus: commode, quoniam loci heliocentrici tanto interuallo ab inuicem distantes vix vmquam ad determinationem orbitae penitus adhuc incognitae adhibebuntur, ex orbitae autem cognitione qualicunque valor approximatus ipsius $g$ nullo propemodum. negotio per aequationem 1 vel 5 art. 89 demanat: denique e valore approximato ipsius $g$ valor correctus, aequationi 12 vel 12 * omni quae desideratur praecisione satisfaciens, semper paucis tentaminibus eruetur. Ceterum quoties duo loci heliocentrici propositi plus vna reuolutione integra complectuntur, memorem esse oportet, quod ab anomalia excentrica totidem reuolutiones completae absolutae ferunt, ita vt anguli $E^{\prime}-E, v^{\prime}-v$ vel ambo iaceant inter o et $360^{\circ}$, vel ambo inter multipla similia totius peripheriae, adeoque $f$ et $g$ vel simul inter o et $180^{\circ}$, vel inter multipla similia semiperipheriae. Quodsi tandem orbita omnino incognita esset, neque adeo constaret, vtrum corpus coeleste, transeundo a radio rectore prima ad secundum, descripserit partem tantum reuolutionis, an insuper reuolutionem integram vnam seu plures, problema nostrum nonnumquam plures solutiones diuersas admitteret: attamen buic casui in praxi vix vmquam occursuro hic non immoramur.

95.

'Transimus ad negotium secundum' puta determinationem elementorum ex innento angulo $g$. Semiaxis maior hic statim habetur per formulas $10,10^{*}$, pro quibus etiam sequentes adhiberi possunt:

$[17] a=\frac{2 m m \cos f \sqrt{ } r r^{\prime}}{y y \sin g^{2}}=-\frac{k k t t}{4 y y r r^{\prime} \cos f^{2} \sin g^{2}}$
$\left[17^{\star}\right] a=\frac{-2 M M \cos f V r r^{\prime}}{Y Y \sin g^{2}}=\frac{k k t t}{4 X Y r r^{\prime} \cos f^{2} \sin g^{2}}$ 
Scmiaxis minor $b=\sqrt{ }$ ap habetur per aequationem 1 , qua eum praecedentibus combinata prodic

[18] $p=\left(\frac{y r r^{\prime} \sin 2 f}{k t}\right)^{2}$

$\left[18^{\star}\right] p=\left(\frac{\operatorname{Ir} r^{\prime} \sin 2 f}{k t}\right)^{2}$

Iam sector ellipticus inter duos radios vectores atque arcum ellipticum contentus fit $=\frac{r}{2} k t \vee p$, triangulum antem inter eosdem radios voctores atque chordam $=\frac{x}{2} r r^{\prime} \sin 2 f$ : quamobrem ratio sectoris ad triangulum est vt $y: 1$ vel $Y: 1$. Haec obseruatio maximi est momenti, simulque aequationes $12,12^{\star}$ pulcherrime illustrat: patet enim hinc, in aequatione 12 partes $m,(l+x)^{\frac{\pi}{2}}, X(l+x)^{\frac{3}{2}}$, in aequatione $12^{*}$ antem partes $M,(L-x)^{\frac{\pi}{2}}, X(L-x)^{\frac{3}{2}}$ respectiue proportionales esse areae sectoris inter radios vectores atque arcum ellipticum), areae trianguli (inter radios vectores atque chordam), areae segmenti (inter arcum atque chordam), quoniam manifesto area prima aequalis est vel summae vel differentiae duarum reliquarum, prout $v^{\prime}-v$ vel inter o et $180^{\circ}$ iacet vel inter $180^{\circ}$ et $360^{\circ}$. In casu eo, vbi $v^{\prime}-v$ maior est quam $560^{\circ}$, areae sectoris nec non areae segmenti aream integrae ellipsis toties adiectam concipere oportet, quot reuolutiones integras ille motus continet.

Quum $b$ sit $=a \cos \varphi$, e combinatione aequationum $x, 10,10^{*}$ porro sequitur

$[19] \cos \varphi=\frac{\sin g \tan g f}{2\left(l+\sin \frac{\pi}{2} g^{2}\right)}$

$\left[19^{*}\right] \cos \varphi=\frac{-\sin g \tan g f}{2\left(L-\sin \frac{r}{2} g^{2}\right)}$

vude substituendo pro $l, L$ valores suos ex art. 89 prodit

$[20] \cos \varphi=\frac{\sin f \sin g}{1-\cos f \cos g+2 \operatorname{tang} 2 \omega^{2}}$

Haec formula ad calculum exactum excentricitatis non est idonea, quoties haecos modica est: sed facile ex ista deducitur formula aptiór sequens

[21] $\operatorname{tang} \frac{\pi}{2} \varphi^{2}=\frac{\sin \frac{\pi}{2}(f-g)^{2}+\operatorname{tang} 2 \omega^{2}}{\sin \frac{\pi}{2}(f+g)^{2}+\operatorname{tang} 2 \omega^{2}}$

cui ctiam forma sequens tribui potest (multiplicando rumeratorem et denominatorem per $\left.\cos \omega^{2}\right)$

[22] $\operatorname{tang} \frac{x}{2} \varphi^{2}=\frac{\sin \frac{x}{2}(f-g)^{2}+\cos \frac{\pi}{2}(f-g)^{2} \sin 2 \omega^{2}}{\sin \frac{x}{2}(f+g)^{2}+\cos \frac{x}{2}(f-g)^{2} \sin 2 \omega^{2}}$ 
Per vtramque formulam (achibitis si placet angulis auxiliaribus quorum tangentes $\frac{\operatorname{tang} 2 \omega}{\sin \frac{T}{2}(f-g)}, \frac{\operatorname{tang} 2 \omega}{\sin \frac{x}{2}(f+g)}$ pro priori, vel $\frac{\sin 2 \omega}{\operatorname{tang} \frac{x}{2}(f-g)}, \frac{\sin 2 \omega}{\operatorname{tang} \frac{x}{2}(f+g)}$ pro posteriori) angulum $\varphi$ omni semper praecisione determinare licebit.

Pro determinatione anguli $G$ adhiberi potest formula sequens, quae sponte demanat e combinatione aequationum 5,7 et sequentis non numeratae:

[25] $\operatorname{tang} G=\frac{\left(r^{\prime}-r\right) \sin g}{\left(r^{\prime}+r\right) \cos g-2 \cos f \sqrt{ } r r^{\prime}}$

e qua, introducendo $\omega$, facile deriuatur

[24] $\tan g G=\frac{\sin g \sin 2 \omega}{\cos 2 \omega^{2} \sin \frac{r}{2}(f-g) \sin \frac{r}{2}(f+g)+\sin 2 \omega^{2} \cos g}$

Ambiguitas hic remanens facile deciditur adiumento aeqrationis 7 , quae docet; $G$ inter o et $180^{\circ}$ vel inter $180^{\circ}$ et $560^{\circ}$ accipi debere, prout numerator in his duabus formulis positiuus fuerit rel negatiuus.

Combinando aequationem $3 \mathrm{cum}$ his, quae protinus demanant ex aequatione II art. 8.

$$
\begin{aligned}
& \frac{1}{r}-\frac{1}{r^{\prime}}=\frac{2 e}{p} \sin f \sin F \\
& \frac{1}{r}+\frac{1}{r^{\prime}}=\frac{2}{p}+\frac{2 e}{p} \cos f \cos F
\end{aligned}
$$

nullo negotio deriuabitur sequens

[25] $\operatorname{tang} F=\frac{\left(r^{\prime}-r\right) \sin f}{2 \cos g \sqrt{r r^{\prime}}-\left(r^{\prime}+r\right) \cos f}$

e qua, introducto angulo $\omega$, prodit

$[26] \operatorname{tang} F=\frac{\sin f \sin 2 \omega}{\cos 2 \omega^{2} \sin \frac{x}{2}(f-g) \sin \frac{x}{2}(f+g)-\sin 2 \omega^{2} \cos f}$

Ambiguitas hic pexinde tollitur vt ante. - Postquan anguli $F$ et $G$ inuenti erunt, habebitur $v=F-f, y=F+f$, vnde positio perihelii nota erit; nec non $E=G-g$, $E^{\prime}=G+g$. Denique motus medius intra tempus $t$ erit $=\frac{k t}{a^{\frac{3}{2}}}=2 g-2 e \cos G \sin g$, quarum expressionum consensus calculo confirmando inscruiet; cpocha autem anomaliae mediae, respondens temporis momento inter duo proposita medio, erit $G-e \sin G \cos g$, quae pro lubitu ad quoduis aliud tempus transferri poterit. Aliquanto adhuc commodius est, anomalias medias pro duobus temporum momentis 
datis per formulas $E-e \sin E, E^{\prime}-e \sin E^{\prime}$ computare, harumque differentia cum $\frac{k t}{a^{\frac{3}{2}}}$ comparanda ad calculi confirmationem $\mathrm{th}$.

96.

Aequationes in art. praec. traditae tanta quiden concinnitate gaudent, vt nihil amplius desiderari posse videatur. Nihilominus eruere licet formulas quasdam alias, per quas elementa orbitae multo adhuc elegantius et commodius determinantur: verum, enolutio harum formularum paullulo magis recondita est.

Resumimus ex art. 8 aequationes sequentes, quas commoditatis gratia numeris nouis distinguimus:

I. $\sin \frac{r}{2} v \frac{r}{a}=\sin \frac{x}{2} E V(1+e)$

II. $\cos \frac{r}{2} v \vee \frac{r}{a}=\cos \frac{r}{2} E V(1-e)$

III. $\sin \frac{1}{2} \varphi^{\prime} \vee \frac{r^{\prime}}{a}=\sin \frac{r}{2} E^{\prime} V(1+e)$

IV. $\cos \frac{3}{2} v^{\prime} V \frac{r^{\prime}}{a}=\cos \frac{r}{2} E^{\prime} V(1-e)$

Multiplicamus I per $\sin \frac{T}{2}(F+g)$, II per $\cos \frac{x}{2}(F+g)$, vade productis additis nanciscimur

$\cos \frac{r}{2}(f+g) V \frac{r}{a}=\sin \frac{r}{2} E \sin \frac{\pi}{2}(F+g) V(1+e)+\cos \frac{r}{2} E \cos \frac{r}{2}(F+g) V(1-e)$

siue propter $V(1+e)=\cos \frac{x}{2} \varphi+\sin \frac{x}{2} \varphi, V(1-e)=\cos \frac{r}{2} \varphi-\sin \frac{x}{2} \varphi_{3}$

$\cos \frac{r}{2}(f+g) \vee \frac{r}{a}=\cos \frac{r}{2} \varphi \cos \left(\frac{x}{2} F-\frac{x}{2} G+g\right)-\sin \frac{r}{2} \varphi \cos \frac{r}{2}(F+G)$

Prorsus simili modo multiplicando III per $\sin \frac{x}{2}(F-g)$, IV per $\cos \frac{x}{2}(F-g)$, prodit productis additis

$\cos \frac{x}{2}(f+g) \vee \frac{r^{\prime}}{a}=\cos \frac{x}{2} \varphi \cos \left(\frac{x}{2} F-\frac{x}{2} G-g\right)-\sin \frac{x}{2} \varphi \cos \frac{x}{2}(F+G)$

Subtrahendo ab hac aequatione praecedentem, oritur

$\cos \frac{\pi}{2}(f+g)\left(\sqrt{ } \frac{r^{\prime}}{a}-\sqrt{ } \frac{r}{a}\right)=2 \cos \frac{r}{2} \varphi \sin g \sin \frac{r}{2}(F-G)$

siue introducendo angulum auxiliarem $\omega$

$[27] \cos \frac{x}{2}(f+g) \tan g 2 \omega=\sin \frac{x}{2}(F-G) \cos \frac{x}{2} \varphi \sin g V^{4} \frac{a a_{4}}{r r^{r}}$ 
Per transformationes prorsus similes, quarum euolutionem lectori perito relinquimus, inuenitur

$[28] \frac{\sin \frac{x}{2}(f+g)}{\cos 2 \omega}=\cos \frac{x}{2}(F-G) \cos \frac{x}{2} \varphi \sin g \stackrel{4}{V} \frac{a \alpha}{r r^{\prime}}$

[29] $\cos \frac{\pi}{2}(f-g) \operatorname{tang} 2 \omega=\sin \frac{\pi}{2}(F+G) \sin \frac{x}{2} \varphi \sin g \stackrel{4}{V} \frac{a a}{r r^{\prime}}$

$[30] \frac{\sin \frac{r}{2}(f-g)}{\cos 2 \omega}=\cos \frac{r}{2}(F+G) \sin \frac{\tau}{2} \varphi \sin g \stackrel{4}{V} \frac{a \alpha}{r r^{\prime}}$

Quum partes primae in his quatuor aequationibus sint quantitates cognitae, ex 27 et 28 determinabuntur $\frac{x}{2}(F-G)$ et $\cos \frac{x}{2} \varphi \sin g \stackrel{4}{V}^{\prime} \frac{a a}{r r^{\prime}}=P$, nec non ex 29 et 50 perinde $\frac{\pi}{2}(F+G)$ et $\sin \frac{x}{2} \varphi \sin g \stackrel{4}{V} \frac{a \alpha}{r r^{\prime}}=Q$; ambiguitas in determinatione anguIorum $\frac{T}{2}(F-G), \frac{T}{2}(F+G)$ ita decidenda est, vt $P$ et $Q$ cum sin $g$ idem signum obtineant. Dein ex $P$ et $Q$ deriuabuntur $\frac{r}{2} \varphi$ et $\sin g \stackrel{4}{V} \frac{a a}{r r^{\prime}}=R$. Ex $R$ deduci potest $a=\frac{R R V r r^{\prime}}{\sin g^{2}}$, nec non $p=\frac{\sin f^{2} V r r^{\prime}}{R R}$, nisi illa quantitate, quae fieri debet $= \pm \sqrt{ }\left(2\left(l+\sin \frac{\pi}{2} g^{2}\right) \cos f\right)= \pm V\left(-2\left(L-\sin \frac{\pi}{2} g^{2}\right) \cos f\right)$, vnice ad calculi confirmationem vti malimus, in quo casu $a$ et $p$ commodissime determinantur per formulas

$b=\frac{\sin f \vee r r^{\prime}}{\sin g}, a=\frac{b}{\cos \varphi}, p=b \cos \varphi$

Possunt etiam, pro lubito, plures aequationum art. 89 et $9^{5}$ ad calculi confirmationem in vsum vocari, quibus sequentes adhuc adiicimus:

$\frac{2 \operatorname{tang} 2 \omega}{\cos 2 \omega} \vee \frac{r r^{\prime}}{a \alpha}=e \sin G \sin g$

$\frac{2 \tan 2 \omega}{\cos 2 \omega} \vee \frac{p p}{r r^{\prime}}=e \sin F \sin f$

$\frac{2 \tan g 2 \omega}{\cos 2 \omega}=\operatorname{tang} \varphi \sin G \sin f=\operatorname{tang} \varphi \sin F \sin g$

Denique motus medius atque epocha anomaliae mediae perinde inuenientur vt in art. praec.

97.

Ad illustrationem methodi inde ab art. 88 expositae duo exempla art. 87 resumemus: anguli auxiliaris $\omega$ significationem hactenus obseruatam, non esse con- 
fundendam cum ea, in qua in art. 87,88 acceptum erat idem signum, vix opus erit monuisse.

I. In exemplo primo habemus $f=3^{\circ} 47^{\prime} 26^{\prime \prime} 865$, porroque $\log \frac{r^{\prime}}{r}=$ $9,9914599, \log \operatorname{tang}\left(45^{\circ}+\omega\right)=9,997864975, \omega=-8^{\prime} 27^{\prime \prime}$ oo6. Hinc per art. 89

\begin{tabular}{|c|c|}
\hline $\log \sin \frac{x}{2} f^{2} \ldots \ldots \ldots \ldots \ldots, 0389972$ & $\log \operatorname{tang} 2 \omega^{2} \ldots \ldots \ldots \ldots 5,3832428$ \\
\hline $\log \cos f \ldots \ldots \ldots \ldots \ldots . \ldots 9,9990488$ & $\log \cos f \ldots \ldots \ldots \ldots \ldots \ldots . . . . .9,9990488$ \\
\hline 7,0399484 & \\
\hline$=\log 0,0010963480$ & $=\log 0,0000242211$ \\
\hline
\end{tabular}

adeoque $l=0,0011205691, \frac{5}{5}+l=0_{2} 8344539$. Porro fit $\log k t=9,5766974$

$2 \log k t \ldots \ldots \ldots \ldots . . .9,1535948$
C. $\frac{3}{2} \log r r^{\prime} \ldots \ldots \ldots . . .9,0205181$
C. $\log 8 \cos f^{3} \ldots . .9,0997656$
$\frac{\log m m \ldots \ldots \ldots \ldots \ldots .7,2756765}{\log \frac{5}{6}+l \ldots \ldots \ldots \ldots .9,9214023}$
7,5522742

Est itaque valor approximatus ipsius $h=0,00225047$, cui in tabula nostra II respondet $\log y y=0,0021633$. Habetur itaque $\log \frac{m m}{y y}=7,2715132$, siue $\frac{m i n}{y y}$ $=0,001868587$, vnde per formulam 16 fit $x=0,0007480179:$ quamobrem quum $\xi$ per tabulam III omnino insensibilis sit, valores inuenti pro $h, y, x$ correctione non indigent. Iam determinatio elementorum ita se habet:

$\log x \ldots \ldots \ldots .6,8739120$

$\log \sin \frac{\pi}{2} g \ldots 8,4369560, \frac{\pi}{2} g=1^{0} 34^{\prime} 2^{\prime \prime} 0286, \frac{x}{2}(f+g)=3^{\circ} 27^{\prime} 45^{\prime \prime} 4611, \quad \frac{\pi}{2}(f-g)=$ $19^{\prime} 41^{\prime \prime} 403 \mathrm{~g}$. Quare ad normam formularum $27,28,29,50$ habetur

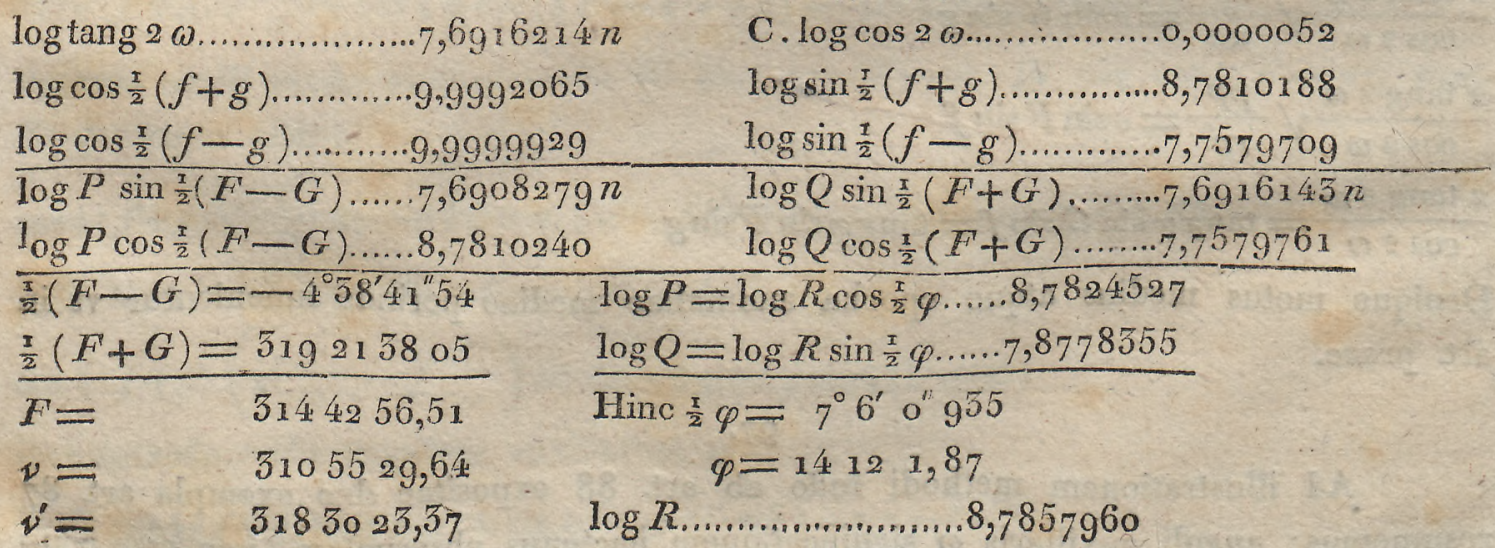




\begin{tabular}{|c|c|}
\hline$G=$ & $324^{\circ}$ ó $^{\prime} 19^{\prime \prime} 59$ \\
\hline & $327 \quad 825,65$ \\
\hline & $\overline { \frac { 7 } { 2 } } \longdiv { \operatorname { l o g } r r ^ { \prime } \ldots \ldots \ldots \ldots , 5 , 3 6 4 9 3 9 }$ \\
\hline & $\log \sin f \ldots \ldots \ldots . .8,8202 g \circ 9$ \\
\hline & C. $\log \sin g \ldots \ldots .1,2621765$ \\
\hline & $\log b \ldots \ldots \ldots \ldots \ldots, 4089613$ \\
\hline & $\log \cos \varphi \ldots \ldots \ldots . .9,9865224$ \\
\hline & $\log p \ldots \ldots \ldots \ldots ., 39,354857$ \\
\hline & $\log a \ldots \ldots \ldots \ldots, 4224389$ \\
\hline & $\begin{array}{l}\log k \ldots \ldots \ldots \ldots \ldots . .5,5500066 \\
\frac{3}{2} \log a \ldots \ldots \ldots . . .6,635658 \\
\end{array}$ \\
\hline & $2,9^{165482}$ \\
\hline & $\log t \ldots \ldots \ldots \ldots \ldots 1,5411160$ \\
\hline & 4,2574642 \\
\hline
\end{tabular}

Est itaque motu's medius diurnus $=82$ ' 7989 . Motus medius Ad calculum confrmandum $\frac{x}{2} \log 2 \cos f$ $. .0,150039$ $\frac{\frac{1}{2} \log (l+x)=\log \frac{m}{y} \ldots 8,6357566}{8,7857960}$ $\log \sin \varphi \ldots \ldots \ldots \ldots \ldots, 9,5897262$ $\log _{206265 \ldots \ldots \ldots \ldots . .5,5144251}$ log ein secundis........4,7041513 $\log \sin E \ldots \ldots \ldots \ldots \ldots . . . . .9,8000767 n$ $\log \sin E^{\prime} \ldots \ldots \ldots \ldots \ldots \ldots . .9,7544714 n$ $\log e \sin E \ldots \ldots \ldots \ldots \ldots, 4,5042280 n$ $\log e \sin E^{\prime} \ldots \ldots \ldots \ldots \ldots . . .4386227 n$ $e \sin E=-51932^{\prime \prime} 14=-8^{\circ} 52^{\prime} 12^{\prime \prime} 14$ $e \sin E^{\prime}=-27455,08=-73755,08$

Hinc anomalia media pro loco primo $=329^{\circ} 44^{\prime} 27^{\prime \prime} 67$ pro secundo $=\quad 5344558,73$ Differentia $=\frac{5151,06}{5}$ intra tempus $t=18091^{\prime \prime} 07$ $=5^{\circ} 1^{\prime} 51^{\prime \prime} 07$

II. In exemplo altero fit $f=5 x^{\circ} 27^{\prime} 38^{\prime \prime} 32, \omega=-21^{\prime} 50^{\circ} 565, l=0,08655659$, $\log m m=9,3530651, \frac{m m}{\frac{5}{6}+l}$ siue valor approximatus ipsius $h=0,2451454 ;$ huic in tabula II respondet $\log y y=0,1722685$, vade deducitur $\frac{m m}{y y}=0,15163408, x=$ 0,06527749 , hine e tabula III sumitur $\xi=0,0002531$. Quo valore adhibito prodeunt valores correcti $h=0,2450779, \log y y=0,1722303, \frac{m m}{y y}=0,15164737, x=$ $0,06529078, \xi=0,0002532$. Quodsi cum hoc valore ipsius $\xi$, vnica tantum vnitate in figura septima a priori diuerso, calculus denuo repeteretur; $h, \log y y, x$ mutationem sensibilem non acciperent, quamobrem valor inuentus ipsius $x$ iam est verus, statimque inde ad determinationem elementorum progredi licet. Cui hic non immoramur, quum nihil ab exemplo praecedente differat.

III. Haud abs re erit, etiam casum alterum vbi $\cos f$ negatiuus est exemplo illustrare. Sit $v^{\prime}-v=224^{\circ} 0^{\prime} o^{\prime \prime}$, siue $f=112^{\circ} o^{\prime} o^{\prime \prime}, \log r=0,1394892, \log r^{\prime}$ 
$=0,397879^{4}, t=206,80919$ dies. Hic inuenitur $\omega=+4^{\circ} 14^{\prime} 45^{\prime \prime} 78, L=1,8942298$, $\log M M=0,67^{24333}$, valor primus approximatus ipsius $\log I I=0,6467603$, vnde per solutionem aequationis $15^{\star}$ obtinetur $Y=1,591432$, ac dein $x=0,037037$, cui respondet, in tabula III, $\xi=0,0000801$. Hinc oriuntur valores correcti $\log I I=$ $0,6467931, Y=1,5915107, x=0,0372195, \xi=0,0000809$. Calculo cum hoc valore ipsius $\xi$ denuo repetito prodit $x=0,0372215$, qui valor, quum $\xi$ inde haud mutata prodeat, nulla amplius correctione indiget. Inuenitur dein $\frac{\pi}{2} g=11^{\circ} 7^{\prime} 25^{\prime \prime} 40$, atque hinc perinde vt in exemplo I

$$
\begin{aligned}
& \frac{x}{2}(F-G)=3^{\circ} 53^{\prime} 53^{\prime \prime} 59 \quad \log P=\log R \cos \frac{x}{2} \varphi \ldots \ldots .9,9700507 \\
& \frac{x}{2}(F+G)=826 \quad 6,58 \quad \log Q=\log R \sin \frac{\pi}{2} \varphi \ldots \ldots . .9,8580552 \\
& F=\quad x 15959,97 \quad \frac{x}{2} \varphi=37^{\circ} 41^{\prime} 34^{\prime \prime} 27 \\
& \nu=-10000,03 \quad \varphi=75238,54 \\
& v^{\prime}=\quad+1255959,97 \\
& \boldsymbol{G}=\quad 45212,79 \\
& \log R \ldots \ldots \ldots \ldots \ldots \ldots \ldots \ldots \ldots, 0717096 \\
& \text { Ad calculi confirmationem eruitur } \\
& E=-172238,01 \\
& \boldsymbol{E}^{\prime}=+2775,59 \\
& \log \frac{M}{Y} V-2 \cos f \ldots \ldots . . .0,0717097
\end{aligned}
$$

In orbitis tam excentricis angulus $\varphi$ paullulo exactius computatur per formulam $19^{\star}$, quae in exemplo nostro dat $\varphi=75^{\circ} 25^{\prime} 8^{\prime \prime} 57$; excentricitas quoque e maiori praecisione determinatur per formulam $1-2 \sin \left(45^{\circ}-\frac{T}{2} \varphi\right)^{2}$ quam per $\sin \varphi$; secundum illam fit $e=0,96764650$.

Per formulam 1 porro inuenitur $\log b=0,6576611$, vnde $\log p=0,0595967$, $\log \alpha=1,2557255$, atque $\log$ arithmus distantiae in perihelio $=\log \frac{p}{1+e}=$ $\log a(1-e)=\log b \operatorname{tang}\left(45^{\circ}-\frac{x}{2} \varphi\right)=9,7656496$.

In orbitis tantopere ad parabolae similitudinem vergentibus loco epochae anomaliae mediae assignari solet tempus transitus per perihelium; interualia inter hoc tempus atque tempora duobus locis propositis respondentia determinari poterunt ex elementis cognitis per methodum in art. $4 x$ traditam, quorum differentia vel summa (prout perihelium vel extra duo loca proposita iacet vel intra) quum consentire debeat cum tempore $t$, calculo confirmando inseruiet. - Ceterum numeri huius tertii exempli superstructi erant elementis in exemplo art. 58 et 43 suppositis, quin adeo istud ipsum exemplum locum nostrum primum suppeditauerat: differentiae leuiusculae elementorum hic erutorum vnice a limitata praecisione tabulam sum logarithmicarum et trigonometricarum originem traxerunt. 
RETATIONTS TNTER LOCOS PLVRES IN ORBTTA.

109

98.

Solutio problematis nostri pro ellipsi in praecc. euoluta etiam ad parabolam et hyperbolam transferri posset, considerando parabolam tamquam ellipsin, in qua $a$ et $b$ essent quantitates infinitae, $\varphi=90^{\circ}$, tandem $\boldsymbol{E}, \boldsymbol{E}^{\prime}, g, G=0$; et perinde hyperbolam tamquam ellipsin in qua a esset negatiua, atque $b, E, E^{\prime}, g$, $G, \varphi$ imaginariae: malumus tamen his suppositionibus abstinere, problemaque pro troque sectionum conicarum genere seorsim tractare. Analogia insignis inter omnia tria genera sic sponte se manifestabit.

Retinendo in Parabora characteres $p, v, v^{\prime}, f, f, r, r^{\prime}, t$ in eadem significatione in qua supra accepti sunt, habemus e theoria motus parabolici:

[1] $\vee \frac{p}{2 r}=\cos \frac{\pi}{2}(F-f)$

$[2] \vee \frac{p}{2 r^{\prime}}=\cos \frac{x}{2}\left(T^{\prime}+f\right)$

$$
\begin{aligned}
& \frac{2 \hbar t}{p^{\frac{3}{2}}}=\operatorname{tang} \frac{x}{2}(F+f)-\operatorname{tang} \frac{x}{2}(F-f)+\frac{2}{3} \operatorname{tang} \frac{x}{2}(F+f)^{3}-\frac{x}{3} \operatorname{tang} \frac{x}{2}(F-f)^{3} \\
& =\left\{\operatorname{tang} \frac{1}{2}(F+f)-\tan g \frac{\pi}{2}(F-f)\right\} \cdot\left\{1+\operatorname{tang} \frac{x}{2}(F+f) \operatorname{tang} \frac{x}{2}(F-f)\right. \\
& \left.+\frac{x}{3}\left(\operatorname{lang} \frac{x}{2}(F+f)-\operatorname{tang} \frac{x}{2}(F-f)\right)^{2}\right\} \\
& =\frac{2 \sin f \vee r r^{\prime}}{p}\left\{\frac{2 \cos f V r r^{\prime}}{p}+\frac{4 \sin f^{2} r r^{\prime}}{3 p p}\right\} \text {, vade }
\end{aligned}
$$

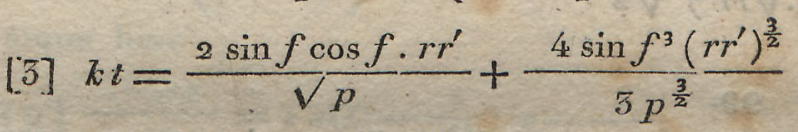

Porro deducitur ex multiplicatione aequationum $i, 2$

[4] $\frac{p}{\sqrt{r} r^{\prime}}=\cos F+\cos f$

nec non ex additione quadratorum

[5] $\frac{p\left(r+r^{\prime}\right)}{2 r r^{\prime}}=x+\cos F \cos f$

Hinc eliminato $\cos F$

[6] $p=\frac{2 r r^{\prime} \sin f^{2}}{r+r^{\prime}-2 \cos f \sqrt{ } r r^{\prime}}$

Quodsi itaque aequationes $9,9^{*}$ art. 88 hic quoque adoptamus, priorem pro cos $f$ positiuo, postexiorem pro negatino, habebinaus 
[7] $p=\frac{\sin f^{2} \vee r r^{\prime}}{2 l \cos f}$

$\left[7^{\star}\right] p=\frac{\sin f^{2} V r r^{\prime}}{-2 L \cos f}$

quibus valoribus in aequatione 3 substitutis, prodibit, retinendo characteres $m, M$ in significatione per aequationes 11,11 art. 88 stabilita,

[8] $m=l^{\frac{2}{2}}+\frac{4}{3} l^{\frac{3}{2}}$

$\left[8^{\star}\right] M=-L^{\frac{1}{2}}+\frac{4}{3} L^{\frac{3}{2}}$

Hae aequationes conueniunt cum $12,12^{*}$ art. 88 , si illic statuatur $g=0$. Hinc colligitur, si duo loci heliocentrici, quibus per parabolam satisfit, ita tractentur, ac si orbita esset elliptica, ex applicatione praeceptorum art. $9 x$ statim resultare debere $x=0$; vice versa facile perspicitur, si per praecepta ista prodeat $x=0$, orbitam pro ellipsi parabolam euadere, quum per aequationes $1,16,17,19,20$ fiat $b=\infty, a=\infty, \varphi=0$. Determinatio elementorum facillime dein absoluitur. Pro $p$ enim adhiberi poterit vel aequatio 7 art. praesentis, vel aequ. 18 art. $9^{5}$ ): pro $F$ autem fit ex aequationibus 1,2 huius art. $\tan g \frac{x}{2} F^{\prime}=\frac{\sqrt{ } r^{\prime}-\sqrt{ } r}{\sqrt{r^{\prime}+\sqrt{ } r}} \operatorname{cotang} \frac{x}{2} f=$ $\sin 2 \omega \operatorname{cotang} \frac{x}{2} f$, si angulus auxiliaris in eadem significatione accipitur, vt in art. 89 .

Hacce occasione adhue obseruamus, si in aequ. 3 pro $p$ substituatur valor eius ex 6 , prodire aequationem satis notam

$k t=\frac{r}{3}\left(r+r^{\prime}+\cos f \cdot V r r^{\prime}\right)\left(r+r^{\prime}-2 \cos f \cdot V r r^{\prime}\right)^{\frac{7}{2}} \sqrt{2}$

\section{9.}

In HYPERBOLA quoque characteres $p, v, v^{\prime}, f, F, r, r^{\prime}, t$ in significatione eadem retinemus, pro semiaxi maiori $a$ autem, qui hic negatiuus est, scribemus $-\alpha$; excentricitatem $e$ perinde vt supra art. 21 etc. statuemus $=\frac{1}{\cos \psi}$. Quantitatem auxiliarem illic per u expressam, statuemus pro loco primo $=\frac{C}{c}$, pro secundo $=C c$, vnde facile concluditur, $c$ semper esse maiorem quam 1 , sed ceteris paribus eo minus differre ab 1 , quo minus duo loci propositi ab inuicem distent. Ex aequationibus in art. $\div 21$ euolutis huc transferimus forma paullulum mutata sextam et septimam.

*) Vnde simul patet, $r$ et $X$ in paxabola easdem rationes exprimere $v t$ in ellipsi, $v$. art. $9^{5}$. 
RELATIONES INTER LOCOS PIVRES IN ORBITA.

I. I

[1] $\cos \frac{\pi}{2} v=\frac{\mathrm{T}}{2}\left(V \frac{C}{c}+V \frac{c}{C}\right) \vee \frac{(e-1) \alpha}{r}$

[2] $\sin \frac{x}{2} p=\frac{1}{2}\left(\vee \frac{C}{c}-\sqrt{ } \frac{c}{C}\right) \vee \frac{(e+1) \alpha}{r}$

[5] $\cos \frac{x}{2} y^{\prime}=\frac{x}{2}\left(V C_{c}+V \frac{1}{C c}\right) V\left(\frac{(e-1) \alpha}{r^{\prime}}\right.$

[4] $\sin \frac{x}{2} v^{\prime}=\frac{x}{2}\left(\sqrt{ } C c-\sqrt{ } \frac{x}{C c}\right) \vee \frac{(e+1) \alpha}{r^{\prime}}$

Hinc statim demanant sequentes:

[5] $\sin F=\frac{x}{2} \alpha\left(C-\frac{1}{C}\right) \vee \frac{e e-i}{r r^{\prime}}$

[6] $\sin f=\frac{r}{2} \alpha\left(c-\frac{1}{c}\right) \vee \frac{e e-i}{r r^{2}}$

[7] $\cos F=\left(e\left(c+\frac{1}{c}\right)-\left(C+\frac{1}{C}\right)\right) \frac{\alpha}{2 \sqrt{ } r r^{\prime}}$

[8] $\cos f=\left(e\left(C+\frac{x}{C}\right)-\left(c+\frac{1}{c}\right)\right) \frac{\alpha}{2 \sqrt{r r}}$

Porro fit per aequ. $\mathrm{X}$ art. 21

$$
\begin{aligned}
& \frac{r}{\alpha}=\frac{r}{2} e\left(\frac{C}{c}+\frac{c}{C}\right)-1 \\
& \frac{r^{\prime}}{\alpha}=\frac{1}{2} e\left(C c+\frac{1}{C c}\right)-1
\end{aligned}
$$

atque hinc

[9] $\frac{r^{\prime}-r}{\alpha}=\frac{x}{2} e\left(C-\frac{x}{C}\right)\left(c-\frac{x}{c}\right)$

$[10] \frac{r^{\prime}+r}{\alpha}=\frac{x}{2} e\left(C+\frac{1}{C}\right)\left(c+\frac{1}{c}\right)-2$

Haec aequatio 10 cum 8 combinata praebet

[i1] $\alpha=\frac{r^{\prime}+r-\left(c+\frac{1}{c}\right) \cos f \cdot V r r^{\prime}}{\frac{x}{2}\left(c-\frac{1}{c}\right)^{2}}$

Statuendo itaque perinde $v t$ in ellipsi $\frac{V \frac{r^{\prime}}{r}+\sqrt{ } \frac{r}{r^{\prime}}}{2 \cos f}=1+2 l$, vel $=1-2 L$, prout cos $f$ est positiuus rel negatiuus, fit 
[12] $\alpha=\frac{8\left(l-\frac{\pi}{4}\left(\sqrt{ } c-\sqrt{ } \frac{1}{c}\right)^{2}\right) \cos f \cdot \sqrt{ } r r^{\prime}}{\left(c-\frac{1}{c}\right)^{2}}$

$\left[12^{\star}\right] \alpha=\frac{-8\left(L+\frac{\pi}{4}\left(\sqrt{ } c-V\left(\frac{1}{c}\right)^{2}\right) \cos f \cdot V r r^{\prime}\right.}{\left(c-\frac{1}{c}\right)^{2}}$

Computus quantitatis $l$ vel $L$ hic perinde vt in ellipsi adiumento anguli auxiliaris as instituetur. Denique fit ex aequatione XI art. 22, (accipiendo logarithmos hyperbolicos)

$$
\begin{aligned}
\frac{k t}{a^{\frac{3}{2}}} & =\frac{r}{2} e\left(C c-\frac{1}{C c}-\frac{C}{c}+\frac{c}{C}\right)-\log C c+\log \frac{C}{c} \\
& =\frac{x}{2} e\left(C+\frac{1}{C}\right)\left(c-\frac{1}{c}\right)-2 \log c
\end{aligned}
$$

siue eliminata $C$ adiumento aequationis 8

$$
\frac{k t}{\alpha^{\frac{3}{2}}}=\frac{\left(c-\frac{1}{c}\right) \cos f \cdot V r r^{\prime}}{\alpha}+\frac{1}{2}\left(c c-\frac{1}{c c}\right)-2 \log c
$$

In hac aequatione pro $\alpha$ substituimus valorem eius ex $12,12^{*}$; dein characterem $m$ vel $M$ in eadem significatione, quam formulae $11,11^{*}$ art. 88 assignant, introducimus; tandemque breuitatis gratia scribimus

$$
\frac{\pi}{4}\left(\sqrt[V]{ } c-\sqrt{ } \frac{1}{c}\right)^{2}=z, \frac{c c-\frac{1}{c c}-4 \log c}{\frac{x}{4}\left(c-\frac{1}{c}\right)^{3}}=Z
$$

quo facto oriuntur aequationes

$[13] m=(l-z)^{\frac{x}{2}}+(l-z)^{\frac{3}{2}} Z$

$\left[15^{\star}\right] M=-(L+z)^{\frac{\pi}{2}}+(L+z)^{\frac{3}{2}} Z$

quae rnicam incognitam $z$ implicant, quum manifesto sit $Z$ functio ipsius $z$ per formulam sequentem expressa

$$
Z=\frac{(1+2 z) V(z+z z)-\log (V(1+z)+V z)}{2(z+z z)^{\frac{3}{2}}}
$$


RELATIONES INTER IOCOS PLVRES IN ORBITA.

$1 \times 3$

$x 00$.

In soluenda aequatione 15 vel $13^{*}$ eum casum primo seorsim considerabimus, vbi $z$ obtinet valorem haud magnum, ita vt $Z$ per seriem secundum potestates ipsius $z$ progredientem celeriterque conuergentem exprimi possit. Iam fit $(1+2 z) \sqrt{ }(z+z z)=z^{\frac{\pi}{2}}+\frac{5}{2} z^{\frac{3}{2}}+\frac{7}{8} z^{\frac{5}{2}} \ldots, \log (\sqrt{ }(1+z)+\sqrt{ } z)=z^{\frac{x}{2}}-\frac{x}{6} z^{\frac{3}{2}}+\frac{3}{4^{\frac{3}{0}}} z^{\frac{5}{2}} \ldots$, adeo que numerator ipsius $Z=\frac{8}{3} z^{\frac{3}{2}}+\frac{4}{5} z^{\frac{5}{2}} \ldots$; denominator autem fit $=2 z^{\frac{3}{2}}+3 z^{5} \ldots$, vnde $Z=\frac{4}{3}-\frac{8}{5} z \ldots$ Vt legem progressionis deiegamus, differentiamus aequationem $2(z+z z)^{\frac{3}{2}} Z=(x+2 z) \sqrt{ }(z+z z)-\log (\sqrt{ }(1+z)+\sqrt{ } z)$ vnde prodit omnibus rite reductis

$$
2(z+z z)^{\frac{3}{2}} \frac{\mathrm{d} Z}{\mathrm{~d} z}+3 Z(1+2 z) V(z+z z)=4 V(z+z z)
$$

siue

$$
(2 z+2 z z) \frac{d Z}{d z}=4-(3+6 z) Z
$$

vnde simili ratione $v t$ in art. 90 deducitur $Z=\frac{4}{3}-\frac{4.6}{3.5} z+\frac{4.6 .8}{3.5 .7} z z-\frac{4.6 .8 \cdot 10 .}{5.5 \cdot 7 \cdot 9 \cdot} z^{3}+\frac{4.6 .8 .10 .12}{5.5 \cdot 7 \cdot 9 \cdot 11} z^{4}-$ etc.

Patet itaque, $Z$ prorsus eodem modo a $Z$ pendere, vt supra in ellipsi $X$ ab $x$ : quamobrem si statuimus

$$
Z=\frac{x}{\frac{3}{4}+\frac{9}{10}(z+\zeta)}
$$

determinabitur etiam $\zeta$ perinde per $-z$ vt supra $\xi$ per $x$, ita vi habeatur.

$$
\text { [14] } \zeta=\frac{\frac{\frac{2}{35} z z}{1-\frac{2}{35} z+\frac{\frac{40}{6} \frac{0}{3} z}{1+\frac{4}{90} z}}}{\frac{1+\frac{70}{x+3} z}{1+\text { etc. }}}
$$

siue

$$
\zeta=\frac{\frac{2}{35} z z}{1+\frac{x 8}{35} z+\frac{\frac{4}{63} z}{1+\frac{\frac{40}{9} z}{1+\frac{18}{1 \frac{8}{4} 3} z}}}
$$

Hoc modo computati sunt valores ipsius $\zeta$ pro $z=0$ vsque ad $z=0,5$ per singulas partes millesimas, quos columna tertia tabulae III exhibet.

15 
101.

Introducendo quantitatem $\zeta$ statuendoque $V(l-z)=\frac{m}{y} \operatorname{vel} V(L-z)=\frac{I I}{Y}$, nec non

$[15] \frac{m m}{\frac{5}{6}+l+\zeta}=h$, vel

$\left[15^{\star}\right] \frac{M M}{L-\frac{5}{6}-\zeta}=I$

aequationes $13,13^{\star}$ hancce formam induunt

[16] $\frac{(y-1) y y}{y+\frac{x}{9}}=h$

$\left[16^{*}\right] \frac{(Y+1) Y Y}{Y-\frac{x}{9}}=H$

adeoque omnino identicae fiunt cum iis ad quas in ellipsi pertuentum est $\left(15,15^{\star}\right.$ art. 91). Hinc igitur, quatenus $h$ vel $I I$ pro cognita haberi potest, $y$ vel $I$ deduci poterit, ac dein erit

$[17] z=l-\frac{m m}{y y}$

$\left[17^{\star}\right] z=\frac{M M}{Y Y}-\tau$

Ex his colligitur, omnes operationes supra pro ellipsi praescriptas perinde etiam pro hyperbola valere, donec e valore approximato ipsius $h$ vel $H$ eruta fuerit quantitas $y$ vel $Y$; dein vero quantitas $\frac{m m}{y y}-l$ vel $L-\frac{M M}{Y Y}$, quae in ellipsi positiua enadere debebat, in parabolaque $=0$, fieri debet negatiua in hyperbola: hoc itaque criterio genus sectionis conicae definietur. Ex inuenta $z$ tabula nostra dabit $\zeta$, hinc orietur valor correctus ipsius $h$ vel $I I$, cum quo calculus repetendus est, donec omnia ex asse conspirent.

Postquam valor verus ipsius $z$ inuentus est, $c$ inde per formulam $c=$ $x+2 z+2 V(z+z z)$ deriuari posset, sed praestat, etiam ad vsus sequentes, angulum auxiliarem $n$ introducere, per aequationem tang $2 n=2 \sqrt[V]{ }(z+z z)$ determinandum; hinc fiet $c=\operatorname{tang} 2 n+V\left(1+\operatorname{tang} 2 n^{2}\right)=\operatorname{tang}\left(45^{\circ}+n\right)$ 
102.

Quum in hyperbola perinde vt in ellipsi $y$ necessario esse debeat positiua, solutio aequationis 16 hic quoque ambiguitati obnoxia esse nequit ${ }^{\star}$ : sed respectu aequationis $16^{\star}$ hic paullo aliter ratiocinandum est quam in ellipsi. Ex aequationum theoria facile demonstratux, pro valore positiuo ipsius $H^{\star \star}$ ) hanc aequationem (siquidem vllam radicem realem positiuam habeat) cum vna radice negatiua duas positiuas habere, quae vel ambae aequales erunt puta $=\frac{x}{6} \sqrt{ } 5-\frac{x}{6}=0,20601$, vel altera hoc limite maior altera minor. Iam in problemate nostro (suppositioni superstructo, $z$ esse quantitatem haud magnam, saltem non maiorem quam 0,3 , ne tabulae tertiae vsu destituamur) necessario semper radicem maiorem accipiendam esse sequenti modo demonstramus. $\mathrm{Si}$ in aequatione $13^{\star}$ pro $M$ substituitur $Y V(L+z)$, prodit $Y+1=(L+z) Z>(x+z) Z$, siue $Y>$ $\frac{x}{3}-\frac{4}{3 \cdot 5} z+\frac{4 \cdot 6}{3 \cdot 5 \cdot 7} z z-\frac{4 \cdot 6 \cdot 8}{3 \cdot 5 \cdot 7 \cdot 9} z^{3}+$ etc., vnde facile concluditur, pro valoribus tam paruis ipsius $z$, quales hie supponimus, semper fieri debere $Y>0,20601$. Reuera calculo facto inuenimus, vt $(1+z) Z$ huic limiti aequalis fiat, esse debere $z=0,79858$ : multum vero abest, quin methodum nostram ad tantos valores ipsius $z$ extendere velimus.

103.

Quoties $z$ valorem maiorem obtinet, tabulae III limites egredientem, aequationes $15,15^{*}$ tuto semper ac commode in forma sua non mutata tentando soluentur, et quidem ob rationes iis similes quas in art. 94 pro ellipsi exposuimus. In tali casu elementa orbitae obiter saltem cognita esse supponere licet: tum vero valor approximatus ipsius $n$ statim habetur per formulam tang $2 n=\frac{\sin f \sqrt{ } r r^{\prime}}{\alpha \sqrt{ }(e e-1)}$, quae sponte demanat ex aequatione 6 art. 99. Ex $n$ autem habebitur $z$ per formulam $z=\frac{x-\cos 2 n}{2 \cos 2 n}=\frac{\sin n^{2}}{\cos 2 n}$, et ex valore approximato ipsius $z$ paucis tentaminibus deriuabitur ille, qui aequationi $x 3$ vel $13^{\star}$ ex asse satisfacit. Possunt quoque illae aequationes in hac forma exhiberi

*) Vix opus erit monere, tabulam nostram If in hyperbola perinde vt in ellipsi ad solutionem huius acquationis adhiberi posse, quandiu $h$ ipsius limites non egrediatur.

**) Quantitas $H$ manifesto fieri nequit negatiua, nisi fuerit $\zeta>\frac{\tau}{6}$; tali autem valori ipsius $\zeta$ responderet valox ipsius z maior quam 2,684, adeoque limites huius methodi longe egrediens. 


$$
\begin{aligned}
& m=\left(l-\frac{\sin n^{2}}{\cos 2 n}\right)^{\frac{1}{2}}+2\left(l-\frac{\sin n^{2}}{\cos 2 n}\right)\left\{\frac{\frac{\operatorname{tang} 2 n}{\cos 2 n}-\log \text { hyp tang }\left(45^{\circ}+n\right)}{\operatorname{tang} 2 n^{3}}\right\} \\
& r=-\left(L+\frac{\sin n^{2}}{\cos 2 n}\right)^{\frac{\pi}{2}}+2\left(L+\frac{\sin n^{2}}{\cos 2 n}\right)^{\frac{3}{2}}\left\{\frac{\frac{\operatorname{tang} 2 n}{\cos 2 n}-\operatorname{loghyp} \operatorname{tang}\left(45^{\circ}+n\right)}{\operatorname{tang} 2 n^{3}}\right\}
\end{aligned}
$$

atque sic, neglecta $z$, statim valor verus ipsius $n$ erui.

\section{4.}

Superest, vt ex $z, n$ vel $c$ elementa ipsa determinemns. Statuendo $\alpha V(e e-1)$ $=\beta$, habebitur ex aequatione 6 art. 99

$[18] \beta=\frac{\sin f \sqrt{ } r r^{\prime}}{\operatorname{tang} 2}$

Combinando hanc formulam cum $12,12^{*}$ art. 99 , eruitur

[19] $V(e e-x)=\operatorname{tang} \psi=\frac{\operatorname{tang} f \operatorname{tang} 2 n}{2(l-z)}$

$\left[19^{\star}\right] \operatorname{tang} \psi=-\frac{\operatorname{tang} f \operatorname{tang} 2 n}{2(L+z)}$

vnde excentricitas commode atque exacte computatur; ex $\beta$ et $V(e e-x)$ prodibit per divisionem $a$, per multiplicationem $p$, ita vt sit

$$
\begin{aligned}
& \alpha=\frac{2(l-z) \cos f \cdot V r r^{\prime}}{\operatorname{tang} 2 n^{2}}=\frac{2 m m \cos f \cdot V r r^{\prime}}{y y \operatorname{tang} 2 n^{2}}=\frac{k \pi t t}{4 Y Y r r^{\prime} \cos f^{2} \operatorname{tang} 2 n^{2}} \\
& =\frac{-2(L+z) \cos f \cdot V r r^{\prime}}{\operatorname{tang} 2 n^{2}}=\frac{-2 I M M \cos f \cdot V r r^{\prime}}{Y Y \operatorname{tang} 2 n^{2}}=\frac{k k t t}{4 y y r r^{\prime} \cos f^{2} \operatorname{tang} 2 n^{2}} \\
& p=\frac{\sin f \cdot \tan g f \cdot V r r^{\prime}}{2(l-z)}=\frac{y y \sin f \cdot \operatorname{tang} f \cdot V r r^{\prime}}{2 m m}=\left(\frac{y r r^{\prime} \sin 2 f}{k t}\right)^{2} \\
& =\frac{-\sin f \cdot \tan g f \cdot V r r^{\prime}}{2(L+z)}=\frac{-Y Y \sin f \cdot \tan g f \cdot V r r^{\prime}}{2 M M}=\left(\frac{Y r r^{\prime} \sin 2 f}{k t}\right)^{2}
\end{aligned}
$$

Expressio tertia et sexta pro $p$, quae omnino identicae sunt cum formulis $18,18^{*}$ art. $9^{5}$, ostendunt, ea quae illic de significatione quantitatum $y, Y$ tradita sunt, etiam pro hyperbola valere.

E combinatione aequationum 6,9 art. 98 deducitur $\left(r^{\prime}-r\right) \sqrt{\frac{e e}{r r^{\prime}}-x}$ $=e \sin f .\left(C-\frac{1}{C}\right) ;$ introducendo itaque $\psi$ et $\omega$, statuendoque $C=\operatorname{tang}\left(45^{\circ}+N\right)$, fit 
[20] $\operatorname{tang} 2 N=\frac{2 \sin \psi \operatorname{tang} 2 \omega}{\sin f \cos 2 \omega}$

Inuento hinc $C$, habebuntur valores quantitatis in art. 21 per $u$ expressae pro vtroque loco; dein fiet per aequationem III art. $2 x$

$\operatorname{tang} \frac{x}{2} v=\frac{C-c}{(C+c) \operatorname{tang} \frac{x}{2} \psi}$

$\operatorname{tang} \frac{x}{2} v^{\prime}=\frac{C c-1}{(C c+1) \operatorname{tang} \frac{1}{2} \psi}$

siue introducendo pro $C, c$ angulos $N, n$

[21] $\operatorname{tang} \frac{x}{2} \nu=\frac{\sin (N-n)}{\cos (N+n) \operatorname{tang} \frac{x}{2} \psi}$

[22] $\operatorname{tang} \frac{x}{2} x^{\prime}=\frac{\sin (N+n)}{\cos (N-n) \operatorname{tang} \frac{x}{2} \psi}$

Hinc determinabuntur anomaliae verae $p, v^{\prime}$, quarum differentia cum $2 f$ comparata simul calculo confirmando inseruiet.

Denique per formulam XI art. 22 facile deducitur, interuallum temporis a perihelio vsque ad tempus loco primo respondens esse

$=\frac{\alpha^{\frac{3}{2}}}{k}\left\{\frac{2 e \cos (N+n) \sin (N-n)}{\cos 2 N \cos 2 n}-\log\right.$ hyp $\left.\frac{\operatorname{tang}\left(45^{\circ}+N\right)}{\operatorname{tang}\left(45^{\circ}+n\right)}\right\}$

et perinde interuallum temporis a perihelio vsque ad tempus loco secundo respondens

$=\frac{\alpha^{\frac{3}{2}}}{k}\left\{\frac{2 e \cos (N-n) \sin (N+n)}{\cos 2 N \cos 2 n}-\log\right.$ hyp tang $\left.\left(45^{\circ}+N\right) \operatorname{tang}\left(45^{\circ}+n\right)\right\}$

Si itaque tempus primum statuitur $=T-\frac{T}{2} t$, adeoque secundum $=T+\frac{T}{2} t$, fit

[23] $T=\frac{\alpha^{\frac{3}{2}}}{k}\left\{\frac{e \operatorname{tang} 2 N}{\cos 2 n}-\log \operatorname{tang}\left(45^{\circ}+N\right)\right\}$

vnde tempus transitus per perihelium innotescet; denique

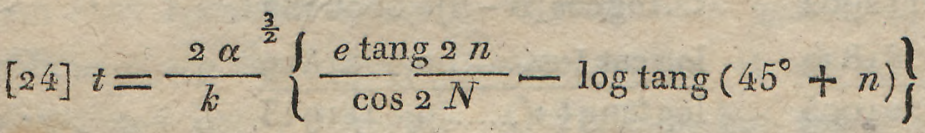

quae aequatio, si placet, ad vltimam calculi confirmationem adhiberi potest.

$$
105 .
$$

Ad illustrationem horum praeceptorum exemplum e duobus locis in artt. $25,24,25,46$ secundum eadem elementa hyperbolica calculatis conficiemus. Sit itaque $y^{\prime}-v=48^{\circ} 12^{\prime} o^{\prime \prime}$ siue $f=24^{\circ} 6^{\prime} 0^{\prime \prime}, \log r=0,0353585, \log r^{\prime}=0,200854 x$, 
$i=5 i, 49788$ dies. Hinc inuenitur $\omega=2^{\circ} 45^{\prime}{ }^{\prime \prime \prime} 47, l=0,05796059, \frac{m m}{\frac{5}{6}+l}$ sine valor approximatus ipsius $h=0,064437 x$; hinc, per tabulam II, $\log y y=0,0560848$, $\frac{m m}{y y}=0,05047454, z=0,00748585$, cui in tabula III respondet $\zeta=0,0000032$.

Hinc fit valor correctus ipsius $h=0,06443691, \log y y=0,0560846, \frac{\mathrm{mm}}{y^{y}}=$ $0,05047456, z=0,00748583$, qui valores, quum $\zeta$ inde non mutetur, nulla amplius correcitione opus habent. Jam calculus elementorum ita se habet:

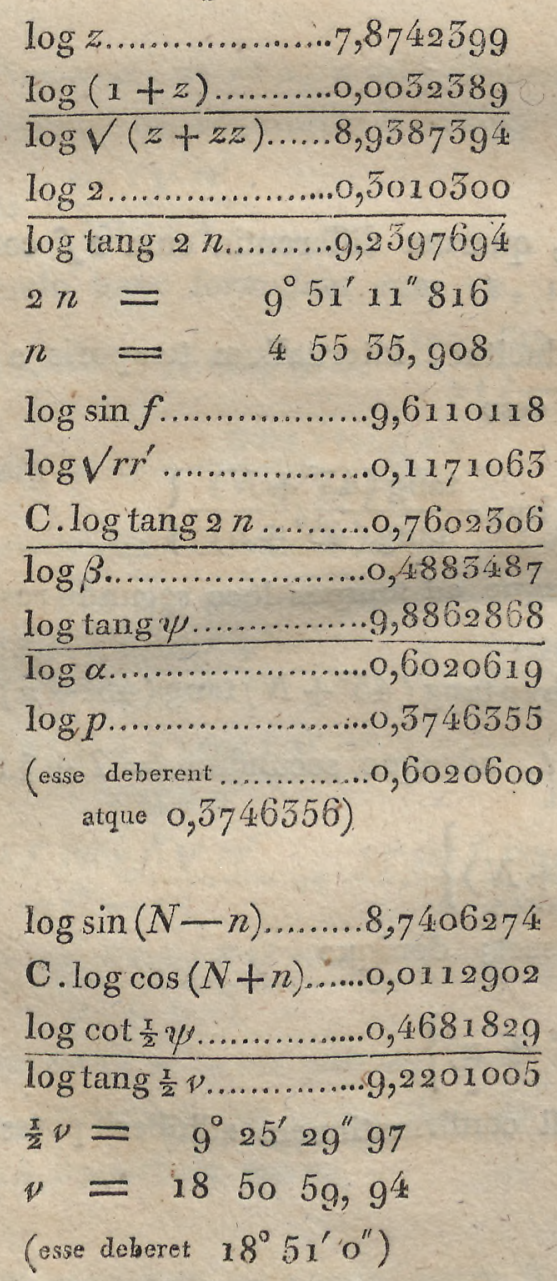

$\log \operatorname{tang} f \ldots \ldots \ldots . . . . . .9,6506199$

$\log \frac{x}{2} \operatorname{tang} 2 n \ldots \ldots \ldots .8,9587394$

C. $\log (l-z) \ldots \ldots \ldots 1,2969275$

$\overline{\log \operatorname{tang} \psi \ldots \ldots \ldots . . .9,8862868}$

$\psi=57^{\circ} 34^{\prime} 59^{\prime \prime} 77$

(esse deberet $=37^{\circ} 55^{\prime} 0^{\prime \prime}$ )

C. $\log \frac{x}{2} \sin f \ldots \ldots o, 6900182$

$\log \operatorname{tang} 2 \omega \ldots \ldots . .8,9848318$

C. $\log \cos 2 \omega \ldots . .0,0020156$

$\log \sin \psi \ldots \ldots \ldots \ldots 9,7852685$

$\log \operatorname{tang} 2 N \ldots \ldots .9,4621541$

$2 N=16^{\circ} 9^{\prime} 46^{\prime \prime} .253$

$N=8455,127$

$N-n=3917,219$

$N+n=13 \circ 29,035$

$\log \sin (N+n) \ldots .9,3523527$

C. $\log \cos (N-n) 0,0006587$

$\frac{\log \cot \frac{r}{2} \psi \ldots \ldots \ldots ., 4681829}{\log \tan g \frac{1}{2} v^{\prime} \ldots \ldots \ldots g, 8211943}$

$\frac{x}{2} v^{\prime}=33^{\circ} 3 x^{\prime} 29^{\prime \prime} 9^{5}$

$\phi^{\prime}=67$ 2 59,86

(esse deberet $67^{\circ} 3^{\prime} 0^{\prime \prime}$ 


\begin{tabular}{|c|c|}
\hline & 0 \\
\hline $\log \operatorname{tang} 2 \mathrm{~N} \ldots \ldots \ldots \ldots, 9,4621541$ & $\log \tan g 2 n \ldots \ldots \ldots \ldots . . .9,2397694$ \\
\hline C. $\log \cos 2 n \ldots \ldots \ldots \ldots, 0064539$ & C. $\log \cos 2 N \ldots \ldots \ldots \ldots, 0175142$ \\
\hline 9,5696064 & \\
\hline mumerus $=0,37119863$ & numerus $=0,2281928$ \\
\hline $\log$ hyp $\operatorname{tang}\left(45^{\circ}+N\right)=\ldots \ldots \ldots$ & $\log$ hyp tang $\left(45^{\circ}+n\right)=$. \\
\hline 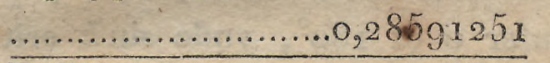 & 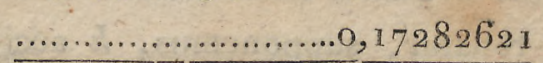 \\
\hline Differentia $=0,08528612$ & Differentia $=0,0555666$ \\
\hline $\log \ldots \ldots \ldots \ldots \ldots \ldots \ldots \ldots \ldots \ldots \ldots \ldots \ldots \ldots$ & 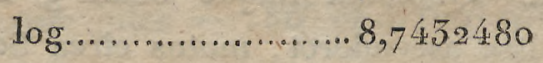 \\
\hline$\frac{3}{2} \log \alpha \ldots \ldots \ldots \ldots \ldots \ldots \ldots, 0,905 \log _{2} 8$ & $\frac{3}{2} \log \alpha \ldots \ldots \ldots \ldots \ldots \ldots \ldots, 9 \circ 5 \circ 0_{2}$ \\
\hline C. $\log k \ldots \ldots \ldots \ldots \ldots \ldots .1,7644186$ & C. $\log k \ldots \ldots \ldots \ldots \ldots \ldots 1,764418$ \\
\hline $\log T \ldots \ldots \ldots \ldots \ldots \ldots \ldots \ldots \ldots \ldots \ldots \ldots \ldots, 5,585897$ & $\log 2 \ldots \ldots \ldots \ldots \ldots \ldots \ldots \ldots, 50 \times 050$ \\
\hline$T=39,66358$ & $\begin{aligned} & \log t \ldots \ldots \ldots \ldots \ldots \ldots 1,711789 \\
& t=51,49788\end{aligned}$ \\
\hline
\end{tabular}

- Distat itaque transitus per perihelium a tempore loco primo respondente 15,91444 diebus, a tempore loco secundo respondente 65,41232 diebus. - Ceterum differentias exiguas elementorum hic erutorum ab is, secundum quae loca proposita calculata fuerant, tabularum praecisioni limitatae tribuere oportet.

106.

In tractatu de relationibus maxime insignibus ad motum corporum coelestium in sectionibus conicis spectantibus, silentio praeterire non possumus expressionem elegantem temporis per semiaxem maiorem, summam $r+r^{\prime}$ atque chordam duo loca iungentem. Haec formula pro parabola quidem primo ab ill. Euler inuenta esse videtur (Miscell. Berolin. T. VII p. 20), qui tamen eam in posterum neglexit, neque etiam ad ellipsin et hyperbolam extendit: errant itaque, qui formulam clar. Lambert tribuunt, etiamsi huic geometrae meritum, hanc expressionem obliuione sepultam proprio marte eruisse et ad reliquas sectiones conicas ampliavisse, non possit denegari. Quamquam hoc argumentum a pluribus geometris iam traclatum sit, tamen lectores attenti expositionem sequentem haud superfluam agnoscent. A motu elliptico initium facimus.

Ante omnia obseruamus, angulum circa Solem descriptum $2 f$ (art. 88, vnde reliqua quoque signa desumimus) infra $360^{\circ}$ supponi posse; patet enim, si iste angulus $560^{\circ}$ gradibus augeatur, tempns vna reuolutione siue $\frac{a^{\frac{3}{2}} \cdot 560^{\circ}}{k}=a^{\frac{3}{2}} \times 565,25$ 
diebus crescere. Iam si chordam per $\rho$ denotamus, manifestum est feri $\rho \varrho=\left(r^{\prime} \cos \vartheta^{\prime}-r \cos \vartheta\right)^{2}+\left(r^{\prime} \sin \vartheta^{\prime}-r \sin \vartheta\right)^{2}$

adeoque per aequationes VIII, IX art. 8

$\varrho \rho=a a\left(\cos E^{\prime}-\cos E\right)^{2}+a a \cos \varphi^{2}\left(\sin E^{\prime}-\sin E\right)^{2}$

$=4 a a \sin g^{2}\left(\sin G^{2}+\cos \varphi^{2} \cos G^{2}\right)=4 a a \sin g^{2}\left(1-e e \cos G^{2}\right)$

Introducamus angulum auxiliarem $h$ talem, vt sit $\cos h=e \cos G$; simul, quo omnis ambiguitas tollatur, supponemns, $h$ accipi inter o et $180^{\circ}$, vnde $\sin h$ erit quantitas positiua. Quoniam itaque etiam $g$ inter eosdem limites iacet (si enim $2 \mathrm{~g}$ ad $560^{\circ} \mathrm{vel}$ vltra ascenderet, motus circa Solem renolutionem integram attingeret vel superaret), ex aequatione praecedente sponte sequitur $\rho=2 a \sin g \sin h$, siquidem. chorda tamquam quantitas positiua consideratur. Qưum porro habeatur $r+r^{\prime}$ $=2 a(1-e \cos g \cos G)=2 a(1-\cos g \cos h)$, patet, si statuatur $h-g=\delta, h+g$ $=\varepsilon$, fieri

[1] $r+r^{\prime}-\rho=2 a(1-\cos \delta)=4 a \sin \frac{1}{2} \delta^{2}$

[2] $r+r+\rho=2 a(1-\cos \varepsilon)=4 a \sin \frac{\pi}{2} \varepsilon^{2}$

Denique habetur $k t=a^{\frac{3}{2}}(2 g-2 e \sin g \cos G)=a^{\frac{3}{2}}(2 g-2 \sin g \cos \hbar)$, siue [3] $k t=\alpha^{\frac{3}{2}}(\varepsilon-\sin \varepsilon-(\delta-\sin \delta))$

Determinari poterunt itaque, secundum aequationes 1,2 , anguli $\delta$ et $\varepsilon$ ex $r+r^{\prime}, \rho$ et $a$ : quamobrem ex iisdem quantitatibus determinabitur, secundum aequationem 5 , tempus $t$. Si magis placet, haec formula ita exhiberi potest:

$$
\begin{aligned}
k t= & a^{\frac{3}{2}}\left\{\arccos \frac{2 a-\left(r+r^{\prime}\right)-e}{2 a}-\sin \arccos \frac{2 a-\left(r+r^{\prime}\right)-e}{2 a}\right. \\
& \left.-\arccos \frac{2 a-\left(r+r^{\prime}\right)+\rho}{2 a}+\sin \arccos \frac{2 a-\left(r+r^{\prime}\right)+\rho}{2 a}\right\}
\end{aligned}
$$

Sed in determinatione angulorum $\delta, \varepsilon$ per cosinus suos ambiguitas remanet, quam propius considerare oportet. Sponte quidem patet, $\delta$ iacere debere inter $-180^{\circ}$ et $+180^{\circ}$, atque $\varepsilon$ inter o et $560^{\circ}$ : sed sic quoque vterque angulus determinationem duplicem, adeoque tempus resultans quadruplicem admittere videtur. Attamen ex aequatione 5 art. 88 habemus $\cos f \cdot V r r^{\prime}=a(\cos g-\cos h)=2 a \sin \frac{1}{2} \delta \sin \frac{r}{2} \varepsilon$ : iam $\sin \frac{\pi}{2} \varepsilon$ necessario fit quantitas positiua, vnde concludimus, $\cos f$ et $\sin \frac{r}{2} \delta$ necessario eodem signo affectos esse, adeoque $\delta$ inter o et $180^{\circ}$, vel inter $-180^{\circ}$ et o accipiendum esse, prout $\cos f$ positiuus fuerit vel negatiuus, i. e. prout motus heliocentricus $2 f$ fucrit infra rel supra $180^{\circ}$. Ceterum sponte patet, pro $2 f=180^{\circ}$ aecessario esse debere $\delta=0$. Hoc itaque modo $\delta$ plene determinatus est. At de- 
ferminatio anguli $\varepsilon$ necessario ambigua manet, ita vt semper pro tempore diuo valores prodeant, quorum quis verus sit, nisi aliunde constet, decidi nequit. Ceterum ratio huius phaenomeni facile perspicitur: constat enim, per duo puncta data describi posse duas ellipses diuersas, quae ambae focum suum habeant in codem puncto dato, simulque eundem semiaxem maiorem *); manifesto autem motns a loco primo ad secundum in his ellipsibus temporibus inaequalibus absoluetur.

107.

Denotando per $z$ arcum quemcunque inter $-180^{\circ}$ et $+180^{\circ}$ situm, et per s sinum arcus $\frac{x}{2} \mathcal{X}$, constat esse

$\frac{x}{2} X=s+\frac{\pi}{3} \cdot \frac{x}{2} s^{3}+\frac{\pi}{5} \cdot \frac{1.3}{2 \cdot 4} s^{5}+\frac{x}{7} \cdot \frac{1.3 .5}{2 \cdot 4 \cdot 6} s^{7}+$ etc.

Porro fit

$\frac{5}{2} \sin \chi=s V(x-s s)=s-\frac{x}{2} s^{3}-\frac{1.1}{2.4} s^{5}-\frac{1 \cdot 1.5}{2.4 .6} s^{7}-$ etc.

adeoque

$\chi-\sin \chi=4\left(\frac{x}{3} s^{5}+\frac{\pi}{5} \cdot \frac{x}{2} s^{5}+\frac{\pi}{7} \cdot \frac{1.3}{2 \cdot 4} s^{7}+\frac{x}{2} \cdot \frac{1 \cdot 5 \cdot 5}{2 \cdot 4 \cdot 6} s^{9}+\right.$ etc.

Substituimus in hac serie pro $s$ deinceps $\frac{x}{2} \vee \frac{r+r^{\prime}-\rho}{a}$, et $\frac{x}{2} \vee \frac{r+r^{\prime}+\rho}{a}$, quaeque inde proueniunt multiplicamus per $a^{\frac{3}{2}}$; ita respective oriuntur series

$$
\begin{aligned}
& \frac{7}{6}\left(r+r^{\prime}-\rho\right)^{\frac{3}{2}}+\frac{r}{80} \cdot \frac{1}{a}\left(r+r^{\prime}-\rho\right)^{\frac{5}{2}}+\frac{3}{7 \frac{3}{2}} \overline{2} \cdot \frac{1}{a a}\left(r+r^{\prime}-\rho\right)^{\frac{7}{2}}+ \\
& \frac{5}{x+\frac{1}{3}=\frac{x}{2}}\left(r+r^{\prime}-\rho\right)^{\frac{9}{2}}+\text { etc. } \\
& \frac{x}{6}\left(r+r^{\prime}+\rho\right)^{\frac{3}{2}}+\frac{x}{30} \cdot \frac{1}{a}\left(r+r^{\prime}+\rho\right)^{\frac{5}{2}}+\frac{3}{7 \frac{3}{2}} \cdot \frac{1}{a a}\left(r+r^{\prime}+\rho\right)^{\frac{7}{2}}+ \\
& \mathbf{T}_{8} \frac{5}{4} \frac{1}{3} \frac{1}{a^{3}}\left(r+r^{\prime}+\rho\right)^{\frac{\rho}{2}}+\text { etc. }
\end{aligned}
$$

quarum summas denotabimus per $T, U$. Iam nullo negotio patet, quum sit $\sin \frac{1}{2} \delta= \pm \vee \frac{r+r^{\prime}-\varrho}{a}$, signo superiori vel inferiori valente pront $2 f$ infra vel supra $180^{\circ}$ est, fieri $a^{\frac{3}{2}}(\delta-\sin \delta)= \pm T$, signo perinde determinato. Eodem

*) Descripto e loco primo circulo radio $a-r$, alioque radio $a-r^{\prime}$ e loco secundo, ellipseos focum alterum in intersectione hortun circulorum iacere patet. Quare quim generaliter loquendo duae semper dentur intersectiones, duae ellipses diuersac prodibunt. 
modo si pro $\varepsilon$ accipitur valor minor infra $180^{\circ}$ situs, fiet $a^{\frac{3}{2}}(\varepsilon-\sin \varepsilon)=U$ accepto vero valore altero, qui est illius complementum ad $360^{\circ}$, manifesto fiet $a^{\frac{3}{2}}(\varepsilon-\sin \varepsilon)=a^{\frac{3}{2}} 360^{\circ}-U$. Hinc itaque colliguntur duo yalores pro tempore $t$ $\frac{U \mp T}{k}$, atque $\frac{a^{\frac{3}{2}} 360^{\circ}}{k}-\frac{U \pm T}{k}$.

108.

Si parabola tamquam ellipsis spectatur, cuius axis maior infinite magnus est, expressio temporis in art. praec. inuenta transit in $\frac{1}{6 k}\left\{\left(r+r^{\prime}+\rho\right)^{\frac{3}{2}} \mp\right.$ $\left.\left(r+r^{\prime}-\rho\right)^{\frac{3}{2}}\right\}$ : sed quum haecce formulae deductio fortasse quibusdam dubiis exposita videri possit, aliam ab ellipsi haud pendentem exponemus.

Statuendo brcuitatis caussa $\operatorname{tang} \frac{r}{2} p=\theta, \operatorname{tang} \frac{r}{2} v^{\prime}=\theta^{\prime}$, fit $r=\frac{r}{2} p(1+\theta \theta)$, $r^{\prime}=\frac{r}{2} p\left(1+\theta^{\prime} \theta^{\prime}\right), \cos v=\frac{1-\theta \theta}{1+\theta \theta}, \cos v^{\prime}=\frac{1-\theta^{\prime} \theta^{\prime}}{1+\theta^{\prime} \theta^{\prime}}, \sin v=\frac{2 \theta}{1+\theta \theta}$, $\sin v^{\prime}=\frac{2 \theta^{\prime}}{1+\theta^{\prime} \theta^{\prime}}$. Hinc fit $r^{\prime} \cos v^{\prime}-r \cos v=\frac{r}{2} p\left(\theta \theta-\theta^{\prime} \theta^{\prime}\right), r^{\prime} \sin v^{\prime}-r \sin v=$ $p\left(\theta^{\prime}-\theta\right)$, adeoque $\varrho \rho=\frac{1}{4} p p\left(\theta^{\prime}-\theta\right)^{2}\left(4+\left(\theta^{\prime}+\theta\right)^{2}\right)$. Jam ficile perspicitur, $\theta^{\prime}-\theta=\frac{\sin f}{\cos \frac{1}{2} \nu \cos \frac{1}{2} \nu^{\prime}}$ esse quantitatem positiuam: statuendo itaque

$\checkmark\left(1+\frac{r}{4}\left(\theta^{\prime}+\theta\right)^{2}\right)=\eta$, erit $\rho=p\left(\theta^{\prime}-\theta\right) \eta$. Porro fit $r+r^{\prime}=\frac{x}{2} p\left(2+\theta \theta+\theta^{\prime} \theta^{\prime}\right)=$ $p\left(\eta \eta+\frac{4}{4}\left(\theta^{\prime}-\theta\right)^{2}\right):$ quamobrem habetur $\frac{r+r^{\prime}+\rho}{p}=\left(\eta+\frac{r}{2}\left(\theta^{\prime}-\theta\right)\right)^{2}$ $\frac{r+r^{\prime}-\rho}{p}=\left(\eta-\frac{x}{2}\left(\theta^{\prime}-\theta\right)\right)^{2}$

Ex aequatione priori sponte deducitur $+V \frac{r+r^{\prime}+\rho}{p}=\eta+\frac{\pi}{2}\left(\theta^{\prime}-\theta\right)$ quoniam $\eta$ et $\theta^{\prime}-\theta$ sunt quantitates positinae; sed quum $\frac{x}{2}\left(\theta^{\prime}-\theta\right)$ minor sit rel

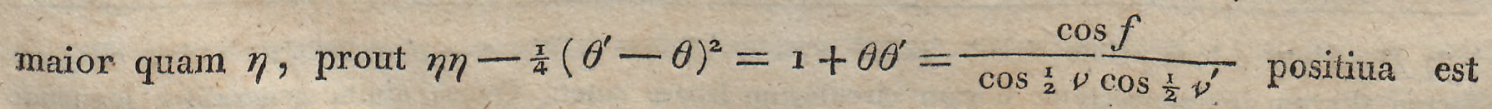
vel negatiua, patet, ex aequatione posteriori concludere oportere 
$\pm v \frac{r+r^{\prime}-\varphi}{p}=\eta-\frac{T}{2}\left(\theta^{\prime}-\theta\right)$, vbi signum superius vel inferius adoptandrum est, prout angulus circa solem descriptus infra $180^{\circ}$ vel supra $180^{\circ}$ fuerit.

Ex aequatione, quae in art. 98 seciandam sequitur, porro habemus

$$
\begin{gathered}
\frac{2 h t}{p^{\frac{3}{2}}}=\left(\theta^{\prime}-\theta\right)\left(\left(x+\theta \theta^{\prime}+\frac{1}{3}\left(\theta^{\prime}-\theta\right)^{2}\right)=\left(\theta^{\prime}-\theta\right)\left(\eta \eta+\frac{1}{x^{2}}\left(\theta^{\prime}-\theta\right)^{2}\right)\right. \\
=\frac{x}{3}\left(\eta+\frac{r}{2}\left(\theta^{\prime}-\theta\right)\right) \cdot-\frac{\pi}{3}\left(\eta-\frac{r}{2}\left(\theta^{\prime}-\theta\right)\right)^{3}
\end{gathered}
$$

rnde sponte sequitur

$$
h t=\frac{x}{6}\left\{\left(r+r^{\prime}+\rho\right)^{\frac{3}{2}} \mp\left(r+r^{\prime}-\rho\right)^{\frac{3}{2}}\right\}
$$

signo superiori vel inferiori valente, prout $2 f$ infra vel supra $180^{\circ}$ est.

$$
109 \text {. }
$$

Si in hyperbola signa $\alpha, C$, $c$ in eadem significatione accipimus, rt in art. 99, habemus ex aequationibus VIII, IX art. 21

$r^{\prime} \cos v^{\prime}-r \cos v=-\frac{1}{2}\left(c-\frac{1}{c}\right)\left(C-\frac{1}{C}\right) \alpha$

$r^{\prime} \sin v^{\prime}-r \sin v=\frac{x}{2}\left(c-\frac{1}{c}\right)\left(c+\frac{1}{C}\right) \alpha V(e e-1)$

adeoque $\rho=\frac{\tau}{2} \alpha\left(c-\frac{1}{c}\right) \nabla\left(e e\left(C+\frac{1}{C}\right)^{2}-4\right)$

Supponamus $\gamma$ esse quantitatem per aequationem $\gamma+\frac{x}{\gamma}=e\left(C+\frac{1}{C}\right)$ determinatam: cui quum manifesto duo valores sibi inuicem reciproci satisfaciant, adoptamus eum qui est maior quam 1. Ita fit

$\rho=\frac{x}{2} \alpha\left(c-\frac{1}{c}\right)\left(\gamma-\frac{1}{\gamma}\right)$

Porro fit $r+r^{\prime}=\frac{x}{2} \alpha\left(e\left(c+\frac{1}{c}\right)\left(C+\frac{i}{C}\right)-4\right)=\frac{x}{2} \alpha\left(\left(c+\frac{1}{c}\right)\left(\gamma+\frac{1}{\gamma}\right)-4\right)$, adeoque

$$
\begin{aligned}
& r+r^{\prime}+\rho=\alpha\left(\vee c \gamma-V \frac{1}{c \gamma}\right)^{2} \\
& r+r^{\prime}-\rho=\alpha\left(\vee \frac{\gamma}{c}-V \frac{c}{\gamma}\right)^{2}
\end{aligned}
$$

Statuendo itaque $\vee \frac{r+r^{\prime}+\rho}{4 a}=m, \vee \frac{r+r^{\prime}-e}{4 a}=n$, erit necessario 
$\checkmark c \gamma-V \frac{x}{c \gamma}=2 m ;$ ad decidendam vero quaestionem, virum $\vee \frac{\gamma}{c}-\vee \frac{c}{\gamma}$ fat $=+2 n$ an $=-2 n$, inquirere oportet, vtrum $\gamma$ maior an minor sit quam $c$ : sed ex aequatione 8 art. 99 facile sequitur, casum priorem locum habere, quoties if $f$ sit infra $180^{\circ}$, posteriorem quoties $2 f$ sit supra $180^{\circ}$. Denique ex eodem art. habemus $\frac{k t}{a^{\frac{3}{2}}}=\frac{x}{2}\left(\gamma+\frac{1}{\gamma}\right)\left(c-\frac{1}{c}\right)-2 \log c=\frac{x}{2}\left(c \gamma-\frac{1}{c \gamma}\right)-\frac{x}{2}\left(\frac{\gamma}{c}-\frac{c}{\gamma}\right)$ $-\log c \gamma+\log \frac{\gamma}{c}=2 m V(1+m m) \mp 2 n V(1+n n)-2 \log (V(1+m m)+m)$ $\pm 2 \log (V(1+n n)+n)$

signis inferioribus semper ad casum $2 f>180^{\circ}$ spectantibus. Iam $\log (V(1+m m)+m)$ facile enoluitur in seriem sequentem $m-\frac{\pi}{3} \cdot \frac{x}{2} m^{3}+\frac{x}{5} \cdot \frac{1.3}{2.4} m^{5}-\frac{x}{7} \cdot \frac{1.3 .5}{2.4 .6} m^{7}+$ etc.

Hoc sponte colligitur ex $d \log (V(1+m m)+m)=\frac{d m}{V(1+m m)}$. Prodit itaque $2 m V(1+m m)-2 \log (V(1+m m)+m)=4\left(\frac{x}{3} m^{3}-\frac{\pi}{5} \cdot \frac{1}{2} m^{5}+\frac{5}{7} \cdot \frac{1.3}{2 \cdot 4} m^{7}-\right.$ etc. $)$ et perinde formula alia prorsus similis, si $m$ cum $n$ permutatur. Hine tandem colligitur, si statuatur

$$
\begin{aligned}
& T=\frac{r}{6}\left(r+r^{\prime}-\rho\right)^{\frac{3}{2}}-\frac{r}{80} \cdot \frac{1}{\alpha}\left(r+r^{\prime}-\rho\right)^{\frac{3}{2}}+\frac{3}{7 \frac{3}{2}} \cdot \frac{1}{\alpha \alpha}\left(r+r^{\prime}-\rho\right)^{\frac{7}{2}}- \\
& \text { T } \frac{5}{4} \frac{5}{3}=\frac{1}{a^{3}}\left(r+r^{\prime}-\rho\right)^{\frac{9}{2}}+\text { etc. } \\
& U=\frac{r}{6}\left(r+r^{\prime}+\rho\right)^{\frac{3}{2}}-\frac{x}{80} \cdot \frac{1}{\alpha}\left(r+r^{\prime}+\rho\right)^{\frac{5}{2}}+\frac{3}{7^{3} \frac{5}{2}} \cdot \frac{1}{\alpha \alpha}\left(r+r^{\prime}+\rho\right)^{\overline{2}}- \\
& \text { I } \frac{5}{4}-\frac{1}{2} \cdot \frac{1}{a^{3}}\left(r+r^{\prime}+\rho\right)^{\frac{2}{2}}+\text { etc. }
\end{aligned}
$$

fieri $k t=U \mp T$, quae expressiones cum iis, quae in art. 107 traditae sunt, omnino coincidunt, si illic $a$ in $-\alpha$ mutetur.

Ceterum hae series tum pro ellipsi tum pro hyperbola ad vsum practictm tunc inprimis sunt commodae, vbi $a$ vel $\alpha$ valorem permagnum obtinet, i. e. vbi sectio conica magnopere ad parabolae similitudinem vergit. In tali casu etiam ad solutionem problematis supra tractati (art. 85 - 105) adhiberi possent: sed quoniam, nostro iudicio, ne tunc quidem breuitatem solutionis supra traditae praebent, huic methodo fusius exponendae non immoramur. 


\section{SECTIO QVARTA \\ Relationes inter locos plures in spatio.}

110.

Relationes in hac Sectione considerandae ab orbitae indole independentes solique suppositioni innixae erunt, omnia orbitae puncta in eodem plano cum Sole iacere. Placuit autem, hic quasdam simplicissimas tantum attingere, aliasque magis complicatas et speciales ad Librum alterum nobis reseruare.

Situs plani orbitae per duos locos corporis coelestis in spatio plene determinatus est, siquidem hi loci non iacent in eadem recta cum Sole. Quare quum duobus potissimum modis locus puncti in spatio assignari possit, duo hine problemata soluenda se offerunt.

Supponemus primo, duos locos dari per longitudines et latitudines heliocentricas resp. per $\lambda, \lambda^{\prime} ; \beta, \beta^{\prime}$ designandas: distantiae a Sole in calculum non ingredientur. Tune si longitudo nodi ascendentis per $\delta$, inclinatio orbitae ad eclipticam per $i$ denotatur, erit

$\operatorname{tang} \beta=\operatorname{tang} i \sin (\lambda-\Omega)$

$\operatorname{tang} \beta^{\prime}=\operatorname{tang} i \sin \left(\hat{\lambda}^{\prime}-\delta\right)$

Determinatio incognitarum $\Omega$, tang $i$ hic ad problema in art. 78 , II consideratum referiur; habemus itaque, ad normam solutionis primae

$\operatorname{tang} i \sin (\lambda-\delta)=\operatorname{tang} \beta$

$\operatorname{tang} i \cos (\lambda-\Omega)=\frac{\tan g \beta^{\prime}-\operatorname{tang} \beta \cos \left(\lambda^{\prime}-\lambda\right)}{\sin \left(\lambda^{\prime}-\lambda\right)}$

ad normam solutionis tertiae autem inuenimus $\Omega$ per aequationem

$\left.\operatorname{tang} \frac{\pi}{2} \lambda+\frac{x}{2} \lambda^{\prime}-\Omega\right)=\frac{\sin \left(\beta^{\prime}+\beta\right) \operatorname{tang} \frac{x}{2}\left(\lambda^{\prime}-\lambda\right)}{\sin \left(\beta^{\prime}-\beta\right)}$

vtique aliquanto commodius, si anguli $\beta, \beta^{\prime}$ immediate dantur, neque vero per logarithmos tangentium: sed ad determinandum $i$, recurrendum erit ad aliquam formularum tang $i=\frac{\operatorname{tang} \beta}{\sin (\lambda-\Omega)}=\frac{\operatorname{tang} \beta^{\prime}}{\sin \left(\lambda^{\prime}-\Omega\right)}$. Ceterum ambiguitas in determinatione anguli $\lambda-\Omega$, vel $\frac{\pi}{2} \lambda+\frac{\pi}{2} \lambda-\delta$ per tangentem suam ita erit decidenda, vt tang $i$ positiua enadat vel negatiua, pront motus ad eclipticam proiectus directus est vel retrogradus: hane incertitudinem itaque tune tantum tollere licet, vbi con- 
stat, a quanam parte corpus cocleste a loco primo ad sccundum peruenerit; quod si ignoraretux, vtique impossibile esset, nodum ascendentem a descendente distinguere. per formulas

Postquam anguli $\Omega, i$ inuenti sunt, eruentur argumenta latitudinum $u^{\prime}$, $z$

$\operatorname{tang} u=\frac{\operatorname{tang}(\lambda-\delta)}{\cos i}, \operatorname{tang} u^{\prime}=\frac{\operatorname{tang}\left(\hat{\lambda}^{\prime}-\delta\right)}{\cos i}$

quae in semicirculo primo vel secundo accipienda sunt, prout latitudines respondentes boreales sunt vel australes. His formulis adhuc sequentes adiicimus, e quibus, si placet, vna wel altera ad calculum confirmandum in vsum. vocari poterit: $\cos u=\cos \beta \cos (\lambda-\Omega), \cos u^{\prime}=\cos \beta^{\prime} \cos \left(\lambda^{\prime}-\Omega\right)$

$\sin u=\frac{\sin \beta}{\sin i}, \sin u^{\prime}=\frac{\sin \beta^{\prime}}{\sin i}$

$\sin \left(u^{\prime}+u\right)=\frac{\sin \left(\lambda+\lambda^{\prime}-2 \delta\right) \cos \beta \cos \beta^{\prime}}{\cos i}, \sin \left(u^{\prime}-u\right)=\frac{\sin \left(\lambda^{\prime}-\lambda\right) \cos \beta \cos \beta}{\cos i}$

111.

Suppomamus sccundo, duos locos dari per distantias suas a tribus planis in Sole sub angulis rectis se secantibus; designemus has distantias pro loro primo per $x, y, z$, pro secundo per $x^{\prime}, y^{\prime}, z^{\prime}$, supponamusque planum terlium esse ipsam eclipticam, plani primi et secundi autem polos positiuos in longitudine $N$ et $90^{\circ}+N$ sitos esse. Ita erit per art. 53, duobus radiis vectoribus per $r, r^{\prime}$ designatis, $x=r \cos u \cos (N-\Omega)+r \sin u \sin (N-\Omega) \cos i$

$y=r \sin u \cos (N-\Omega) \cos i-r \cos u \sin (N-\Omega)$

$z=r \sin u \sin i$

$x^{\prime}=r^{\prime} \cos u^{\prime} \cos (N-\Omega)+r^{\prime} \sin u^{\prime} \sin (N-\delta) \cos i$

$y^{\prime}=r^{\prime} \sin u^{\prime} \cos (N-\Omega) \cdot \cos i-r^{\prime} \cos u^{\prime} \sin (N-\Omega)$

$z^{\prime}=r^{\prime} \sin u^{\prime} \sin i$

Hinc sequitur

$z y^{\prime}-y z^{\prime}=r r^{\prime} \sin \left(u^{\prime}-u\right) \sin (N-\Omega) \sin i$

$x z^{\prime}-z x^{\prime}=r r^{\prime} \sin \left(u^{\prime}-u\right) \cos (N-\Omega) \sin i$

$x y^{\prime}-y x^{\prime}=r r^{\prime} \sin \left(u^{\prime}-u\right) \cos i$

E combinatione formulae primae cum secunda habebitur $N-\delta$ atque $r r^{\prime} \sin u \sin i$, hinc et ex formula tertia prodibit $i$ atque $r r^{\prime} \sin \left(u^{\prime}-u\right)$.

Quatenus locus, cui coordinatae $x, y, z$ respondent, tempore posterior supponitur, $u$ maior quam $u$ fieri debet: quodsi itaque insuper constat, vtrum angu- 
Ius inter locum primum et secundum circa Solem descriplus duobus rectis minor an maior sit, $r r^{\prime} \sin \left(u^{\prime}-u\right) \sin i$ atque $r r^{\prime} \sin \left(u^{\prime}-u\right)$ esse debent quantitates positivae in casu primo, negatiuae in secundo: tunc itaque $N-\Omega$ sine ambiguitate determinatur, simulque ex signo quantitatis $x y^{\prime}-y x^{\prime}$ deciditur, vtrum motus directus sit, an retrogradus. Vice versa, si de motus directione constat, e signo quantitatis $x y^{\prime}-y x^{\prime}$ decidere licebit, vtrum $u^{\prime}-u$ minor an maior quam $180^{\circ}$ accipiendus sit. Sin vero tum motus directio, tum indoles anguli circa Solem descripti plane incognitae sunt, manifestum est, inter nodum ascendentem ac descendentem distinguere non licere.

Ceterum facile perspicitur, sicuti cos $i$ est cosinus inclinationis plani orbitae versus planum tertium, ita $\sin (N-\Omega) \sin i, \cos (N-\Omega) \sin i$ ešse resp. cosinus inclinationum plani orbitae versus planum primum et secundum; nec non exprimere $r^{\prime} \sin \left(u^{\prime}-u\right)$ duplam aream trianguli inter duos radios vectores inclusi, atque $z y^{\prime}-y^{\prime} z^{\prime}$; $x z^{\prime}-z x^{\prime}, x y^{\prime}-y x^{\prime}$ duplam aream proiectionum eiusdem trianguli ad singula plana.

Denique patet, planum tertium pro ecliptica quoduis aliud planum esse posse, si modo omnes magnitudines per relationes suas ad eclipticam definitae perinde ad planum tertium, quidquid sit, referantux.

\section{2.}

Sint $x^{\prime \prime}, y^{\prime \prime}, z^{\prime \prime}$ coordinatae alicuins loci tertii, atque $u^{\prime \prime}$ eius argumentum latitudinis, $r^{\prime \prime}$ radius vector. Designabimus quantitates $r^{\prime} r^{\prime \prime} \sin \left(u^{\prime \prime}-u^{\prime}\right), r r^{\prime \prime} \sin \left(u^{\prime \prime}-u\right)$, $r r^{\prime} \sin \left(u^{\prime}-u\right)$, quae sunt areae duplae triangulorum inter radium vectorem secundum et tertium, prinum et tertium, primun et secundum, resp. per $n, n^{\prime}, n^{\prime \prime}$. Habebuntur itaque pro $x^{\prime \prime}, y^{\prime \prime}, z^{\prime \prime}$ expressiones iis similes, guas in art. praec. pro $x, y, z$ et $x^{\prime}, y^{\prime}, z^{\prime}$ tradidimus, vnde adiumento lemmatis I art. $7^{8}$ facile deducuntur aequationes sequentes:

$$
\begin{aligned}
& 0=n x-n^{\prime} x^{\prime}+n^{\prime \prime} x^{\prime \prime} \\
& 0=n y-n^{\prime} y^{\prime}+n^{\prime \prime} y^{\prime \prime} \\
& 0=n z-n^{\prime} z^{\prime}+n^{\prime \prime} z^{\prime \prime}
\end{aligned}
$$

Sint iam longitudines geocentricae corporis coelestis tribus illis locis respondentes $\alpha, \alpha^{\prime}, \alpha^{\prime \prime}$; latitudines geocentricae $\beta, \beta^{\prime}, \beta^{\prime \prime}$; distantiae a terra ad eclipticam proiectae $\delta, \delta^{\prime}, \delta^{\prime \prime}$; porro respondentes longitudines heliocentricae terrae $L, L^{\prime}, L^{\prime \prime}$; latitndines $B, B^{\prime}, B^{\prime \prime}$, quas non statuimus $=0$, vt liceat, tum parallaxis rationem habere, tum, si placet, pro ecliptica quoduis aliud planum adoptare; denique 
$D, D^{\prime}, D^{\prime \prime}$ distantiae terrac a Sole ad eclipticam proiectae. Quodsi tunc $x, y, z$ per $L, B, D, \alpha, \beta, \delta$ exprimuntur, similiterque coordinatae ad locum secundum et tertium spectantes, aequationes praecedentes sequentem formam induunt:

[1] $0=n(\delta \cos \alpha+D \cos L)-n^{\prime}\left(\delta^{\prime} \cos \alpha^{\prime}+D^{\prime} \cos L^{\prime}\right)+n^{\prime \prime}\left(\delta^{\prime \prime} \cos \alpha^{\prime \prime}+D^{\prime \prime} \cos L^{\prime \prime}\right)$

[2] $0=n(\delta \sin \alpha+D \sin L)-n^{\prime}\left(\delta^{\prime} \sin \alpha^{\prime}+D^{\prime} \sin L^{\prime}\right)+n^{\prime \prime}\left(\delta^{\prime \prime} \sin \alpha^{\prime \prime}+D^{\prime \prime} \sin L^{\prime \prime}\right)$

[3] $0=n(\delta \tan \beta+D \operatorname{tang} B)-n^{\prime}\left(\delta^{\prime} \operatorname{tang} \beta^{\prime}+D^{\prime} \operatorname{tang} B^{\prime}\right)+n^{\prime \prime}\left(\delta^{\prime \prime} \operatorname{tang} \beta^{\prime \prime}+D^{\prime \prime} \operatorname{tang} Z^{\prime \prime}\right)$

Si hic $\alpha, \beta, D, L, B$, quantitatesque analogae pro duobus reliquis locis, tamquam cognitae spectantur, aequationesque per $n$, vel per $n^{\prime}$, vel per $n^{\prime \prime}$ diuiduntur, quinque incognitae remanent, e quibus itaque duas eliminare, siue per duas quascunque tres reliquas determinare licet. Hoc modo illae tres aequationes ad conclizsiones plurimas granissimas viam sternunt, e quibus quasdam imprimis insignes hio euoluemus.

115.

Ne formularum prolixitate nimis obruamur, sequentibus abbreuiationibus vti placet. Primo designamus quantitatem $\operatorname{tang} \beta \sin \left(\alpha^{\prime \prime}-\alpha^{\prime}\right)+\operatorname{tang} \beta^{\prime} \sin \left(\alpha-\alpha^{\prime \prime}\right)+\operatorname{tang} \beta^{\prime \prime} \sin \left(\alpha^{\prime}-\alpha\right)$ per $(0.1 .2)$ : si in expressione illa pro longitudine et latitudine loco cuiuis geocentrico respondentibus substituuntur longitudo et Iatitudo cuilibet trium locorum heliocentricorum terrae respondentes, in signo (0.1.2) numerum illi respondentem cum numero romano eo commutamus, qui posteriori respondet. Ita e. g. character (o.1.I) exprimet quantitatem

$\operatorname{tang} \beta \sin \left(L^{\prime}-\alpha^{\prime}\right)+\operatorname{tang} \beta^{\prime} \sin \left(\alpha-L^{\prime}\right)+\operatorname{tang} B^{\prime} \sin \left(\alpha^{\prime}-\alpha\right)$

nec non character $(0 . \mathrm{O} .2)$ hanc

$\operatorname{tang} \beta \sin \left(\alpha^{\prime \prime}-L\right)+\operatorname{tang} B \sin \left(\alpha-\alpha^{\prime \prime}\right)+\operatorname{tang} \beta^{\prime \prime} \sin (L-\alpha)$

Simili modo characterem mutamus, si in expressione prima pro duabus longitudinibus et latitudinibus geocentricis duae quaecunque heliocentricae terrae substituuntur. Si duae longitudines et latitudines in eandem expressionem ingredientes tantummodo inter se permutantur, etiam in charactere numeros respondentes permutare oportet: hine autem valor ipse non mutatur, sed tantummodo e positiuo negatiuus, e negatiuo positiuus cuadit. Ita e. g. fit $(0.1 .2)=-(0.2 .1)=(1.2 .0)=$ $-(1.0 .2)=(2.0 .1)=-(2.1 .0)$. Omnes itaque quantitates hoc modo oriundae ad sequentes 19 reducuntur

$(0.1 .2)$

$(0.1 .0),(0.1 . I),(0.1 . I I),(0.0 .2),(0 . I .2),(0 . I I .2),(0.1 .2),($ I. 1.2), (II. 1.2). 
(0.O.I), (o.O.II), (o.I.II), (1.O.I), (1.O.II); (1.I.II), (2.O.I), (2.O.II), (2.I.II) quibus accedit vigesima (O.I.II).

Ceterum facile demonstratur, singulas has expressiones, per productum e tribus cosinibus latitudinum ipsas ingredientium multiplicatas, aequales fieri volumini sextuplo pyramidis, cuius vertex est in Sole, basis vero triangulum formatum inter tria sphaerae coelestis puncta, quae locis expressionem illam ingredientibus respondent, statuto sphaerae radio $=1$. Quoties itaque hi tres loci in eodem circulo maximo iacent, valor expressionis fieri debet $=0$; quod quum in tribus locis heliocentricis terrae semper locum habeat, quoties ad parallaxes et latitudines terrae a perturbationibus ortas non respicimus, i. e. quoties terram in ipso eclipticae plano constituimus, semper, hacce suppositione valente, erit (O.I.II) $=0$, quae quidem aequatio identica est, si pro plano tertio ecliptica ipsa accepta fuit. Ceterum quoties tum $B$, tum $B^{\prime}$, tum $B^{\prime \prime}=0$, omnes istae expressiones, prima excepta, multo simpliciores fiunt; singulae scilicet a secunda vsque ad decimam binis partibus conflatae erunt, ab vndecima autem vsque ad vndeuigesimam vnico termino constabunt.

114.

Multiplicando aequationem [1] per $\sin \alpha^{n} \operatorname{tang} B^{\prime \prime}-\sin L^{n} \operatorname{tang} \beta^{\prime \prime}$, aequationem [2] per $\cos L^{\prime \prime} \operatorname{tang} \beta^{\prime \prime}-\cos \alpha^{\prime \prime} \operatorname{tang} \mathcal{B}^{\prime \prime}$, aequationem [3] per $\sin \left(L^{\prime \prime}-\alpha^{\prime \prime}\right)$, addendoque producta, prodit

[4] o $=n\left\{(0.2 . \mathrm{II}) \delta+(\right.$ O. 2.II) $D\}-n^{\prime}\left\{(1.2 . \mathrm{II}) \delta^{\prime}+(\mathrm{I} .2 . \mathrm{II}) D^{\prime}\right\}$

similique modo, vel commodius per solam locorum inter se permutationem

$[5] \circ=n\{(0.1 . I) \delta+($ O. I.I $) D\}+n^{\prime \prime}\left\{(2.1 . I) \delta^{\prime \prime}+\right.$ (II. 1.I $\left.) D^{\prime \prime}\right\}$

[6] $0=n^{\prime}\left\{(1.0 .0) \delta^{\prime}+\left(\right.\right.$ I. o. O) $\left.D^{\prime}\right\}-n^{\prime \prime}\left\{(2.0 .0) \delta^{\prime \prime}+\left(\right.\right.$ II. o. O) $\left.D^{\prime \prime}\right\}$

Quodsi itaque ratio quantitatum $n, n^{\prime}$ data est, adiumento aequationis 4 ex $\delta$ determinare licebit $\delta^{\prime}$, vel $\delta$ ex $\delta^{\prime}$; similiterque de aequationibus 5,6 . E combinatione aequationum $4,5,6$ oritur haec

[7] $\frac{(0.2 . \mathrm{II}) \delta+(\mathrm{O} .2 . \mathrm{II}) D}{(0.1 . \mathrm{I}) \delta+(\mathrm{O} .1 . \mathrm{I}) D} \times \frac{(1.0 .0) \delta^{\prime}+(\text { I. } 0.0) D^{\prime}}{(1.2 . \mathrm{II}) \delta^{\prime}+(\text { I. 2.II }) D^{\prime}} \times \frac{(2.1 .1) \delta^{\prime \prime}+\left(\text { II. 1.I) } D^{\prime \prime}\right.}{(2.0 .0) \delta^{\prime \prime}+\left(\text { II.0.0) } D^{\prime \prime}\right.}=-1$, per, quam e duabus distantiis corporis coelestis a terra determinare licet tertiam. Ostendi potest autem, hanc aequationem 7 fieri identicam, adeoque ad determinationem vnius distantiae e duabus reliquis ineptam, quoties fuerit 
$\left.\begin{array}{r}\operatorname{tang} \beta^{\prime} \operatorname{tang} \beta^{\prime \prime} \sin (L-\alpha) \sin \left(L^{\prime \prime}-L^{\prime}\right) \\ +\operatorname{tang} \beta^{\prime \prime} \operatorname{tang} \beta \sin \left(L^{\prime}-\alpha^{\prime}\right) \sin \left(L-L^{\prime \prime}\right) \\ +\operatorname{tang} \beta \operatorname{tang} \beta^{\prime} \sin \left(L^{\prime \prime}-\alpha^{\prime \prime}\right) \sin \left(L^{\prime}-L\right)\end{array}\right\}=0$.

$A b$ hoc incommodo libera est formula sequens, ex aequationibus $s, 2,3$ facile demanans:

[8] (0.1.2) $\delta \delta^{\prime} \delta^{\prime \prime}+(0.1 .2) D \delta^{\prime} \delta^{\prime \prime}+$ (o.I.2) $D^{\prime} \delta \delta^{\prime \prime}+$ (o. 1.II) $D^{\prime \prime} \delta \delta^{\prime}+$ (o.I.II) $D^{\prime} D^{\prime \prime} \delta+$ (O. 1. II) $D D^{\prime \prime} \delta^{\prime}+$ (O.I. 2) $D D^{\prime} \delta^{\prime \prime}+$ (O.I. II) $D D^{\prime} D^{\prime \prime}=0$.

Multiplicando aequationem 1 per $\sin \alpha^{\prime} \operatorname{tang} \beta^{\prime \prime}-\sin \alpha^{\prime \prime} \operatorname{tang} \beta^{\prime}$, aequationem 2 per $\cos \alpha^{\prime \prime} \operatorname{tang} \beta^{\prime}-\cos \alpha^{\prime} \operatorname{tang} \beta^{\prime \prime}$, aequationem 3 per $\sin \left(\alpha^{\prime \prime}-\alpha^{\prime}\right)$, addendoque producta, prodit

[9] $0=n\{(0.1 .2) \delta+(0.1 .2) D\}-n^{\prime}\left(\right.$ I. 1.2) $D^{\prime}+n^{\prime \prime}\left(\right.$ II. 1.2) $D^{\prime \prime}$

et perinde

[10] $0=n(0.0 .2) D-n^{\prime}\left\{(0.1 .2) \delta^{\prime}+(0\right.$. I. 2$\left.) D^{\prime}\right\}+n^{\prime \prime}\left(\right.$ o.II.2.) $D^{\prime \prime}$

$[11] 0=n(0.1 .0) D-n^{\prime}(0.1 .1) D^{\prime}+n^{\prime \prime}\left\{(0.1 .2) \delta^{\prime \prime}+(0.1\right.$. II $\left.) D^{\prime \prime}\right\}$

Adiumento harum aequationum e ratione inter quantitates $n, n^{\prime}, n^{\prime \prime}$ cognita eruere licebit distantias $\delta, \delta^{\prime}, \delta^{\prime \prime}$. Sed haecce conclusio generaliter tantum loquendo valet, exceptionemque patitur, quoties fit $(0.1 .2)=0$. Ostendi enim potest, in hocce casu ex aequationibus 8,9 , to nihil aliud sequi, nisi relationem necessariam inter quantitates $n, n^{\prime}, n_{*}^{\prime \prime}$, et quidem e singulis tribus eandem. Restrictiones analogae circa aequationes 4, 5, 6 lectori perito sponte se offerent.

Ceterum omnes conclusiones hic euolutae nullius sunt vstrs, quoties planum orbitae cum ecliptica coincidit. Si enim $\beta, \beta^{\prime}, \beta^{\prime \prime}, B, B^{\prime}, B^{\prime \prime}$ omnes sunt $=0$, aequatio 3 identica est, ac proin omnes quoque sequentes. 


\section{LIBER SECVNDVS}

\section{INVESTIGATIO ORBITARVM CORPORVM COELESTIVM EX OBSERVATIONIBVS GEOCENTRICIS.}

\section{SECTIO PRIMA}

\section{Determinatio orbitae e tribus obseruationibus completis.}

\section{5.}

Ad determinationem completam motus corporis coelestis in orbita sua requiruntur elementa septem, quorum autem numerus vno minor enadit, si corporis massa vel cognita est vel negligitur; haec licentia vix euitari poterit in determinatione orbitae penitus adhuc incognitae, vbi omnes quantitates ordinis perlurbationum tantisper seponere oportet, donec massae a quibus pendent aliunde innotuerint. Quamobrem in disquisitione praesente massa corporis neglecta elementorum numerum ad sex reducimus, patetque adeo, ad determinationem orbitae incognitae totidem quantitates ab elementis pendentes ab inuicem vero independentes requiri. Quae quantitates nequeunt esse nisi loca corporis coelestis e terra obseruata, quae singula quum bina data subministrent, puta longitudinem et latitudinem, vel ascensionem rectam et decinationem, simplicissimum vtique erit, tria loca geocentrica adoptare, quae generaliter loquendo sex elementis incognitis determinandis sufficient. Hoc problema tamquam grauissimum huius operis spectandum erit, summaque ideo cura in hac sectione pertractabitur.

Verum enim vero in casu speciali, vbi planum orbitae cum ecliptica coincidit, adeoque omnes latitudines tum heliocentricae tum geocentricae natura sua euanescunt, tres latitudines geocentricas euanescentes haud amplius considerare licet tamquam tria data $\mathrm{ab}$ inuicem independentia: tunc igitur problema istud indeterminatum maneret, tribusque locis geocentricis per orbitas infinite multas satisfieri posset. In tali itaque casu necessario quatuor longitudines geocentricas datas esse oportet, vt quatuor elementa incognita reliqua (excidentibus inclinatione orbi- 
tae et longitudine nodi) determinare liceat. Etiamsi vero per principium indiscernibilium haud exspectandum sit, talem casum in rerum natura vmquam se oblaturum esse, tamen facile praesumitur, problema, quod in orbita cum plano eclipticae omnino coincidente absolute indeterminatum fit, in orbitis perparum ad eclipticam inclinatis propter obseruationum praecisionem limitatam tantum non indeterminatum manere debere, vbi vel leuissimi obseruationum errores incognitarum determinationem penitus turbare valent. Quamobrem vt huic quoque casui consulamus, alia sex data eligere oportebit: ad quem finem in sectione secunda orbitam incognitam e quatuor obseruationibus determinare docebimus, quarum duae quidem completae sint, duae reliquae autem incompletae, latitudinibus rel declinationibus deficientibus.

Denique quum omnes obseruationes nostrae propter instrumentorum sensuumque imperfectionem non sint nisi approximationes ad veritatem, orbita, sex tantum datis absolute necessariis superstructa, erroribus considerabilibus adhuc obnoxia esse poterit. Quos vt quantum quidem licet extenuemus, summamque adeo praecisionem possibilem attingamus, via alia non dabitur, nisi vt obseruationes perfectissimas quam plurimas congeramus, elementaque ita perpoliamus, vt non quidem his vel illis praecisione absoluta satisfaciant, sed cum cunctis quam optime conspirent. Quonam pacto talem consensum, si nullibi absolutum tamen vbique quam arctissimum, secundum principia calculi probabilitatis obtinere liceat, in sectione tertia ostendemus.

Hoc itaque modo determinatio orbitarum, quaienus corpora coelestia secundum leges Kepleri in ipsis mouentur, ad omnem quae desiderari potest perfectionem euecta exit. Vltimam quidem expolitionem tunc demum suscipere licebit, vbi etiam perturbationes, quas planetae reliqui motui indricunt, ad calculum erunt reuocatae: quarum rationem quomodo habere oporteat, quantum quidem ad institutum nostrum pertinere videbitur, in sectione quarta breuiter indicabimus.

116.

Antequam determinatio alicuius orbitae ex obseruationibus geocentricis suscipitur, his quaedam reductiones applicandae erunt, propter nutationem, praecessionem, parallaxin et aberrationem, siquidem summa praecisio requiritur: in crassiori enim calculo has minutias negligere licebit.

Planetarum et cometarum obseruationes vulgo expressae proferuntur per ascensiones rectas et declinationes apparentes, i. e. ad situm aequatoris apparen- 
tem relatas. Qui situs quum propter nutationem et praecessionem variabilis adeoque pro diuersis obseruationibus diuersus sit, ante omnia loco plani variabilis planum aliquod fixum introducere conueniet, ad quem finem vel aequator situ suo medio pro aliqua epocha, vel ecliptica adoptari poterit: planum posterius plerumque adhiberi solet, sed prius quoque commodis peculiaribus haud spernendis se commendat.

Quoties itaque planum aequatoris eligere placuit, ante omnia obseruationes a nutatione purgandae, ac dein adhibita praecessione ad epocham quandam arbitrariam reducendae sunt: haec operalio prorsus conuenit cum ea, per quam e loco stellae fixae obseruato eiusdem positio media pro epocha data deriuatur, adeoque explicatione hic non indiget. Sin vero planum eclipticae adoptare constitutum est, duplex methodus patebit: scilicet vel ex ascensionibus rectis et declinationibus ab nutationem et praecessionem correctis deduci poterunt longitudines et latitudines adiumento obliquitatis mediae, vnde longitudines iam ad aequinoctium medium relatae prodibunt; vel commodius ex ascensionibus rectis et declinationibus apparentibus adiumento obliquitatis apparentis computabuntur longitudines et latitudines, ac dein illae a nutatione et praecessione purgabuntur.

Loci terrae singulis obseruationibus respondentes per tabulas solares cdmputantur, manifesto autem ad idem planum referendi eruni, ad quod obseruationes corporis coelestis relatae sunt. Quamobrem in computo longitudinis Solis negligetur nutatio; dein vero haec longitudo adhibita praecessione ad epocham fixam reducetur, atque 180 gradibus augebitur; latitudini Solis, siquidem eius rationem habere operae pretium videtur, signum oppositum tribuetur: sic positio terrae heliocentrica habebitur, quam, si aequator pro plano fundamentali electus est, adiumento obliquitatis mediae in ascensionem rectam et declinationem transformare licebit.

\section{x 7 .}

Positio terras hoc modo e tabulis computata ad terrae centrum referenda est, locus obseruatus autem corporis coelestis ad punctum in terrae superficie spectat: huic dissensui tribus modis remedium afferre licet. Potest scilicet vel observatio ad centrum terrae reduci, siue a parallaxi liberari; vel locus heliocentricus terrae ad locum ipsum obseruationis reduci, quod efficitur, si loco Solis e tabulis computato parallaxis rite applicatur; vel denique vtraque positio ad punctum aliquod tertium transferri, quod commodissime in intersectione radii visus cum plano eclipticae assumitur: obseruatio ipsa tunc immutata manet, reductionemque loci 
terrae ad hoc punctum in art. 72 docuimus. Methodus prima adhiberi nequit, nisi corporis coelestis distantia a terra proxime saltem nota fuerit: tunc autem satis commoda est, praesertím quoties obseruatio in ipso meridiano instifuta est, vbi sola declinatio parallaxi afficitur. Ceterum praestabit, hanc reductionem loco obseruato immediate applicare, antequam transformationes art. praec. adeantur. Si vero distantia a terra penitus adhuc incognita est, ad methodum secundam vel tertiam confugiendum est, et quidem illa in vsum vocabitur, quoties aequator pro plano fundamentali accipitur, tertia autem praeferetur, quoties omnes positiones ad eclipticam referre placuit.

118.

Si corporis coclestis distantia a terra alicui obseruationi respondens proxime iam nota est, hanc ab effectu aberrationis liberare licet pluribus modis, qui methodis diuersis in art. 70 traditis innituntur. Sit $t$ tempus verum obseruationis; $\theta$ interuallum temporis, intra quod lumen a corpore coelesti ad terram descendit, quod prodit ducendo $495^{\prime}$ in distantiam; $l$ locus obseruatus, $l^{\prime}$ idem, locus adiumento motus geocentrici diurni ad tempus $t+\theta$ reductus; $l^{\prime \prime}$ locus $l$ ab ea aberrationis parte purgatus, quae planetis cum fixis communis est; $L$ locus terrae verus tempori $t$ respondens (i. e. tabularis $20^{\prime \prime} 25$ auctus); denique ' $L$ locus terrae verus tempori $t-\theta$ respondens. His ita factis erit

I. $l$ locus verus corporis coelestis ex ' $L$ visus tempore $t-\theta$

II. $l^{\prime}$ locus verus corporis coelestis ex $L$ visus tempore $t$

III. $l^{\prime \prime}$ locus verus corporis coelestis ex $L$ visus tempore $t-\theta$

Per methodum I itaque locus obsertiatus immutatus retinetur, pro tempore vero autem fictum $t-\theta$ substituitur, loco terrae pro eodem computato; methodus II soli obseruationi mutationem applicat, quae autem praeter distantiam insuper motum diurnum requirit; in methodo III obseruatio correctionem patitur a distantia non pendentem, pro tempore vero fictum $t-\theta$ substituitur, sed retento loco terrae tempori vero respondente. Ex his methodis prima longe commodissima est, quoties distantia eatenus iam nota est, vt reductio temporis $\theta$ praecisione suficiente computari possit.

Quodsi autem haec distantia penitus adhuc incognita est, nulla harum methodorum immediate applicari potest: in prima scilicet habetur quidem corporis coelestis locus geocentricus, sed desideratur tempus et positio terrae a distantia incognita pendentia; in secunda e contrario adsunt haec, deest ille; denique in ter- 
tia habetur locus geocentricus corporis coelestis atque positio terrae; sed tempus deest cum illis datis iungendum.

Quid faciendum est itaque in problemate nostro, ssi in tali casu solutio respectu aberrationis quoque exacta postulatur? Simplicissimum vtique est, orbitam primo neglecta aberratione determinare, quae quum effectum considerabilem numquam producere possit, distantiae hinc ea certe praecisione demanabunt, vt iam obseruationes per aliquam methodorum modo expositarum ab aberratione purgare, orbitaeque determinationem accuratius iterare liceat. Iam in hocce negotio methodus tertia ceteris longe praeferenda erit: in methodo enim prima omnes operationes a positione terrae pendentes ab ouo rursus inchoandae sunt: in secunda (quae ne applicabilis quidem est, nisi tanta obseruationum copia adsit, vt motus diurnus inde elici possit) omnes operationes a loco geocentrico corporis coelestis pendentes denuo instituere oportet: contra in tertia (siquidem iam calculus primus superstructus fuerat locis geocentricis ab aberratione fixarum purgatis) omnes operationes praeliminares a positione terrae et loco geocentrico corporis coelestis pendentes, in computo nouo inuariatae retineri poterunt. Quin adeo hoc modo primo statim calculo aberrationem complecti licebit; si methodus ad determinationem orbitae adhibita ita comparata est, vt valores distantiartum prodeant prius, quam tempora correcta in calculum introducere opus fuerit. Tunc aberrationis quidem caussa calculus duplex haud necessarius erit, vti in tractatione ampliori problematis nostri: clarius apparebit.

I19.

Haud difficile esset, e nexu inter problematis nostri data atque incognitas, eius statum ad sex aequationes reducere, vel adeo ad pauciores, quum vnam alteramue incognitam satis commode eliminare liceret: sed quoniam nexus ille complicatissimus est, hae aequationes maxime intractabiles euaderent; incognitarum separatio talis, vt tandem aequatio vnicam tantummodo continens prodeat, generaliter 'loquendo *) pro impossibili haberi potest, multoque adeo minus problematis solutionem integram per solas operationes directas absoluere licebit.

Sed ad duarum aequationum solutionem $X=0, Y=0$, in quibus duae tantum incognitae $x, y$ intermixtae remanserunt, vtique reducére licet problema no-

*) Quoties obseruationes ab inuicem tam parum remotae sunt, vt temporum interualla tamquam: quantitates infinite paruas tractare liceat, huiusmodi separatio vtique succedit, totumque problema ad solutionem aequationis algebraicae septimi octauiue gradus reducitur. 
strum, et quidem variis modis. Haud equidem necesse est, vt $x, y$ sint duo ex elementis ipsis: esse poterunt quantitates qualicunque modo cum elementis connexae, si modo illis inuentis elementa inde commode deriuare licet. Praeterea manifesto haud opus est, vt $X, Y$ per functiones explicitas ipsartum $x, y$ exhibeantur: sufficit, si cum illis per systema aequationum ita iunctae sunt, vt a valoribus datis ipsarum $x, y$ ad valores respondentes ipsarum $X, Y$ descendere in po= testate sit.

\section{0.}

Quoniam itaque problematis natura reductionem vlteriorem non permittit; quam ad duas aequationes, duas incognitas mixtim implicantes, rei summa primo quidem in idonea harum incognitarum electione aequationumque adornatione versabitur, vt tum $X$ et $Y$ quam simplicissime ab $x, y$ pendeant, tum ex harum valoribus inuentis elementa ipsa quam commodissime demanent: dein vero circumspiciendum erit, quo pacto incognitarum valores aequationibus satisfacientes per operationes non nimis operosas eruere liceat. Quod si coecis quasi tentaminibus tantum efficiendum esset, ingens sane ac vix tolerandus labor requireretur, qualem fere nihilominus saepius susceperunt astronomi, qui cometarum orbitas per methodum quam indirectam vocant determinauerunt: magnopere vtique in tali negotio labor subletzatur eo, quod in tentaminibus primis calculi crassiores sufficiunt, donec ad valores approximatos incognitarum peruentum fuerit. Quamprimum vero determinatio approximata iam habetur, rem tutis semper expeditisque methodis ad finem perdueere licebit, quas antequam viterius progrediamur hic explicauisse iuiabit.

Aequationibus $X=0, Y=0$, si pro $x, y$ valores veri ipsi accipiuntur, ex asse sponte satisfiet: contra si pro $x, y$ valores a veris diuersi substituuntur, $X$ et $Y$ inde valores a a diuersos nanciscentur. Quo propitus vero illi ad veros accedunt, eo minores quoque valores ipsarum $X, Y$ emergere debebunt, quoties. que illorum differentiae a veris perexiguae sunt, supponere licebit, variationes in valoribus ipsarum $X, Y$ proxime proportionales esse variationi ipsins $x$, si $y$, vel variationi ipsius $y$, si $x$ non mutetur. Quodsi itaque valores veri ipsarum $x, y$ resp. designantur per $\xi, \eta$, valores ipsarum $X, Y$ suppositioni $x=\xi+\lambda, y=\eta+\mu$ respondentes per formam $X=\alpha \lambda+\beta \mu, Y=\gamma \lambda+\delta \mu$ exhibebuntur, ita vt coëfficientes $\alpha, \beta, \gamma, \delta$ pro constantibus haberi queant, dum $\lambda$ et $\mu$ perexiguae manent. Hinc concluditur, si pro tribus systematibus valorum ipsarum $x, y$, a veris parum 
diuersorum, valores respondentes ipsarum $X, Y$ determinati sint, valores veros ipsarum $x, y$ inde deriuari posse, quatenus quidem suppositionem istam admittere licet. Statuamus

$$
\begin{aligned}
& \text { pro } x=a, y=b \text { fieri } X=A, Y=B \\
& x=a^{\prime}, y=b^{\prime} \quad X=A^{\prime}, Y=B^{\prime} \\
& x=a^{\prime \prime}, y=b^{\prime \prime} \quad X=A^{\prime \prime}, Y=B^{n}
\end{aligned}
$$

habebimusque

$A=\alpha(a-\xi)+\beta(b-\eta), B=\gamma(a-\xi)+\delta(b-\eta)$

$A^{\prime}=\alpha\left(a^{\prime}-\xi\right)+\beta\left(b^{\prime}-\eta\right), B^{\prime}=\gamma\left(a^{\prime}-\xi\right)+\delta\left(b^{\prime}-\eta\right)$

$A^{\prime \prime}=\alpha\left(a^{\prime \prime}-\xi\right)+\rho\left(b^{\prime \prime}-\eta\right), b^{\prime \prime}=\gamma\left(a^{\prime \prime}-\xi\right)+\delta\left(b^{\prime \prime}-\eta\right)$

Hinc fit, eliminatis $\alpha, \beta, \gamma, \delta$

$\xi=\frac{a\left(A^{\prime} B^{\prime \prime}-A^{\prime \prime} B^{\prime}\right)+a^{\prime}\left(A^{\prime \prime} B-A B^{\prime \prime}\right)+a^{\prime \prime}\left(A B^{\prime}-A^{\prime} B\right)}{A^{\prime} B^{\prime \prime}-A^{\prime \prime} B^{\prime \prime}+A^{\prime \prime} B-A B^{\prime \prime}+A B^{\prime}-A^{\prime} B}$

$\eta=\frac{b\left(A^{\prime} B^{\prime \prime}-A^{\prime \prime} B^{\prime}\right)+b^{\prime}\left(A^{\prime \prime} B-A B^{\prime \prime}\right)+b^{\prime \prime}\left(A B^{\prime}-A^{\prime} B\right)}{A^{\prime} B^{\prime \prime}-A^{\prime \prime} B^{\prime}+A^{\prime \prime} B-A B^{\prime \prime}+A B^{\prime}-A^{\prime} B}$

sine in forma ad calculum commodiori

$\xi=a+\frac{\left(a^{\prime}-a\right)\left(A^{\prime \prime} B-A B^{\prime \prime}\right)+\left(a^{\prime \prime}-a\right)\left(A B^{\prime}-A^{\prime} B\right)}{A^{\prime} B^{\prime \prime}-A^{\prime \prime} B^{\prime}+A^{\prime \prime} B-A B^{\prime \prime}+A B^{\prime}-A^{\prime} B}$

$\eta=b+\frac{\left(b^{\prime}-b\right)\left(A^{\prime \prime} B-A B^{\prime \prime}\right)+\left(b^{\prime \prime}-b\right)\left(A B^{\prime}-A^{\prime} B\right)}{A^{\prime} B^{\prime \prime}-A^{\prime \prime} B^{\prime}+A^{\prime \prime} B-A B^{\prime \prime}+A B^{\prime}-A^{\prime} B}$

Manifesto quoque in his formulis quantitates $a, b, A, B$ cum $a^{\prime}, b^{\prime}, A^{\prime}, B^{\prime}$, vel cum his $a^{\prime \prime}, b^{\prime \prime}, A^{\prime \prime}, B^{\prime \prime}$ permutare licet.

Ceterum denominator communis omnium harum expressionum, quem etiam sub formam $\left(A^{\prime}-A\right)\left(B^{\prime \prime}-B\right)-\left(A^{\prime \prime}-A\right)\left(B^{\prime}-B\right)$ ponere licet, fit $=(\alpha \delta-\beta \gamma)$ $\left\{\left(a^{\prime}-a\right)\left(b^{\prime \prime}-b\right)-\left(a^{\prime \prime}-a\right)\left(b^{\prime}-b\right)\right\}:$ wnde patet, $a, a^{\prime}, a^{\prime \prime}, b, b^{\prime}, b^{\prime \prime}$ ita accipi debere, vt non fiat $\frac{a^{\prime \prime}-a}{b^{\prime \prime}-b}=\frac{a^{\prime}-a}{b^{\prime}-b}$, alioquin enim haec methodus hand applicabilis esset, sed pro $\xi$ et $\eta$ valores fractos suggereret, quorum numeratores et denominatores simul euanescerent. Simul hinc manifestum est, si forte fiat $\alpha \delta-\beta \gamma=0$, eundem defectum methodi vsum omnino destruere, quomodocunque $a, a^{\prime}, a^{\prime \prime}, b$, $b^{\prime}, b^{\prime \prime}$ accipiantur. In tali casu pro valoribus ipsius $\boldsymbol{X}$ formam talem supponere oporteret $\alpha \lambda+\beta \mu+\varepsilon \lambda \lambda+\zeta \lambda \mu+\theta \mu \mu$, similemque pro valoribus ipsius $Y$, quo facto analysis methodos praecedenti analogas suppeditaret, e valoribus ipsarum $X, Y$ pro quatuor systematibus valorum ipsarum $x, y$ computatis harum valores veros eruendi. 
LIBR. II. SECT. I.

Hoc vero modo calculus permolestus euaderet, praetereaque ostendi potest, in tali casu orbitae determinationem praecisionem necessariam per ipsius rei naturam non admittere: quod incommodum quum aliter enitaxi nequeat, nisi nouis obseruationibus magis idoneis adscitis, huic argumento hic non immoramur.

121.

Quoties itaque incognitarum valores approximati iam in potestate sunt, veri inde per methodum modo explicatam omni quae desideratur praecisione deriuari possunt. Primo 'scilicet computabuntur valores ipsarum $X, Y$ istis valoribus approximatis $(a, b)$ respondentes: qui nisi sponte iam euanescunt, calculus duobus aliis valoribus ab illis parum diuersis $\left(a^{\prime}, b^{\prime}\right)$ répetetur, ac dein tertio systemate $a^{\prime \prime}, b^{\prime \prime}$, nisi fortuito ex secundo $X$ et $Y$ ellanuerunt. Tunc per formulas art. praec. valores veri elicientur, quatenus suppositio, cui illae formulae innituntur, a veritate haud sensibiliter discrepat. De qua re quo melius iudicium ferri possit, calculus valorum ipsarum $X, Y$ cum illis valoribus correctis repetetur: qui si aequationibus $X=0, Y=0$ nondum satisfieri monstrat, certe valores multo minores ipsarum $\boldsymbol{X}, \boldsymbol{Y}$ inde prodibunt, quam per tres priores hypotheses, adeoque elementa orbitae hinc resultantia longe exactiora erunt, quam ea, quae primis hypothesibus respondent. Quibus si acquiescere nolumus, consultissimum erit, omissa ea hypothesi quae maximas differentias produxerat, duas reliquas cum quarta denuo iungere, atque sic ad normam art. praec. quintum systema valorum ipsarum $x, y$ formare: eodemque modo, vbi operae pretium videbitur, ad hypothesin sextam etc. progredi licebit, donec aequationibus $X=0, Y=0$ tam exacte satisfactum fuerit, quam tabulae logarithmicae et trigonometricae permittunt. Rarissime tamen opus erit, vltra systema quartum progredi, nisi hypotheses primae nimis adhuc a veritate aberrantes suppositae fuerint.

122.

Quum incognitarum valores in hypothesi secunda et tertia supponendi quodammodo arbitrarii sint, si modo ab hypothesi prima non nimis differant, praetereaque caueatur, ne ratio $\left(a^{\prime \prime}-a\right):\left(b^{\prime \prime}-b\right)$ ad aequalitatem huius $\left(a^{\prime}-a\right):\left(b^{\prime}-b\right)$ conuergat, plerumque statui solet $a^{\prime}=a, b^{n}=b$. Duplex hinc lucrum deriuatur: namque non solum formulae pro $\xi, \eta$ paullo adhuc simpliciores euadunt, sed pars quoque calculi primi eadem manebit in hypothesi secunda, aliaque pars in tertia. 
Est tamen casus, vbi aliae rationes ab hac consuetudine discedere suadent: fingamus enim, $X$ habere formam $X^{\prime}-x$, atque $Y$ hanc $Y^{\prime}-y$, functionesque $X^{\prime}, Y^{\prime}$ per problematis naturam ita comparatas esse, vt erroribus mediocribus in valoribus ipsarum $x, y$ commissis perparum afficiantur, siue $v t\left(\frac{d X^{\prime}}{d x}\right),\left(\frac{d X^{\prime}}{d y}\right)$, $\left(\frac{d Y^{\prime}}{d x}\right),\left(\frac{d Y^{\prime}}{d y}\right)$ sint quantitates perexiguae, patetque, differentias inter valores istarum functionum systemati $x=\xi, y=\eta$ respondentes, eosque qui ex $x=a, y=b$ prodeunt, ad ordinem quasi altiorem referri posse, quan differentias $\xi-a, \eta-b$; at valores illi sunt $X^{\prime}=\xi, Y^{\prime}=\eta$, hi vero $X^{\prime}=a+A, Y^{\prime}=b+B$, vnde sequitur, $a+A, b+B$ esse valores multo exactiores ipsarum $x, y$, quam $a, b$. Quibus si hypothesis secunda superstruitur, persaepe aequationibus $X=0, Y=0$ tam exacte iam satisfit, vt viterius progredi haud opus sit; sin secus, eodem modo ex hypothesi secunda tertia formabitur faciendo $a^{\prime \prime}=a^{\prime}+A^{\prime}=a+A+A^{\prime}, b^{\prime \prime}=b^{\prime}+B^{\prime}$ $=b+B+B^{\prime}$, vnde tandem, si nondum satis praecisa reperitur, quarta ad normam art. 119 elicie'ur.

\section{3.}

In praec. supposuimus, valores approximatos incognitarum $x, y$ alicunde iam haberi. Quoties quidem totius orbitae dimensiones approximatae in potestate sunt (ex ahiis forte obseruationibus per calculos anteriores deductae iamque per novas corrigendae), conditioni illi absque difficultate satisfieri poterit, quamcunque significationem incognitis tribuamus. Contra in determinatione prima orbitae penitus adhuc ignotae (quae est problema longe difficillimum) neutiquam indifferens est, quasnam incognitas adhibeamus; arte potius talique modo eligendac sunt, vt valores approximatos ex ipsius problematis natura haurire liceat. Quod exoptatissime succedit, quoties tres obseruationes ad orbitae inuestigationem adhibitae motum heliocentricum corporis coelestis non nimis magnum complectuntur. Huiusmodi itaque obseruationes ad determinationem primam semper adhibendae sunt, quam dein per obseruationes magis ab inuicem remotas ad lubitum corrigere conueniet. Nullo enim negotio perspicitur, obseruationum errores ineuitabiles calculum eo magis turbare, quo propiores obseruationes adhibeantur. Hinc colligitur, obseruationes ad determinationem primam haud temere eligendas, sed cauendum esse, primo ne sint nimis sibi inuicem vicinae, dein vero etiam ne nimis ab inuicem distent: in primo enim casu calculus elementorum obseruationibus satisfacientium expeditissime 
quidem absolneretur, sed his elementis ipsis parum fidendum foret, quinimo erroribus tam enormiter deprauata euadere possent, vt ne approximationis quidem vice fungi valerent; in casu altero vero artificiis, quibus ad determinationem approximatam incognitarum vtendum est, destitueremus, neque inde aliam deriuaremus, , nisi vel crassissimam vbi hypotheses multo plures, vel omnino ineptam, vbi tentamina fastidiosissima haud euitare liceret. Sed de hisce methodi limitibus scite iudicare melius per vsum frequentem quam per praecepta ediscitur: exempla infra tradenda ostendent, ex obseruationibus Iunonis 22 tantum diebus ab inuicem dissitis motumque heliocentricum $7^{\circ} 55^{\prime}$ complectentibus elementa multa iam praecisione gaudentia deriuari, ac vicissim, methodum nostram optimo etiamnum successu ad obseruationes Cereris applicari, quae 260 diebus ad inuicem distant, motumque heliocentricum $62^{\circ} 55^{\prime}$ includunt, quatuorque hypothesibus seu potius approximatiotionibus successiuis adhibitis elementa optime cum obseruationibus conspirantia producere.

124.

Progredimur iam ad enumerationem methodorum maxime idonearum principiis praecedentibus innixarum, quarum quidem praecipua momenta in libro primo exposita sunt, atque hic tantum instituto nostro accommodari debent.

Methodus simplicissima esse videtur, si pro $x, y$ distantiae corporis coelestis a terra in duabus obseruationibus accipiantur, aut potius vel logarithmi harum distantiarum vel logarithmi distantiarum ad eclipticam siue aequatorem proiectarum. Hinc per art. $64, \mathrm{~V}$ elicientur loca heliocentrica et distantiae a Sole ad eadem loca pertinentia; hinc porro per art. 110 situs plani orbitae-atque longitudines heliocentricae in ea; hinc atque ex radiis vectoribus temporibusque respondentibus per problema in art. $85 \ldots 105$ copiose pertractalum cuncta reliqua elementa, per quae illas obseruationes exacte repraesentari manifestum est, quicunque valores ipsis $x, y$ tributi fuerint. Quodsi iam per haec elementa locus geocentricus pro tempore obseruationis tertiae computatur, huius consensus cum obsernato vel dissensus decidet, vtrum valores suppositi veri fuerint, an ab iis discrepent; vnde quum comparatio duplex deriuetur, differentia altera (in longitudine vel ascensione recta) accipi poterit pro $X$, alteraque (in latitudine vel declinatione) pro $\boldsymbol{Y}$. Nisi jgitur valores harum differentiarum $X, Y$ sponte prodeunt $=0$, valores veros ipsarum $x, y$ per methodum in art. 120. sqq. descriptam eruere licebit. Ceterum per se arbitrarium est, a quibusnam trium obseruationum proficiscamur: plerumque 
tamen praestat, primam et postremam adoptare, casu speciali de quo statim dicemus excepto.

Haecce methodus plerisque post explicandis eo nomine praeferenda est, quod applicationem maxime generalem patitur. Excipere oportet casum, vbi duae obseruationes extremae motum heliocentricum 180 vel 560 vel 540 etc, graduum complectuntur; tunc enim positio plani orbitae e duabus locis heliocentricis doterminari nequit (art. 110.). Perinde methodum applicare haud conueniet, quoties motus heliocentricus inter duas obseruationes extremas perparum differt ab $180^{\circ}$ vel $360^{\circ}$ etc. quoniam in hoc easu determinatio positionis orbitae accurata obtineri nequit, siue potius, quoniam variationes leuissimae in valoribus suppositis incognitarum tantas variationes in positione orbitae et proin etiam in valoribus ipsarum $X, Y$ producerent, vt hae illis non amplitis proportionales censeri possent. Verumtamen remedium hic praesto est; scilicet in tali casu non proproficiscemur a duabus obseruationibus extremis, sed a prima et media, vel a media et vlima, adeoque pro $X, Y$, accipiemus differentias inter computum et obsernationem in loco tertio vel primo. Quodsi autem tum locus secundus a primo tum teriius a secundo propemodum 180 gradibus distarent, incommodum illud hoc modo tollere non liceret; sed praestat, huiusmodi obseruationes, e quibus per rci naturam determinatio accurata situs orbitae erui omnino nequit, ad calculum clementorum haud adhibere.

Practerea haec methodus eo quoque se commendat, quod nullo negotio aestimari potest, quantas variationes elementa patiantur, si manentibus locis extremis medins paullulum mutetur: hoc itaque modo iudicium ferri poterit qualecunque de gradu praecisionis elementis inuent's tribuendae.

\section{5.}

Leui mutatione applicata e methodo praecedente secundam eliriemus. A distantiis in duabus obseruationibus profect, perinde vt in illa, cuncta elementa determinabimus; ex his vero non locum geocentricum pro obseruatione tertia computabimus, sed tantummodo vsque ad locum heliocentricum in orbita progrediemur; ex altera parte eundem locum heliocentricum per problema in artt. $7^{4}, 7^{5}$ tractatum e loco geocentrico obseruato atque situ plani orbitae deriuabimus; hae duae determinationes inter se differentes (nisi forte valores veri ipsarum $x, y$ suppositae fuerint), ipsas $X, Y$ nobis suppeditabunt, accepta pro $X$ differentia inter duos valores longitudinis in orbita, atque pro $I$ differentia inter duos valores radii recto- 
ris, aut potius logarithmi eits. Haecce methodus isdem monitionibus obnoxia est, quas in art. praec. attigimus: adiungere oportet aliam, scilicet, quod locus helioeentricus in orbita e geocentrico deduci nequit, quoties locus torrae in alterutrum. nodorum orbitae incidit; tunc itaque hanc methodum applicare non licet. Sed in eo quoque casu, vbi locus terrae ab alterutro nodorum perparum distat, hac methodo abstinere conueniet, quoniam suppositio, variationibus páruis ipsarum $x, y$ respondere variationes proportionales ipsarum $X, Y$, nimis erronea enaderet, per rationem ei quam in art. praec. attigimus similem. Sed hic quoque remedium o permutatione loci medii cum aliquo extremortim, cui locus tenrae a nodis magis remotus respondeat, petere licebit, nisi forte in ommibus tribus obseruationibus ferra in nodorum viciniis versata fuerit.

126.

Methodus praecedens ad tertiam illico sternit viam. Determinentur, perinde vt ante, e distantiis corporis coelestis a terra in obseruationibus extremis longitudines respondentes in orbita cum radiis vectoribus. Adiumento positionis plani orbitae, quam hic calculus suppeditanerit, eruatur ex obseruatione media longitudo in orbita atque radius vector. Tunc autem computentur ex his tribus locis heliocentricis elementa reliqua per problema in artt. 82,83 tractatum, quae operatio ab obseruationum temporibus independens erit. Hoc itaque modo innotescent tres anomaliae mediae atque motus diurnus, vnde ipsa temporum interualla inter obseruationem primam et secundam, atque inter secundam et tertiam computare licebit. Horum differentiae ab interuallis veris pro $X$ et $Y$ accipientur.

Haec methodus minus idonea esset, quoties motus heliocentricus arcum exiguum tantum complectitur. In tali enim casu ista orbitae determinatio ( $v t$ inm in art. 82 monumus) a quantitatibus tertii ordinis pendet, adeoque praecisionem sufficientem non admiltit. Variationes leuissimae in valoribus ipsarum $x, y$ producere possent variationes permagnas in elementis adeoque etiam in valoribus ipsarum $X, Y$ neque has illis proportionales supponere liceret. Quoties autem tres loci motum heliocentricum considerabilem subtendunt, methodi vsus vtique succedet optime, siquidem exceptionibus in artt. praec. explicatis haud turbetur, ad quas manifesto in hac quoque methodo respiciendum erit.

$12 \%$

Postquam tres loci heliocentrici co quem in art. praec. descripsimus modo eruti sunt, sequenti quoque modo procedi poterit. Determinentur elementa reliqua 
pex problema in artt. $85 \ldots 105$ tractatum primo e loco primo et secundo cum interuallo temporis respondente, dein vero eodem modo e loco secundo et tertio temporisque interuallo respondente: ita pro singulis elementis duo valores prodibunt, e quorum differentiis duas ad libitum pro $X$.et $Y$ accipere licebit. Magnopere hanc methodum commendat commodum haud spernendum, quod in hypothesibus primis elementa reliqua, praeter duo ea quae ad stabiliendum $X$ et $Y$ eliguntur, omnino negligere licet, quae in vltimo demum calculo, valoribus correctis ipsarum $x, y$ superstructo, determinabuntur siue e sola combinatione prima, siue e sola secunda, siue quod plerumque praeferendum est e combinatione loci primi cum tertio. $\mathrm{Ce}-$ terum electio illorum duorum elementorum, quae generaliter loquendo arbitraria est, magnam solutionum varietatem suppeditat; adoptari poterunt e. g. logarithmus semiparametri cum logarithmo semiaxis maioris, vel prior cum excentricitate, vel cum eadem posterior, vel cum aliquo horum elementorum longitudo perihelii : combinari quoque poterit aliquod horum quatuor elèmentorum cum anomalia excentrica loco medio in vtroque calculo respondente, siquidem orbita elliptica euaserit, vbi formulae $27-30$ art. $9^{6}$ calculum maxime expeditum afferent. In casibus specialibus autem haec electio quadam circumspectione indiget; ita e. g. in orbitis ad parabolae similitudinem vergentibus semiaxis maior a ipsiusue logarithmus minus idonei forent, quippe quorum variationes immodicae variationibus ipsarum $x, y$ haud proportionales censeri possent: in tali casu magis e re esset eligere $\frac{1}{a}$. Sed his cautelis co minus immoramur, quum methodus quinta in art. seq. explicanda quatuor hactenus expositis in omnibus fere casibus palmam praeripiat.

128 .

Designemus tres radios rectores eodem modo erutos $v t$ in artt. 125,126 per $r, r^{\prime}, r^{\prime \prime}$; motum angularem heliocent:icum in orbita a loco secundo ad tertium per $2 f$, a primo ad tertium per $2 f^{\prime}$, a primo ad secundum per $2 f^{\prime \prime}$, i a vt habeatur $f^{\prime}=f+f^{\prime \prime}$; sit porro $r^{\prime} r^{\prime \prime} \sin 2 f=n, r r^{\prime \prime} \sin 2 f^{\prime}=n^{\prime}, r r^{\prime} \sin 2 f^{\prime \prime}=n^{\prime \prime} ;$ denique producta quantitatis constantis $k$ (art. 2 ) in temporis interualla ab obseruatione secunda ad tertiam, a prima ad tertiam, a prima ad secundam resp. $\theta, \theta^{\prime}, \theta^{\prime \prime}$. Incipiatur computus duplex elementorum (perinde vt in art. praec.) tum ex $r, r^{\prime}, f^{\prime \prime}$ et $\theta^{\prime \prime}$, tum ex $r^{\prime}, r^{\prime \prime}, f, \theta$ : in vtroque vero calculo non ad elementa ipsa progredieris, sed subsistes, quamprimum quantitas ea, quae rationem sectoris elliptici ad triangulum exprimit, supraque (art. 91) per $y$ vel $-Y$ denotata est, eruta fuerit. Sit valor huius quantitatis 
in calculo primo $\eta^{\prime \prime}$, in secundo $\eta$. Habebimus itaque per formulam 18 art. 95 pro semiparametro $p$ valorem duplicem:

$$
\sqrt{ } p=\frac{\eta^{\prime \prime} n^{\prime \prime}}{\theta^{\prime \prime}}, \text { atque } \vee p=\frac{\eta n}{\theta}
$$

Sed per art. 82 habemus insuper valorem tertium

$$
p=\frac{4 r r^{\prime} r^{\prime \prime} \sin f \sin f^{\prime} \sin f^{\prime \prime}}{n-n^{\prime}+n^{\prime \prime}}
$$

qui tres valores manifesto identici esse deberent, si pro $x, y$ ab initio valores veri accepti fuissent. Quamobrem esse deberet

$$
\begin{aligned}
& \frac{\theta^{\prime \prime}}{\theta}=\frac{\eta^{\prime \prime} n^{\prime \prime}}{\eta n} \\
& n-n^{\prime}+n^{\prime \prime}=\frac{4 \theta \theta^{\prime \prime} r r^{\prime} r^{\prime \prime} \sin f \sin f^{\prime} \sin f^{\prime \prime}}{\eta \eta^{\prime \prime} n l^{\prime \prime}}=\frac{n^{\prime} \theta \theta^{\prime \prime}}{2 \eta \eta^{\prime \prime} r r^{\prime} r^{\prime \prime} \cos f \cos f^{\prime} \cos f^{\prime \prime}}
\end{aligned}
$$

Nisi itaque his aequationibus iam in primo calculo sponte satisfit, statuere licebit.

$$
\begin{aligned}
& X=\log \frac{\eta n \theta^{\prime \prime}}{\eta^{\prime \prime} n^{\prime \prime} \theta} \\
& Y=n-n^{\prime}+n^{\prime \prime}-\frac{n^{\prime} \theta \theta^{\prime \prime}}{2 \eta \eta^{\prime \prime} r r^{\prime} r^{\prime \prime} \cos f \cos f^{\prime} \cos f^{\prime \prime}}
\end{aligned}
$$

Haec methodus applicationem aeque generalem patitur, ac secunda in art. 124 explicata, magnum vero lucrum est, quod in hacce quinta hypotheses primae enolutionem elementorum ipsorum non requirunt, sed in media quasi via subsistunt. Ceterum simulatque in hac operatione eo peruentum est, vt praeuideri possit, hypothesin nouam a veritate haud sensibiiiter discrepaturam esse, in hac elementa ipsa vel duntaxat ex $r, r^{\prime}, f^{\prime \prime}, \theta^{\prime \prime}$, vel ex $r^{\prime}, r^{\prime \prime}, f, \theta$, rel quod praestat ex $r, r^{\prime \prime}, f^{\prime} \theta^{\prime}$, determinare sufficiet.

129.

Quinque methodi hactenus expositae protinus ad totidem alias viam sternunt, quae $a b$ illis eo tantum differunt, quod pro $x$ et $y$ loco distantiarum a terra, inclinatio orbitae atque longitudo nodi ascendentis accipiuntur. Hae igitur methodi nouae ita se habent:

I. Determinantur ex $x$ et $y$ duobusque locis geocentricis extremis secundum artt. 74,75 longitudines heliocentricae in orbita radiique vectores, atque hinc et ex temporibus respondentibus omnia reliqua elementa; ex his denique locus geocentricus pro tempore obseruationis mediae, cuius differentiac a loco obseruato in longitudinem et latitudinem ipsas $X$ et $Y$ suppeditabunt. 
Quatuor reliquae methodi in eo conueniunt, quod e positione plani orbitae: locisque geocentricis omnes tres longitudines heliocentricae in orbita radiique rectores respondentes computantur. Dein autem

II. elementa reliqua determinantur e duobus locis extremis tantum atque temporibus respondentibus; secundum haec elementa calculantur pro tempore observationis mediae longitudo in orbita atque radius vector, quarum quantitatum differentiae a valoribus prius inuentis, i. e. e loco geocentrico deductis, ipsas $\boldsymbol{X}, \boldsymbol{Y}$ exhibebunt.

III. Aut deriuantur orbitae dimensiones reliquae ex ommibus tribus locis heliocentricis ( $\operatorname{artt.} 82,83$ ), in quem calculum tempora non ingrediuntur: dein temporum interualla eruuntur, quae in orbita ita inuenta inter obseruationem primam et secundam, atque inter hanc et tertiam elapsa esse deberent, et quorum differentiae a veris ipsas $X, Y$ nobis suggerent.

IV. Calculantur elementa reliqua duplici modo, puta tum e combinatione loci primi cum secundo, tum e combinatione secundi cum tertio, adhibitis temporum interuallis respondentibus. Comparatis hisce duobus elementorum systematibus inter se, e differentiis duae quaecunque pro $X, Y$ accipi poterunt.

$V$. Siue denique idem calculus duplex tantummodo vsque ad valores quantitatis in art. 91 per $y$ denotatae producitur, ac dein pro $X, Y$ expressiones in art. praec. traditae adoptantur.

Vt quatuor vltimis harum methodorum tuto vti liceat, loci terrae pro omnibus tribus obseruationibus orbitae nodis non nimis vicini esse debent: contra vsus methodi primae tantummodo requirit, vt eadem conditio in duabus obseruationibus extremis locum habeat, siue potius, (quoniam locum medium pro aliquo extremorum substituere licet) vt e tribus locis terrae non plures quam vnus in nodorum viciniis versentur.

130.

Decem methodi inde ab art. 124 explicatae innituntur suppositioni, valores approximatos distantiarum corporis coelestis a terra, ant positionis plani orbitae, iam in potestate esse. Quoties quidem id agitur, vt dimensiones orbitae, quarum valores approximati iam alicunde innotuerunt, puta per calculum anteriorem obseruationibus aliis innixum, per obseruationes magis ab inuicem remotas corrigantur, postulatum illud nullis manifesto difficultatibus obnoxium erit. Sed hine nondum liquet, quonam modo calculum primum aggredi liceat, vbi omnes orbitae dimen- 
siones penitus adhuc incognitae sunt: hic vero problematis nostri casus longe gravissimus atque difficillimus est, vti iam ex problemate analogo in theoria cometarum praesumi potest, quod quamdiu geometras torserit, quotque tentaminibus irritis originem dederit satis constat. Vt problema nostrum recte solutum censeri possit, manifesto conditionibus sequentibus satisfieri oportet, siquidem solutio ad instar normae inde ab art. 119 explicatae exhibetur: Primo quantitates $x, y$ tali modo sunt eligendae, vt valores ipsarum approximatos ex ipsa problematis natura petere liceat, saltem, quamdiu corporis coelestis motus heliocentricus intra observationes non nimis magnus est. Secundo autem requiritur, vit variationibus exiguis quantitatum $x, y$ variationes non nimis magnae in quantitatibus inde deriuandis respondeant, ne errores in illarum valoribus suppositis forte commissi impediant, quominus has quoque pro approximatis habere liceat. Denique tertio postulamus, vt operationes, per quas a quantitatibus $x, y$ successiue vsque ad $X, Y$ progrediendum est, non nimis prolixae euadant.

Hae conditiones criterium subministrabunt, secundum quod de cuiusuis methodi praestantia iudicium ferri poterit: adhuc euidentius quidem ea applicationibus frequentibus se manifestabit. Methodus ea, quam exponere iam accingimur, et quae quodammodo támquam pars grauissima huius operis consideranda est, illis conditionibus ita satisfacit, vt nihil amplius desiderandum relinquere videatur. Quam antequam in forma ad praxin commodissima explicare aggrediamur, quasdam considerationes praeliminares praemittemus, aditumque quasi ad illam, qui alias forsan obscurior minusque obuius videri possit, illustrabimus atque aperiemus.

151.

In art. 114 ostensum est, si ratio inter quantitates illic atque in art. 128 per $n, n^{\prime}, n^{\prime \prime}$ denotatas cognita fuerit, corporis coelestis distantias a terra per formulas persimplices. determinari posse. Quodsi itaque pro $x, y$ assumerentur quotientes $\frac{n}{n^{\prime}}, \frac{n^{\prime \prime}}{n^{\prime}}$, pro his quantitatibus in eo casu, vbi motus heliocentricus inter obseruationes haud ita magnus est, statim valores, approximati $\frac{\theta}{\theta^{\prime}}, \frac{\theta^{n}}{\theta^{\prime}}$ se offerrent (accipiendo, characteres $\theta, \theta^{\prime}, \theta^{\prime \prime}$ in eadem significalione $v t$ in art. 128): hinc itaque solutio obuia problematis nostri demanare videtur, si ex $x$ et $y$ distantiae duae a terra eliciantur, ac dein ad instar alicuius ex quinque methodis artt. 124-128 procedatur. Reuera, acceptis quoque characteribus $\eta, \eta^{\prime \prime}$ in significatione art. 128 , 
DETERMINATIO ORBITAE E TRIBVS ODSERVATIONIBVS COMPLETIS. 747 designatoque analogice per $\eta^{\prime}$ quotiente orto ex diuisione sectoris inter duos radios vectores contenti per aream trianguli inter eosdem, erit $\frac{n}{n^{\prime}}=\frac{\theta}{\theta^{\prime}} \cdot \frac{\eta^{\prime}}{\eta}, \frac{n^{\prime \prime}}{n^{\prime}}=\frac{\theta^{\prime \prime}}{\theta^{\prime}} \cdot \frac{\eta^{\prime}}{\eta^{\prime \prime}}$, patetque facile, si $n, n^{\prime}, n^{\prime \prime}$ tamquam quantitates paruae primi ordinis spectentur, esse generaliter loquendo $\eta-1, \eta^{\prime}-1, \eta^{\prime \prime}-1$ quantitates secundi ordinis, adeoque valores ipsarum $x, y$ approximatos $\frac{\theta}{\theta^{\prime}}, \frac{\theta^{\prime \prime}}{\theta^{\prime}}$ a veris differre tantummodo quantitatibus secundi ordinis. Nihilominus re propius considerata methodus haecce omnino inepta inuenitur, cuius phaenomeni rationem paucis explicabimus. Leui scilicet negotio perspicitur, quantitatem $(0,1,2)$, per quam distantiae in formulis $9,10,11$ art. 114 multiplicatae sunt, ad minimum tertii ordinis fieri, contra e. g. in aequ. 9 quantitates (O.1.2), (I.1.2), (II.1.2) primi ordinis; hine antem facile sequitur, errorem secundi ordinis in valoribus quantitatum $\frac{n}{n^{\prime}}, \frac{n^{\prime \prime}}{n^{\prime}}$ commissum producere in valoribus distantiarum êrrorem ordinis o. Quamobrem, secundum vulgarem loquendi vsum, distantiae tunc quoque errore finito affectae prodirent, quando temporum interualla infinite parua sunt, adeoque neque has distantias neque reliquas quantitates inde deriuandas ne pro approximatis quidem habere liceret, naethodusque conditioni secundae art. praec. aduersaretur.

152.

Statuendo breuitatis gratia $(0.1 .2)=a,(0.1 .2) D^{\prime}=-b,($ o. O.2) $D=-c$, (o. II. 2) $D^{\prime \prime}=-d$, ita vt aequatio to art. 114 fiat $a \delta^{\prime}=b+c \cdot \frac{n}{n^{\prime}}+d \cdot \frac{n^{\prime \prime}}{n^{\prime}}$, coëfficientes $c$ et $d$ quidem erunt primi ordinis, facile vero ostendi potest, differentiam $c-d$ ad secundum ordinem referendam esse. Hinc vero sequitur, valorem quantitatis $\frac{c n+d n^{\prime \prime}}{n+n^{\prime \prime}}$ ex suppositione approximata $n: n^{\prime \prime}=\theta: \theta^{\prime \prime}$ prodeuntem errore quarti tantum ordinis affectum esse, quin adeo quinti tantum, quoties obseruatio media $a b$ extremis aequalibus interuallis distat. Fit enim iste error

$=\frac{c \theta+d \theta^{\prime \prime}}{\theta+\theta^{\prime \prime}}-\frac{c n+d n^{\prime \prime}}{n+n^{\prime \prime}}=\frac{\theta \theta^{\prime \prime}(d-c)\left(\eta^{\prime \prime}-\eta\right)}{\left(\theta+\theta^{\prime \prime}\right)\left(\eta^{\prime \prime} \theta+\eta \theta^{\prime \prime}\right)}$

vbi denominator secundi ordinis est, numeratorisque factor alter $\theta \theta^{\prime \prime}(d-c)$ quarti, alter $\eta^{\prime \prime}-\eta$ secundi, vel in casu isto speciali tertii ordinis. Exhibita itaque aequatione illa in hacce forma $a d^{\prime}=b+\frac{c n+d n^{n}}{n+n^{\prime \prime}} \cdot \frac{n+n^{\prime \prime}}{n^{\prime}}$, manifestum est, vitium 
methodi in art. praec. propositae non inde oriri, quod quantitates $n, n^{n}$ hisce $\theta, \theta^{\prime \prime}$ proportionales suppositae sunt, sed inde, quod insuper $n^{\prime}$ ipsi $\theta^{\prime}$ proportionalis statuta est. Hoc quippe modo loco factoris $\frac{n+n^{\prime \prime}}{n^{\prime}}$, valor minus exactus $\frac{\theta+\theta^{\prime \prime}}{\theta^{\prime}}=1$ introducitur, a quo verus $=1+\frac{\theta \theta^{\prime \prime}}{2 \eta \eta^{\prime \prime} r r^{\prime} r^{\prime \prime} \cos f \cos f^{\prime} \cos f^{\prime \prime}}$ quantitate ordinis secundi discrepat (art. 128).

\section{3.}

Quum cosinus angulorum $f, f^{\prime}, f^{\prime \prime}$, perinde vt quantitates $\eta, \eta^{\prime \prime}$ ab vnitate differentia secundi ordinis discrepent, patet, si pro $\frac{n+n^{\prime \prime}}{n^{\prime}}$ valor approximatus $1+\frac{\theta \theta^{\prime \prime}}{2 r r^{\prime} r^{\prime \prime}}$ introducatur, errorem quarti ordinis committi. Quodsi itaque loco aequationis art. 114. haecce adhibetur

$$
a \mathrm{~d}^{\prime}=b+\frac{c \theta+d \theta^{\prime \prime}}{\theta^{\prime}}\left(1+\frac{\theta \theta^{\prime \prime}}{2 r r^{\prime} r^{\prime \prime}}\right)
$$

in valorem distantiae $d^{\prime}$ redundabit error secundi ordinis, quando obseruationes extremae a media aequidistant, vel primi ordinis in casibus reliquis. Sed haecce noua aequationis illius forma ad determinationem ipsius $d^{\prime}$ haud idonea est, quia quantitates adhuc incognitas $r, r^{\prime}, r^{\prime \prime}$ inuoluit.

Ian generaliter loquendo quantitates $\frac{r}{r^{\prime}}, \frac{r^{\prime \prime}}{r^{\prime}}$ ab vnitate differentia primi ordinis distant, et perinde etiam productum $\frac{r r^{\prime \prime}}{r^{\prime} r^{\prime}}$ : in casu speciali saepius commemorato facile perspicitur, hoc productum differentia secundi ordinis tantum $a b$ vnitate discrepare. Quin adeo quoties orbita ellipsis parum excentrica est, ita vt excentricitatem tamquam quantitatem primi ordinis spectare liceat, differentia $\frac{r r^{\prime \prime}}{r^{\prime} r^{\prime}}$ ad ordinem vno gradu adhuc altiorem referri poterit. Manifestum est itaque, errorem illum eiusdem ordinis vt antea manere, si in aequatione nostra pro $\frac{\theta \theta^{\prime \prime}}{2 r r^{\prime \prime}}$ substituatur $\frac{\theta \theta^{\prime \prime}}{2 r^{\prime 3}}$, vade nanciscitur formam sequentem

$$
a \delta^{\prime}=b+\frac{c \theta+d \theta^{\prime \prime}}{\theta^{\prime}}\left(x+\frac{\theta \theta^{\prime \prime}}{2 r^{\prime 3}}\right)
$$


Continet quidem haec aequatio etiamnum quantitatem incognitam $r$, quam tamen eliminari posse patet, quum tantummodo a $\delta^{\prime}$ atque quantitatibus cognitis pendeat. Quodsi dein aequatio rite ordinaretur, ad octauum gradum ascenderet.

Ex praecedentibus iam ratio percipietur, cur in methodo nostra pro $x, y$ resp. quantitates $\frac{n^{\prime \prime}}{n}=P$ atque $2\left(\frac{n+n^{\prime \prime}}{n^{\prime}}-1\right) r^{\prime 3}=Q$ accepturi simus. Patet enim primo, si $P$ et $Q$ tamquam cognitae spectentur, $\delta^{\prime}$ inde per aequationem.

$$
a \delta^{\prime}=b+\frac{c+d P}{1+P}\left(1+\frac{Q}{2 r^{\prime 3}}\right)
$$

determinari posse, ac dein $\delta$ et $\delta^{\prime \prime}$ per aequationes 4,6 art. 114, quum habeatur $\frac{n}{n^{\prime}}=\frac{x}{1+P}\left(1+\frac{Q}{2 r^{\prime 3}}\right), \frac{n^{\prime \prime}}{n^{\prime}}=\frac{P}{1+P}\left(1+\frac{Q}{2 r^{\prime 3}}\right)$. Secundo manifestum est, in hypothesi prima pro quantitatibus $P, Q$, quarum valores exacte veri sunt $\frac{\theta^{\prime \prime}}{\theta} \cdot \frac{\eta}{\eta^{\prime \prime}}, \frac{r r^{\prime \prime} \theta \theta^{\prime \prime}}{r^{\prime} r^{\prime} \eta \eta^{\prime \prime} \cos f \cos f^{\prime} \cos f^{\prime \prime}}$, statim obuios esse valores approximatos $\frac{\theta^{\prime \prime}}{\theta}$, $\theta \theta^{\prime \prime}$, ex qua hypothesi in determinationem ipsius $\delta^{\prime}$ et proin etiam ipsarum $\delta, \delta^{\prime \prime}$, redundabunt errores primi ordinis, vel secundi in cast speciali pluries commemorato. Ceterum etiamsi his conclusionibus, generaliter loquendo, tutissime fidendum sit, tamen in casu quodam speciali vim suam perdere possunt, scilicet-quoties quantitas (o.1.2), quae in genere est ordinis tertii, fortuito fit $=0$, vel tam parua, vt ad altiorem ordinem referri debeat. Hoc enenit, quoties motus geocentricus in sphaera coelesti prope locum medium punctum inflexionis sistit. Denique apparet, rt methodus nostra in vsum vocari possit, necessario requiri, vt molus heliocentricus inter tres obseruationes non nimis magnus sit: sed haec restrictio, per problematis complicatissimi naturam, nullo modo euitari potest, neque etiam pro incommodo habenda est, quoniam semper in votis erit, determinationem primam orbitae incognitae corporis coelestis noui quam primum licet suscipere. Praeterea restricio illa sensu satis lato accipi potest, vti exempla infra tradenda ostendent.

\section{5.}

Disquisitiones praecedentes eum in finem allatae sunt, $v t$ principia, quibus methodus nostra innititur, verusque eius quasi neruus eo clarius perspiciantur: tractatio ipsa autem methodum in forma prorsus dinersa exhibebit, quam post appli- 
cationes frequentissimas tamquam commodissimam inter plures alias a nobis tentatas commendare possumus. Quum in determinanda orbita incognita e tribus obseruationibus totum negotium semper ad aliquot hypotheses, ant potius approximationes successiuas reducatur, pro lucro eximio habendum erit, si calculum ita adornare successerit, vt iam ab initio operationes quam plurimas, quae non a $P$ et $Q$ sed vnice a combinatione quantitatum cognitarum pendeant, ab ipsis hypothesibus separare liceat. Tunc manifesto has operationes praeliminares, singulis hypothesibus communes, semel tantum exsequi oportet, hypothesesque ipsae ad operationes quam paucissimas reducuntur. Perinde maximi momenti erit, si in singulis hypothesibus vsque ad ipsa elementa progredi haud opus fucrit, horumque computum vsque ad hypothesin postremam reseruare liceat. Vtroque respectu methodus nostra, quam exponere iam aggredimur, nihil desiderandum relinquere videtur.

136.

Ante omnia tres locos heliocentricos terrae in sphaera coelesti $A, A^{\prime}, A^{n}$ (fig. 4) cum tribus locis geocentricis respondentibus corporis coelestis $B, \mathcal{B}^{\prime}, B^{n}$ per circulos maximos iungere, atque tum positionem horum circulorum maximorum respectu eclipticae (siquidem eclipticam pro plano fundanentali adoptamus), tum situm punctorum $B, B^{\prime}, B^{\prime \prime}$ in ipsis computare oportet. Sint $\alpha, \alpha^{\prime}, \alpha^{\prime \prime}$ tres corporis coelestis longitudines geocentricae; $\beta, \beta^{\prime}, \beta^{\prime \prime}$ latitudines, $l, l^{\prime}, l^{\prime \prime}$ longitudines heliocentricae terrae, cuius latitudines statuimus $=0$ (artt. 117, 72). Sint porro $\gamma, \gamma^{\prime}, \gamma^{\prime \prime}$, circulorum maximorum ab $A, A^{\prime}, A^{\prime \prime}$ resp. ad $B, B^{\prime}, B^{\prime \prime}$ ductorum inclinationes ad eclipticam: quas inclinationes, $v t$ in ipsarum determinatione normam fixam sequamur, perpetuo respectu eius eclipticae partis mensurabimus, quae a punctis $A, A^{\prime}, A^{\prime \prime}$ secundum ordinem signorum sita est, ita vt ipsarum magnitudo a o vsque ad $360^{\circ}$ numeretur, sine quod eodem redit, in parte boreali a o rsque ad $180^{\circ}$, in australi a o rsque ad $-180^{\circ}$. Arcus $A B, A^{\prime} B^{\prime}, A^{\prime \prime} B^{\prime \prime}$, quos semper intra $o$ et $180^{\circ}$ statuere licebit, designamus per $\delta, \delta^{\prime}, \delta^{\prime \prime}$. Ita pro determinatione ipsarum $\gamma, \delta$ habemus formulas .

[1] $\operatorname{tang} \gamma=\frac{\operatorname{tang} \beta}{\sin (\alpha-l)}$

[2] $\operatorname{tang} \delta=\frac{\operatorname{tang}(\alpha-\eta)}{\cos \gamma}$

quibus si placet ad calculi confirmationem adiici possunt sequentes: 
$\sin \delta=\frac{\sin \beta}{\sin \gamma}, \cos \delta=\cos \beta \cos (\alpha-\lambda)$

Pro determinandis $\gamma^{\prime}, \delta^{\prime}, \gamma^{\prime \prime}, \delta^{\prime \prime}$, manifesto formulae prorsus analogae habentur. Quodsi simul fuerit $\beta=0, \alpha-\lambda=0$ vel $=180^{\circ}$, i. e. si corpus coeleste simul in oppositione vel coniunctione atque in ecliptica fuerit, $\gamma$ fieret indeterminata: at supponemus, hunc casum in nulla trium obseruationum locum habere.

Si loco eclipticae aequator tamquam planum fundamentale adoptatum est, ad positionem trium circulorum maximorum respectu aequatoris determinandam praeter inclinationes insuper requirentur rectascensiones intersectionum cum aequatore: nec non praeter distantias punctorum $B, B^{\prime}, B^{\prime \prime}$ ab his intersectionibus etiam distantias punctorum $A, A^{\prime}, A^{\prime \prime}$ ab iisdem computare oportebit. Quae quum a problemate in art. 110 tractato pendeant, formularum eloolutioni hic non immoramur.

\section{7.}

Negotium secundum erit determinatio situs relativi illorum trimm circulorum maximorum inter se, qui pendebit a situ intersectionum mutuarum et ab inclinationibus. Quae si absque ambiguitate ad notiones claras ac generales reducere cupimus, ita vt non opus sit pro singulis casibus diuersis ad figuras peculiares recurrere, quasdam dilucidationes praeliminares praemittere oportebit. Primo scilicet in quouis circulo maximo duae directiones oppositae aliquo modo distinguendae sunt, quod fiet, dum alteram tamquam progressiuam seu positiuam, alteram tamquam retrogradam seu nega iuam consideramus. Quod quum per se prorsus arbitrarium sit, vt normam certam stabiliamus, semper directiones ab $A, A^{\prime}, A^{\prime \prime}$ versus $B, B^{\prime}, B^{\prime \prime}$ ceu positiuas considerabimus; ita e. g. si intersectio circuli primi cum secundo per/distantiam positiuam a puncto $A$ exhibetur, haec capienda subintelligetur ab $A$ versus $B$ (vt $D^{\prime \prime}$ in figura nostra); si vero negatiua esset, ipsam ab altera parte ipsius $A$ sumere oporteret. Secundo vero etiam duo haemisphaeria, in quae omnis circulus maximus sphaeram integram dirimit, denominationibus idoneis distinguenda sunt: et quidem hemisphaerium superius rocabimus, quod in superficie interiori sphaerae circulum maximum directione progressiua permeanti ad dextram est, alterum inferius. Plaga itaque superior analoga erit hemisphaerio boreali respectu eclipticae vel aequatoris, inferior australi.

His rite intellectis, ambas duorum circulorum maximorum intersectiones eommode ab inuicem distinguere licebit: in vna scilicet circulus primus e secundi 
regione inferiori in superiorem tendit, vel quod idem est secundus e primi regione superiori in inferiorem; in altera intersectione opposita locum habent. Per se quidem prorsus arbitrarium est, quasnam intersectiones in problemate nostro eligere velimus: sed vt hic quoque iuxta normam inuariabilem procedamus, eas semper adoptabimus, $\left(D, D^{\prime}, D^{\prime \prime}\right.$ in fig. 4), vbi resp. circulus terius $A^{\prime \prime} B^{\prime \prime}$ in secundi $A^{\prime} B^{\prime}$, tertius in primi $A B$, secundus in primi plagam superiorem transit. Situs harum intersectionum determinabitur per ipsarum distantias a punctis $A^{\prime}$ et $A^{\prime \prime}, A$ et $A^{\prime \prime}$, $A$ et $A^{\prime}$, quas simpliciter per $A^{\prime} D, A^{\prime \prime} D, A D^{\prime}, A^{\prime \prime} D^{\prime}, A D^{\prime \prime}, A^{\prime} D^{\prime \prime}$ designabimus. Quibus ita factis circulorum inclinationes mutuae erunt anguli, qui resp. in his intersectionum punctis $D, D^{\prime}, D^{\prime \prime}$ inter circulorum se secantium partes eas continentur, quae in directione progressiua iacent: has inclinationes, semper inter o et $180^{\circ}$ accipiendas, per $\varepsilon, \varepsilon^{\prime}, \varepsilon^{\prime \prime}$ denotabimus. Determinatio harum nouem quantitatum incognitarum e cognitis manifesto ab eodem problemate pendet, quod in art. 55 tractauimus: habemus itaque aequationes sequentes:

[5] $\sin \frac{1}{2} \varepsilon \sin \frac{x}{2}\left(A^{\prime} D+A^{\prime \prime} D\right)=\sin \frac{x}{2}\left(l^{\prime \prime}-l^{\prime}\right) \sin \frac{x}{2}\left(\gamma^{\prime \prime}+\gamma^{\prime}\right)$

[4] $\sin \frac{\pi}{2} \varepsilon \cos \frac{r}{2}\left(A^{\prime} D+A^{\prime \prime} D\right)=\cos \frac{x}{2}\left(l^{\prime \prime}-l^{\prime}\right) \sin \frac{r}{2}\left(\gamma^{\prime \prime}-\gamma^{\prime}\right)$

[5] $\cos \frac{x}{2} \varepsilon \sin \frac{x}{2}\left(A^{\prime} D-A^{\prime \prime} D\right)=\sin \frac{x}{2}\left(l^{\prime \prime}-l^{\prime}\right) \cos \frac{x}{2}\left(\gamma^{\prime \prime}+\gamma^{\prime}\right)$

[6] $\cos \frac{\pi}{2} \varepsilon \cos \frac{1}{2}\left(A^{\prime} D-A^{\prime \prime} D\right)=\cos \frac{\pi}{2}\left(l^{\prime \prime}-l^{\prime}\right) \cos \frac{1}{2}\left(\gamma^{\prime \prime}-\gamma^{\prime}\right)$

Ex aequationibus 3 et 4 innotescent $\frac{1}{2}\left(A^{\prime} D+A^{\prime \prime} D\right)$ et $\sin \frac{x}{2} \varepsilon$, e duabus reliquis $\frac{\pi}{2}\left(A^{\prime} D-A^{\prime \prime} D\right)$ et $\cos \frac{1}{2} \varepsilon$; hinc $A^{\prime} D, A^{\prime \prime} D$ et $\varepsilon$. Ambiguitas determinationi arcuum $\frac{x}{2}\left(A^{\prime} D+A^{\prime \prime} D\right), \frac{\pi}{2}\left(A^{\prime} D-A^{\prime \prime} D\right)$ per tangentes adhaerens conditione ea decidetur, quod $\sin \frac{\pi}{2} \varepsilon$ et $\cos \frac{\pi}{2} \varepsilon$ positiui euadere debent, consensusque inter $\sin \frac{x}{2} \varepsilon$ et $\cos \frac{T}{2} \varepsilon$ toti calculo confirmando inseruiet.

Determinatio quantitatum $A D^{\prime}, A^{\prime \prime} D^{\prime}, \varepsilon^{\prime}, A D^{\prime \prime}, A^{\prime} D^{\prime \prime}, \varepsilon^{\prime \prime}$ prorsus simili modo perficietur, neque opus erit octo aequationes ad hunc calculum adhibendas. huc transscribere, quippe quae ex aequ. $3-6$ sponte prodeunt, si

$$
\begin{array}{r|c|c|c|c|c}
A^{\prime} D & A^{\prime \prime} D & \varepsilon & l^{\prime \prime}-l^{\prime} & \gamma^{\prime \prime} & \gamma^{\prime} \\
\operatorname{cum} A D^{\prime} & A^{\prime \prime} D^{\prime} & \varepsilon^{\prime} & l^{\prime \prime}-l & \gamma^{\prime \prime} & \gamma \\
\operatorname{cum} A D^{\prime \prime} & A^{\prime} D^{\prime \prime} & \varepsilon^{\prime \prime} & l^{\prime}-l & \gamma^{\prime} & \gamma
\end{array}
$$

resp. commutantur.

Noua adhuc totius calculi confirmatio deriuari potest e relatione mutua inter latera angulosque trianguli sphaerici inter puncta $D, D^{\prime}, D^{\prime \prime}$ formati, vnde demanant aequationes generalissime verae, quamcunque situm haec puncta habeant: $\frac{\sin \left(A D^{\prime}-A D^{\prime \prime}\right)}{\sin \varepsilon}=\frac{\sin \left(A^{\prime} D-A^{\prime} D^{\prime \prime}\right)}{\sin \varepsilon^{\prime}}=\frac{\sin \left(A^{\prime \prime} D-A^{\prime \prime} D^{\prime}\right)}{\sin \varepsilon^{\prime \prime}}$ 
Denique si loco eclipticae aequator tamquam planum fundamentale electus est, calculus mutationem non subit, nisi quod pro terrae locis heliocentricis $A, A^{\prime}, A^{\prime \prime}$ substituere oportet ea aequatoris puncta, vbi a circulis $A B, A^{\prime} B^{\prime}, A^{\prime \prime} B^{n}$ secatur; accipiendae sunt itaque pro $l, l^{\prime}, l^{\prime \prime}$ ascensiones rectae harum intersectionum, nec non pro $A^{\prime} D$ distantia puncti $D$ ab intersectione secunda etc.

158.

Negotium tertium iam in eo consistit, vt duo loci geocentrici extremi corporis coelestis, i. e. puncta $B, B^{\prime \prime}$, per circulum maximum iungantur, huiusque intersectio cum circulo maximo $A^{\prime} B^{\prime}$ determinetur. Sit $B^{*}$ haec intersectio, atque $\delta^{\prime}-\sigma$ eius distantia a puncto $A^{\prime}$, nec non $\alpha^{\star}$ eirs longitudo, $\beta^{\star}$ latitudo. Habemus itaque, propterea quod $B, B^{\star}, B^{\prime \prime}$ in codem circulo maximo iacent, aequationem satis notam

$$
0=\operatorname{tang} \beta \sin \left(\alpha^{\prime \prime}-\alpha^{\star}\right)-\operatorname{tang} \beta^{\star} \sin \left(\alpha^{\prime \prime}-\alpha\right)+\operatorname{tang} \beta^{\prime \prime} \sin \left(\alpha^{*}-\alpha\right)
$$

quae, substituendo $\operatorname{tang} \gamma^{\prime} \sin \left(\alpha^{\star}-l^{\prime}\right)$ pro $\operatorname{tang} \beta^{\star}$, sequentem formam induit

$$
o=\left\{\begin{array}{l}
\cos \left(\alpha^{*}-l^{\prime}\right)\left\{\operatorname{tang} \beta \sin \left(\alpha^{\prime \prime}-l^{\prime}\right)-\operatorname{tang} \beta^{\prime \prime} \sin \left(\alpha-l^{\prime}\right)\right\} \\
-\sin \left(\alpha^{\star}-l^{\prime}\right)\left\{\operatorname{tang} \beta \cos \left(\alpha^{\prime \prime}-l^{\prime}\right)+\operatorname{tang} \gamma^{\prime} \sin \left(\alpha^{\prime \prime}-\alpha\right)-\operatorname{tang} \beta^{\prime \prime} \cos \left(\alpha-l^{\prime}\right)\right\}
\end{array}\right.
$$

Quare quum sit $\operatorname{tang}\left(\alpha^{\star}-l^{\prime}\right)=\cos \gamma^{\prime} \operatorname{tang}\left(\delta^{\prime}-\sigma\right)$ habebimus

$$
\operatorname{tang}\left(\delta^{\prime}-\sigma\right)=\frac{\operatorname{tang} \beta \sin \left(\alpha^{\prime \prime}-l^{\prime}\right)-\operatorname{tang} \beta^{\prime \prime} \sin \left(\alpha-l^{\prime}\right)}{\cos \gamma^{\prime}\left(\operatorname{tang} \beta \cos \left(\alpha^{\prime \prime}-l^{\prime}\right)-\operatorname{tang} \beta^{\prime \prime} \cos \left(\alpha-l^{\prime}\right)\right)+\sin \gamma^{\prime} \sin \left(\alpha^{\prime \prime}-\alpha\right)}
$$

Hinc deriuantur formulae sequentes, ad calculum numericum magis accommodatae. Statuatur

[7] $\operatorname{tang} \beta \sin \left(\alpha^{\prime \prime}-l^{\prime}\right)-\operatorname{tang} \beta^{\prime \prime} \sin \left(\alpha-l^{\prime}\right)=S$

[8] $\operatorname{tang} \beta \cos \left(\alpha^{\prime \prime}-l^{\prime}\right)-\operatorname{tang} \beta^{\prime \prime} \cos \left(\alpha-l^{\prime}\right)=T^{\prime} \sin t$

[9] $\sin \left(\alpha^{\prime \prime}-\alpha\right)=T \cos t$

(art. 14, II), eritque

[10] $\operatorname{tang}\left(\delta^{\prime}-\sigma\right)=\frac{s}{T \sin \left(t+\gamma^{\prime}\right)}$

Ambiguitas in determinatione arcus $\delta^{\prime}-\sigma$ per tangentem inde oritur, quod circuli maximi $A^{\prime} B^{\prime}, B B^{n}$ in duobus punctis se intersecant: nos pro $B^{\star}$ semper adoptabimus intersectionem puncto $B^{\prime}$ proximam, ita $\mathrm{vt} \sigma$ semper cadat inter limites $-90^{\circ}$ et $+90^{\circ}$, vnde ambiguitas illa tollitur. Plerumque tunc valor arcus $\sigma$ (qui pendet a curtiatura motus geocentrici) quantitas satis modica erit, et quidem gene- 
raliter loquendo secundi ordinis, si temporum interualla tamquam quantitates primi ordinis spectantur.

Quaenam modificationes calculo applicandae sint, si pro ecliptica aequator tamquam planum fundamentale electum est, ex annotatione art. praec. sponte patebit.

Ceterum manifestum est, situm puncti $B^{\star}$ indeterminatum manere, si circuli $B B^{\prime \prime}, A^{\prime} B^{\prime \prime}$ ommino coinciderent: hunc casum, vbi quatuor puncta $A^{\prime}, B, B^{\prime}, B^{\prime \prime}$. in eodem circulo maximo iacerent, a disquisitione nostra excludimus. Conueniet autem in eligendis obseruationibus eum quoque casum euitare, vbi situs horum quatuor punctorum a circulo maximo parum distat: tunc enim situs puncti $B^{\star}$, qui in operationibus sequentibus magni momenti est, per leuissimos obseruationum errores nimis afficeretur, nec praecisione necessaria determinari posset. - Perinde punctum $B^{\star}$ indeterminatum manere patet, quoties puncta $B, B^{\prime \prime}$ in vnum coincidunt *), in quo casu ipsius circuli $B B^{\prime \prime}$ positio indeterminata fieret. Quamobrem hunc quoque casum excludemus, quemadmodum, per rationes praecedentibus similes, talibus quioque obseruationibus abstinendum erit, vbi locus geocentricus primus et vltimus in puncta sphaerae sibi proxima cadunt.

\section{9.}

Sint in sphaera coelesti $C, C^{\prime}, C^{\prime \prime}$ tria corporis coelestis loca heliocentrica, quae resp. in circulis maximis $A B, A^{\prime} B^{\prime}, A^{\prime \prime} B^{\prime \prime}$, et quidem inter $A$ et $B, A^{\prime}$ et $B^{\prime}, A^{\prime \prime}$ et $B^{\prime \prime}$ sita erunt (art. 64, III): praeterea puncta $C, C^{\prime}, C^{\prime \prime}$ in eodem circulo maximo iacebunt, puta in eo, quem planum orbitae in sphaera coelesti proiicit. Designabimus per $r, r^{\prime}, r^{\prime \prime}$ tres corporis coelestis distantias a Sole; per $\rho, \rho^{\prime}, \varrho^{\prime \prime}$ eiusdem distantias a terra; per $R, R^{\prime}, R^{\prime \prime}$ terrae distantias a Sole. Porro statuimus arcus $C^{\prime} C^{\prime \prime}, C C^{\prime \prime}, C C^{\prime}$ resp. $=2 f, 2 f^{\prime \prime}, 2 f^{\prime \prime}$, atque $r^{\prime} r^{\prime \prime} \sin 2 f=n, r r^{\prime \prime} \sin 2 f^{\prime}$ $=n^{\prime}, r r^{\prime} \sin 2 f^{\prime \prime}=n^{\prime \prime}$. Habemus itaque $f^{\prime}=f+f^{\prime \prime}, A C+C B=\delta, A^{\prime} C^{\prime}+C^{\prime} B^{\prime}=\delta^{\prime}$, $A^{\prime \prime} C^{\prime \prime}+C^{\prime \prime} B^{\prime \prime}=\delta^{\prime \prime}$, nec non

$$
\begin{aligned}
& \frac{\sin \delta}{r}=\frac{\sin A C}{\rho}=\frac{\sin C B}{R} \\
& \frac{\sin \delta^{\prime}}{r^{\prime}}=\frac{\sin A^{\prime} C^{\prime}}{\rho^{\prime}}=\frac{\sin C^{\prime} B^{\prime}}{R^{\prime}} \\
& \frac{\sin \delta^{\prime \prime}}{r^{\prime \prime}}=\frac{\sin A^{\prime \prime} C^{\prime \prime}}{\rho^{\prime \prime}}=\frac{\sin C^{\prime \prime} B^{n}}{R^{\prime \prime}}
\end{aligned}
$$

*) Sine etiam quoties sibi opposita sunt, sed de hoc casu non loquimur, quum methodus nostra ad obseruationes tantum interuallum complectentes non sit extendenda. 
DETERMINATIO ORBITAE E TRIBVS OBSERVATIONIBVS COMPLETIS. $\quad 55$

Hinc patet, simulac situs punctorum $C, C^{\prime}, C^{\prime \prime}$ innotuerit, quantitates $r, r^{\prime}, r^{m}$ $\varrho, \rho^{\prime}, \rho^{\prime \prime}$ determinabiles fore. Iam ostendemus, quomodo ille e quantitatibus $\frac{n^{n}}{n}$ $=P, 2\left(\frac{n+n^{\prime \prime}}{n^{\prime}}-1\right) r^{\prime 3}=Q$ elici possit, a quibus methodum nostram proficisci iam supra declaranimus.

140.

Primo obseruamus, si $N$ fuerit punctum quodcunque circuli maximi $C C^{\prime} C^{m}$, distantiaeque punctorum $C, C^{\prime}, C^{\prime \prime}$ a puncto $N$ secundum directionem eandem numerentur, quae tendit a $C$ ad $C^{\prime \prime}$, ita vt generaliter fiat

$N C^{\prime \prime}-N C^{\prime}=2 f, N C^{\prime \prime}-N C=2 f^{\prime}, N C^{\prime}-N C=2 f^{\prime \prime}$, haberi aequationem $0=\sin 2 f \sin N C-\sin 2 f^{\prime} \sin N C^{\prime}+\sin 2 f^{\prime \prime} \sin N C^{n}$.

Iam stpponemus, $N$ accipi in intersectione circulorum maximorum $B B^{\star} B^{\prime \prime}, C C^{\prime} C^{\prime \prime}$, quasi in nodo ascendente prioris supra posteriorem. Designemus per $\mathfrak{E}, \mathfrak{S}^{\prime}, \mathfrak{C}^{\prime \prime}$, $\mathfrak{D}, \mathfrak{D}^{\prime}, \mathfrak{D}^{\prime \prime}$ resp. distantias punctorum $C, C^{\prime}, C^{\prime \prime}, D, D^{\prime}, D^{\prime \prime}$ a circulo maximo $B B^{\star} B^{\prime \prime}$, ab alterutrá ipsius parte positiue, ab altera opposita negatiue acceptas. Hinc manifesto $\sin \mathfrak{C}, \sin \mathfrak{C}^{\prime}, \sin \mathfrak{C}^{\prime \prime}$ resp. proportionales erunt ipsis $\sin N C, \sin N C^{\prime}$, $\sin N C^{\prime \prime}$, vnde aequatio (I) sequentem induit formam

$0=\sin 2 f \sin \mathbb{C}-\sin 2 f^{\prime} \sin \mathbb{C}^{\prime}+\sin 2 f^{\prime \prime} \sin \mathbb{C}^{\prime \prime}$

siue multiplicando per $r r^{\prime \prime} r^{\prime \prime}$

$0=n r \sin \Subset-n^{\prime} r^{\prime} \sin \bigodot^{\prime}+n^{\prime \prime} r^{\prime \prime} \sin \bigodot^{\prime \prime}$.

Porro patet, esse $\sin \subseteq$ ad $\sin D^{\prime}$, vt sinum distantiae puncti $C$ a $B$ ad distantiam puncti $D^{\prime}$ a $B$, vtraque distantia secundum eandem directionem mensurata. 'Habetur itaque

$$
-\sin C=\frac{\sin D^{\prime} \sin C B}{\sin \left(A D^{\prime}-\delta\right)}
$$

prorsusque simili modo eruitur

$$
\begin{aligned}
& -\sin C=\frac{\sin D^{\prime \prime} \sin C B}{\sin \left(A D^{\prime \prime}-\delta\right)} \\
& -\sin C^{\prime}=\frac{\sin D \sin C^{\prime} B^{\star}}{\left(\sin A^{\prime} D-\delta^{\prime}+\sigma\right)}=\frac{\sin D^{\prime \prime} \sin C^{\prime} B^{\star}}{\sin \left(A^{\prime} D^{\prime \prime}-\delta^{\prime}+\sigma\right)} \\
& -\sin C^{\prime \prime}=\frac{\sin D^{\prime} \sin C^{\prime \prime} B^{\prime \prime}}{\sin \left(A^{\prime \prime} D-\delta^{\prime \prime}\right)}=\frac{\sin D^{\prime} \sin C^{\prime \prime} B^{\prime \prime}}{\sin \left(A^{\prime \prime} D^{\prime}-\delta^{\prime \prime}\right)}
\end{aligned}
$$


Dinidendo itaque aequationem (II) per $r^{\prime \prime} \sin \mathbb{C}^{*}$, prodit $0=n \cdot \frac{r \sin C B}{r^{\prime \prime} \sin C^{\prime \prime} B^{\prime \prime}}+\frac{\sin \left(A^{\prime \prime} D^{\prime}-\delta^{\prime \prime}\right)}{\sin \left(A D^{\prime}-\delta\right)}-n^{\prime} \cdot \frac{r^{\prime} \sin C^{\prime} B^{\star}}{r^{\prime \prime} \sin C^{\prime \prime} B^{\prime \prime}} \cdot \frac{\sin \left(A^{\prime \prime} D-\delta^{\prime \prime}\right)}{\sin \left(A^{\prime} D-\delta^{\prime}+\sigma\right)}+n^{\prime \prime}$ Quodsi hic arcum $C^{\prime} B^{\prime}$ per $z$ designamus, pro $r, r^{\prime}, r^{\prime \prime}$ valores suos ex art. praec. substituimus, breuitatisque caussa ponimus

[11] $\frac{R \sin \delta \sin \left(A^{\prime \prime} D^{\prime}-\delta^{\prime \prime}\right)}{R^{\prime \prime} \sin \delta^{\prime \prime} \sin \left(A D^{\prime}-\delta\right)}=a$

[12] $\frac{R^{\prime} \sin \delta^{\prime} \sin \left(A^{\prime \prime} D-\delta^{\prime \prime}\right)}{R^{\prime \prime} \sin \delta^{\prime \prime} \sin \left(A^{\prime} D-\delta^{\prime}+\sigma\right)}=6$

aequatio nostra ita se habebit

$0=a n-b n^{\prime} \cdot \frac{\sin (z-\sigma)}{\sin z}+n^{\prime \prime}$

Coëfficientem $b$ etiam per formulam sequentem computare licet, quae ex aequationibus modo allatis facile deducitur:

[13] $a \times \frac{R^{\prime} \sin \delta^{\prime} \sin \left(A D^{\prime \prime}-\delta\right)}{R \sin \delta \sin \left(A^{\prime} D^{\prime \prime}-\delta^{\prime}+\sigma\right)}=b$

Calculi confirmandi caussa haud inutile erit, vtraque formula 12 et 15 vti. Quoties $\sin \left(A^{\prime} D^{\prime \prime}-\delta^{\prime}+\sigma\right)$ maior est quam $\sin \left(A^{\prime} D-\delta^{\prime}+\sigma\right)$, formula posterior a tabularum erroribus ineuitabilibus minus afficietur, quam prior, adeoque huic praeferenda erit, si forte paruula discrepantia illine explicanda in valoribus ipsius $b$ se prodiderit; contra formulae priori magis fidendum erit, quoties $\sin \left(A^{\prime} D^{\prime \prime}-\delta^{\prime}+\sigma\right)$ minor est quam $\sin \left(A^{\prime} D-\delta^{\prime}+\sigma\right)$ : si magis placet, medium idoneum inter ambos valores adoptabitur.

Calculo examinando sequentes quoque formulae inseruire possunt, quarum tamen deriuationem non ita difficilem breuitatis caussa supprimimus:

$$
\begin{aligned}
& 0=\frac{a \sin \left(l^{\prime \prime}-l^{\prime}\right)}{R}-\frac{b \sin \left(l^{\prime \prime}-l\right)}{R^{\prime}} \cdot \frac{\sin \left(\delta^{\prime}-\sigma\right)}{\sin \delta^{\prime}}+\frac{\sin \left(l^{\prime}-l\right)}{R^{\prime \prime}} \\
& b=\frac{R^{\prime} \sin \delta^{\prime}}{R^{\prime \prime} \sin \delta^{\prime \prime}} \cdot \frac{U \cos \beta \cos \beta^{\prime \prime}}{\sin \left(A D^{\prime}-\delta\right) \sin \varepsilon^{\prime}}
\end{aligned}
$$

vbi $U$ exprimit quotientem $\frac{S}{\sin \left(\delta^{\prime}-\sigma\right)}=\frac{T \sin \left(t+y^{\prime}\right)}{\cos \left(\delta^{\prime}-\sigma\right)}($ art. 158. aequ. 10).

141.

Ex $P=\frac{n^{\prime \prime}}{n^{\prime}}$, atque aequatione III. art. praec. sequitur $\left(n+n^{\prime \prime}\right) \frac{P+a}{P+1}$

$=b n^{\prime} \frac{\sin (z-\sigma)}{\sin z} ;$ hinc vero et ex $Q=2\left(\frac{n+n^{n}}{n}-1\right) r^{\prime 3}$ atque 
$r^{\prime}=\frac{R^{\prime} \sin \delta^{\prime}}{\sin z}$ elicitur

$\sin z+\frac{Q \sin z^{4}}{2 R^{\prime 3} \sin \delta^{3}}=b \frac{P+1}{P+a} \sin (z-\sigma)$, sine

$\frac{Q \sin z^{4}}{2 R^{\prime 3} \sin \delta^{\prime 3}}=\left(b \frac{P+1}{P+a}-\cos \sigma\right) \sin (z-\sigma)-\sin \sigma \cos (z-\sigma)$

Statuendo itaque breuitatis caussa

[14] $\frac{1}{2 R^{\prime 3} \sin \delta^{\prime 3} \sin \sigma}=c$

introducendoque angulum auxiliarem $\omega$ talem vt fiat

$\tan \omega=\frac{\sin \sigma}{b \frac{P+1}{P+a}-\cos \sigma}$

prodit aequatio (IV)

$c Q \sin \omega \sin z^{4}=\sin (z-\omega-\sigma)$

ex qua incognitam $z$ eruere oportebit. Vt angulus $\omega$ commodius compuletur, formulam praecedentem pro tang $\omega$ ita exhibere conueniet

$$
\operatorname{tang} \omega=\frac{(P+a) \operatorname{tang} \sigma}{P\left(\frac{b}{\cos \sigma}-1\right)+\left(\frac{b}{\cos \sigma}-a\right)}
$$

Quamobrem statuendo

$$
\begin{aligned}
& {[15] \frac{\frac{b}{\cos \sigma}-a}{\frac{b}{\cos \sigma}-1}=a} \\
& {[16] \frac{\operatorname{tang} \sigma}{\frac{b}{\cos \sigma}-1}=e}
\end{aligned}
$$

habebimus ad determinandum $\omega$ formulam simplicissimam $\operatorname{tang} \omega=\frac{e(P+a)}{P+d}$

Computum quantitatum $a, b, c, d, e$ per formulas $x 1==16$, a solis quantitatibus datis pendentem, tamquam negotium quartum consideramus. Quantitates $b, c$, e ipsae non, erunt necessariae, verum soli ipsarum logarithmi.

Ceterum datur casus specialis, vbi haec praecepta aliqua mutatione indigent. Quoties scilicet circulus maximus $B B^{\prime \prime}$ cum $A^{\prime \prime} B^{\prime \prime}$ coincidit, adeoque puncta $B, B^{\text {* }}$ 
resp. cum $D^{\prime}, D$, quantitates $a, b$ valores infinitos nanciscerentur. Statuendo in hoc casu

$$
\frac{R \sin \delta \sin \left(A^{\prime} D^{\prime \prime}-\delta^{\prime}+\sigma\right)}{R^{\prime} \sin \delta^{\prime} \sin \left(A D^{\prime \prime}-\delta\right)}=\pi
$$

habebimus loco aequationis III hancee: $o=\pi n-\frac{n^{\prime} \sin (z-\sigma)}{\sin z}$, rnde faciendo $\operatorname{tang} \omega=\frac{\pi \sin \sigma}{P+(1-\pi \cos \sigma)}$, eadem aequatio IV elicitur.

Perinde in casu speciali, vbi $\sigma=0$, fit $c$ infinita atque $\omega=0$, vnde factor $c \sin \omega$ in aequatione IV indeterminatus esse videtur: nihilominus reuera determinatus est, ipsiusque valor $=\frac{P+a}{2 R^{\prime 3} \sin \delta^{\prime 3}(b-1)(P+d)}$, vti leuis attentio docebit. In hoc itaque casu fit $\sin z=R^{\prime} \sin \delta^{\prime} V^{\prime} \frac{2(b-1)(P+d)}{Q(P+a)}$.

\section{2.}

Aequatio IV, quae euoluta ad ordinem octaum ascenderet, in forma sta non mutata expeditissime tentando soluitur. Ceterum e theoria aequationum facile ostendi potest, (quod tamen fusius enoluere breuitatis caussa hic supersedemus), hanc aequationem vel duas vel quatuor solutiones per valores reales admittere. In casu priori valor alter ipsius sinz positiuus erit, alterum negatiuum reiicere oportebit, quia per problematis naturam $r^{\prime}$ negatiuus euadere nequit. In casu posteriori inter valores ipsius $\sin z$ vel vnus positiuus erit, tresque reliqui negatiui - vbi igitm haud ambiguum erit, quemnam adoptare oporteat - vel tres positiui cum vno-negatiuo; in hoc casu e valoribus positinis ii quoque si qui adsunt reiici debent, vbi $z$ maior euadit quam $\delta^{\prime}$, quoniam per aliam problematis conditionem essentialem $\varrho^{\prime}$ adeoque etiam $\sin \left(\delta^{\prime}-z\right)$ quantitas positiua esse debet.

Quoties obseruationes mediocribus temporum interuallis ab inuicem distant, plerumque casus postremus locum habebit, vt tres valores positiui ipsius sin z aequationi satisfaciant. Inter has solutiones praeter veram reperiri solet aliqua, vbi $z$ parum differt a $\delta$, modo excessu, modo defectu: hoc phaenomenon sequenti modo explicandum est. Problematis nostri tractatio analytica ei soli conditioni superstructa est, quod tres corporis coelestis in spatio loci iacere debent in rectis, quarum situs per locum absolutum terrae positionemque obseruatam determinatur. Iam per ipsius rei naturam loci illi iacere quidem debent in iis rectarum partibus, 
DETERMINATIO ORBITAE E TRIBVS OBSERVATIONIBVS COMPLETIS. $\quad 159$

inde lumen ad terram descendit: sed aequationes analyticae hanc restrictionem non agnoscunt, omniaque locorum systemata, qui quidem cum Kepleri legibus consentiunt, perinde complecti debent, siue ab hac terrae parte in illis rectis iaceant, siue ab illa, sine denique cum ipsa terra coincidant. Iam hic vltimus casus vtique problemati nostro satisfaciet, quum terra ipsa ad normam illarum legum moueatur. Hinc patet, aequationes comprehendere debere solutionem, in qua puncta, $C, C^{\prime}$, $C^{\prime \prime}$ cum punctis $A, A^{\prime}, A^{\prime \prime \prime}$ coincidant (quatenus variationes minutissimas locis terrae ellipticis a perturbationibus et parallaxi inductas negligimus): aequatio itaque IV semper admittere deberet solutionem $z=\delta$, si pro $P$ et $Q$ valores veri locis terrae respondentes acciperentur. Quatenus autem illis quantitatibus valores tribuuntur ab his non multum discrepantes (quod semper supponere licet, quoties temporum interualla modica sunt), inter solutiones aequationis IV necessario aliqua reperiri debet, quae proxime ad valorem $z=\delta^{\prime}$ accedit.

Plerumque quidem in eo casu, vbi aequatio IV tres solutiones per valores positiuos ipsius sin $z$ admittit, tertia ex his (praeter reram eamque de qua modo diximus) valorem ipsius $z$ maiorem quam $\delta^{\prime}$ sistet, adeoque analytice tantum possibilis, physice vero impossibilis erit: tunc itaque quamnam adoptare oporteat ambiguum esse nequit. Attamen contingere vtique potest, vt aequatio illa duas solutiones idoneas diuersas admittat, adeoque problemati nostro per duas orbitas prorsus diuersas satisfacere liceat. Ceterum in tali casu orbita vera a falsa facile dignoscetur, quamprimum obseruationes alias magis remotas ad examen reuocare licuerit.

143.

Simulac angulus $z$ erutus est, statim habetur $r^{\prime}$ per aequationem $r^{\prime}=\frac{R^{\prime} \sin \delta^{\prime}}{\sin z}$. Porro ex aequationibus $P=\frac{n^{\prime \prime}}{n}$ atque IV elicimus $\frac{n^{\prime} r^{\prime}}{n}=\frac{(P+a) R^{\prime} \sin \delta^{\prime}}{b \sin (z-\sigma)}$ $\frac{n^{\prime} r^{\prime}}{n^{\prime \prime}}=\frac{1}{P} \cdot \frac{n^{\prime} r^{\prime}}{n}$

Iam vt formulas, secundum quas situs punctorum $C, C^{n}$ e situ puncti $C^{\prime}$ determinandus est, tali modo tractemus, vt ipsarum veritas generalis pro iis quoque casibus, quos figura 4 non monstrat, statim eluceat, obseruamus, sinum distantiae puncti $C^{\prime}$ a circulo maximo $C B$ (positize sumtae in regione superiori, ne- 
gatiue in inferiori) aequalem ficri producto ex $\sin \varepsilon^{\prime \prime}$ in sinum distantiae puncti $C^{\prime}$ a $D^{u}$ secundum directionem progressiuam mensuratae, adeoque $=-\sin \varepsilon^{\prime \prime} \sin C^{\prime} D^{\prime \prime}=$ $-\sin \varepsilon^{\prime \prime} \sin \left(z+A^{\prime} D^{\prime \prime}-\delta^{\prime}\right)$; perinde fit sinus distantiae puncti $C^{\prime \prime}$ ab eodem circulo maximo $=-\sin \varepsilon^{\prime} \sin C^{\prime \prime} D^{\prime}$. Manifesto autem ridem sinus sunt vt $\sin C C^{\prime}$ ad $\sin C C^{\text {b }}$, siue vt $\frac{n^{\prime \prime}}{r r^{\prime}}$ ad $\frac{n^{\prime}}{r r^{\prime \prime}}$, sine vt $n^{\prime \prime} r^{\prime \prime}$ ad $n^{\prime} r^{\prime}$. Statuendo itaque $C^{\prime \prime} D^{\prime}=b^{\prime \prime}$, habemus V. $r^{\prime \prime} \sin \zeta^{\prime \prime}=\frac{n^{\prime} r^{\prime}}{n^{\prime \prime}} \cdot \frac{\sin \varepsilon^{\prime \prime}}{\sin \varepsilon^{\prime}} \sin \left(z+A^{\prime} D^{\prime \prime}-\delta^{\prime}\right)$

Prorsus simili modo statuendo $C D^{\prime}=\zeta$ eruitur

VI. $r \sin \zeta=\frac{n^{\prime} r^{\prime}}{n} \cdot \frac{\sin \varepsilon}{\sin \varepsilon} \sin \left(z+A^{\prime} D-\delta^{\prime}\right)$

VII. $r \sin \left(\zeta+A D^{\prime \prime}-A D^{\prime}\right)=r^{\prime \prime} P \cdot \frac{\sin \varepsilon}{\sin \varepsilon^{\prime \prime}} \sin \left(\zeta^{\prime \prime}+A^{\prime \prime} D-A^{\prime \prime} D^{\prime}\right)$

Combinando aequationes $\mathrm{V}$ et VI cum sequentibus ex art. 139 transscriptis VIII. $r^{\prime \prime} \sin \left(\zeta^{\prime \prime}-A^{\prime \prime} D^{\prime}+\delta^{\prime \prime}\right)=R^{\prime \prime} \sin \delta^{\prime \prime}$

IX. $r \sin \left(\zeta-A D^{\prime}+\delta\right)=R \sin \delta$ quantitates $\zeta, \zeta^{\prime \prime}, r, r^{\prime \prime}$ ad normam art. 78 inde deriuabuntur. Qui calculus quo commodius absoluatur, formulas ipsas huc attulisse haud ingratum erit. Statuatur [17] $\frac{R \sin \delta}{\sin \left(A D^{\prime}-\delta\right)}=x$

[17] $\frac{R^{\prime \prime} \sin \delta^{\prime \prime}}{\sin \left(A^{\prime \prime} D^{\prime}-\delta^{\prime \prime}\right)}=x^{\prime \prime}$.

[19] $\frac{\cos \left(A D^{\prime}-\delta\right)}{R \sin \delta}=\lambda$

$[20] \frac{\cos \left(A^{\prime \prime} D^{\prime}-\delta^{\prime \prime}\right)}{R^{\prime \prime} \sin \delta^{\prime \prime}}=\lambda^{\prime \prime}$

Computus harum quantitatum, aut potius logarithmorum earum, a $P$ et $Q$ etiamnum independens, tamquam negotium quintum et vltimum in operationibus quasi praeliminaribus spectandum est, commodeque statim cum computo ipsarum $a, b$ siue cuin negotio quarto absoluitur, vbi fit $a=\frac{x}{x^{\prime \prime}}$. Faciendo dein $\frac{n^{\prime} r^{\prime}}{n} \cdot \frac{\sin \varepsilon}{\sin \varepsilon^{\prime}} \cdot \sin \left(z+A^{\prime} D-\delta^{\prime}\right)=p$ $\frac{n^{\prime} r^{\prime}}{n^{\prime \prime}} \cdot \frac{\sin \varepsilon^{\prime \prime}}{\sin \varepsilon^{\prime}} \cdot \sin \left(z+A^{\prime} D^{\prime \prime}-\delta^{\prime}\right)=p^{n}$ 
$x(\lambda p-1)=q$

$x^{\prime \prime}\left(\lambda^{\prime \prime} p^{\prime \prime}-1\right)=q^{\prime \prime}$

eliciemus $\zeta$ et $r$ ex $r \sin \zeta=p, r \cos \zeta=q$, atque $\zeta^{\prime \prime}$ et $r^{\prime \prime}$ ex $r^{\prime \prime} \sin \zeta^{\prime \prime}=p^{\prime \prime}, r^{\prime \prime} \cos \zeta^{\prime \prime}=q^{\prime \prime}$. Ambiguitas in determinandis $\zeta$ et $\zeta^{\prime \prime}$ hic adesse nequit, quia $r$ et $r^{\prime \prime}$ necessario euadere debent quantitates positiuae. Calculus perfectus per aequationem VII si lubet confirmari poterit.

Sunt tamen duo casus, vbi aliam methodum sequi oportet. Quoties scilicet punctum $D^{\prime}$ cum $B$ vel coincidit vel ipsi in sphaera oppositum est, siue quoties $A D^{\prime}-\delta$ $=0 \mathrm{vel}=180^{\circ}$, aequationes VI et IX necessario identicae esse debent, fieretque $x=\infty, \lambda p-1=0$, adeoque $q$ indeterminata. In hoc casu $\zeta^{\prime \prime}$ et $r^{\prime \prime}$ quidem eo quo docuimus modo determinabuntur, dein vero $\zeta$ et $r$ e combinatione aequationis VII cum V vel IX elicere oportebit. Formulas ipsas ex art. 78 desumendas huc transscribere supersedemus; obseruamus tantummodo, quod in eo quoquo casu, vbi est $A D^{\prime}-\delta$ non quidem $=0$ neque $=180^{\circ}$, attamen arcus valde paruus, eandem methodum sequi praestat, quoniam tunc methodus prior praecisionem necessariam non admitteret. Fit quidem adoptabitur combinatio aequationis VII cum V vel cum IX, prout $\sin \left(A D^{\prime \prime}-A D^{\prime}\right)$ maior vel minor est quam $\sin \left(A D^{\prime}-\delta\right)$.

Perinde in casu, vbi punctum $D^{\prime}$, vel ipsi oppositum, cum $B^{\prime \prime}$ vel coincidit. vel parum ab eodem distat, determinatio ipsarum $\zeta^{\prime \prime}, r^{\prime \prime}$ per methodum praecedentem vel impossibilis vel parum tata foret. Tunc itaque $\zeta$ et $r$ quidem per illam methodum determinabuntur, dein vero $\zeta^{\prime \prime}$ et $r^{n}$ e combinatione aequationis VII vel cum VI vel cum IX, prout $\sin \left(A^{\prime \prime} D-A^{\prime \prime} D^{\prime}\right)$ maior vel minor est quam $\sin \left(A^{\prime \prime} D^{\prime}-\delta^{\prime \prime}\right)$ Ceterum haud metuendum est, ne simul $D^{\prime}$ cum punctis $B, B^{\prime \prime}$ vel cum punctis oppositis coincidat, vel parum ab ipsis distet: casum enim eum, vbi $B$ cum $B^{\prime \prime}$ coincidit, vel perparum ab ea distat, iam supra art. 138 a disquisitione nostra exclusimus.

144.

Arcubus $\zeta, \zeta^{\text {qa }}$ inuentis, punctorum $C, C^{\prime \prime}$ positio data erit, poteritque distantia $C C^{\prime \prime}=2 f^{\prime}$ ex $\zeta, \zeta^{\prime \prime}$ et $\varepsilon^{\prime}$ determinari. Sint $u, u^{n}$ inclinationes circuloram maximorum $A B, A^{\prime \prime} B^{\prime \prime}$ ad circulum maximum $C C^{\prime \prime}$ (quae in figura 4 resp. erunt anguli $C^{\prime \prime} C D^{\prime}$ et $180^{\circ}-C C^{\prime \prime} D^{\prime}$ ), habebimusque aequationes sequentes, aequationibus $3-6$ art. 137 prorsus, analogas: 


$$
\begin{aligned}
& \sin f^{\prime \prime} \sin \frac{x}{2}\left(u^{u}+u\right)=\sin \frac{x}{2} \varepsilon \sin \frac{x}{2}\left(\zeta+\zeta^{\prime \prime}\right) \\
& \sin f^{\prime} \cos \frac{x}{2}\left(u^{\prime \prime}+u\right)=\cos \frac{x}{2} \varepsilon \sin \frac{x}{2}\left(\zeta-\zeta^{\prime \prime}\right) \\
& \cos f^{\prime} \sin \frac{1}{2}\left(u^{\prime \prime}-u\right)=\sin \frac{x}{2} \varepsilon \cos \frac{\pi}{2}\left(\zeta+\zeta^{\prime \prime}\right) \\
& \cos f^{\prime} \cos \frac{x}{2}\left(u^{\prime \prime}-u\right)=\cos \frac{x}{2} \varepsilon \cos \frac{x}{2}\left(\zeta-\zeta^{\prime \prime}\right)
\end{aligned}
$$

Duae priores dabunt $\frac{x}{2}\left(u^{\prime \prime}+u\right)$ et $\sin f^{\prime}$, duae posteriores $\frac{x}{2}\left(u^{u}-u\right)$ et $\cos f^{\prime}$; ex $\sin f^{\prime}$ et $\cos f^{\prime}$ habebitur $f^{\prime}$. Angulos $\frac{x}{2}\left(u^{\prime \prime}+u\right)$ et $\frac{x}{2}\left(u^{\prime \prime}-u\right)$, qui in ultima demum hypothesi ad determinandum situm plani orbitae adhibebuntur, in hypothesibus primis negligere licebit.

Prorsus simili modo $f$ ex $\varepsilon, C^{\prime} D$ et $C^{\prime} D$, nec non $f^{\prime \prime}$ ex $\varepsilon^{\prime \prime}, C D^{\prime \prime} C^{\prime} D^{\prime \prime}$ deriuari possent: sed multo commodius ad hune finem formulae sequentes adhibentur.

$$
\begin{aligned}
& \sin 2 f=r \sin 2 f^{\prime} \cdot \frac{n}{n^{\prime} r^{\prime}} \\
& \sin 2 f^{\prime \prime}=r^{\prime \prime} \sin 2 f^{\prime} \cdot \frac{n^{\prime \prime}}{n^{\prime} r^{\prime}}
\end{aligned}
$$

vbi Iogarithmí quantitatum $\frac{n}{n^{\prime} r^{\prime}}, \frac{n^{\prime \prime}}{n^{\prime} r^{\prime}}$ iam e calculis praecedentibus adsunt. Totus denique calculus confirmationem nouam inde nanciscetur, quod fieri debet $2 f+2 f^{*}$ $=2 f^{\prime}$ : si qua forte differentia prodeat, nullius certe momenti esse poterit, siquidem omnes operationes quam accuratissime peractae fuerint. Interdum tamen, calculo vbique septem figuris decimalibus subducto, ad aliquot minuti secundi partes decimas assurgere poterit, quam si operae pretium videtur facillimo negotio inter $2 f$ $2 f^{\prime \prime}$ ita dispertiemur, vt logarithmi sinuum aequaliter vel augeantur vel diminuantur, quo pacto aequationi $p=\frac{r \sin 2 f^{\prime \prime}}{r^{\prime \prime} \sin 2 f}=\frac{n^{\prime \prime}}{n}$ omni quam tabulae permittunt praecisione satisfactum erit. Quoties $f$ et $f^{\prime}$ parum differunt, differentiam illam inter $2 f$ et $2 f^{\prime \prime}$ aequaliter distribuisse sufficiet.

145.

Postquam hoc modo corporis coelestis positiones in orbita determinatae sunt, duplex elementorum calculus tum e combinatione loci secundi cum tertio, tum e combinatione primi cum secundo, vna cum temporum interuallis respondentibus, inchoabitur. Antequam vero haec operatio suscipiatur, ipsa temporum interualla quadam correctione opus habent, siquidem constitutum fuerit, secundum methodum tertiam art. 118, aberrationis rationem habere. In hocce scilicet casu pro tempori- 
bus reris ficta substituenda sunt, illis resp. $495 \rho, 495 \rho^{\prime}, 495 \varrho^{\prime \prime}$ minutis secundis anteriora. Pro computandis distantiis $\rho, \rho^{\prime}, \rho^{\prime \prime}$ habemus formulas

$$
\begin{aligned}
& \rho=\frac{R \sin \left(A D^{\prime}-\zeta\right)}{\sin \left(\zeta-A D^{\prime}+\delta\right)}=\frac{r \sin \left(A D^{\prime}-\zeta\right)}{\sin \delta} \\
& \rho^{\prime}=\frac{R^{\prime} \sin \left(\delta^{\prime}-z\right)}{\sin z}=\frac{r^{\prime} \sin \left(\delta^{\prime}-z\right)}{\sin \delta^{\prime}} \\
& \rho^{\prime \prime}=\frac{R^{\prime \prime} \sin \left(A^{\prime \prime} D^{\prime}-\zeta^{\prime \prime}\right)}{\sin \left(\zeta^{\prime \prime}-A^{\prime \prime} D^{\prime}-+\delta^{\prime \prime}\right)}=\frac{r^{\prime \prime} \sin \left(A^{\prime \prime} D^{\prime}-\zeta^{\prime}\right)}{\sin \delta^{\prime \prime}}
\end{aligned}
$$

Ceterum si obseruationes ab initio statim per methodum primam vel secundam art. $118 \mathrm{ab}$ aberratione purgatae fuissent, hicce calculus omittendus, neque adeo necessarium foret, valores distantiarum $\rho, \rho^{\prime}, \rho^{\prime \prime}$ eruere, nisi forte ad confirmandum, an ii, quibus calculus aberrationum superstructus erat, satis exacti fuerint. Denique sponte patet, totum istum calculum tunc quoque supprimendum esse, quando aberrationem omnino negligere placuerit.

146.

Calculus elementorum, hinc ex $r^{\prime}, r^{\prime \prime}, 2 f$ atque temporis interiallo correcto inter obseruationem secundam et tertiam, cuius productum in quantitatem $k$ (art. 1 ) per $\theta$ denotamus, illine ex $r, r^{\prime}, 2 f^{\prime \prime}$ atque temporis interuallo inter obseruationem primam et secundam, cuius productum per $k$ esto $=\theta^{\prime \prime}$, secundum methodum in artt. 88- 105 expositam tantummodo vsque ad quantitatem illic per $y$ denotatam. producendus est, cuius valorem in combinatione priori per $\eta$, in posteriori per $\eta^{\prime \prime}$ denotabimus. Fiat deinde

$$
\frac{\theta^{\prime \prime} \eta}{\theta \eta^{\prime \prime}}=P^{\prime}, \frac{r^{\prime} r^{\prime}}{r r^{\prime \prime} \eta \eta^{\prime \prime} \cos f} \frac{\theta \theta^{\prime \prime}}{\cos f^{\prime} \cos f^{\prime \prime}}=Q^{\prime}
$$

patetque, si valores quantitatum $P, Q$, quibus totus hucusque calculus superstructus erat, ipsi veri fuerint, euadere debere $P^{\prime}=P, Q^{\prime}=Q$. Vice versa facile perspicitur, si prodeat $P^{\prime}=P, Q^{\prime}=Q$, duplicem elementorum calculum, si vtrimque ad finem perducatur, numeros prorsus aequales suppeditaturum esse, per quos itaque omnes tres obseruationes exacte repraesentabuntur, adeoque problemati ex asse satisfiet. Quoties autem non fit $P^{\prime}=P, Q^{\prime}=Q$, accipientur $P^{\prime}-P, Q^{\prime}-Q$ pro $X$ et $Y$, siquidem $P$ et $Q$ pro $x$ et $y$ acceptae fuerint: adhuc magis commodum erit statucre $\log P=x, \log Q=y, \log P^{\prime}-\log P=X, \log Q^{\prime}-\log Q=Y$. Dein calculus cum aliis valoribus ipsarum $x, y$ repetendus erit. 
147.

Proprie quidem etiam hic, sicuti in decem methodis supra traditis, arbitrarium esset, quosnam valores nouos pro $x$ et $y$ in hypothesi secunda supponamus, si modo conditionibus generalibus supra explicatis non aduersentur: attamen quum. manifesto pro lucro magna habendum sit, si statim a valoribus magis exactis proficisci liceat, in methodo hacce parum prudenter ageres, si valores secundos temere quasi adoptares, quum ex ipsa rei natura facile perspiciatur, si valores primi ipsarum $P, Q$ leuibus erroribus affecti fuerint, ipsas $P^{\prime}, Q^{\prime}$ valores multo exactiores exhibituras esse, siquidem motus heliocentricus, fuerit modicus. Quamobrem semper ipsas $P^{\prime}, Q^{\prime}$ pro valoribus secundis ipsarum $P, Q$ adoptabimus, siue $\log P^{\prime}$, $\log Q^{\prime}$ pro valoribus secundis ipsarum $x, y$ si $\log P, \log Q$ primos designare suppositi sint.

Iam in hac hypothesi secunda, vbi omnes operationes praeliminares per formulas $1-20$ exhibitae inuariatae retinendae sunt, calculus prorsus simili modo repetetur. Primo scilicet determinabitur angulus $\omega$; dein $z, r^{\prime}, \frac{n^{\prime} r^{\prime}}{n}, \frac{n^{\prime} r^{\prime}}{n^{\prime \prime}}, \zeta$, $r, \zeta^{\prime \prime}, r^{\prime \prime}, f^{\prime}, f, f^{\prime \prime}$. E differentia plus minusue considerabili inter valores nouos harum quantitatum atque primos facile aestimabitur, vtrum operae pretium sit, necne, correctionem quoque temporum propter aberrationent denuo computare: in casu posteriori temporum interualla, adeoque etiam quantitates $\theta$ et $\theta^{\prime \prime}$ eaedem manebunt vt ante. Denique ex $f, r^{\prime}, r^{\prime \prime} ; f^{\prime \prime}, r, r^{\prime}$ temporumque interuallis eruentur $\eta, \eta^{\prime \prime}$ atque hinc valores noui ipsarum $P^{\prime}, Q^{\prime}$, qui plerumque ab iis, quos hypothesis prima suppeditauerat, multo minus different, quam hi ipsi a valoribus primis ipsarum $P, Q$. Valores secundi ipsarum $X, Y$ itaque multo minores erunt, quam primi, valoresque secundi ipsarnm $P^{\prime}, Q^{\prime}$ tamquam valores tertii ipsarum $P$, $Q$ adoptabuntur, et cum his calculus denuo repetetur. Hoc igitur modo sicuti ex hypothesi secunda numeri exactiores resultauerant, quam ex prima, ita e tertia iterum exactiores resultabunt, quam e secunda, possentque valores tertii ipsarum $P^{\prime}, Q^{\prime}$ tamquam quarti ipsarum $P, Q$ adoptari, atque sic calculus toties repeti, vsque dum ad hypothesin perueniatur, in qua $X$ et $Y$ pro enanescentibus habere liceret: sed quoties hypothesis tertia nondum sufficiens videatur, valores ipsarum $P$, $Q$ in hypothesi quarta adoptandos secundum methodum in artt. 120121 explicatam e tribus primis deducere praestabit, quo pacto approximatio celerior obtinebitur, raroque opus erit, ad hypothesin quintam progredi. 
148.

Quoties elementa e tribus obseruationibus deriuanda adhuc penitus incognita sunt (cui casui methodus nostra imprimis accommodata est), in hypothesi prima vt iam monuimus pro $P$ et $Q$ valores approximati $\frac{\theta^{\prime \prime}}{\theta_{i}}$ et $\theta \theta^{\prime \prime}$ accipientur, vbi $\theta$ et $\theta^{\prime \prime}$ aliquantisper ex interuallis temporum non correctis deriuandae sunt. Quorum ratione ad interualla correcta per $\mu: 1$ et $\mu^{\prime \prime}: 1$ resp. expressa, habebimus in hypothesi prima

$X=\log \mu-\log \mu^{\prime \prime}+\log \eta-\log \eta^{\prime \prime}$

$Y=\log \mu+\log \mu^{\prime \prime}-\log \eta-\log \eta^{\prime \prime}+$ Comp. $\log \cos f+$ Comp. $\log \cos f^{\prime}$

+ Comp. $\log \cos f^{\prime \prime}+2 \log r^{\prime}-\log r-\log r^{\prime \prime}$

Logarithmi quantitatum $\mu, \mu^{\prime \prime}$ respectu partinm reliquarum nullius sunt momenti; $\log \eta$ et $\log \eta^{\prime \prime}$, qui ambo sunt positiui, in $X$ aliquatenus se inuicem destruunt, praesertim quoties temporum interualla fere aequalia sunt, vade $X$ valorem exiguum modo positiuum modo negatiuum obtinet; contra in $Y$ e partibus negatiuis $\log \eta$ et $\log \eta^{\prime \prime}$ compensatio quidem aliqua partium positiuarum Comp. $\log \cos f$, Comp. $\log$ $\cos f^{\prime}$, Comp. $\log \cos f^{n}$ oritur, sed minus perfecta, plerumque enim hae illas notabiliter superant. De signo ipsius $\log \frac{r^{\prime} r^{\prime}}{r r^{\prime \prime}}$ in genere nihil determinare licet.

Iam quoties motus heliocentricus inter obseruationes modicus est, raro opus erit, vsque ad hypothesin quartam progredi: plerumque tertia, saepius iam secunda praecisionem sufficientem praestabit, quin adeo interdum numeris ex ipsa hypothesi prima resultantibus acquiescere licebit. Iuuabit semper, ad maiorem minoremue. praecisionis gradum, qua obseruationes gaudent, respicere: ingratum enim foret opus, in calculo praecisionem affectare centies milliesue maiorem ea quam obseruationes permittunt. In his vero rebus iudicium per exercitationem frequentem practicam melius quam per praecepta acuitur, peritique facile acquirent facultatem quandam, vbi consistere conueniat recte diiudicandi.

149 .

In vitima demum hypothesi elementa ipsa calculabuntur, vel ex $f, r^{\prime}, r^{\prime \prime}$, vel ex $f^{\prime \prime}, r, r^{\prime}$, perducendo scilicet ad finem calculum alterutrum, quem in hypothesibus antecedentibus tantummodo vsque ad $\eta$ vel $\eta^{\prime \prime}$ prosequi oportuerat: si vtrumque perficere placuerit, harmonia numerorum resultantium nouam totius la- 
boris confirmationem suppeditabit. Attamen praestat; quam primum $f, f^{\prime}, f^{\prime \prime}$ crutae sunt, elementa e sola combinatione loci primi cum tertio deriuare, puta ex $\therefore, r, r^{\prime \prime}$ atque temporis interuallo, tandemque ad maiorem calculi certitudinem locum medium in orbita secundum elementa inuenta determinare.

Hoc itaque modo sectionis conicae dimensiones innotescent, puta excentricitas, semiaxis maior siue semiparameter, positio perihelii respectu locorum heliocentricorum $C, C^{\prime}, C^{\prime \prime}$, motus medius, atque anomalia media pro epocha arbitraria, siquidem orbita elliptica est, vel tempus transitus per perihelium, si orbita fit hyperbolica vel parabolica. Superest itaque tantummodo, vt positio locorum heliocentricorum in orbita respectn nodi ascendentis, positio huius nodi respectu puncti aequinoctialis, atque inclinatio orbitae ad eclipticam (vel aequatorem) determinentur. Haec omnia per solutionem vnius trianguli sphaerici efficere licet. Sit $\delta$ longitudo nodi ascendentis; $i$ inclinatio orbitae; $g$ et $g^{\prime \prime}$ argumenta latitudinis in obseruatione prima et tertia; denique $l-\Omega=h, l^{\prime \prime}-\Omega=h^{\prime \prime}$. Exprimente iam in fig. quarta $\Omega$ nodum ascendentem, trianguli $\Omega A C$ latera erunt $A D^{\prime}-\zeta, \delta, h$, angulique his resp. oppositi $i, 180^{\circ}-\gamma, u$. Habebimus itaque

$\sin \frac{r}{2} i \sin \frac{x}{2}(g+h)=\sin \frac{x}{2}\left(A D^{\prime}-\zeta\right) \sin \frac{x}{2}(\gamma+u)$

$\sin \frac{x}{2} i \cos \frac{T}{2}(g+h)=\cos \frac{\pi}{2}\left(A D^{\prime}-\zeta\right) \sin \frac{x}{2}(\gamma-u)$

$\cos \frac{\pi}{4} i \sin \frac{x}{2}(g-h)=\sin \frac{x}{2}\left(A D^{\prime}-\zeta\right) \cos \frac{x}{2}(\gamma+u)$

$\cos \frac{x}{2} i \cos \frac{r}{2}(g-h)=\cos \frac{x}{2}\left(A D^{\prime}-\zeta\right) \cos \frac{x}{2}(\gamma-u)$

Duae primae aequationes dabunt $\frac{x}{2}(g+h)$ et $\sin \frac{x}{2} i$, duae reliquae $\frac{\tau}{2}(g-h)$ et $\cos \frac{\pi}{2} i ;$ ex $g$ innotescet situs perihelii respectu nodi ascendentis, ex $h$ situs nodi in ecliptica; denique innotescet $i$, sinu et cosinu se mutuo confirmantibus. Ad eundem scopum peruenire possumus adiumento trianguli $\delta A^{\prime \prime} C^{\prime \prime}$, Tbi tantummodo in formulis praecedentibus characteres $g, h, A, \zeta, \gamma, u$ in $g^{\prime \prime}, \hbar^{\prime \prime}, A^{\prime \prime}, \zeta^{\prime \prime}, \gamma^{\prime \prime}, u^{\prime \prime}$ mutare oportet. Vt toti labori adhuc alia confirmatio concilietur, haud abs re erit, calculum vtroque modo perficere: vnde si quae leuiusculae differentiae inter valores ipsius $i, \Omega$ atque longitudinis perihelii in orbita prodeunt, valores medios adoptare conueniet. Raro tamen hae differentiae ad o"1 vel o"2 ascendent, siquidem omnes ca culi septem figuris decimalibus accurate elaborati fuerant.

Ceterum quoties loco eclipticae aequator tamquam planum fundamentale adoptatum est, nulla hinc in calculo differentia orietur, nisi quod loco punctorum $A, A^{\prime \prime}$ intersectiones aequatoris cum circulis maximis $A B, A^{\prime \prime} B^{\prime \prime}$ accipiendae sunt. 


\section{DETERMINATIO OREITAE E TRIBVS OBSERVATIONIBVS COMPIETIS. I67}

150.

Progredimur iam ad illustrationem huius methodi per aliquot exempla copiose explicanda, quae simul euidentissime ostendent, quam late pateat, et quam commode et expedite semper ad finem exoptatum perducat ${ }^{*}$ ).

Exemplum primum planeta nouus Iuno nobis suppeditabit, ad quem finem obseruationes sequentes Grenouici factas et a cel. Maskelyne nobiscum communicatas eligimus.

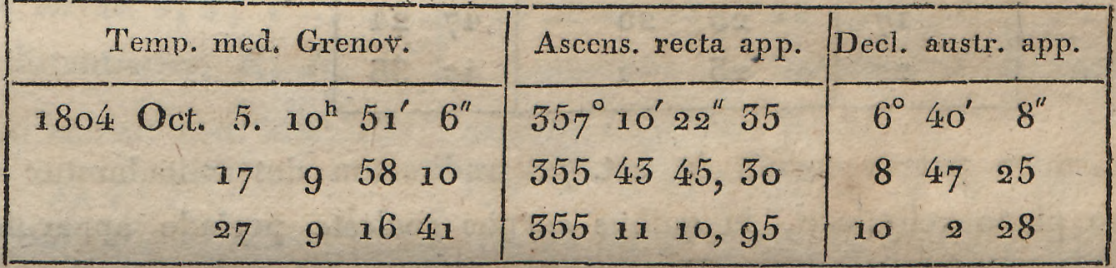

E tabulis Solaribus pro iisdem temporibus inuenitur

\begin{tabular}{|c|c|c|c|c|c|}
\hline & $\begin{array}{l}\text { longit. Solis ab ae- } \\
\text { quin. appar. }\end{array}$ & nutatio & $\begin{array}{c}\text { distantia a } \\
\text { terra }\end{array}$ & $\begin{array}{l}\text { latitudo } \\
\text { Solis }\end{array}$ & $\begin{array}{c}\text { obliquitas appar. } \\
\text { eclipticae }\end{array}$ \\
\hline Oct. 5 & $192^{\circ} 28^{\prime} 53^{\mu} 7^{2}$ & $+15^{\prime \prime} 43$ & o,9988859 & $-0^{\prime \prime} 49$ & $25^{\circ} 27^{\prime} 59^{\prime \prime} 48$ \\
\hline 17 & $204 \quad 20 \quad 21,54$ & $+15,51$ & 0,9953968 & $+0,79$ & 59,26 \\
\hline 27 & $214 \quad 16 \quad 52,21$ & $+15,60$ & 0,9928340 & $-0,15$ & 59,06 \\
\hline
\end{tabular}

Calculum ita adstruemus, ac si orbita adhuc penitus incognita esset: quamobrem loca Iunonis a parallaxi liberare non licebit, sed hanc ad loca terrae transferre oportebit. Primo itaque ipsa loca obseruata ab aequatore ad eclipticam reducimus, adhibita obliquitate apparente, vnde prodit:

\begin{tabular}{|c|c|c|}
\hline & $\begin{array}{l}\text { Longit. appar. } \\
\text { Iunonis }\end{array}$ & $\begin{array}{l}\text { Latit. appar. } \\
\text { Innonis }\end{array}$ \\
\hline Oct. 5 & $354^{\circ} 44^{\prime} 54^{\prime \prime} 27$ & $-4^{\circ} 5 g^{\prime} 3 x^{\prime \prime} 59$ \\
\hline 17 & $352 \quad 34 \quad 44,51$ & $-62156,25$ \\
\hline 27 & 5515451,57 & $-71752,70$ \\
\hline
\end{tabular}

*) Male loquuntur, qui methodum aliquam alia magis minusue exactam pronunciaint. Ea enim sola methodus problema soluisse censeri potest, per quam quemuis praecisionis gradum attingere saltem in potestate est. Quamobrem methodus alia alii co tantum nomine palmam praeripit, quod eundem praecisionis gradum per aliam celerius minorique labore, per aliam tardius gratuorique opera assequi licet. 
Cum hoc calculo statim iungimus determinationem longitudinis et latitudinis ipsius zenith loci obseruationis in tribus obseruationibus: rectascensio quidem cuin rectascensione Iunonis conuenit (quod obseruationes in ipso meridiano sunt factae), declinatio autem aequalis est altitudini poli $=51^{\circ} 28^{\prime} 59^{\prime \prime}$. Ita obtinemus

\begin{tabular}{|c|c|c|}
\hline & Long. ipsius zenith & latitudo \\
\hline Oct. 5 & $24^{\circ} \quad 29^{\prime}$ & $46^{\circ} 53^{\prime}$ \\
\hline 17 & $25 \quad 25$ & $47 \quad 24$ \\
\hline 27 & 23 & $47 \quad 36$ \\
\hline
\end{tabular}

Iam ad normam praeceptorum in art. 72 traditorum determinabuntur terrae loci ficti in ipso plano eclipticae, in quibus corpus coeleste perinde apparuisset, atque in locis veris obseruationum. Hoc modo prodit, statuendo parallaxin Solis mediam $=8^{\prime \prime} 6$

\begin{tabular}{|r|r|r|r|}
\hline & Reductio longit. & Reductio distantiae & Reductio temporis \\
\hline Oct. 5 & $-22^{\prime \prime} 59$ & $+0,0005856$ & $-0^{\prime \prime} 19$ \\
17 & $-27,21$ & $+0,0002529$ & $-0,12$ \\
27 & $-35,82$ & $+0,0002085$ & $-0,12$ \\
\hline
\end{tabular}

Reductio temporis ideo tantum adiecta est, vt appareat, eam omnino insensibilem esse.

Deinde omnes longitudines tum planetae tum terrae reducendae sunt ad aequinoctium vernale medium pro aliqua epocha, pro qua adoptabimus initium anni 1805 ; subducta itaque nutatione adhuc adiicienda est praecessio, quae pro tribus obseruationibus resp. est $11^{\prime \prime} 87,10^{\prime \prime} 23,8^{\prime \prime} 86$, ita vt pro obseruatione prima addere oporteat $-3^{\prime \prime} 56$, pro secunda $-5^{\prime \prime} 2.8$, pro tertia $-6^{\prime \prime} 74$.

Denique longitudines et latitudines Iunonis 'ab aberratione fixarum purgandae sunt; sic per regulas notas inuenitur, a longitudinibus resp. subtrahi debere $19^{\prime \prime} 12,17^{\prime \prime} 11,14^{\prime \prime} 82$, latitudinibus vero addi $\mathrm{o}^{\prime \prime} 55,1^{\prime \prime} 18,1^{\prime \prime} 75$, per quam additionem valores absoluti diminutionem patientur, quoniam latitudines australes tamquam negatiuae spectantur. 
151.

Omnibus hisce reductionibus rite applicatis, rera problematis data ita se hrabent:

Obseruationum tempora ad meridia-

num Parisinum reducta..

$\left|\begin{array}{r}\text { Oct. } 5,458644 \\ 554^{\circ} 44^{\prime} 51^{\prime \prime} 60 \\ -45951,06 \\ 122827,76 \\ 9,9996826\end{array}\right|$

\begin{tabular}{|c|c|}
\hline $17,42 \times 885$ & 27,303079 \\
\hline $552^{\mathrm{C}} 34^{\prime} 22^{\prime \prime} \times 2$ & $351^{\circ} 34^{\prime} 30^{\prime \prime}$ or \\
\hline$-62155,07$ & $-71750,9^{5}$ \\
\hline $\begin{array}{c}241949,05 \\
9,9980979\end{array}$ & $\begin{array}{c}54 \quad 16 \quad 9,65 \\
9,9969678\end{array}$ \\
\hline
\end{tabular}

Irnonis longitudines $\alpha, \alpha^{\prime}, \alpha^{\prime \prime}$.

Jatitudines $\beta, \beta^{\prime}, \beta^{\prime \prime}$

longitudines terrae $l, l^{\prime}, l^{\prime \prime} \ldots \ldots .$.

logarithmi distantiarum $R, R^{\prime}, R^{\prime \prime}$

9,9996826

9,9980979

9,9969678

Hinc calculi artt. 156,157 numeros sequentes producunt

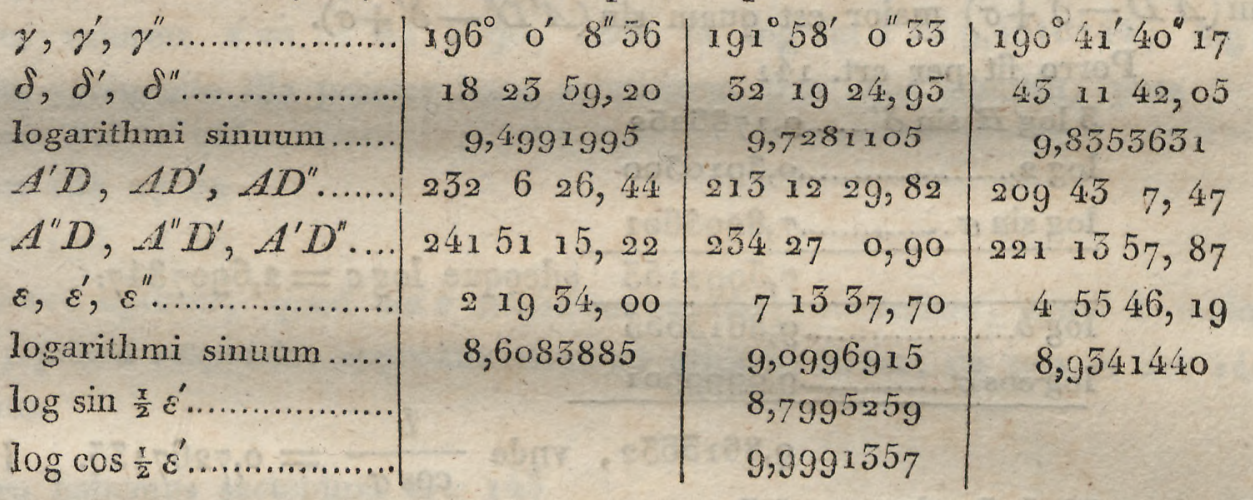

Porro secundum art. 158 habemus

\begin{tabular}{ll}
$\log \operatorname{tang} \beta \ldots \ldots \ldots \ldots . . .1,9412494 n$ & $\log \tan g \beta^{\prime \prime} \ldots \ldots \ldots \ldots . . \ldots, 1074080 n$ \\
$\log \sin \left(\alpha^{\prime \prime}-l^{\prime}\right) \ldots \ldots .9,7552591 n$ & $\log \sin \left(\alpha-l^{\prime}\right) \ldots \ldots .9,6955181 n$ \\
$\log \cos \left(\alpha^{\prime \prime}-l^{\prime}\right) \ldots \ldots .9,9247904$ & $\log \cos \left(\alpha-l^{\prime}\right) \ldots \ldots .9,9595180$ \\
\hline
\end{tabular}

Hinc

$\log \left(\operatorname{tang} \beta \cos \left(\alpha^{\prime \prime}-l^{\prime}\right)-\tan g \beta^{\prime \prime} \cos \left(\alpha-l^{\prime}\right)\right)=\log T \sin t$

8,5786513

$\log \sin \left(\alpha^{\prime \prime}-\alpha\right)=\log T \cos t$

Hinc $t=145^{\circ} 32^{\prime} 57^{\prime \prime} 78, \quad \log T$......

8,8260683

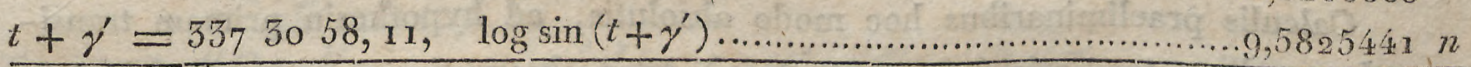

Denique

$\log \left(\operatorname{tang} \beta \sin \left(\alpha^{\prime \prime}-l^{\prime}\right)-\operatorname{tang} \beta^{\prime \prime} \sin \left(\alpha-l^{\prime}\right)\right)=\log S \ldots \ldots \ldots \ldots \ldots \ldots \ldots \ldots . \ldots \ldots, 2055519 n$

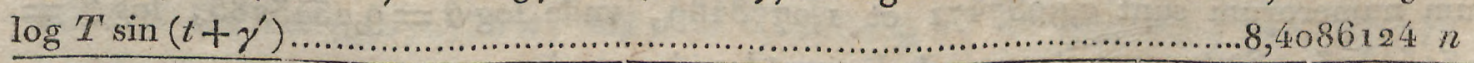

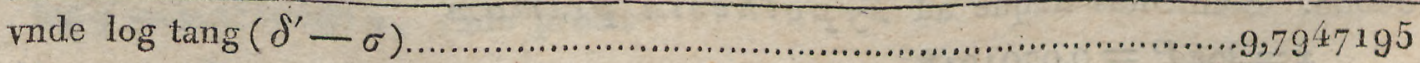

$\delta^{\prime}-\sigma=31^{\circ} 56^{\prime} 11^{\prime \prime} 81$, adeoque $\sigma=0^{\circ} 25^{\prime} 15^{\prime \prime} 12$.

Secundum art. 140 fit 


$$
\begin{aligned}
& A^{\prime \prime} D^{\prime}-\delta^{\prime \prime}=191^{\circ} 15^{\prime} 18^{\prime \prime} 85 \log \sin \ldots 9,2904352 n \log \cos \ldots 9,9915661 n \\
& A D^{\prime}-\delta=1944830,62--\ldots 9,4075427 n-\ldots 9,9855301 n \\
& A^{\prime \prime} D-\delta^{\prime \prime}=1983935,17-\ldots 9,5050667 n \\
& A^{\prime} D-\delta^{\prime}+\sigma=2001014,63-\ldots 9,5375909 n \\
& A D^{\prime \prime}-\delta=191198,27-\ldots 9,2928554 n \\
& A^{\prime} D^{\prime \prime}-\delta^{\prime}+\sigma=1891746,06-\ldots 9,2082723 n
\end{aligned}
$$

Hinc sequitur

$$
\log a \ldots . .9,5494457, a=+0,3543592
$$

$\log b \ldots . .9,8613533$

Formula 13 produceret $\log b=9,86 \mathbf{b} 5531$, sed valorem illum praeferimus, quoniam $\sin \left(A^{\prime} D-\delta^{\prime}+\sigma\right)$ maior est quam $\sin \left(A^{\prime} D^{\prime \prime}-\delta^{\prime}+\sigma\right)$.

Porro fit per art. 141

$3 \log R^{\prime} \sin \delta^{\prime} \ldots . .9,1786252$

$\log 2 \ldots \ldots \ldots \ldots \ldots \ldots . . \ldots, 3010300$

$\frac{\log \sin \sigma \ldots \ldots \ldots \ldots .7,8295601}{7,309^{2155}}$ adeoque $\log c=2,6907847$

$\log b \ldots \ldots \ldots \ldots \ldots . . . . . .9,8615533$

$\log \cos \sigma \ldots \ldots \ldots \ldots . . . .9,9999901$

$$
9,8613652 \text {, vnde } \frac{b}{\cos \sigma}=0,7267135 \text {. Hinc eruitur }
$$

$d=-1,3625052, \log e=8,3929518$.

Denique per formulas art. 143 eruitur

$\log x \ldots \ldots \ldots \ldots . \ldots, 0915394 n$
$\log x^{\prime \prime} \ldots \ldots \ldots . . .0,5418957 n$
$\log \lambda \ldots \ldots \ldots \ldots .0,4864480 n$
$\log \lambda^{\prime \prime} \ldots \ldots \ldots \ldots 0,1592352 n$

152.

Calculis praeliminaribus hoc modo absolutis, ad hypothesin primam transimus. Intertallum temporis (non correctum) inter obseruationem secundam et tertiam est dierum 9,97192 , inter primam et secundam 11,963241 . Logarithmi horum numerorum sunt $0,9987_{471}$ et $1 \xi 0778489$, vade $\log \theta=9,2343285, \log \theta^{\prime \prime}=$ 9,3134303 . Statuemus itaque ad hypothesin primam

$$
\begin{aligned}
& x=\log P=0,0791018 \\
& y=\log Q=8,5477588
\end{aligned}
$$


Hinc fit $P=1,1997804, P+a=1,5541396, P+d=-0,1627248$;

$\log e$ $8,3929518 n$

$\log (P+a)$ ...0, 1914900

C. $\log (P+d) \ldots . .0,7885463 n$

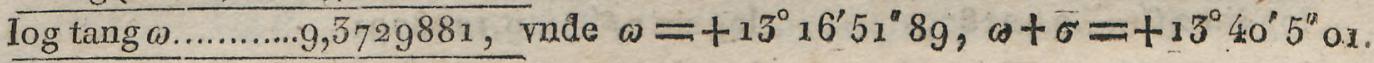
$\log Q \ldots \ldots \ldots \ldots \ldots . . . . ., 54775588$

$\log c \ldots \ldots \ldots \ldots \ldots \ldots . . . .2,6907847$

$\frac{\log \sin \omega \ldots \ldots \ldots . .9,5612147}{\log Q c \sin \omega \ldots \ldots . .0,5997582}$

Aequationi $Q c \sin \omega \sin z^{4}=\sin \left(z-13^{\circ} 40^{\prime} 5^{\prime \prime} 01\right)$ paucis tentaminibus factis satisfieri inuenitur per valorem $z=14^{\circ} 35^{\prime} 4^{\prime \prime} 90$, vnde fit $\log \sin z=9,4010744, \log r_{\text {, }}^{\prime}$ $=0,3251340$. Aequatio illa praeter hane solutionem tres alias admittit, puta

$$
\begin{aligned}
& z=32^{\circ} \quad 2^{\prime} 28^{\prime \prime} \\
& z=137 \quad 2759 \\
& z=193 \quad 4 \quad 18
\end{aligned}
$$

Tertiam reiicere oportet, quod $\sin z$ negatiuus euadit; secundam, quod $z$ maior fit quam $\delta^{\prime}$; prima respondet approximationi ad orbitam terrae, de qua in art. 142 loquati sumus.

Porro habemus secundum art. 145

$$
\begin{aligned}
& \log \frac{R^{\prime} \sin \delta^{\prime}}{b} \ldots \ldots \ldots . . .9,8648511 \\
& \log (P+a) \ldots \ldots \ldots \ldots . . .0,1914900 \\
& \text { C. } \log \sin (z-\sigma) \ldots \ldots, 6103578 \\
& \log \frac{n^{\prime} r^{\prime}}{n} \ldots \ldots \ldots \ldots . . . . . . . .0,6667029 \\
& \underline{\log P \ldots \ldots \ldots \ldots \ldots \ldots \ldots \ldots \ldots \ldots}, 0791018 \\
& \log \frac{n^{\prime} r^{\prime}}{n^{\prime \prime}} \ldots \ldots \ldots \ldots \ldots . . . . . .0,5876011 \\
& z+A^{\prime} D-\delta^{\prime}=z+199^{\circ} 47^{\prime} 1^{\prime \prime} 51=214^{\circ} 22^{\prime} 6^{\prime \prime} 41 ; \log \sin =9,7516756 n \\
& z+A^{\prime} D^{\prime \prime}-\delta^{\prime}=z+1885432,94=2032937,84 ; \log \sin =9,6005925 n
\end{aligned}
$$

Hinc fit $\log p=9,9270735 n, \log p^{n}=0,0226459 n$, ac dein $\log q=0,2930977 n$, $\log q^{\prime \prime}=0,2580086 n$, vnde prodit

$$
\begin{array}{ll}
\zeta=203^{\circ} 17^{\prime} 31^{\prime \prime} 22 & \log r=0,3300178 \\
\zeta^{\prime \prime}=210 \text { 10 58,88 } & \log r^{\prime \prime}=0,5212819
\end{array}
$$


Denique per art. 144 obtinemus

$$
\begin{aligned}
& \frac{r}{2}\left(u^{\prime \prime}+u\right)=205^{\circ} 18^{\prime} 10^{\prime \prime} 53 \\
& \frac{1}{2}\left(u^{\prime \prime}-u\right)=-5 \quad 14 \quad 2,02 \\
& f^{\prime}=\quad 54814,66
\end{aligned}
$$

$\log \sin 2 f^{\prime} \ldots . .9,121879^{1} \quad c^{\prime} \quad \log \sin 2 f^{\prime} \ldots . .9,121879^{7}$

$\log r \ldots \ldots \ldots \ldots \ldots, 0,5300178 \quad \log r^{\prime \prime} \ldots \ldots \ldots \ldots ., 3212819$

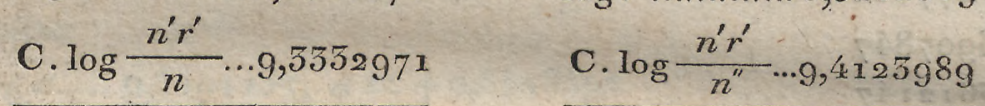

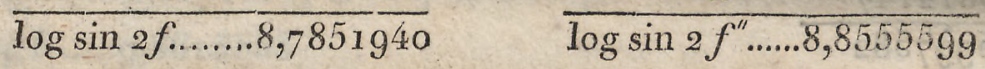

$2 f=5^{\circ} 29^{\prime} 46^{\prime \prime} 03 \quad 2 f^{\prime \prime}=4^{\circ} 6^{\prime} 45^{\prime \prime} 28$

Aggregatum $2 f+2 f^{\prime \prime}$ hic a $2 f^{\prime}$ tantummodo o"a 1 differt.

Iam vt tempora propter aberrationem corrigantur, distantias $\rho, \rho^{\prime}, \rho^{\prime \prime}$ per formulas art. 145 computare, ac dein per ipsas tempus $495^{\prime \prime}$ vel $0^{d}, 005706$ multiplicare oportet. Ecce calculum

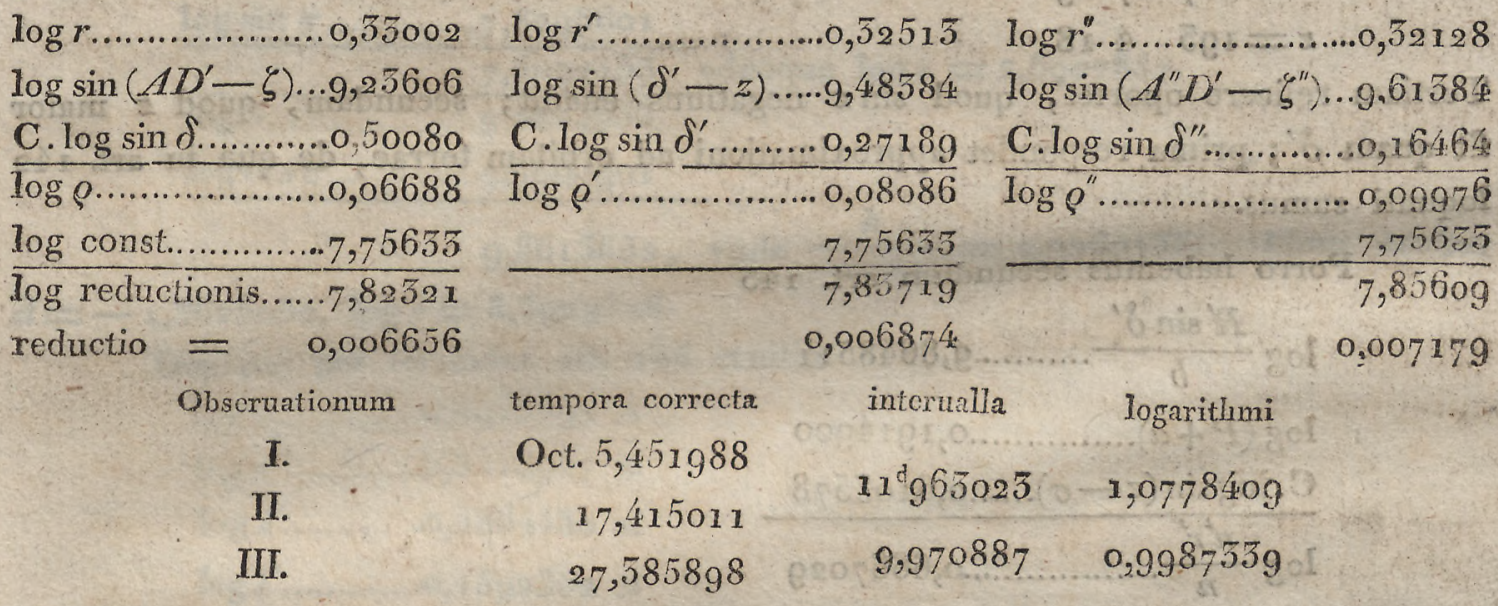

Fiunt itaque logarithmi quantitatum $\theta ; \theta^{\prime \prime}$ correcti 9,2545155 et 9,5134225 . Incipiendo iam determinationem elementorum ex $f, r^{\prime}, r^{\prime \prime}, \theta$ prodit $\log \eta=0,0002285$, perinde ex $f^{\prime \prime}, r, r^{\prime}, \theta^{\prime \prime}$ fit $\log \eta^{\prime \prime}=0,0003191$. Hunc calculum in Libri primi

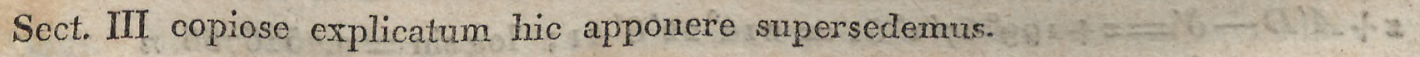

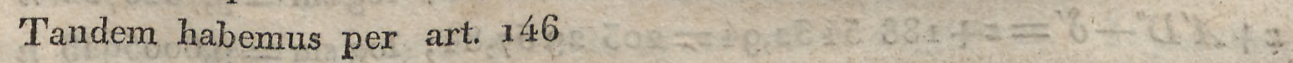


DETERMINATIO ORBITAE 2 TRIBVS OBSERVATIONTBVS COMPLETXS. IJ

\begin{tabular}{|c|c|}
\hline $\log \theta^{\prime \prime} \ldots \ldots \ldots \ldots, 9,5154225$ & $2 \log r^{\prime} \ldots \ldots \ldots \ldots \ldots, 6502680$ \\
\hline C. $\log \theta \ldots \ldots \ldots \circ, 7656847$ & C. $\log r r^{\prime \prime} \ldots \ldots \ldots \ldots, 9,5487005$ \\
\hline $\log \eta \ldots \ldots \ldots \ldots . .0,0002285$ & $\log \theta \theta^{\prime \prime} \ldots \ldots \ldots \ldots . . .8,5477576$ \\
\hline C. $\log \eta^{\prime \prime} \ldots \ldots . .99,9996809$ & C. $\log \eta \eta^{\prime \prime} \ldots \ldots \ldots . . .99,9994524$ \\
\hline $\log P^{\prime} \ldots \ldots \ldots \ldots . . .0,0790164$ & C. $\log \cos f \ldots \ldots \ldots 0,0002022$ \\
\hline & C. $\log \cos f^{\prime} \ldots \ldots \ldots, 0,0009579$ \\
\hline & $\frac{\mathrm{C} \cdot \log \cos f^{\prime \prime} \ldots \ldots \mathrm{0}, 0002797}{\log Q^{\prime} \ldots \ldots \ldots \ldots \ldots . .8,5475981}$ \\
\hline
\end{tabular}

E prina itaque hypothesi resultat $X=-0,0000854, X=-0,000160 \%$.

155.

In hypothesi secunda ipsis $P, Q$ eos ipsos valores tribuemus, quos in prima pro $P^{\prime}, Q^{\prime}$ inuenimus. Statuemus itaque

$$
\begin{aligned}
& x=\log P=0,0790164 \\
& y=\log Q=8,5475981
\end{aligned}
$$

Quum calculus hic prorsus codem modo tractandus sit, vt in hypothesi prima, praecipua eius momenta hic apposuisse sufficiet:

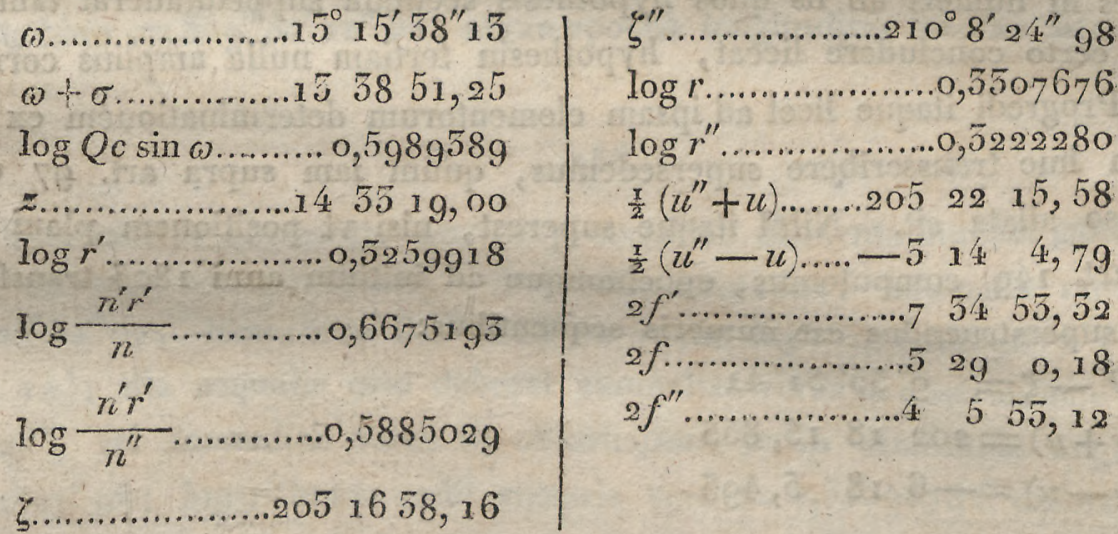

Reductiones temporum propter aberrationem denuo compufare operae haud pretium esset, vix enim $\mathbf{l}^{\prime \prime}$ ab iis quas in hypothesi prima eruimus differunt. deducitur

Calculi vlteriores praebent $\log \eta=0,000227^{\circ}, \log \eta^{\prime \prime}=0,000517^{5}$, vnde

$$
\begin{array}{ll}
\log P^{\prime}=0,0790167, & X=+0,0000005 \\
\log Q^{\prime}=8,5476110, & Y=+0,0000129
\end{array}
$$

Hinc patet, quanto adhuc magis exacta sit hypothesis secunda quam prima. 
154.

Ne quidquam desiderandum relinquatur, adhuc tertiam hypothesin extruemus, vbi rursus valores ipsarum $P^{\prime}, Q^{\prime}$ in hypothesi secunda erutos tamquam. valores ipsarum $P, Q$ adoptabimus. Statuendo itaque

$$
\begin{aligned}
& x=\log P=0,0790167 \\
& y=\log Q=8,5476110
\end{aligned}
$$

praecipua calculi momenta haec inueniuntur :

$$
\begin{aligned}
& \text { a.................1 } 5^{\circ} 15^{\prime} 38^{\prime \prime} 39 \\
& \zeta^{\prime \prime} \ldots \ldots \ldots \ldots \ldots \ldots . .210^{\circ} 8^{\prime} 25^{\prime \prime} 65 \\
& \omega+\sigma \ldots \ldots \ldots \ldots 15 \quad 5851,51 \\
& \log Q c \sin \omega \ldots \ldots \ldots . .0,5989542 \\
& \text { z............... } 1453 \text { i } 9,50 \\
& \log r^{\prime} \ldots \ldots \ldots \ldots \ldots \ldots . . .0,5259878 \\
& \log \frac{n^{\prime} r^{\prime}}{n} \ldots \ldots \ldots \ldots, 6675154 \\
& \log \frac{n^{\prime} r^{\prime}}{n^{\prime \prime}} \ldots \ldots \ldots \ldots . . .0,5884987 \\
& \text { G................205 } 1658,41
\end{aligned}
$$

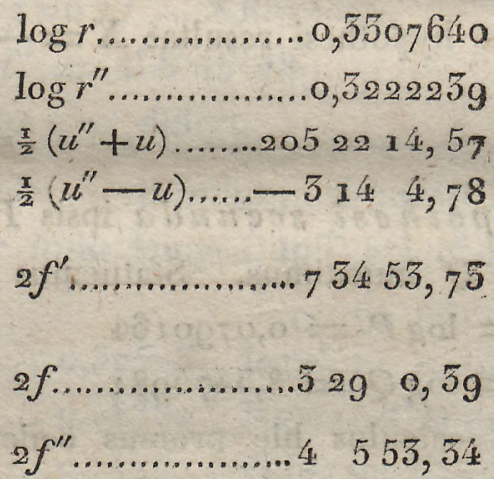

Omnes hi numeri ab is quos hypothesis secunda suppeditauerat tam parum differunt, vt certo concludere liceat, hypothesin tertiam nulla amplius correctione indigere ${ }^{*}$ ). Progredi itaque licet ad ipsam elementorum determinationem ex $2 f^{\prime}, r$; $r^{\prime \prime}, \theta^{\prime}$, quam huc transscribere supersedemus, quum iam supra art. 97 exempli loco in extenso allata sit. Nihil itaque superest, nisi vt positionem plani orbitae ad normam art. 149 computemus, epochamque ad initium anni 1805 transferamus. Calculus ille superstruendus est numeris sequentibus:

$$
\begin{aligned}
& A D^{\prime}-\zeta=9^{\circ} 55^{\prime} 5 x^{\prime \prime} 4 x \\
& \frac{x}{2}(\gamma+u)=202 \quad 18 \quad 13,855 \\
& \frac{\pi}{2}(\gamma-u)=-6 \quad 18 \quad 5,495
\end{aligned}
$$

vnde deriuamus

$$
\begin{aligned}
& \frac{x}{2}(g+h)=196^{\circ} 43^{\prime} 14^{\prime \prime} 62 \\
& \frac{x}{2}(g-h)=-43724,41 \\
& \frac{x}{2} i=65322,05
\end{aligned}
$$

\footnotetext{
*) Si calculus perinde $\mathrm{vt}$ in hypothesibus antecedentibus ad finem perduceretur, prodiret $X \approx O_{\vartheta}$ $Y=+0,0000003$, qui valor tamquam euanescens considerandus est, et vix smpra incerlitudinem figurae decimali vltimae semper inhaerentem exsurgit.
} 
DETERWIYATIO ORBITAE F TRIBVS OBSERVATIONIBVS COMPLETIS. I75

Fit igitur $h=201^{\circ} 20^{\prime} 59^{\prime} 05$, adeoque $\Omega=l-h=171^{\circ} 7^{\prime} 48^{n} 73 ;$ porro $g=$ $192^{\circ} 5^{\prime} 50^{\prime \prime} 21$, et proin, quum anomalia vera pro obseruatione prima in art. 97 inuenta sit $=310^{\circ} 55^{\prime} 29^{\prime \prime} 64$, distantia perihelii a nodo ascendente in orbita $=241^{\circ} 10^{\prime} 20^{\prime \prime} 57$, longitudoque perihelii $=52^{\circ} 18^{\prime} 9^{\prime \prime} 30$; denique inclinatio orbitae $=13^{\circ} 6^{\prime} 44^{\prime \prime} 10$. - Si ad eundem calculum a loco tertio proficisci malumus, habemus

$$
\begin{aligned}
& A^{\prime \prime} D^{\prime}-\zeta^{\prime \prime}=24^{\circ} 18^{\prime} 35^{\prime \prime}{ }_{2} 5 \\
& \frac{x}{2}\left(\gamma^{\prime \prime}+u^{\prime \prime}\right)=1962454,98 \\
& \frac{x}{2}\left(\gamma^{\prime \prime}-u^{\prime \prime}\right)=-54314,81
\end{aligned}
$$

Hinc elicitur

$$
\begin{aligned}
& \frac{7}{2}\left(g^{\prime \prime}+h^{\prime \prime}\right)=211^{\circ} 24^{\prime} 52^{\prime \prime} 45 \\
& \frac{1}{2}\left(g^{\prime \prime}-h^{\prime \prime}\right)=-114348,48 \\
& \frac{x}{2} i=65522,05
\end{aligned}
$$

atque hinc longitudo nodi ascendentis $=l^{\prime \prime}-h^{\prime \prime}=171^{\circ} 7^{\prime} 48^{\prime \prime} 72$, Iongitudo perihelii $=52^{\circ} 18^{\prime} 9^{\prime \prime} 50$, inclinatio orbitae $=15^{\circ} 6^{\prime} 44^{\prime \prime} 10$, prorsus eaedem vt ante.

Interuallum temporis ab obseruatione vltima rsque ad initium anni 1805 est dierum 64,614102; cui respondet motus heliocentricus medius $53293^{\prime \prime} 66$ $=14^{\circ} 48^{\prime} 15^{\prime \prime} 66$; hinc fit epocha anomaliae mediae pro initio anni 1805 in meridiano Parisino $=549^{\circ} 54^{\prime} 12^{\prime \prime} 38$, atque epocha longitudinis mediae $=41^{\circ} 52^{\prime} 21^{\prime \prime} 68$.

155

Quo clarius elucescat, quanta praecisione elementa intuenta gaudeant, locum. medium ex ipsis compuiabimus. Pro Oct. 17,415011 anomalia media inuenitur $=552^{\circ} 28^{\prime} 54^{\prime \prime} 77$, hinc vera $315^{\circ} x^{\prime} 23^{\prime \prime}$ o2 atque $\log r^{\prime}=0,5259877$ (vid. exempla artt. 15,14$)$; illa aequalis esse deberet anomaliae verae in obseruatione prima auctae angulo $2 f^{\prime \prime}$, vel anomaliae verae in obseruatione tertia diminutae angulo $2 f$, $i$. e. $=515^{\circ} 1_{22}^{\prime \prime} 98$; logarithmus radii vectoris vero $=0,5259878$ : differentiae pro nihilo habendae sunt. Si calculus pro obseruatione media vsque ad locum geocentricum continuatur, numeri resultant ab obseruatione paucis tantum minuti secundi partibus centesimis deuiantes (art. 63), quales differentiae ab erroribus ineuitabilibus e tabularum praecisione limitata oriundis quasi absorbentur.

Exemplum praecedens summa praecisione ideo tractauimus, vt appareat, quam facile per methodum nostram solutio quam accuratissima obtineri possit. In ipsa praxi raro opus erit, hunc typum aeque anxie imitari: plerumque sufficiet, 
sex figuras decimales vbique adhibere, et in exemplo nostro secunda iam hypothesis praecisionem haud minorem, primaque praecisionem abunde sufficientem suppeditauisset. Haud ingratán fore lectoribus censemus comparationem elementorum ex hypothesi tertia erutorum cum iis, quae prodeunt, si hypothesis secunda rol adco prima perinde ad eundem scopum adhibitae fuissent. Haec tria elementorum systemata in schemate sequente exhibemus:

\begin{tabular}{|c|c|c|c|}
\hline & ex lypothesi III & ex hypothesi II & ex hypothesi I \\
\hline Epocha longit. med. 1805 & $4 x^{\circ} \quad 52^{\prime} 2 x^{\prime \prime} 68$ & $41^{\circ} 52^{\prime} 18^{\prime \prime} 10$ & $42^{\circ} 12^{\prime} 57^{\prime \prime} 85$ \\
\hline Motus medius diurnus & $824^{\prime \prime} 7989$ & $824^{\prime \prime} 7985$ & $825^{\prime \prime} 5025$ \\
\hline Perihelium & $52 \quad 18 \quad 9,50$ & $52 \quad 18 \quad 6,66$ & $5241 \quad 9,81$ \\
\hline$\varphi$ & $14 \quad 12 \quad 1,87$ & $14 \times 1 \times 5,9^{4}$ & 142127,49 \\
\hline Logar, semiaxis maioris & 0,4224389 & $0,422459^{2}$ & $0,422894 \mathbf{1}$ \\
\hline Nodus ascendens & $748,7^{3}$ & 171749,15 & 171548,86 \\
\hline Inclinatio orbitae & 15644,10 & 15645,12 & 15257,50 \\
\hline
\end{tabular}

Computando locum heliocentricum in orbita pro obseruatione media per secundum elementorum systema, inuenitur error logarithmi radii vectoris $=0$, error Iongitudinis in orbita $=\mathrm{o}^{\prime \prime} \mathrm{o} 3$; computando vero istum locum per systema ex hypothesi prima deriuatum prodit error logarithmi radii vectoris $=0,0000002$, error longitudinis in orbita $=x^{\prime \prime} 31$. Continuando vero calculum vsque ad locum geocentricum inuenitur

\begin{tabular}{|l|r|r|}
\hline & ex hypothesi II & ex hypothesi I \\
\hline $\begin{array}{l}\text { longitudo geocentrica } \\
\text { error }\end{array}$ & $552^{\circ} 34^{\prime} 22^{\prime \prime} 26$ & $552^{\circ} 54^{\prime} 19^{\prime \prime} 97$ \\
$\begin{array}{l}\text { latitudo geocentrica } \\
\text { error }\end{array}$ & 0,14 & 2,15 \\
\hline
\end{tabular}



156.

Exemplum secundum a Pallade sumemus, cuius obseruationes sequentes Mediolani factas e Commercio literario clar. de Zach., Vol. XIV. pag. go excerpimus.

\begin{tabular}{|c|c|c|}
\hline Tempus medium Mediol. & Asc. recta app. & Declin. app. \\
\hline 1805 Nov. 5 14." $14^{\prime} 4^{\prime \prime}$ & $78^{\circ} 20^{\prime} 57^{\prime \prime} 8$ & $27^{\circ} 16^{\prime} 56^{\prime \prime} 7$ Austr. \\
\hline Dec. $\quad 6 \quad 11 \quad 51$ & $\begin{array}{lll}73 & 8 & 48,8\end{array}$ & $52 \quad 52 \quad 44,5$ \\
\hline 1806 Jan. 15 & 671411,1 & $28 \quad 38 \quad 8, x$ \\
\hline
\end{tabular}

Loco eclipticae hic aequatorem tamquam planum fundamentale accipiemus, calcu'oque ita defungemur, ac si orbita penitus adhuc incognita esset. Primo e tabulis Solis pro temporibus propositis sequentia petimus:

\begin{tabular}{|c|c|c|c|}
\hline & $\begin{array}{l}\text { Iongitudo Solis ab } \\
\text { aequin. med. }\end{array}$ & distantia a terra & $\begin{array}{l}\text { Tatitudo } \\
\text { Solis }\end{array}$ \\
\hline Nov. 5 & $223^{\circ} \quad 14^{\prime} \quad 7^{\prime \prime} 61$ & 0,9804511 & $+o^{\prime \prime} 59$ \\
\hline Dec. 6 & $254 \quad 28 \quad 42,59$ & 0,9846753 & $+0,12$ \\
\hline Jan. 15 & $295 \quad 5 \quad 47,62$ & o, 9838153 & $-0,19$ \\
\hline
\end{tabular}

Longitudines Solis, adiectis praecessionibus $+7^{\prime \prime} 59,+3^{\prime \prime} 56,-2^{\prime \prime} 11$ ad initium anmi 1806 reducimus, ac dein, adhibita obliquitate media $23^{\circ} 27^{\prime} 55^{\prime \prime} 55$ latitudinumque ratione rite habita, ascensiones rectas et declinationes inde deducimus. Ita inuenimus

\begin{tabular}{|c|c|c|}
\hline & ascensio recta Solis & declinatio Solis \\
\hline Nov. 5 & $220^{\circ} 46^{\prime} 44^{\prime \prime} 65$ & $15^{\circ} 49^{\prime} 45^{\prime \prime} 9^{4}$ Austr. \\
\hline Dec. 6 & $\begin{array}{lll}253 & 9 & 23,26\end{array}$ & $22 \quad 33 \quad 59,45$ \\
\hline Jan. 15 & 251,11 & $8 \quad 12,98$ \\
\hline
\end{tabular}

Hae positiones ad centrum terrae referuntur, adeoque parallaxi adiecta ad locum obseruationis reducendae sunt, quum positiones planetae a parallaxi purgare non liceat. Rectascensiones ipsius zenith in hoc calculo adhibendae cum rectascensionibus planetae conueniunt (quoniam obseruationes in ipso meridiano sunt institutae), declinatio vero vbique erit altitudo poli $=45^{\circ} 28^{\prime}$. Hinc eruuntur numeri sequentes: 


\begin{tabular}{|c|c|c|c|}
\hline & Asc. recta terrae & declinatio terrae & log. dist. a Sole \\
\hline Nov. 5 & $40^{\circ} 46^{\prime} 48^{\prime \prime} 51$ & $15^{\circ} 49^{\prime} 18^{\prime \prime} 5 \mathrm{~g}$ Bor. & 9,9958575 \\
\hline Dec. 6 & $75 \quad 925,26$ & $22.5342,83$ & 9,9955099 \\
\hline Ian. 15 & 117246,09 & $21 \quad 8 \times 7,29$. & $9,99^{2} 9259$ \\
\hline
\end{tabular}

Loca obseruata Palladis a nutatione et aberratione fixarum liberanda, ac dein adiecta praecessione ad initium anni 1806 reducenda sunt. Hisce titulis sequentes correctiones positionibus obseruatis applicare oportebit:

\begin{tabular}{|c|c|c|c|c|c|c|}
\hline & \multicolumn{2}{|c|}{ obseruatio I } & \multicolumn{2}{|c|}{ obseruatio II } & \multicolumn{2}{|c|}{ obseruatio III } \\
\hline & Asc. R. & decl. & asc. $x$. & decl. & asc. $\mathrm{r}$. & decl. \\
\hline Nutatio & $-12^{\prime \prime} 86$ & $-5^{\prime \prime} 08$ & $-13^{\prime \prime} 68$ & $-5,42$ & $-15^{\prime \prime} \circ 6$ & $-5^{\prime \prime} 75$ \\
\hline Aberratio & $-18,15$ & $-9,89$ & $-2 x, 5 x$ & $-1,65$ & $-15,60$ & $+9,76$ \\
\hline Praecessio & $+5,45$ & $+0,62$ & $+2,55$ & $+0,39$ & $-x, 51$ & $-0,33$ \\
\hline Summa & $-25,56$ & $-12,55$ & $-32,64$ & $-4,66$ & $-50,17$ & $+5,68$ \\
\hline
\end{tabular}

Hinc prodeunt positiones sequentes Palladis, calculo substruendae:

\begin{tabular}{|r|c|c|}
\hline T. m. Parisinum & asc. recta & declinatio \\
\hline Nov. 5,574074 & $78^{\circ} 20^{\prime} 12^{\prime \prime} 24$ & $-27^{\circ} 17^{\prime} 9^{\prime \prime} 05$ \\
36,175055 & $7^{3} 816,16$ & $-325248,9^{6}$ \\
76,349444 & 671340,95 & $-2858 \quad 2,42$ \\
\hline
\end{tabular}

157

Primo nunc situm circulorum maximorum a locis heliocentricis terrae ad locos geocentricos planetae ductorum determinabimus. Intersectionibus horum circulorum cum aequatore, aut si mauis illorum nodis ascendentibus, characteres $\mathfrak{x}, \mathfrak{Z}^{\prime}, \mathfrak{A}^{\prime \prime}$ adscriptos concipimus, distantiasque punctorum $B, B^{\prime}, B^{\prime \prime}$ ab his punetis per $\Delta, \Delta^{\prime}, \Delta^{\prime \prime}$ designamus. In maiori operationum parte pro $\mathcal{A}, \mathcal{A}^{\prime}, \mathcal{L}^{\prime \prime}$ iam x, $\mathfrak{a}^{\prime}, \mathfrak{A}^{\prime \prime}$, et pro $\delta, \delta^{\prime}, \delta^{\prime \prime}$ iam $\Delta, \Delta^{\prime}, \Delta^{\prime \prime}$ substituere oportebit; vbi vero $A$, $A^{\prime}, A^{\prime \prime}, \delta, \delta^{\prime}, \delta^{\prime \prime}$ retinere oporteat, lector attentus vel nobis non monentibus facile intelliget. 
Calculo facto iam inuenimus

\begin{tabular}{|c|c|c|c|}
\hline $\begin{array}{l}\text { Ascens. recta punctorum } \\
\qquad \mathfrak{I}, \mathfrak{A}^{\prime}, \mathfrak{A}^{\prime \prime}\end{array}$ & $233^{\circ} 54^{\prime} 57^{\prime \prime} 10$ & $253^{\circ}-8^{\prime} 57^{\prime \prime} \mathrm{O} 1$ & $276^{\circ} 40^{\prime} 25^{\prime \prime} 87$ \\
\hline$\gamma, \gamma^{\prime}, \gamma^{\prime \prime}$ & $\begin{array}{lll}51 & 17 & 15,7^{4}\end{array}$ & $90 \quad 3,19$ & 1315958,05 \\
\hline$\Delta, \Delta^{\prime}, \Delta^{\prime \prime}$ & 2155849,27 & 2125248,96 & $220 \quad 9 \times 2,9^{6}$ \\
\hline$\delta, \delta^{\prime}, \delta^{\prime \prime}$ & $56 \quad 2654, x 9$ & $55 \quad 2631,79$ & 69 10 57,84 \\
\hline $\mathfrak{H}^{\prime} D, \mathfrak{X} D^{\prime}, \mathfrak{x} D^{\prime \prime}$ & 255452,15 & 3o $\quad 18 \quad 3,25$ & 29843,52 \\
\hline $\mathfrak{A}^{\prime \prime} D, \mathfrak{A}^{\prime \prime} D^{\prime}, \mathfrak{A}^{\prime} D^{\prime \prime}$ & $35 \quad 3 \quad 26,55$ & 315921,14 & $2220 \quad 6,9^{1}$ \\
\hline$\varepsilon, \varepsilon^{\prime}, \varepsilon^{\prime \prime}$ & $47 \quad 154,69$ & 893457,17 & 423341,17 \\
\hline logarithmi sinuum & 9,8645525 & 9,9999885 & 9,8301910 \\
\hline $\log \sin \frac{x}{2} \varepsilon^{\prime}$ & & $9,847897^{1}$ & \\
\hline $\log \cos \frac{\pi}{2} \varepsilon^{\prime}$ & & 9,8510614 & \\
\hline
\end{tabular}

In calculo art. 158 pro $l^{\prime}$ ascensio recta puncti $\mathfrak{l}^{\prime}$ adhibebitur. Sic inuenitur $\log T \sin t \ldots . .8,4868256 n$ $\log T \cos t \ldots \ldots 9,2848162 n$

Hinc $t=189^{\circ} 2^{\prime} 48^{\prime \prime} 83, \log T=9,2902527 ;$ porro $t+\gamma^{\prime}=279^{\circ} 5^{\prime} 52^{\prime \prime}$ o2, $\log S$. $9,0110566 n$ $\log T \sin \left(t+\gamma^{\prime}\right) \ldots . . .9,2847950 n$ vnde $\Delta^{\prime}-\sigma=208^{\circ} 1^{\prime} 55^{\prime \prime} 64$, atque $\sigma=4^{\circ} 50^{\prime} 53^{\prime \prime} 32$.

In formulis art. 140 pro $a, b$ et $\frac{b}{a}$ ipsos $\sin \delta, \sin \delta^{\prime}, \sin \delta^{\prime \prime}$ retinere oportet, et perinde in formulis art. 1/42. Ad hos calculos habemus

$$
\begin{aligned}
& \mathfrak{U}^{\prime \prime} D^{\prime}-\Delta^{\prime \prime}=171^{\circ} 50^{\circ} 8^{\prime \prime} 18 \quad \log \sin \ldots \ldots .9,1525506 \quad \log \cos \ldots . . .9,995575 \mathrm{~g} n
\end{aligned}
$$

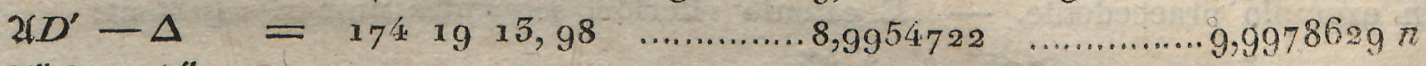

$$
\begin{aligned}
& \mathfrak{A}^{\prime \prime} D-\Delta^{\prime \prime}=17254 \quad 15,59 \quad \ldots \ldots \ldots \ldots \ldots . . .6,0917972 \\
& \mathfrak{q}^{\prime} D-\Delta^{\prime}+\sigma=\quad \begin{array}{lllll}
175 & 52 & 56,49 & \ldots \ldots \ldots \ldots \ldots .8,8561520
\end{array} \\
& 2\left(D^{\prime \prime}-\Delta=\begin{array}{llll}
773 & 9 & 54,05 \quad \ldots \ldots \ldots \ldots \ldots . . .9,0755844
\end{array}\right. \\
& \mathfrak{i}^{\prime} D^{\prime \prime}-\Delta^{\prime}+\sigma=174 \quad 18 \quad 11,27 \quad \ldots \ldots \ldots \ldots . . .8,9967978
\end{aligned}
$$

\section{Hinc elicimus}

$$
\begin{aligned}
& \log x=0,9211850, \quad \log \lambda=0,0812057 n \\
& \log x^{\prime \prime}=0,8112762, \quad \log \lambda^{\prime \prime}=0,0519691 n \\
& \log a=0,1099088, \quad a=+1,2879790 \\
& \log b=0,1810404 \\
& \log \frac{b}{a}=0,0711514, \text { vnde fit } \log b=0,1810402 . \text { Inter hos duos valores }
\end{aligned}
$$


tantum non aequales medium $\log b=0,1810405$ adoptabimus., Denique prodit

$$
\begin{aligned}
& \log c=1,045029^{5} \\
& d=+0,4489906 \\
& \log e=9,210289^{4}
\end{aligned}
$$

quo pacto calculi pracliminares absoluti sunt.

Temporis interuallum inter obseruationem secundam et tertiam est dierum 59,874409 , inter primam et secundam dierum 50,900961 : hine fit $\log \theta=9,8562757$ $\log \theta^{\prime \prime}=9,7255553$. Statuimus itaque ad hypothesin primam

$$
\begin{aligned}
& x=\log P=9,8892776 \\
& y=\log Q=9,561829 \circ
\end{aligned}
$$

Praecipua dein calculi momenta haec prodeunt:

$$
\begin{aligned}
& \omega+\sigma=20^{\circ} 8^{\prime} 46^{\prime \prime} 72 \\
& \log Q c \sin \omega=0,0282028
\end{aligned}
$$

Hinc fit valor verus ipsius $z=21^{\circ} 11^{\prime} 24^{\prime \prime} 30$, atque $\log r^{\prime}=0,5509379$. Tres reliqui valores ipsius $z$ aequationi IV art. 141 satisfacientes in hoc casu fiunt

$$
\begin{aligned}
& z=63^{\circ} 41^{\prime} \cdot 12^{\prime \prime} \\
& z=1011258 \\
& z=199247
\end{aligned}
$$

e quibus primus tamquam approximatio ad orbitam terrestrem spectandus est, cuins quidem aberratio, propter nimium temporis interuallum, longe hic maior est, quam in exemplo praecedente. - $\mathrm{E}$ calculo vlteriori sequentes numeri resultant:

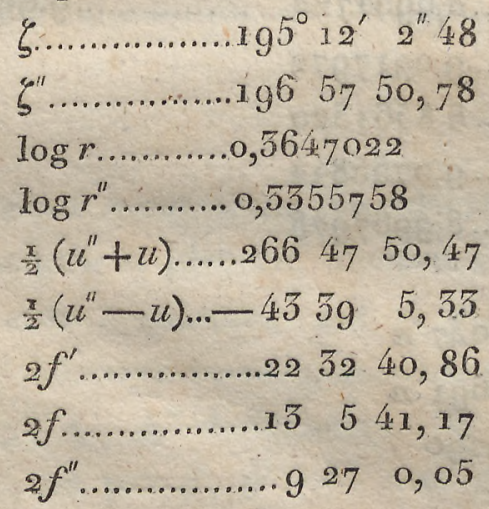

Differentiam inter $2 f^{\prime}$ et $2 f+2 f^{\prime \prime}$, quae hic est $0^{\prime \prime} 56$, inter $2 f$ et $2 f^{\prime \prime}$ ita dispertiemur, vt statuamus $2 f=15^{\circ} 5^{\prime} 40^{\prime \prime} 9^{6}, 2 f^{\prime \prime}=9^{\circ} 26^{\prime} 5 g^{\prime \prime} g^{\circ}$. 
DETERMINATIO ORBITAE TE TRIBVS OBSERVATIONIBVS COMPLETIS. 181

Corrigenda iam sunt tempora propter aberrationem, vbi in formulis art. 145 statuenduim est $A D^{\prime}-\zeta=2\left(D^{\prime}-\Delta+\delta-\zeta, A^{\prime \prime} D^{\prime}-\zeta^{\prime \prime}=2 I^{\prime \prime} D^{\prime}-\Delta^{\prime \prime}+\delta^{\prime \prime}-\zeta^{\prime \prime}\right.$. Habemus itaque

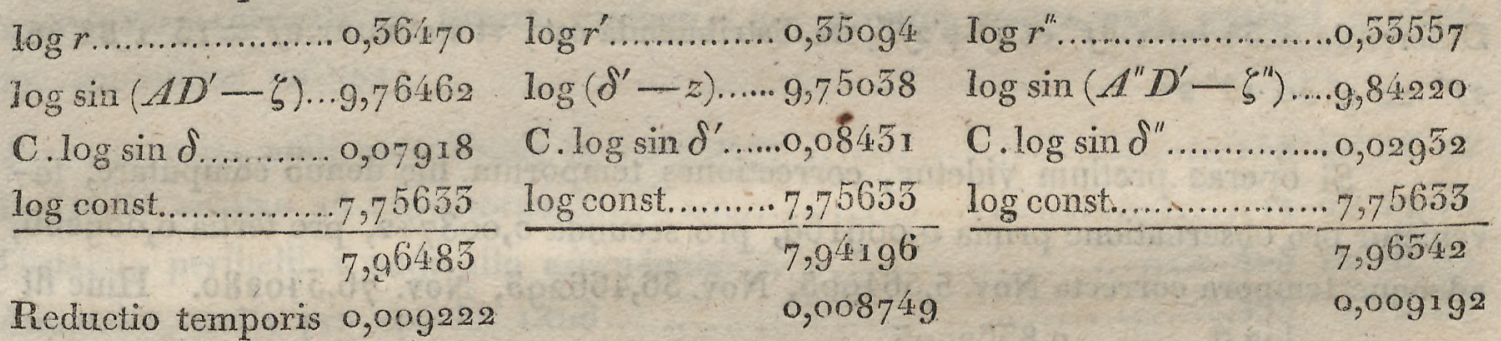

Hinc prodeunt

\begin{tabular}{r|c|c} 
tempora correcta & interualla & logarithmi \\
Nov. 5,564852 & 30,901454 & $x, 4899785$ \\
56,466286 & 39,875966 & $x, 6006894$ \\
76,540252 &
\end{tabular}

vnde deriuantur logarithmi correcti quantitatum $\theta$, $\theta^{\prime \prime}$ resp. 9,8362708 atque 9,7255599 . Incipiendo dein calculum elementorum ex $r^{\prime}, r^{\prime \prime}, 2 f, \theta$, prodit $\log \eta=0,0051 g^{21}$, sicuti ex $r, r^{\prime}, 2 f^{\prime \prime}, \theta^{\prime \prime}$ obtinemus $\log \eta^{\prime \prime}=0,0017500$. Hinc colligitur $\log P^{\prime \prime}$ $=9,8907512, \log Q^{\prime}=9,5712864$, adeoque

$$
X=+0,0014756, \quad Y=+0,0094574
$$

Praecipua momenta hypothesis secundae, in qua statuimus

$$
\begin{aligned}
& x=\log P=9,8907512, \\
& y=\log Q=9,5712864
\end{aligned}
$$

haec sunt:

$$
\begin{aligned}
& \omega+\sigma \ldots \ldots \ldots \ldots . . .20^{\circ} 8^{\prime} a^{\prime \prime} 87 \\
& \log Q c \sin 6 \ldots . . .0,0573071 \\
& \text { z.................21 } 12 \quad 6,09 \\
& \log r^{\prime} \ldots . . . \ldots \ldots \ldots . . . . .0,5507110 \\
& \text { G............... } 9^{5} 1659,90 \\
& \zeta^{\prime \prime} \ldots \ldots \ldots \ldots \ldots . . .196 \quad 5240,65 \\
& \log r \ldots \ldots \ldots \ldots \ldots . . . .0,5650642 \\
& \log r^{\prime \prime} \ldots \ldots \ldots \ldots \ldots . . \ldots, 3569708 \\
& \frac{x}{2}(u+u) \ldots \ldots . .267 \quad 610,7^{5} \\
& \frac{x}{2}\left(u^{\prime \prime}-u\right) \ldots=4559 \quad 4,00
\end{aligned}
$$




$$
\begin{aligned}
& 2 f^{\prime} \ldots \ldots \ldots \ldots \ldots . \ldots 22^{\circ} 52^{\prime} 8^{\prime \prime} 6 g \\
& 2 f \ldots \ldots \ldots \ldots \ldots . \ldots 13 \quad \text { i } 54,65 \\
& 2 f^{\prime \prime} \ldots \ldots \ldots \ldots \ldots \ldots . . \ldots 50 \quad 14,58
\end{aligned}
$$

Differentia o" 54 inter $2 f^{\prime}$ et $2 f+2 f^{\prime \prime}$, ita distribuenda est, vt statuatur $2 f=15^{\circ} 1^{\prime} 54^{\prime \prime} 45$, $2 f^{\prime \prime}=9^{\circ} 30^{\prime} 14^{\prime \prime} 24$.

Si operae pretium videtur, correctiones temporum hic denuo computare, invenietur pro obseruatione prima $0,009_{1} 69$, pro secunda 0,008742 , pro tertia $0,009_{2} 36$, adeoque tempora correcta Nov. 5,564905 , Nov. 36,466293 , Nov. 76,540280 . Hinc fit

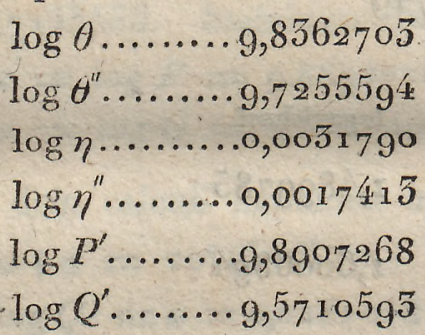

Hoc itaque modo ex hypothesi secunda resultat

$$
X=-0,0000244, \quad Y=-0,0002271
$$

\begin{tabular}{|c|c|}
\hline$\omega+\sigma \ldots \ldots \ldots 20^{\circ} 8^{\prime} 1^{\prime \prime} 62$ & $\log r^{\prime \prime} \ldots \ldots \ldots \ldots, 0,3369536$ \\
\hline $\log Q c \sin \omega \ldots \ldots ., 0370857$ & $\frac{x}{2}\left(u^{\prime \prime}+u\right) \ldots \ldots 267553,09$ \\
\hline $2 \ldots \ldots \ldots \ldots \ldots 2112 \quad 4,60$ & $\frac{1}{2}\left(u^{\prime \prime}-u\right) \ldots-45594,19$ \\
\hline $\log r \ldots \ldots \ldots \ldots ., 5,507191$ & $2 f^{\prime} \ldots \ldots \ldots \ldots 2252 \quad 7,67$ \\
\hline 6........... 1951654,08 & $2 f \ldots \ldots \ldots \ldots \ldots 15 \quad 157,42$ \\
\hline ...........196 5244,45 & 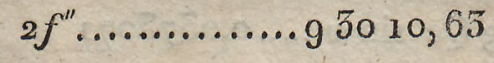 \\
\hline$\ldots \ldots \ldots \ldots, 0,3$ & \\
\hline
\end{tabular}

Denique in hypothesi tertia, in qua statuimus

$$
\begin{aligned}
& x=\log P=9,8907268 \\
& y=\log Q=9,5710593
\end{aligned}
$$

praecipua calculi momenta ita se habent:

Differentia $o^{\prime \prime} 58$ hic ita distribuetur, vt statuatur $\left.2 f=13^{\circ}{ }_{1}^{\prime} 57^{\prime \prime} 20,2 f^{\prime \prime}=9^{\circ} 50^{\prime} 10^{\prime \prime} 47^{\star}\right)$.

Quum differentiae omnium horum numerorum ab iis, quos hypothesis secunda suppeditauerat, - leuissimae sint, tuto iam concludere licebit, hypothesin ter-

*) Haecce differentia maiuscula, in omnibusque hypothesibus tantum non aequalis, ad maximam partem inde orta est, quod $\sigma$ duabus fere partibus centesimis minuti secundi iusto ninor, logarithmusque ipsius $b$ aliquot vnitatibus iusto maior exutus erat. 
tiam nulla amplius correctione opus habituram, adeoque hypothesin nouam superfluam esse. Quocirca nunc ad calculum elementorum ex $2 f^{\prime}, \theta^{\prime}, r, r^{\prime \prime}$ progredi licebit: qui quum operationibus supra amplissime iam explicatis contineatur, elementa ipsa inde resultantia in corum gratiam, qui proprio marte eum exsequi cupient, hic apposuisse sufficiet:

Ascensio recta nodi ascendentis in aequatore .................... $158^{\circ} 40^{\prime} 58^{\prime \prime} 9^{5}$

Inclinatio orbitae ad aequatorem............................. 1 $42 \quad 49,15$

Distantia perihelii a nodo illo ascendente....................... 523 14 56, 92

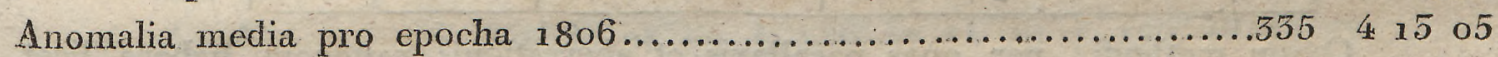

Motus medius (sidereus) diurnus.............................. $770^{11} 2662$

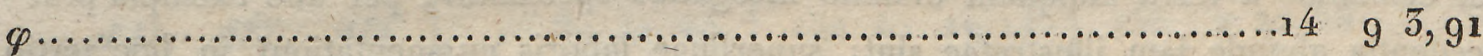

Logarithmus semiaxis maioris...................................., 4422438

158.

Duo exempla praccedentia occasionem nondum suppeditauerunt, methodum art. 120 in vsum vocandi: hypotheses enim successiuae tam rapide conuergebant, Wt iam in secunda subsistere licuisset, tertiaque a veritate vix sensibiliter aberraret. Reuera hocce commodo semper fruemur, quartaque hypothesi supersedere poterimus, quoties motus heliocentricus modicus est, tresque radii vectores non nimis inaequales sunt, praesertim si insuper temporum interualla parum inter se discrepant. Quanto magis autem problematis conditiones hinc recedunt, tanto fortius valores primi suppositi quantitatum $P, Q$ a veris different, tantoque lentius valores sequentes ad veros conuergent. In tali itaque casu tres quidem primae hypotheses ita absoluendae sunt, vti duo exempla praecedentia monstrant (ea sola differentia, quod in hypothesi tertia non elementa ipsa, sed, perinde vt in hypothesi prima et secunda, quantitates $\eta, \eta^{\prime \prime}, F^{\prime}, Q^{\prime}, X, Y$ computare oportet): dein vero haud amplius valores postremi ipsarum $P^{\prime}, Q^{\prime}$ tamquam valores noui quantitatum $P, Q$ in hypothesi quarta accipientur, sed hi per methodum art. 120 e combinatione trium primarum hypothesium eruentur. Rarissime tunc opus erit, ad hypothesin quintam secundum praccepta art. 121 progredi. - Iam hos quoque calculos exemplo illustrabimus, ex quo simul elucebit, quam late methodus nostra pateat.

159 .

Ad exemplum tertium obseruationes sequentes Cereris eligimus, quarum 
prima Bremae a clar. Olbers, secunda Gottingae a clar. Harding, tertia Lilienthalii a clar. Bessel instituta est.

\begin{tabular}{|c|c|c|}
\hline $\begin{array}{l}\text { Tempus medium loci } \\
\text { obseruationis }\end{array}$ & Asc. recta & Declin. boreal. \\
\hline 1805 Sept. $5.15^{\mathrm{h}} 8^{\prime} 54^{\prime \prime}$ & $95^{\circ} 59^{\prime} 25^{\prime \prime}$ & $22^{\circ} 2 x^{\prime} 25^{\prime \prime}$ \\
\hline $1806 \mathrm{Jan} .17 \cdot x \cdot 58.5 x$ & 101 $18 \quad 40,6$ & $30 \quad 21 \quad 22,3$ \\
\hline 1806 Maii 23. 10. 25. 55 & $12156 \quad 7$ & 245 \\
\hline
\end{tabular}

Quum methodi, per quas parallaxis et aberrationis rationem habere licet, si distantiae a terra tamquam omnino incognitae spectantur, per duo exempla praecedentia abunde iam illustratae sint: superfluae laboris augmentationi in hoc tertio exemplo renunciabimus, distantiasque approximatas e Commercio litterario clar. de Zach (Vol. XI p. 284) eum in finem excerpemus, vt obseruationes ab effectu parallaxis et aberrationis purgentur. Has distantias vna cum reductionibus inde derivatis tabula sequens exhibet:

Distantia Cereris a terra

Tempus, intra quod lumen ad terram descendit

Tempus obseruationis reductum

Tempus sidereum in gradibus

Parallaxis ascensionis rectae

Parallaxis declinationis

\begin{tabular}{|r|r|r}
2,899 & 1,638 & 2,964 \\
$25^{\prime} 49^{\prime \prime}$ & $15^{\prime} 28^{\prime \prime}$ & $24^{\prime} 21^{\prime \prime}:$ \\
$12^{\mathrm{h}} 45^{\prime} 5^{\prime \prime}$ & $10^{\mathrm{h}} 45^{\prime} 25^{\prime \prime}$ & $9^{\mathrm{h}} 59^{\prime} 32^{\prime \prime}$ \\
$355^{\circ} 55^{\prime}$ & $97^{\circ} 59^{\prime}$ & $210^{\circ} 41^{\prime}$ \\
$+1^{\prime \prime} 90$ & $+0^{\prime \prime} 22$ & $-1^{\prime \prime} 97$ \\
$-2,08$ & $-1,90$ & $-2,04$
\end{tabular}

Problematis itaque data, postquam a parallaxi et aberratione liberata, temporaque ad meridianum Parisinum reducta sunt, ita se habent:

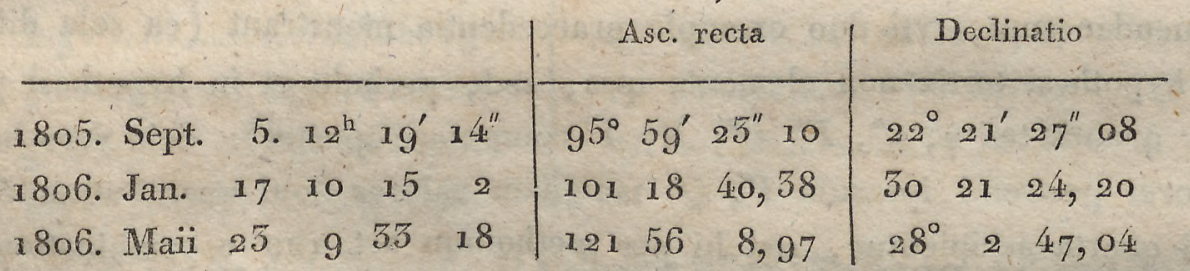

Ex his ascensionibus' rectis et declinationibus deductae sunt longitudines et latitudines adhibita obliquitate eclipticae $25^{\circ} 27^{\prime} 55^{\prime \prime} 90,25^{\circ} 27^{\prime} 54^{\prime \prime} 59,25^{\circ} 27^{\prime}$ $55^{\prime \prime} 27$; dein longitudines 'a nutatione purgatae sunt, quae resp. fuit $+17^{\prime \prime} 31$, $+17^{\prime \prime} 88,+18^{\prime \prime} 00$, posteaque ad initium anni 1806 reductae, applicata praecessione $+15^{\prime \prime} 98,-2^{\prime \prime} 39,-19^{\prime \prime} 68$. Denique pro temporibus, reductis e tabulis excerpta sunt loca Solis, vbi in longitudinibus nutatio praetermissa, contra prae- 
cessio perinde $\mathrm{vt}$ longitudinibus Cereris adiecta est. Latitudo Solis omnino neglecta. Hoc modo numeri sequentes in calculo adhibendi resultauerunt:

\begin{tabular}{|c|c|c|c|}
\hline 5. & 5,51556 & 159,42711 & $265,598 \mathrm{r} 3$ \\
\hline & $9^{5^{\circ}} 52^{\prime} 18^{\prime \prime} 56$ & $99^{\circ} 49^{\prime} 5^{\prime \prime}, 87$ & $118^{\circ} \quad 5^{\prime} \quad 28^{\prime \prime} 85$ \\
\hline , $\beta^{\prime \prime}$ & - o 5934,06 & $+7 \quad 1656,80$ & +758 \\
\hline & 3425456, oо & $117 \quad 1243,25$ & 5850 \\
\hline 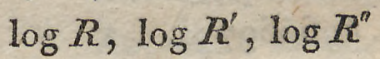 & 0,003151 & - 9,9929861 & \\
\hline
\end{tabular}

- Iam calculi praeliminares in artt. $156-140$ explicati sequentia suppeditant:

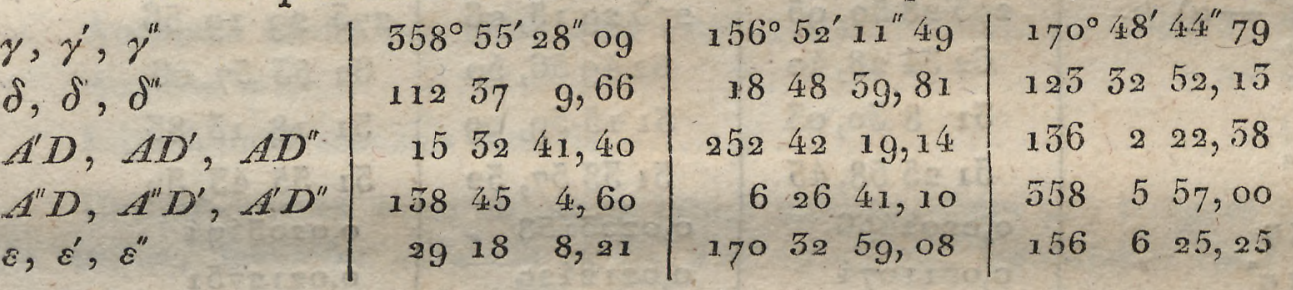

$\sigma=8^{\circ} 52^{\prime} 4^{\prime \prime} 05$

$\log a=0,1840193 n \quad a=-1,5276340$

$\log b=0,0040987$

$\log c=2,0066755$

$d=117,50873$

$\log e=0,8568244$

$\log x=0,1611012$

$\log x=9,9770819 n$

$\log \lambda=9,9164090 n$

$\log \lambda^{\prime \prime}=9,7320127 n$

Interuallum temporis inter obseruationem primam et secundam est dierum 133,91375, inter secundam et tertiam 125,97102: hinc fit $\log \theta=0,5558520$, $\log \theta^{\prime \prime}=0,3624066, \log \frac{\theta^{\prime \prime}}{\theta}=0,0265546, \log \theta \theta^{n}=0,6982586$. Iam praecipua momenta hypothesium trium primarum deinceps formatarum in conspectn sequenti exhibemus:

\begin{tabular}{l|l|l|l} 
& \multicolumn{1}{|c|}{ I } & \multicolumn{1}{|c|}{ II } & \multicolumn{1}{|c}{ III } \\
\hline \begin{tabular}{l|l}
$\log P=x$ \\
$\log Q=y$
\end{tabular} & 0,0265546 & 0,0256968 & 0,0256275 \\
$\omega+\sigma$ & 0,6982586 & 0,7590190 & 0,7481055 \\
$7^{\circ} 15^{\prime} 13^{\prime \prime} 523$ & $7^{\circ} 1^{\prime} 47^{\prime \prime} 159$ & $7^{0} 1 t^{\prime} 45^{\prime \prime}$ o $1 x$
\end{tabular}




\begin{tabular}{|c|c|c|c|}
\hline $\log Q_{c} \sin \omega$ & $\begin{array}{l}1,1546650 n \\
7^{\circ} 5^{\prime} 5 a^{\prime \prime} \text { ox } 8\end{array}$ & $\begin{array}{l}x, 973925 n \\
7^{\circ} 2^{\prime} 32^{\prime \prime} 870\end{array}$ & $\begin{array}{l}1,2066327 n \\
7^{\circ} 2^{\prime} 16^{\prime \prime} \text { g0o }\end{array}$ \\
\hline $\log r^{\prime}$ & 0,4114726 & 0,4129571 & 0,4152107 \\
\hline$\zeta$ & 1601046,74 & $16020 \quad 7,82$ & $160 \quad 22 \quad 9,42$ \\
\hline$\zeta^{t h}$ & 26261,05 & 2621218,26 & 262 14 19,4 \\
\hline $\log r$ & 0,4523954 & 0,4291773 & 0,4284841 \\
\hline $\begin{array}{l}\log r^{\prime \prime} \\
\frac{x}{2}\left(u^{\prime \prime}+u\right)\end{array}$ & $\begin{array}{l}0,4094712 \\
2625525,22\end{array}$ & $\begin{array}{l}0,4071975 \\
26257 \quad 6,85\end{array}$ & 0,4064697 \\
\hline$\frac{\tau}{2}\left(u u^{\prime \prime}-u\right)$ & $27528.50,95$ & $27329.15,06$ & $\begin{array}{lll}262 & 57 & 31,17 \\
275 & 29 & 19,56\end{array}$ \\
\hline $2 f^{\prime}$ & 623428,40 & 624956,50 & 625357,06 \\
\hline $2 f$ & 5 I 850,05 & $51155 \mathrm{~g}, \mathrm{og}$ & $\begin{array}{lll}51 & 18 & 15,83\end{array}$ \\
\hline & 312558,45 & $3 x 3557,52$ & 315545,52 \\
\hline $\log \eta$ & 0,0202496 & 0,0203158 & $0,020349^{4}$ \\
\hline $\log \eta^{11}$ & 0,0211074 & 0,0212429 & $0,021275 x$ \\
\hline $\log P^{\prime \prime}$ & 0,0256968 & 0,0256275 & $0,025628 \mathrm{~g}$ \\
\hline $\log Q^{\prime \prime}$ & 0,7390190 & $0,7481.055$ & 0,7502357 \\
\hline$X$ & $-0,0008578$ & $-0,0000695$ & $+0,0000014$ \\
\hline$\gamma$ & $+0,0407604$ & $+0,0090865$ & $+0,0021288$ \\
\hline
\end{tabular}

Iann designando tres valores ipsius $X$ per $A, A^{\prime \prime}, A^{\prime \prime}$; tres valores ipsius $Y$ per $B, B^{\prime \prime}, B^{\prime \prime} ;$ quotientes e diuisione quantitatum $A^{\prime} B^{\prime \prime}-A^{\prime \prime} B^{\prime}, A^{\prime \prime} B-A B^{\prime \prime}$, $A B^{\prime}-A^{\prime} B$ per eorundem aggregatum ortas resp. per $k, k^{\prime}, k^{\prime \prime}$, ita vt habeatur $k+k^{\prime}+k^{\prime \prime}=1$, denique valores ipsorum $\log P^{\prime}$ et $\log Q^{\prime}$ in hypothesi tertia per $\mathbb{M}$ et $N$ (qui forent valores noui ipsarum $x, y$, si hypothesin quartam perinde e tertia deriuare conueniret, vt tertia $\mathrm{c}$ secunda derinata fuerat): e formulis art. 120 facile colligitur, valorem correctum ipsius $x$ fieri $=M-k\left(A^{\prime}+A^{\prime \prime}\right)-k^{\prime} A^{\prime \prime}$, valoremque correctum ipsius $y=N-k\left(B^{\prime}+B^{\prime \prime}\right)-k^{\prime} B^{\prime \prime}$. Calculo facto prior eruitur $=0,0256551$, posterior $=0,750 g 143$. Hisce valoribus correctis iam hypothesin quartam superstruimus, cuius praecipua momenta haec sunt:

\begin{tabular}{|c|c|}
\hline 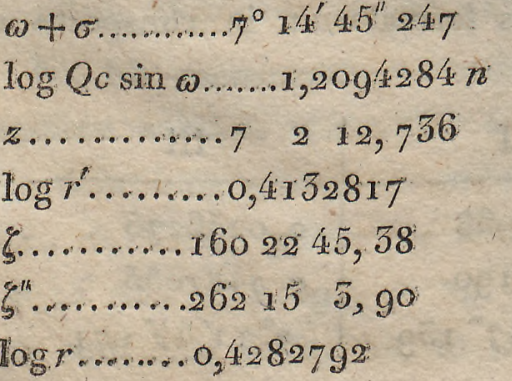 & 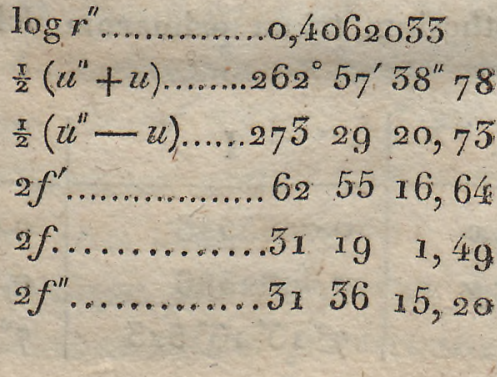 \\
\hline
\end{tabular}


DETERMINATIO ORBITAE E TRIBVS OBSERVATIONTBVS COMPLETIS. $\quad 187$

Inter $2 f^{\prime}$ et $2 f+2 f^{\prime \prime}$ differentia $o^{\prime \prime}$ o5 emergit, quam ita distribuemus, vt statuamus $2 f=51^{\circ} 19^{\prime} 1^{\prime \prime} 47,2 f^{\prime \prime}=51^{\circ} 56^{\prime} 15^{\prime \prime} 17$. Quodsi iam e duobus locis extremis elementa ipsa determinantur, sequentes numeri resultant:

Anomalia vera pro loco primo........................28 $89^{\circ} 7^{\prime} 39^{n} 7^{5}$

Anomalia vera pro loco tertio.........................552 2 56,59

Anomalia media pro loco primo......................297 4135,65

Anomalia media pro loco tertio......................... $555 \quad 1522,49$

Motus medius diurnus sidereus.............................. 769,6755

Anomalia media pro initio anni $1806 \ldots \ldots \ldots \ldots \ldots \ldots \ldots . \ldots \ldots 2255 \quad 52,51$

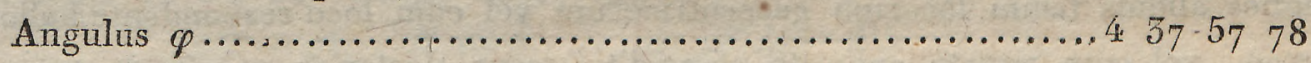

Logarithmus semiaxis maioris............................ $442466 x$

Computando ex hisce elementis locum heliocentricum pro tempore obseruationis mediae, inuenitur anomalia media $526^{\circ} 19^{\prime} 25^{\prime \prime} 7^{2}$, logarithmus radii vectoris 0,4132825 , anomalia vera $520^{\circ} 43^{\prime} 54^{\prime \prime} 87$ : haecce distare deberet ab anomalia vera pro loco primo differentia $2 f^{\prime \prime}$, siue ab anomalia vera pro loco tertio differentia $2 f$, adeoque fieri deberet $=320^{\circ} 45^{\prime} 54_{2}^{\prime \prime} 9^{2}$, siruti logarithmus radii vectoris $=0,4152817$ : differentia $0^{\prime \prime} 05$ in anomalia vera, octoque vnitatum in isto logarithmo nullius momenti censenda est.

Si hypothesis quarta eodem modo ad finem perduceretur, vt tres praecedentes, prodiret $X=0, Y=-0,0000168$, vnde valores correcti ipsarum $x, y$ hi colligerentur

$$
\begin{aligned}
& x=\log P=0,025635 x \text { (idem vt in hypothesi quarta) } \\
& y=\log Q=0,7508917
\end{aligned}
$$

Quibus valoribus si hypothesis quinta superstrueretur, solutio vltimam quam tabulae permittunt praecisionem nanciscerelur: sed elementa hinc resultantia vix sensibiliter $a b$ is discreparent, quae hypothesis quarta suggessit.

Vt elementa completa habeantur, nihil iam superest, nisi vt situs plani orbitae computetur. Ad normam praeceptorum art. 142 hic prodit

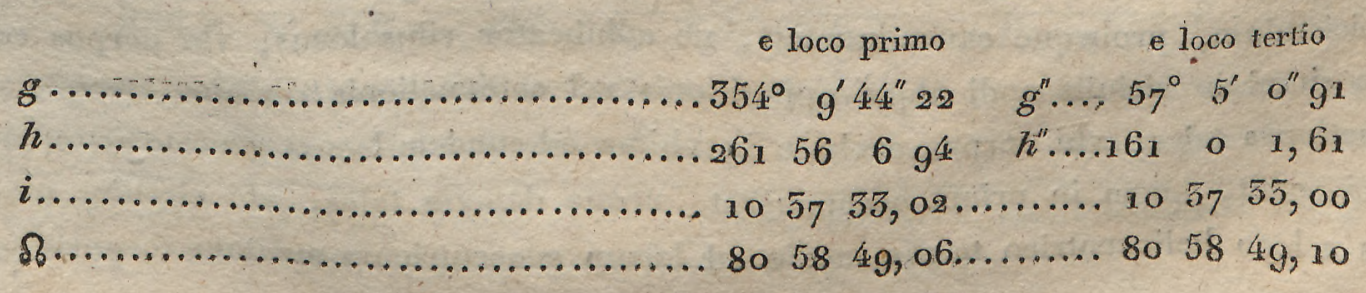


distantia perihelii a nodo ascendente... $65 \quad 2 \quad 4,47 \ldots \ldots \ldots .65 \quad 2 \quad 4,52$

longitudo perihelii...................146 o $55,55 \ldots \ldots \ldots 146$ o 55,62

Sumto itaque medio statuetur $i=10^{\circ} 37^{\prime} 35^{\prime \prime}$ or, $\delta=80^{\circ} 58^{\prime} 49^{\prime \prime} 08$, longitudo perihelii $=1460^{\prime} 53^{\prime \prime} 57$. Denique longitudo media pro initio anni 1806 erit $=108^{\circ} 56^{\prime} 46^{\prime \prime} 08$.

160.

In expositione methodi, cui disquisitiones praecedentes dicatae fuerunt, in quosdam casus speciales incidimus, vbi applicationem non patitur, saltem non in forma ea, in qua a nobis exhibita est. Hune defectum locum habere vidimus primo, quoties aliquis trium locorum geocentricorum vel cum loco respondente heliocentrico terrae, vel cum puncto opposito coincidit (casus posterior manifesto tunc tantum occurrere potest, vbi corpus coeleste inter Solem et terram transiit):

secundo, quoties locus geocentricus primus corporis coelestis cum tertio coincidit:

tertio, quoties omnes tres loci geocentrici vna cum loco heliocentrico terrae secundo in eodem circulo maximo siti sunt.

In casu primo situs alicuius circilorum maximorum $A B, A^{\prime} B^{\prime}, A^{\prime \prime} B^{\prime \prime}$ indeterminatus manebit, in secundo atque tertio situs puncti $B^{\star}$. "In hisce itaque casibus methodi supra expositae, per quas, si quantitates $P, Q$ tamquam cognitae spectantur, e locis geocentricis heliocentricos determinare docuimus, vim suam perdunt: attamen discrimen essentiale hic notandum est, scilicet in casu primo hic defectus soli methodo attribuendus erit, in casu secundo et tertio autem ipsius problematis naturae; in casu primo itaque ista determinatio vtique effici poterit, si modo methodus apte varietur, in secundo et tertio autem absolute impossibilis erit, locique heliocentrici indeterminati manebunt. Hand pigebit, hasce relationes paucis euolvere: omnia vero, quae ad hoc argumentum pertinent exhaurire eo minus e re esset, quod in omnibus his casibus specialibus orbitae determinatio exacta impossibilis est, vbi a letrissimis obseruationum erroribus enormiter afficeretur. Idem defectus etiamnum valebit, quoties obseruationes haud quidem exacte, attamen proxime ad aliquem horum casuum referuntur: quamobrem in eligendis obseruationibus hue respiciendum, probeque cauendum est, ne adhibeatur vllus locus, vbi corpus coeleste simul in viciniis nodi atque oppositionis vel coniunctionis versatur, neque obseruationes tales, vbi corpus coeleste in vltima ad eundem locum geocentricum prosime rediit, quem in prima occupanerat, neque demum tales, vbi circulus maximus a loco heliocentrico terrae medio ad locum geocentricum medium corporis coe- 
DETERMINATIO ORBITAE E TRTBVS OBSERVATTONIBVS COMPIETIS.

lestis ductus angulum acutissimum cum directione motus geocentrici format, atque locum primum et tertium quasi stringit.

161.

Casus primi tres subdiuisiones faciemus.

I. Si punctum $B$ cum $A$ vel cum puncto opposito coincidit, erit $\delta=0$ vel $=180^{\circ} ; \gamma, \varepsilon^{\prime}, \varepsilon^{\prime \prime}$ atque puncta $D^{\prime}, D^{\prime \prime}$ indeterminata ertunt; contra $\gamma^{\prime}, \gamma^{\prime \prime}$, $\varepsilon$ atque puncta $D, B^{\star}$ determinata; punctum $C$ necessario coincidet cum $A$. Per ratiocinia, iis, quae in art, 140 tradita sunt, analoga, facile elicietur aequatio haecce:

$$
0=n^{\prime} \frac{\sin (z-\sigma)}{\sin z} \cdot \frac{R^{\prime} \sin \delta^{\prime}}{R^{\prime \prime} \sin \delta^{\prime \prime}} \cdot \frac{\sin \left(A^{\prime \prime} D-\delta^{\prime \prime}\right)}{\sin \left(A^{\prime} D-\delta^{\prime}+\sigma\right)}-n^{\prime \prime}
$$

Omnia itaque, quae in artt. 141, 142 exposita sunt, etiam huc transferre licebit, si modo statuatur $a=0$, atque $b$ per ipsam aequationem 12 art. 140 determinetur, quantitatesque $z, r^{\prime}, \frac{n^{\prime} r^{\prime}}{n}, \frac{n^{\prime} r^{\prime}}{n^{4}}$ perinde vt supra computabuntur. Iam simulac $z$ adeoque situs puncti $C^{\prime}$ innotuit, assignare licebit situm circuli maximi $C C^{\prime}$, huius intersectionem cum circulo maximo $A^{\prime \prime} B^{\prime \prime}$ i. e. punctum $C^{\prime \prime}$, et proin arcus $C C^{\prime}$, $C C^{\prime \prime}, C^{\prime} C^{\prime \prime}$ siue $2 f^{\prime \prime}, 2 f^{\prime}, 2 f$ : hinc denique habebitur $r=\frac{n^{\prime} r^{\prime}}{n} \cdot \frac{\sin 2 f}{\sin 2 f^{\prime}}$, $r^{\prime \prime}=\frac{n^{\prime} r^{\prime}}{n^{\prime \prime}} \cdot \frac{\sin 2 f^{\prime \prime}}{\sin 2 f^{\prime \prime}}$.

II. Ad casum eum, vbi punctum $B^{\prime \prime}$ cum $A^{\prime \prime}$ vel cum puncto opposito coincidit, omnia quae modo tradidimus transferre licet, si modo omnia, quae ad locum primum spectant, cum iis, quae ad tertium referuntur, permutantur.

III. Paullo aliter vero casum cum tractare oportet, vbi $B^{\prime}$ vel cum $A^{\prime}$ vel cum puncto opposito coincidit. Hic punctum $C^{\prime}$ cum $A^{\prime}$ coincidet; $\gamma^{\prime}, \varepsilon, \varepsilon^{\prime \prime}$ punctaque $D, D^{\prime \prime}, B^{\star}$ indeterminata erunt: contra assignari poterit intersectio circuli maximi $B B^{\prime \prime}$ cum ecliptica ${ }^{*}$ ), cuius Iongitudo ponatur $=l^{\prime}+\pi$. Per ratiocinia, iis, quae in art. 1 to euoluta sunt, similia, eruetur aequatio

$$
0=n \frac{R \sin \delta \sin \left(A^{\prime \prime} D^{\prime}-\delta^{\prime \prime}\right)}{R^{\prime \prime} \sin \delta^{\prime \prime} \sin \left(A D^{\prime}-\delta\right)}+n^{\prime} r^{\prime} \frac{\sin \pi}{R^{\prime \prime} \sin \left(l^{\prime \prime}-l^{\prime}-\pi\right)}+n^{\prime \prime}
$$

Designemus coëfficientem ipsius $n$, qui conuenit cum $a$ art. 140 , per eundem chaxacterem $a$, coëflicientemque ipsius $n^{\prime} r^{\prime}$ per $\beta$ : ipsum $a$ hic etiam per formulam

\footnotetext{
*) Generalius, cum circulo maximo $A A^{\prime \prime}$ : sod breuitatis caussa eum tantummodo casum hic consideramus, vbi ecliptica tamquam planum fuudamentale accipitur.
} 
$a=-\frac{R \sin \left(l^{\prime}+\pi-l\right)}{R^{\prime \prime} \sin \left(l^{\prime \prime}-l^{\prime}-\pi\right)}$ determinare licet. Habemus itaque $0=a n+\beta n^{\prime} r^{\prime}+n^{\prime \prime}$, qua aequatione cum his combinata $P=\frac{n^{\prime \prime}}{n}, Q=2\left(\frac{n+n^{\prime \prime}}{n^{\prime}}-1\right) r^{\prime 3}$, emergit $\frac{\beta(P+1)}{P+a} r^{\prime 4}+r^{\prime 3}+\frac{r}{2} Q=0$

vnde distantiam $r^{\prime}$ elicere poterimus, siquidem non fuerit $\beta=0$, in quo casu nihil aliud illine sequeretur, nisi $P=-a$. Ceterum etiamsi non fuerit $\beta=0$ (v.bi ad casum tertium in art. sequ. considerandum delaberemur), tamen semper $\beta$ quantitas perexigua erit, adeoque $P$ parum a $-a$ differre debebit: hinc vero manifestum est, determinationem coëfficientis $\frac{\beta(P+1)}{P+\sigma}$ valde lubricam fieri, neque adeo $r$. vlla praecisione determinabilem esse.

Porro habebimus $\frac{n^{\prime} r^{\prime}}{n}=-\frac{P+a}{\beta}, \frac{n^{\prime} r^{\prime}}{n^{n}}=-\frac{P+a}{\beta P}$ : dein simili modo $\mathrm{vt}$ in art. 143 facile euoluentur aequationes

$$
\begin{aligned}
& r \sin \zeta=\frac{n^{\prime} r^{\prime}}{n} \cdot \frac{\sin \gamma^{\prime \prime}}{\sin \zeta^{\prime}} \sin \left(l^{\prime \prime}-l^{\prime}\right) \\
& r^{\prime} \sin \zeta^{\prime \prime}=-\frac{n^{\prime} r^{\prime}}{n^{\prime \prime}} \cdot \frac{\sin \gamma}{\sin \varepsilon^{\prime}} \sin \left(l^{\prime}-l\right) \\
& r \sin \left(\zeta-A D^{\prime}\right)=r^{\prime \prime} P \frac{\sin \gamma^{\prime \prime}}{\sin \gamma} \sin \left(\zeta^{\prime \prime}-A^{\prime \prime} D^{\prime}\right)
\end{aligned}
$$

- quarum combinatione cum aequatt. VIII et IX art. 147 , quantitates $r, \zeta, r^{u}, \zeta^{n}$ determinare licebit. Calculi operationes reliquae cum supra descriptis conuenient.

162.

In casu secundo, vbi $B^{\prime \prime}$ cum $B$ coincidit, etiam $D^{\prime}$ cum iisdem vel cum puncto opposito coincidet. Erunt itaque $A D^{\prime}-\delta$ et $A^{\prime \prime} D^{\prime}-\delta^{\prime \prime}$ vel $=0$ vel $=\mathbf{1} 80^{\circ}$ : vnde ex aequationibus art. 146 deriuamus

$$
\begin{aligned}
& \frac{n^{\prime} r^{\prime}}{n}= \pm \frac{\sin \varepsilon^{\prime}}{\sin \varepsilon} \cdot \frac{R \cdot \sin \delta}{\sin \left(z+A^{\prime} D-\delta^{\prime}\right)} \\
& \frac{n^{\prime} r^{\prime}}{n^{\prime \prime}}= \pm \frac{\sin \varepsilon^{\prime}}{\sin \varepsilon^{\prime \prime}} \cdot \frac{R^{\prime \prime} \sin \delta^{\prime \prime}}{\sin \left(z+A^{\prime} D^{\prime \prime}-\delta^{\prime}\right)}
\end{aligned}
$$

$R \sin \delta \sin \varepsilon^{\prime \prime} \sin \left(z+A^{\prime} D^{\prime \prime}-\delta^{\prime}\right)=P R^{\prime \prime} \sin \delta^{\prime \prime} \sin \varepsilon \sin \left(z+A^{\prime} D-\delta^{\prime}\right)$

Hine manifestum est, $z$, independenter a $Q$, per solam $P$ determinabilem esse (nisi forte fuerit $A^{\prime} D^{\prime \prime}=A^{\prime} D$ vel $=A^{\prime} D \pm 180^{\circ}$, vbi ad casum tertium delaberemur): inuenta autem $z$, innotescet etiam $r^{\prime}$, et proin adiumento valorum quantita- 
DETERMINATIO ORBITAE E TRIBVS OBSERVATIONIBVS COMPLETIS. T9I tum $\frac{n^{\prime} r^{\prime}}{n}, \frac{n^{\prime} r^{\prime}}{n^{\prime \prime}}$ etiam $\frac{n}{n^{\prime}}$ et $\frac{n^{\prime \prime}}{n^{\prime}}$; hinc denique etiam $Q=2\left(\frac{n}{n^{\prime}}+\frac{n^{\prime \prime}}{n^{\prime}}-1\right) r^{\prime 3}$. Manifesto igitur, $P$ et $Q$ tamquam data $a b$ inuicem independentia considerari nequeunt, sed vel rnicum tantummodo datum exhibebunt, vel data incongrua. Situs punctorum $C, C^{\prime \prime}$ in hoc casu arbitrarius manebit, si modo in eodem circulo maximo cum $C^{p}$ capiantur:

In casu terlio, vbi $A^{\prime}, B, B^{\prime}, B^{\prime \prime}$ in eodem circulo maximo iacent, $D$ e é $D^{\prime \prime}$ resp. cum punctis $B^{\prime \prime}, B$, vel cum punctis oppositis coincident: hinc e combinatione aequationum VII, VIII, IX art. 143 colligitur $P=\frac{R^{\prime \prime} \sin \delta^{\prime \prime} \sin \varepsilon}{R \sin \delta \sin \varepsilon^{\prime \prime}}=\frac{R^{\prime \prime} \sin \left(l^{\prime \prime}-l^{\prime}\right)}{R^{\prime \prime} \sin \left(l^{\prime}-l\right)}$. In hoc itaque casu valor ipsius $P$, per ipsa problematis data iam habetur, adeoque positio punctorum $C, C^{\prime}, C^{\prime \prime}$ indeterminata manebit.

$x 65$.

Methodus, quam inde ab art. 136 exposuimus, praecipue quidem determinationi primae orbitae penitus adhuc incognitae accommodata est: attamen successu aeque felici tunc quoque in vsum vocatur, vbi de correctione orbitae proxime iam cognitae per tres obseruationes quantumuis ab inuicem distantes agitur. In tali autem casu quaedam immutare conueniet. Scilicet quoties obseruationes motum heliacentricum pernagnum complectuntur, haud amplius licebit, $\frac{\theta^{\prime \prime}}{\theta}$ atque $\theta \theta^{\prime \prime}$ tamquam valores approximatos quantitatum $P, Q$ considerare: quin potius ex elementis proxime cognitis valores multo magis exacti elici poterunt. Calculabuntur itaque reui calamo per ista elementa pro tribus obseruationum temporibus loca heliocentrica in orbita, vnde designando anomalias veras per $v, v^{\prime}, v^{\prime \prime}$, radios vectores per $r, r^{\prime}, r^{\prime \prime}$, semiparametrum per $p$, prodibunt valores approximati sequentes:

$$
P=\frac{r \sin \left(v^{\prime}-v\right)}{r^{\prime \prime} \sin \left(v^{\prime \prime}-v^{\prime}\right)}, Q=\frac{4 r^{\prime 4} \sin \frac{r}{2}}{p \cos \frac{r}{2}\left(v^{\prime \prime}-v\right) \sin \frac{r}{2}\left(v^{\prime \prime}-v^{\prime}\right)}
$$

His itaque hypothosis prima superstruetur, paullulumque ad libitum immutatis secunda et tertia: haud enim e re esset, $P^{\prime \prime}$ et $Q^{\prime}$ hic pro notis valoribus adoptare (vii supra fecimus), quum hos valores magis exactos euadere haud amplius supponcre liceat. Hac ratione omnes tres hypotheses commodissime simul absolui poterunt: quarta. dein secundum praecepta art. 120 formabitur. Ceternm haud abnuemas, si quis vnam alteramue decem methodorum in artt. $124-129$ expositarum in tali casu si non magis tamen aeque fere expeditam existimet, ideoque in vsum vocare malit. 


\section{SECTIO SECVNDA}

\section{Determinatio orbitae e quatuor obseruationibus, quarum duae tantum completae sunt.}

164.

Iam in ipso limine Libri secundi (art. 115) declarauimus, vsum problematis in Sect. praec. pertractati ad eas orbitas limitari, quarum inclinatio nec euanescit, nec nimis exigua est, determinationemque orbitarum parum inclinatarum necessario quatuor obseruationibus superstrui debere. Quatuor autem obseruationes completae, quum octo aequationibus aequiualeant, incognitarumque numerus ad sex tantum ascendat, problema plus quam determinatum redderent: quapropter a duabus obseruationibus latitudines (siue declinationes) seponere oportebit, vt datis reliquis exacte satisfieri possit. Sic oritur problema, cui haec Sectio dicata erit: solutio autem, quam hic trademus, non solum ad orbitas parum inclinatas patebit, sed etiam ad orbitas inclinationis quantumuis magnae pari successu applicari poterit. Etiam lije, perinde vt in problemate Sect. praec., casum eum, vbi orbitae dimensiones approximatae iam in potestate sunt, segregare oportet a determinatione prima orbitae penitus adhuc incognitae: ab illo initium faciemus.

165.

Methodus simplicissima, orbitam proxime iam cognitam quatuor obseruationibus adaptandi, haec esse videtur. Sint $x, y$ distantiae approximatae corporis coelestis a terra in duabus obseruationibus completis: harum adiumento computentur loci respondentes heliocentrici, atque hinc ipsa elementa: ex his dein elementis longitudines vel ascensiones rectae geocentricae pro duabus reliquis obseruationibus. Quae si forte cum obseruatis conueniunt, elementa nulla amplins correctione egebunt: sin minus, differentiae $X, Y$ notabuntur, idemque calculus iterum bis repetetur, valoribus ipsarum $x, y$ paullulum mutatis. Ita prodibunt tria systemata valorum quantitatum $x, y$ atque differentiarum $X, Y$, vnde per praecepta art. 120 valores correcti quantitatum $x, y$ eruentur, quibus valores $X=0, Y=0$ respondebunt. Calculo itaque simili huic quarto systemati superstructo elementa emergent, per quae ommes quatuor obseruationes rite repraesentabuntur.

Ceterum, siquidem eligendi potestas datur, eas obseruationes completas retinere praestabit, e quibus situm orbitae maxima praecisione determinare licet, proin 
duas obseruationes extremas, quoties motum heliocentricum go graduum minoremue complectuntur. Sin vero praecisione aequali non gaudent, earum latitudines vel declinationes sepones, quas minus exactas esse suspicaberis.

166.

Ad determinationem primam orbitae penitus adhuc incognitae e quatuor obseruationibus necessario eiusmodi positiones adhibendae erunt, quae motum heliocentricum non nimis magnum complectuntur: alioquin enim careremus subsidiis ad approximationem primam commode formandam. Methodus tamen ea quam statim trademus extensione tam lata gaudet, vt absque haesitatione obseruationes motum heliocentricum 30 vel 40 graduum complectentes in vstum vocare liceat, si modo distantiae a Sole non nimis inaequales fuerint: quoties eligendi copia datur, temporum interualla inter primam et secundam, secundam et tertiam, tertiam et quartam $a b$ aequalitate parum recedentia accipere iuuabit. Sed hoc quoque respectu anxietate nimia haud opus erit, vti exemplum subnexum monstrabit, vbi temporum interualla sunt 48,55 et 59 dierum, motusque heliocentricus vltra $50^{\circ}$.

Porro solutio nostra requirit, vt completae sint obseruatio secunda et tertia, adeoque latitudines vel declinationes in obseruationibus extremis negligantur. Supra quidem monuimus, praecisionis maioris gratia plerumque praestare, si elementa duabus obseruationibus extremis completis, atque intermediarum longitudinibus vel ascensionibus rectis accommodentur: attamen in prima orbitae determinatione huic lucro renuntiauisse haud poénitebit, quum approximatio expeditissima longe maioris momenti sit, iacturamque illam, quae praecipue tantum in longitudinem nodi atque inclinationem orbitae cadit, elementaque reliqua vix sensibiliter af ficiat, postea facile explere liceat.

Breuitatis caussa methodi expositionem ita adornabimus, vt omnes locos ad eclipticam referamus, adeoque quatuor longitudines cum duabus latitudinibus datas esse supponemus: attamen quoniam in formulis nostris ad terrae latitudinem qroque respicietur, sponte ad eum casum transferri poterunt, vbi aequator tamquam planum fundamentale accipitur, si modo ascensiones rectae ad declinationes in locum longitudinum et, latitudinum substituuntur.

Ceterum respectu nutationis, praecessionis et parallaxis, nec non aberrationis, omnia quae in Sectione praec. exposuimus etiam hic valent: nisi itaque distantiae approximatae a terra aliunde iam innotuerunt, vt respectu aberrationis methodum $I$ art $x 18$ in vsum vocare liceat, loca obseruata initio tantum ab aber- 
ratione fxarum purgabuntur, temporaque corrigentur, quamprimum inter calculi decursum distantiarum determinatio approximata in potestatem venit, vti infra clarius elucebit.

$$
167 .
$$

nobis

Solutionis expositioni signorum praecipuorum indicem praemittimus. Erunt

$t, t^{\prime}, t^{\prime \prime}, t^{\prime \prime \prime}$ quatuor obseruationum tempora

$\alpha, \alpha^{\prime}, \alpha^{\prime \prime}, \alpha^{\prime \prime \prime}$ corporis coelestis longitudines geocentricae

$\beta, \beta^{\prime}, \beta^{\prime \prime}, \beta^{\prime \prime \prime}$ eiusdem latitudines

$r, r^{\prime}, r^{\prime \prime}, r^{\prime \prime}$ distantiae a Sole

$\varrho, \rho^{\prime}, \rho^{\prime \prime}, \rho^{\prime \prime \prime}$ distantiae a terra

$l, l^{\prime}, l^{\prime \prime}, l^{\prime \prime}$ terrae longitudines heliocentricae

$B, B^{\prime}, B^{n}, B^{\prime \prime}$ terrae latitudines heliocentricae

$\boldsymbol{R}, \boldsymbol{R}^{\prime}, \boldsymbol{R}^{\prime \prime}, \boldsymbol{R}^{\prime \prime \prime}$ terrae distantiae a Sole.

$(n 01),(n 12),(n 25),(n 02),(n 15)$ areae duplicatae triangulorum, quae resp. inter Solem atque corporis coelestis locum primum et secundum, secundum et tertium, tertium et quartum, primum et tertium, secundum et quartum continentuir. $(\eta 01),(\eta 12),(\eta 25)$ quoticntes e diuisione arearum $\frac{x}{2}(n 0 x), \frac{x}{2}(n 12), \frac{x}{2}(n 25)$ pere areas sectorum respondentium oriundi.

$P^{\prime}=\frac{(n 12)}{(n 01)}, P^{n}=\frac{(n 12)}{(n 25)}$

$Q^{\prime}=\left(\frac{(n 01)+(n 12)}{(n 02)}-1\right) r^{\prime 3}, Q^{\prime \prime}=\left(\frac{(n 12)+(n 25)}{(n+5)}-1\right) r^{\prime \prime}$

$v, v^{\prime}, v^{\prime \prime}, v^{\prime \prime \prime}$ corporis coelestis longitudines in orbita a puncto arbitrario numeratae. Denique pro obseruatione secunda et tertia locos heliocentricos terrae in sphaera coelesti per $A^{\prime}, A^{\prime \prime}$ denotabimus, locos geocentricos corporis coelestis per $B^{\prime}, B^{\prime \prime}$, eiusdemque locos heliocentricos per $C^{\prime}, C^{\prime \prime}$.

His ita intellectis negotium primum perinde $v t$ in problemate Sect. praec. (art. 136) consistet in determinatione situs circulorum maximorum $A^{\prime} C^{\prime} B^{\prime}, A^{\prime \prime} C^{\prime \prime} B^{\prime \prime}$, quorum inclinationes ad eclipticam per $\gamma^{\prime}, \gamma^{\prime \prime}$ designamus: cum hoc calculo simul iungetur determinatio arcuum $A^{\prime} B^{\prime}=\delta^{\prime}, A^{\prime \prime} B^{\prime \prime}=\delta^{\prime \prime}$. Hinc manifesto erit

$$
\begin{aligned}
& r^{\prime}=V\left(\rho^{\prime} \varrho^{\prime}+2 \rho^{\prime} R^{\prime} \cos \delta^{\prime}+R^{\prime} R^{\prime}\right) \\
& \left.r^{\prime \prime}=V \rho^{\prime \prime} \varrho^{\prime \prime}+2 \rho^{\prime \prime} R^{\prime \prime} \cos \delta^{\prime \prime}+R^{\prime \prime} R^{\prime \prime}\right)
\end{aligned}
$$

siue statuendo $\rho^{\prime}+R^{\prime} \cos \delta^{\prime \prime}=x^{\prime}, \rho^{\prime \prime}+R^{\prime \prime} \cos \delta^{\prime \prime}=x^{\prime \prime}, R^{\prime} \sin \delta^{\prime}=a, R^{\prime \prime} \sin \delta^{\prime \prime}=a^{\prime \prime}$, 


$$
\begin{aligned}
& r^{\prime}=V\left(x^{\prime} x^{\prime}+a^{\prime} a^{\prime}\right) \\
& r^{\prime \prime}=V\left(x^{\prime \prime} x^{\prime \prime}+a^{\prime \prime} a^{\prime \prime}\right)
\end{aligned}
$$

168.

Combinando aequationes 1 et 2 art. 112 , prodeunt in signis disquisitionis praesentis aequationes sequentes:

$0=(n 12) R \cos B \sin (l-\alpha)-(n 02)\left(\rho^{\prime} \cos \beta^{\prime} \sin \left(\alpha^{\prime}-\alpha\right)+R^{\prime} \cos B^{\prime} \sin \left(l^{\prime}-\alpha\right)\right)$

$$
+(n 02)\left(\rho^{\prime \prime} \cos \beta^{\prime \prime} \sin \left(\alpha^{\prime \prime}-\alpha\right)+R^{\prime \prime} \cos B^{\prime \prime} \sin \left(l^{\prime \prime}-\alpha\right)\right)
$$

$0=(n 25)\left(\rho^{\prime} \cos \beta^{\prime} \sin \left(\alpha^{\prime \prime \prime}-\alpha^{\prime}\right)+R^{\prime} \cos B^{\prime} \sin \left(\alpha^{\prime \prime}-l^{\prime}\right)\right)-(n 15)\left(\varrho^{\prime \prime} \cos \beta^{\prime \prime} \sin \left(\alpha^{\prime \prime \prime}-\alpha^{\prime \prime}\right)\right.$

$$
\left.+R^{\prime \prime} \cos B^{\prime \prime} \sin \left(\alpha^{\prime \prime \prime}-l^{\prime \prime}\right)\right)+(n 12) R^{\prime \prime \prime} \cos B^{\prime \prime \prime} \sin \left(\alpha^{\prime \prime \prime}-l^{\prime \prime}\right)
$$

Hae aequationes, statuendo

$$
\begin{aligned}
& \frac{R^{\prime} \cos B^{\prime} \sin \left(l^{\prime}-\alpha\right)}{\cos \beta^{\prime} \sin \left(\alpha^{\prime}-\alpha\right)}-R^{\prime} \cos \delta^{\prime}=b^{\prime} \\
& \frac{R^{\prime \prime} \cos B^{\prime \prime} \sin \left(\alpha^{\prime \prime \prime}-l^{\prime \prime}\right)}{\cos \beta^{\prime \prime} \sin \left(\alpha^{\prime \prime \prime}-\alpha^{\prime \prime}\right)}-R^{\prime \prime} \cos \delta^{\prime}=b^{\prime \prime} \\
& \frac{R^{\prime} \cos B^{\prime} \sin \left(\alpha^{\prime \prime}-l^{\prime}\right)}{\cos \beta^{\prime} \sin \left(\alpha^{\prime \prime \prime}-\alpha^{\prime}\right)}-R^{\prime} \cos \delta^{\prime}=\varkappa^{\prime} \\
& \frac{R^{\prime \prime} \cos B^{\prime \prime} \sin \left(l^{\prime \prime}-\alpha\right)}{\cos \beta^{\prime \prime} \sin \left(\alpha^{\prime \prime}-\alpha\right)}-R^{\prime \prime} \cos \delta^{\prime \prime}=x^{\prime \prime} \\
& \frac{R \cos B \sin (l-\alpha)}{\cos \beta^{\prime \prime} \sin \left(\alpha^{\prime \prime}-\alpha\right)}=\lambda \\
& \frac{R^{\prime \prime \prime} \cos B^{\prime \prime \prime} \sin \left(\alpha^{\prime \prime \prime}-l^{\prime \prime}\right)}{\cos \beta^{\prime} \sin \left(\alpha^{\prime \prime}-\alpha^{\prime}\right)} \lambda^{\prime \prime \prime} \\
& \frac{\cos \beta^{\prime} \sin \left(\alpha^{\prime}-\alpha\right)}{\cos \beta^{\prime \prime} \sin \left(\alpha^{\prime \prime}-\alpha\right)}=\mu^{\prime} \\
& \frac{\cos \beta^{\prime \prime} \sin \left(\alpha^{\prime \prime}-\alpha^{\prime \prime}\right)}{\cos \beta \sin \left(\alpha^{\prime \prime \prime}-\alpha^{\prime}\right)}=\mu^{\prime \prime}
\end{aligned}
$$

omnibusque rite reductis, transeunt in sequentes

$$
\begin{aligned}
& \frac{\mu^{\prime}\left(1+P^{\prime}\right)\left(x^{\prime}+b^{\prime}\right)}{Q^{\prime}}=x^{\prime \prime}+x^{\prime \prime}+\lambda P^{\prime} \\
& \frac{\mu^{\prime \prime}\left(1+P^{\prime \prime}\right)\left(x^{\prime \prime}+b^{\prime \prime}\right)}{\left(x^{\prime} x^{\prime}+a^{\prime} a^{\prime}\right)^{\frac{3}{2}}} \\
& 1+\frac{Q^{\prime \prime}}{\left(x^{\prime \prime} x^{\prime \prime}+a^{\prime \prime} a^{\prime \prime}\right)^{\frac{3}{2}}}
\end{aligned}
$$


siue, statuendo insuper

in hasce

$$
\begin{aligned}
& -x^{\prime \prime}-\lambda P^{\prime}=c^{\prime}, \quad \mu^{\prime}\left(1+P^{\prime}\right)=d^{\prime} \\
& -x^{\prime}-\lambda^{\prime \prime} P^{\prime \prime}=c^{\prime \prime}, \mu^{\prime \prime}\left(1+P^{\prime \prime}\right)=d^{\prime \prime}
\end{aligned}
$$

$$
\begin{aligned}
& \text { I. } x^{\prime \prime}=c^{\prime}+\frac{d^{\prime}\left(x^{\prime}+b^{\prime}\right)}{1+\frac{Q^{\prime}}{\left(x^{\prime} x^{\prime}+a^{\prime} a^{\prime}\right)^{\frac{3}{2}}}} \\
& \text { II. } x^{\prime}=c^{\prime \prime}+\frac{d^{\prime \prime}\left(x^{\prime \prime}+b^{\prime \prime}\right)}{1+\frac{Q^{\prime \prime}}{\left(x^{\prime \prime} x^{\prime \prime}+a^{\prime \prime} a^{\prime \prime}\right)^{\frac{3}{2}}}}
\end{aligned}
$$

Adiumento harum duarum aequationum $x^{\prime}$ et $x^{\prime \prime}$ ex $a^{\prime}, b^{\prime}, c^{\prime}, d^{\prime}, Q^{\prime}, a^{\prime \prime}, b^{\prime \prime}, c^{\prime \prime}$, $d^{\prime \prime}, Q^{\prime \prime}$, determinari poterunt. Quodsi quidem $x^{\prime}$ vel $x^{\prime \prime}$ inde eliminanda esset, ad aequationem ordinis permagni delaberemur: attamen per methodos indirectas incognitarum $x^{\prime}, x^{\prime \prime}$ valores ex illis aequationibus forma non mutata satis expedite elicientur. Plerumque valores incognitarum approximati iam prodeunt, si primo $Q^{\prime}$ atque $Q^{\prime \prime}$ negliguntur; scilicet

$$
\begin{aligned}
& x^{\prime}=\frac{c^{\prime \prime}+d^{\prime \prime}\left(b^{\prime \prime}+c^{\prime}\right)+d^{\prime} d^{\prime \prime} b^{\prime}}{1-d^{\prime} d^{\prime \prime}} \\
& x^{\prime \prime}=\frac{c^{\prime}+d^{\prime}\left(b^{\prime}+c^{\prime \prime}\right)+d^{\prime} d^{\prime \prime} b^{\prime \prime}}{1-d^{\prime} d^{\prime \prime}}
\end{aligned}
$$

Quamprimum attem valor approximatus alterutrius incognitae habetur, valores aequationibus exacte satisfacientes facillime elicientur. Sit scilicet $\xi$ valor approximatus ipsius $x^{\prime}$, quo in aequatione I substituto prodeat $x^{\prime \prime}=\xi^{\prime \prime}$; perinde substituto $x^{\prime \prime}=\xi^{\prime \prime}$ in aequatione II prodeat inde $x^{\prime}=X^{\prime}$; repetantur eaedem operationes, substituendo pro $x^{\prime}$ in I valorem alium $\xi^{\prime}+\nu^{\prime}$, vnde prodeat $x^{\prime \prime}=\xi^{\prime \prime}+\nu^{\prime \prime}$, quo valore in II substituto prodeat inde $x^{\prime}=X^{\prime}+N$. 'Tum valor correctus ipsius $x^{\prime}$ erit $=\xi^{\prime}+\frac{\left(\xi^{\prime}-X^{\prime}\right) \nu^{\prime}}{N^{\prime}-\nu^{\prime}}=\frac{\xi^{\prime} N^{\prime}-X^{\prime} \nu^{\prime}}{N^{\prime}-\nu^{\prime}}$, valorque correctus ipsius $x^{\prime \prime}=\xi^{\prime \prime}+$ $\frac{\left(\xi-X^{\prime}\right) \nu^{\prime \prime}}{N^{\prime}-\nu^{\prime}}$. Si operae pretium videtur, cum valore correcto ipsius $x^{\prime}$ alioque leuius mutato eaedem operationes repetentur, donec valores ipsarum $x^{\prime}, x^{\prime \prime}$ aequationibus I, II exacte satisfacientes prodierint. Ceterum analystae vel mediocriter. tantum exercitato subsidia calculum contrahendi haud deerunt.

In his operationibus quantitates irrationales $\left(x^{\prime} x^{\prime}+a^{\prime} a^{\prime}\right)^{\frac{3}{2}},\left(x^{\prime \prime} x^{\prime \prime}+a^{\prime \prime} a^{\prime \prime}\right)^{\frac{3}{2}}$ commode calculantur per introductionem arcuum $z^{\prime}, z^{\prime \prime}$, quortum tangentes resp. 
sunt $\frac{a^{\prime}}{x^{\prime}}, \frac{a^{\prime \prime}}{x^{\prime \prime}}$, vnde fit

$$
\begin{aligned}
& \vee\left(x^{\prime} x^{\prime}+a^{\prime} a^{\prime}\right)=r^{\prime}=\frac{a^{\prime}}{\sin z^{\prime}}=\frac{x^{\prime}}{\cos z^{\prime}} \\
& V\left(x^{\prime \prime} x^{\prime \prime}+a^{\prime \prime} a^{\prime \prime}\right)=r^{\prime \prime}=\frac{a^{\prime \prime}}{\sin z^{\prime \prime}}=\frac{x^{\prime \prime}}{\cos z^{\prime \prime}}
\end{aligned}
$$

Hi arcus auxiliares, quos inter o et $180^{\circ}$ accipere oportet, vt $r^{\prime}, r^{\prime \prime}$ positiui euadant, manifesto-cum arcubus $C^{\prime} B^{\prime}, C^{\prime \prime} B^{\prime \prime}$ identici erunt, vnde patet, hacce ratione non modo $r^{\prime}$ et $r^{\prime \prime}$, sed etiam situm punctorum $C^{\prime}, C^{\prime \prime}$ innotescere.

Haecce determinatio quantitatum $x^{\prime}, x^{\prime \prime}$ requirit, vt $a^{\prime}, a^{\prime \prime}, b^{\prime}, b^{\prime \prime}, c^{\prime}, c^{\prime \prime}$, $d^{\prime}, d^{\prime \prime}, Q^{\prime}, Q^{\prime \prime}$ cognitae sint, quarum quantitatum quatuor primae quidem per problematis data habentur, quatuor sequentes autem a $P^{\prime}, P^{\prime \prime}$ pendent. Jam quantitates $P^{\prime}, P^{\prime}, Q^{\prime}, Q^{\prime \prime}$, exacte quidem nondum determinari possunt; attamen guum habeatur

III. $P^{\prime}=\frac{t^{\prime \prime}-t^{\prime}}{t^{\prime}-t} \cdot \frac{(\eta 01)}{(\eta 12)}$

IV. $P^{\prime \prime}=\frac{t^{\prime \prime}-t^{\prime}}{t^{\prime \prime}-t^{\prime \prime}} \cdot \frac{(\eta 25)}{(\eta 12)}$

V. $Q^{\prime}=\frac{\mathrm{r}}{2} k k\left(t^{\prime}-t\right)\left(t^{\prime \prime}-t^{\prime}\right) \frac{r^{\prime} r^{\prime}}{r r^{\prime \prime}} \cdot \frac{x}{(\eta \mathrm{OI})(\eta 12) \cos \frac{\mathrm{r}}{2}\left(v^{\prime}-v\right) \cos \frac{\mathrm{T}}{2}\left(v^{\prime \prime}-v\right) \cos \frac{\mathrm{x}}{2}\left(v^{\prime \prime}-v^{\prime}\right)}$

VI. $Q^{\prime \prime}=\frac{x}{2} k k\left(t^{\prime \prime}-t^{\prime}\right)\left(t^{\prime \prime \prime}-t^{\prime \prime}\right) \cdot \frac{r^{\prime \prime} r^{\prime \prime}}{r^{\prime} r^{\prime \prime \prime}} \cdot \frac{1}{(\eta 12)(\eta 23) \cos \frac{1}{2}\left(v^{\prime \prime}-v^{\prime}\right) \cos \frac{x}{2}\left(v^{\prime \prime \prime}-v^{\prime}\right) \cos \frac{\pi}{2}\left(v^{\prime \prime \prime}-v^{\prime \prime}\right)}$ statim adsunt valores approximati

$$
\begin{aligned}
& P^{\prime}=\frac{t^{\prime \prime}-t^{\prime}}{t^{\prime}-t}, P^{\prime \prime}=\frac{t^{\prime \prime}-t^{\prime}}{t^{\prime \prime \prime}-t^{\prime \prime}} \\
& \left.Q^{\prime}=\frac{1}{2} k k\left(t^{\prime}-t\right)\left(t^{\prime \prime}-t^{\prime}\right), Q^{\prime \prime}=k k\left(t^{\prime \prime}-t^{\prime}\right) t^{\prime \prime}-t^{\prime \prime}\right)
\end{aligned}
$$

quibus calculus primus superstruetur.

$$
169 .
$$

Absoluto calculo art. praec. ante omnia arcum $C^{\prime} C^{\prime \prime}$ determinare oportebit. Quod fiet commodissime, si antea perinde vt in art. 137 intersectio $D$ circulorum maximorum $\mathscr{A}^{\prime} C^{\prime} B^{\prime}, A^{\prime \prime} C^{\prime \prime} B^{\prime \prime}$, mutuaque inclinatio $\varepsilon$ eruta fuerit: inuenietur dein ex $\varepsilon, C^{\prime} D=z^{\prime}+B^{\prime} D$, atque $C^{\prime \prime} D=z^{\prime \prime}+B^{\prime \prime} D$, per formulas easdem quas in art. $14 \pm$ tradidimus, non modo $C^{\prime} C^{\prime \prime}=v^{\prime \prime}-v^{\prime}$, sed etiam anguli $\left(u^{\prime}\right.$, $\left.u^{\prime \prime}\right)$, sub quibus circuli maximi $A^{\prime} B^{\prime}, A^{\prime \prime} B^{\prime \prime}$ circulum maximum $C^{\prime} C^{\prime \prime}$ secant. 
Postquan arcus $v^{\prime \prime}-v^{\prime}$ inuentus est, $v^{\prime}-v$ et $r$ eruentur e combinatione aequationum

$$
\begin{aligned}
& r \sin \left(v^{\prime}-v\right)=\frac{r^{\prime \prime} \sin \left(v^{\prime \prime}-v^{\prime}\right)}{P^{\prime}} \\
& r \sin \left(v^{\prime}-v+v^{\prime \prime}-v^{\prime}\right)=\frac{1+P^{\prime}}{P^{\prime}} \cdot \frac{r^{\prime} \sin \left(v^{\prime \prime}-v^{\prime}\right)}{1+\frac{Q^{\prime}}{r^{\prime 3}}}
\end{aligned}
$$

et perinde $r^{\prime \prime \prime}$ atque $v^{\prime \prime \prime}-v^{\prime \prime}$ e combinatione harum

$$
\begin{aligned}
& r^{\prime \prime \prime} \sin \left(v^{\prime \prime \prime}-v^{\prime \prime}\right)=\frac{r^{\prime} \sin \left(v^{\prime \prime}-v^{\prime}\right)}{P^{\prime \prime}} \\
& r^{\prime \prime \prime} \sin \left(v^{\prime \prime \prime}-v^{\prime \prime}+v^{\prime \prime}-v^{\prime}\right)=\frac{1+P^{\prime \prime}}{P^{\prime \prime}} \cdot \frac{r^{\prime \prime} \sin \left(v^{\prime \prime}-v^{\prime}\right)}{x+\frac{Q^{\prime \prime}}{r^{\prime \prime}}}
\end{aligned}
$$

Omnes numeri hoc modo inuenti exacti forent, si ab initio a valoribus veris ipsarum $P^{\prime}, P^{\prime \prime}, Q^{\prime}, Q^{\prime \prime}$ proficisci licuisset: tumque situm plani orbitae perinde vt in art. 149 vel ex $A^{\prime} C^{\prime}$, $u^{\prime}$ et $\gamma^{\prime}$, vel ex $A^{\prime \prime} C^{\prime \prime}$, $u^{\prime \prime}$ et $\gamma^{\prime \prime}$ determinare conueniret, ipsasque orbitae dimensiones vel ex $r^{\prime}, r^{\prime \prime}, t^{\prime}$, $t^{\prime \prime}$, et $v^{\prime \prime}-v^{\prime}$, vel, quod exactius est, ex $r, r^{\prime \prime}, t, t^{\prime \prime \prime}$, et $v^{\prime \prime \prime}-v$. Sed in calculo primo haec omnia praeteribimus, atque in id potissimum incumbemus, vt valores magis approximatos pro quantitatibus $P^{\prime}, P^{\prime \prime}, Q^{\prime}, Q^{\prime \prime}$ obtineamus. Hunc finem assequemur, si per methodum inde ab art. 88 expositam

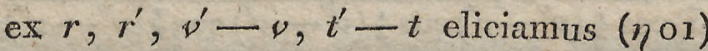

$$
\begin{aligned}
& r^{\prime}, r^{\prime \prime}, v^{\prime \prime}-v^{\prime}, t^{\prime \prime}-t^{\prime} \ldots \ldots \ldots \ldots(\eta 12) \\
& r^{\prime \prime}, r^{\prime \prime}, v^{\prime \prime \prime}-v^{\prime \prime}, t^{\prime \prime \prime}-t^{\prime \prime} \ldots \ldots \ldots \ldots(\eta 25)
\end{aligned}
$$

Has quantitates, nce non valores ipsarum $r, r^{\prime}, r^{\prime \prime}, r^{\prime \prime}, \cos \frac{1}{2}\left(v^{\prime}-v\right)$ ete. in formulis III-VI substituemus, vnde valores ipsarum $P^{\prime}, Q^{\prime}, P^{\prime \prime}, Q^{\prime \prime}$ resultabunt multo magis exacti quam ii, quibus hypothesis prima superstructa erat. Cum illis itaque hypothesis secunda formabitur, quae si prorsus eodem modo vt prima ad finem perducitur, valores ipsarum $P^{\prime}, Q^{\prime}, P^{\prime \prime}, Q^{\prime \prime}$ multo' adhuc exactiores suppeditabit, atque sic ad hypothesin tertiam deducet. Hae operationes tam diu iterabuntur, donec valores ipsarum $P^{\prime}, Q^{\prime}, P^{\prime \prime}, Q^{\prime \prime}$ nulla amplius correctione opus habere videantur, quod recte iudicare exercitatio frequens mox docebit. Quoties motus heliocentricus partuus est, plerumque prima hypothesis illos valores iam satis exacte subministrat: si vero ille arcum maiorem complectitur, si insuper temporum interualla ab aequalitate notabiliter recedunt, hypothesibus pluries repetitis opus erit; in tali vero casu 
hypotheses primae magnam calculi praecisionem haud postulant. In vltima denique hypothesi elementa ipsa ita vt modo indicauimus determinabuntur.

170.

In hypothesi prima quidem temporibus non correctis $t, t^{\prime}, t^{\prime}, t^{\prime \prime \prime}$ vti oportebit, quum distantias a terra computare nondum liceat: simulac vero valorēs approximati quantitatum $x^{\prime}, x^{\prime \prime}$ innotuerunt, illas distantias quoque proxime determinare poterimus. Attamen quum formulae pro $\rho$ et $\rho^{\prime \prime \prime}$ hic paullo complicatiores euadant, computum correctionis temporum eousque differre conueniet, vbi distantiarum valores satis praecisi euaserunt, ne calculo repetito opus sit. Quamóbrem e re erit, hanc operationem iis valoribus quantitatum $x^{\prime}, x^{\prime \prime}$ superstruere, ad quas hypothesis penvltima produxit, ita vt vltima demum hypothesis a valoribus correctis temporum atque quantitatum $P^{\prime}, P^{\prime \prime}, Q^{\prime}, Q^{\prime \prime}$ proficiscatur. Ecce formulas, ad hunc finem in vsum vocandas:

VII. $\rho^{\prime}=x^{\prime}-R^{\prime} \cos \delta^{\prime}$

VIII. $\rho^{\prime \prime}=x^{\prime \prime}-R^{\prime \prime} \cos \delta^{\prime \prime}$

IX. $\rho \cos \beta=-R \cos B \cos (\alpha-l)+\frac{1+P^{\prime}}{P^{\prime}\left(1+\frac{Q^{\prime}}{r^{\prime 3}}\right)}\left(\rho^{\prime} \cos \beta^{\prime} \cos \left(\alpha^{\prime}-\alpha\right)+R^{\prime} \cos B^{\prime} \cos \left(l^{\prime}-\alpha\right)\right)$

$$
-\frac{1}{P^{\prime}}\left(\rho^{\prime \prime} \cos \beta^{\prime \prime} \cos \left(\alpha^{\prime \prime}-\alpha\right)+R^{\prime \prime} \cos B^{\prime \prime} \cos \left(l^{\prime \prime}-\alpha\right)\right)
$$

X. $\rho \sin \beta=-R \sin B+\frac{1+P^{\prime}}{P^{\prime}\left(1+\frac{Q^{\prime}}{r^{3}}\right)}\left(\rho^{\prime} \sin \beta^{\prime}+R^{\prime} \sin B^{\prime}\right)-\frac{1}{P^{\prime}}\left(\rho^{\prime \prime} \sin \beta^{\prime \prime}+R^{\prime \prime} \sin B^{\prime \prime}\right)$

XI. $\rho^{\prime \prime \prime} \cos \beta^{\prime \prime \prime}=-R^{\prime \prime \prime} \cos B^{\prime \prime \prime} \cos \left(\alpha^{\prime \prime \prime}-l^{\prime \prime \prime}\right)+\frac{1+P^{\prime \prime}}{P^{\prime \prime}\left(1+\frac{Q^{\prime \prime}}{r^{\prime \prime}}\right)}\left(\rho^{\prime \prime} \cos \beta^{\prime \prime} \cos \left(\alpha^{\prime \prime \prime}-\alpha^{\prime \prime}\right)+\right.$

$$
\left.R^{\prime \prime} \cos B^{\prime \prime} \cos \left(\alpha^{\prime \prime \prime}-l^{\prime \prime}\right)\right)-\frac{1}{P^{\prime \prime}}\left(\rho^{\prime} \cos \beta^{\prime} \cos \left(\alpha^{\prime \prime \prime}-\alpha^{\prime}\right)+R^{\prime} \cos B^{\prime} \cos \left(\alpha_{-}^{\prime \prime \prime}-l^{\prime}\right)\right)
$$

XII. $\rho^{\prime \prime \prime} \sin \beta^{\prime \prime \prime}=-R^{\prime \prime \prime} \sin B^{\prime \prime \prime}+\frac{1+P^{\prime \prime}}{P^{\prime \prime}\left(1+\frac{Q^{\prime \prime}}{r^{\prime \prime 3}}\right)}\left(\rho^{\prime \prime} \sin \beta^{\prime \prime}+R^{\prime \prime} \sin B^{\prime \prime}\right)$

$$
-\frac{1}{P^{\prime \prime}}\left(\rho^{\prime} \sin \beta^{\prime}+R^{\prime} \sin B^{\prime}\right)
$$

Formulae IX-XII nullo negotio ex aequationibus 1, 2, 3 art. 112 deriuantur, si modo characteres illic adbibiti in eos quibus hic vtimur rite conuertuntur. Mani- 
festo formulae multo simpliciores enadunt, si $B, B^{\prime}, B^{n}$ euanescunt. $\mathbf{E}$ combinatione formularum IX et $\mathbf{X}$ non modo $\rho$ sed etiam $\beta$, et perinde ex XI et XII praeter $r^{\prime \prime \prime}$ etiam $\beta^{\prime \prime \prime}$ demanat: valores harum latitudinum cum obseruatis (calculum non ingredientibus), siquidem datae sunt, comparati ostendent, quonam praecisionis gradu latitudines extremae per elementa sex reliquis datis adaptata repraesentari possint.

$$
77 x .
$$

Exemplum ad illustrationem huius disquisitionis a Vesta desumere conneniet, quae inter omnes planetas recentissime detectos inclinatione ad eclipticam minima gaudet *). Eligimus obseruationes sequentes Bremae, Parisiis, Lilienthalii et Mediolani ab astronomis clarr. Olbers, Bouvard, Bessel et Oriani institutas:

\begin{tabular}{|c|c|c|}
\hline Tempus med. loci obseruationis & Ascensio recta & Declinatio \\
\hline 1807 Martii 50, $12^{\mathrm{h}} 55^{\prime} 17^{\prime \prime}$ & $185^{\circ} 52^{\prime} 40^{\prime \prime} 8$ & $11^{\circ} 54^{\prime} 27^{\prime \prime}$ \\
\hline Maii $17,8 \quad 16 \quad 5$ & $178 \quad 3642,3$ & 113946,8 - \\
\hline Iulii $11,1030 \quad 19$ & $18949 \quad 7,7$ & $3910,1-$ \\
\hline Sept. $\quad 8, \quad 722 \quad 16$ & $21250 \quad 5,4$ & 858 17, o Austr. \\
\hline
\end{tabular}

Pro iisdem temporibus e tabulis motuum Solis inuenimus

\begin{tabular}{|c|c|c|c|c|c|}
\hline & $\begin{array}{l}\text { longit. Solis ab } \\
\text { aequin. app. }\end{array}$ & nutatio & distantia a terra & $\begin{array}{l}\text { Latitudo } \\
\text { Solis }\end{array}$ & $\begin{array}{l}\text { obliquitas eclipt. } \\
\text { apparens }\end{array}$ \\
\hline Martii 30 & $9^{\circ} 21^{\prime} 59^{\prime \prime} 5$ & $+16,8$ & 0,9996448 & $+\mathrm{O}_{2} 3$ & $25^{\circ} 27^{\prime} 50^{\prime \prime} 82$ \\
\hline Maii 17 & 5556 20, о & $+16,2$ & 1,0119789 & $-0,63$ & 49,83 \\
\hline Iulii 11 & 1085453,3 & $+17,3$ & 1,0165795 & $-0,46$ & 49,79 \\
\hline Sept. 8 & 165857,1 & $+x 6,7$ & 1,0067421 & $+0,29$ & 49,26 \\
\hline
\end{tabular}

Iam loca obseruata planetae, adhibita eclipticae obliquitate apparente, in longitudines et latitudines conuersa, a nutatione et aberratione fixarum purgata,

*) Nihilominus haec inclinatio etiamnum satis considerabilis est, vt orbitae determinationem satis tuto atque exacte tribus obseruationibus superstruere liceat: renera elementa prina, quae hoc modo ex obseruationibus 19 tantum diebus ad inuicem distantibus deducta crant (vid. Von Zach Monatl. Corresp. Vol. XV. p. 595), proxime iam accedunt ad ea, quae hic ex obseruationibus quatuor, 162 diebus ad inuicem dissitis, deriuabuntur. 
tandemque demta praecessione ad initium anni 1807 reducta sunt, dein elocis Solis ad normam praeceptorum art. 72 deriuata sunt loca terrae ficta (vt parallaxis ratio habeatur), longitudinesque demta nutatione et praecessione ad eandem epocham translatae; tandem tempora ab initio anni numerata et ad meridianum Parisinum reducta. Hoc modo orti sunt numeri sequentes:

\begin{tabular}{|c|c|c|c|c|}
\hline$x^{\prime} \quad t^{\prime \prime} \quad t^{\prime \prime \prime}$ & 89,505162 & 157,544502 & 192 & \\
\hline$\alpha, \alpha^{\prime}$, & $178^{\circ} 43^{\prime} 38^{\prime \prime} 87$ & $174^{\circ} \quad l^{\prime} 30^{\prime \prime} 08$ & $187^{\circ} 45^{\prime} 42^{\prime \prime} 23$ & $215^{\circ} 54^{\prime} 15^{\prime \prime} 63$ \\
\hline & $1227 \quad 6,16$ & $108 \quad 7,80$ & 64725,51 & 42021,65 \\
\hline $\begin{array}{l}l, l^{\prime}, l^{\prime \prime}, l^{\prime \prime \prime} \\
\log R, R^{\prime}, R^{\prime \prime}, R^{\prime}\end{array}$ & $\begin{array}{r}1892135,71 \\
9,9997990\end{array}$ & $\begin{array}{c}235 \quad 56 \quad 0,63 \\
0,005 \wedge 376\end{array}$ & $\begin{array}{r}288 \quad 3520,32 \\
0,0071739\end{array}$ & $\begin{array}{c}345 \quad 9 \quad 18,69 \\
0,0030625\end{array}$ \\
\hline
\end{tabular}

Hinc deducimus

$y^{\prime}=168^{\circ} 32^{\prime} 4 x^{\prime \prime} 34, \delta^{\prime}=62^{\circ} 23^{\prime} x^{\prime \prime} 88, \log a^{\prime}=9,9526104$

$\gamma^{\prime \prime}=173 \quad 5 \quad 15,68, \delta^{\prime \prime}=100 \quad 451,40, \log a^{\prime \prime}=9,9994859$

$b^{\prime}=-11,009449, x^{\prime}=-1,085306, \log \lambda=0,0728800, \quad \log \mu^{\prime}=9,7159702 n$

$b^{\prime \prime}=-2,082056, x^{\prime \prime}=+6,322006, \log \lambda^{\prime \prime \prime}=0,0798512 n, \log \mu^{\prime \prime}=9,8587061$

$A^{\prime} D=37^{\circ} 17^{\prime} 51^{\prime \prime} 50, A^{\prime \prime} D=89^{\circ} 24^{\prime} 11^{\prime \prime} 84, \varepsilon=9^{\circ} 5^{\prime} 5^{\prime \prime} 48$

$B^{\prime} D=-25 \quad 5 \quad 13,38, B^{\prime \prime} D=-112049,56$

His calculis praeliminaribus absolutis, hypothesin primam aggredimur. E temporum interuallis elicimus

$$
\begin{aligned}
& \log k\left(t^{\prime}-t\right)=9,9155666 \\
& \log k\left(t^{\prime \prime}-t^{\prime}\right)=9,9765359 \\
& \log k\left(t^{\prime \prime \prime}-t^{\prime \prime}\right)=0,005465 t
\end{aligned}
$$

atque hinc valores primos approximatos

hinc porro

$$
\begin{aligned}
& \log P^{\prime}=0,06117, \quad \log \left(1+P^{\prime}\right)=0,33269, \quad \log Q^{\prime}=9,59087 \\
& \log P^{\prime \prime}=9,97107, \log \left(1+P^{\prime \prime}\right)=0,28681, \quad \log Q^{\prime \prime}=9,68097
\end{aligned}
$$

$$
\begin{aligned}
& c^{\prime}=-7,68361, \log d^{\prime}=0,04666 n \\
& c^{\prime \prime}=+2,20771, \log d^{\prime \prime}=0,12552
\end{aligned}
$$

Hisce valoribus, paucis tentaminibus factis, solutio sequens aequationum I, II elicitur:

$$
\begin{aligned}
& x^{\prime}=2,04856 ; z^{\prime}=23^{\circ} 58^{\prime} \cdot 17^{\prime \prime}, \quad \log r^{\prime}=0,34951 \\
& x^{\prime \prime}=1,95745, z^{\prime \prime}=27 \quad 2 \quad 0, \quad \log r^{\prime \prime}=0,34194
\end{aligned}
$$

Ex $z^{\prime}, z^{\prime \prime}$ atque $\varepsilon$ eruimus $C^{\prime} C^{\prime \prime}=v^{\prime \prime}-v^{\prime}=17^{\circ} 7^{\prime} 5^{\prime \prime}$ : hinc $v^{\prime}-v, r, v^{\prime \prime \prime}-v^{\prime \prime}, r^{\prime \prime}$. per aequationes sequentes determinandae erunt: 
vnde eruimus

$\log r \sin \left(v^{\prime}-v\right)=9,74942, \log r \sin \left(w^{\prime}-v+17^{\circ} 7^{\prime} 5^{\prime \prime}\right)=0,07500$

$\log r^{\prime \prime \prime} \sin \left(y^{\prime \prime \prime}-v^{\prime \prime}\right)=9,847^{2} 9, \log r^{\prime \prime} \sin \left(\psi^{\prime \prime \prime}-v^{\prime \prime}+17^{\circ} 7^{\prime} 5^{\prime \prime}\right)=0,10753$

$$
\begin{aligned}
& v^{\prime}-v=14^{\circ} 14^{\prime} 52^{\prime \prime}, \log r=0,55865 \\
& v^{\prime \prime \prime}-v^{\prime \prime}=184833, \log r^{\prime \prime \prime}=0,53887
\end{aligned}
$$

Denique inuenitur $\log (n 01)=0,00426, \log (n 12)=0,00599, \log (n 25)=0,00711$, atque hinc valores correcti ipsarum $P^{\prime}, P^{\prime \prime}, Q^{\prime}, Q^{\prime \prime}$.

$\log P^{\prime}=0,05944, \log Q^{\prime}=9,60574$

$\log P^{\prime \prime}=9,97219, \log Q^{\prime \prime}=9,69581$

quibus hypothesis secunda superstruenda erit. Huius praecipua momenta ita se - habent:

$$
\begin{aligned}
& c^{\prime}=-7,67820, \log d^{\prime}=0,045756 n \\
& c^{\prime \prime}=+2,21061, \log d^{\prime \prime}=0,126054 \\
& x^{\prime}=2,05308, z^{\prime}=25^{\circ} 47^{\prime} 5 i^{\prime \prime}, \log r^{\prime}=0,546747 \\
& x^{\prime \prime}=1,94290, z^{\prime \prime}=271225, \log r^{\prime \prime}=0,35957^{5} \\
& C^{\prime} C^{\prime \prime}=v^{\prime \prime}-v^{\prime}=17^{\circ} 8^{\prime} o^{\prime \prime} \\
& v^{\prime}-v=14^{\circ} 21^{\prime} 36^{\prime \prime}, \log r=0,554687 \\
& v^{\prime \prime}-v^{\prime \prime}=185045, \log r^{\prime \prime}=0,554564 \\
& \log (n 01)=0,004559, \log (n 12)=0,006102, \log (n 23)=0,007280
\end{aligned}
$$

Hinc prodeunt valores denuo correcti ipsarum $P^{\prime}, P^{\prime \prime}, Q^{\prime}, Q^{\prime \prime}$.

$$
\begin{aligned}
& \log P^{\prime}=0,059^{426}, \quad \log Q^{\prime}=9,604749 \\
& \log P^{\prime \prime}=9,972249, \quad \log Q^{\prime \prime}=9,697564
\end{aligned}
$$

quibus si ad tertiam hypothesin progredimur, numeri sequentes resultant:

$$
\begin{aligned}
& c^{\prime}=-7,67815, \log d^{\prime}=0,045729 n \\
& c^{\prime \prime}=+2,21076, \log d^{\prime \prime}=0,126082 \\
& x^{\prime}=2,05255, z^{\prime}=23^{\circ} 48^{\prime} 14^{\prime \prime}, \log r^{\prime}=0,546655 \\
& x^{\prime \prime}=1,94235, z^{\prime \prime}=271249, \log r^{\prime \prime}=0,559276 \\
& C^{\prime} C^{\prime \prime}=v^{\prime \prime}-v^{\prime}=17^{\circ} 8^{\prime} 4^{\prime \prime} \\
& v^{\prime}-v=14^{\circ} 21^{\prime} 49^{\prime \prime}, \log r=0,554522 \\
& y^{\prime \prime}-v^{\prime \prime}=1851 \quad 7, \log r^{\prime \prime \prime}=0,554290 \\
& \log (n 01)=0,004365, \log (n 12)=0,006106, \log (n 25)=0,007290
\end{aligned}
$$

Quodsi iam ad normam praeceptorum art. praec. distantiae a terra supputantur, prodit:

$$
\rho^{\prime}=1,5635, \rho^{\prime \prime}=2,1319
$$




$$
\begin{array}{ll}
\log \rho \cos \beta=0,09876 & \log \varrho^{\prime \prime \prime} \cos \beta^{\prime \prime \prime}=0,42842 \\
\log \rho \sin \beta=9,44252 & \log \varrho^{\prime \prime \prime} \sin \beta^{\prime \prime \prime}=9,50905 \\
\beta=12^{\circ} 26^{\prime} 40^{\prime \prime} & \beta^{\prime \prime \prime}=4^{\circ} 20^{\prime} 59^{\prime \prime} \\
\log \varphi=0,10909 & \log \varrho^{\prime \prime \prime}=\quad 0,42967
\end{array}
$$

Hinc inueniuntur

\begin{tabular}{l|c|c} 
& $\begin{array}{c}\text { Correctiones tem- } \\
\text { porim }\end{array}$ & tempora correcta. \\
\hline I & 0,007555 & 89,497827 \\
II & 0,008921 & 155,355581 \\
III & 0,012165 & 192,407537 \\
IV & 0,015346 & 251,272756
\end{tabular}

vade prodeunt valores quantitatum $P^{\prime}, P^{\prime \prime}, Q^{\prime}, Q^{\prime \prime}$ denuo correcti

$\log P^{\prime}=0,059^{415}, \log Q^{\prime}=9,604782$

$\log P^{\prime \prime}=9972255, \log Q^{\prime \prime}=9,697687$

Tandem si hisce valoribus nouis hypothesis quarta formatur, numeri sequentes prodeunt:

$$
\begin{aligned}
& c^{\prime}=-7,678116, \log d^{\prime}=0,045725 \\
& c^{\prime \prime}=+2,210773, \log d^{\prime \prime}=0,126084 \\
& x^{\prime}=2,032475, z^{\prime}=23^{\circ} 48^{\prime} 16^{\prime \prime} 7, \log r^{\prime}=0,546658 \\
& x^{\prime \prime}=1,942281, z^{\prime \prime}=271251,7, \log r^{\prime \prime}=0,559265 \\
& v^{\prime \prime}-v^{\prime}=17^{\circ} 8^{\prime} 5^{\prime \prime} 1, \frac{1}{2}\left(u^{\prime \prime}+u^{\prime}\right)=176^{\circ} 7^{\prime} 50^{\prime \prime} 5, \frac{x}{2}\left(u^{\prime \prime}-u^{\prime}\right)=4^{\circ} 55^{\prime} 25^{\prime \prime} 6 \\
& v^{\prime}-v=142151,9, \log r=0,554503 \\
& v^{\prime \prime \prime}-v^{\prime \prime}=1851 \quad 9,5, \log r^{\prime \prime}=0,554265
\end{aligned}
$$

Hi numeri ab iis, quos hypothesis tertia suppeditanerat, tam parum differunt, vt iam tuto ad ipsorum elementorum determinationem progredi liceat. Primo situm plani orbitac eruimus. Per praecepta art. 149 inuenitur ex $\gamma^{\prime}, u^{\prime}$ atque $\mathcal{A}^{\prime} C^{\prime}=\delta^{\prime}-z^{\prime}$, inclinatio orbitae $=7^{\circ} 8^{\prime} 14^{\prime \prime} 8$, longitudo nodi ascendentis $105^{\circ} 16^{\prime} 37^{\prime \prime} 2$, argumentum latitudinis in obseruatione secunda $94^{\circ} 36^{\prime} 4^{\prime \prime} 9$, adeoque longitudo in orbita $197^{\circ} 52^{\prime} 42^{\prime \prime} 1$; perinde ex $\gamma^{\prime \prime}, u^{\prime \prime}$ atque $A^{\prime \prime} C^{\prime \prime}=\delta^{\prime \prime}-z^{\prime \prime}$ elicitur inclinatio orbitae $=7^{\circ} 8^{\prime} 14^{\prime \prime} 8$, longitudo modi ascendentis $105^{\circ} 16^{\prime} 57^{\prime \prime} 5$, argumentum latitudinis in obseruatione tertia $111^{\circ} 44^{\prime} 9^{\prime \prime} 7$, adeoque longitudo in orbita $215^{\circ} 0^{\prime} 47^{\prime \prime}-2$. Hine erit longitudo in orbita pro obseruatione prima $185^{\circ} 50^{\prime} 50^{\prime \prime} 2$, pro quarta $255^{\circ} 51^{\prime}$ $56^{\prime \prime} 7$. Quodsi iam ex $t^{\prime \prime \prime}-t, r, r^{\prime \prime \prime}$ atque $v^{\prime \prime \prime}-\nu=50^{\circ} 2 x^{\prime} 6^{\prime \prime} 5$ orbitae dimensiones determinantur, prodit 
Anomalia vera pro loco primo............................29 $95^{\circ} 55^{\prime} 45^{\prime \prime} 7$

Anomalia vera pro loco quarto..........................5 $45.54 \quad 50,2$

Hinc longitudo perihelii.................................249 $57 \quad 6,5$

Anomalia media pro loco primo.......................502 55 52, 6

Anomalia media pro loco quario....................... $34652 \quad 25,2$

Motus medius diurnus sidereus.............................. $978^{\prime \prime} 7216$

Anomalia media pro initio anni $1807 \ldots \ldots \ldots \ldots \ldots \ldots . . .278 \quad 15 \quad 39$, 1

Longitudo media pro eadem epocha....................168 10 45,6

Angulus $\varphi$

5258,1

Logarithmus semiaxis maioris.:

0,572898

Si secundum haecce elementa pro temporibus $t, t^{\prime}, t^{\prime \prime}, t^{\prime \prime \prime}$ correctis loca planetae geocentrica computantur, quatuor longitudines $\operatorname{cum} \alpha, \alpha^{\prime}, \alpha^{\prime \prime}, \alpha^{\prime \prime \prime}$, duaeque latitudines intermediae cum $\beta^{\prime}, \beta^{\prime \prime}$ ad vnam minuti secundi partem decimam conspirant; latitudines extremae vero prodeunt $12^{\circ} 26^{\prime} 45^{\prime \prime} 7$ atque $4^{\circ} 20^{\prime} 40^{\prime \prime} 1$, illa $22^{\prime \prime} 4$ errans defectu, haec $18^{\prime \prime} 5$ excessu. Attamen si manentibus elementis reliquis tantummodo inclinatio orbitae 6 " augeatur, longitudoque nodi $44^{\prime} 40^{\prime \prime}$ diminuatur, errores inter omnes latitudines distributi ad pauca minuta secunda deprimentur, longitudinesque Ieuissimis tantum erroribus afficientur, qui et ipsi propemodun ad nihilum reducentur, si insuper epocha longitudinis $2^{\prime \prime}$ diminuatur. 


\section{SECTIO TERTIA}

\section{Determinatio orbitae obseruationibus quotcunque quam proxime satisfacientis.}

172.

Si obseruationes astronomicae ceterique numeri, quibus orbitarum computus innititur, absoluta praecisione gauderent, elementa quoque, siue tribus obseruationibus siue quatuor superstructa fuerint, absolute exacta statim prodirent (quatenus quidem motus secundum leges Kepleri exacte fieri supponitur), adeoque accitis aliis aliisque obseruationibus confirmari tantum possent, haud corrigi. Verum enim vero quum omnes mensurationes atque obseruationes nostrae nihil sint nisi approximationes ad veritatem, idemque de omnibus calculis illis innitentibus valere debeat, scopum summum omnium computorum circa phaenomena concreta institutorum in eo ponere oportebit, vt ad veritatem quam proxime fieri potest accedamus. Hoc autem aliter fieri nequit, nisi per idoneam combinationem obscruationum plurium, quam quot ad determinationem quantitatum incognitarum absolute requiruntur. Hoc negotium tunc demum suscipere licebit, quando orbitae cognitio approximata iam innotuit, quae dein ita rectificanda est, vt omnibus obseruationibus quam exactissime satisfaciat. Etiamsi haec expressio aliquid vagi implicare videatur, tamen infra principia tradentur, secundum quae problema solutioni legitimae ac methodicae subiicietur.

Praecisionem summam ambire tunc tantummodo operae pretium esse potest, quando orbitae determinandae postrema quasi manus apponenda est. Contra quamdiu spes affuget, mox nouas obseruationes nouis correctionibus occasionem daturas esse, prout res fert plus minusue ab extrema praecisione remittere conuenict, si tali modo operationum prolixitatem notabiliter sublenare licet. Nos vtrique casui consulere studebimus.

$$
175 .
$$

Maximi imprimis momenti est, vt singulae corporis coelestis positiones geocentricae, quibus orbitam, superstruere propositum est, non ex obseruationibus solitariis petitae sint, sed si fieri potest e pluribus ita combinatis, vt errores forte commissi quantum licet sese mutuo destruxerint. Obseruationes scilicet tales, quae paucorum dierum interuallo ab inuicem distant - vel adeo prout res fert inter- 
vallo 15 aut 20 dierum - in calculo non adhibendae erunt tamquam totidem positiones diuersae, sed potius positio ynica inde derinabitur, quae inter cunctas quasi media est, adeoque praecisionem longe maiorem admittit, quam obseruationes singulae seorsim consideratae. Quod negotium sequentibus principiis innititur.

Corporis coelestis loca geocentrica ex elementis approximatis calculata a locis veris parum discrepare, differentiaeque inter haec et illa mutationes lentissimas tantum subire debent, ita vt intra pancorum dierum decursum propemodum pro constantibus haberi queant, vel saltem variationes tamquam temporibus proportionales spectandae sint. Si itaque obseruationes ab omni errore immunes essent, differentiae inter locos obseruatos temporibus $t, t^{\prime}, t^{\prime \prime}, t^{\prime \prime}$ etc. respondentes, eosque qui ex elementis computati sunt, i. e. differentiae tum longitudinum tum latitudinum, sine tum ascensionum rectarum tum declinationum, obsexuatarum a computatis, forent quantitates vel sensibiliter aequales, vel saltem vniformiter lentissimeque increscentes aut decrescentes. Respondeant e. g. illis temporibus ascensiones rectae obseruatae $\alpha, \alpha^{\prime}, \alpha^{\prime \prime}, \alpha^{\prime \prime \prime}$ etc., computatae autem $\sin t \alpha+\delta, \alpha^{\prime}+\delta^{\prime}, \alpha^{\prime \prime}+\delta^{\prime \prime}, \alpha^{\prime \prime \prime}+\delta^{\prime \prime \prime}$ etc.; tunc differentiae $\delta, \delta^{\prime}, \delta^{\prime \prime}, \delta^{\prime \prime \prime}$ etc. a veris elementorum deviationibus eatenus tantun discrepabunt, qualenus obseruationes ipsae sunt erroneae: si itaque illas deuiationes pro omnibus istis obseruationibus tamquam constantes spectare licet, exhibobunt quantitates $\delta, \delta^{\prime}, \delta^{\prime \prime}, \delta^{\prime \prime \prime}$ etc. lotidem determinationes diuersas eiusdem magnitudinis, pro cuius valore correcto ilaque assiminore conueniet medium arithmeticum inter illas determinationes, quatenus quidem nulla adest ratio, cur vnam alteramue praeferamus. Sin vero obseruationibus singulis idem praecisionis gradus haud attribuendus videtur, supponamus praecisionis gradum in singulis resp. proportionalem aestimandum esse numeris $e, e^{\prime}, e^{\prime \prime}, e^{\prime \prime \prime}$ etc., $i$. e. errores his numeris reciproce proportionales in obseruationibus aeque facile committi potuisse; tum sccundum principia infra tradenda valor medius maxime probabilis hand amplius erit medium arithmeticum simplex, sed $=\frac{e e \delta+e^{\prime} e^{\prime} \delta^{\prime}+e^{\prime \prime} e^{\prime \prime} \delta^{\prime \prime}+e^{\prime \prime \prime} e^{\prime \prime \prime} \delta^{\prime \prime \prime}+\text { etc. }}{e e+e^{\prime} e^{\prime}+e^{\prime \prime} e^{\prime \prime}+e^{\prime \prime \prime} e^{\prime \prime}+\text { etc. }}$. Statuendo iam hunc valorem medium $=\Delta$, pro ascensionibus rectis veris assumere licebit resp. $\alpha+\delta-\Delta, \alpha^{\prime}+\delta^{\prime}-\Delta, \alpha^{\prime \prime}+\delta^{\prime \prime}-\Delta, \alpha^{\prime \prime \prime}+\delta^{\prime \prime}-\Delta$, tumque arbitrarium erit, quanam in calculo vtamur. Quodsi vero vel obseruationes temporis interuallo nimis magno ab inuicem distant, aut si orbitae elementa satis approximata nondum inuotuerant, ita $\mathrm{vt}$ non licuerit, horum deuiationes tamquam constantes pro obseruationibus cunctis spectare, facile perspicietur, aliam linc diférentiam tion oriri, nisi quod deuiatio media sic inuenta non tam omnibus obseruationibus 
communis supponenda erit, quam potius ad tempus medium quoddam referenda, quod perinde e singulis temporum momentis deriuare oportet, vt $\Delta$ ex singulis deuiationibus, adeoque generaliter ad tempus $\frac{e e t+e^{\prime} e^{\prime} t^{\prime}+e^{\prime \prime} e^{\prime \prime} t^{\prime \prime}+e^{\prime \prime \prime} e^{\prime \prime \prime} t^{\prime \prime}+\text { etc. }}{e e+e^{\prime} e^{\prime}+e^{\prime \prime} e^{\prime \prime}+e^{\prime \prime \prime} e^{\prime \prime}+\text { etc. }}$. Si itaque summam praecisionem appetere placet, pro eodem tempore locum geocentricum ex elementis computare, ac dein ab errore medio $\Delta$ liberare oportebit, vt positio quam accuratissima emergat: plerumque tamen abunde sufficiet, si error medius ad obseruationem tempori medio proximam referatur. Quae hic de ascensionibus rectis diximus, perinde de declinationibus, aut si mauis de longiludinibus et latitudinibus valent: attamen semper praestabit, immediate ascensiones rectas et declinationes ex elementis computatas cum obseruatis comparare; sic enim non modo calculum magis expeditum lucramur, praesertim si methodis in artt. 53-6o expositis vtimur, sed eo insuper titulo illa ratio se commendat, quod obseruationes incompletas quoque in vsum vocare licet, praetereaque si omnia ad longitudines et latitudines referrentur metuendum esset, ne obseruatio quoad ascensionem recte, quoad declinationem male instituta (vel vice versa) ab vtraque parte deprauetur, atque sic prorsus inutilis euadat. - Ceterum gradus praecisionis medio ita inuento attribuendus secundum principia mox explicanda erit $=V\left(e e+e^{\prime} e^{\prime}+e^{\prime \prime} e^{\prime \prime}\right.$ $+e^{\prime \prime \prime} e^{m}+$ etc. $)$, ita vt quatuor vel nonem obseruationes aeque exactae requirantur, si medium praecisione dupla vel tripla gaudere debet, et sic porro.

$$
174 .
$$

Si corporis coelestis orbita secundum methodos in Sectionibus praecc. traditas e tribus quatuorue positionibus geocentricis talibus determinata est, quae ipsae singulae ad normam art. praec. e compluribus obseruationibus petitae fuerant, orbita ista inter omnes hasce obseruationes medium quasi tenebit, neque in differentiis inter locos obseruatos et calculatos vllum ordinis vestigium remanebit, quod per elementorum correctionem tollere vel sensibiliter extenuare liceret. Iam quoties tota obseruationum copia interuallum temporis non nimis magnum complectitur, hoc modo consensum exoptatissimum elementorum cum omnibus obseruationibus assequi licebit, si modo tres quatuorue positiones quasi normales scite eligantur. In determinandis orbitis cometarum planetarumue nouorum, quorum obsernationes annum vrum nondum egrediuntur, ista ratione plerumque tantum proficiemus, quantum ipsa rei natura permittit. Quoties itaque orbita determinanda angulo considerabili versus eclipticam inclinata est, in genere tribus obseruationibus 
superstructur, quas quam remotissimas ab innicem eligemus: si vero hoc pacto in aliquem casuum supra exclusorum (arit. $160-162$ ) fortuito incideremus, aut quoties orbitae inclinatio nimis parua videtur, determinationem ex poșitionibus quatuor praefcremus, quas itidem quam remotissimas ab inuicem accipiemus.

Quando autem iam adest obseruationum series longior plures annos complectens, plures inde positiones normales deriuari poterunt: quamobrem praecisioni maximae male consuleremus, si ad orbitae determinationem tres tantum quatuorue positiones excerperemus, omnesque reliquas omnino negligeremus. Quin potitis in tali casu, si summam praccisionem assequi propositum est, operam dabimus, vt positiones exquisitas quam plurimas congeramus, atque in vsum rocemus. Tunc itaque aderunt data plura, quas, ad incognitarum determinationem requiruntur: sed omnia ista data erroribus vtut exiguis obnoxia erunt, +ita vt generaliter impossibile sit, omnibus ex asse satisfacere. Iam quum nulla adsit ratio, cur ex hisco datis sex hacc vel illa tamquam absolute exacta consideremus, sed potius, secundum probabilitatis principia, in cunctis promiscue errores maiores vel minores aeque possibiles supponere oporteat; porro quum generaliter loquendo errores leuiores saepius committantur quam grauiores; manifestum est, orbitam talem, quae dum sex datis ad amussim satisfacit a reliquis plus minusue deuiat, principiis calcali probabilitatis minus consentaneam censendam esse, quam aliam, quae dum ab illis quoque sex datis aliquantulum discrepat, consensum tanto meliorem cum reliquís praestat. Inuestigatio orbitae, sensu stricto maximam probabilitatem prae se ferentis a cognitione legis pendebit, secundum quam errorum crescentium probabilitas decrescit: illa vero a tot considerationibus ragis vel dubiis - physiologicis quoque - pendet, quae calculo subiici nequeunt, vt huiusmodi legem vix ac ne vix quidem in vllo astronomiae practicae casu rite assignare liceat. Nihilominus indagatio nexus inter hanc legem orbitamque maxime probabilem, quam summa iam generalitate suscipiemus, neutiquam pro speculatione sterili habenda erit.

$$
175 .
$$

Ad hunc finem a problemate nostro speciali ad disquisitionem generalissimam in omni calculi ad philosophiam naturalem applicatione foecundissimam ascendemus. Sint $V, V^{\prime}, V^{\prime \prime}$ etc. functiones incognitarum $p, q, r, s$ etc., $\mu$ multitudo illarum functionum, $\nu$ multitudo incognitarum, supponamusque, per obseruationes, immediatas valores functionum ita inuentos esse $V=M, V^{\prime}=M^{\prime}, V^{\prime \prime}=M^{\prime \prime}$ etc. Generaliter itaque loquendo euolutio valorum incognitarum constituet problema indeter- 
minatum, determinatum, vel plus quam determinatum, prout fuerit $\mu<\nu, \mu=\nu$, vel $\mu>\nu^{*}$ ). Hic de vltimo tantum casu sermo erit, in quo manifesto exacta cunctarum obseruationum repraesentatio tunc tantum possibilis foret, vbi illae omnes ab erroribus absolute immunes essent. Quod quum in rerum natura locum non habeat, omne systema valorum incognitarum $p, q, r, s$ etc. pro possibili habendum erit, ex quo valores functionum $V-M, V^{\prime}-I^{\prime}, V^{\prime \prime}-I^{\prime \prime}$ etc. oriuntur, limitibus errorum, qui in istis obseruationibus committi potuerunt, non maiores: quod tamen neutiquam ita intelligendum est, ac si singula haec systemata possibilia aequali probabilitatis_gradu gauderent.

Supponemus primo, etm rerum statum fuisse in omnibus obseruationibus; vt nulla ralio adsit, cur aliam alia minus exactam esse suspicemur, siue vt errores aeque magnos in singulis pro aeque probabilibus habere oporteat. Probabilitas itaque cuilibet exrori $\Delta$ tribuenda exprimetur per functionem ipsitus $\Delta$, quam per $\varphi \Delta$ denotabimus. Iam etiamsi hanc functionem praccise assignare non liceat, saltem affirmare possumus, eius valorem fieri debere maximum pro $\Delta=0$, plerumque aequalem esse pro valoribus aequalibus oppositis ipsius $\Delta$, denique euanescere, si pro $\Delta$ accipiatur error maximus vel maior valor. Proprie itaque $\varphi \Delta$ ad functionum discontinuarum genus referre oportet, et si quam functionem analyticam istius loco substituere ad vsus practicos nobis permittimus, haec ita comparata esse debebit, vt vtrimque a $\Delta=0$ asymptotice quasi ad o conuergat, ita vt vitra istum limitem tamquam vere euanescens considerari possit. Porro probabilitas, errorem iacere inter limites $\Delta$ et $\Delta+d \Delta$ differentia infinite parua $\mathrm{d} \Delta$ ab inuicem distantes, exprimenda erit per $\varphi \Delta \cdot d \Delta$; proin generaliter probabilitas, errorem iacere inter $D$ et $D^{\prime}$, exhibebitur per integrale $\int \varphi \Delta \cdot d \Delta$, a $\Delta=D$ rsque ad $\Delta=D^{\prime}$. extensum. Hoc integrale a valore maximo negatino ipsius $\Delta$ vsque ad valorem maximum positiuum, siue generalius a $\Delta=-\infty$ vsque ad $\Delta=+\infty$ sumtum, ne cessario fieri debet $=1$.

Supponendo igitur, systema aliquod determinatum valorum quantitatum $p$, $q, r, s$ etc. locum habere, probabilitas, pro $V$ ex obseruatione proditurum esse valorem $M$, exprimetur per $\varphi(M-V)$, substitulis in $V$ pro $p, q, r, s$ etc. valo-

*) Si in casu tertio functiones $V, V^{\prime}, V^{\prime \prime}$, etc. ita comparatae essent, vt $\mu+1-\nu$ ex ipsis vel plures tamquam functiones reliquarum spectare liceret, problema respectu harum functionum etiamnum plus quam determinatum foret, respectu quantitatum $p, q, r, s$ etc. autem indeterminatum : harum scilicet valores ne tunc quidem determinare liceret, quando valores functionum $V, V^{\prime}, V^{\prime \prime}$ etc, absolute exacti dati essent: sed hune casum a disquisitione nostra excludemus. 
ribus suis; perinde $\varphi\left(M^{\prime}-V^{\prime}\right), \varphi\left(M^{\prime \prime}-V^{\prime \prime}\right)$ etc. expriment probabilitates, ex obseruationibus resultaturos esse functionum $V^{\prime}, V^{\prime \prime}$ etc. valores $M^{\prime}$, $M^{\prime \prime}$ etc. Quamobrem quandoquidem omnes obseruationes tamquam euentus ab inuicem independentes spectare licet, productum

$$
\varphi(M-V) \cdot \varphi\left(M^{\prime}-V^{\prime}\right) \cdot \varphi\left(M^{\prime \prime}-V^{\prime \prime}\right) \text { etc. }=\Omega
$$

exprimet exspectationem seu probabilitatem, omnes istos valores simul ex obseruationibus prodituros esse.

$$
176 .
$$

Iam perinde, vt positis valoribus incognitarum determinatis quibuscunque; cuiuis systemati valorum functionum $V, V^{\prime}, V^{\prime \prime}$ etc. ante obseruationem factam probabilitas determinata competit, ita vice versa, postquam ex obseruaiionibus valores determinati functionum prodierunt, ad singula systemata valorum incognitarum, e quibus illi demanare potuerunt, probabilitas determinata redundabit: manifesto enim systemata ea pro magis probabilibus habenda erunt, in quibus euentus eius qui prodiit exspectatio maior affuerat. Huiusce probabilitatis aestimatio sequenti theoremati innititur:

Si posita hypothesi aliqua $\mathbf{H}$ probabilitas alicuius euentus determinati $\mathbf{E}$ est $=\mathrm{h}$, posita autem hypothesi alia $\mathrm{H}^{\prime}$ illan excludente et per se aeque probabili eiusdem euentus probabilitas est $=h^{\prime}$ : tum dico, quando euentus $\mathrm{E}$ reuera apparuerit, probabilitatem, quod H fuerit vera hypothesis, fore ad probabilitatem, quod $\mathrm{H}^{\prime}$ fuerit hypothesis vera, vt had $\mathrm{h}^{\prime}$.

Ad quod demonstrandum supponamus, per distinctionem omnium circumstantiarum, a quibus pendet, num $H$ aut $H^{\prime}$ aut alia hypothesis locum habeat, vtrum euentus $E$ an alius emergere debeat, formari systema quoddam casuum diuersorum, qui singuli per se (i.e. quamdiu incertum est, vtrum euentus $E$ an alius proditurus sit) tamquam aeque probabiles considerandi sint, hosque casus ita distribui,

\begin{tabular}{c|c|c}
$\begin{array}{c}\text { vt inter ipsos } \\
\text { reperiantur }\end{array}$ & $\begin{array}{c}\text { vbi locum habere } \\
\text { debet hypothesis }\end{array}$ & $\begin{array}{c}\text { cum modificationibus talibus } \\
\text { vt prodire debeat euentus }\end{array}$ \\
\hline$m$ & $H$ & $E$ \\
$m^{\prime}$ & $H$ & ab diversus \\
$n^{\prime}$ & $H^{\prime}$ & $E$ \\
$m^{m}$ & $H^{\prime}$ & ab $E$ diuersus \\
$n^{n}$ & ab $H$ et $H^{\prime}$ diuersa & $E$ \\
ab $H$ et $H^{\prime}$ diuersa & ab $E$ diuersus
\end{tabular}


Tunc exit $h=\frac{m}{m+n}, h^{\prime}=\frac{m^{\prime}}{m^{\prime}+n^{\prime}} ;$ porro ante euentum cognitum probabili tas hypothesis $H$ erat $=\frac{m+n}{m+n+m^{\prime}+n^{\prime}+m^{\prime \prime}+n^{\prime \prime}}$, post enentum cognitum autem, vbi casus $n, n^{\prime}, n^{\prime \prime}$ e possibilium numero abeunt, eiusdem hypothesis probabilitas erit $=\frac{m}{m+m^{\prime}+m^{\prime \prime}} ;$ perinde hypothesis $H^{\prime}$ probabilitas ante et post euentum resp. exprimetur per $\frac{m^{\prime}+n^{\prime}}{m+n+m^{\prime}+n^{\prime}+m^{\prime \prime}+n^{\prime \prime}}$ et $\frac{m^{\prime}}{m+m^{\prime}+m^{\prime \prime}}$ : quoniam itaque hypothesibus $H$ et $H^{\prime}$ ante euentum cognitum eadem probabilitas supponitur, erit $m+n=m^{\prime}+n^{\prime}$, vnde theorematis veritas sponte colligitur.

Iam quatenus supponimus, praeter obseruationes $V=M, V^{\prime}=M^{\prime}, V^{\prime \prime}=M^{*}$ etc. nulla alia data ad incognitarum determinationem adesse, adeoque omnia systemata valorum harum incognitarum ante illas obseruationes aeque probabilia fuisse, manifesto probabilitas cuiusuis systematis determinati post illas obseruationes ipsi $\Omega$ proporlionalis erit. Hoc ita intelligendum est, probabilitatem, quod valores incognitarum resp. iaceant inter limites infinite vicinos $p$ et $p+\mathrm{d} p, q$ et $q+\mathrm{d} q, r$ et $r$ $+\mathrm{d} r, s$ et $s+\mathrm{d} s$ etc., exprimi per $2 \Omega \mathrm{d} p \mathrm{~d} q \mathrm{~d} r \mathrm{~d} s$ etc. vbi $\lambda$ erit quantitas constans a $p, q, r, s$ etc. independens. Et quidem manifesto erit $\frac{1}{\lambda}$ valor integralis ordinis $\nu^{t i} \rho^{\nu} \Omega \mathrm{d} p \mathrm{~d} q \mathrm{~d} r \mathrm{~d} s . . . .$, , singulis variabilibus $p, q, r, s$ etc. a valore $-\infty$ vsque ad valorem $+\infty$ extensis.

$777^{\circ}$

Hine iam sponte sequitur, systema maxime probabile valorum quantitatum $p, q, r, s$ etc. id fore, in quo $\Omega$ valorem maximum obtineat, adeoque ex $\nu$ aequationibus $\frac{\mathrm{d} \Omega}{\mathrm{d} p}=0, \frac{\mathrm{d} \Omega}{\mathrm{d} q}=0, \frac{\mathrm{d} \Omega}{\mathrm{d} r}=0, \frac{\mathrm{d} \Omega}{\mathrm{d} s}=0$ etc. eruendum esse. Hae aequationes, statuendo $V-M=v, V^{\prime}-M^{\prime}=v^{\prime}, V^{\prime \prime}-M^{\prime \prime}=v^{\prime \prime}$ etc., atque $\frac{d \varphi \Delta}{\varphi \Delta \cdot d \Delta}=\varphi^{\prime} \Delta$, formam sequentem nanciscuntur:

$$
\begin{aligned}
& \frac{\mathrm{d} v}{\mathrm{~d} p} \varphi^{\prime} v+\frac{\mathrm{d} v^{\prime}}{\mathrm{d} p} \varphi^{\prime} v^{\prime}+\frac{\mathrm{d} v^{\prime \prime}}{\mathrm{d} p} \varphi^{\prime} v^{\prime \prime}+\text { etc. }=0 \\
& \frac{\mathrm{d} \psi}{\mathrm{d} q} \varphi^{\prime} v+\frac{\mathrm{d} v^{\prime}}{\mathrm{d} q} \varphi^{\prime} v^{\prime}+\frac{\mathrm{d} v^{\prime \prime}}{\mathrm{d} q} \varphi^{\prime} v^{\prime \prime}+\text { etc. }=0
\end{aligned}
$$




$$
\begin{aligned}
& \frac{d v}{d r} \varphi^{\prime} v+\frac{d v^{\prime}}{d r} \varphi^{\prime} v^{\prime}+\frac{d v^{\prime \prime}}{d r} \varphi^{\prime} v^{\prime \prime}+\text { etc. }=0 \\
& \frac{d}{d s} \varphi^{\prime} v+\frac{d v^{\prime}}{d s} \varphi^{\prime} v^{\prime}+\frac{d v^{\prime \prime}}{d s} \varphi^{\prime} v^{\prime \prime}+\text { etc. }=0
\end{aligned}
$$

Hinc itaque per eliminationem problematis solutio plene determinata derinari poterit, quamprimum functionis $\varphi^{\prime}$ indoles innotuit. Quae quoniam a priori definiri nequit, rem ab altera parte aggredientes inquiremus, cuinam fnnctioni, tacite quasi pro basi acceptae, proprie innixum sit principium triuium, cuius praestantia generaliter agnoscitur. Axiomatis scilicet loco haberi solet hypothesis, si quae quantitas per plures obseruationes immediatas, sub aequalibus circumstantiis aequalique cura institutas, determinata fuerit, medium arithmeticum inter omnes valores obseruatos exhibere valorem maxime probabilem, si non absoluto rigore, tamen proxime saltem, ita vt semper tutissimum sit illi inhaerere. Statuendo itaque $V=V^{\prime}=V^{\prime \prime}$ etc. $=p$, generaliter esse debebit $\varphi^{\prime}(M-p)+\varphi^{\prime}\left(M^{\prime}-p\right)+$ $\varphi^{\prime}\left(M^{\prime \prime}-p\right)+$ etc. $=0$, si pro $p$ substituitur valor $\frac{1}{\mu}\left(M+M^{\prime}+M^{\prime \prime}+\right.$ etc. $)$, quemcunque integrum positiuum exprimat $\mu$. Supponendo itaque $M^{\prime}=M^{\prime \prime}=$ etc. $=I I-\mu N$, erit generaliter, i. e pro quouis valore integro positiuo ipsius $\mu$, $\varphi^{\prime}(\mu-1) N=(1-\mu) \varphi^{\prime}(-N)$, vnde facile colligitur, generaliter esse debere $\frac{\varphi^{\prime} \Delta}{\Delta}$ quantitatem constantem, quam per $k$ designabimus. Hinc fit $\log \varphi \Delta=$ $\frac{\pi}{2} k \Delta \Delta+$ Const., siue designando basin logaxithmortum hyperbolicorum per e, supponendoque Const. $=\log x$,

$$
\Delta \varphi=x e^{\frac{1}{2} k \Delta \Delta}
$$

Porro facile perspicitur, $k$ necessario negatiuam esse debere, quo $\Omega$ reuera fieri possit maximum, quamobrem statuemus $\frac{\pi}{2} k=-h h$; et quum per theorema elogans primo ab ill. Laplace invientum, integrale $\int e^{-h h \Delta s} d \Delta$, a $\Delta=-\infty$ vsque ad $\Delta=+\infty$, fiat $=\frac{\sqrt{ } \pi}{h}$, (denotando per $\pi$ semaicircumferentiam circuli cuius radius 1), functio nostra fiet

$$
\varphi \Delta=\frac{h}{\sqrt{\pi}} e^{-\operatorname{hh} \Delta \Delta} \quad 178
$$

Functio modo eruta omni quidem rigore errorum probabilitates exprimere certo non potest: quum enim errores possibiles semper limitibus certis coërceantur, 
errorum maiorum probabilitas semper euadere deberet $=0$, dum formula nostra semper valorem finitum exhibet. Attamen hic defectus, quo omnis functio analytica natura sua laborare debet, ad omnes vsus practicos nullius momenti est, quum valor functionis nostrae tam rapide de̋crescat, quamprimum $h \Delta$ valorem considerabilem acquisiuit, vt tuto ipsi o aequiualens censeri possit. Praeterea ipsos errorum limites absoluto rigore assignare, rei natura numquam permittet.

Ceterum constans $h$ tamquam mensura praecisionis obseruationum considerari poterit. Si enim probabilitas erroris $\Delta$ in aliquo obseruationum systemate per $\frac{h}{\sqrt{\pi}} e^{-\mathrm{hh \Delta \Delta}}$, in alio rero systemate obseruationum magis minusue exactarum per $\frac{h^{\prime}}{\sqrt{\pi}} e^{-h^{\prime} h^{\prime} \Delta \Delta}$ exprimi concipitur, exspectatio, in obseruatione aliqua e systemate priori errorem inter limites $-\delta$ et $+\delta$ contineri, exprimetur per integrale $\int \frac{h}{\sqrt{\pi}} e^{-\operatorname{th} \Delta \Delta} \mathrm{d} \Delta$ a $\Delta=-\delta$ vsque ad $\Delta=+\delta$ sumtum, et perinde exspectatio, errorem alicuius obseruationis e systemate posteriori limites $-\delta^{\prime}$ et $+\delta^{\prime}$ non egredi, exprimetur per integrale $\int \frac{h^{\prime}}{\sqrt{\pi}} e^{-h^{\prime} h^{\prime} \Delta \Delta} \mathrm{d} \Delta$ a $\Delta=-\delta^{\prime}$ vsque ad $\Delta=+\delta^{\prime \prime}$ extensum: ambo autem integralia manifesto aequalia fiunt, quoties habeiur $h \delta=h^{\prime} \delta^{\prime}$. Quodsi igitur e. g. $h^{\prime}=2 h$, aeque facile in systemate priori error duplex committi poterit, ac simplex in posteriori, in quo casu obseruationibus posterioribus secundum vulgarem loquendi morem praecisio duplex tribuitur.

\section{9.}

Iam ca quae ex hac lege sequuntur euoluemus. Sponte patet, vt produ$\operatorname{ctum} \Omega=h^{\mu} \pi^{-\frac{T}{2} \mu} e^{-\operatorname{lih}\left(v \nu+v^{\prime} v^{\prime}+v^{\prime \prime} \nu^{\prime \prime}+\cdots\right)}$ fiat maximum, aggregatum $v v+v^{\prime} v^{\prime}+v^{\prime \prime} v^{\prime \prime}$ + etc. minimum fieri debere. Systema itaque maxime probabile valorum incognitarum $\mathrm{p}, \mathrm{q}, \mathrm{r}$, s etc. id erit, in quo quadrata differentiarum inter functionum $\mathrm{V}, \mathrm{V}^{\prime}, \mathrm{V}^{\prime \prime}$ etc. valores obseruatos et computatos summam minimam efficiunt, siquidem in omn bus obseruationibus idem praecisionis gradus praesumendus est.

Hocce principium, quod in omnibus applicationibus mathesis ad philosophiam. naturalem vsum frequentissimum offert, vbique axiomatis loco eodem iure valere debet, quo medium arithmeticum inter plures valores obseruatos eiusdem quantitatis tamquam valor maxime probabilis adoptatur. 
Ad obseruationes praecisionis inaequalis principium nullo iam negotio extendi potest. Scilicet si mensura praecisionis obseruationum, per quas inuentum est $V=M, V^{\prime}=M^{\prime}, V^{\prime \prime}=M^{\prime \prime}$ etc. resp. per $h, h^{\prime}, h^{\prime \prime}$ etc. exprimitur, i. e. si supponitur, errores his quantitatibus reciproce proportionales in istis obseruationibus aeque facile committi potuisse, manifesto hoc idem erit, ac si per obseruationes praecisionis aequalis (cuius mensura $=1$ ) valores functionum $h V, h^{\prime} V^{\prime}, h^{\prime \prime} V^{\prime \prime}$ etc. immediate inuenti essent $=h I M, h^{\prime} I M^{\prime}, h^{\prime \prime} I^{\prime \prime}$ etc.: quamobrem systema maxime probabile valorum pro quantitatibus $p, q, r, s$ etc, id erit, vbi aggregatum $h h v v+$ $h^{\prime} h^{\prime} v^{\prime} v^{\prime}+h^{\prime \prime} h^{\prime \prime} v^{\prime \prime} v^{\prime \prime}+$ etc. i. e. vbi summa quadratorum differentiarum inter valores reuera obseruatos et computatos per numeros qui praecisionis gradum metiuntur multiplicatarum fit minimum. Hoc pacto ne necessarium quidem est, vt functiones $V, V^{\prime}, V^{\prime \prime}$ etc. ad quantitates homogeneas referantur, sed heterogeneas quoque (e. g. minuta secunda arcuum et temporis) repraesentare poterunt, si modo rationem errorum, qui in singulis aeque facile committi potuerunt, aestimare licet.

180.

Principium in art. praec. expositum eo quoque nomine se commendat, quod determinatio incognitarum numerica ad algorithmum expeditissimum reducitur, quoties functiones $V, V^{\prime}, V^{\prime \prime}$ etc, lineares sunt. Supponamus esse

$$
\begin{aligned}
& V-I M=v=-m+a p+b q+c r+d s+\text { etc. } \\
& V^{\prime}-M^{\prime}=v^{\prime}=-m^{\prime}+a^{\prime} p+b^{\prime} q+c^{\prime} r+d^{\prime} s+\text { etc. } \\
& V^{\prime \prime}-I^{\prime \prime}=v^{\prime \prime}=-m^{\prime \prime}+a^{\prime \prime} p+b^{\prime \prime} q+c^{\prime \prime} r+d^{\prime \prime} s+\text { etc. }
\end{aligned}
$$

etc., statuamusque

$$
\begin{aligned}
& a v+a^{\prime} v^{\prime}+a^{\prime \prime} v^{\prime \prime}+\text { etc. }=P \\
& b v+b^{\prime} v^{\prime}+b^{\prime \prime} v^{\prime \prime}+\text { etc. }=Q \\
& c v+c^{\prime} v^{\prime}+c^{\prime \prime} v^{\prime \prime}+\text { etc. }=R \\
& d v+d^{\prime} v^{\prime}+d^{\prime \prime} v^{\prime \prime}+\text { etc. }=S
\end{aligned}
$$

etc. Tunc $y$ aequationes art. 177 , e quibus incognitarum valores determinare oportet, manifesto hae erunt:

$$
P=0, Q=0, R=0, S=0 \text { etc. }
$$

siquidem obseruationes aeque bonas supponimus, ad quem casum reliquos reducere in art. praec. docuimus. Adsunt itaque totidem aequationes lineares, quot incognitae determinandae sunt, vnde harum valores per eliminationem vulgarem elicientur.

Videamus nunc, vtrum haec eliminatio semper possibilis sit, an vmquam solutio indeterminata vel adeo impossibilis euadere possit. Ex eliminationis theoria 
constat, casum secundum vel tertium tunc locum habiturum esse, quando ex aequationibus $P=0, Q=0, R=0, S=0$ etc., omissa vna, aequatio conflari potest vel identica cum omissa vel eidem repugnans, siue quod eodem redit, quando assignare licet functionem linearem $\alpha P+\beta Q+\gamma R+\delta S+$ etc., quae fit identice vel $=0$ vel saltem ab omnibus incognitis $p, q, r, s$ etc. libera. Supponamus itaque fieri $\alpha P+\beta Q+\gamma R+\delta S+$ etc. $=\varkappa$. Sponte habetur aequatio identica

$$
(v+m) v+\left(v^{\prime}+m^{\prime}\right) v^{\prime}+\left(v^{\prime \prime}+m^{\prime \prime}\right) v^{\prime \prime}+\text { etc. }=p P+q Q+r R+s S+\text { etc. }
$$

Quodsi itaque per substitutiones $p=\alpha x, q=\beta x, r=\gamma x, s=\delta x$ etc. functiones $\nu$, $\dot{v}^{\prime}, \nu^{\prime \prime}$ etc. resp. in $-m+\lambda x,-m^{\prime}+\lambda^{\prime} x, m^{\prime \prime}+\lambda^{\prime \prime} x$ etc. transire supponimus, manifesto aderit aequatio identica

\section{$\left(\lambda \lambda+\lambda^{\prime} \lambda^{\prime}+\lambda^{\prime \prime} \lambda^{\prime \prime}+\right.$ etc. $) x x-\left(\lambda m+\lambda^{\prime} m^{\prime}+\lambda^{\prime \prime} m^{\prime \prime}\right.$ etc. $) x=x x$}

i. e. erit $\lambda \lambda+\lambda^{\prime} \lambda^{\prime}+\lambda^{\prime \prime} \lambda^{\prime \prime}+$ etc. $=0, x+2 m+\lambda^{\prime} m^{\prime}+\lambda^{\prime \prime} m^{\prime \prime}+$ etc. $=0$ : hinc vero necessario esse debebit $\lambda=0, \lambda^{\prime}=0, \lambda^{\prime \prime}=0$ etc. atque $x=0$. Hinc patet, functiones omnes $V, V^{\prime}, V^{\prime \prime}$ etc. ita comparatas esse, vt valores ipsarum non mutentur, si quantiates $p, q, r, s$ etc. capiant incrementa vel decrementa quaecunque numeris $\alpha, \beta, \gamma, \delta$ etc. proportionalia: huiusmodi antem casus, in quibus manifesto determinatio incognitarum ne tunc quidem possibilis esset, si ipsi veri valores functionum $V, V^{\prime}, V^{\prime \prime}$ etc. darentur, huc non pertinere iam supra monuimus.

Ceterum ad casum hic consideratum omnes reliquos, vbi functiones $V, V^{\prime}$, $I^{\prime \prime}$ etc. non sunt lineares, facile reducere possumus. Scilicet designantibus $\pi, \chi$, $\rho, \sigma$ etc. valores approximatos incognitarum $p, q, r, s$ etc. (quos facile eliciemus, si ex $\mu$ aequationibus $V=\mathbb{M}, V^{\prime}=\mathbb{M}^{\prime}, V^{\prime \prime}=\mathbb{M}^{\prime \prime}$ etc. primo $\nu$ tantum in vsum rocamus), introducemus incognitarum loco alias $p^{\prime}, q^{\prime}, r^{\prime}, s^{\prime}$ etc., statuendo $p=\pi+p^{\prime}$, $q=x+q^{\prime}, r=\rho+r^{\prime}, s=\sigma+s^{\prime}$ etc.: manifesto harum nouarum incognitarum valores tam parui erunt, vt quadrata productaque negligere liceat, quo pacto aequationes sponte euadent lineares. Quodsi dein calculo absoluto contra exspectationem. valores incognitarum $p^{\prime}, q^{\prime}, r^{\prime}, s$ etc. tanti emergerent, vt parum tutum videatur, quadrata productaque neglexisse, eiusdem operationis repetitio (acceptis loco ipsarum $\pi, \chi, \rho, \sigma$ etc. valoribus correctis ipsarum $p, q, r, s$ etc.) remedium promtum afferet.

181.

Quoties itaque vnica tantum incognita $p$ adest, ad cuirus determinationem valores functionum $a p+n, a^{\prime} p+n^{\prime}, a^{\prime \prime} p+n^{\prime \prime}$ etc. resp. inuenti sunt $=M, M^{\prime}, M^{\prime \prime}$ 
etc. et quidem per obseruationes aeque exactas, valor maxime probabilis ipsius $p$ erit

$$
=\frac{a m+a^{\prime} m^{\prime}+a^{\prime \prime} m^{\prime \prime}+e t c .}{a a+a^{\prime} a^{\prime}+a^{\prime \prime} a^{\prime \prime}+\text { etc. }}=\mathcal{A}
$$

scribendo $m, m^{\prime}, m^{\prime \prime}$ etc. resp. pro $M-n, M^{\prime}-n^{\prime}, M^{\prime \prime}-n^{\prime \prime}$ etc.

Iam vt gradus praecisionis in hoc valore praesumendae aestimetur, supponemus, probabilitatem erroris $\Delta$ in obseruationibus exprimi per $\frac{\hbar}{\sqrt{ } \pi} e^{- \text {hh } \Delta \Delta}$. Hinc probabilitas, quod valorem verum ipsius $p$ esse $=A+p^{\prime}$, proportionalis erit functioni

$$
e^{-h h\left((a p-m)^{2}+\left(a^{\prime} p-m^{\prime}\right)^{2}+\left(a^{\prime \prime} p-m^{\prime \prime}\right)^{2}+\text { etc. }\right)}
$$

si pro $p$ substituitur $A+p^{\prime}$. Exponens huius functionis reduci potest ad formam - $h h\left(a a+a^{\prime} a^{\prime}+a^{\prime \prime} a^{\prime \prime}+\right.$ etc. $)(p p-2 p A+B)$, vbi $B$ a $p$ independens est: proin fun= ctio ipsa proportionalis erit huic

$$
e^{-h h\left(a c+a^{\prime} a^{\prime}+a^{\prime \prime} a^{\prime \prime}+\text { etc. }\right) p^{\prime} p^{\prime}}
$$

Patet itaque, valori $A$ eundem praccisionis gradum tribuendum esse, ac si inuentus esset per obseruationem immediatam, cuius praecisio ad praecisionem obseruationum primitiuarum esset vt $h V\left(a a+a^{\prime} a^{\prime}+a^{\prime \prime} a^{\prime \prime}+\right.$ etc. $)$ ad $h$, sime vt $V\left(a a+a^{\prime} a^{\prime}+a^{\prime \prime} a_{-}^{\prime \prime}\right.$ etc. $)$ ad 1.

\section{2.}

Disquisitioni de gradu praecisionis incognitarum valoribus tribuendo; quoties plures adsunt, praemittere oportebit considerationem accuratiorem functionis $v v+v^{\prime} v^{\prime}+v^{\prime \prime} v^{\prime \prime}+$ etc., quam per $W$ denotabimus.

I. Statuamus $\frac{x}{2} \frac{d W}{d p}=p^{\prime}=\lambda+\alpha p+\beta q+\gamma r+\delta s+$ etc., atque $W-\frac{p^{\prime} p^{\prime}}{\alpha}$ $=W^{\prime}$, patetque fieri $p^{\prime}=P$, et, quum sit $\frac{\mathrm{d} W^{\prime}}{\mathrm{d} p}=\frac{\mathrm{d} W}{\mathrm{~d} p}-\frac{2 p^{\prime}}{\alpha} \cdot \frac{\mathrm{d} p^{\prime}}{\mathrm{d} p}=0$, functionem $W^{\prime}$ a $p$ liberam fore. Coëfliciens $\alpha=a a+a^{\prime} a^{\prime}+a^{\prime \prime} a_{-}^{\prime \prime}+$ etc. manifesto semper érit quantitas positiua.

II. Perinde statuemus $\frac{r}{2} \cdot \frac{d W^{\prime}}{d q}=q^{\prime}=\lambda^{\prime}+\beta^{\prime} q+\gamma^{\prime} r+\delta^{\prime} s+$ etc., atque $W^{\prime}-\frac{q^{\prime} q^{\prime}}{\beta^{\prime}}=W^{\prime \prime}$, eritque $q^{\prime}=\frac{\mathrm{r}}{2} \cdot \frac{\mathrm{d} \mathscr{W}}{\mathrm{d} q}-\frac{p^{\prime}}{\alpha} \cdot \frac{\mathrm{d} p^{\prime}}{\mathrm{d} q}=Q-\frac{\beta}{\alpha} \cdot p^{\prime}$, atque $\frac{\mathrm{d} W^{\prime \prime}}{\mathrm{d} q}=0$, vnde patet, functionem $W^{n}$ tum a $p$ tum a $q$ liberam fore. Haec locum non haberent, si fieri posset $\beta^{\prime}=0$. Sed patet, $W_{-4}^{\prime}$ oriri ex $v v+v^{\prime} v^{\prime}+v^{\prime \prime} v^{\prime \prime}+$ etc., elimi- 
nata quantitate $p \operatorname{ex} v, v^{\prime}, v^{\prime \prime}$ etc. adiumento aequationis $p^{\prime}=0$; hinc $\beta^{\prime}$ erit summa coëfficientium ipsius $q q$ in $v v, v^{\prime} v^{\prime}, v^{\prime \prime} v^{\prime \prime}$ etc. post illam eliminationem, hi vero singuli coëfficientes ipsi sunt quadrata, neque omnes simul euanescere possunt, nisi in casu supra excluso, vbi incognitae indeterminatae manent. Patet itaque, $\beta^{\prime}$ esse debere quantitatem positiuam.

III. Statuendo denuo $\frac{x}{2} \cdot \frac{d W^{\prime \prime}}{d r}=r^{\prime}=\lambda^{\prime \prime}+\gamma^{\prime \prime} r+\delta^{n} s+$ etc., atque $W^{\prime \prime}-\frac{r^{\prime} r^{\prime}}{\gamma^{\prime \prime}}$ $=\mathbb{W}^{10}$, erit $r^{\prime}=R-\frac{\gamma}{\alpha} p^{\prime}-\frac{\gamma^{\prime}}{\beta^{\prime}} q^{\prime}$, atque $W^{\prime \prime}$ libera tum a $p$, tum a $q$, tum a $r$. Ceterum coëfficientem $\gamma^{\prime \prime}$ necessario positiuum fieri, simili modo probatur, vt in II. Facile scilicet perspicitur, $\gamma^{\prime \prime}$ esse summam coëfficientium ipsius $r r$ in $v v, v^{\prime} v^{\prime}, v^{\prime \prime} v^{\prime \prime}$ etc., postquam quantitates $p$ et $q$ adiumento aequationum $p^{\prime}=0, q^{\prime}=0$ ex $v, v^{\prime}, v^{\prime \prime}$ etc. eliminatae sunt.

IV. Eodem modo statuendo $\frac{x}{2} \frac{d W^{\prime n}}{d s}=s^{\prime}=\lambda^{\prime \prime \prime}+\delta^{\prime \prime \prime} s+$ etc., $W^{i r}=W^{\prime \prime \prime}-\frac{s^{\prime} s^{\prime}}{\delta \text {, }}$ erit $s^{\prime}=S-\frac{\delta}{\alpha} p^{\prime}-\frac{\delta^{\prime}}{\beta^{\prime}} q^{\prime}-\frac{\delta^{\prime \prime}}{\gamma^{\prime \prime}} r^{\prime}, W^{i v}$ a $p, q, r, s$ libera, atque $\delta^{\prime \prime \prime}$ quantitas positiua.

V. Hoc modo, si praeter $p, q, r, s$ adhuc aliae incognitae adsunt, vlterius progredi licebit, ita vt tandem habeatur

$$
W=\frac{1}{\alpha} p^{\prime} p^{\prime}+\frac{1}{\beta^{\prime}} q^{\prime} q^{\prime}+\frac{1}{\gamma^{\prime \prime}} r^{\prime} r^{\prime}+\frac{1}{\delta^{\prime \prime \prime}} s^{\prime} s^{\prime}+\text { etc. + Const. }
$$

vbi omnes coëfficientes $\alpha, \beta^{\prime}, \gamma^{\prime \prime}, \delta^{\prime \prime \prime}$ etc. erunt quantitates positiuae.

VI. Iam probabilitas alicuius systematis valorum determinatorum pro quantitatibus $p, q, r, s$ etc. proportionalis est functioni $e^{-h h W}$; quamobrem, manente valore quantitatis $p$ indeterminato, probabilitas systematis valorum determinatorum pro reliquis, proportionalis erit integrali $\int e-h h W \mathrm{~d} p$ a $p=-\infty$ vsque ad $p=+\infty$ ex-

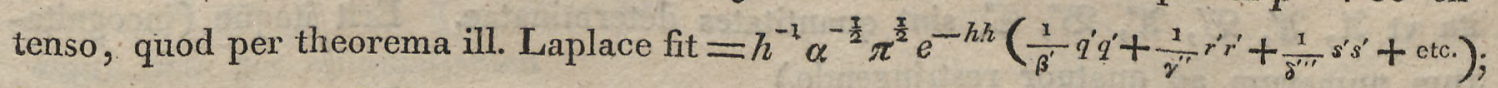
haecce itaque probabilitas proportionalis erit functioni $e^{-h h} W^{\prime}$. Perinde si insuper $q$ tamquam indeterminata tractatur, probabilitas systematis valorum determinatorum pro $r$, s etc. proportionalis erit integrali $\int e-h h W^{\prime} \mathrm{d} q$ a $q=-\infty$ rsque ad $q=+\infty$ extenso, quod fit $=h^{-1} \beta^{\prime-\frac{x}{2}} \pi^{\frac{1}{2}} e^{-h h}\left(\frac{1}{\gamma^{\prime \prime}} r^{\prime} r^{\prime}+\frac{1}{\delta^{\prime \prime \prime}} s^{\prime} s^{\prime}+\right.$ etc. $)$; siue proportionalis functioni $e^{-h h W^{\prime \prime}}$. Prorsus simili modo, si etiam $r$ tamquam indeterminata conside-ratur, probabilitas valorum determinatorum pro reliquis $s$ etc. proportionalis erit functioni $e^{-h W^{\prime \prime \prime}}$ et sic porro. Supponamus, incognitarum numerum ad quatuor 
ascendere, eadem enim conclusio valebit, si maior vel minor est. Valor maxime probabilis ipsius $s$ hic erit $=-\frac{\lambda^{\prime \prime \prime}}{\delta^{m \prime \prime}}$, probabilitasque, hunc a vero differentia $\sigma$ distare, proportionalis erit functioni $e^{-\frac{h h o \sigma}{\partial^{\prime \prime}}}$, vnde concludimus, mensuram praecisionis relatiuae isti determinationi tribuendae exprimi per $\sqrt{ } \frac{1}{\delta_{-}^{11}}$, si mensura praecisionis obseruationibus primitiuis tribuendae statuatur $=x$.

185.

Per methodum art. praec. mensura praecisionis pro ea sola incognita commodé exprimitur, cui in eliminationis negotio vltimus locus assignatus est, quod incommodum vt euitemus, coëfficientem $\delta^{\prime \prime \prime}$ alio modo exprimere conueniet. Ex aequationibus

$$
\begin{aligned}
& P=p^{\prime} \\
& Q=q^{\prime}+\frac{\beta}{\alpha} p^{\prime} \\
& R=r^{\prime}+\frac{\gamma^{\prime}}{\beta^{\prime}} q^{\prime}+\frac{\gamma}{\alpha} p^{\prime} \\
& S=s^{\prime}+\frac{\delta^{\prime \prime}}{\gamma^{\prime \prime}} r^{\prime \prime}+\frac{\delta^{\prime}}{\beta^{\prime}} q^{\prime}+\frac{\delta}{\alpha} p^{\prime}
\end{aligned}
$$

sequitur, ipsas $p^{\prime}, q^{\prime}, r^{\prime}, s^{\prime}$ per $P, Q, R, S$ ita exprimi posse

$$
\begin{aligned}
& p^{\prime}=P \\
& q^{\prime}=Q+\mathfrak{2} P \\
& r^{\prime}=R+\mathfrak{B}^{\prime} Q+\mathfrak{I}^{\prime} P \\
& s^{\prime}=S+\mathbb{C}^{\prime \prime} R+\mathfrak{B}^{\prime \prime} Q+\mathfrak{l}{ }^{\prime \prime} P
\end{aligned}
$$

ita vt $\mathfrak{A}, \mathfrak{I}^{\prime}, \mathfrak{S}^{\prime}, \mathfrak{l}^{\prime \prime}, \mathfrak{S}^{\prime \prime}, \mathfrak{C}^{\prime \prime}$ sint quantitates determinatae. Erit itaque (incognitarum numerum ad quatuor restringendo)

$$
s=-\frac{\lambda^{\prime \prime \prime}}{\delta^{\prime \prime \prime}}+\frac{2^{\prime \prime}}{\delta^{\prime \prime \prime}} P+\frac{\mathfrak{D}^{\prime \prime}}{\delta^{\prime \prime \prime}} Q+\frac{\mathfrak{C}^{\prime \prime}}{\delta^{\prime \prime \prime}} R+\frac{x}{\delta^{\prime \prime \prime}} S
$$

Hinc conclusionem sequentem deducimus. Valores maxime probabiles incognitarum $p, q, r, s$ etc. per eliminationem ex aequationibus $P=0, Q=0, R=0, S=0$ etc. deducendi, manifesto, si aliquantisper $P, Q, R, S$ etc. tamquam indeterminatae spectentur, secundum eandem eliminationis operationem in forma lineari per $P$, $Q, R, S$ etc. exprimentur, ita vt habeatur 


$$
\begin{aligned}
& p=L+A P+B Q+C R+D S+\text { etc. } \\
& q=L^{\prime}+A^{\prime} P+B^{\prime} Q+C^{\prime} R+D^{\prime} S+\text { etc. } \\
& r=L^{\prime \prime}+A^{\prime \prime} P+B^{\prime \prime} Q+C^{\prime \prime} R+D^{\prime \prime} S+\text { etc. } \\
& s=L^{\prime \prime \prime}+A^{\prime \prime} P+B^{\prime \prime} Q+C^{\prime \prime \prime} R+D^{\prime \prime \prime} S+\text { etc. }
\end{aligned}
$$

etc.

His ita factis, valores maxime probabiles ipsarum $p, q, r$, s etc. manifesto erunt resp. $L, L^{\prime}, L^{\prime \prime}, L^{\prime \prime}$ etc., mensuraque praecisionis his determinationibus tribuendae resp. exprimetur per $\sqrt{ } A, \sqrt{ } B^{\prime}, \sqrt{ } C^{\prime \prime}, \sqrt{ } D^{\prime \prime \prime}$ etc., posita praecisione obseruationum primitiuarum $=1$. Quae enim de determinatione incognitae $s$ ante demonstrauimus (pro qua $\frac{x}{\delta^{\prime \prime}}$ respondet ipsi $D^{\prime \prime}$ ), per solam incognitarum permutationem ad omnes reliquas transferre licebit,

184.

Vt disquisitiones praecedentes per exemplum illustrentur, supponamus, per obseruationes, in quibus praecisio aequalis praesumenda sit, inuentum esse

$$
\begin{aligned}
& p-q+2 r=3 \\
& 5 p+2 q-5 r=5 \\
& 4 p+q+4 r=21
\end{aligned}
$$

per quartam vero, eui praecisio dimidia tantum tribuenda est, prodiisse

$$
-2 p+6 q+6 r=28
$$

Loco aequationis vltimae itaque hanc substituemus

$$
-p+5 q+3 r=14
$$

hancque ex obseruatione prioribus praecisione aequali profienisse supponemus. Hinc fit

$$
\begin{aligned}
& P=27 p+6 q \quad-88 \\
& Q=6 p+15 q+r \quad-70 \\
& R=r+54 r-107
\end{aligned}
$$

atque hinc per eliminationem

$$
\begin{aligned}
19899 P & =49154+809 P-324 Q+6 R \\
737 q & =2617-12 P+54 Q-R \\
39798 r & =76242+12 P-54 Q+1473 R
\end{aligned}
$$

Incognitarum itaque valores maxime probabiles erunt

$$
\begin{aligned}
& p=2,470 \\
& q=3,551 \\
& r=1,916
\end{aligned}
$$


atque praecisio relatiua his determinationibus tribuenda, posita praecisione obseruationum primitiuarum $=1$,

$$
\begin{aligned}
& \text { pro } p \ldots . . \vee \frac{19899}{809}=4,96 \\
& \text { pro } q \ldots \ldots \vee \frac{737}{54}=3,69 \\
& \text { pro } r \ldots . . \vee \frac{15266}{49 x}=5,20
\end{aligned}
$$

185.

Argumentum hactenus pertractatum pluribus disquisitionibus analyticis elegantibus occasionem dare posset, quibus tamen hic non immoramur, ne nimis ab instituto nostro distrahamur. Fadem ratione expositionem artificiorum, per quae calculus numericus ad algorithmum magis expeditum reduci potest, ad aliam occasionem nobis reseruare debemus. Vnicam obseruationem hic adiicere liceat. Quoties multitudo functionum seu aequationum propositarum considerabilis est, calculus ideo potissimum paullo molestior euadit, quod coëfficientes per quos aequationes primitivae multiplicandae sunt vt $P, Q, R, S$ etc. obtineantur, plerumque fractiones decimales parum commodas inuoluunt. Si in hoc casu operae pretium non videtur, has multiplicationes adiumento tabularum logarithmicarum quam accuratissime perficere, in plerisque casibus sufficiet, horum multiplicatorum loco alios ad calculum commodiores adhibere, qui ab illis parum differant. Haecce licentia errores sensibiles producere nequat, eo tantummodo casu excepto, vbi mensura praecisionis in reterminatione incognitarum multo minor euadit, quam praecisio obseruationum primitinarum fuerat.

186.

Ceterum principium, quod quadrata differentiarum inter quantitates observatas et computatas summam quam minimam producore debeant, etiam independenter a calculo probabilitatis sequenti modo considerari poterit.

Quoties multitudo incognitarum multitudini quantitatum obseruatarum inde pendentium aequalis est, illas ita determinare licet, vt his exacte satisfat. Quoties autem multitudo illa hac minor est, consensus absolute exactus obtineri nequit, quatenus obseruationes praecisione absoluta non gaudent. In hoc itaque casu operam dare oportet, vt consensus quam optimus stabiliatur, sine vt differentiae quan- 
tum fieri potest extenuentur. Haec vero notio natura sua aliquid vagi inuoluit. Etiamsi enim systema valorum pro incognitis, quod omnes differentias resp. minores reddit quam aliud, procul dubio huic praeferendum sit, nihilominus optio inter duo systemata, quorum alterum in aliis obseruationibus consensum meliorem offert, alterum in aliis, arbitrio nostro quodammodo relinquitur, manifestoque innumera principia diuersa proponi possunt, per quae, conditio prior impletur. Designando differentias inter obseruationes et calculum per $\Delta, \Delta^{\prime}, \Delta^{\prime \prime}$ etc., conditioni priori non modo satisfiet, si $\Delta \Delta+\Delta^{\prime} \Delta^{\prime}+\Delta^{\prime \prime} \Delta^{\prime \prime}+$ etc. fit minimum (quod est principium nostrum ), sed etiam si $\Delta^{4}+\Delta^{\prime 4}+\Delta^{\prime \prime 4}+$ etc., vel $\Delta^{6}+\Delta^{\prime 6}+\Delta^{\prime \prime 6}+$ etc, vel generaliter summa potestatum exponentis cuiuscunque paris in minimum abit. Sed ex omnibus his principiis nostrum simplicissimum est, dum in reliquis ad calculos complicatissimos deferremur. Ceterum principium nostrum, quo iam inde ab anno 1795 vsi sumus, nuper etiam a clar. Legendre in opere Nowelles methodes pour la determination des orbites des cometes, Paris 1806 prolatum est, vbi plures aliae proprietates huius principii expositae sunt, quas hic breuitatis caussa supprimimus.

Si potestatem exponentis paris infinite magni adoptaremus, ad systema id reduceremur, in quo differentiae maximae fiunt quam minimae.

Ill. Laplace ad solutionem aequationum linearium, quartm multitudo maior est quam multitudo quantitatum incognitarum, principio alio vtitur, quod olim iam a clar. Boscovich propositum erat, scilicet vt differentiae ipsae sed omnes positive sumfae summam minimam conficiant. Facile ostendi potest, systema valorum incognitarum, quod ex hoc solo principio exutum sit, necessario *) tot aequationibus e propositarum numero exacte satisfacere debere, quot sint-incognitae, ita vt reliquae aequationes eatenus tanfum in considerationem veniant, quaterus ad optionem decidendam conferunt: si ilaque e. g. aequatio $V=M$ est ex earum numero, quibus non satisfit, systema valorum secundum illud principium inuentorum nihil mutaretur, etiamsi loco ipsius $M$ valor quicunque alius $N$ obseruatus esset, si modo designando per $n$ valorem computatum, differentiae $M-n$, et $N-n$ eodem signo affectae sint. Ceterum ill. Laplace principium istud per adiectionem conditionis novae quodammodo temperat: postulat scilicet, vt summa differentiarum ipsa, signis non mutatis, fiat $=0$. Hinc efficitur, vt multitudo aequationum exacte repraesentatarum vnitate minor fiat quam multitudo quantitatum incognitarum, verumtamen quod ante obseruauimus etiamnum locum habebit, siquidem duae saltem incognitae affuerint.

*) Casibus specialibus exceptis, vbi solutio qnodammodo indeterminata manet. 
187.

Renertimur ab his disquisitionibus generalibus ad propositum nostrum proprium, cuins caussa illae susceptae fuerant. Antequam determinationem quam exactissimam orbitae ex obseruationibus pluribus, qnam quot necessario requiruntur, aggredi liceat, determinatio approximata iam adesse debet, quae ab omnibus obseruationibus datis haud multum discrepet. Correctiones his elementis approximatis adhuc applicandae, vt consensus quam accuratissimus efficiatur, tamquam problematis quacsita considerabuntur. Quaș quum tam exiguas euasuras esse supponi possit, vt quadrata productaque negligere liceat, variationes, quas corporis coelestis loca geocentrica computata inde nanciscuntur, per formulas differentiales in Sect. secunda Libri primi traditas computari poterunt. Loca igitar secundum elementa correcta quae quaerimus computata, exhibebuntur per functiones lineares correctio$n$ num elementorum, illorumque comparatio cum locis obseruatis secundum principia supra exposita ad determinationem valorum maxime probabilium perducet, Hae operationes tanta simplicitate gaudent, vt vlteriori illustratione opus non habeant, sponteque patet, obseruationes quotcunque et quantumuis ab inuicem remotas in vsum yocari posse. - Eadem methodo etiam ad correctionem orbitarum parabolicarum cometarum wti licet, si forte obseruationum series longior adest, consensusque quam optimus postulatur.

\section{8.}

Methodus praecedens iis potissimum casibus adaptata est, vbi praecisio summa desideratur: saepissime autem occurrunt casus, vbi sine haesitatione paullulum ab illa remitti potest, si hoc modo calculi prolixitatem considerabiliter contrahere licet, praesertim quando obseruationes magnum temporis interuallum nondum includunt; adeoque de orbitae determinatione vt sic dicam definitita nondum cogitatur. In talibus casibus methodus sequens lucro notabili in vsum wocari poterit.

Eligantur e tota obseruationum copia duo loca completa $L$, et $L^{\prime}$, computenturque pro temporibus respondentibus ex elementis approximatis corporis coelestis distantiae a terra. Formentur dein respectu harum distantiarum tres hypotheses, retentis in prima valoribus computatis, mutataque in hypothesi secunda distantia prima, secundaque in hypothesi tertia; wtraque mutatio pro ratione, incertitudinis, quae in illis distantiis remanere praesumitur, ad lubitum accipi poterit. Secundum has tres hypotheses, quas in schemate sequente exhibemus, 


\begin{tabular}{|l|c|c|c|}
\hline & Hyp. I & Hyp. II & Hyp. III \\
\hline Distantia *) loco primo respondens & $D$ & $D+\delta$ & $D$ \\
$D^{\prime}$ & $D^{\prime}$ & $D^{\prime}+\delta^{\prime}$ \\
\hline
\end{tabular}

computentur e duobus locis $L, L^{\prime}$ per methodos in Libro primo explicatas tria elementorum systemata, ac dein ex his singulis loca geocentrica corporis coelestis temporibus omnium reliquarum obseruationum respondentia. Sint haec (singulis longitudinibus et latitudinibus, vel ascensionibus rectis et declinationibus seorsim denotatis)

in systemate primo.........M, $\boldsymbol{M}^{\prime}, \quad M^{\prime \prime}$ etc. in systemate secundo......M $M+\alpha, M^{\prime}+\alpha^{\prime}, M^{\prime \prime}+\alpha^{\prime \prime}$ etc. in systemate tertio $\ldots . . . . . M+\beta+\beta, M^{\prime}+\beta^{\prime}, M^{\prime \prime}+\beta^{\prime \prime}$ etc.

Sint porro resp.

loca obseruata...............N, $\quad N^{\prime}, \quad N^{*} \quad$ etc.

Iam quatenus mutationibus paruis distantiarum $D, D^{\prime}$ respondent mutationes proportionales singulorum elomentorum, nec non locorrm geocentricorum ex his computatorum; supponere licebit, loca geocentrica e quarto elementorum systemate computata, quod distantiis a terra $D+x \delta, D^{\prime}+y \delta^{\prime}$ superstructum sit, resp. fore $M+\alpha x+\beta y, M^{\prime}+\alpha^{\prime} x+\beta^{\prime} y, M^{\prime \prime}+\alpha^{\prime \prime} x+\beta^{\prime \prime} y$ etc. Hinc dein, secundum disquisitiones praecedentes, quantitates $x, y$ ita determinabuntur, vt illae quantitates cum $N, N^{\prime}, N^{\prime}$ etc. resp. quam optime consentiant (ratione praecisionis relatiuae obseruationum habita). Systema elementorum correctum ipsum vel perinde ex $L$, $L^{\prime}$ et distantiis $D+x \delta, D^{\prime}+x \delta^{\prime}$, vel secundum regulas notas e tribus elementorum systematibus primis per simplicem interpolationem deriuari poterit.

$$
189 \text {. }
$$

Methodus haecce a praecedente in co tantum differt, quod duobus locis geocentricis exacte, ac dein reliquis quam exactissime satisfit, dum secundum methodum alteram obseruatio nulla reliquis praefertur, sed exrores quantum fieri potest inter omnes distribuuntur. Methodus art. praec. itaque priori eatenus tantum postponenda erit, quatenus locis $L, L^{\prime}$ aliquam errorum partem recipientibus errores in locis reliquis notabiliter diminuere licet: attamen plerumque per idoneam

*) Adhuc commodius erit, loco distantiarum ipsarum logarithmis distantiarum curtatarum vii. 
electionem obseruationum $L, L^{\prime}$ facile caueri potest, ne haec differentia magni momenti euadere possit. Operam scilicet dare oportebit, vt pro $L, L$ tales obseruationes adoptentur, quae non solum exquisita praecisione gaudeant, sed ita quoque comparatae sint, vt elementa ex ipsis distantiisque deriuata a variationibus paruis ipsarum positionum geocentricarum non nimis afficiantur. Parum prudenter itaque ageres, si obseruationes paruo temporis interuallo ab inuicem distantes eligeres, talesue, quibus loci heliocentrici proxime oppositi vel coincidentes responderent. 


\section{SECTIO QVARTA}

De determinatione orbitarum, habita ratione perturbationum.

$$
190 .
$$

Perturbationes, quas planetarum motus per actionem planetarum reliquorum patiuntur, tam exiguae lentarque sunt, vt post longius demum temporis interuallum sensibiles fiant: intra tempus breuius - vel adeo, prout circumstantiae sunt, per rewolutionem integram vnam pluresue - motus tam parum differet a motu in ellipsi perfecta secundum leges Kepleri exacte descripta, vt obseruationes deuiationem indicare non valeant. Quamdiu res ita se habet, operae haud pretium esset, calculum praematurum perturbationum suscipere, sed potius sufficiet, sectionem conicam quasi osculatricem obseruationibus adaptare: dein vero, postquam planeta per tempus longius accurate obseruatus est, effectus perturbationum tandem ita se manifestabit, vt non amplius possibile sit, omnes obseruationes per motum pure ellipticum exacte conciliare; tunc itaque harmonia completa et stabilis parari non poterit, nisi perturbationes cum motu elliptico rite iungantur.

Quum determinatio elementorum ellipticorum, cum quibus perturbationes iungendae sunt, vt obseruationes exacte repraesententur, illarum cognitionem supponat, vicissim vero theoria perturbationum accurate stabiliri nequeat, nisi elementa iam proxime cognita sint: natura rei non permittit, arduum hoc negotium primo statim conatu perfectissime absoluere, sed potius perturbationes et elementa per correctiones alternis demum vicibus pluries repetitas ad summum praecisionis fastigium euehi poterunt. Prima itaque perturbationum theoria superstruetur elementis pure ellipticis, quae obseruationibus proxime adaptata fuerant: dein orbita noua inuestigabitur, quae cum his perturbationibus iuncta obseruationibus quam proxime satisfaciat. Quae si a priori considerabiliter discrepat, iterata perturbationum euolutio ipsi superstruenda erit, quae correctiones alternis vicibus toties repetentur, donec obseruationes, elementa et perturbationes quam arctissime consentiant.

$$
191 .
$$

Quum euolutio theoriae perturbationum ex elementis datis ab instituto nostro aliena sit, hic tantummodo ostendendum erit, quomodo orbita approximata ita corrigi possit, vt cum perturbationibus datis iuncta obseruationibus satisfaciat quam proxime. Simplicissime hoc negotium absoluitur per methodum üs quas in 
artt. $124,165,188$ exposuimus analogam. Pro temporibus omnium obseruationum quibus ad hunc finem vti propositum est, et quae prout res fert esse poterunt vel tres, vel quatuor vel plures, computabuntur ex aequationibus perturbationum harum valores numerici, tum pro longitudinibus in orbita, tum pro radiis vectoribus, tum pro latitudinibus heliocentricis: ad hunc calculum argumenta desumentur ex elementis ellipticis approximatis, quibus perturbationum theoria superstructa erat. Dein ex omnibus obseruationibus eligentur duae, pro quibus distantiae a terra ex iisdem elementis approximatis computabuntur: hae hypothesin primam constituent; hypothesis secunda et tertia formabuntur, distantiis illis paullulum mutatis. In singulis dein hypothesibus e duobus locis geocentricis determinabuntur positiones heliocentricae distantiaeque a Sole; ex illis, postquam latitudines a perturbationibus purgatae fuerint, deducentur longitudo nodi ascendentis, inclinatio orbitae, longitudinesque in orbita. In hoc calculo methodus art. 110 aliqua modificatione opus habet, siquidem ad variationem secularem longitudinis nodi et inclinationis respicere operae pretium videtur. Scilicet designantibus $\beta, \beta^{\prime}$ latitudines heliocentricas a perturbationibus periodicis purgatas; $\lambda, \lambda^{\prime}$ longitudines heliocentricas; $\Omega, \Omega+\Delta$ longitudines nodi ascendentis; $i, i+\delta$ inclinationes orbitae; aequationes in hac forma exhibere conueniet:

$$
\begin{aligned}
& \operatorname{tang} \beta=\operatorname{tang} i \sin (\lambda-\beta) \\
& \frac{\operatorname{tang} i}{\operatorname{tang}(i+\delta)} \operatorname{tang} \beta^{\prime}=\operatorname{tang} i \sin \left(\lambda^{\prime}-\Delta-\Omega\right)
\end{aligned}
$$

Hic valor ipsius $\frac{\operatorname{tang} i}{\operatorname{tang}(i+\delta)}$ omni praecisione necessaria obtinetur, substituendo pro $i$ valorem approximatum: dein $i$ et $\delta$ per methodos vulgares erui poterunt.

A duabus porro longitudinibus in orbita, nec non a duobus radiis vectoribus aggregata perturbationum subbtrahentur, vt valores pure elliptici prodeant. Hic vero etiam effectus, quem variationes seculares positionis perihelii et excentricitatis in longitudinem in orbita radiumque vectorem exserunt, et qui per formulas differentiales Sect. I libri primi determinandus est, statim cum perturbationibus periodicis iungendus est, siquidem obseruationes satis ab inuicem distant, vt illius rationem habere operae pretium videatur. Ex his longitudinibus in orbita radiisque vectoribus correctis, vna cum temporibus respondentibus, elementa reliqua determinabuntur: tandemque ex his elementis positiones geocentricae pro omnibus reliquis obseruationibus calculabuntur. Quibus cum obseruatis comparatis, eodem modo quem 
in art. 188 explicauimus systema id distantiarum elicietur, ex quo elementa omnibus reliquis obseruationibus quam optime satisfacientia demanabunt.

192.

Methodus in art. praec. exposita praecipue determinationi primae orbitae perturbationes implicantis accommodata est: quamprimum vero tum elementa media elliptica tum aequationes perturbationum proxime iam sunt cognitae, determinatio exactissima adiumento obseruationum quam plurimarum commodissime per methodum art. 187, absoluetur, quae hic explicatione peculiari opus non habebit. Quodsi hic obseruationum praestantissimarum copia satis magna est, magnumque temporis interuallum complectitur, haec methodus in pluribus casibus simul determinationi exactiori massarum planetarum perturbantium, saltem maiorum, inseruire poterit. Scilicet, si massa cuiusdam planetae perturbantis in ealculo perturbationum supposita nondum satis certa videtur, introducetur, praeter sex incognitas a correctionibus elementorum pendentes, adhuc alia $\mu$, statuendo rationem massae correctae ad massam suppositam vt $1+\mu$ ad $\mathbf{x}$; supponere tune licebit, perturbationes ipsas in eadem ratione mutari, vnde manifesto in singulis positionibus calculatis terminus nouus linearis ipsam es continens producetur, cuius euolutio nulbi difficultati obnoxia erit. Comparatio positionum calculatarum cum abseruatis secundum principia supra exposita, simul cum correctionibus elementorum etiam correctionem $\mu$ suppeditabit. Quinadeo hoc modo massae pluritum planetarum exactius determinari poterunt, qui quidem perfurbationes satis considerabiles exercent. Nullum dubium est, quin motus planetarum nouorum, praesertim Palladis et Iunonis, qui tantas a Ioue perturbationes patiuntur, post aliquot deceninia hoc modo determinationem exactissimam massae Iouis allaturi sint: quinadeo forsan ipsam massam vnius alteriusue horum planetarum nonorum ex perturbationibus, quas in reliquos exercet, aliquando cognoscere licebit. 


\section{E R R A T A}

Pag. 54112 a calce pro illa 1. illi:

- 76 in formula $V^{\star}$ pro $\operatorname{tang} b$ statui debet $\sin 6 \cos b$; errox ron modo in computo numerico huius formulae p. 79 propagatus, sed idem etiam in computo numerico formulae VIII commissus est, rbi itaque pro tang $b$ adhibere oportet $\cos b \sin b$.

- 871.5 pro vitimo $n 1 . n^{\prime \prime}$

- 102 ]. 2 a calce pro $\cos \omega^{2}$ 1. $\cos 2 \omega^{2}$

- 1101.13 pro $\varphi=0$ 1. $\varphi=90^{\circ}$.

- 121 1. 5 a calce pro $\sin \frac{x}{2} \delta 1.2 \sin \frac{x}{2} \delta$.

- 1261.2 a calce pro $x, y, z$ 1. $x^{\prime}, y^{\prime}, z^{\prime}$.

- 140 1. 5 pro destitueremus 1. destitueremur.

Ibid. 1. 12 pro ab inuicem 1. ad inuicem.

- 144 1. vlt. pro longitudinem et latitudinem 1. longitudine et latitudine.

- 148 1. 11, I2 et 14 pro $d^{\prime} 1, \delta^{\prime}$.

- 149 1. 12 pro $\frac{\eta^{\prime \prime} \theta \theta^{\prime \prime}}{r^{\prime} r^{\prime} \eta \eta^{\prime \prime} \cos f \cos f^{\prime} \cos f^{\prime \prime}}$ 1. $\frac{r^{\prime} r^{\prime} \theta \theta^{\prime \prime}}{r r^{\prime \prime} \eta \eta^{\prime \prime} \cos f \cos f^{\prime} \cos f^{\prime \prime}}$

- 1561.2 a calce pro $P=\frac{n^{\prime \prime}}{n^{\prime}}$ 1. $P=\frac{n^{\prime \prime}}{n}$

Ibid. 1. vlt. 1. $Q=2\left(\frac{n+n^{\prime \prime}}{n^{\prime}}-1\right) r_{3}^{\prime 3}$

- 1621.21 pro $p$ 1. $P$

- 1631.6 pro $-+\delta^{\prime \prime} 1 .+\delta^{\prime \prime}$.

-1951.15 pro $\lambda^{\prime \prime \prime}$. $1 .=\lambda^{\prime \prime \prime}$. 


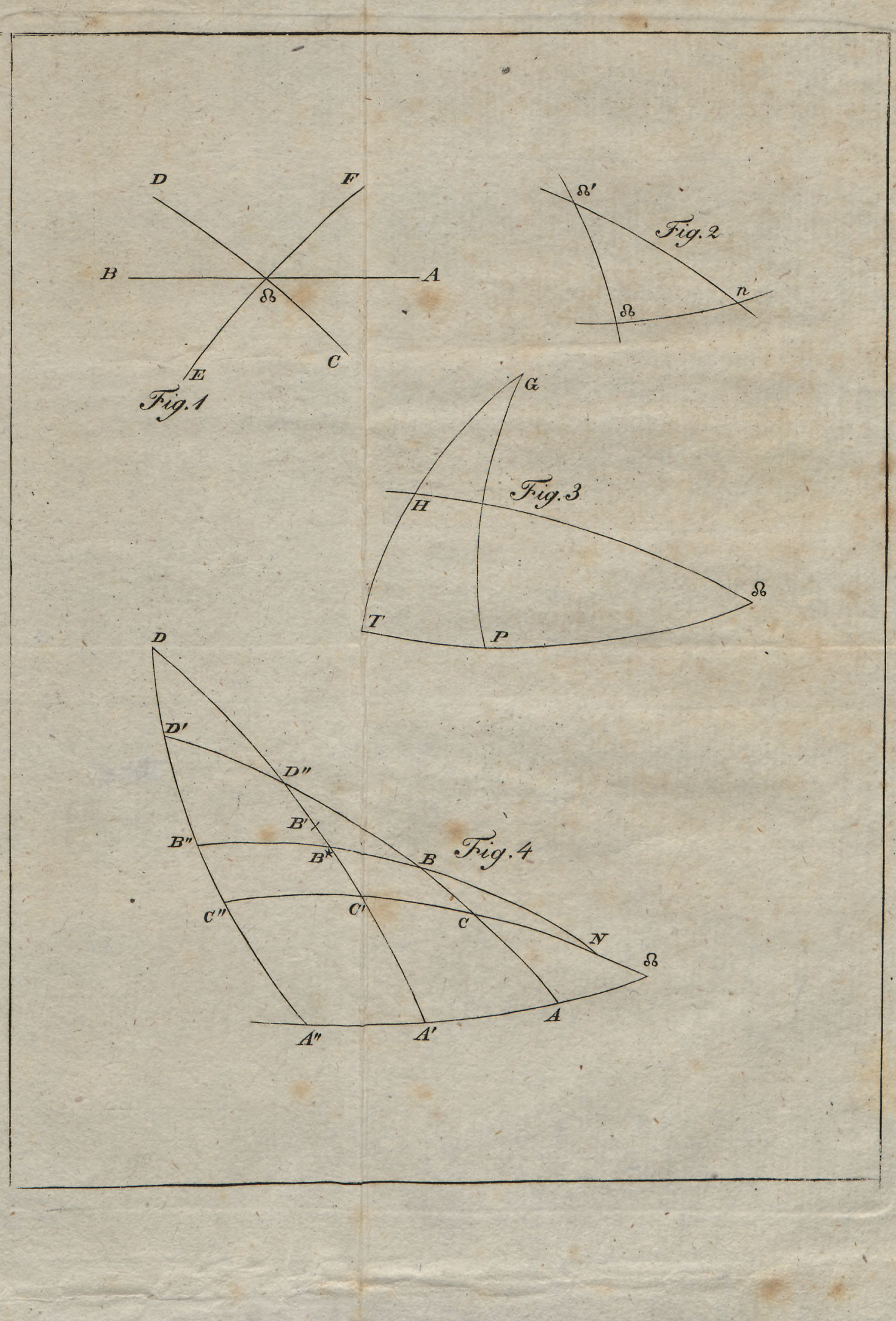




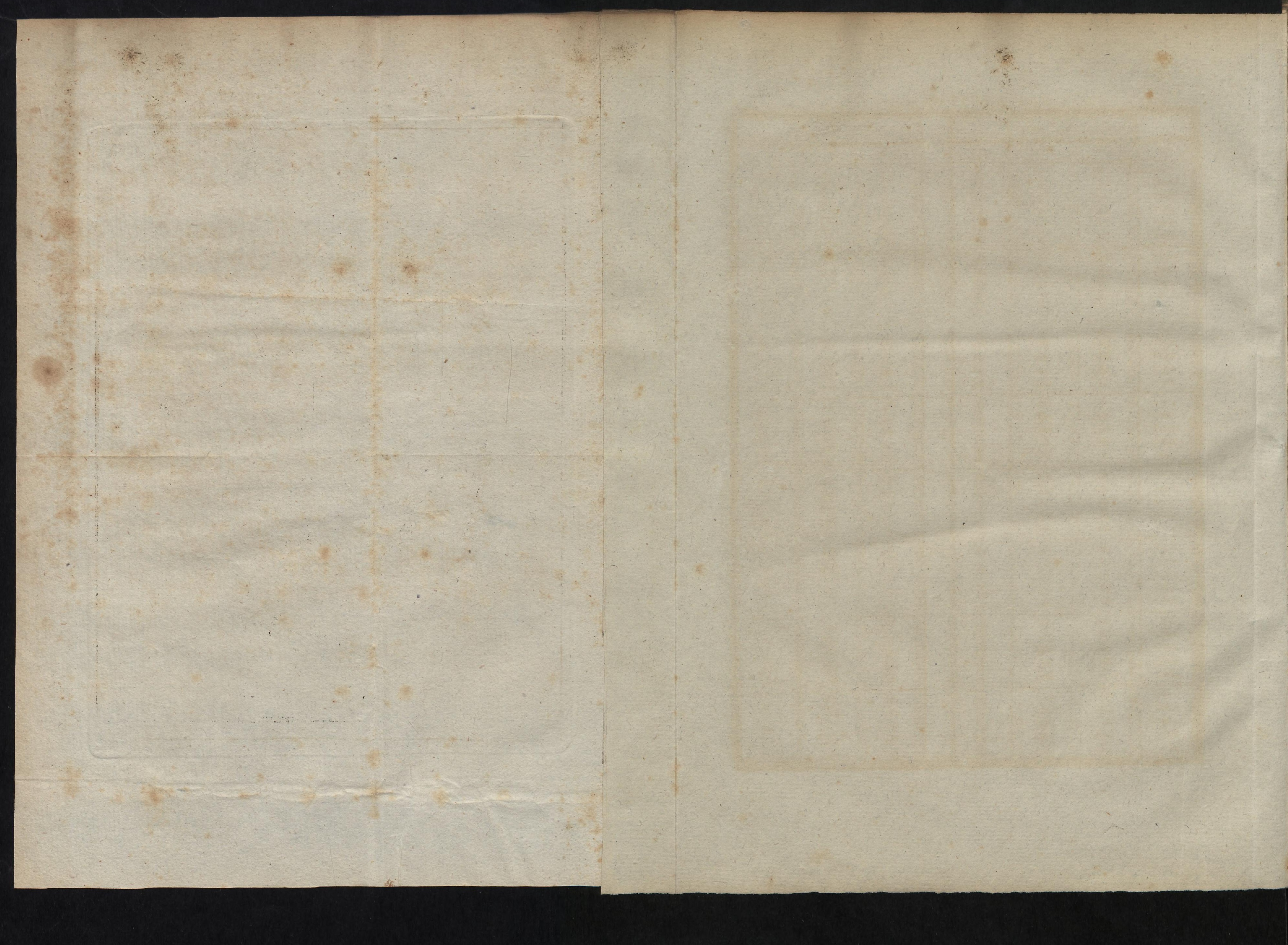


TABVLA I (v. artt. 42, 45)

\begin{tabular}{|c|c|c|c|c|c|c|}
\hline & \multicolumn{3}{|c|}{ Ellipsis } & \multicolumn{3}{|c|}{ Hyperbola } \\
\hline$A$ & $\log B$ & $C$ & $T$ & $\log B$ & $C$ & $T$ \\
\hline 0,000 & 0 & o & 0,00000 & 0 & 0 & 0,0000 \\
\hline 0,001 & 0 & 0 & 100 & 0 & 0 & 100 \\
\hline 0,002 & 0 & 2 & 200 & 0 & & 200 \\
\hline 0,003 & $i$ & 4 & 301 & $x$ & 4 & 299 \\
\hline 0,004 & 1 & 7 & 401 & 1 & 7 & $3_{99}$ \\
\hline 0,005 & 2 & 11 & 502 & 2 & $x x$ & 498 \\
\hline 0,006 & 3 & 16 & 603 & 3 & 16 & 597 \\
\hline 0,007 & 4 & 22 & 704 & 4 & 22 & $6 g^{6}$ \\
\hline 0,008 & 5 & 29 & 805 & 5 & 29 & 795 \\
\hline 0,009 & 6 & 37 & 0,00907 & 6 & 3 & 894 \\
\hline 0,010 & 7 & 46 & 0,01003 & 7 & 46 & $0,0099^{2}$ \\
\hline 0,011 & 9 & 56 & 110 & 9 & 55 & 0,01090 \\
\hline 0,012 & 11 & 66 & 212 & $x$ & 66 & 189 \\
\hline $0,0 \times 3$ & 13 & $7^{8}$ & 314 & 13 & 77 & 287 \\
\hline 0,014 & 15 & 90 & 416 & 15 & 89 & 384 \\
\hline 0,015 & 27 & 103 & 518 & 17 & 102 & 482 \\
\hline 0,016 & 19 & 118 & 621 & 19 & $116^{\circ}$ & 580 \\
\hline $0,0 \times 7$ & 22 & +33 & 723 & 21 & 33 & 677 \\
\hline 0,018 & 24 & 349 & $\therefore 826$ & 24 & $\times 47$ & $77^{4}$ \\
\hline 0,019 & 27 & 366 & 0,01929 & 27 & 164 & 872 \\
\hline 0,020 & 30 & 184 & 0,02032 & 30 & 182 & 0,01963 \\
\hline 0,021 & 33 & 203 & 136 & 33 & 200 & 0,02065 \\
\hline 0,022 & 36 & 223 & 239 & ․ 36 & 220 & 162 \\
\hline 0,023 & 40 & 244 & 343 & 39 & 240 & 258 \\
\hline 0,024 & 43 & 265 & 447 & $=43$ & 261 & 355 \\
\hline 0,025 & 47 & 238 & 551 & 46 & 2.83 & 451 \\
\hline 0,026 & 51 & 312 & 655 & 50 & 306 & $\therefore 547$ \\
\hline 0,027 & 55 & 336 & 760 & 54 & 330 & 643 \\
\hline 0,028 & 59 & 362 & 864 & 58 & 355 & $7^{39} 9$ \\
\hline 0,029 & 65 & 388 & 0,02969 & 62 & 531 & 834 \\
\hline 0,030 & 67 & 416 & 0,03074 & 67 & 407 & $0 ; 02950$ \\
\hline 0,031 & 72 & 444 & 179 & 71 & 435 & 0,05025 \\
\hline 0,052 & 77 & 473 & 284 & $7^{6}$ & 463 & 220 \\
\hline 0,033 & 82 & 503 & 589 & -80 & 492 & 215 \\
\hline 0,034 & 87 & 535 & 495 & 85 & 523 & 510 \\
\hline 0,055 & 92 & 567 & 601 & 91 & 554 & 404 \\
\hline 0,056 & 97 & 600 & . 707 & $9^{6}$ & 585 & $\therefore 499$ \\
\hline 0,037 & 103 & 634 & 813 & 101 & 619 & $5 g^{3}$ \\
\hline 0,058 & 108 & 669 & 0,03919 & 107 & 652 & 688 \\
\hline o,o59 & .114 & 704 & 0,04025 & 112 & $686^{-}$ & 782 \\
\hline 0,040 & 120 & 741 & 132 & 118 & 722 & 876 \\
\hline
\end{tabular}


TABVLA I

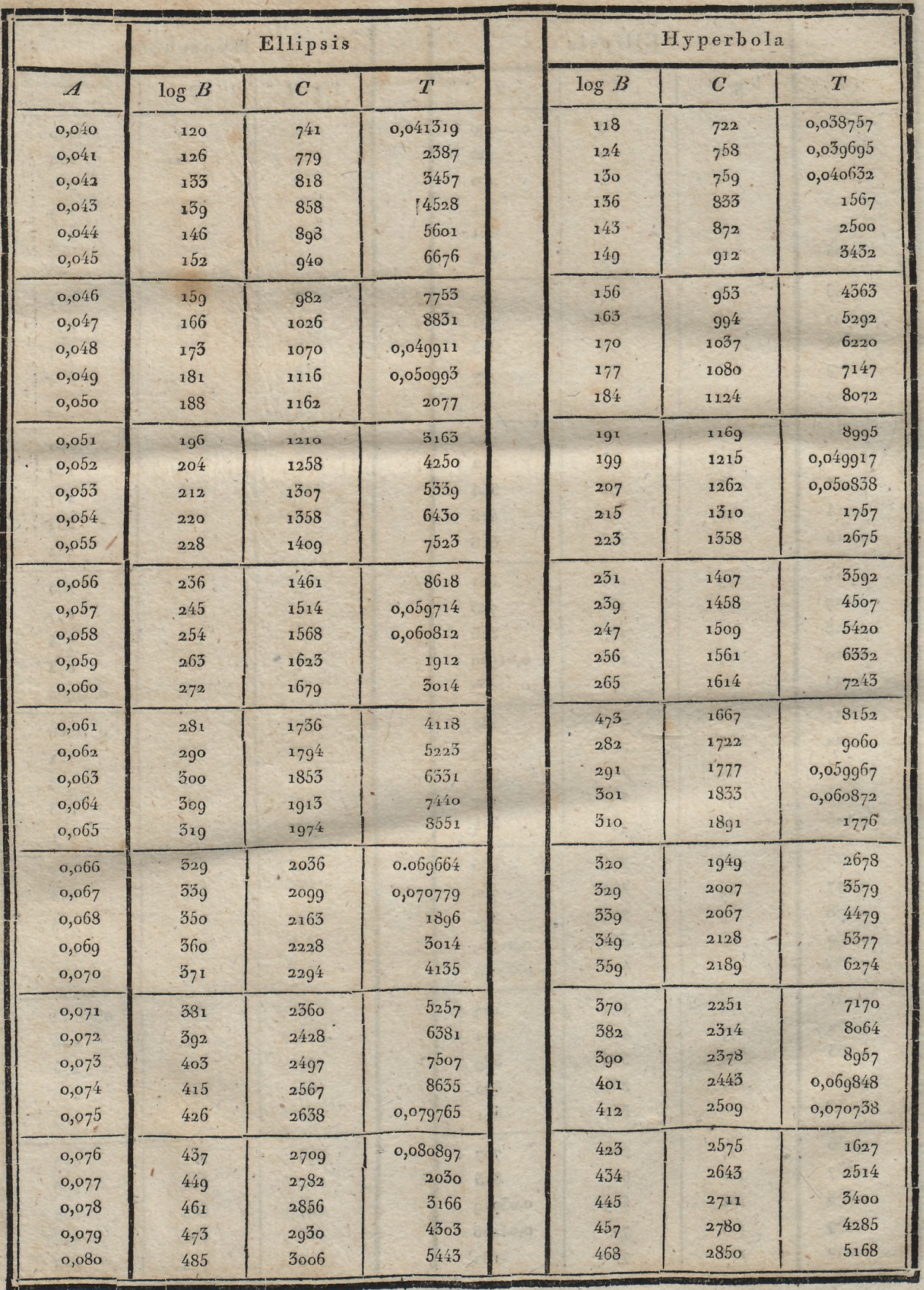


TABVLA $\mathrm{I}$

\begin{tabular}{|c|c|c|c|c|c|c|}
\hline & \multicolumn{3}{|c|}{ Ellipsis } & \multicolumn{3}{|c|}{ Hypexbola } \\
\hline$A$ & $\log B$ & $C$ & $T$ & $\log B$ & $C$ & $T$ \\
\hline $\begin{array}{l}0,080 \\
0,081 \\
0,082 \\
0,083 \\
0,084 \\
0,085\end{array}$ & $\begin{array}{l}485 \\
498 \\
510 \\
523 \\
535 \\
548\end{array}$ & $\begin{array}{l}3006 \\
3083 \\
3160 \\
3239 \\
3319 \\
3399\end{array}$ & $\begin{array}{r}0,085443 \\
6584 \\
7727 \\
0,08887^{2} \\
0,090019 \\
1168\end{array}$ & $\begin{array}{r}468 \\
480 \\
492 \\
504 \\
516 \\
528\end{array}$ & $\begin{array}{l}2850 \\
2921 \\
2992 \\
3065 \\
3138 \\
3212\end{array}$ & $\begin{array}{r}0,075 \times 68 \\
6050 \\
6930 \\
7810 \\
8688 \\
0,079564\end{array}$ \\
\hline $\begin{array}{l}0,086 \\
0,087 \\
0,088 \\
0,089 \\
0,090\end{array}$ & $\begin{array}{l}561 \\
575 \\
588 \\
602 \\
615\end{array}$ & $\begin{array}{l}3481 \\
3564 \\
3647 \\
3752 \\
3818\end{array}$ & $\begin{array}{l}2319 \\
3472 \\
4627 \\
5784 \\
6943\end{array}$ & $\begin{array}{l}540 \\
553 \\
566 \\
573 \\
591\end{array}$ & $\begin{array}{l}3287 \\
3363 \\
3410 \\
3517 \\
3595\end{array}$ & $\begin{array}{r}0,080439 \\
1313 \\
2186 \\
3057 \\
3927\end{array}$ \\
\hline $\begin{array}{l}0,091 \\
0,092 \\
0,09^{3} \\
0,09^{4} \\
0,095\end{array}$ & $\begin{array}{l}629 \\
645 \\
658 \\
672 \\
687\end{array}$ & $\begin{array}{l}3904 \\
3092 \\
4081 \\
4170 \\
4261\end{array}$ & $\begin{array}{r}8104 \\
0,099266 \\
0,100431 \\
1598 \\
2,66\end{array}$ & $\begin{array}{r}604 \\
618 \\
65 x \\
645 \\
658\end{array}$ & $\begin{array}{l}367^{4} \\
3754 \\
3835 \\
39 \times 7 \\
3999\end{array}$ & $\begin{array}{l}479^{6} \\
5663 \\
6529 \\
739^{4} \\
8257\end{array}$ \\
\hline $\begin{array}{l}0,096 \\
0,097 \\
0,093 \\
0,099 \\
0,100\end{array}$ & $\begin{array}{l}701 \\
716 \\
731 \\
746 \\
762\end{array}$ & $\begin{array}{l}4353 \\
4446 \\
4539 \\
4634 \\
4730\end{array}$ & $\begin{array}{l}3937 \\
5110 \\
6234 \\
746 x \\
8640\end{array}$ & $\begin{array}{r}672 \\
686 \\
700 \\
714 \\
728\end{array}$ & $\begin{array}{l}4083 \\
4167 \\
4252 \\
4333 \\
4424\end{array}$ & $\begin{array}{r}9119 \\
0,089930 \\
0,090340 \\
1698 \\
2555\end{array}$ \\
\hline $\begin{array}{l}0,101 \\
0,102 \\
0,105 \\
0,104 \\
0,105\end{array}$ & $\begin{array}{l}777 \\
79^{3} \\
809 \\
825 \\
84 x\end{array}$ & $\begin{array}{l}4826 \\
4924 \\
5023 \\
5123 \\
5224\end{array}$ & $\begin{array}{r}0,109820 \\
0,111003 \\
2188 \\
3575 \\
4563\end{array}$ & $\begin{array}{l}7^{43} \\
758 \\
77^{2} \\
787 \\
302\end{array}$ & $\begin{array}{l}4512 \\
4600 \\
4689 \\
4779 \\
4820\end{array}$ & $\begin{array}{l}3410 \\
4265 \\
5118 \\
5969 \\
6820\end{array}$ \\
\hline $\begin{array}{l}0,106 \\
0,107 \\
0,108 \\
0,109 \\
0,110\end{array}$ & $\begin{array}{l}857 \\
873 \\
890^{\circ} \\
907 \\
924\end{array}$ & $\begin{array}{l}53_{22} \\
5428 \\
5532 \\
5637 \\
5743\end{array}$ & $\begin{array}{r}5754 \\
6947 \\
8142 \\
0,119339 \\
0,120538\end{array}$ & $\begin{array}{l}817 \\
833 \\
848 \\
864 \\
880\end{array}$ & $\begin{array}{l}4962 \\
5054 \\
5148 \\
5242 \\
5337\end{array}$ & $\begin{array}{r}7669 \\
8517 \\
0,099364 \\
0,100209 \\
1053\end{array}$ \\
\hline $\begin{array}{l}0,111 \\
0,112 \\
0,113 \\
0,3114 \\
0,115\end{array}$ & $\begin{array}{c}9^{41} \\
9^{58} \\
97^{5} \\
99^{3} \\
1011\end{array}$ & $\begin{array}{l}5850 \\
5958 \\
6067 \\
6177 \\
6288\end{array}$ & $\begin{array}{l}1739 \\
2942 \\
4148 \\
5355 \\
6564\end{array}$ & $\begin{array}{l}89^{5} \\
911 \\
9^{28} \\
9^{44} \\
9^{60}\end{array}$ & $\begin{array}{l}5432 \\
5529 \\
5626 \\
5724 \\
5823\end{array}$ & $\begin{array}{l}1896 \\
2738 \\
3578 \\
4417 \\
5255\end{array}$ \\
\hline $\begin{array}{l}0,116 \\
0,117 \\
0,118 \\
0,119 \\
0,120\end{array}$ & $\begin{array}{l}1029 \\
1047 \\
1065 \\
1033 \\
1102\end{array}$ & $\begin{array}{l}6400 \\
6513 \\
6627 \\
6742 \\
6858\end{array}$ & $\begin{array}{r}777^{6} \\
0,128989 \\
0,130205 \\
1423 \\
2643\end{array}$ & $\begin{array}{r}977 \\
994 \\
1010 \\
1027 \\
1045\end{array}$ & $\begin{array}{l}5923 \\
6024 \\
6125 \\
6228 \\
6331\end{array}$ & $\begin{array}{l}60 g^{2} \\
69_{2} 7 \\
77^{61} \\
859^{4} \\
9426\end{array}$ \\
\hline
\end{tabular}


TABVLA I

\begin{tabular}{|c|c|c|c|c|c|c|}
\hline & \multicolumn{3}{|c|}{ Ellipsis } & \multicolumn{3}{|c|}{ Hyperbola } \\
\hline$A$ & $\log B$ & $C$ & $T$ & $\log B$ & $C$ & $T$ \\
\hline $\begin{array}{l}0,120 \\
0,121 \\
0,122 \\
0,123 \\
0,124 \\
0,125\end{array}$ & $\begin{array}{l}1102 \\
1121 \\
1139 \\
1158 \\
1178 \\
1197\end{array}$ & $\begin{array}{l}6358 \\
6976 \\
7094 \\
7213 \\
7334 \\
7455\end{array}$ & $\begin{array}{r}0,132643 \\
3865 \\
5089 \\
6315 \\
7543 \\
0,138774\end{array}$ & $\begin{array}{l}1045 \\
1062 \\
1079 \\
1097 \\
1114 \\
1132\end{array}$ & $\begin{array}{l}6331 \\
6435 \\
6539 \\
6645 \\
6751 \\
6858\end{array}$ & $\begin{array}{r}0,109426 \\
0,110256 \\
1085 \\
1913 \\
2740 \\
3566\end{array}$ \\
\hline $\begin{array}{l}0,126 \\
0,127 \\
0,128 \\
0,129 \\
0,130\end{array}$ & $\begin{array}{l}1217 \\
1256 \\
1256 \\
1276 \\
1296\end{array}$ & $\begin{array}{l}7577 \\
7701 \\
7825 \\
7951 \\
8077\end{array}$ & $\begin{array}{r}0,140007 \\
1241 \\
2478 \\
3717 \\
4959\end{array}$ & $\begin{array}{l}1150 \\
1168 \\
1186 \\
1205 \\
1223\end{array}$ & $\begin{array}{l}6966 \\
707^{5} \\
7185 \\
7295 \\
7406\end{array}$ & $\begin{array}{l}4390 \\
5213 \\
6035 \\
6855 \\
7675\end{array}$ \\
\hline $\begin{array}{l}0,131 \\
0,132 \\
0,133 \\
0,134 \\
0,135\end{array}$ & $\begin{array}{l}1317 \\
1337 \\
1358 \\
1578 \\
1399\end{array}$ & $\begin{array}{l}8205 \\
8334 \\
8463 \\
8594 \\
8726\end{array}$ & $\begin{array}{r}6202 \\
7443 \\
8695 \\
0,149945 \\
0,151197\end{array}$ & $\begin{array}{l}1242 \\
1261 \\
1280 \\
1299 \\
1318\end{array}$ & $\begin{array}{l}7518 \\
7631 \\
7745 \\
7859 \\
7974\end{array}$ & $\begin{array}{r}849^{3} \\
0,119^{3} 10 \\
0,120126 \\
0940 \\
1754\end{array}$ \\
\hline $\begin{array}{l}0,136 \\
0,137 \\
0,138 \\
0,139 \\
0,140\end{array}$ & $\begin{array}{l}1421 \\
1442 \\
1463 \\
1435 \\
1507\end{array}$ & $\begin{array}{l}8859 \\
899^{3} \\
9128 \\
9264 \\
9401\end{array}$ & $\begin{array}{l}2452 \\
3708 \\
4967 \\
6228 \\
7491\end{array}$ & $\begin{array}{l}1337 \\
1357 \\
1376 \\
1396 \\
1416\end{array}$ & $\begin{array}{l}8090 \\
8207 \\
8325 \\
8443 \\
8562\end{array}$ & $\begin{array}{l}2566 \\
3377 \\
4186 \\
4995 \\
5802\end{array}$ \\
\hline $\begin{array}{l}0,141 \\
0,142 \\
0,143 \\
0,144 \\
0,145\end{array}$ & $\begin{array}{l}1529 \\
1551 \\
1573 \\
1596 \\
1618\end{array}$ & $\begin{array}{r}9^{539} \\
9^{6} 78 \\
9819 \\
9960 \\
10102 \\
\end{array}$ & $\begin{array}{r}0,158756 \\
0,160024 \\
1294 \\
2566 \\
3840\end{array}$ & $\begin{array}{l}1436 \\
1456 \\
1476 \\
1497 \\
1517\end{array}$ & $\begin{array}{l}8682 \\
8803 \\
8925 \\
9047 \\
9170\end{array}$ & $\begin{array}{r}6609 \\
7414 \\
8217 \\
9020 \\
0,129822\end{array}$ \\
\hline $\begin{array}{l}0,145 \\
0,147 \\
0,148 \\
0,149 \\
0,150\end{array}$ & $\begin{array}{l}1641 \\
1664 \\
1687 \\
1710 \\
1734\end{array}$ & $\begin{array}{l}10246 \\
10390 \\
10536 \\
10683 \\
10850\end{array}$ & $\begin{array}{r}5116 \\
63395 \\
7676 \\
0,168959 \\
0,170245\end{array}$ & $\begin{array}{l}1538 \\
1559 \\
1580 \\
1601 \\
1622\end{array}$ & $\begin{array}{l}9^{2} 9^{4} \\
9^{419} \\
9^{545} \\
9^{6} 71 \\
979^{8}\end{array}$ & $\begin{array}{r}0,130622 \\
1421 \\
2219 \\
3016 \\
3812\end{array}$ \\
\hline $\begin{array}{l}0,151 \\
0,152 \\
0,153 \\
0,154 \\
0,155\end{array}$ & $\begin{array}{l}1757 \\
1731 \\
1805 \\
1829 \\
1854\end{array}$ & $\begin{array}{l}10979 \\
11129 \\
11280 \\
11432 \\
11585\end{array}$ & $\begin{array}{l}1533 \\
2825 \\
4115 \\
5410 \\
6707\end{array}$ & $\begin{array}{l}1643 \\
1665 \\
1686 \\
1708 \\
1730\end{array}$ & $\begin{array}{r}9926 \\
10055 \\
10185 \\
10315 \\
10446\end{array}$ & $\begin{array}{r}4606 \\
5399 \\
6191 \\
6982 \\
+7772\end{array}$ \\
\hline $\begin{array}{l}0,156 \\
0,157 \\
0,158 \\
0,159 \\
0,160\end{array}$ & $\begin{array}{l}1878 \\
1903 \\
1927 \\
1952 \\
1977\end{array}$ & $\begin{array}{l}117^{3} 9 \\
11894 \\
12051 \\
12208 \\
12366\end{array}$ & $\begin{array}{r}8006 \\
0,179308 \\
0,180612 \\
1918 \\
3226\end{array}$ & $\begin{array}{l}1752 \\
1774 \\
1797 \\
1819 \\
1842\end{array}$ & $\begin{array}{l}10578 \\
10711 \\
10844 \\
10978 \\
11113\end{array}$ & $\begin{array}{r}8561 \\
0,139349 \\
0,140135 \\
0920 \\
1704\end{array}$ \\
\hline
\end{tabular}


TABVLA I

\begin{tabular}{|c|c|c|c|c|c|c|}
\hline & $e^{2}$ & Ellipsi & & & yperb & \\
\hline$A$ & $\log B$ & $C$ & $T$ & $\log B$ & $C$ & $T$ \\
\hline $\begin{array}{l}0,160 \\
0,161 \\
0,162 \\
b, 163 \\
0,164 \\
0,165\end{array}$ & $\begin{array}{l}1977 \\
2003 \\
2028 \\
2054 \\
2030 \\
2106\end{array}$ & $\begin{array}{l}12366 \\
12526 \\
12686 \\
12848 \\
13011 \\
1317^{5}\end{array}$ & $\begin{array}{r}0,183226 \\
4537 \\
5850 \\
7166 \\
8484 \\
0,189804\end{array}$ & $\begin{array}{l}1842 \\
1864 \\
1887 \\
1910 \\
1933 \\
1956\end{array}$ & $\begin{array}{l}11115 \\
11249 \\
1 \times 386 \\
11523 \\
11661 \\
11800\end{array}$ & $\begin{array}{r}0,141704 \\
2487 \\
3269 \\
4050 \\
4829 \\
5608\end{array}$ \\
\hline $\begin{array}{l}0,166 \\
0,167 \\
0,168 \\
0,169 \\
0,170\end{array}$ & $\begin{array}{l}2132 \\
2158 \\
2184 \\
2311 \\
2238\end{array}$ & $\begin{array}{l}13340 \\
13506 \\
13673 \\
13841 \\
14010\end{array}$ & $\begin{array}{r}0,191127 \\
2452 \\
3779 \\
5109 \\
6441\end{array}$ & $\begin{array}{l}1980 \\
2003 \\
2027 \\
2051 \\
2075\end{array}$ & $\begin{array}{l}11940 \\
12081 \\
12222 \\
12364 \\
12507\end{array}$ & $\begin{array}{r}6385 \\
7 x 61 \\
7937 \\
87 \times 0 \\
0,149483\end{array}$ \\
\hline $\begin{array}{l}0,171 \\
0,172 \\
0,17^{3} \\
0,17^{4} \\
0,17^{5}\end{array}$ & $\begin{array}{l}2265 \\
2292 \\
2319 \\
2347 \\
2374\end{array}$ & $\begin{array}{l}14181 \\
14352 \\
14525 \\
14699 \\
14873\end{array}$ & $\begin{array}{r}777^{5} \\
0,199112 \\
0,200451 \\
179^{3} \\
3137\end{array}$ & $\begin{array}{l}2099 \\
2123 \\
2147 \\
2172 \\
2196\end{array}$ & $\begin{array}{l}12651 \\
12795 \\
x 2940 \\
x 3086 \\
x 3233\end{array}$ & $\begin{array}{r}0,150255 \\
1026 \\
1795 \\
2564 \\
3331\end{array}$ \\
\hline $\begin{array}{l}0,176 \\
0,177 \\
0,178 \\
0,179 \\
0,180\end{array}$ & $\begin{array}{l}2402 \\
2430 \\
2458 \\
2486 \\
2515\end{array}$ & $\begin{array}{r}15049 \\
15226 \\
15404 \\
15583 \\
15764\end{array}$ & $\begin{array}{r}4484 \\
5832 \\
7184 \\
8538 \\
0,209394\end{array}$ & $\begin{array}{l}2221 \\
2246 \\
2271 \\
2296 \\
2321\end{array}$ & $\begin{array}{l}13380 \\
13529 \\
13678 \\
13827 \\
13978\end{array}$ & $\begin{array}{l}4097 \\
4862 \\
5626 \\
6389 \\
7151\end{array}$ \\
\hline $\begin{array}{l}0,181 \\
0,182 \\
0,183 \\
0,184 \\
0,185\end{array}$ & $\begin{array}{l}2543 \\
2572 \\
2601 \\
2630 \\
2660\end{array}$ & $\begin{array}{l}15945 \\
16128 \\
16311 \\
16496 \\
16682\end{array}$ & $\begin{array}{r}0,211253 \\
2614 \\
3977 \\
5343 \\
6712\end{array}$ & $\begin{array}{l}2346 \\
2372 \\
2398 \\
2423 \\
2449\end{array}$ & $\begin{array}{l}14129 \\
14281 \\
14434 \\
14588 \\
14742\end{array}$ & $\begin{array}{r}79^{1 x} \\
867 x \\
0,159429 \\
0,160137 \\
0943\end{array}$ \\
\hline $\begin{array}{l}0,186 \\
0,187 \\
0,188 \\
0,189 \\
0,190\end{array}$ & $\begin{array}{l}2689 \\
2719 \\
2749 \\
2779 \\
2809\end{array}$ & $\begin{array}{l}16868 \\
17057 \\
17246 \\
17436 \\
17627\end{array}$ & $\begin{array}{r}8083 \\
0,219456 \\
0,220852 \\
2211 \\
3592\end{array}$ & $\begin{array}{l}24.75 \\
2502 \\
2528 \\
2554 \\
258 \mathrm{i}\end{array}$ & $\begin{array}{l}14893 \\
15054 \\
\times 52 \times 0 \\
\times 5568 \\
\times 5526\end{array}$ & $\begin{array}{l}1698 \\
2453 \\
3206 \\
5958 \\
4709\end{array}$ \\
\hline $\begin{array}{l}0,191 \\
0,19^{2} \\
0,19^{3} \\
0,19^{4} \\
0,19^{5}\end{array}$ & $\begin{array}{l}2839 \\
2870 \\
2900 \\
2931 \\
2962\end{array}$ & $\begin{array}{l}17820 \\
18013 \\
18208 \\
18404 \\
18601\end{array}$ & $\begin{array}{r}4975 \\
6361 \\
7750 \\
0,229141 \\
0,230535\end{array}$ & $\begin{array}{l}2608 \\
2634 \\
266 x \\
2688 \\
2716\end{array}$ & $\begin{array}{l}15685 \\
15845 \\
16005 \\
16167 \\
16329\end{array}$ & $\begin{array}{l}5458 \\
6207 \\
6055 \\
7702 \\
8447\end{array}$ \\
\hline $\begin{array}{l}0,19^{6} \\
0,197 \\
0,198 \\
0,199 \\
0,200\end{array}$ & $\begin{array}{l}299^{3} \\
3025 \\
3056 \\
3088 \\
3120\end{array}$ & $\begin{array}{l}18799 \\
18998 \\
19198 \\
19400 \\
19602\end{array}$ & $\begin{array}{l}1931 \\
3329 \\
4731 \\
6135 \\
7541\end{array}$ & $\begin{array}{l}2743 \\
277 x \\
2798 \\
2826 \\
2854\end{array}$ & $\begin{array}{l}x 649 x \\
x 6655 \\
x 68 x 9 \\
x 6984 \\
x 750\end{array}$ & $\begin{array}{r}9^{192} \\
0,169935 \\
0,170678 \\
1419 \\
2159\end{array}$ \\
\hline
\end{tabular}


TABVLA I

\begin{tabular}{|c|c|c|c|c|c|c|}
\hline & od to & Ellipsi & & ela sifis & ypexbo & \\
\hline$A$ & $\log B$ & $C$ & $T$ & $\log B$ & $C$ & $T$ \\
\hline $\begin{array}{l}0,200 \\
0,201 \\
0,202 \\
0,203 \\
0,204 \\
0,205\end{array}$ & $\begin{array}{l}3_{120} \\
315_{2} \\
3_{184} \\
3_{21} 6 \\
3_{249} \\
3_{282}\end{array}$ & $\begin{array}{l}19602 \\
19806 \\
20011 \\
20217 \\
20424 \\
20632\end{array}$ & $\begin{array}{r}0,237541 \\
0,233950 \\
0,240361 \\
1776 \\
3192 \\
4612\end{array}$ & $\begin{array}{l}2854 \\
2882 \\
2910 \\
2938 \\
2967 \\
2995\end{array}$ & $\begin{array}{l}17150 \\
17317 \\
17484 \\
17652 \\
17821 \\
17991\end{array}$ & $\begin{array}{r}0,172159 \\
2899 \\
5637 \\
4374 \\
5110 \\
5845\end{array}$ \\
\hline $\begin{array}{l}0,206 \\
0,207 \\
0,208 \\
0,209 \\
0,210\end{array}$ & $\begin{array}{l}3315 \\
3348 \\
3381 \\
3414 \\
3448\end{array}$ & $\begin{array}{l}20842 \\
21052 \\
21264 \\
21477 \\
21690\end{array}$ & $\begin{array}{r}6034 \\
7458 \\
0,248885 \\
0,250315 \\
1748\end{array}$ & $\begin{array}{l}3024 \\
3053 \\
3082 \\
3111 \\
3140\end{array}$ & $\begin{array}{l}18161 \\
18332 \\
18504 \\
18677 \\
18850\end{array}$ & $\begin{array}{r}6579 \\
7312 \\
8044 \\
8775 \\
0,179^{505}\end{array}$ \\
\hline $\begin{array}{l}0,211 \\
0,212 \\
0,213 \\
0,214 \\
0,215\end{array}$ & $\begin{array}{l}3482= \\
3516 \\
3550 \\
3584 \\
3618\end{array}$ & $\begin{array}{l}21905 \\
22 \times 22 \\
22539 \\
22557 \\
22777\end{array}$ & $\begin{array}{r}5133 \\
4620 \\
6061 \\
7504 \\
0,258950\end{array}$ & $\begin{array}{l}3169 \\
3_{199} \\
3_{228} \\
3_{258} \\
3_{288}\end{array}$ & $\begin{array}{l}1902 k \\
19^{1} 99 \\
19^{\prime} 375 \\
19^{5} 51 \\
19728\end{array}$ & $\begin{array}{r}0,180254 \\
0962 \\
1688 \\
2414 \\
3139\end{array}$ \\
\hline $\begin{array}{l}0,216 \\
0,217 \\
0,218 \\
0,219 \\
0,220\end{array}$ & $\begin{array}{l}3653 \\
3688 \\
3723 \\
3758 \\
3795\end{array}$ & $\begin{array}{l}22998 \\
23220 \\
23443 \\
23667 \\
2389^{2}\end{array}$ & $\begin{array}{r}0,260398 \\
1849 \\
3303 \\
4759 \\
6218 \\
\end{array}$ & $\begin{array}{l}3318 \\
3348 \\
3373 \\
3409 \\
3439\end{array}$ & $\begin{array}{l}19906 \\
2008 / 4 \\
20264 \\
20444 \\
20625\end{array}$ & $\begin{array}{r}3863 \\
4585 \\
5307 \\
6028 \\
6747\end{array}$ \\
\hline $\begin{array}{l}0,221 \\
0,222 \\
0,223 \\
0,224 \\
0,225\end{array}$ & $\begin{array}{l}3829 \\
3865 \\
3900 \\
5936 \\
397^{3}\end{array}$ & $\begin{array}{l}24119 \\
24347 \\
24576 \\
24306 \\
25037\end{array}$ & $\begin{array}{r}7680 \\
0,269145 \\
0,270612 \\
2082 \\
3555\end{array}$ & $\begin{array}{l}3470 \\
3500 \\
3551 \\
3562 \\
559^{4}\end{array}$ & $\begin{array}{l}20806 \\
20988 \\
21172 \\
21555 \\
21540\end{array}$ & $\begin{array}{r}7466 \\
8184 \\
8900 \\
0,189616 \\
0,190331\end{array}$ \\
\hline $\begin{array}{l}0,226 \\
0,227 \\
0,228 \\
0,229 \\
0,230\end{array}$ & $\begin{array}{r}4009 \\
4046 \\
4032 \\
4119 \\
4156\end{array}$ & $\begin{array}{l}25269 \\
25502 \\
25737 \\
25973 \\
26210\end{array}$ & $\begin{array}{r}5031 \\
6509 \\
7990 \\
0,279^{4} 74 \\
0,280960\end{array}$ & $\begin{array}{l}3625 \\
3656 \\
5688 \\
3719 \\
375 x\end{array}$ & $\begin{array}{l}21725 \\
21911 \\
22098 \\
22285 \\
22473\end{array}$ & $\begin{array}{r}1044 \\
1757 \\
2468 \\
3179 \\
3889\end{array}$ \\
\hline $\begin{array}{l}0,231 \\
0,232 \\
0,233 \\
0,234 \\
0,235\end{array}$ & $\begin{array}{l}4194= \\
4231 \\
4269 \\
4306 \\
4344\end{array}$ & $\begin{array}{l}26448 \\
26687 \\
26928 \\
27169 \\
27412\end{array}$ & $\begin{array}{l}2450 \\
3942 \\
5437 \\
6955 \\
8435\end{array}$ & $\begin{array}{l}3783 \\
3815 \\
3847 \\
3880 \\
3912\end{array}$ & $\begin{array}{l}22662 \\
22852 \\
23042 \\
23234 \\
23425\end{array}$ & $\begin{array}{l}4597 \\
5305 \\
6012 \\
6717 \\
7^{422}\end{array}$ \\
\hline $\begin{array}{l}0,236 \\
0,237 \\
0,238 \\
0,239 \\
0,240\end{array}$ & $\begin{array}{l}4382 \\
4421 \\
4459 \\
4498 \\
4537\end{array}$ & $\begin{array}{l}27656 \\
27901 \\
28148 \\
28395 \\
28644\end{array}$ & $\begin{array}{r}0,289939 \\
0,291445 \\
2954 \\
4466 \\
5080\end{array}$ & $\begin{array}{l}3945 \\
3977 \\
4010 \\
4043 \\
4076\end{array}$ & $\begin{array}{l}23618 \\
23811 \\
24005 \\
24200 \\
24396\end{array}$ & $\begin{array}{r}8126 \\
8829 \\
0,199530 \\
0,20023 x \\
0931\end{array}$ \\
\hline
\end{tabular}


TABVLA I

\begin{tabular}{|c|c|c|c|c|c|c|}
\hline$\therefore$ & 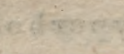 & Ellips & & Y3ag का & ypexb & \\
\hline$A$ & $\log B$ & C & l & $\log B$ & $C$ & $T$ \\
\hline $\begin{array}{l}0,240 \\
0,241 \\
0,242 \\
0,243 \\
0,244 \\
0,245\end{array}$ & $\begin{array}{l}4537 \\
4576 \\
4615 \\
4654 \\
4694 \\
4734\end{array}$ & $\begin{array}{l}28644 \\
28894 \\
29145 \\
29397 \\
29651 \\
29905\end{array}$ & $\begin{array}{r}0,295980 \\
7498 \\
0,299018 \\
0,300542 \\
2068 \\
3597\end{array}$ & $\begin{array}{l}407^{6} \\
4110 \\
4143 \\
4176 \\
4210 \\
4244\end{array}$ & $\begin{array}{l}24396 \\
24592 \\
24789 \\
24987 \\
25185 \\
25384\end{array}$ & $\begin{array}{r}0,200931 \\
1630 \\
2328 \\
3025 \\
3721 \\
4416\end{array}$ \\
\hline $\begin{array}{l}0,246 \\
0,247 \\
0,248 \\
0,249 \\
0,250\end{array}$ & $\begin{array}{l}4774 \\
4814 \\
4854 \\
4894 \\
4955\end{array}$ & $\begin{array}{l}3016 x \\
30413 \\
30676 \\
3 \circ 935 \\
31196\end{array}$ & $\begin{array}{r}5129 \\
6664 \\
8202 \\
0,507743 \\
0,311286\end{array}$ & $\begin{array}{l}4277 \\
431 x \\
4346 \\
4380 \\
4414\end{array}$ & $\begin{array}{l}25584 \\
25785 \\
25986 \\
26183 \\
263 \mathrm{gl}\end{array}$ & $\begin{array}{l}5110 \\
5303 \\
6495 \\
7 \times 86 \\
7876\end{array}$ \\
\hline $\begin{array}{l}0,251 \\
0,252 \\
0,255 \\
0,254 \\
0,255\end{array}$ & $\begin{array}{l}4976 \\
5017 \\
5058 \\
5099 \\
5141\end{array}$ & $\begin{array}{l}31458 \\
31721 \\
31985 \\
32250 \\
32517\end{array}$ & $\begin{array}{r}2833 \\
4382 \\
5935 \\
7^{4} 40 \\
0,319048\end{array}$ & $\begin{array}{l}4449 \\
4483 \\
45 \times 3 \\
4553 \\
4588\end{array}$ & $\begin{array}{l}26594 \\
26799 \\
27004 \\
27209 \\
27416\end{array}$ & $\begin{array}{r}8565 \\
9254 \\
0,209941 \\
0,210627 \\
1313\end{array}$ \\
\hline $\begin{array}{l}0,256 \\
0,257 \\
0,258 \\
0,259 \\
0,260\end{array}$ & $\begin{array}{l}5182 \\
5224 \\
5266 \\
5309 \\
5351\end{array}$ & $\begin{array}{l}32784 \\
33053 \\
33525 \\
33595 \\
33867\end{array}$ & $\begin{array}{r}0,320610 \\
217^{4} \\
3741 \\
5312 \\
6885\end{array}$ & $\begin{array}{l}4623 \\
4653 \\
4644 \\
4729 \\
4765\end{array}$ & $\begin{array}{l}27623 \\
27830 \\
28039 \\
28248 \\
28458\end{array}$ & $\begin{array}{l}1997 \\
2681 \\
3564 \\
4045 \\
4726\end{array}$ \\
\hline $\begin{array}{l}0,261 \\
0,262 \\
0,263 \\
0,264 \\
0,265\end{array}$ & $\begin{array}{l}5394 \\
5436 \\
5479 \\
5522 \\
5556\end{array}$ & $\begin{array}{l}34141 \\
344: 16 \\
34692 \\
34970 \\
35248\end{array}$ & $\begin{array}{r}0,328461 \\
0,330041 \\
1623 \\
3208 \\
4797\end{array}$ & $\begin{array}{l}4801 \\
4838 \\
4873 \\
4909 \\
4945\end{array}$ & $\begin{array}{l}28669 \\
28880 \\
29092 \\
29305 \\
29519\end{array}$ & $\begin{array}{l}5406 \\
6085 \\
6763 \\
7440 \\
8116\end{array}$ \\
\hline $\begin{array}{l}0,266 \\
0,267 \\
0,268 \\
0,269 \\
0,270\end{array}$ & $\begin{array}{l}5609 \\
5653 \\
5697 \\
5741 \\
5785\end{array}$ & $\begin{array}{l}55528 \\
35809 \\
36092 \\
6375 \\
36659\end{array}$ & $\begin{array}{r}6388 \\
7983 \\
0,339580 \\
0,341181 \\
2785 \\
\end{array}$ & $\begin{array}{l}4981 \\
5013 \\
5055 \\
509 x \\
5128\end{array}$ & $\begin{array}{l}29733 \\
29948 \\
30164 \\
30380 \\
30597\end{array}$ & $\begin{array}{r}8791 \\
0,219465 \\
0,220138 \\
0811 \\
1482\end{array}$ \\
\hline $\begin{array}{l}0,271 \\
0,272 \\
0,273 \\
0,274 \\
0,275\end{array}$ & $\begin{array}{l}5829 \\
5874 \\
5919 \\
5964 \\
6009\end{array}$ & $\begin{array}{l}36945 \\
37232 \\
37521 \\
37810 \\
38101\end{array}$ & $\begin{array}{r}4392 \\
6002 \\
7615 \\
0,549231 \\
0,350850\end{array}$ & $\begin{array}{l}5165 \\
5202 \\
5240 \\
5277 \\
5315\end{array}$ & $\begin{array}{l}30815 \\
31033 \\
31253 \\
31473 \\
31693\end{array}$ & $\begin{array}{l}2153 \\
2822 \\
3491 \\
4159 \\
4826\end{array}$ \\
\hline $\begin{array}{r}0,276 \\
0,277 \\
0,278 \\
0,279 \\
0,280\end{array}$ & $\begin{array}{l}6054 \\
6100 \\
6145 \\
619 x \\
6237\end{array}$ & $\begin{array}{l}33393 \\
38686 \\
38981 \\
39277 \\
39573\end{array}$ & $\begin{array}{l}247^{3} \\
409^{8} \\
5727 \\
7^{35} 59 \\
899^{4}\end{array}$ & $\begin{array}{l}5352 \\
5390 \\
5428 \\
5466 \\
5504\end{array}$ & $\begin{array}{l}3_{1915} \\
3_{2137} \\
3_{2359} \\
3_{2583} \\
3_{2807}\end{array}$ & $\begin{array}{l}5492 \\
6157 \\
6821 \\
7484 \\
8147\end{array}$ \\
\hline
\end{tabular}


TABVLA I

\begin{tabular}{|c|c|c|c|c|c|c|}
\hline & \multicolumn{3}{|c|}{ Ellips is } & \multicolumn{3}{|c|}{ Hyperbola } \\
\hline$A$ & $\log B$ & $C$ & $T$ & $\log B$ & $\bar{C}$ & $T$ \\
\hline $\begin{array}{l}0,280 \\
0,281 \\
0,282 \\
0,283 \\
0,284 \\
0,285\end{array}$ & $\begin{array}{l}6237 \\
6283 \\
6330 \\
6376 \\
6423 \\
6470\end{array}$ & $\begin{array}{l}3957^{3} \\
39872 \\
40171 \\
40472 \\
4077^{4} \\
41077\end{array}$ & $\begin{array}{r}0,35899^{4} \\
9,360632 \\
2274 \\
3918 \\
5566 \\
7217\end{array}$ & $\begin{array}{l}5504 \\
5542 \\
558 x \\
5619 \\
5658 \\
5697\end{array}$ & $\begin{array}{l}32807 \\
33032 \\
33257 \\
33484 \\
33711 \\
33938\end{array}$ & $\begin{array}{r}0,228147 \\
8808 \\
0,229469 \\
0,230 \times 28 \\
0787 \\
1445\end{array}$ \\
\hline $\begin{array}{l}0,286 \\
0,287 \\
0,288 \\
0,289 \\
0,290\end{array}$ & $\begin{array}{l}6517 \\
6564 \\
6612 \\
6660 \\
6708\end{array}$ & $\begin{array}{l}41381 \\
41687 \\
41994 \\
42302 \\
42611\end{array}$ & $\begin{array}{r}0,368871 \\
0,370529 \\
2189 \\
3853 \\
5521\end{array}$ & $\begin{array}{l}5736 \\
5775 \\
5814 \\
5853 \\
5893\end{array}$ & $\begin{array}{l}34167 \\
34396 \\
34626 \\
34856 \\
35087\end{array}$ & $\begin{array}{r}2102 \\
2758 \\
3413 \\
4068 \\
4721\end{array}$ \\
\hline $\begin{array}{l}0,291 \\
0,292 \\
0,293 \\
0,294 \\
0,295\end{array}$ & $\begin{array}{l}6756 \\
6804 \\
6852 \\
6901 \\
6950\end{array}$ & $\begin{array}{l}42922 \\
43233 \\
43547 \\
43861 \\
44177\end{array}$ & $\begin{array}{r}7191 \\
0,378865 \\
0,380542 \\
2222 \\
3906\end{array}$ & $\begin{array}{l}5932 \\
597^{2} \\
6012 \\
6052 \\
6092\end{array}$ & $\begin{array}{l}35319 \\
35552 \\
35785 \\
36019 \\
36253\end{array}$ & $\begin{array}{l}537^{4} \\
6025 \\
6676 \\
75_{26} 6 \\
797^{5}\end{array}$ \\
\hline $\begin{array}{l}0,296 \\
0,297 \\
0,298 \\
0,299 \\
0,200\end{array}$ & $\begin{array}{l}6999 \\
7048 \\
7097 \\
7147 \\
7196\end{array}$ & $\begin{array}{l}4449^{3} \\
44812 \\
4513 x \\
45452 \\
4577^{4}\end{array}$ & $\begin{array}{r}559^{3} \\
7^{283} \\
0,388977 \\
0,39067^{3} \\
257^{4}\end{array}$ & $\begin{array}{l}6132 \\
6172 \\
6213 \\
6253 \\
6294\end{array}$ & $\begin{array}{l}36489 \\
36725 \\
36961 \\
37199 \\
37437\end{array}$ & $\begin{array}{r}8623 \\
9^{271} \\
0,239917 \\
0,240563 \\
1207\end{array}$ \\
\hline
\end{tabular}


TABVI.A. II (vid. art. $9^{5}$ )

\begin{tabular}{|c|c|c|c|c|c|}
\hline$h$ & $\log y y$ & $h$ & $\log y y$ & $h$ & $\log y y$ \\
\hline 0,0000 & 0,0000000 & 0,0040 & 0,0038332 & 0,0080 & $0,007^{6133}$ \\
\hline $0 x$ & 0965 & & 0,0039284 & 81 & $707^{1}$ \\
\hline $\mathrm{O}_{2}$ & 1930 & 42 & 0,0040235 & 82 & 8009 \\
\hline 03 & $289^{4}$ & $x:=43$ & 1186 & 83 & 8947 \\
\hline 04 & 3853 & 44 & 2136 & 84 & 0,0079884 \\
\hline o5 & 4821 & .45 & 3086 & 85 & $0,008082 x$ \\
\hline o6 & 5784 & 46 & 4036 & .86 & ${ }^{17} 58$ \\
\hline 07 & 6747 & 47 & 4985 & 87 & $\quad 26 g^{4}$ \\
\hline o8 & 7710 & $2=48$ & $\therefore 593$ & & 3630 \\
\hline 99 & 8672 & 349 & 6883 & 89 & 4566 \\
\hline $\begin{array}{r}10 \\
\end{array}$ & 0,0009634 & 50 & 7832 & $9^{\circ}$ & 5502 \\
\hline 11 & 0,0010595 & 51 & 8780 & $9^{I}$ & 6437 \\
\hline$\therefore 12$ & $\mathbf{1 5 5 7}$ & 52 & 0,0049728 & $9^{2}$ & $7^{3} 7^{2}$ \\
\hline & 2517 & 53 & 0,0050675 & $9^{5}$ & 8306 \\
\hline 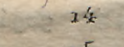 & 3478 & 54 & $\quad 1622$ & 9 í & 0,0089240 \\
\hline $\begin{array}{r}35 \\
\end{array}$ & $\begin{array}{r}4 \\
4\end{array}$ & 55 & $\begin{array}{r}2569 \\
\end{array}$ & $9^{5}$ & $0,00901,4$ \\
\hline 36 & 2द 5398 & 56 & 5515 & $9^{6}$ & $\therefore \quad x 108$ \\
\hline$x 7$ & 6357 & 57 & 4462 & 97 & 2041 \\
\hline$x=18$ & 7516 & 58 & 5407 & $\begin{array}{l}98 \\
78\end{array}$ & $297^{4}$ \\
\hline 19 & 8275 & $\because 59$ & .6353 & 0,0099 & $\therefore 506$ \\
\hline 20 & $0,0019^{234}$ & 60 & $7^{295}$ & 0,0100 & $\begin{array}{r}4839 \\
\end{array}$ \\
\hline 21 & 0,0020192 & $6 x$ & 8243 & 01 & 5770 \\
\hline 22 & $\therefore 150$ & 62 & $0,005 g_{187}$ & 02 & 6702 \\
\hline $2 x=23$ & 2107 & 63 & 0,0060131 & o'3 & 7633 \\
\hline 24 & 3064 & - 64 & 1075 & 04 & 8564 \\
\hline 25 & $402 x$ & 65 & 2.019 & 05 & $0,0099^{4} 5$ \\
\hline 26 & 4977 & 66 & 2962 & o6 & 0,0100425 \\
\hline 27 & $5 g 33$ & 67 & 3905 & 07 & $\because \quad 3356$ \\
\hline 28 & 6889 & 68 & $48 \pm 7$ & os & 2285 \\
\hline 29 & 7845 & 69 & 5790 & 09 & 3215 \\
\hline 30 & $\begin{array}{r}8800 \\
\end{array}$ & 70 & $\begin{array}{r}6732 \\
\end{array}$ & 10 & 4144 \\
\hline 31 & 0,0029755 & 71 & 7673 & 11 & $507^{3}$ \\
\hline $3 x$ & 0,0030709 & $7^{2}$ & 8614 & 12 & 6001 \\
\hline 33 & 1663 & $=7^{3}$ & 0,0069555 & 13 & $6 g^{2} 9$ \\
\hline 34 & 2617 & $7^{4}$ & $0,0070 \pm 96$ & 14 & 7857 \\
\hline 35 & 3570 & $7^{5}$ & 1456 & 15 & 8785 \\
\hline 36 & 4523 & $7^{6}$ & $237^{6}$ & $x 6$ & $0,01097^{12}$ \\
\hline 37 & $\quad 5476$ & 77 & $\therefore 3316$ & 17 & 0,0110639 \\
\hline 38 & 6428 & & 4255 & 18 & $\therefore 1565$ \\
\hline$\therefore 39$ & 7381 & $\begin{array}{r}79 \\
-\quad 7\end{array}$ & $519^{4}$ & $\begin{array}{r}19 \\
\end{array}$ & $\begin{array}{r}2491 \\
\end{array}$ \\
\hline 0,0040 & 0,0058332 & 0,0030 & 0,0076133 & 0,0120 & $0,0110+17$ \\
\hline
\end{tabular}


TABVLA II

\begin{tabular}{|c|c|c|c|c|c|}
\hline$h$ & $\log y y$ & $h^{\prime}$ & $\log y y$ & $h$ & $\log y y$ \\
\hline 0,0120 & 0,0113417 & 0,0160 & 0,0150202 & 0,0200 & 0,0186501 \\
\hline$=21$ & 4343 & 61 & 1115 & 01 & $7^{403}$ \\
\hline 22 & 5268 & 62 & 2028 & $: 02$ & 8304 \\
\hline 23 & 6193 & 63 & $29^{41}$ & 03 & 0,0189205 \\
\hline 24 & 7118 & 64 & 3854 & 04 & 0,0190105 \\
\hline 25 & 8043 & 65 & 4766 & 05 & 1005 \\
\hline 26 & 8967 & 66 & 5678 & o6 & 1905 \\
\hline 27 & 0,0119890 & 67 & 6589 & 07 & 2805 \\
\hline-28 & 0,0120814 & 68 & 7500 & os & 3704 \\
\hline 29 & 1737 & 69 & 8411 & og & 4603 \\
\hline 30 & 2660 & 70 & 0,0159322 & 10 & 5502 \\
\hline $3 i$ & -3582 & 71 & $0,016023_{2}$ & 11 & 4601 \\
\hline 32 & 4505 & 72 & 1142 & 12 & 7299 \\
\hline 33 & 5427 & $7^{3}$ & 2052 & $=x$ & 8197 \\
\hline 34 & 6348 & 74 & $29^{61}$ & $65 \quad 14$ & 9094 \\
\hline 35 & $7^{269}$ & 75 & 3870 & 15 & $0,019999^{2}$ \\
\hline 36 & $819^{\circ}$ & $7^{6}$ & 4779 & 16 & 0,0200889 \\
\hline 37 & $0,0129 i 1 x$ & 77 & 5688 & 17 & 1785 \\
\hline 38 & $0,013003_{2}$ & 78 & 6596 & 18 & 2682 \\
\hline 39 & og 52 & 79 & $7^{504}$ & 19 & 3578 \\
\hline 40 & 1871 & 80 & (1) 8412 & 20 & 4474 \\
\hline 41 & 2791 & 81 & 0,0169519 & 21 & 5369 \\
\hline 42 & $3_{710}$ & 82 & 0,0170226 & 22 & 6264 \\
\hline 43 & 4629 & 83 & 1133 & 23 & 7159 \\
\hline 44 & 5547 & 84 & 2039 & 24 & 8054 \\
\hline 45 & 6466 & 85 & 2945 & 25 & 8948 \\
\hline 46 & 7333 & 86 & 3351 & 26 & 0,0209843 \\
\hline 47 & 8301 & 87 & 4757 & 27 & 0,0210736 \\
\hline$\rightarrow 48$ & 0,0139218 & 88 & 5662 & 28 & 1630 \\
\hline 49 & 0,0140135 & 89 & 6567 & 29 & 2523 \\
\hline 50 & 1052 & 90 & 7471 & 30 & 3416 \\
\hline 51 & 1968 & $9^{1}$ & 8376 & $3 x$ & 4309 \\
\hline 52 & 2884 & 92 & 0,0179280 & 32 & 5201 \\
\hline 53 & 3800 & $9^{3}$ & 0,0180183 & 33 & $\because 60 g^{3}$ \\
\hline 54 & 4716 & $9^{4}$ & $\quad 3087$ & 34 & 6985 \\
\hline 55 & 5631 & $9^{5}$ & 1990 & 35 & 7876 \\
\hline 56 & 6546 & 96 & 2893 & 56 & 8768 \\
\hline 57 & $7^{460}$ & 97 & 3796 & 37 & 0,0219659 \\
\hline 58 & 8375 & $9^{8}$ & 4698 & 38 & 0,0220549 \\
\hline 59 & 0,0149288 & 0,0199 & 5600 & 39 & 1440 \\
\hline 0,0160 & 0,0150202 & 0,0200 & 0,0186501 & 0,0240 & 0,0222330 \\
\hline
\end{tabular}


TABVLA II

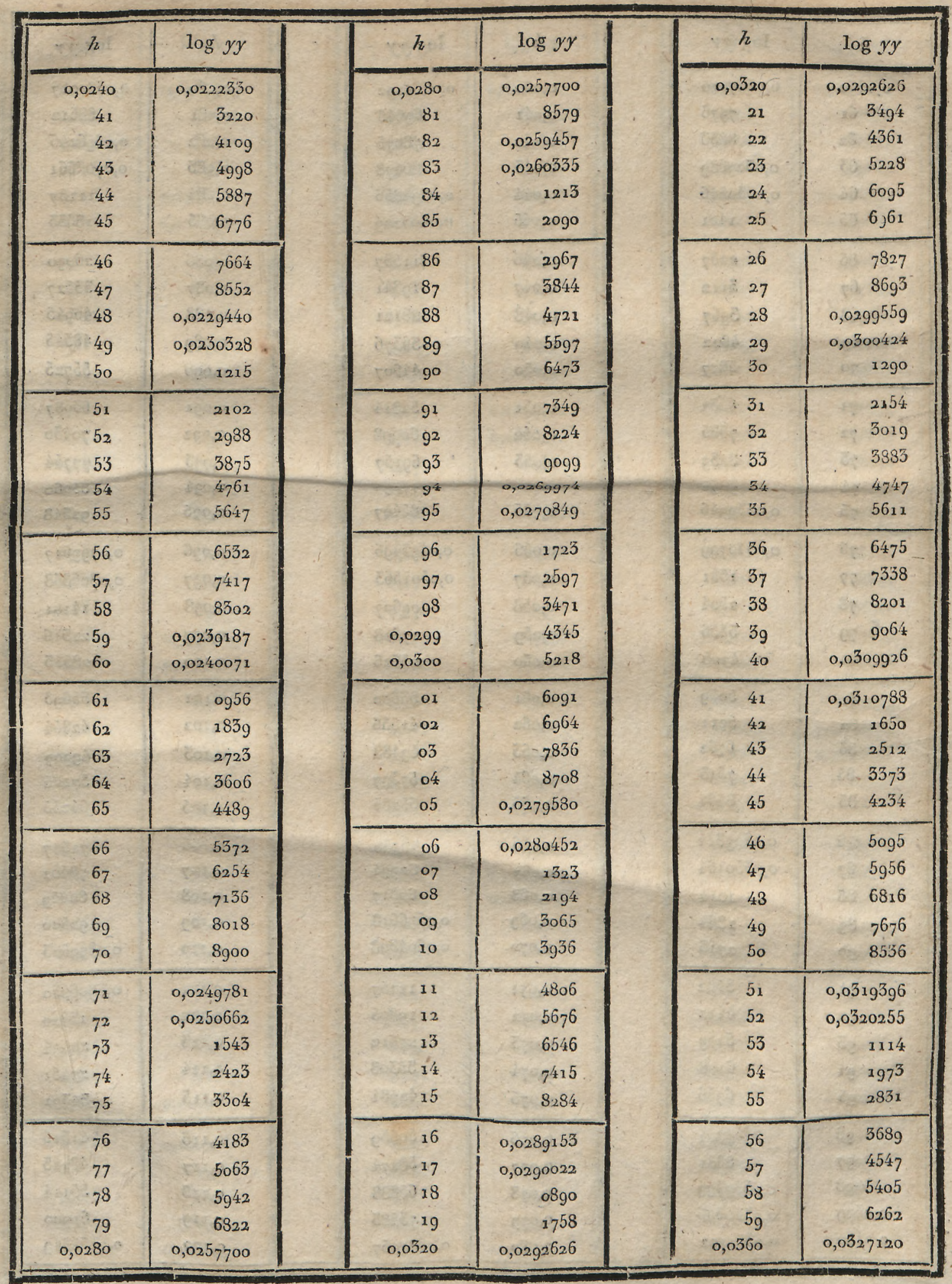


TABVLA II

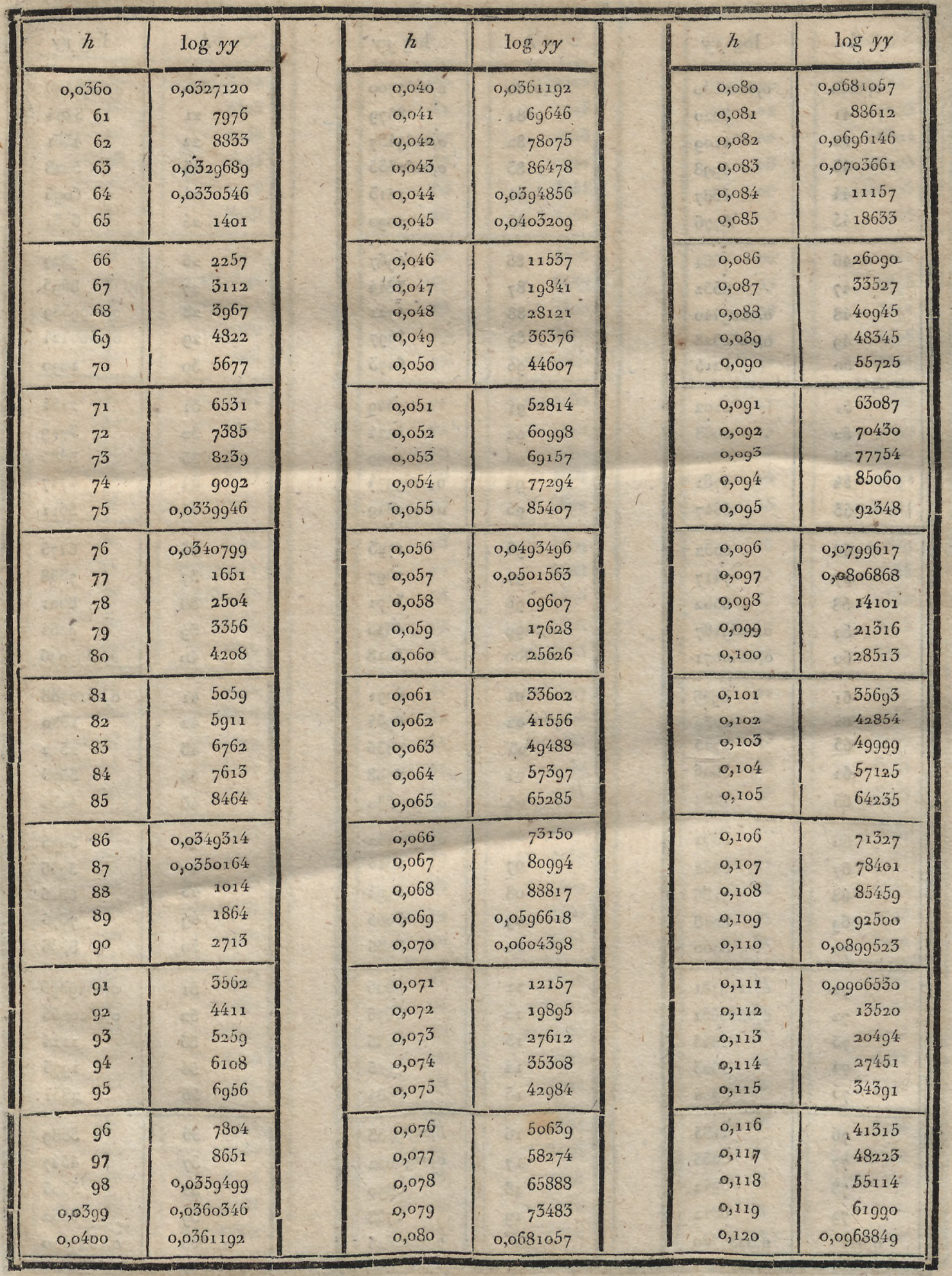


TABVLA II

\begin{tabular}{|c|c|c|c|c|c|}
\hline$h$ & $\log y y$ & $h$ & $\log y y$ & $h$ & $\log y y$ \\
\hline 0,120 & 0,0968849 & $0,16 a$ & $0,1.2309^{2} 7$ & 0,200 & 0,1471869 \\
\hline 0,121 & 75692 & 0,161 & 37192 & 0,201 & 77653 \\
\hline 0,122 & 82.520 & 0,162 & 43444 & 0,202 . & $83+27$ \\
\hline 0,123 & 89331 & 0,163 & 40682 & 0,203 & 89189 \\
\hline 0,124 & $0,099^{6127}$ & 0,164 & $559 \circ 8$ & 0,204 & 0,1494940 \\
\hline 0,125 & 0,1002907 & 0,165 & 62121 & 0,205 & 0,1500681 \\
\hline 0,126 & $\operatorname{og}^{6} 7^{2}$ & 0,166 & 68521 & 0,206 & 064 II \\
\hline 0,127 & $16 \pm 21$ & 0,167 & 74508 & 0,207 & 12130 \\
\hline 0,128 & 23154 & 0,168 & 80683 & 0,208 & 17838 \\
\hline 0,129 & $2987^{3}$ & 0,169 & 86845 & 0,209 & 23535 \\
\hline 0,130 & 36576 & 0,170 & $9299^{4}$ & 0,210 & 29222 \\
\hline $0,13 x$ & 43264 & 0,171 & 0,1299131 & $0,21 x$ & 34899 \\
\hline 0,132 & 49956 & $0,17^{2}$ & 0,1305255 & 0,212 & 40564 \\
\hline 0,133 & $5650^{4}$ & 0,173 & 11567 & 0,213 & 46220 \\
\hline 0,134 & 63237 & 0,17 & $x 7 * 66$ & 0,214 & $5 \times 365$ \\
\hline 0,135 & 69865 & 0,175 & 23553 & 0,215 & 57499 \\
\hline 0,136 & $7^{64} 7^{8}$ & $0,17^{6}$ & 29628 & 0,216 & 63123 \\
\hline 0,137 & $8307^{6}$ & 0,177 & 35690 & 0,217 & 68737 \\
\hline 0,138 & 89660 & $0,17^{8}$ & 41740 & 0,218 & 74340 \\
\hline 0,139 & 0,1096229 & 0,179 & 47778 & 0,219 & 79935 \\
\hline 0,140 & 0,1102783 & 0,180 & 53804 & 0,220 & 85516 \\
\hline 0,141 & $\operatorname{og}_{2} 3$ & 0,181 & 59818 & 0,221 & $9^{1089}$ \\
\hline 0,142 & $158 \div 9$ & 0,182 & 65821 & 0,222 & 0,1596652 \\
\hline 0,143 & 22360 & 0,183 & 71811 & 0,223 & 0,1602204 \\
\hline $0, x 44$ & 28357 & 0,184 & 77789 & 0,224 & 07747 \\
\hline 0,145 & 35340 & 0,135 & 83755 & 0,225 & 13279 \\
\hline 0,146 & 41809 & 0,286 & 89710 & 0,226 & 18802 \\
\hline $0, x 47$ & 48264 & 0,187 & 0,1595653 & 0,227 & 24315 \\
\hline 0,148 & 54704 & 0,188 & 0,1401585 & 0,228 & 29817 \\
\hline 0,149 & 61351 & 0,189 & 07504 & 0,229 & 35310 \\
\hline 0,150 & 67544 & 0,190 & 13412 & 0,250 & 40793 \\
\hline $0,15 x$ & 73943 & $0,19^{1}$ & 19309 & $0,23_{1}$ & 46267 \\
\hline 0,152 & 80329 & $0,19^{2}$ & $2519^{4}$ & 0,232 & 51730 \\
\hline 0,153 & 86701 & $0,19^{3}$ & 31068 & 0,233 & 57184 \\
\hline 0,154 & 93059 & $0,19^{4}$ & $36 g 31$ & 0,254 & 62628 \\
\hline 0,155 & 0,1199404 & $0,19^{5}$ & 42782 & 0,235 & 68063 \\
\hline 0,156 & 0,1205735 & $0,19^{6}$ & 48622 & 0,236 & $7^{3488}$ \\
\hline 0,157 & 12053 & 0,197 & 54450 & 0,237 & $7^{8903}$ \\
\hline 0,158 & 18557 & 0,198 & $6 o 268$ & 0,238 & $845 \circ 9$ \\
\hline 0,159 & 24649 & 0,199 & $6607^{4}$ & 0,239 & 89705 \\
\hline 0,160 & 0,1230927 & 0,200 & 0,1471869 & 0,210 & 0,1605092 \\
\hline
\end{tabular}


TABVLA II

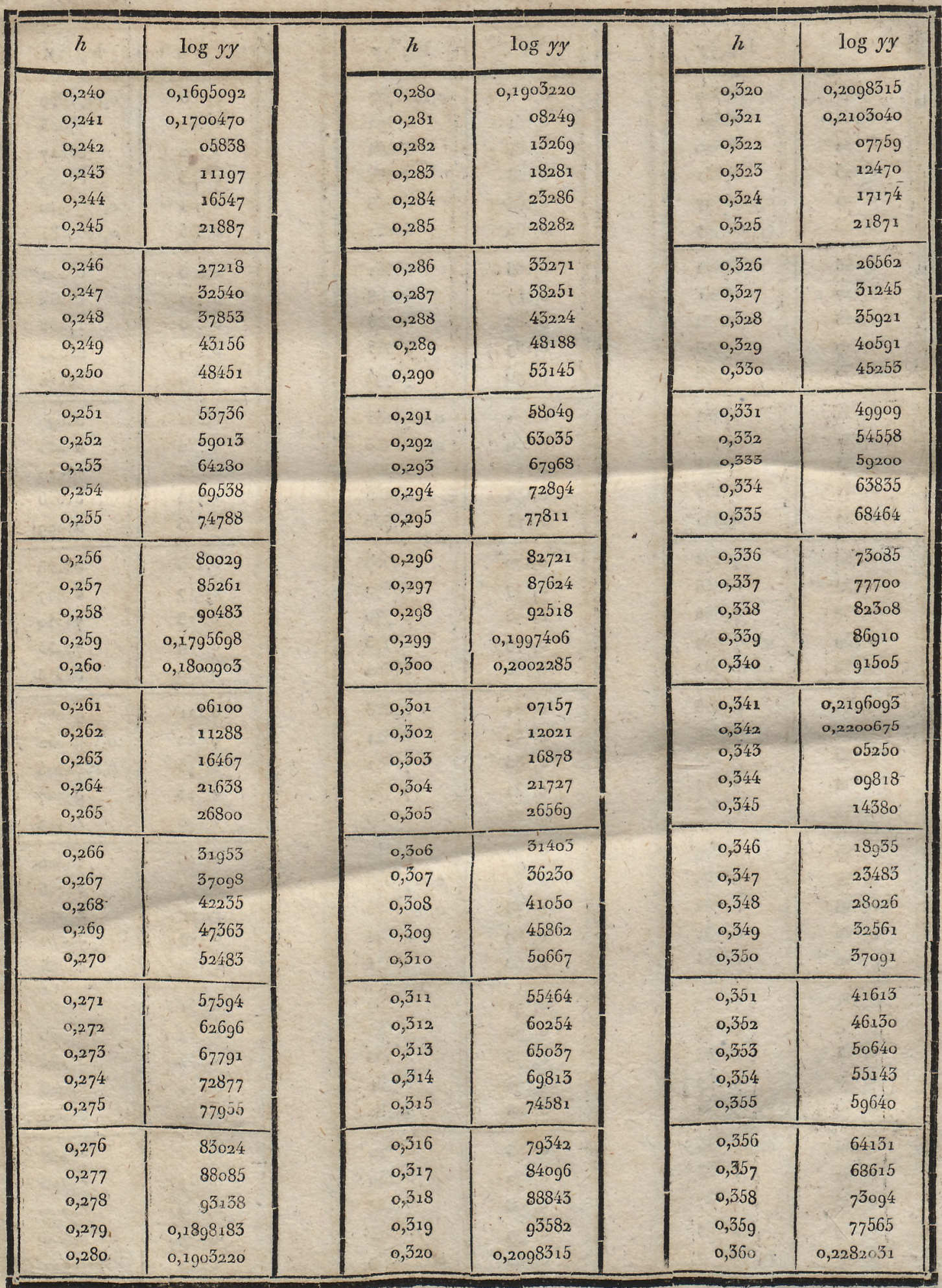


TABVLA II

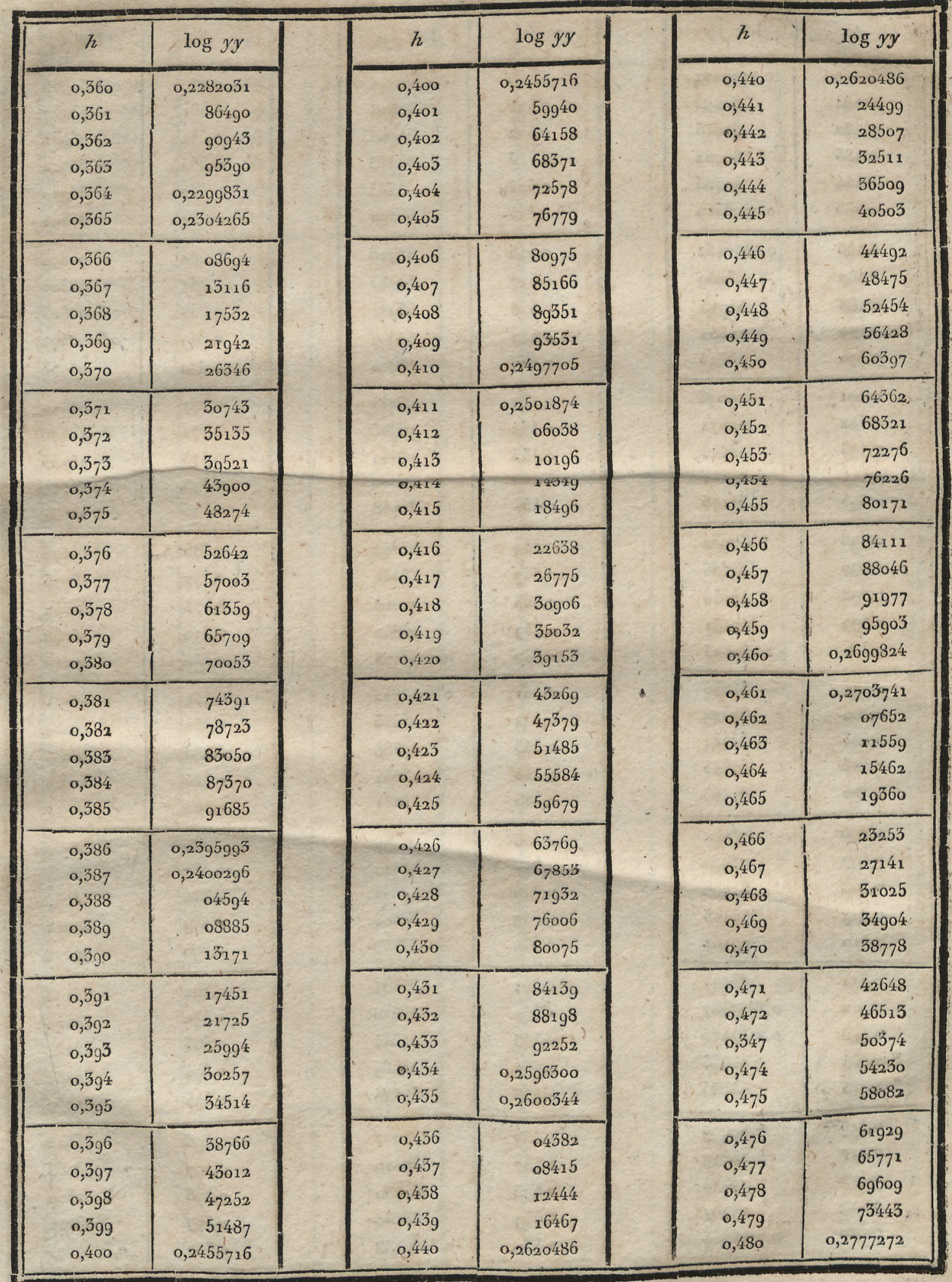


TABVI A II

\begin{tabular}{|c|c|c|c|c|c|}
\hline$h$ & $\log y y$ & $h$ & $\log y y$ & $h$ & $\log y y$ \\
\hline $\begin{array}{l}0,480 \\
0,481 \\
0,482 \\
0,483 \\
0,484 \\
0,485\end{array}$ & $\begin{array}{r}0,2777272 \\
81096 \\
84916 \\
88732 \\
92543 \\
0,2796349\end{array}$ & $\begin{array}{l}0,520 \\
0,521 \\
0,522 \\
0,52.5 \\
0,524 \\
0,525\end{array}$ & $\begin{array}{r}0,2926364 \\
30518 \\
54168 \\
57813 \\
41455 \\
45092\end{array}$ & $\begin{array}{l}0,560 \\
0,561 \\
0,562 \\
0,563 \\
0,564 \\
0,565\end{array}$ & $\begin{array}{r}0,30699^{38} \\
75437 \\
79931 \\
80422 \\
83910 \\
87394\end{array}$ \\
\hline $\begin{array}{l}0,486 \\
0,487 \\
0,483 \\
0,489 \\
0,490\end{array}$ & $\begin{array}{r}0,2800152 \\
03949 \\
07743 \\
11532 \\
15316\end{array}$ & $\begin{array}{l}0,526 \\
0,527 \\
0,528 \\
0,529 \\
0,550\end{array}$ & $\begin{array}{l}48726 \\
52855 \\
55981 \\
59602 \\
63220\end{array}$ & $\begin{array}{l}0,566 \\
0,567 \\
0,568 \\
0,569 \\
0,570\end{array}$ & $\begin{array}{r}90874 \\
94350 \\
0,3097823 \\
0,3101292 \\
04758\end{array}$ \\
\hline $\begin{array}{l}0,49^{1} \\
0,49^{2} \\
0,49^{5} \\
0,49^{4} \\
0,49^{5}\end{array}$ & $\begin{array}{l}19096 \\
22872 \\
26644 \\
30411 \\
34173\end{array}$ & $\begin{array}{l}0,531 \\
0,532 \\
0,533 \\
0,534 \\
0,535\end{array}$ & $\begin{array}{l}66833 \\
70443 \\
74049 \\
77650 \\
81248\end{array}$ & $\begin{array}{l}0,571 \\
0,572 \\
0,57^{3} \\
0,57^{4} \\
0,575\end{array}$ & $\begin{array}{r}08220 \\
11678 \\
15133 \\
18584 \\
22031\end{array}$ \\
\hline $\begin{array}{l}0,496 \\
0,497 \\
0,498 \\
0,499 \\
0,500\end{array}$ & $\begin{array}{l}37932 \\
41686 \\
45436 \\
49281 \\
52923\end{array}$ & $\begin{array}{l}0,536 \\
0,537 \\
0,538 \\
0,539 \\
0,540\end{array}$ & $\begin{array}{r}84842 \\
88432 \\
9^{2018} \\
95600 \\
0,2999^{1} 78\end{array}$ & $\begin{array}{l}0,576 \\
0,577 \\
0,573 \\
0,579 \\
0,580\end{array}$ & $\begin{array}{l}25475 \\
28915 \\
32352 \\
35785 \\
39215\end{array}$ \\
\hline $\begin{array}{l}0,501 \\
0,502 \\
0,503 \\
0,504 \\
0,505\end{array}$ & $\begin{array}{l}56660 \\
60392 \\
64121 \\
67845 \\
71565\end{array}$ & $\begin{array}{l}0,541 \\
0,542 \\
0,543 \\
0,544 \\
0,545\end{array}$ & $\begin{array}{r}0,3002752 \\
06323 \\
09888 \\
13452 \\
17011\end{array}$ & $\begin{array}{l}0,581 \\
0,582 \\
0,583 \\
0,584 \\
0,585\end{array}$ & $\begin{array}{l}42641 \\
46064 \\
49483 \\
52398 \\
56310\end{array}$ \\
\hline $\begin{array}{l}0,506 \\
0,507 \\
0,508 \\
0,509 \\
0,510\end{array}$ & $\begin{array}{l}75281 \\
78992 \\
82700 \\
86403 \\
90102\end{array}$ & $\begin{array}{l}0,546 \\
0,547 \\
0,548 \\
0,549 \\
0,550\end{array}$ & $\begin{array}{l}20566 \\
24117 \\
27664 \\
31203 \\
34748\end{array}$ & $\begin{array}{l}0,586 \\
0,587 \\
0,588 \\
0,589 \\
0,590\end{array}$ & $\begin{array}{l}59719 \\
63124 \\
66525 \\
69925 \\
73318\end{array}$ \\
\hline $\begin{array}{l}0,511 \\
0,512 \\
0,513 \\
0,514 \\
0,515\end{array}$ & $\begin{array}{r}9^{3} 797 \\
0,2897487 \\
0,2901174 \\
04356 \\
08535\end{array}$ & $\begin{array}{l}0,551 \\
0,552 \\
0,553 \\
0,554 \\
0,555\end{array}$ & $\begin{array}{r}38234 \\
41816 \\
45344 \\
48869 \\
52590\end{array}$ & $\begin{array}{l}0,591 \\
0,592 \\
0,59^{3} \\
0,59^{4} \\
0,59^{5}\end{array}$ & $\begin{array}{l}76709 \\
80096 \\
8348 x \\
86861 \\
90239\end{array}$ \\
\hline $\begin{array}{l}0,516 \\
0,517 \\
0,518 \\
0,519 \\
0,520\end{array}$ & $\begin{array}{r}12209 \\
15879 \\
39545 \\
23207 \\
0,2926864\end{array}$ & $\begin{array}{l}0,556 \\
0,557 \\
0,558 \\
0,559 \\
0,560\end{array}$ & $\begin{array}{r}55907 \\
59420 \\
62930 \\
66436 \\
0,3069938\end{array}$ & $\begin{array}{l}0,596 \\
0,597 \\
0,598 \\
0,599 \\
0,600\end{array}$ & $\begin{array}{r}93612 \\
0,3196983 \\
0,3200350 \\
03714 \\
0,3207074\end{array}$ \\
\hline
\end{tabular}


TABVLA III (v. artt. 90, 100)

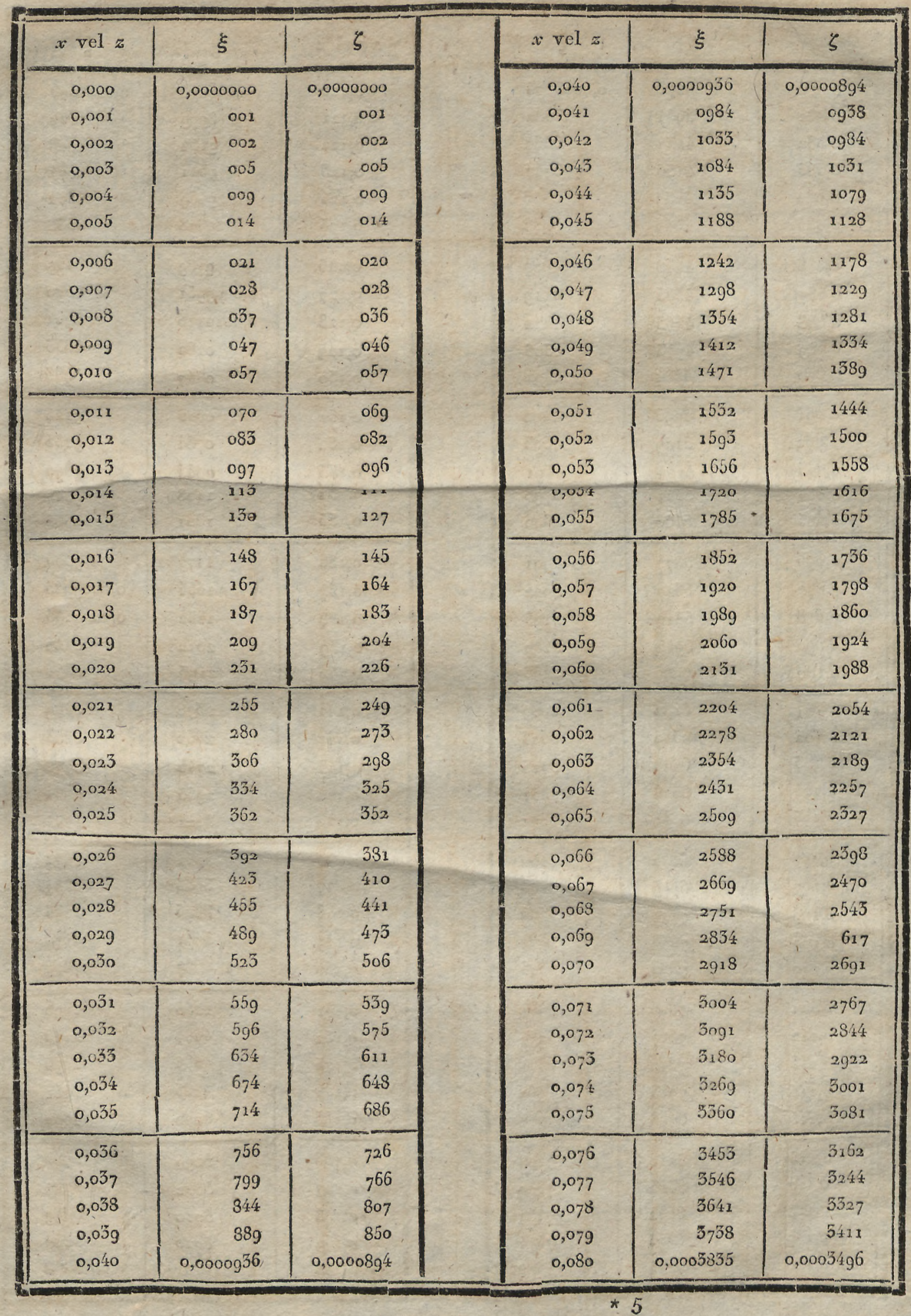


TABVLA III

\begin{tabular}{|c|c|c|c|c|c|}
\hline$x$ rel $z$ & $\xi$ & $\zeta$ & $x$ vel $z$ & $\xi$ & $\zeta$ \\
\hline 0,080 & 0,0003835 & $0,000^{\prime 3} 496$ & 0,120 & 0,0008845 & 0,0007693 \\
\hline 0,081 & $3 g^{3} 34$ & 3582 & 0,121 & 8999 & 7822 \\
\hline 0,082 & 4034 & 3669 & 0,122 & 9154 & $79^{48}$ \\
\hline 0,083 & 4136 & 3757 & 0,123 & $9^{3311}$ & 8074 \\
\hline 0,084 & 4239 & 3846 & 0,124 & $9^{469}$ & 8202 \\
\hline 0,035 & 4343 & 3936 & 0,125 & .9628 & 8330 \\
\hline 0,086 & 4448 & 4027 & 0,126 & 9789 & 8459 \\
\hline 0,087 & 4555 & 4119 & 0,127 & 0,0009953 & $859^{\circ}$ \\
\hline 0,088 & 4663 & 4212 & 0,128 & 0,0010115 & 8721 \\
\hline 0,089 & $477^{3}$ & 4306 & 0,129 & 0280 & 8853 \\
\hline 0,090 & 4884 & 4401 & 0,130 & 0447 & 8986 \\
\hline 0,091 & $499^{6}$ & 4496 & 0,131 & 0615 & 9120 \\
\hline 0,092 & 5109 & $459^{3}$ & 0,132 & 0784 & $9^{255}$ \\
\hline 0,093 & 5224 & $469 x$ & 0,133 & $\therefore \quad \operatorname{og} 55$ & 9390 \\
\hline 0,094 & 5341 & 4790 & 0,134 & 1128 & $9^{527}$ \\
\hline 0,095 & 5458 & 4890 & 0,135 & 1301 & 9665 \\
\hline 0,096 & $\quad 5577$ & 4991 & 0,136 & 1477 & 9803 \\
\hline 0,097 & 5697 & $5 \circ \operatorname{og}_{2}$ & 0,137 & 1654 & 0,0009943 \\
\hline 0,098 & 5819 & 5195 & 0,138 & 1832 & 0,0010083 \\
\hline 0,099 & 5942 & 5299 & 0,139 & 2012 & 0224 \\
\hline 0,100 & $x^{2}=6066$ & 5403 & 0,140 & $219^{3}$ & o366 \\
\hline 0,102 & $619^{2}$ & 5509 & 0,141 & 2376 & 0509 \\
\hline 0,102 & $6: 6319$ & 5616 & 0,142 & 2560 & 0653 \\
\hline 0,103 & 6448 & $57_{2} 3$ & 0,143 & 2745 & 0798 \\
\hline 0,104 & 6578 & 5832 & 0,144 & 2933 & $\operatorname{og} 44$ \\
\hline 0,105 & 6709 & 5941 & 0,145 & 3121 & $\log x$ \\
\hline 0,106 & 6842 & 6052 & 0,146 & 3311 & 1238 \\
\hline 0,107 & 6976 & 6163 & 0,147 & 3503 & 1387 \\
\hline 0,108 & $=7112$ & 6275 & 0,148 & 3696 & 1536 \\
\hline 0,109 & 7248 & 6389 & 0,149 & $379^{2}$ & 1689 \\
\hline 0,110 & 7386 & $650^{\prime} 3$ & 0,150 & 4087 & 1838 \\
\hline 0,111 & $7^{526}$ & 6618 & 0,151 & 4285 & 1990 \\
\hline 0,112 & $\quad 7667$ & 6734 & 0,152 & 4484 & 2143 \\
\hline 0,113 & 7809 & 6851 & 0,153 & 4684 & 2296 \\
\hline 0,114 & $-79^{53}$ & 6969 & 0,154 & 4886 & 2451 \\
\hline 0,115 & 8098 & 7088 & 0,155 & 5090 & 2607 \\
\hline 0,116 & $\quad 8245$ & 7203 & 0,156 & 5295 & 2763 \\
\hline 0,117 & $83 g^{3}$ & $7^{329}$ & 0,157 & 5502 & 29.21 \\
\hline 0,118 & 8542 & 7451 & 0,158 & 5710 & 3079 \\
\hline $0,1: 19$ & $86 g^{3}$ & $7^{5} 7^{4}$ & 0,159 & 5920 & 3238 \\
\hline 0,120 & 0,0008845 & 0,0007608 & 0,160 & 0,0016131 & $0,00133,8$ \\
\hline
\end{tabular}


TABVIA III

\begin{tabular}{|c|c|c|c|c|c|}
\hline$x$ vel $z$ & $\xi$ & $\zeta$ & $x$ vel $z$ & $\xi$ & $\zeta$ \\
\hline $\begin{array}{l}0,160 \\
0,161 \\
0,162 \\
0,163 \\
0,164 \\
0,165\end{array}$ & $\begin{array}{r}0,0016131 \\
6344 \\
6559 \\
677^{5} \\
699^{2} \\
7211\end{array}$ & $\begin{array}{r}0,0013398 \\
3559 \\
3721 \\
3883 \\
4047 \\
4211\end{array}$ & $\begin{array}{l}0,200 \\
0,201 \\
0,202 \\
0,203 \\
0,204 \\
0,205\end{array}$ & $\begin{array}{r}0,0025877 \\
6154 \\
6433 \\
6713 \\
699^{5} \\
7278\end{array}$ & $\begin{array}{r}0,0020507 \\
0702 \\
0897 \\
1094 \\
1292 \\
1490\end{array}$ \\
\hline $\begin{array}{l}0,166 \\
0,167 \\
0,168 \\
0,169 \\
0,170\end{array}$ & $\begin{array}{l}7^{432} \\
7654 \\
7878 \\
8103 \\
8330\end{array}$ & $\begin{array}{r}4377 \\
4543 \\
4710 \\
4873 \\
5047\end{array}$ & $\begin{array}{l}0,206 \\
0,207 \\
0,208 \\
0,209 \\
0,210\end{array}$ & $\begin{array}{l}7564 \\
7851 \\
8139 \\
8429 \\
8722\end{array}$ & $\begin{array}{l}1689 \\
1889 \\
2090 \\
2291 \\
2494\end{array}$ \\
\hline $\begin{array}{l}0,171 \\
0,172 \\
0,17^{3} \\
0,17^{4} \\
0,17^{5}\end{array}$ & $\begin{array}{r}8558 \\
8788 \\
9020 \\
2253 \\
9487\end{array}$ & $\begin{array}{l}5216 \\
5387 \\
5558 \\
5730 \\
5903\end{array}$ & $\begin{array}{l}0,211 \\
0,212 \\
0,213 \\
0,214 \\
0,215\end{array}$ & $\begin{array}{r}9015 \\
9^{311} \\
9608 \\
0,0029907 \\
0,0030207\end{array}$ & $\begin{array}{r}2697 \\
2901 \\
3106 \\
3311 \\
3518\end{array}$ \\
\hline $\begin{array}{l}0,176 \\
0,177 \\
0,178 \\
0,179 \\
0,180\end{array}$ & $\begin{array}{r}9724 \\
0,0019961 \\
0,0020201 \\
0442 \\
0685\end{array}$ & $\begin{array}{l}6077 \\
6252 \\
6428 \\
6604 \\
6782\end{array}$ & $\begin{array}{l}0,216 \\
0,217 \\
0,218 \\
0,219 \\
0,220\end{array}$ & $\begin{array}{l}0509 \\
0814 \\
1119 \\
1427 \\
1736\end{array}$ & $\begin{array}{l}3725 \\
3932 \\
4142 \\
4352 \\
4562\end{array}$ \\
\hline $\begin{array}{l}0,181 \\
0,182 \\
0,183 \\
0,184 \\
0,185\end{array}$ & $\begin{array}{l}0929 \\
1175 \\
1422 \\
1671 \\
1922\end{array}$ & $\begin{array}{l}6960 \\
7139 \\
7319 \\
7500 \\
7681\end{array}$ & $\begin{array}{l}0,221 \\
0,222 \\
0,223 \\
0,224 \\
0,225\end{array}$ & $\begin{array}{l}2047 \\
2359 \\
2674 \\
299^{\circ} \\
3308\end{array}$ & $\begin{array}{l}477^{4} \\
4986 \\
5199 \\
5412 \\
5627\end{array}$ \\
\hline $\begin{array}{l}0,186 \\
0,187 \\
0,188 \\
0,189 \\
0,190\end{array}$ & $\begin{array}{l}217^{4} \\
2428 \\
2683 \\
2941 \\
3199\end{array}$ & $\begin{array}{l}7864 \\
8047 \\
8231 \\
8426 \\
8602\end{array}$ & $\begin{array}{l}0,226 \\
0,227 \\
0,228 \\
0,229 \\
0,230\end{array}$ & $\begin{array}{l}3627 \\
3949 \\
4272 \\
4597 \\
4924\end{array}$ & $\begin{array}{l}5842 \\
6058 \\
6275 \\
6493 \\
6711\end{array}$ \\
\hline $\begin{array}{l}0,19^{1} \\
0,19^{2} \\
0,19^{5} \\
0,19^{4} \\
0,19^{5}\end{array}$ & $\begin{array}{l}3460 \\
3722 \\
3985 \\
4251 \\
4518\end{array}$ & $\begin{array}{l}8789 \\
3976 \\
9165 \\
9354 \\
9544\end{array}$ & $\begin{array}{l}0,231 \\
0,232 \\
0,233 \\
0,234 \\
0,235\end{array}$ & $\begin{array}{l}5252 \\
5582 \\
5914 \\
6248 \\
6584\end{array}$ & $\begin{array}{l}693 i \\
7151 \\
737 x \\
7593 \\
7816\end{array}$ \\
\hline $\begin{array}{l}0,196 \\
0,197 \\
0,198 \\
0,199 \\
0,200\end{array}$ & $\begin{array}{r}4786 \\
5056 \\
5328 \\
5602 \\
0,0025877\end{array}$ & $\begin{array}{r}97^{35} \\
0,0019926 \\
0,0020119 \\
0312 \\
0,0020507\end{array}$ & $\begin{array}{l}0,236 \\
0,237 \\
0,238 \\
0,239 \\
0,240\end{array}$ & $\begin{array}{r}6921 \\
7260 \\
7601 \\
7944 \\
0,0038289\end{array}$ & $\begin{array}{r}8039 \\
8263 \\
8487 \\
8713 \\
0,0028039\end{array}$ \\
\hline
\end{tabular}


TABVLA III

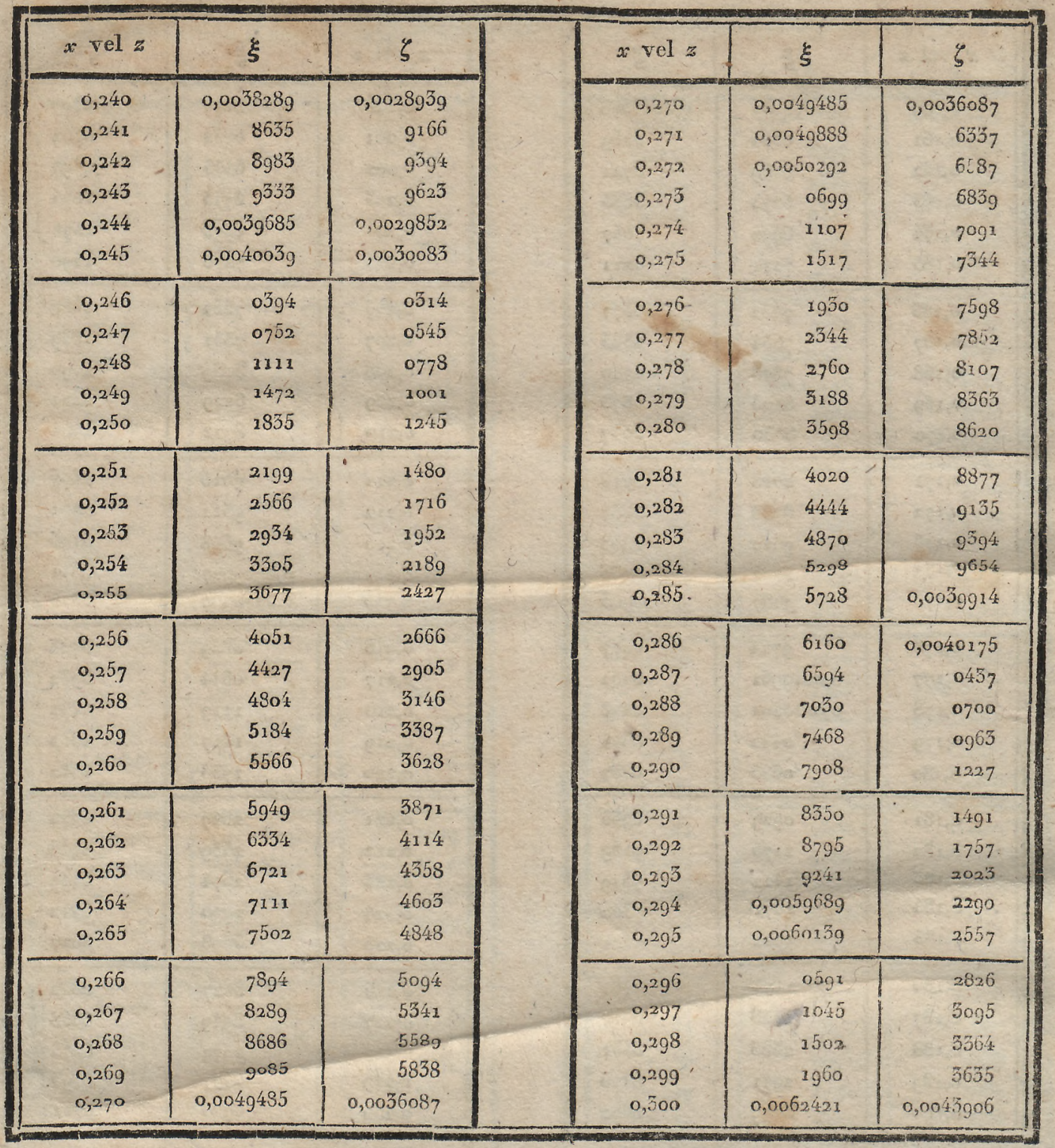





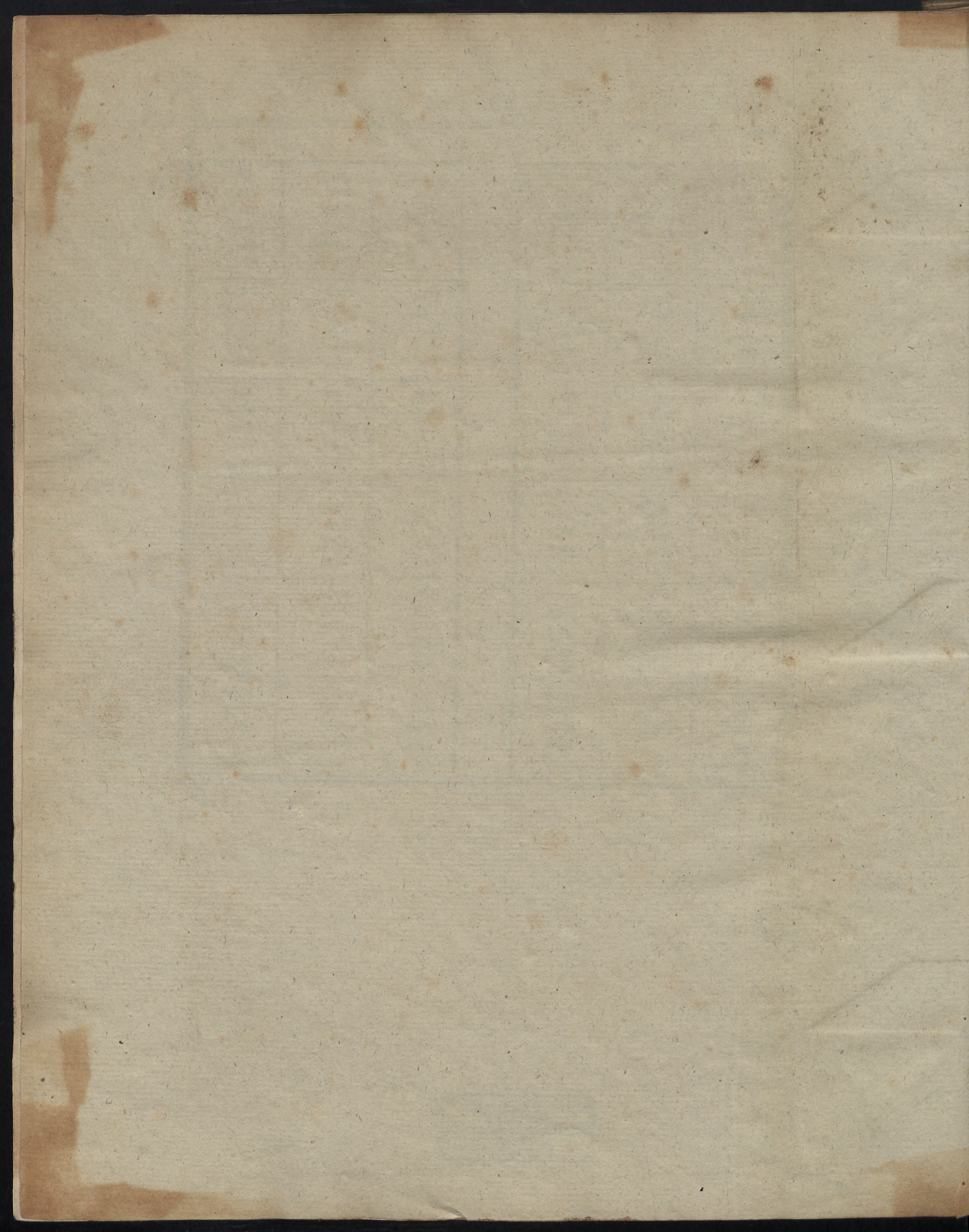


(3)

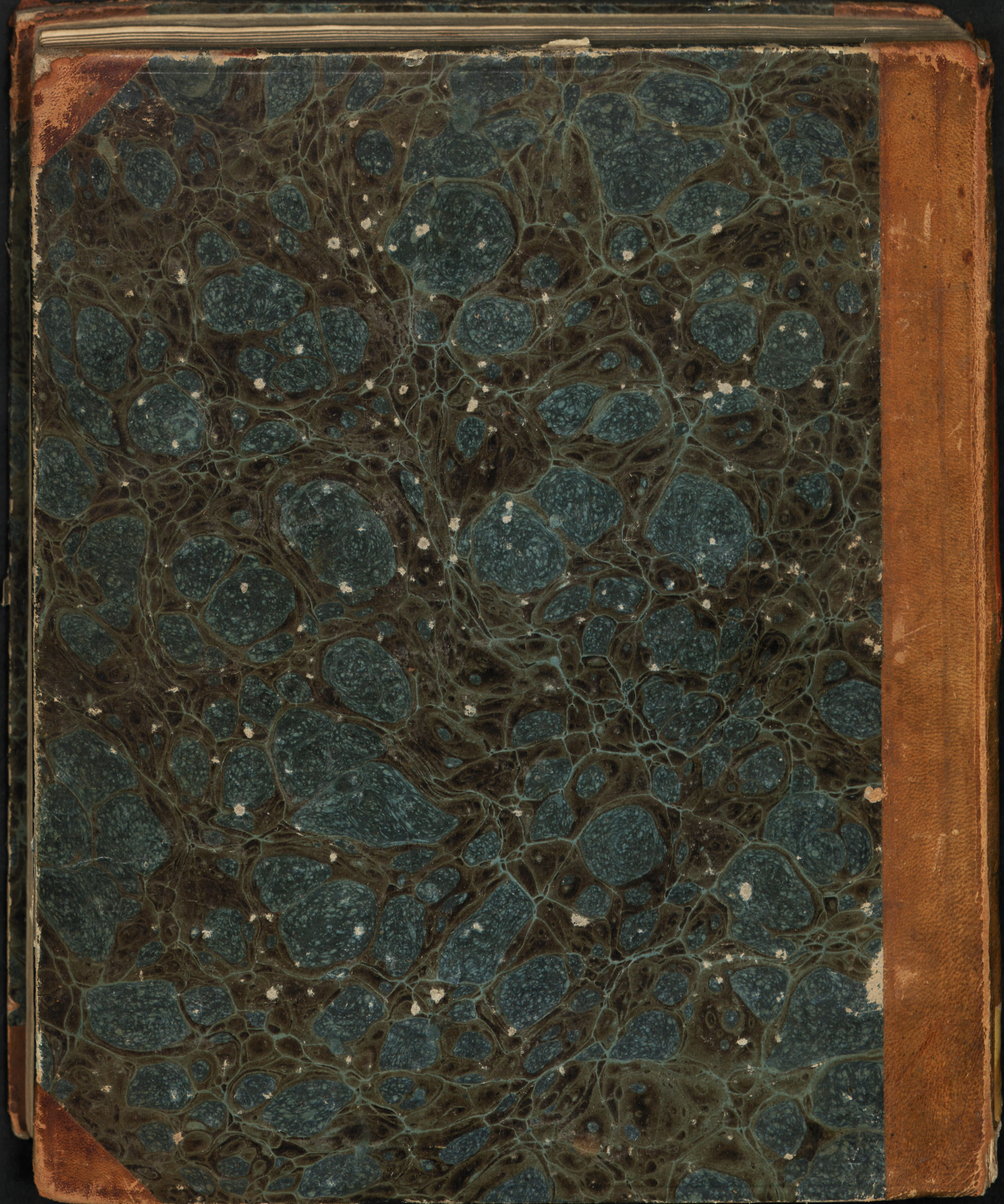

